Supporting Information

\title{
Silver-Catalyzed Dibromotrifluoromethoxylation of Terminal Alkynes
}

\author{
Feng Wang, Yuecong Guo, Yutong Zhang and Pingping Tang*
}

State Key Laboratory and Institute of Elemento-Organic Chemistry, College of Chemistry, Nankai University, Tianjin 300071, China

Tianjin 300071, China

E-mail:ptang@nankai.edu.cn 


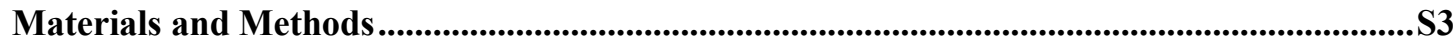

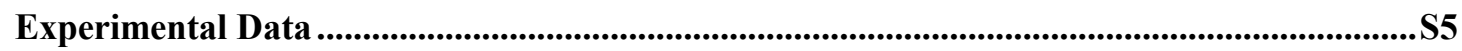

Optimization of the reaction condition for the trifluoromethoxylation ..........................S5

Optimization of solvents on the reaction...............................................................S5

Optimization of reaction concentration on the reaction ............................................S5

Optimization of $\mathrm{Br}^{+}$reagents on the reaction ............................................................S6

Optimization of silver salts on the reaction..........................................................S7

Effect of fluorine sources on the reaction................................................................S8

Effect of " $\mathrm{OCF}_{3}$ " sources on the reaction..............................................................S9

Effect of temperatures on the reaction ................................................................S10

Effect of atmospheres on the reaction ................................................................S10

Effect ofsizes of sealed tube on the reaction.....................................................S11

Experimental Procedures and Compound Characterization ........................................S11

Hex-5-yn-1-yl 3-methoxybenzoate (1b) .............................................................S11

Pent-4-yn-1-yl 4-phenoxybenzoate (1c) ...............................................................S12

Hex-5-yn-1-yl [1,1'-biphenyl]-4-carboxylate (1d) .................................................S12

Hex-5-yn-1-yl 3-methylbenzoate (1e)............................................................................S13

Hex-5-yn-1-yl 4-(tert-butyl)benzoate (1f) ...................................................S13

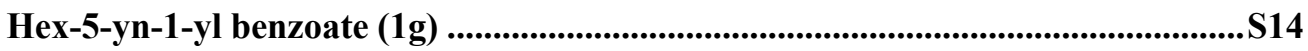

Hex-5-yn-1-yl 4-fluorobenzoate (1h).............................................................S14

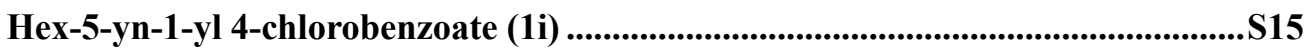

Pent-4-yn-1-yl 4-bromobenzoate (1j).....................................................................S15

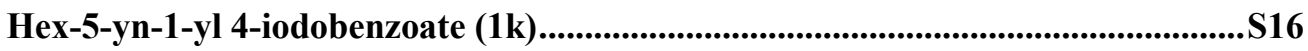

Hex-5-yn-1-yl 4-(trifluoromethyl)benzoate (11) ..............................................S16

Hex-5-yn-1-yl 4-(trifluoromethoxy)benzoate (1 m) ..................................................S17

Hex -5-yn-1-yl 4-benzoylbenzoate (1n) .......................................................S17

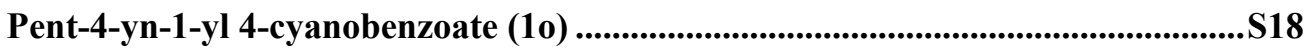

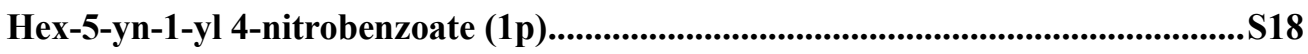

Pent-4-yn-1-yl 4-(methylsulfonyl)benzoate (1q) ......................................................S19

Oxiran-2-ylmethyl 4-(prop-2-yn-1-yloxy)benzoate (1u) .....................................S19

2-(Trimethylsilyl)ethyl 4-(prop-2-yn-1-yloxy)benzoate (1v) ...............................S20

4-Chlorobutyl 4-(prop-2-yn-1-yloxy)benzoate (1w) ........................................S20

N-(hex-5-yn-1-yl)-Saccharin (1x) ..........................................................................S21

$N$-butyl- $N$-(hex-5-yn-1-yl)benzenesulfonamide (1y)...............................................S21

Hex-5-yn-1-yl 1-naphthoate (1z) ...............................................................S21

Hex-5-yn-1-yl benzofuran-2-carboxylate (1 aa)...................................................S22

Hex-5-yn-1-yl 5-bromothiophene-2-carboxylate (1bb) ............................................S22

Hex-5-yn-1-yl quinoline-2-carboxylate (1cc)....................................................S23

Hex-5-yn-1-yl 6-fluoropicolinate (1dd) ................................................................S23

Celecoxib derivative(111) ...................................................................................S24

Podophyllotoxin derivative $(1 \mathrm{~mm})$...................................................................S25

Mycophenolic acid derivative(1nn).................................................................S26

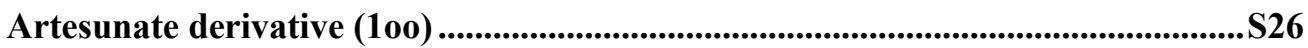




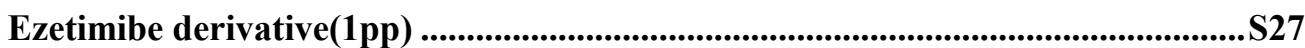

(IS)-(-)-camphanic acid derivative (1qq) ..........................................................................S28

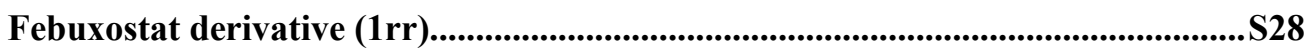

L-phenylalanine derivative (1ss) ........................................................................S29

Lithocholic acid derivative $(1 \mathrm{tt})$..................................................................................529

Arbutin derivative (1uu) .........................................................................................................530

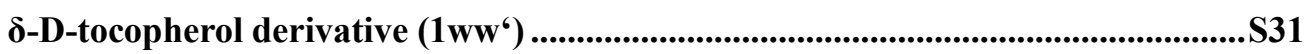

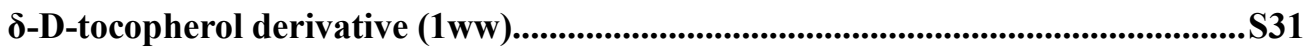

1,1-Dibromo-2-(trifluoromethoxy)dodec-1-ene (3a)..............................................S32

6,6-Dibromo-5-(trifluoromethoxy)hex-5-en-1-yl 3-methoxybenzoate (3b)..............S33

5,5-Dibromo-4-(trifluoromethoxy)pent-4-en-1-yl 4-phenoxybenzoate (3c).............S33

6,6-Dibromo-5-(trifluoromethoxy)hex-5-en-1-yl [1,1'-biphenyl]-4-carboxylate (3d)S34

6,6-Dibromo-5-(trifluoromethoxy)hex-5-en-1-yl 3-methylbenzoate (3e).................S34

6,6-Dibromo-5-(trifluoromethoxy)hex-5-en-1-yl 4-(tert-butyl)benzoate (3f)..........S35

6,6-Dibromo-5-(trifluoromethoxy)hex-5-en-1-yl benzoate (3g).................................S35

6,6-Dibromo-5-(trifluoromethoxy)hex-5-en-1-yl 4-fluorobenzoate (3h)...................S36

6,6-Dibromo-5-(trifluoromethoxy)hex-5-en-1-yl 4-chlorobenzoate (3i)...................S36

5,5-Dibromo-4-(trifluoromethoxy)pent-4-en-1-yl 4-bromobenzoate (3j) .................S37

6,6-Dibromo-5-(trifluoromethoxy)hex-5-en-1-yl 4-iodobenzoate (3k) .....................S37

6,6-Dibromo-5-(trifluoromethoxy)hex-5-en-1-yl 4-(trifluoromethyl)benzoate (3I)S38

5,5-Dibromo-4-(trifluoromethoxy)pent-4-en-1-yl 4-(trifluoromethoxy)benzoate (3m)

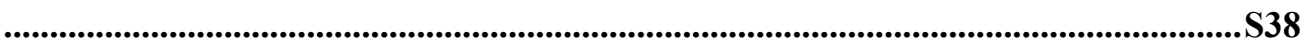

5,5-Dibromo-4-(trifluoromethoxy)hex-5-en-1-yl 4-benzoylbenzoate (3n) ...............S39

6,6-Dibromo-5-(trifluoromethoxy)hex-5-en-1-yl 4-cyanobenzoate (3o) ...................S39

6,6-Dibromo-5-(trifluoromethoxy)hex-5-en-1-yl 4-nitrobenzoate (3p).....................S40

6,6-Dibromo-5-(trifluoromethoxy)hex-5-en-1-yl 4-(methylsulfonyl)benzoate (3q)S40

4-((3,3-Dibromo-2-(trifluoromethoxy)allyl)oxy)benzaldehyde (3r) ...........................S41

4-((3,3-Dibromo-2-(trifluoromethoxy)allyl)oxy)benzoic acid (3s)...........................S41

4-Chlorobutyl 4-((3,3-dibromo-2-(trifluoromethoxy)allyl)oxy)benzoate (3t) .........S42

Oxiran-2-ylmethyl 4-((3,3-dibromo-2-(trifluoromethoxy)allyl)oxy)benzoate (3u) S42

2-(Trimethylsilyl)ethyl 4-((3,3-dibromo-2-(trifluoromethoxy)allyl)oxy)benzoate (3v)S43

2-(6,6-Dibromo-5-(trifluoromethoxy)hex-5-en-1-yl)isoindoline-1,3-dione (3w).....S43

2-(6,6-Dibromo-5-(trifluoromethoxy)hex-5-en-1-yl)benzo[d]isothiazol-3(2H)-one

1,1-dioxide (3x) .544

$N$-butyl- $N$-(6,6-dibromo-5-(trifluoromethoxy)hex-5-en-1-yl)benzenesulfonamide

6,6-Dibromo-5-(trifluoromethoxy)hex-5-en-1-yl 1-naphthoate (3z)

6,6-Dibromo-5-(trifluoromethoxy)hex-5-en-1-yl benzofuran-2-carboxylate (3aa) S45 6,6-Dibromo-5-(trifluoromethoxy)hex-5-en-1-yl 5-bromothiophene-2-carboxylate (3bb) S46

6,6-Dibromo-5-(trifluoromethoxy)hex-5-en-1-yl quinoline-2-carboxylate (3cc)....S46

6,6-Dibromo-5-(trifluoromethoxy)hex-5-en-1-yl 6-fluoropicolinate (3dd) ..............S47

1-(2,2-Dibromo-1-(trifluoromethoxy)vinyl)-4-methoxybenzene (3ee) .......................S47

Gram

scale

synthesis

of 
1-(2,2-dibromo-1-(trifluoromethoxy)vinyl)-4-methoxybenzene (3ee)....................S48

4-(2,2-Dibromo-1-(trifluoromethoxy)vinyl)-1,1'-biphenyl (3ff) ...........................S48

1-(Tert-butyl)-4-(2,2-dibromo-1-(trifluoromethoxy)vinyl) benzene (3gg)...............S49

(2,2-Dibromo-1-(trifluoromethoxy)vinyl)benzene (3hh) .........................................S49

1-Chloro-4-(2,2-dibromo-1-(trifluoromethoxy)vinyl)benzene (3ii)........................S50

1-(2,2-Dibromo-1-(trifluoromethoxy)vinyl)-4-nitrobenzene (3jj) .........................S50

(1-Bromo-2-(trifluoromethoxy)ethene-1,2-diyl)dibenzene $(Z)+(E)(3 \mathrm{kk})$.............S51

Celecoxib derivative (311) .......................................................................551

Epipodophyllotoxin derivative (3mm) .................................................................S52

Mycophenolic acid derivative (3nn)..........................................................552

Artesunate derivative (300) ...................................................................................S53

Ezetimibe derivative (3pp) .......................................................................554

(1S)-(-)-camphanic acid derivative (3qq) .............................................................S54

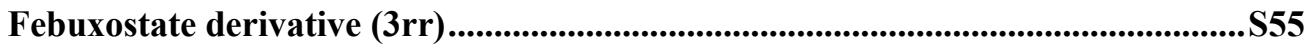

L-phenylalanine derivative (3ss) ......................................................................S55

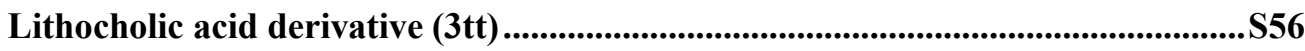

Arbutin derivative (3uu) ...................................................................................556

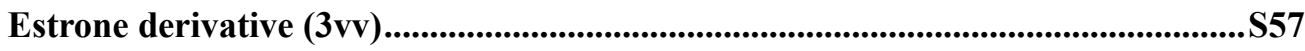

ס-D-tocopherol derivative (3ww).........................................................................S58

(E)-6-bromo-7-(4-methoxyphenyl)-7-(trifluoromethoxy)hept-6-en-4-yn-1-ol (4ee)S58

(E)-1-(2-bromo-2-phenyl-1-(trifluoromethoxy)vinyl)-4-methoxybenzene (5ee) ....S59

(4-Fluorophenyl)(2-(4-methoxyphenyl)-2-(trifluoromethoxy)vinyl)sulfane

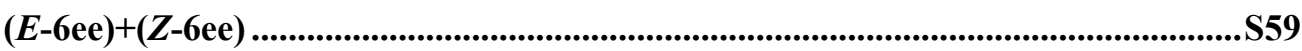

(E)-1-(2-bromo-1-(trifluoromethoxy)vinyl)-4-methoxybenzene (7ee).....................S60

(E)-1-bromo-2-(trifluoromethoxy)dodec-1-ene $(E-7 a)$..........................................S60

(Z)-1-bromo-2-(trifluoromethoxy)dodec-1-ene $(Z-7$ a)............................................S61

Mechanism Studies .......................................................................................................S61

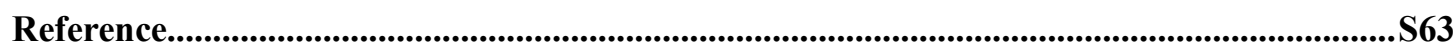

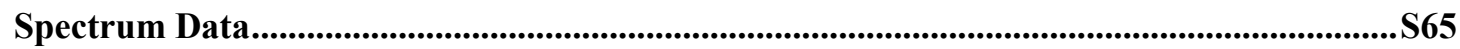




\section{Materials and Methods}

MeCN, EA, DCE, DMC, 1,4-dioxane, $\mathrm{Et}_{2} \mathrm{O}, \mathrm{HMPA}$, DMSO and DCM were dried by distillation over $\mathrm{CaH}_{2}$. THF and toluene were dried by distillation over sodium/benzophenone. $\mathrm{CDCl}_{3}$ was purchased from Sigma-Aldrich.CsF was purchased from TCI. "OCF 3 "reagentswas prepared according to the reported literatures ${ }^{[1]}$. TLC was performed on silica gel Huanghai $\mathrm{HSGF}_{254}$ plates and visualized by quenching of UV fluorescence $\left(\lambda_{\max }=254 \mathrm{~nm}\right)$. Preparative TLC was performed on silica gel Xinnuo $\mathrm{HSGF}_{254}$ preparative TLC plates. Silica gel (200-300 mesh) was purchased from Qingdao Haiyang Chemical Co., China. Unless otherwise noted, all other reagents and starting materials were purchased from commercial sources and used without further purification. ${ }^{1} \mathrm{H}$ NMR, ${ }^{13} \mathrm{C}$ NMR and ${ }^{19} \mathrm{~F}$ NMR were recorded on a Varian NMR $400(400 \mathrm{MHz}, 101 \mathrm{MHz}$ and $376 \mathrm{MHz}$ ). Signal positions were recorded in ppm with the abbreviations s, d, t, q, dd, dt and $\mathrm{m}$ denoting singlet, doublet, triplet, quadruplet, doublet of doublets, doublet of triplets and multiplet respectively. All NMR chemical shifts were referenced to residual solvent peaks or to $\mathrm{Si}\left(\mathrm{CH}_{3}\right)_{4}$ as an internal standard. For ${ }^{1} \mathrm{H}$ NMR: $\mathrm{CDCl}_{3}=\delta 7.26 \mathrm{ppm}, \mathrm{Si}\left(\mathrm{CH}_{3}\right)_{4}=\delta 0$ ppm. For ${ }^{13} \mathrm{C} \mathrm{NMR}$ : $\mathrm{CDCl}_{3}=\delta 77.16 \mathrm{ppm}$. Mass spectra were acquired on Agilent 6520 Q-TOF LC/MS, Bruker APEX II FT-ICR-MS and Waters GCT Premier. Preparative HPLC was performed on an UltiMate 3000 liquid chromatography with an Innoval ODS-2, $21.2 \mathrm{~mm} \times 25 \mathrm{~cm}$ column. 


\section{Experimental Data}

\section{Optimization of the reaction condition for the trifluoromethoxylation}

Optimization of solvents on the reaction

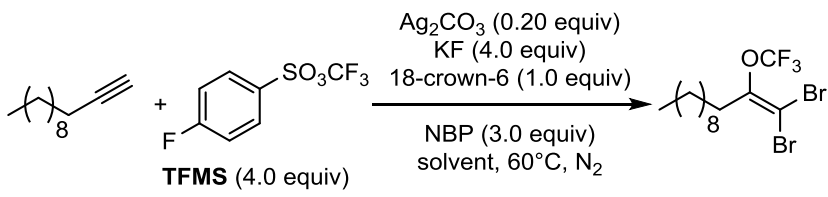

In a glove box, to a $2.0 \mathrm{~mL}$ sealed tube were added in sequence 1-dodecyne (1a) (8.3 mg, 0.0500 mmol, 1.00 equiv), $0.10 \mathrm{~mL}$ solvent, $\mathrm{Ag}_{2} \mathrm{CO}_{3}$ (2.8 mg, $0.0100 \mathrm{mmol}, 0.200$ equiv), $\mathrm{KF}$ (11.6 mg, $0.200 \mathrm{mmol}, 4.00$ equiv), 18 -crown-6 (13.2 mg, $0.050 \mathrm{mmol}, 1.00$ equiv), NBP (33.9 mg, 0.150 mmol, 3.00 equiv). The mixture was stirring at room temperature for $5 \mathrm{mins}$, then added TFMS (48.9 mg, $0.200 \mathrm{mmol}, 4.00$ equiv). After stirring for $4.0 \mathrm{~h}$ at $60^{\circ} \mathrm{C}$, trifluoromethylthiobenzene (7 $\mu \mathrm{L}, 0.0500 \mathrm{mmol}$ ) was added. The yield of 1,1-dibromo-2-(trifluoromethoxy)dodec-1-ene (3a) was determined by comparing the integration of the ${ }^{19} \mathrm{~F}$ NMR resonance of 1,1-dibromo-2-(trifluoromethoxy)dodec-1-ene (3a) $\quad(-55.81 \quad \mathrm{ppm})$ with that of trifluoromethylthiobenzene $(-42.80 \mathrm{ppm})$. Yields are reported in Table S1.

Table S1: Optimization of solventson the reaction

\begin{tabular}{cccc}
\hline Solvent & $\begin{array}{r}\text { Yield [\%] } \\
\left({ }^{19} \text { F NMR }\right)\end{array}$ & Solvent & $\begin{array}{r}\text { Yield [\%] } \\
\left({ }^{19} \text { F NMR }\right)\end{array}$ \\
\hline MeCN & 80 & THF & 13 \\
Acetone & 24 & 1,4 -Dioxane & 80 \\
DCM & 55 & Anisole & 42 \\
DCE & 61 & Hexane & 34 \\
EtOAc & 49 & Toluene & 75 \\
DMC & 69 & DMA & 26 \\
\hline
\end{tabular}

\section{Optimization of reaction concentration on the reaction}

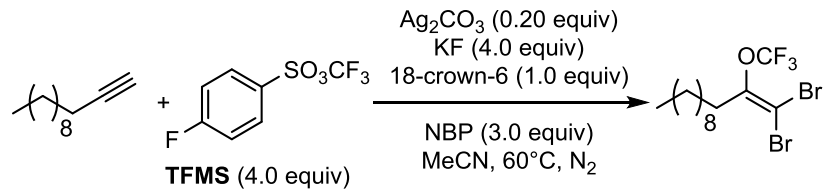

In a glove box, to a $2.0 \mathrm{~mL}$ sealed tube were added in sequence 1-dodecyne (1a) (8.3 $\mathrm{mg}, 0.0500$ mmol, 1.00 equiv), $\mathrm{n} \mathrm{mL} \mathrm{MeCN}, \mathrm{Ag}_{2} \mathrm{CO}_{3}$ (2.8 mg, $0.0100 \mathrm{mmol}, 0.200$ equiv), $\mathrm{KF}$ (11.6 mg, $0.200 \mathrm{mmol}, 4.00$ equiv), 18-crown-6 (13.2 mg, $0.050 \mathrm{mmol}, 1.00$ equiv), NBP (33.9 mg, 0.150 mmol, 3.00 equiv). The mixture was stirring at room temperature for 5 mins, then added TFMS (48.9 mg, $0.200 \mathrm{mmol}, 4.00$ equiv). After stirring for $4.0 \mathrm{~h}$ at $60^{\circ} \mathrm{C}$, trifluoromethylthiobenzene $(7$ $\mu \mathrm{L}, 0.0500 \mathrm{mmol}$ ) was added. The yield of 1,1-dibromo-2-(trifluoromethoxy)dodec-1-ene (3a) was determined by comparing the integration of the ${ }^{19} \mathrm{~F}$ NMR resonance of 
1,1-dibromo-2-(trifluoromethoxy)dodec-1-ene $\quad$ (3a) $\quad\left(\begin{array}{lll}-55.81 & \mathrm{ppm}\end{array}\right)$ with that of trifluoromethylthiobenzene (-42.80 ppm). Yields are reported in Table S2.

Table S2: Optimization of reaction concentration on the reaction

\begin{tabular}{cc}
\hline Concentration $(\mathrm{M})$ & $\begin{array}{c}\text { Yield }[\%] \\
\left({ }^{19} \mathrm{~F} \text { NMR }\right)\end{array}$ \\
\hline 0.5 & 80 \\
0.25 & 68 \\
0.125 & 33 \\
0.0625 & 19 \\
\hline
\end{tabular}

\section{Optimization of $\mathrm{Br}^{+} / \mathrm{Cl}^{+}$reagents on the reaction}

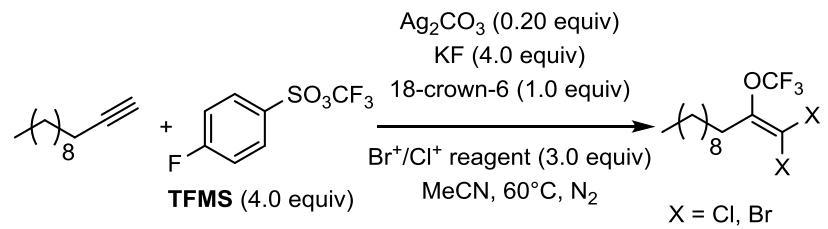

In a glove box, to a $2.0 \mathrm{~mL}$ sealed tube were added in sequence 1-dodecyne (1a) $(8.3 \mathrm{mg}, 0.0500$ mmol, 1.00 equiv), $0.10 \mathrm{~mL} \mathrm{MeCN}, \mathrm{Ag}_{2} \mathrm{CO}_{3}$ ( $2.8 \mathrm{mg}, 0.0100 \mathrm{mmol}, 0.200$ equiv), $\mathrm{KF}$ (11.6 mg, $0.200 \mathrm{mmol}, 4.00$ equiv), 18 -crown-6 (13.2 $\mathrm{mg}, 0.050 \mathrm{mmol}, 1.00$ equiv), $\mathrm{Br}^{+}$reagent $(0.150 \mathrm{mmol}$, 3.00 equiv). The mixture was stirring at room temperature for $5 \mathrm{mins}$, then added TFMS ( $48.9 \mathrm{mg}$, $0.200 \mathrm{mmol}, 4.00$ equiv). After stirring for $4.0 \mathrm{~h}$ at $60{ }^{\circ} \mathrm{C}$, trifluoromethylthiobenzene $(7 \mu \mathrm{L}$, $0.0500 \mathrm{mmol}$ ) was added. The yield of 1,1-dihalo-2-(trifluoromethoxy)dodec-1-ene was determined by comparing the integration of the ${ }^{19} \mathrm{~F}$ NMR resonance of 1,1-dibromo-2-(trifluoromethoxy)dodec-1-ene (3a) $\quad(-55.81 \quad \mathrm{ppm})$ with that of trifluoromethylthiobenzene (-42.80 ppm). Yields are reported in Table S3.

Table S3: Optimization of $\mathrm{Br}^{+}$reagents on the reaction

\begin{tabular}{cccc}
\hline Br $^{+}$reagent & $\begin{array}{r}\text { Yield [\%] } \\
\left({ }^{19} \text { F NMR }\right)\end{array}$ & $\mathrm{Br}^{+}$reagent & $\begin{array}{c}\text { Yield [\%] } \\
\left({ }^{19} \mathrm{~F} \mathrm{NMR}\right)\end{array}$ \\
\hline NBS & 9 & NBP $(2.0$ eq $)$ & 73 \\
NBP & 80 & NBP (4.0 eq $)$ & 80 \\
DBDMH (1.5 eq $)$ & 53 & NCS (3.0 eq $)$ & 0 \\
$\mathrm{Br}_{2}$ & 0 & & \\
\hline
\end{tabular}




\section{Optimization of crown ethers on the reaction}

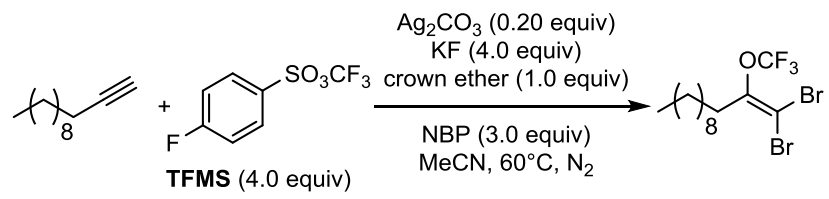

In a glove box, to a $2.0 \mathrm{~mL}$ sealed tube were added in sequence 1-dodecyne (1a) $(8.3 \mathrm{mg}, 0.0500$ mmol, 1.00 equiv), $0.10 \mathrm{~mL} \mathrm{MeCN}, \mathrm{Ag}_{2} \mathrm{CO}_{3}$ ( $2.8 \mathrm{mg}, 0.0100 \mathrm{mmol}, 0.200$ equiv), $\mathrm{KF}$ (11.6 mg, $0.200 \mathrm{mmol}, 4.00$ equiv), crown ether ( $0.050 \mathrm{mmol}, 1.00$ equiv), NBP ( $33.9 \mathrm{mg}, 0.150 \mathrm{mmol}, 3.00$ equiv). The mixture was stirring at room temperature for 5 mins, then added TFMS (48.9 $\mathrm{mg}$, $0.200 \mathrm{mmol}, 4.00$ equiv). After stirring for $4.0 \mathrm{~h}$ at $60{ }^{\circ} \mathrm{C}$, trifluoromethylthiobenzene $(7 \mu \mathrm{L}$, $0.0500 \mathrm{mmol}$ ) was added. The yield of 1,1-dibromo-2-(trifluoromethoxy)dodec-1-ene (3a) was determined by comparing the integration of the ${ }^{19} \mathrm{~F}$ NMR resonance of 1,1-dibromo-2-(trifluoromethoxy)dodec-1-ene (3a) (-55.81 $\mathrm{ppm})$ with that of trifluoromethylthiobenzene $(-42.80 \mathrm{ppm})$. Yields are reported in Table S4.

Table S4: Optimization of crown ethers on the reaction

\begin{tabular}{cccc}
\hline crown ether & $\begin{array}{r}\text { Yield [\%] } \\
\left({ }^{19} \text { F NMR }\right)\end{array}$ & crown ether & $\begin{array}{r}\text { Yield [\%] } \\
\left({ }^{19} \text { F NMR }\right)\end{array}$ \\
\hline 18-crown-6 & 80 & Dibenzo-18-crown-6 & 58 \\
15 -crown-5 & 74 & Dibenzo-24-crown-8 & 69 \\
12 -crown-4 & 48 & Benzo-21-crown-7 & 75 \\
Benzo-18-crown-6 & 73 & No & 44 \\
Dicyclohexano-18-crown-6 & 59 & & \\
\hline
\end{tabular}

\section{Optimization of silver salts on the reaction}

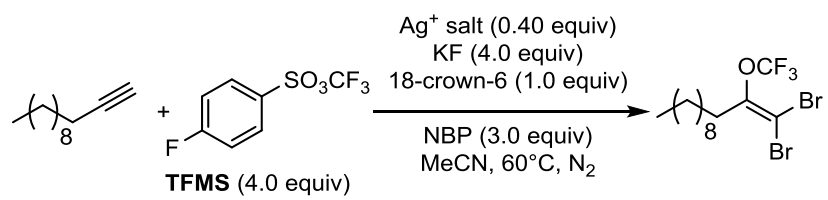

In a glove box, to a $2.0 \mathrm{~mL}$ sealed tube were added in sequence 1-dodecyne (1a) $(8.3 \mathrm{mg}, 0.0500$ mmol, 1.00 equiv), $0.10 \mathrm{~mL} \mathrm{MeCN}$, silver salts ( $0.0200 \mathrm{mmol}, 0.400$ equiv), $\mathrm{KF}$ (11.6 mg, 0.200 mmol, 4.00 equiv), 18 -crown-6 (13.2 mg, $0.050 \mathrm{mmol}, 1.00$ equiv), NBP (33.9 mg, $0.150 \mathrm{mmol}$, 3.00 equiv). The mixture was stirring at room temperature for $5 \mathrm{mins}$, then added TFMS ( $48.9 \mathrm{mg}$, $0.200 \mathrm{mmol}, 4.00$ equiv). After stirring for $4.0 \mathrm{~h}$ at $60{ }^{\circ} \mathrm{C}$, trifluoromethylthiobenzene $(7 \mu \mathrm{L}$, $0.0500 \mathrm{mmol}$ ) was added. The yield of 1,1-dibromo-2-(trifluoromethoxy)dodec-1-ene (3a) was determined by comparing the integration of the ${ }^{19} \mathrm{~F}$ NMR resonance of 1,1-dibromo-2-(trifluoromethoxy)dodec-1-ene (3a) (-55.81 $\quad$ ppm) with that of trifluoromethylthiobenzene $(-42.80 \mathrm{ppm})$. Yields are reported in Table S5.

Table S5: Optimization of silver salts on the reaction

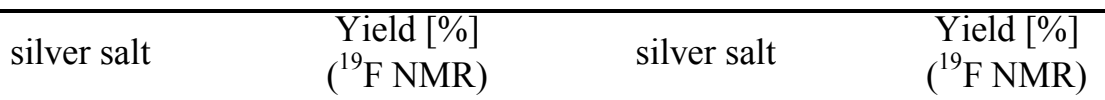




\begin{tabular}{cccc}
\hline $\mathrm{AgSbF}_{6}$ & 69 & $\mathrm{AgBF}_{4}$ & 52 \\
$\mathrm{AgNO}_{3}$ & 28 & $\mathrm{AgBr}$ & 30 \\
$\mathrm{AgF}$ & 83 & $\mathrm{No}$ & 33 \\
$\mathrm{Ag}_{2} \mathrm{CO}_{3}$ & 81 & $\mathrm{Ag}_{2} \mathrm{CO}_{3}$ & 50 \\
$(0.20$ equiv $)$ & 77 & $\begin{array}{c}(0.10 \text { equiv }) \\
\mathrm{Ag}_{2} \mathrm{CO}_{3}\end{array}$ & 88 \\
$\mathrm{Ag}_{2} \mathrm{O}$ & $(0.25$ equiv $)$ & \\
$(0.20$ equiv $)$ & 65 & & \\
$\mathrm{AgOCOCF}_{3}$ & & & \\
\hline
\end{tabular}

\section{Effect of fluorine sources on the reaction}

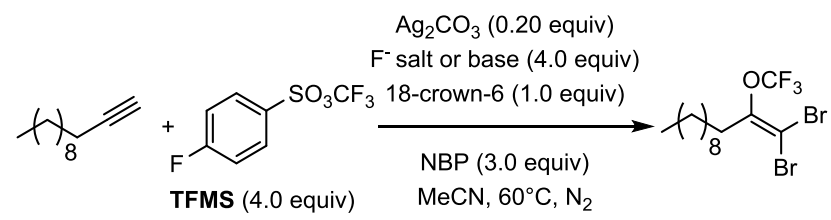

In a glove box, to a $2.0 \mathrm{~mL}$ sealed tube were added in sequence 1-dodecyne (1a) $(8.3 \mathrm{mg}, 0.0500$ mmol, 1.00 equiv), $0.10 \mathrm{~mL} \mathrm{MeCN}, \mathrm{Ag}_{2} \mathrm{CO}_{3}$ ( $2.8 \mathrm{mg}, 0.0100 \mathrm{mmol}, 0.400$ equiv), $\mathrm{F}^{-}$salts $(0.200$ mmol, 4.00 equiv), 18-crown-6 (13.2 mg, $0.050 \mathrm{mmol}, 1.00$ equiv), NBP (33.9 mg, $0.150 \mathrm{mmol}$, 3.00 equiv). The mixture was stirring at room temperature for $5 \mathrm{mins}$, then added TFMS (48.9 $\mathrm{mg}$, $0.200 \mathrm{mmol}, 4.00$ equiv). After stirring for $4.0 \mathrm{~h}$ at $60{ }^{\circ} \mathrm{C}$, trifluoromethylthiobenzene $(7 \mu \mathrm{L}$, $0.0500 \mathrm{mmol}$ ) was added. The yield of 1,1-dibromo-2-(trifluoromethoxy)dodec-1-ene (3a) was determined by comparing the integration of the ${ }^{19} \mathrm{~F}$ NMR resonance of 1,1-dibromo-2-(trifluoromethoxy)dodec-1-ene (3a) $\quad(-55.81 \quad \mathrm{ppm})$ with that of trifluoromethylthiobenzene (-42.80 ppm). Yields are reported in Table S6.

Table S6: Effect of fluorine sources on the reaction

\begin{tabular}{cccc}
\hline Fluorine salt & $\begin{array}{c}\text { Yield [\%] } \\
\left({ }^{19} \mathrm{~F} \text { NMR }\right)\end{array}$ & Fluorine salt & $\begin{array}{c}\text { Yield [\%] } \\
\left({ }^{19} \mathrm{~F} \text { NMR }\right)\end{array}$ \\
\hline $\mathrm{KF}$ & 79 & $\begin{array}{c}\mathrm{AgF}_{2} \\
(2.00 \text { equiv })\end{array}$ & 21 \\
$\mathrm{RbF}$ & 7 & $\mathrm{No}$ & 0 \\
$\mathrm{CsF}$ & 64 & $\mathrm{KF}$ & 67 \\
$\mathrm{NaF}$ & 6 & $\mathrm{KF}$ & $(3.00$ equiv $)$ \\
$\mathrm{LiF}$ & 0 & $\mathrm{KCl}$ & 72 \\
$\mathrm{FeF}$ & 6 & $\mathrm{KOAc}_{3}$ & 7 \\
$(1.33$ equiv $)$ & 0 & $\mathrm{~K}_{2} \mathrm{CO}_{3}$ & 0 \\
$\mathrm{MgF}_{2}$ & 0 & $\mathrm{~K}_{3} \mathrm{PO}_{4}$ & 9 \\
$(2.00$ equiv $)$ & & & 0 \\
$\mathrm{CuF}_{2}$ & & & \\
$(2.00$ equiv $)$ & & &
\end{tabular}




\section{Effect of "OCF 3 " sources on the reaction}

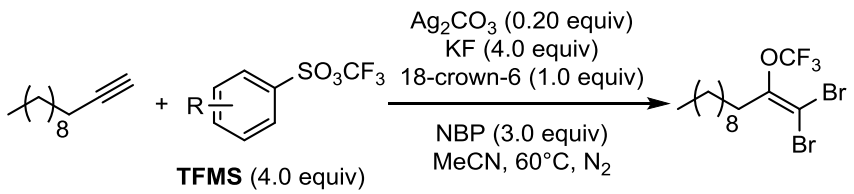

In a glove box, to a $2.0 \mathrm{~mL}$ sealed tube were added in sequence 1-dodecyne (1a) $(8.3 \mathrm{mg}, 0.0500$ mmol, 1.00 equiv), $0.10 \mathrm{~mL} \mathrm{MeCN}, \mathrm{Ag}_{2} \mathrm{CO}_{3}$ ( $2.8 \mathrm{mg}, 0.0100 \mathrm{mmol}, 0.400$ equiv), $\mathrm{KF}$ (11.6 mg, $0.200 \mathrm{mmol}, 4.00$ equiv), 18 -crown-6 (13.2 mg, $0.050 \mathrm{mmol}, 1.00$ equiv), NBP (33.9 mg, 0.150 mmol, 3.00 equiv). The mixture was stirring at room temperature for 5 mins, then added TFMS $\left(0.200 \mathrm{mmol}, 4.00\right.$ equiv). After stirring for $4.0 \mathrm{~h}$ at $60^{\circ} \mathrm{C}$, trifluoromethylthiobenzene $(7 \mu \mathrm{L}$, $0.0500 \mathrm{mmol}$ ) was added. The yield of 1,1-dibromo-2-(trifluoromethoxy)dodec-1-ene (3a) was determined by comparing the integration of the ${ }^{19} \mathrm{~F}$ NMR resonance of 1,1-dibromo-2-(trifluoromethoxy)dodec-1-ene (3a) $\quad(-55.81 \quad \mathrm{ppm})$ with that of trifluoromethylthiobenzene (-42.80 ppm). Yields are reported in Table S7.

Table S7: Effect of " $\mathrm{OCF}_{3}$ " sources on the reaction

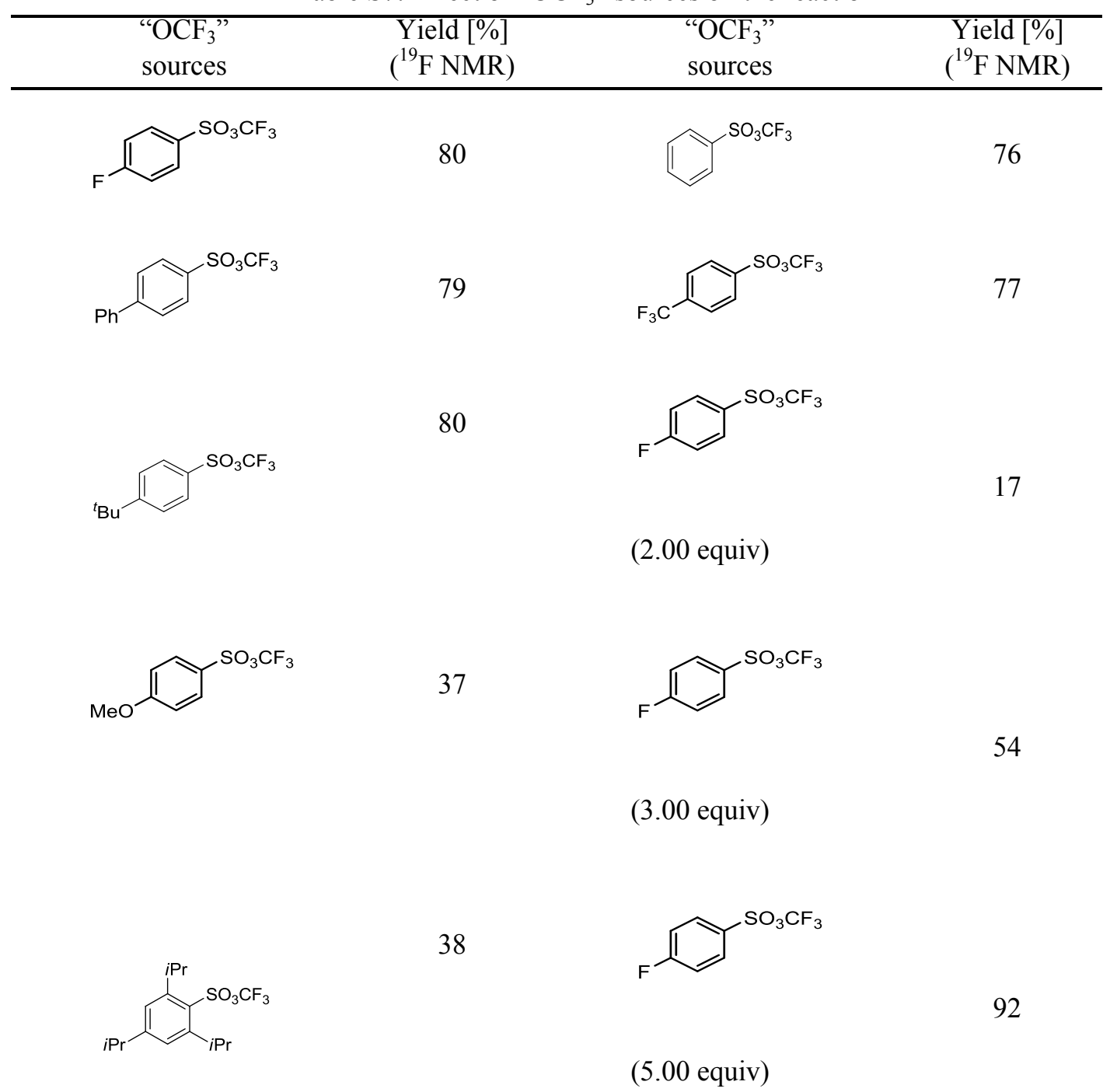




\section{Effect of temperatures on the reaction}

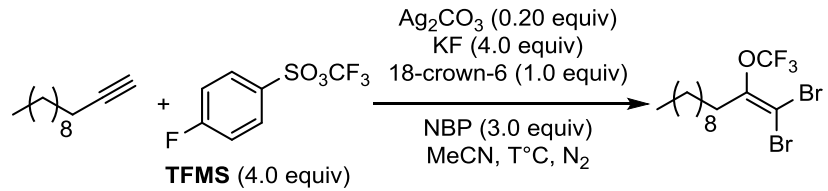

In a glove box, to a $2.0 \mathrm{~mL}$ sealed tube were added in sequence 1-dodecyne (1a) $(8.3 \mathrm{mg}, 0.0500$ mmol, 1.00 equiv), $0.10 \mathrm{~mL} \mathrm{MeCN}, \mathrm{Ag}_{2} \mathrm{CO}_{3}$ ( $2.8 \mathrm{mg}, 0.0100 \mathrm{mmol}, 0.400$ equiv), $\mathrm{KF}$ (11.6 mg, $0.200 \mathrm{mmol}, 4.00$ equiv), 18-crown-6 (13.2 mg, $0.050 \mathrm{mmol}, 1.00$ equiv), NBP (33.9 mg, 0.150 mmol, 3.00 equiv). The mixture was stirring at room temperature for 5 mins, then added TFMS (48.9 mg, $0.200 \mathrm{mmol}, 4.00$ equiv). After stirring for $4.0 \mathrm{~h}$ at different temperature, trifluoromethylthiobenzene $(7 \mu \mathrm{L}, \quad 0.0500 \mathrm{mmol})$ was added. The yield of 1,1-dibromo-2-(trifluoromethoxy)dodec-1-ene (3a) was determined by comparing the integration of the ${ }^{19} \mathrm{~F}$ NMR resonance of 1,1-dibromo-2-(trifluoromethoxy)dodec-1-ene (3a) (-55.81 ppm) with that of trifluoromethylthiobenzene $(-42.80 \mathrm{ppm})$. Yields are reported in Table S8.

Table S8: Effect of temperatures on the reaction

\begin{tabular}{cc}
\hline Temperature & $\begin{array}{r}\text { Yield }[\%] \\
\left({ }^{19} \mathrm{~F} \mathrm{NMR}\right)\end{array}$ \\
\hline $40^{\circ} \mathrm{C}$ & 71 \\
$50{ }^{\circ} \mathrm{C}$ & 78 \\
$60^{\circ} \mathrm{C}$ & 81 \\
$70^{\circ} \mathrm{C}$ & 80 \\
$80^{\circ} \mathrm{C}$ & 81 \\
\hline
\end{tabular}

\section{Effect of atmospheres on the reaction}

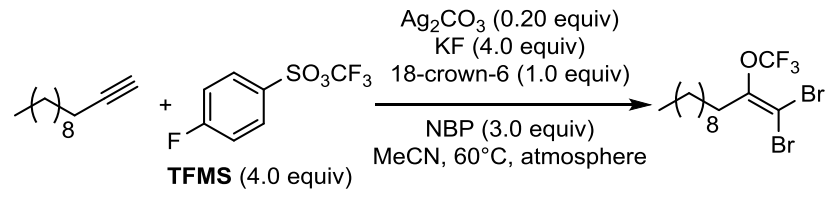

In different atmosphere, to a $2.0 \mathrm{~mL}$ sealed tube were added in sequence 1-dodecyne (1a) $(8.3 \mathrm{mg}$, $0.0500 \mathrm{mmol}, 1.00$ equiv), $0.10 \mathrm{~mL} \mathrm{MeCN}, \mathrm{Ag}_{2} \mathrm{CO}_{3}$ ( $2.8 \mathrm{mg}, 0.0100 \mathrm{mmol}, 0.400$ equiv), $\mathrm{KF}$ (11.6 mg, $0.200 \mathrm{mmol}, 4.00$ equiv), 18 -crown-6 (13.2 mg, $0.050 \mathrm{mmol}, 1.00$ equiv), NBP (33.9 $\mathrm{mg}, 0.150 \mathrm{mmol}, 3.00$ equiv). The mixture was stirring at room temperature for $5 \mathrm{mins}$, then added TFMS (48.9 mg, $0.200 \mathrm{mmol}, 4.00$ equiv). After stirring for $4.0 \mathrm{~h}$ at $60{ }^{\circ} \mathrm{C}$, trifluoromethylthiobenzene $(7 \mu \mathrm{L}, \quad 0.0500 \quad \mathrm{mmol})$ was added. The yield of 1,1-dibromo-2-(trifluoromethoxy)dodec-1-ene (3a) was determined by comparing the integration of the ${ }^{19} \mathrm{~F}$ NMR resonance of 1,1-dibromo-2-(trifluoromethoxy)dodec-1-ene (3a) (-55.81 ppm) with that of trifluoromethylthiobenzene $(-42.80 \mathrm{ppm})$. Yields are reported in Table S9.

Table S9: Effect of atmospheres on the reaction 


\begin{tabular}{cc}
\hline Atmospheres & $\begin{array}{c}\text { Yield [\%] } \\
\left({ }^{19} \mathrm{~F} \mathrm{NMR}\right)\end{array}$ \\
\hline $\mathrm{N}_{2}$ & 80 \\
$\mathrm{O}_{2}$ & 80 \\
air & 78 \\
\hline
\end{tabular}

\section{Effect ofsizes of sealed tube on the reaction}

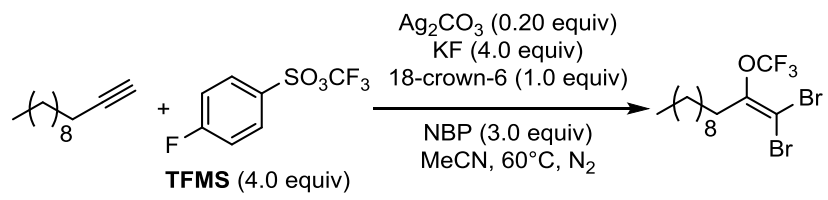

In a glove box, to different sizes sealed tube were added in sequence 1-dodecyne (1a) $(8.3 \mathrm{mg}$, $0.0500 \mathrm{mmol}, 1.00$ equiv), $0.10 \mathrm{~mL} \mathrm{MeCN}, \mathrm{Ag}_{2} \mathrm{CO}_{3}$ ( $2.8 \mathrm{mg}, 0.0100 \mathrm{mmol}, 0.400$ equiv), $\mathrm{KF}$ (11.6 mg, $0.200 \mathrm{mmol}, 4.00$ equiv), 18 -crown-6 (13.2 mg, $0.050 \mathrm{mmol}, 1.00$ equiv), NBP (33.9 $\mathrm{mg}, 0.150 \mathrm{mmol}, 3.00$ equiv). The mixture was stirring at room temperature for $5 \mathrm{mins}$, then added TFMS (48.9 mg, $0.200 \mathrm{mmol}, 4.00$ equiv). After stirring for $4.0 \mathrm{~h}$ at $60{ }^{\circ} \mathrm{C}$, trifluoromethylthiobenzene $(7 \mu \mathrm{L}, \quad 0.0500 \quad \mathrm{mmol})$ was added. The yield of 1,1-dibromo-2-(trifluoromethoxy)dodec-1-ene (3a) was determined by comparing the integration of the ${ }^{19} \mathrm{~F}$ NMR resonance of 1,1-dibromo-2-(trifluoromethoxy)dodec-1-ene (3a) (-55.81 ppm) with that of trifluoromethylthiobenzene $(-42.80 \mathrm{ppm})$. Yields are reported in Table S10.

Table S10: Effect of sizes of sealed tube on the reaction

\begin{tabular}{cc}
\hline sizes of sealed tube & $\begin{array}{c}\text { Yield [\%] } \\
\left({ }^{19} \mathrm{~F} \mathrm{NMR}\right)\end{array}$ \\
\hline $2 \mathrm{~mL}$ & 80 \\
$4 \mathrm{~mL}$ & 62 \\
$5 \mathrm{~mL}$ & 55 \\
\hline
\end{tabular}

\section{Experimental Procedures and Compound Characterization}

\section{Hex-5-yn-1-yl 3-methoxybenzoate (1b)}<smiles>C#CCCCCOC(=O)c1cccc(OCCN(C)C(=O)O)c1</smiles>

To a solution of 3-methoxybenzoic acid (1.52 g, $10.0 \mathrm{mmol}, 1.00$ equiv), DMAP (4-dimethylaminepyridine) (244 mg, $2.00 \mathrm{mmol}, 0.200$ equiv) and $\mathrm{Et}_{3} \mathrm{~N}(3.03 \mathrm{~g}, 30.0 \mathrm{mmol}, 3.00$ equiv) in $\mathrm{CH}_{2} \mathrm{Cl}_{2}(50.0 \mathrm{~mL})$ at room temperature were added EDCI (1-ethyl-(3-(3-dimethylamino)propyl)-carbodiimide hydrochloride) (3.83 g, $20.0 \mathrm{mmol}, 2.00$ equiv) and hex-5-yn-1-ol (1.18 g, $12.0 \mathrm{mmol}, 1.20$ equiv). The reaction mixture was stirred at room temperature for $6 \mathrm{~h}$ before quenched with $\mathrm{H}_{2} \mathrm{O}(30.0 \mathrm{~mL})$ and extracted 3 times with $\mathrm{CH}_{2} \mathrm{Cl}_{2}$ $(30.0 \mathrm{~mL})$. The combined organic layer was dried over $\mathrm{MgSO}_{4}$. The filtrate was concentrated in 
vacuo and the residue was purified by chromatography on silica gel, eluting with hexanes/EtOAc 20:1 (v/v) to afford $1.81 \mathrm{~g}$ hex-5-yn-1-yl 3-methoxybenzoate (1b) as a colourless liquid (78\% yield).

$\mathrm{R}_{f}=0.15$ (hexanes/EtOAc 40:1 (v/v)). NMR Spectroscopy: ${ }^{1} \mathrm{H}$ NMR $\left(400 \mathrm{MHz}, \mathrm{CDCl}_{3}\right) \delta 7.65-$ $7.61(\mathrm{~m}, 1 \mathrm{H}), 7.57-7.54(\mathrm{~m}, 1 \mathrm{H}), 7.34(\mathrm{t}, J=7.9 \mathrm{~Hz}, 1 \mathrm{H}), 7.09(\mathrm{dd}, J=8.2,2.0 \mathrm{~Hz}, 1 \mathrm{H}), 4.34(\mathrm{t}$, $J=6.4 \mathrm{~Hz}, 2 \mathrm{H}), 3.85(\mathrm{~s}, 3 \mathrm{H}), 2.28(\mathrm{td}, J=7.0,2.6 \mathrm{~Hz}, 2 \mathrm{H}), 1.97(\mathrm{t}, J=2.6 \mathrm{~Hz}, 1 \mathrm{H}), 1.94-1.86$ $(\mathrm{m}, 2 \mathrm{H}), 1.74-1.64(\mathrm{~m}, 2 \mathrm{H}) .{ }^{13} \mathrm{C} \mathrm{NMR}\left(101 \mathrm{MHz}, \mathrm{CDCl}_{3}\right) \delta 166.6,159.7,131.8,129.5,122.1$, 119.4, 114.2, 84.0, 68.9, 64.7, 55.6, 27.9, 25.2, 18.2. Mass Spectrometry: HRMS-ESI (m/z): Calcd for $\mathrm{C}_{14} \mathrm{H}_{17} \mathrm{O}_{3}[\mathrm{M}+\mathrm{H}]^{+}, 233.1178$. Found, 233.1168.

\section{Pent-4-yn-1-yl 4-phenoxybenzoate (1c)}

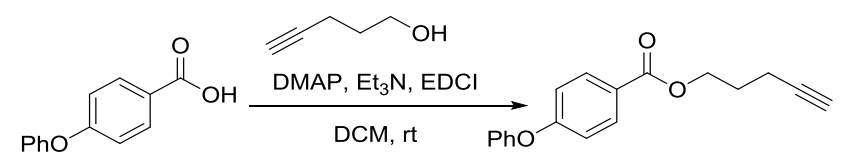

To a solution of 4-phenoxybenzoic acid (2.14 g, $10.0 \mathrm{mmol}, 1.00$ equiv), DMAP (4-dimethylaminepyridine) (244 mg, $2.00 \mathrm{mmol}, 0.200$ equiv) and $\mathrm{Et}_{3} \mathrm{~N}(3.03 \mathrm{~g}, 30.0 \mathrm{mmol}, 3.00$ equiv) in $\mathrm{CH}_{2} \mathrm{Cl}_{2}(50.0 \mathrm{~mL})$ at room temperature were added EDCI (1-ethyl-(3-(3-dimethylamino)propyl)-carbodiimide hydrochloride) (3.83 g, $20.0 \mathrm{mmol}, 2.00$ equiv) and pent-4-yn-1-ol (1.01 g, $12.0 \mathrm{mmol}, 1.20$ equiv). The reaction mixture was stirred at room temperature for $6 \mathrm{~h}$ before quenched with $\mathrm{H}_{2} \mathrm{O}(30.0 \mathrm{~mL})$ and extracted 3 times with $\mathrm{CH}_{2} \mathrm{Cl}_{2}$ $(30.0 \mathrm{~mL})$. The combined organic layer was dried over $\mathrm{MgSO}_{4}$. The filtrate was concentrated in vacuo and the residue was purified by chromatography on silica gel, eluting with hexanes/EtOAc 20:1 (v/v) to afford 2.27 g pent-4-yn-1-yl 4-phenoxybenzoate (1c) as a colorless liquid (81\% yield).

$\mathrm{R}_{f}=0.23$ (hexanes/EtOAc 40:1 (v/v)). NMR Spectroscopy: ${ }^{1} \mathrm{H}$ NMR $\left(400 \mathrm{MHz}, \mathrm{CDCl}_{3}\right) \delta 8.03-$ $7.98(\mathrm{~m}, 2 \mathrm{H}), 7.42-7.36(\mathrm{~m}, 2 \mathrm{H}), 7.22-7.16(\mathrm{~m}, 1 \mathrm{H}), 7.10-7.04(\mathrm{~m}, 2 \mathrm{H}), 7.01-6.96(\mathrm{~m}, 2 \mathrm{H})$, $4.41(\mathrm{t}, J=6.2 \mathrm{~Hz}, 2 \mathrm{H}), 2.38$ (td, $J=7.0,2.6 \mathrm{~Hz}, 2 \mathrm{H}), 2.03-1.96(\mathrm{~m}, 3 \mathrm{H}) .{ }^{13} \mathrm{C}$ NMR $(101 \mathrm{MHz}$, $\left.\mathrm{CDCl}_{3}\right) \delta 166.1,162.0,155.7,131.8,130.2,124.6,120.2,117.4,83.2,69.2,63.5,27.8,15.5$. Mass Spectrometry: HRMS-ESI (m/z): Calcd for $\mathrm{C}_{18} \mathrm{H}_{17} \mathrm{O}_{3}[\mathrm{M}+\mathrm{H}]^{+}$, 281.1178. Found, 281.1169.

\section{Hex-5-yn-1-yl [1,1'-biphenyl]-4-carboxylate (1d)}

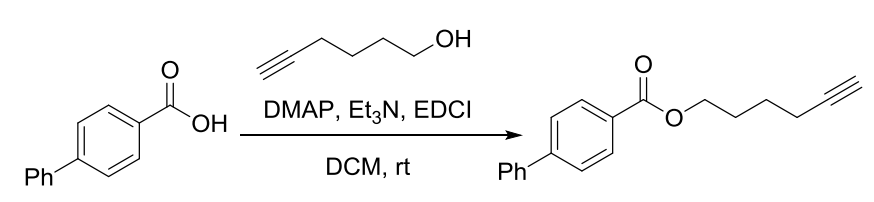

To a solution of [1,1'-biphenyl]-4-carboxylic acid (1.98 g, $10.0 \mathrm{mmol}, 1.00$ equiv), DMAP (4-dimethylaminepyridine) (244 mg, $2.00 \mathrm{mmol}, 0.200$ equiv) and $\mathrm{Et}_{3} \mathrm{~N}(3.03 \mathrm{~g}, 30.0 \mathrm{mmol}, 3.00$ equiv) in $\mathrm{CH}_{2} \mathrm{Cl}_{2}(50.0 \mathrm{~mL})$ at room temperature were added EDCI (1-ethyl-(3-(3-dimethylamino)propyl)-carbodiimide hydrochloride) (3.83 g, $20.0 \mathrm{mmol}, 2.00$ equiv) and hex-5-yn-1-ol (1.18 g, $12.0 \mathrm{mmol}, 1.20$ equiv). The reaction mixture was stirred at room temperature for $6 \mathrm{~h}$ before quenched with $\mathrm{H}_{2} \mathrm{O}(30.0 \mathrm{~mL})$ and extracted 3 times with $\mathrm{CH}_{2} \mathrm{Cl}_{2}$ $(30.0 \mathrm{~mL})$. The combined organic layer was dried over $\mathrm{MgSO}_{4}$. The filtrate was concentrated in vacuo and the residue was purified by chromatography on silica gel, eluting with hexanes/EtOAc 
20:1 (v/v) to afford $2.28 \mathrm{~g}$ hex-5-yn-1-yl [1,1'-biphenyl]-4-carboxylate (1d) as a white solid (82\% yield).

$\mathrm{R}_{f}=0.19$ (hexanes/EtOAc 40:1 (v/v)). NMR Spectroscopy: ${ }^{1} \mathrm{H}$ NMR $\left(400 \mathrm{MHz}, \mathrm{CDCl}_{3}\right) \delta 8.15-$ 8.09 (m, 2H), $7.72-7.60(\mathrm{~m}, 4 \mathrm{H}), 7.51-7.44(\mathrm{~m}, 2 \mathrm{H}), 7.43-7.37$ (m, 1H), 4.38 (t, $J=6.4 \mathrm{~Hz}$, 2H), $2.31(\mathrm{td}, J=7.0,2.6 \mathrm{~Hz}, 2 \mathrm{H}), 2.00(\mathrm{t}, J=2.6 \mathrm{~Hz}, 1 \mathrm{H}), 1.98-1.89(\mathrm{~m}, 2 \mathrm{H}), 1.78-1.70(\mathrm{~m}$, $2 \mathrm{H}) .{ }^{13} \mathrm{C}$ NMR $\left(101 \mathrm{MHz}, \mathrm{CDCl}_{3}\right) \delta 166.6,145.7,140.1,130.2,129.2,129.0,128.2,127.4,127.1$, 84.0, 68.9, 64.6, 27.9, 25.2, 18.3. Mass Spectrometry: HRMS-ESI (m/z): Calcd for $\mathrm{C}_{19} \mathrm{H}_{19} \mathrm{O}_{2}[\mathrm{M}+$ $\mathrm{H}]^{+}, 279.1385$. Found, 279.1376 .

\section{Hex-5-yn-1-yl 3-methylbenzoate (1e)}

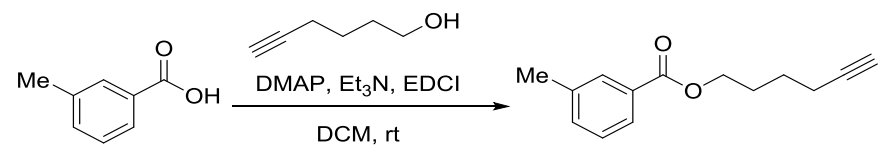

To a solution of 3-methylbenzoic acid (1.36 g, $10.0 \mathrm{mmol}, 1.00$ equiv), DMAP (4-dimethylaminepyridine) (244 mg, $2.00 \mathrm{mmol}, 0.200$ equiv) and $\mathrm{Et}_{3} \mathrm{~N}(3.03 \mathrm{~g}, 30.0 \mathrm{mmol}, 3.00$ equiv) in $\mathrm{CH}_{2} \mathrm{Cl}_{2}(50.0 \mathrm{~mL})$ at room temperature were added EDCI (1-ethyl-(3-(3-dimethylamino)propyl)-carbodiimide hydrochloride) (3.83 g, $20.0 \mathrm{mmol}, 2.00$ equiv) and hex-5-yn-1-ol (1.18 g, $12.0 \mathrm{mmol}, 1.20$ equiv). The reaction mixture was stirred at room temperature for $6 \mathrm{~h}$ before quenched with $\mathrm{H}_{2} \mathrm{O}(30.0 \mathrm{~mL})$ and extracted 3 times with $\mathrm{CH}_{2} \mathrm{Cl}_{2}$ $(30.0 \mathrm{~mL})$. The combined organic layer was dried over $\mathrm{MgSO}_{4}$. The filtrate was concentrated in vacuo and the residue was purified by chromatography on silica gel, eluting with hexanes/EtOAc 30:1 (v/v) to afford $1.57 \mathrm{~g}$ hex-5-yn-1-yl 3-methylbenzoate (1e) as a colourless liquid (73\% yield). $\mathrm{R}_{f}=0.34$ (hexanes/EtOAc 40:1 (v/v)). NMR Spectroscopy: ${ }^{1} \mathrm{H}$ NMR $\left(400 \mathrm{MHz}, \mathrm{CDCl}_{3}\right) \delta 7.89-$ $7.80(\mathrm{~m}, 2 \mathrm{H}), 7.38-7.28(\mathrm{~m}, 3 \mathrm{H}), 4.34(\mathrm{t}, J=6.4 \mathrm{~Hz}, 2 \mathrm{H}), 2.40(\mathrm{~s}, 3 \mathrm{H}), 2.28(\mathrm{td}, J=7.0,2.6 \mathrm{~Hz}$, $2 \mathrm{H}), 1.98(\mathrm{t}, J=2.6 \mathrm{~Hz}, 1 \mathrm{H}), 1.96-1.86(\mathrm{~m}, 2 \mathrm{H}), 1.74-1.65(\mathrm{~m}, 2 \mathrm{H}) .{ }^{13} \mathrm{C}$ NMR $(101 \mathrm{MHz}$, $\left.\mathrm{CDCl}_{3}\right) \delta 166.9,138.2,133.8,130.4,130.2,128.4,126.8,84.0,68.9,64.5,27.9,25.2,21.4,18.2$. Mass Spectrometry: HRMS-ESI (m/z): Calcd for $\mathrm{C}_{14} \mathrm{H}_{17} \mathrm{O}_{2}[\mathrm{M}+\mathrm{H}]^{+}$, 217.1229. Found, 217.1219.

\section{Hex-5-yn-1-yl 4-(tert-butyl)benzoate (1f)}

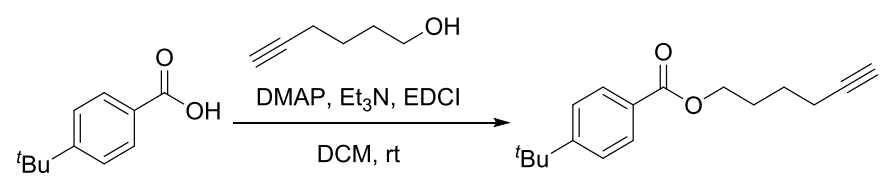

To a solution of 4-(tert-butyl)benzoic acid (1.78 g, $10.0 \mathrm{mmol}, 1.00$ equiv), DMAP (4-dimethylaminepyridine) (244 mg, $2.00 \mathrm{mmol}, 0.200$ equiv) and $\mathrm{Et}_{3} \mathrm{~N}$ (3.03 g, $30.0 \mathrm{mmol}, 3.00$ equiv) in $\mathrm{CH}_{2} \mathrm{Cl}_{2}(50.0 \mathrm{~mL})$ at room temperature were added EDCI (1-ethyl-(3-(3-dimethylamino)propyl)-carbodiimide hydrochloride) (3.83 g, $20.0 \mathrm{mmol}, 2.00$ equiv) and hex-5-yn-1-ol (1.18 g, $12.0 \mathrm{mmol}, 1.20$ equiv). The reaction mixture was stirred at room temperature for $6 \mathrm{~h}$ before quenched with $\mathrm{H}_{2} \mathrm{O}(30.0 \mathrm{~mL})$ and extracted 3 times with $\mathrm{CH}_{2} \mathrm{Cl}_{2}$ $(30.0 \mathrm{~mL})$. The combined organic layer was dried over $\mathrm{MgSO}_{4}$. The filtrate was concentrated in vacuo and the residue was purified by chromatography on silica gel, eluting with hexanes/EtOAc 30:1 (v/v) to afford $1.80 \mathrm{~g}$ hex-5-yn-1-yl 4-(tert-butyl)benzoate (1f) as a colourless liquid (70\% yield). 
$\mathrm{R}_{f}=0.27$ (hexanes/EtOAc 40:1 (v/v)). NMR Spectroscopy: ${ }^{1} \mathrm{H}$ NMR $\left(400 \mathrm{MHz}, \mathrm{CDCl}_{3}\right) \delta 8.01-$ $7.95(\mathrm{~m}, 2 \mathrm{H}), 7.49-7.43(\mathrm{~m}, 2 \mathrm{H}), 4.34(\mathrm{t}, J=6.4 \mathrm{~Hz}, 2 \mathrm{H}), 2.28(\mathrm{td}, J=7.0,2.6 \mathrm{~Hz}, 2 \mathrm{H}), 1.97$ (t, $J=2.6 \mathrm{~Hz}, 1 \mathrm{H}), 1.94-1.85(\mathrm{~m}, 2 \mathrm{H}), 1.74-1.65(\mathrm{~m}, 2 \mathrm{H}), 1.34(\mathrm{~s}, 9 \mathrm{H}) .{ }^{13} \mathrm{C}$ NMR $(101 \mathrm{MHz}$, $\left.\mathrm{CDCl}_{3}\right) \delta 166.7,156.6,129.6,127.7,125.4,84.0,68.9,64.3,35.2,31.2,27.9,25.2$, 18.3. Mass Spectrometry: HRMS-ESI (m/z): Calcd for $\mathrm{C}_{17} \mathrm{H}_{23} \mathrm{O}_{2}[\mathrm{M}+\mathrm{H}]^{+}$, 259.1698. Found, 259.1689.

\section{Hex-5-yn-1-yl benzoate (1g)}

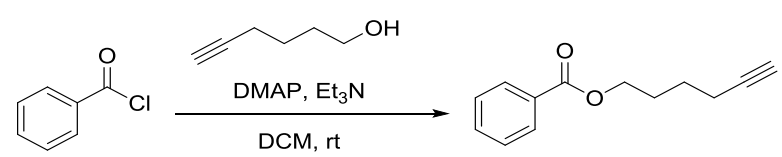

To a solution of hex-5-yn-1-ol (1.08 g, $11.0 \mathrm{mmol}, 1.10$ equiv), DMAP (4-dimethylaminepyridine) (244 mg, $2.00 \mathrm{mmol}, 0.200$ equiv) and $\mathrm{Et}_{3} \mathrm{~N}$ (1.21 g, $12.0 \mathrm{mmol}, 1.20$ equiv) in $\mathrm{CH}_{2} \mathrm{Cl}_{2}(20.0 \mathrm{~mL})$ at $0{ }^{\circ} \mathrm{C}$ was added benzoyl chloride $(1.40 \mathrm{~g}, 10.0 \mathrm{mmol}, 1.00$ equiv). The reaction mixture was warmed to room temperature and stirred for $2 \mathrm{~h}$ before quenched with $\mathrm{H}_{2} \mathrm{O}(20.0 \mathrm{~mL})$ and extracted 3 times with $\mathrm{CH}_{2} \mathrm{Cl}_{2}(20.0 \mathrm{~mL})$. The combined organic layer was dried over $\mathrm{MgSO}_{4}$. The filtrate was concentrated in vacuo and the residue was purified by chromatography on silica gel, eluting with hexanes/EtOAc 20:1 (v/v) to afford $1.96 \mathrm{~g}$ hex-5-yn-1-yl benzoate (1g) as a colourless liquid (97\% yield).

$\mathrm{R}_{f}=0.26$ (hexanes/EtOAc 40:1 (v/v)). NMR Spectroscopy: ${ }^{1} \mathrm{H}$ NMR (400 MHz, $\left.\mathrm{CDCl}_{3}\right) \delta 8.06-$ $8.02(\mathrm{~m}, 2 \mathrm{H}), 7.57-7.53(\mathrm{~m}, 1 \mathrm{H}), 7.47-7.41(\mathrm{~m}, 2 \mathrm{H}), 4.35(\mathrm{t}, J=6.4 \mathrm{~Hz}, 2 \mathrm{H}), 2.28(\mathrm{td}, J=7.0$, $2.6 \mathrm{~Hz}, 2 \mathrm{H}), 1.98(\mathrm{t}, J=2.6 \mathrm{~Hz}, 1 \mathrm{H}), 1.94-1.87(\mathrm{~m}, 2 \mathrm{H}), 1.75-1.66(\mathrm{~m}, 2 \mathrm{H}) .{ }^{13} \mathrm{C}$ NMR $(101$ $\left.\mathrm{MHz}, \mathrm{CDCl}_{3}\right) \delta 166.7,133.0,130.5,129.7,128.5,84.0,68.9,64.5,27.9,25.2,18.3$. Mass Spectrometry: HRMS-ESI (m/z): Calcd for $\mathrm{C}_{13} \mathrm{H}_{15} \mathrm{O}_{2}[\mathrm{M}+\mathrm{H}]^{+}$, 203.1072. Found, 203.1063.

\section{Hex-5-yn-1-yl 4-fluorobenzoate (1h)}

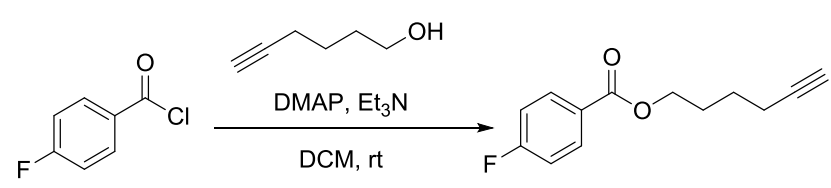

To a solution of hex-5-yn-1-ol (1.08 g, $11.0 \mathrm{mmol}, 1.10$ equiv), DMAP (4-dimethylaminepyridine) (244 mg, $2.00 \mathrm{mmol}, 0.200$ equiv) and $\mathrm{Et}_{3} \mathrm{~N}\left(1.21 \mathrm{~g}, 12.0 \mathrm{mmol}, 1.20\right.$ equiv) in $\mathrm{CH}_{2} \mathrm{Cl}_{2}(20.0 \mathrm{~mL})$ at $0{ }^{\circ} \mathrm{C}$ was added 4-fluorobenzoyl chloride ( $1.58 \mathrm{~g}, 10.0 \mathrm{mmol}, 1.00$ equiv). The reaction mixture was warmed to room temperature and stirred for $2 \mathrm{~h}$ before quenched with $\mathrm{H}_{2} \mathrm{O}(20.0 \mathrm{~mL})$ and extracted 3 times with $\mathrm{CH}_{2} \mathrm{Cl}_{2}(20.0 \mathrm{~mL})$. The combined organic layer was dried over $\mathrm{MgSO}_{4}$. The filtrate was concentrated in vacuo and the residue was purified by chromatography on silica gel, eluting with hexanes/EtOAc 30:1 (v/v) to afford $2.09 \mathrm{~g}$ hex-5-yn-1-yl 4-fluorobenzoate (1h) as a colourless liquid (95\% yield).

$\mathrm{R}_{f}=0.48$ (hexanes/EtOAc 20:1 (v/v)). NMR Spectroscopy: ${ }^{1} \mathrm{H}$ NMR $\left(400 \mathrm{MHz}, \mathrm{CDCl}_{3}\right) \delta 8.07-$ $8.03(\mathrm{~m}, 2 \mathrm{H}), 7.13-7.08(\mathrm{~m}, 2 \mathrm{H}), 4.34(\mathrm{t}, J=6.4 \mathrm{~Hz}, 2 \mathrm{H}), 2.28(\mathrm{td}, J=7.0,2.6 \mathrm{~Hz}, 2 \mathrm{H}), 1.98(\mathrm{t}, J$ $=2.6 \mathrm{~Hz}, 1 \mathrm{H}), 1.93-1.86(\mathrm{~m}, 2 \mathrm{H}), 1.73-1.64(\mathrm{~m}, 2 \mathrm{H}) .{ }^{13} \mathrm{C} \mathrm{NMR}\left(101 \mathrm{MHz}, \mathrm{CDCl}_{3}\right) \delta 165.9(\mathrm{~d}$, $J=253.5 \mathrm{~Hz}), 165.8,132.2(\mathrm{~d}, J=9.1 \mathrm{~Hz}), 126.7(\mathrm{~d}, J=2.3 \mathrm{~Hz}), 115.6(\mathrm{~d}, J=22.1 \mathrm{~Hz}), 83.9$, 77.5, 77.2, 76.8, 69.0, 64.7, 27.9, 25.2, 18.3. ${ }^{19} \mathrm{~F} \mathrm{NMR}\left(376 \mathrm{MHz}, \mathrm{CDCl}_{3}\right) \delta-110.78--110.85(\mathrm{~m}$, 
1F). Mass Spectrometry: HRMS-ESI (m/z): Calcd for $\mathrm{C}_{13} \mathrm{H}_{14} \mathrm{FO}_{2}[\mathrm{M}+\mathrm{H}]^{+}, 221.0978$. Found, 221.0971.

\section{Hex-5-yn-1-yl 4-chlorobenzoate (1i)}

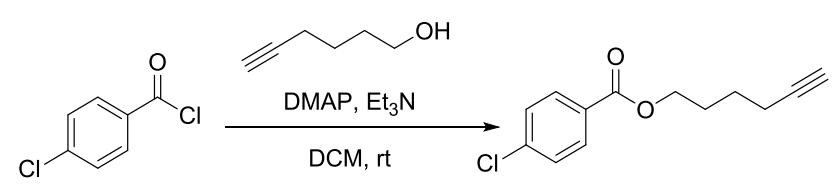

To a solution of hex-5-yn-1-ol (1.08 g, $11.0 \mathrm{mmol}, 1.10$ equiv), DMAP (4-dimethylaminepyridine) (244 mg, $2.00 \mathrm{mmol}, 0.200$ equiv) and $\mathrm{Et}_{3} \mathrm{~N}$ (1.21 g, $12.0 \mathrm{mmol}, 1.20$ equiv) in $\mathrm{CH}_{2} \mathrm{Cl}_{2}(20.0 \mathrm{~mL})$ at $0{ }^{\circ} \mathrm{C}$ was added 4-chlorobenzoyl chloride $(1.74 \mathrm{~g}, 10.0 \mathrm{mmol}, 1.00$ equiv). The reaction mixture was warmed to room temperature and stirred for $2 \mathrm{~h}$ before quenched with $\mathrm{H}_{2} \mathrm{O}(20.0 \mathrm{~mL})$ and extracted 3 times with $\mathrm{CH}_{2} \mathrm{Cl}_{2}(20.0 \mathrm{~mL})$. The combined organic layer was dried over $\mathrm{MgSO}_{4}$. The filtrate was concentrated in vacuo and the residue was purified by chromatography on silica gel, eluting with hexanes/EtOAc 30:1 (v/v) to afford $2.31 \mathrm{~g}$ hex-5-yn-1-yl 4-chlorobenzoate (1i) as a colourless liquid ( $98 \%$ yield).

$\mathrm{R}_{f}=0.32$ (hexanes/EtOAc 40:1 (v/v)). NMR Spectroscopy: ${ }^{1} \mathrm{H}$ NMR $\left(400 \mathrm{MHz}, \mathrm{CDCl}_{3}\right) \delta 8.00-$ $7.94(\mathrm{~m}, 2 \mathrm{H}), 7.44-7.38(\mathrm{~m}, 2 \mathrm{H}), 4.34$ (t, $J=6.5 \mathrm{~Hz}, 2 \mathrm{H}), 2.28$ (td, $J=7.0,2.6 \mathrm{~Hz}, 2 \mathrm{H}), 1.98$ (t, $J=2.6 \mathrm{~Hz}, 1 \mathrm{H}), 1.94-1.83(\mathrm{~m}, 2 \mathrm{H}), 1.73-1.63(\mathrm{~m}, 2 \mathrm{H}) .{ }^{13} \mathrm{C} \mathrm{NMR}\left(101 \mathrm{MHz}, \mathrm{CDCl}_{3}\right) \delta 165.8$, 139.5, 131.1, 128.9, 128.8, 83.9, 69.0, 64.8, 27.8, 25.1, 18.2. Mass Spectrometry: HRMS-ESI (m/z): Calcd for $\mathrm{C}_{13} \mathrm{H}_{14} \mathrm{ClO}_{2}[\mathrm{M}+\mathrm{H}]^{+}$, 237.0682. Found, 237.0673.

\section{Pent-4-yn-1-yl 4-bromobenzoate (1j)}

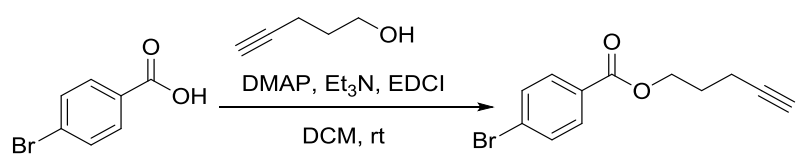

To a solution of 4-phenoxybenzoic acid (2.01 g, $10.0 \mathrm{mmol}, 1.00$ equiv), DMAP (4-dimethylaminepyridine) (244mg, $2.00 \mathrm{mmol}, 0.200$ equiv) and $\mathrm{Et}_{3} \mathrm{~N}$ (3.03 g, $30.0 \mathrm{mmol}, 3.00$ equiv) in $\mathrm{CH}_{2} \mathrm{Cl}_{2}(50.0 \mathrm{~mL})$ at room temperature were added EDCI (1-ethyl-(3-(3-dimethylamino)propyl)-carbodiimide hydrochloride) (3.83 g, $20.0 \mathrm{mmol}, 2.00$ equiv) and pent-4-yn-1-ol (1.01 g, $12.0 \mathrm{mmol}, 1.20$ equiv). The reaction mixture was stirred at room temperature for $6 \mathrm{~h}$ before quenched with $\mathrm{H}_{2} \mathrm{O}(30.0 \mathrm{~mL})$ and extracted 3 times with $\mathrm{CH}_{2} \mathrm{Cl}_{2}$ $(30.0 \mathrm{~mL})$. The combined organic layer was dried over $\mathrm{MgSO}_{4}$. The filtrate was concentrated in vacuo and the residue was purified by chromatography on silica gel, eluting with hexanes/EtOAc 20:1 (v/v) to afford 2.08 g pent-4-yn-1-yl 4-bromobenzoate (1j) as a colorless liquid (78\% yield).

$\mathrm{R}_{f}=0.26$ (hexanes/EtOAc 40:1 (v/v)). NMR Spectroscopy: ${ }^{1} \mathrm{H}$ NMR $\left(400 \mathrm{MHz}, \mathrm{CDCl}_{3}\right) \delta 7.92-$ $7.87(\mathrm{~m}, 2 \mathrm{H}), 7.60-7.55(\mathrm{~m}, 2 \mathrm{H}), 4.42(\mathrm{t}, J=6.3 \mathrm{~Hz}, 2 \mathrm{H}), 2.37(\mathrm{td}, J=7.0,2.5 \mathrm{~Hz}, 2 \mathrm{H}), 2.06-$ $1.95(\mathrm{~m}, 3 \mathrm{H}) .{ }^{13} \mathrm{C} \mathrm{NMR}\left(101 \mathrm{MHz}, \mathrm{CDCl}_{3}\right) \delta 165.9,131.8,131.2,129.2,128.2,83.0,69.3,63.9$, 27.7, 15.5. Mass Spectrometry: HRMS-ESI (m/z): Calcd for $\mathrm{C}_{12} \mathrm{H}_{12} \mathrm{BrO}_{2}[\mathrm{M}+\mathrm{H}]^{+}, 267.0021$. Found, 267.0011. 


\section{Hex-5-yn-1-yl 4-iodobenzoate(1k)}

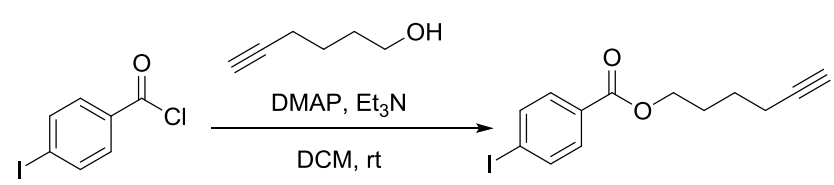

To a solution of hex-5-yn-1-ol (1.08 g, $11.0 \mathrm{mmol}, 1.10$ equiv), DMAP (4-dimethylaminepyridine) (244 mg, $2.00 \mathrm{mmol}, 0.200$ equiv) and $\mathrm{Et}_{3} \mathrm{~N}$ (1.21 g, $12.0 \mathrm{mmol}, 1.20$ equiv) in $\mathrm{CH}_{2} \mathrm{Cl}_{2}(20.0 \mathrm{~mL})$ at $0{ }^{\circ} \mathrm{C}$ was added 4-iodobenzoyl chloride $(2.66 \mathrm{~g}, 10.0 \mathrm{mmol}, 1.00$ equiv). The reaction mixture was warmed to room temperature and stirred for $2 \mathrm{~h}$ before quenched with $\mathrm{H}_{2} \mathrm{O}(20.0 \mathrm{~mL})$ and extracted 3 times with $\mathrm{CH}_{2} \mathrm{Cl}_{2}(20.0 \mathrm{~mL})$. The combined organic layer was dried over $\mathrm{MgSO}_{4}$. The filtrate was concentrated in vacuo and the residue was purified by chromatography on silica gel, eluting with hexanes/EtOAc 30:1 (v/v) to afford 3.11g hex-5-yn-1-yl 4-iodobenzoate (1k) as a colourless liquid ( $95 \%$ yield).

$\mathrm{R}_{f}=0.28$ (hexanes/EtOAc 40:1 (v/v)). NMR Spectroscopy: ${ }^{1} \mathrm{H}$ NMR $\left(400 \mathrm{MHz}, \mathrm{CDCl}_{3}\right) \delta 7.81-$ $7.75(\mathrm{~m}, 2 \mathrm{H}), 7.75-7.69(\mathrm{~m}, 2 \mathrm{H}), 4.33(\mathrm{t}, J=6.4 \mathrm{~Hz}, 2 \mathrm{H}), 2.27$ (td, $J=7.0,2.6 \mathrm{~Hz}, 2 \mathrm{H}), 1.97$ (t, $J=2.6 \mathrm{~Hz}, 1 \mathrm{H}), 1.93-1.83(\mathrm{~m}, 2 \mathrm{H}), 1.72-1.62(\mathrm{~m}, 2 \mathrm{H}) .{ }^{13} \mathrm{C} \mathrm{NMR}\left(101 \mathrm{MHz}, \mathrm{CDCl}_{3}\right) \delta 166.2$, $137.8,131.1,129.9,100.8,83.9,69.0,64.8,27.8,25.1,18.2$. Mass Spectrometry: HRMS-ESI (m/z): Calcd for $\mathrm{C}_{13} \mathrm{H}_{14} \mathrm{IO}_{2}[\mathrm{M}+\mathrm{H}]^{+}, 329.0038$. Found, 329.0031 .

\section{Hex-5-yn-1-yl 4-(trifluoromethyl)benzoate(11)}

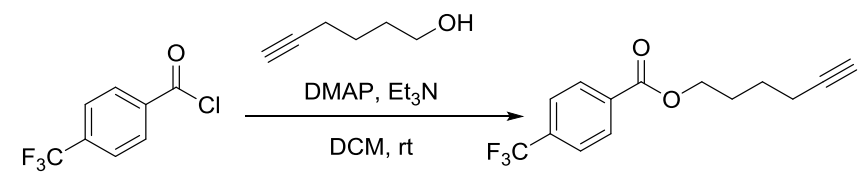

To a solution of hex-5-yn-1-ol (1.08 g, $11.0 \mathrm{mmol}, 1.10$ equiv), DMAP (4-dimethylaminepyridine) (244 mg, $2.00 \mathrm{mmol}, 0.200$ equiv) and $\mathrm{Et}_{3} \mathrm{~N}$ (1.21 g, $12.0 \mathrm{mmol}, 1.20$ equiv) in $\mathrm{CH}_{2} \mathrm{Cl}_{2}(20.0 \mathrm{~mL}$ ) at $0{ }^{\circ} \mathrm{C}$ was added 4-(trifluoromethyl)benzoyl chloride $(2.08 \mathrm{~g}, 10.0 \mathrm{mmol}, 1.00$ equiv). The reaction mixture was warmed to room temperature and stirred for $2 \mathrm{~h}$ before quenched with $\mathrm{H}_{2} \mathrm{O}$ $(20.0 \mathrm{~mL})$ and extracted 3 times with $\mathrm{CH}_{2} \mathrm{Cl}_{2}(20.0 \mathrm{~mL})$. The combined organic layer was dried over $\mathrm{MgSO}_{4}$. The filtrate was concentrated in vacuo and the residue was purified by chromatography on silica gel, eluting with hexanes/EtOAc $30: 1(\mathrm{v} / \mathrm{v})$ to afford $2.51 \mathrm{~g}$ hex-5-yn-1-yl 4-(trifluoromethyl)benzoate (11) as a colourless liquid (93\% yield).

$\mathrm{R}_{f}=0.35$ (hexanes/EtOAc 40:1 (v/v)). NMR Spectroscopy: ${ }^{1} \mathrm{H}$ NMR (400 MHz, $\left.\mathrm{CDCl}_{3}\right) \delta 8.15(\mathrm{~d}$, $J=8.1 \mathrm{~Hz}, 2 \mathrm{H}), 7.70(\mathrm{~d}, J=8.2 \mathrm{~Hz}, 2 \mathrm{H}), 4.38$ (t, $J=6.5 \mathrm{~Hz}, 2 \mathrm{H}), 2.29$ (td, $J=7.0,2.6 \mathrm{~Hz}, 2 \mathrm{H})$, $1.98(\mathrm{t}, J=2.6 \mathrm{~Hz}, 1 \mathrm{H}), 1.97-1.88(\mathrm{~m}, 2 \mathrm{H}), 1.74-1.65(\mathrm{~m}, 2 \mathrm{H}) .{ }^{13} \mathrm{C} \mathrm{NMR}\left(101 \mathrm{MHz}, \mathrm{CDCl}_{3}\right) \delta$ 165.5, 134.5 (q, $J=32.7 \mathrm{~Hz}), 133.7,130.1,125.5$ (q, $J=3.7 \mathrm{~Hz}), 123.8$ (q, $J=272.7 \mathrm{~Hz}), 83.8$, 69.0, 65.2, 27.8, 25.1, 18.2. ${ }^{19} \mathrm{~F}$ NMR $\left(376 \mathrm{MHz}, \mathrm{CDCl}_{3}\right) \delta-62.71$ (s, 3F). Mass Spectrometry: HRMS-ESI (m/z): Calcd for $\mathrm{C}_{14} \mathrm{H}_{14} \mathrm{~F}_{3} \mathrm{O}_{2}[\mathrm{M}+\mathrm{H}]^{+}$, 271.0946. Found, 271.0937. 


\section{Hex-5-yn-1-yl 4-(trifluoromethoxy)benzoate (1 m)}

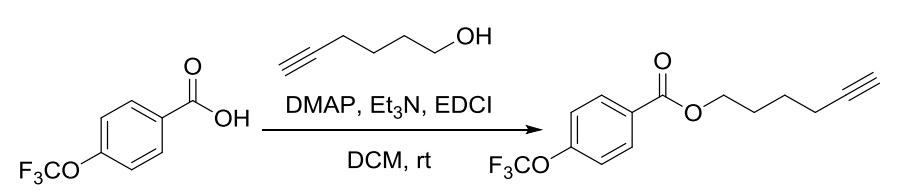

To a solution of 4-(trifluoromethoxy)benzoic acid (2.06 g, $10.0 \mathrm{mmol}, 1.00$ equiv), DMAP (4-dimethylaminepyridine) (244 mg, $2.00 \mathrm{mmol}, 0.200$ equiv) and $\mathrm{Et}_{3} \mathrm{~N}(3.03 \mathrm{~g}, 30.0 \mathrm{mmol}, 3.00$ equiv) in $\mathrm{CH}_{2} \mathrm{Cl}_{2}(50.0 \mathrm{~mL})$ at room temperature were added EDCI (1-ethyl-(3-(3-dimethylamino)propyl)-carbodiimide hydrochloride) (3.83 g, $20.0 \mathrm{mmol}, 2.00$ equiv) and hex-5-yn-1-ol (1.18 g, $12.0 \mathrm{mmol}, 1.20$ equiv). The reaction mixture was stirred at room temperature for $6 \mathrm{~h}$ before quenched with $\mathrm{H}_{2} \mathrm{O}(30.0 \mathrm{~mL})$ and extracted 3 times with $\mathrm{CH}_{2} \mathrm{Cl}_{2}$ $(30.0 \mathrm{~mL})$. The combined organic layer was dried over $\mathrm{MgSO}_{4}$. The filtrate was concentrated in vacuo and the residue was purified by chromatography on silica gel, eluting with hexanes/EtOAc 20:1 (v/v) to afford $1.77 \mathrm{~g}$ hex-5-yn-1-yl 4-(trifluoromethoxy)benzoate (1m) as a colorless liquid (65\% yield).

$\mathrm{R}_{f}=0.33$ (hexanes/EtOAc 40:1 (v/v)). NMR Spectroscopy: ${ }^{1} \mathrm{H}$ NMR (400 MHz, $\left.\mathrm{CDCl}_{3}\right) \delta 8.18-$ $8.06(\mathrm{~m}, 2 \mathrm{H}), 7.30-7.24(\mathrm{~m}, 2 \mathrm{H}), 4.36(\mathrm{t}, J=6.4 \mathrm{~Hz}, 2 \mathrm{H}), 2.29$ (td, $J=7.0,2.6 \mathrm{~Hz}, 2 \mathrm{H}), 1.98(\mathrm{t}$, $J=2.6 \mathrm{~Hz}, 1 \mathrm{H}), 1.96-1.87(\mathrm{~m}, 2 \mathrm{H}), 1.75-1.66(\mathrm{~m}, 2 \mathrm{H}) .{ }^{13} \mathrm{C} \mathrm{NMR}\left(101 \mathrm{MHz}, \mathrm{CDCl}_{3}\right) \delta 165.5$, $152.8,131.7,128.9,120.4$ (q, $J=258.8 \mathrm{~Hz}), 120.4,83.9,69.0,64.9,27.9,25.2,18.3 .{ }^{19} \mathrm{~F} \mathrm{NMR}$ $\left(376 \mathrm{MHz}, \mathrm{CDCl}_{3}\right) \delta-57.76(\mathrm{~s}, 3 \mathrm{~F})$. Mass Spectrometry: HRMS-ESI (m/z): Calcd for $\mathrm{C}_{14} \mathrm{H}_{14} \mathrm{~F}_{3} \mathrm{O}_{3}$ $[\mathrm{M}+\mathrm{H}]^{+}, 287.0890$. Found, 287.0888.

\section{Pent-4-yn-1-yl 4-benzoylbenzoate (1n)}

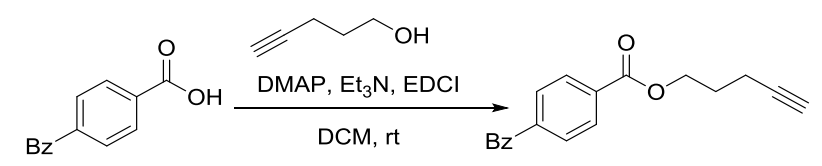

To a solution of 4-benzoylbenzoic acid (2.26 g, $10.0 \mathrm{mmol}, 1.00$ equiv), DMAP (4-dimethylaminepyridine) (244 mg, $2.00 \mathrm{mmol}, 0.200$ equiv) and $\mathrm{Et}_{3} \mathrm{~N}(3.03 \mathrm{~g}, 30.0 \mathrm{mmol}, 3.00$ equiv) in $\mathrm{CH}_{2} \mathrm{Cl}_{2}(50.0 \mathrm{~mL})$ at room temperature were added EDCI (1-ethyl-(3-(3-dimethylamino)propyl)-carbodiimide hydrochloride) (3.83 g, $20.0 \mathrm{mmol}, 2.00$ equiv) and hex-5-yn-1-ol (1.01 g, $12.0 \mathrm{mmol}, 1.20$ equiv). The reaction mixture was stirred at room temperature for $6 \mathrm{~h}$ before quenched with $\mathrm{H}_{2} \mathrm{O}(30.0 \mathrm{~mL})$ and extracted 3 times with $\mathrm{CH}_{2} \mathrm{Cl}_{2}$ $(30.0 \mathrm{~mL})$. The combined organic layer was dried over $\mathrm{MgSO}_{4}$. The filtrate was concentrated in vacuo and the residue was purified by chromatography on silica gel, eluting with hexanes/EtOAc 10:1 (v/v) to afford 2.28 g pent-4-yn-1-yl 4-benzoylbenzoate (1n) as a white solid (78\% yield).

$\mathrm{R}_{f}=0.10$ (hexanes/EtOAc 40:1 (v/v)). NMR Spectroscopy: ${ }^{1} \mathrm{H}$ NMR $\left(400 \mathrm{MHz}, \mathrm{CDCl}_{3}\right) \delta 8.15(\mathrm{~d}$, $J=8.4 \mathrm{~Hz}, 2 \mathrm{H}), 7.86-7.78(\mathrm{~m}, 4 \mathrm{H}), 7.64-7.58(\mathrm{~m}, 1 \mathrm{H}), 7.53-7.47(\mathrm{~m}, 2 \mathrm{H}), 4.48(\mathrm{t}, J=6.3 \mathrm{~Hz}$, $2 \mathrm{H}), 2.40(\mathrm{td}, J=7.0,2.6 \mathrm{~Hz}, 2 \mathrm{H}), 2.08-1.98(\mathrm{~m}, 3 \mathrm{H}) .{ }^{13} \mathrm{C} \mathrm{NMR}\left(101 \mathrm{MHz}, \mathrm{CDCl}_{3}\right) \delta 196.1$, 165.9, 141.5, 137.1, 133.4, 133.1, 130.2, 129.9, 129.6, 128.6, 83.0, 69.4, 64.1, 27.7, 15.5. Mass Spectrometry: HRMS-ESI (m/z): Calcd for $\mathrm{C}_{19} \mathrm{H}_{17} \mathrm{O}_{3}[\mathrm{M}+\mathrm{H}]^{+}$, 293.1178. Found, 293.1168. 


\section{Hex-5-yn-1-yl 4-cyanobenzoate (10)}

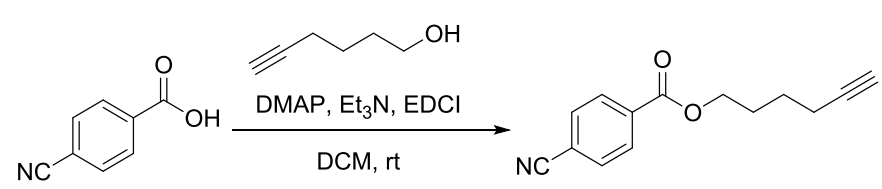

To a solution of 4-cyanobenzoic acid (1.47 g, $10.0 \mathrm{mmol}, 1.00$ equiv), DMAP (4-dimethylaminepyridine) (244 mg, $2.00 \mathrm{mmol}, 0.200$ equiv) and $\mathrm{Et}_{3} \mathrm{~N}(3.03 \mathrm{~g}, 30.0 \mathrm{mmol}, 3.00$ equiv) in $\mathrm{CH}_{2} \mathrm{Cl}_{2}(50.0 \mathrm{~mL})$ at room temperature were added EDCI (1-ethyl-(3-(3-dimethylamino)propyl)-carbodiimide hydrochloride) (3.83 g, $20.0 \mathrm{mmol}, 2.00$ equiv) and hex-5-yn-1-yl (1.18 g, $12.0 \mathrm{mmol}, 1.20$ equiv). The reaction mixture was stirred at room temperature for $6 \mathrm{~h}$ before quenched with $\mathrm{H}_{2} \mathrm{O}(30.0 \mathrm{~mL})$ and extracted 3 times with $\mathrm{CH}_{2} \mathrm{Cl}_{2}$ $(30.0 \mathrm{~mL})$. The combined organic layer was dried over $\mathrm{MgSO}_{4}$. The filtrate was concentrated in vacuo and the residue was purified by chromatography on silica gel, eluting with hexanes/EtOAc 4:1 (v/v) to afford $1.52 \mathrm{~g}$ hex-5-yn-1-yl 4-cyanobenzoate (10) as a colorless liquid (72\% yield).

$\mathrm{R}_{f}=0.50$ (hexanes/EtOAc 20:1 (v/v)). NMR Spectroscopy: ${ }^{1} \mathrm{H}$ NMR $\left(400 \mathrm{MHz}, \mathrm{CDCl}_{3}\right) \delta 8.19-$ 8.09 (m, 2H), $7.79-7.69$ (m, 2H), 4.37 (t, $J=6.5 \mathrm{~Hz}, 2 \mathrm{H}), 2.27$ (td, $J=7.0,2.6 \mathrm{~Hz}, 2 \mathrm{H}), 1.97$ (t, $J=2.6 \mathrm{~Hz}, 1 \mathrm{H}), 1.95-1.86(\mathrm{~m}, 2 \mathrm{H}), 1.74-1.61(\mathrm{~m}, 2 \mathrm{H}) .{ }^{13} \mathrm{C} \mathrm{NMR}\left(101 \mathrm{MHz}, \mathrm{CDCl}_{3}\right) \delta 165.0$, $134.2,132.3,130.2,118.1,116.4,83.8,69.1,65.4,27.7,25.0,18.2$. Mass Spectrometry: HRMS-ESI (m/z): Calcd for $\mathrm{C}_{14} \mathrm{H}_{14} \mathrm{NO}_{2}[\mathrm{M}+\mathrm{H}]^{+}, 228.1025$. Found, 228.1017.

\section{Hex-5-yn-1-yl 4-nitrobenzoate (1p)}

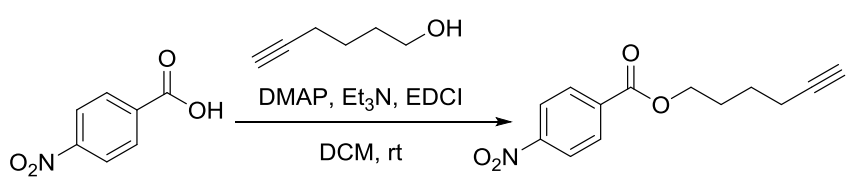

To a solution of 4-nitrobenzoic acid (1.67 $\mathrm{g}, 10.0 \mathrm{mmol}, 1.00$ equiv), DMAP (4-dimethylaminepyridine) (244 mg, $2.00 \mathrm{mmol}, 0.200$ equiv) and $\mathrm{Et}_{3} \mathrm{~N}(3.03 \mathrm{~g}, 30.0 \mathrm{mmol}, 3.00$ equiv) in $\mathrm{CH}_{2} \mathrm{Cl}_{2}(50.0 \mathrm{~mL})$ at room temperature were added EDCI (1-ethyl-(3-(3-dimethylamino)propyl)-carbodiimide hydrochloride) (3.83 g, $20.0 \mathrm{mmol}, 2.00$ equiv) and hex-5-yn-1-ol (1.18 g, $12.0 \mathrm{mmol}, 1.20$ equiv). The reaction mixture was stirred at room temperature for $6 \mathrm{~h}$ before quenched with $\mathrm{H}_{2} \mathrm{O}(30.0 \mathrm{~mL})$ and extracted 3 times with $\mathrm{CH}_{2} \mathrm{Cl}_{2}$ $(30.0 \mathrm{~mL})$. The combined organic layer was dried over $\mathrm{MgSO}_{4}$. The filtrate was concentrated in vacuo and the residue was purified by chromatography on silica gel, eluting with hexanes/EtOAc 15:1 (v/v) to afford $1.70 \mathrm{~g}$ hex-5-yn-1-yl 4-nitrobenzoate (1p) as a yellow solid (56\% yield).

$\mathrm{R}_{f}=0.31$ (hexanes/EtOAc 20:1 (v/v)). NMR Spectroscopy: ${ }^{1} \mathrm{H}$ NMR $\left(400 \mathrm{MHz}, \mathrm{CDCl}_{3}\right) \delta 8.30-$ $8.25(\mathrm{~m}, 2 \mathrm{H}), 8.22-8.18(\mathrm{~m}, 2 \mathrm{H}), 4.40$ (t, $J=6.5 \mathrm{~Hz}, 2 \mathrm{H}), 2.29$ (td, $J=7.0,2.6 \mathrm{~Hz}, 2 \mathrm{H}), 1.98$ (t, $J=2.6 \mathrm{~Hz}, 1 \mathrm{H}), 1.97-1.89(\mathrm{~m}, 2 \mathrm{H}), 1.73-1.66(\mathrm{~m}, 2 \mathrm{H}) .{ }^{13} \mathrm{C} \mathrm{NMR}\left(101 \mathrm{MHz}, \mathrm{CDCl}_{3}\right) \delta 164.8$, 150.6, 135.8, 130.8, 123.7, 83.8, 69.1, 65.6, 27.7, 25.1, 18.2. Mass Spectrometry: HRMS-ESI (m/z): Calcd for $\mathrm{C}_{13} \mathrm{H}_{14} \mathrm{NO}_{4}[\mathrm{M}+\mathrm{H}]^{+}$, 248.0923. Found, 248.0914. 


\section{Hex-5-yn-1-yl 4-(methylsulfonyl)benzoate (1q)}

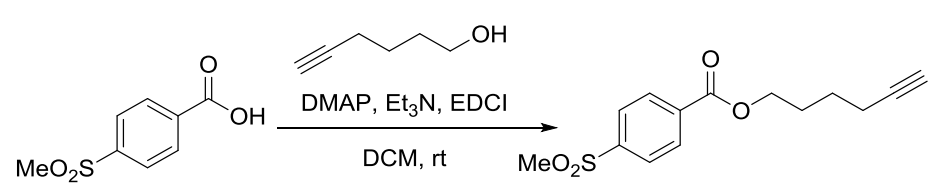

To a solution of 4-(methylsulfonyl)benzoic acid (2.00 g, $10.0 \mathrm{mmol}, 1.00$ equiv), DMAP (4-dimethylaminepyridine) (244 mg, $2.00 \mathrm{mmol}, 0.200$ equiv) and $\mathrm{Et}_{3} \mathrm{~N}(3.03 \mathrm{~g}, 30.0 \mathrm{mmol}, 3.00$ equiv) in $\mathrm{CH}_{2} \mathrm{Cl}_{2}(50.0 \mathrm{~mL})$ at room temperature were added EDCI (1-ethyl-(3-(3-dimethylamino)propyl)-carbodiimide hydrochloride) (3.83 g, $20.0 \mathrm{mmol}, 2.00$ equiv) and hex-5-yn-1-yl (1.18 g, $12.0 \mathrm{mmol}, 1.20$ equiv). The reaction mixture was stirred at room temperature for $6 \mathrm{~h}$ before quenched with $\mathrm{H}_{2} \mathrm{O}(30.0 \mathrm{~mL})$ and extracted 3 times with $\mathrm{CH}_{2} \mathrm{Cl}_{2}$ $(30.0 \mathrm{~mL})$. The combined organic layer was dried over $\mathrm{MgSO}_{4}$. The filtrate was concentrated in vacuo and the residue was purified by chromatography on silica gel, eluting with hexanes/EtOAc 8:1 (v/v) to afford $2.28 \mathrm{~g}$ hex-5-yn-1-yl 4-(methylsulfonyl)benzoate (1q) as a white solid (78\% yield).

$\mathrm{R}_{f}=0.08$ (hexanes/EtOAc 20:1 (v/v)). NMR Spectroscopy: ${ }^{1} \mathrm{H}$ NMR $\left(400 \mathrm{MHz}, \mathrm{CDCl}_{3}\right) \delta 8.21(\mathrm{~d}$, $J=7.7 \mathrm{~Hz}, 2 \mathrm{H}), 8.01$ (d, $J=7.7 \mathrm{~Hz}, 2 \mathrm{H}), 4.38$ (t, $J=6.3 \mathrm{~Hz}, 2 \mathrm{H}), 3.07$ (s, 3H), 2.28 (t, $J=6.9 \mathrm{~Hz}$, $2 \mathrm{H}), 2.00-1.96(\mathrm{~m}, 1 \mathrm{H}), 1.96-1.87(\mathrm{~m}, 2 \mathrm{H}), 1.73-1.64(\mathrm{~m}, 2 \mathrm{H}) .{ }^{13} \mathrm{C}$ NMR (101 MHz, $\left.\mathrm{CDCl}_{3}\right)$ $\delta$ 165.0, 144.3, 135.2, 130.6, 127.6, 83.8, 69.1, 65.4, 44.4, 27.7, 25.0, 18.2. Mass Spectrometry: HRMS-ESI (m/z): Calcd for $\mathrm{C}_{14} \mathrm{H}_{17} \mathrm{O}_{4} \mathrm{~S}[\mathrm{M}+\mathrm{H}]^{+}, 281.0848$. Found, 281.0838.

\section{Oxiran-2-ylmethyl 4-(prop-2-yn-1-yloxy)benzoate(1u)}

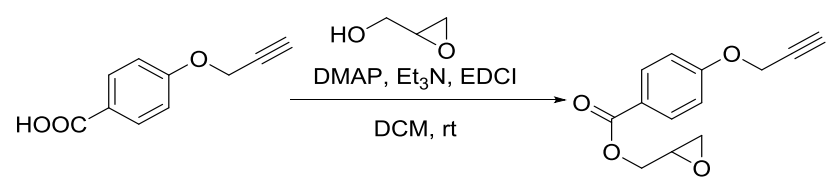

To a solution of 4-(prop-2-yn-1-yloxy)benzoic acid ${ }^{[2]}$ (704 mg, $4.00 \mathrm{mmol}, 1.00$ equiv), DMAP (4-dimethylaminepyridine) $\left(122 \mathrm{mg}, 1.00 \mathrm{mmol}, 0.250\right.$ equiv) and $\mathrm{Et}_{3} \mathrm{~N}(1.21 \mathrm{~g}, 12.0 \mathrm{mmol}, 3.00$ equiv) in $\mathrm{CH}_{2} \mathrm{Cl}_{2}(20.0 \mathrm{~mL})$ at room temperature were added EDCI (1-ethyl-(3-(3-dimethylamino)propyl)-carbodiimide hydrochloride) (1.91 g, $10.0 \mathrm{mmol}, 2.50$ equiv) and glycidol (370 $\mathrm{mg}, 5.00 \mathrm{mmol}, 1.25$ equiv). The reaction mixture was stirred at room temperature for $6 \mathrm{~h}$ before quenched with $\mathrm{H}_{2} \mathrm{O}(30.0 \mathrm{~mL})$ and extracted 3 times with $\mathrm{CH}_{2} \mathrm{Cl}_{2}(30.0$ $\mathrm{mL}$ ). The combined organic layer was dried over $\mathrm{MgSO}_{4}$. The filtrate was concentrated in vacuo and the residue was purified by chromatography on silica gel, eluting with hexanes/EtOAc 8:1 (v/v) to afford 672 mg oxiran-2-ylmethyl 4-(prop-2-yn-1-yloxy)benzoate (1u) as a colorless liquid (72\% yield).

$\mathrm{R}_{f}=0.10$ (hexanes/EtOAc 20:1 (v/v)). NMR Spectroscopy: ${ }^{1} \mathrm{H}$ NMR $\left(400 \mathrm{MHz}, \mathrm{CDCl}_{3}\right) \delta 8.06-$ $7.95(\mathrm{~m}, 2 \mathrm{H}), 7.02-6.96(\mathrm{~m}, 2 \mathrm{H}), 4.74(\mathrm{~d}, J=2.4 \mathrm{~Hz}, 2 \mathrm{H}), 4.61(\mathrm{dd}, J=12.3,3.0 \mathrm{~Hz}, 1 \mathrm{H}), 4.13$ (dd, $J=12.3,6.2 \mathrm{~Hz}, 1 \mathrm{H}), 3.31$ (td, $J=6.5,2.9 \mathrm{~Hz}, 1 \mathrm{H}), 2.87$ (t, $J=4.5 \mathrm{~Hz}, 1 \mathrm{H}), 2.71$ (dd, $J=4.9$, $2.6 \mathrm{~Hz}, 1 \mathrm{H}), 2.55(\mathrm{t}, J=2.4 \mathrm{~Hz}, 1 \mathrm{H}) .{ }^{13} \mathrm{C}$ NMR $\left(101 \mathrm{MHz}, \mathrm{CDCl}_{3}\right) \delta 165.9,161.5,131.9,123.0$, 114.7, 77.9, 76.3, 65.3, 55.9, 49.6, 44.8. Mass Spectrometry: HRMS-ESI (m/z): Calcd for $\mathrm{C}_{13} \mathrm{H}_{13} \mathrm{O}_{4}[\mathrm{M}+\mathrm{H}]^{+}$, 233.0814. Found, 233.0806. 
2-(Trimethylsilyl)ethyl 4-(prop-2-yn-1-yloxy)benzoate (1v)

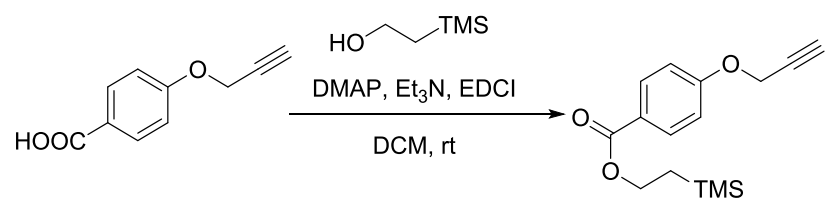

To a solution of 4-(prop-2-yn-1-yloxy)benzoic acid ( $880 \mathrm{mg}, 5.00 \mathrm{mmol}, 1.00$ equiv), DMAP (4-dimethylaminepyridine) $\left(122 \mathrm{mg}, 1.00 \mathrm{mmol}, 0.200\right.$ equiv) and $\mathrm{Et}_{3} \mathrm{~N}(1.51 \mathrm{~g}, 15.0 \mathrm{mmol}, 3.00$ equiv) in $\mathrm{CH}_{2} \mathrm{Cl}_{2}(20.0 \mathrm{~mL})$ at room temperature were added EDCI (1-ethyl-(3-(3-dimethylamino)propyl)-carbodiimide hydrochloride) (1.91 g, $10.0 \mathrm{mmol}, 2.00$ equiv) and 2-(trimethylsilyl)ethan-1-ol (708 $\mathrm{mg}, 6.00 \mathrm{mmol}, 1.20$ equiv). The reaction mixture was stirred at room temperature for $6 \mathrm{~h}$ before quenched with $\mathrm{H}_{2} \mathrm{O}(30.0 \mathrm{~mL})$ and extracted 3 times with $\mathrm{CH}_{2} \mathrm{Cl}_{2}(30.0 \mathrm{~mL})$. The combined organic layer was dried over $\mathrm{MgSO}_{4}$. The filtrate was concentrated in vacuo and the residue was purified by chromatography on silica gel, eluting with hexanes/EtOAc 20:1 $(\mathrm{v} / \mathrm{v}) \quad$ to afford $1.03 \quad \mathrm{~g}$ 2-(trimethylsilyl)ethyl 4-(prop-2-yn-1-yloxy)benzoate (1v) as a colorless liquid (74\% yield).

$\mathrm{R}_{f}=0.25$ (hexanes/EtOAc 40:1 (v/v)). NMR Spectroscopy: ${ }^{1} \mathrm{H}$ NMR $(400 \mathrm{MHz}, \mathrm{CDCl} 3) \delta 8.03-$ $7.98(\mathrm{~m}, 2 \mathrm{H}), 7.03-6.96(\mathrm{~m}, 2 \mathrm{H}), 4.74(\mathrm{~d}, \mathrm{~J}=2.4 \mathrm{~Hz}, 2 \mathrm{H}), 4.44-4.34(\mathrm{~m}, 2 \mathrm{H}), 2.55(\mathrm{t}, \mathrm{J}=2.4$ $\mathrm{Hz}, 1 \mathrm{H}), 1.17-1.06(\mathrm{~m}, 2 \mathrm{H}), 0.08$ (s, 9H). ${ }^{13} \mathrm{C} \mathrm{NMR}\left(101 \mathrm{MHz}, \mathrm{CDCl}_{3}\right) \delta$ 166.5, 161.2, 131.6, 124.1, 114.6, 78.0, 76.2, 63.1, 55.9, 17.6, -1.3. Mass Spectrometry: HRMS-ESI (m/z): Calcd for $\mathrm{C}_{15} \mathrm{H}_{20} \mathrm{NaO}_{3} \mathrm{Si}[\mathrm{M}+\mathrm{Na}]^{+}$, 299.1079. Found, 299.1070.

\section{4-Chlorobutyl 4-(prop-2-yn-1-yloxy)benzoate (1w)}

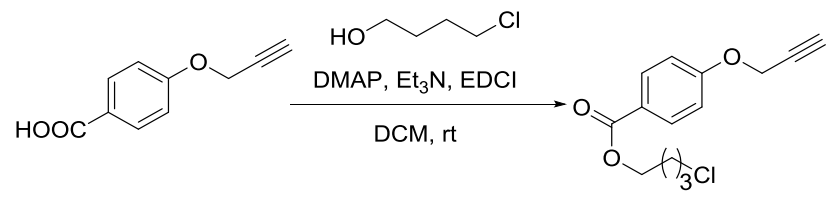

To a solution of 4-(prop-2-yn-1-yloxy)benzoic acid ( $880 \mathrm{mg}, 5.00 \mathrm{mmol}, 1.00$ equiv), DMAP (4-dimethylaminepyridine) $\left(122 \mathrm{mg}, 1.00 \mathrm{mmol}, 0.200\right.$ equiv) and $\mathrm{Et}_{3} \mathrm{~N}(1.51 \mathrm{~g}, 15.0 \mathrm{mmol}, 3.00$ equiv) in $\mathrm{CH}_{2} \mathrm{Cl}_{2}(20.0 \mathrm{~mL})$ at room temperature were added EDCI (1-ethyl-(3-(3-dimethylamino)propyl)-carbodiimide hydrochloride) (1.91 g, $10.0 \mathrm{mmol}, 2.00$ equiv) and 4-chlorobutan-1-ol (650 mg, $6.00 \mathrm{mmol}, 1.20$ equiv). The reaction mixture was stirred at room temperature for $6 \mathrm{~h}$ before quenched with $\mathrm{H}_{2} \mathrm{O}(30.0 \mathrm{~mL})$ and extracted 3 times with $\mathrm{CH}_{2} \mathrm{Cl}_{2}(30.0 \mathrm{~mL})$. The combined organic layer was dried over $\mathrm{MgSO}_{4}$. The filtrate was concentrated in vacuo and the residue was purified by chromatography on silica gel, eluting with hexanes/EtOAc 20:1 (v/v) to afford $492 \mathrm{mg}$ 4-chlorobutyl 4-(prop-2-yn-1-yloxy)benzoate (1w) as a colorless liquid ( $37 \%$ yield).

$\mathrm{R}_{f}=0.09$ (hexanes/EtOAc 40:1 (v/v)). NMR Spectroscopy: ${ }^{1} \mathrm{H}$ NMR $\left(400 \mathrm{MHz}, \mathrm{CDCl}_{3}\right) \delta 8.04-$ $7.96(\mathrm{~m}, 2 \mathrm{H}), 7.03-6.97(\mathrm{~m}, 2 \mathrm{H}), 4.75(\mathrm{~d}, J=2.4 \mathrm{~Hz}, 2 \mathrm{H}), 4.33$ (t, $J=5.9 \mathrm{~Hz}, 2 \mathrm{H}), 3.61$ (t, $J=$ $6.2 \mathrm{~Hz}, 2 \mathrm{H}), 2.55(\mathrm{t}, J=2.4 \mathrm{~Hz}, 1 \mathrm{H}), 1.98-1.86(\mathrm{~m}, 4 \mathrm{H}) .{ }^{13} \mathrm{C} \mathrm{NMR}\left(101 \mathrm{MHz}, \mathrm{CDCl}_{3}\right) \delta 166.3$, $161.3,131.7,123.6,114.7,77.9,76.2,64.0,56.0,44.7,29.4,26.3$. Mass Spectrometry: HRMS-ESI (m/z): Calcd for $\mathrm{C}_{14} \mathrm{H}_{16} \mathrm{ClO}_{3}[\mathrm{M}+\mathrm{H}]^{+}$, 267.0782. Found, 267.0782. 


\section{$N$-(hex-5-yn-1-yl)-Saccharin (1x)}

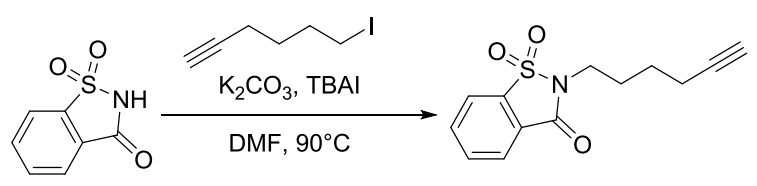

To a round-bottom flask were added Saccharin $\left(915 \mathrm{mg}, 5.00 \mathrm{mmol}, 1.00\right.$ equiv), $\mathrm{K}_{2} \mathrm{CO}_{3}(828 \mathrm{mg}$, $6.00 \mathrm{mmol}, 1.20$ equiv), TBAI ( $370 \mathrm{mg}, 1.00 \mathrm{mmol}, 1.00$ equiv) and DMF (5.00 mL). To this solution was added 6-iodohex-1-yne (1.56 g, $7.50 \mathrm{mmol}, 1.50$ equiv), and the mixture was stired at $90{ }^{\circ} \mathrm{C}$ for $16 \mathrm{~h}$. The reaction was cooked to room temperature, quenched with $2 \mathrm{M} \mathrm{HCl} \mathrm{(aq)}(10$ $\mathrm{mL})$, and extracted with EtOAc $(20 \mathrm{~mL}) 3$ times. The combined organic layer were dried over $\mathrm{MgSO}_{4}$, concentrated, and purified by column chromatography, eluting with hexanes/EtOAc 4:1 (v/v) to afford $1.16 \mathrm{~g} N$-(hex-5-yn-1-yl)-Saccharin (1x) as a colorless liquid (88\% yield).

$\mathrm{R}_{f}=0.19$ (hexanes/EtOAc 8:1 (v/v)). NMR Spectroscopy: ${ }^{1} \mathrm{H}$ NMR $\left(400 \mathrm{MHz}, \mathrm{CDCl}_{3}\right) \delta 8.07-$ $8.01(\mathrm{~m}, 1 \mathrm{H}), 7.93-7.89(\mathrm{~m}, 1 \mathrm{H}), 7.89-7.79(\mathrm{~m}, 2 \mathrm{H}), 3.80(\mathrm{t}, J=7.3 \mathrm{~Hz}, 2 \mathrm{H}), 2.26(\mathrm{td}, J=7.0$, $2.6 \mathrm{~Hz}, 2 \mathrm{H}), 2.02-1.91(\mathrm{~m}, 3 \mathrm{H}), 1.70-1.60(\mathrm{~m}, 2 \mathrm{H}) .{ }^{13} \mathrm{C} \mathrm{NMR}\left(101 \mathrm{MHz}, \mathrm{CDCl}_{3}\right) \delta 159.1$, 137.8, 134.8, 134.4, 127.5, 125.3, 121.0, 83.7, 69.1, 39.0, 27.6, 25.7, 18.0. Mass Spectrometry: HRMS-ESI (m/z): Calcd for $\mathrm{C}_{13} \mathrm{H}_{14} \mathrm{NO}_{3} \mathrm{~S}[\mathrm{M}+\mathrm{H}]^{+}, 264.0694$. Found, 264.0686.

\section{$N$-butyl- $N$-(hex-5-yn-1-yl)benzenesulfonamide (1y)}

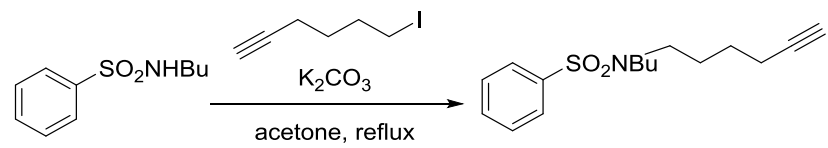

Under $\mathrm{N}_{2}$ atmosphere, to a solution of $N$-butylbenzenesulfonamide ${ }^{[3]}(2.13 \mathrm{~g}, 10.0 \mathrm{mmol}$, 1.00 equiv) and 6-iodohex-1-yne ${ }^{[4]}(4.16 \mathrm{~g}, 20.0 \mathrm{mmol}, 2.00$ equiv) in acetone $(50.0 \mathrm{~mL})$ were added $\mathrm{K}_{2} \mathrm{CO}_{3}(2.76 \mathrm{~g}, 20.0 \mathrm{mmol}, 2.00$ equiv) at room temperature. The reaction mixture was then refluxed for $12 \mathrm{~h}$. After cooling to room temperature, the reaction mixture was filtered through a pad of Celite and concentrated in vacuo. The residue was purified by chromatography on silica gel, eluting with hexanes/EtOAc 8:1 (v/v) to afford $1.38 \mathrm{~g}$ $N$-butyl- $N$-(hex-5-yn-1-yl)benzenesulfonamide (1y) as a colorless liquid (47\% yield).

$\mathrm{R}_{f}=0.45$ (hexanes/EtOAc 8:1 (v/v)). NMR Spectroscopy: ${ }^{1} \mathrm{H}$ NMR (400 MHz, $\left.\mathrm{CDCl}_{3}\right) \delta 7.84$ $-7.75(\mathrm{~m}, 2 \mathrm{H}), 7.58-7.52(\mathrm{~m}, 1 \mathrm{H}), 7.52-7.46(\mathrm{~m}, 2 \mathrm{H}), 3.17-3.08(\mathrm{~m}, 4 \mathrm{H}), 2.20(\mathrm{td}, J=6.9$, $2.6 \mathrm{~Hz}, 2 \mathrm{H}), 1.93(\mathrm{t}, J=2.6 \mathrm{~Hz}, 1 \mathrm{H}), 1.70-1.60(\mathrm{~m}, 2 \mathrm{H}), 1.57-1.44(\mathrm{~m}, 4 \mathrm{H}), 1.33-1.23(\mathrm{~m}$, $2 \mathrm{H}), 0.88(\mathrm{t}, J=7.3 \mathrm{~Hz}, 3 \mathrm{H}) .{ }^{13} \mathrm{C}$ NMR $\left(101 \mathrm{MHz}, \mathrm{CDCl}_{3}\right) \delta 140.1,132.4,129.1,127.2,84.0$, 68.9, 48.2, 47.8, 30.8, 27.7, 25.5, 20.1, 18.1, 13.8. Mass Spectrometry: HRMS-ESI (m/z): Calcd for $\mathrm{C}_{16} \mathrm{H}_{24} \mathrm{NO}_{2} \mathrm{~S}[\mathrm{M}+\mathrm{H}]^{+}$, 294.1522. Found, 294.1518.

\section{Hex-5-yn-1-yl 1-naphthoate (1z)}

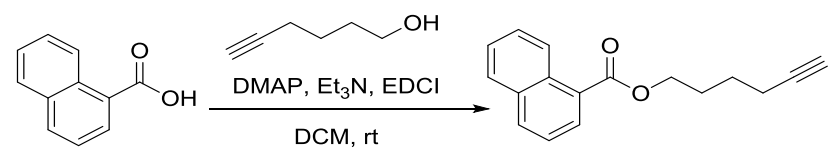

To a solution of 1-naphthoic acid $(1.72 \mathrm{~g}, 10.0 \mathrm{mmol}, 1.00$ equiv), DMAP (4-dimethylaminepyridine) (244 mg, $2.00 \mathrm{mmol}, 0.200$ equiv) and $\mathrm{Et}_{3} \mathrm{~N}(3.03 \mathrm{~g}, 30.0 \mathrm{mmol}, 3.00$ 
equiv) in $\mathrm{CH}_{2} \mathrm{Cl}_{2}(50.0 \mathrm{~mL})$ at room temperature were added EDCI (1-ethyl-(3-(3-dimethylamino)propyl)-carbodiimide hydrochloride) (3.83 g, $20.0 \mathrm{mmol}, 2.00$ equiv) and hex-5-yn-1-ol (1.18 g, $12.0 \mathrm{mmol}, 1.20$ equiv). The reaction mixture was stirred at room temperature for $6 \mathrm{~h}$ before quenched with $\mathrm{H}_{2} \mathrm{O}(30.0 \mathrm{~mL})$ and extracted 3 times with $\mathrm{CH}_{2} \mathrm{Cl}_{2}$ $(30.0 \mathrm{~mL})$. The combined organic layer was dried over $\mathrm{MgSO}_{4}$. The filtrate was concentrated in vacuo and the residue was purified by chromatography on silica gel, eluting with hexanes/EtOAc 20:1 (v/v) to afford $2.01 \mathrm{~g}$ hex-5-yn-1-yl 1-naphthoate (1z) as a colorless liquid (80\% yield).

$\mathrm{R}_{f}=0.25$ (hexanes/EtOAc 40:1 (v/v)). NMR Spectroscopy: ${ }^{1} \mathrm{H}$ NMR (400 MHz, $\left.\mathrm{CDCl}_{3}\right) \delta 8.92(\mathrm{~d}$, $J=8.7 \mathrm{~Hz}, 1 \mathrm{H}), 8.19(\mathrm{dd}, J=7.3,1.3 \mathrm{~Hz}, 1 \mathrm{H}), 8.02(\mathrm{~d}, J=8.2 \mathrm{~Hz}, 1 \mathrm{H}), 7.89(\mathrm{~d}, J=8.1 \mathrm{~Hz}, 1 \mathrm{H})$, $7.65-7.59$ (m, 1H), $7.57-7.47$ (m, 2H), 4.45 (t, $J=6.5 \mathrm{~Hz}, 2 \mathrm{H}), 2.31$ (td, $J=7.0,2.6 \mathrm{~Hz}, 2 \mathrm{H}$ ), $2.01-1.93(\mathrm{~m}, 3 \mathrm{H}), 1.70-1.70(\mathrm{~m}, 2 \mathrm{H}) .{ }^{13} \mathrm{C} \mathrm{NMR}\left(101 \mathrm{MHz}, \mathrm{CDCl}_{3}\right) \delta$ 167.7, 134.0, 133.4, 131.5, 130.2, 128.7, 127.9, 127.4, 126.3, 125.9, 124.6, 84.0, 69.0, 64.7, 28.0, 25.3, 18.3. Mass Spectrometry: HRMS-ESI (m/z): Calcd for $\mathrm{C}_{17} \mathrm{H}_{17} \mathrm{O}_{2}[\mathrm{M}+\mathrm{H}]^{+}$, 253.1229. Found, 253.1218.

\section{Hex-5-yn-1-yl benzofuran-2-carboxylate (1aa)}

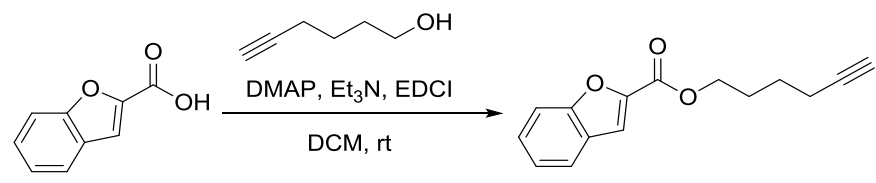

To a solution of benzofuran-2-carboxylic acid (1.62 g, $10.0 \mathrm{mmol}, 1.00$ equiv), DMAP (4-dimethylaminepyridine) (244 mg, $2.00 \mathrm{mmol}, 0.200$ equiv) and $\mathrm{Et}_{3} \mathrm{~N}(3.03 \mathrm{~g}, 30.0 \mathrm{mmol}, 3.00$ equiv) in $\mathrm{CH}_{2} \mathrm{Cl}_{2}(50.0 \mathrm{~mL})$ at room temperature were added EDCI (1-ethyl-(3-(3-dimethylamino)propyl)-carbodiimide hydrochloride) (3.83 g, $20.0 \mathrm{mmol}, 2.00$ equiv) and hex-5-yn-1-ol (1.18 g, $12.0 \mathrm{mmol}, 1.20$ equiv). The reaction mixture was stirred at room temperature for $6 \mathrm{~h}$ before quenched with $\mathrm{H}_{2} \mathrm{O}(30.0 \mathrm{~mL})$ and extracted 3 times with $\mathrm{CH}_{2} \mathrm{Cl}_{2}$ $(30.0 \mathrm{~mL})$. The combined organic layer was dried over $\mathrm{MgSO}_{4}$. The filtrate was concentrated in vacuo and the residue was purified by chromatography on silica gel, eluting with hexanes/EtOAc 30:1 (v/v) to afford $1.38 \mathrm{~g}$ hex-5-yn-1-yl benzofuran-2-carboxylate (1aa) as a colourless liquid (57\% yield).

$\mathrm{R}_{f}=0.14$ (hexanes/EtOAc 40:1 (v/v)). NMR Spectroscopy: ${ }^{1} \mathrm{H}$ NMR $\left(400 \mathrm{MHz}, \mathrm{CDCl}_{3}\right) \delta 7.68(\mathrm{~d}$, $J=7.8 \mathrm{~Hz}, 1 \mathrm{H}), 7.59$ (d, $J=8.4 \mathrm{~Hz}, 1 \mathrm{H}), 7.53(\mathrm{~s}, 1 \mathrm{H}), 7.48-7.42(\mathrm{~m}, 1 \mathrm{H}), 7.30$ (t, $J=7.4 \mathrm{~Hz}$, $1 \mathrm{H}), 4.41(\mathrm{t}, J=6.5 \mathrm{~Hz}, 2 \mathrm{H}), 2.29$ (td, $J=7.0,2.6 \mathrm{~Hz}, 2 \mathrm{H}), 1.98(\mathrm{t}, J=2.6 \mathrm{~Hz}, 1 \mathrm{H}), 1.97-1.89$ (m, 2H), $1.76-1.65(\mathrm{~m}, 2 \mathrm{H}) .{ }^{13} \mathrm{C}$ NMR $\left(101 \mathrm{MHz}, \mathrm{CDCl}_{3}\right) \delta 159.7,155.9,145.7,127.7,127.1$, 123.9, 122.9, 114.0, 112.5, 83.9, 69.0, 65.1, 27.8, 25.0, 18.2. Mass Spectrometry: HRMS-ESI $(\mathrm{m} / \mathrm{z})$ : Calcd for $\mathrm{C}_{15} \mathrm{H}_{14} \mathrm{NaO}_{3}[\mathrm{M}+\mathrm{Na}]^{+}$, 265.0841. Found, 265.0833 .

\section{Hex-5-yn-1-yl 5-bromothiophene-2-carboxylate (1bb)}

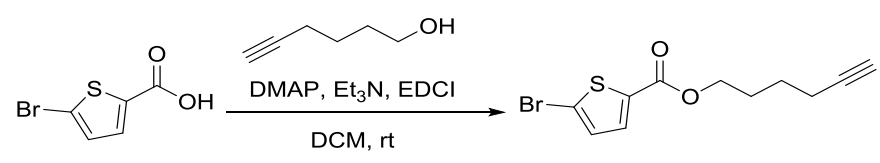

To a solution of 5-bromothiophene-2-carboxylic acid (2.06 g, $10.0 \mathrm{mmol}, 1.00$ equiv), DMAP (4-dimethylaminepyridine) (244 mg, $2.00 \mathrm{mmol}, 0.200$ equiv) and $\mathrm{Et}_{3} \mathrm{~N}(3.03 \mathrm{~g}, 30.0 \mathrm{mmol}, 3.00$ 
equiv) in $\mathrm{CH}_{2} \mathrm{Cl}_{2} \quad(50.0 \mathrm{~mL})$ at room temperature were added EDCI (1-ethyl-(3-(3-dimethylamino)propyl)-carbodiimide hydrochloride) (3.83 g, $20.0 \mathrm{mmol}, 2.00$ equiv) and hex-5-yn-1-ol (1.18 g, $12.0 \mathrm{mmol}, 1.20$ equiv). The reaction mixture was stirred at room temperature for $6 \mathrm{~h}$ before quenched with $\mathrm{H}_{2} \mathrm{O}(30.0 \mathrm{~mL})$ and extracted 3 times with $\mathrm{CH}_{2} \mathrm{Cl}_{2}$ $(30.0 \mathrm{~mL})$. The combined organic layer was dried over $\mathrm{MgSO}_{4}$. The filtrate was concentrated in vacuo and the residue was purified by chromatography on silica gel, eluting with hexanes/EtOAc 20:1 (v/v) to afford $2.01 \mathrm{~g}$ hex-5-yn-1-yl 5-bromothiophene-2-carboxylate (1bb) as a colourless liquid ( $70 \%$ yield).

$\mathrm{R}_{f}=0.25$ (hexanes/EtOAc 40:1 (v/v)). NMR Spectroscopy: ${ }^{1} \mathrm{H}$ NMR $\left(400 \mathrm{MHz}, \mathrm{CDCl}_{3}\right) \delta 7.54(\mathrm{~d}$, $J=4.0 \mathrm{~Hz}, 1 \mathrm{H}), 7.06$ (d, $J=4.0 \mathrm{~Hz}, 1 \mathrm{H}), 4.30$ (t, $J=6.4 \mathrm{~Hz}, 2 \mathrm{H}), 2.26$ (td, $J=7.0,2.6 \mathrm{~Hz}, 2 \mathrm{H})$, $1.97(\mathrm{t}, J=2.6 \mathrm{~Hz}, 1 \mathrm{H}), 1.93-1.79(\mathrm{~m}, 2 \mathrm{H}), 1.70-1.60(\mathrm{~m}, 2 \mathrm{H}) .{ }^{13} \mathrm{C} \mathrm{NMR}\left(101 \mathrm{MHz}, \mathrm{CDCl}_{3}\right) \delta$ 161.2, 135.0, 133.7, 131.0, 120.3, 83.9, 69.0, 65.0, 27.8, 25.0, 18.2. Mass Spectrometry: HRMS-ESI (m/z): Calcd for $\mathrm{C}_{11} \mathrm{H}_{12} \mathrm{BrO}_{2} \mathrm{~S}[\mathrm{M}+\mathrm{H}]^{+}$, 286.9741. Found, 286.9732.

\section{Hex-5-yn-1-yl quinoline-2-carboxylate (1cc)}

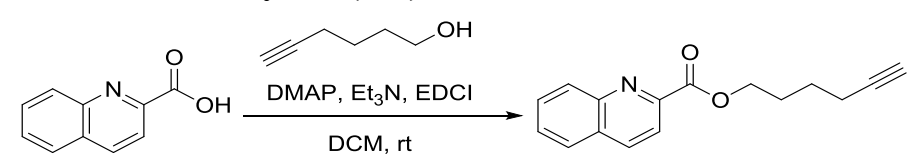

To a solution of quinoline-2-carboxylic acid (1.73 g , $10.0 \mathrm{mmol}, 1.00 \mathrm{equiv})$, DMAP (4-dimethylaminepyridine) (244 mg, $2.00 \mathrm{mmol}, 0.200$ equiv) and $\mathrm{Et}_{3} \mathrm{~N}(3.03 \mathrm{~g}, 30.0 \mathrm{mmol}, 3.00$ equiv) in $\mathrm{CH}_{2} \mathrm{Cl}_{2}(50.0 \mathrm{~mL})$ at room temperature were added EDCI (1-ethyl-(3-(3-dimethylamino)propyl)-carbodiimide hydrochloride) (3.83 g , $20.0 \mathrm{mmol}, 2.00$ equiv) and hex-5-yn-1-ol (1.18 g, $12.0 \mathrm{mmol}, 1.20$ equiv). The reaction mixture was stirred at room temperature for $6 \mathrm{~h}$ before quenched with $\mathrm{H}_{2} \mathrm{O}(30.0 \mathrm{~mL})$ and extracted 3 times with $\mathrm{CH}_{2} \mathrm{Cl}_{2}$ $(30.0 \mathrm{~mL})$. The combined organic layer was dried over $\mathrm{MgSO}_{4}$. The filtrate was concentrated in vacuo and the residue was purified by chromatography on silica gel, eluting with hexanes/EtOAc $6: 1(\mathrm{v} / \mathrm{v})$ to afford $1.42 \mathrm{~g}$ hex-5-yn-1-yl quinoline-2-carboxylate (1cc) as a colourless liquid (56\% yield).

$\mathrm{R}_{f}=0.20$ (hexanes/EtOAc 8:1 (v/v)). NMR Spectroscopy: ${ }^{1} \mathrm{H}$ NMR $\left(400 \mathrm{MHz}, \mathrm{CDCl}_{3}\right) \delta 8.32-$ $8.26(\mathrm{~m}, 2 \mathrm{H}), 8.15(\mathrm{~d}, J=8.5 \mathrm{~Hz}, 1 \mathrm{H}), 7.88-7.84(\mathrm{~m}, 1 \mathrm{H}), 7.79-7.74(\mathrm{~m}, 1 \mathrm{H}), 7.65-7.60(\mathrm{~m}$, $1 \mathrm{H}), 4.50(\mathrm{t}, J=6.7 \mathrm{~Hz}, 2 \mathrm{H}), 2.29(\mathrm{td}, J=7.0,2.6 \mathrm{~Hz}, 2 \mathrm{H}), 2.04-1.93(\mathrm{~m}, 3 \mathrm{H}), 1.75-1.67(\mathrm{~m}$, 2H). ${ }^{13} \mathrm{C}$ NMR $\left(101 \mathrm{MHz}, \mathrm{CDCl}_{3}\right) \delta 165.4,148.2,147.7,137.3,130.9,130.3,129.4,128.7,127.6$, 121.1, 83.9, 69.0, 65.7, 27.9, 25.0, 18.2. Mass Spectrometry: HRMS-ESI (m/z): Calcd for $\mathrm{C}_{16} \mathrm{H}_{16} \mathrm{NO}_{2}[\mathrm{M}+\mathrm{H}]^{+}, 254.1181$. Found, 254.1172.

\section{Hex-5-yn-1-yl 6-fluoropicolinate (1dd)}

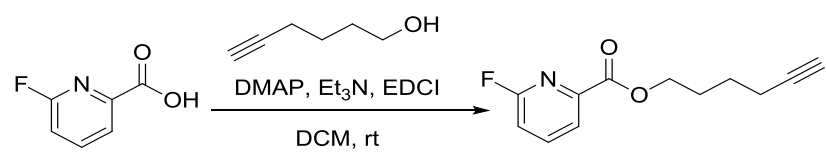

To a solution of 6-fluoropicolinic acid (1.44 g, $10.0 \mathrm{mmol}, 1.00$ equiv), DMAP 
(4-dimethylaminepyridine) (244 mg, $2.00 \mathrm{mmol}, 0.200$ equiv) and $\mathrm{Et}_{3} \mathrm{~N}(3.03 \mathrm{~g}, 30.0 \mathrm{mmol}, 3.00$ equiv) in $\mathrm{CH}_{2} \mathrm{Cl}_{2}(50.0 \mathrm{~mL})$ at room temperature were added EDCI (1-ethyl-(3-(3-dimethylamino)propyl)-carbodiimide hydrochloride) (3.83 g, $20.0 \mathrm{mmol}, 2.00$ equiv) and hex-5-yn-1-ol (1.18 g, $12.0 \mathrm{mmol}, 1.20$ equiv). The reaction mixture was stirred at room temperature for $6 \mathrm{~h}$ before quenched with $\mathrm{H}_{2} \mathrm{O}(30.0 \mathrm{~mL})$ and extracted 3 times with $\mathrm{CH}_{2} \mathrm{Cl}_{2}$ $(30.0 \mathrm{~mL})$. The combined organic layer was dried over $\mathrm{MgSO}_{4}$. The filtrate was concentrated in vacuo and the residue was purified by chromatography on silica gel, eluting with hexanes/EtOAc 6:1 (v/v) to afford $1.13 \mathrm{~g}$ hex-5-yn-1-yl 6-fluoropicolinate (1dd) as a colorless liquid (51\% yield). $\mathrm{R}_{f}=0.26$ (hexanes/EtOAc 8:1 (v/v)). NMR Spectroscopy: ${ }^{1} \mathrm{H}$ NMR (400 MHz, $\left.\mathrm{CDCl}_{3}\right) \delta 8.01$ (ddd, $J=7.4,2.2,0.8 \mathrm{~Hz}, 1 \mathrm{H}), 7.94(\mathrm{dd}, J=15.5,7.6 \mathrm{~Hz}, 1 \mathrm{H}), 7.14$ (ddd, $J=8.0,2.9,0.8 \mathrm{~Hz}, 1 \mathrm{H}$ ), $4.42(\mathrm{t}, J=6.6 \mathrm{~Hz}, 2 \mathrm{H}), 2.26(\mathrm{td}, J=7.0,2.6 \mathrm{~Hz}, 2 \mathrm{H}), 1.99-1.89(\mathrm{~m}, 3 \mathrm{H}), 1.72-1.63(\mathrm{~m}, 2 \mathrm{H})$. ${ }^{13} \mathrm{C}$ NMR $\left(101 \mathrm{MHz}, \mathrm{CDCl}_{3}\right) \delta 164.0,163.0(\mathrm{~d}, J=242.7 \mathrm{~Hz}), 146.6(\mathrm{~d}, J=12.7 \mathrm{~Hz}), 142.2(\mathrm{~d}, J$ $=7.6 \mathrm{~Hz}), 122.8(\mathrm{~d}, J=3.8 \mathrm{~Hz}), 114.0(\mathrm{~d}, J=36.9 \mathrm{~Hz}), 83.9,69.0,65.8,27.8,25.0,18.2 .{ }^{19} \mathrm{~F}$ NMR (376 MHz, $\left.\mathrm{CDCl}_{3}\right) \delta-64.40--64.60(\mathrm{~m}, 1 \mathrm{~F})$. Mass Spectrometry: HRMS-ESI (m/z): Calcd for $\mathrm{C}_{12} \mathrm{H}_{13} \mathrm{FNO}_{2}[\mathrm{M}+\mathrm{H}]^{+}$, 222.0930. Found, 222.0920.

\section{Celecoxib derivative(11I)}

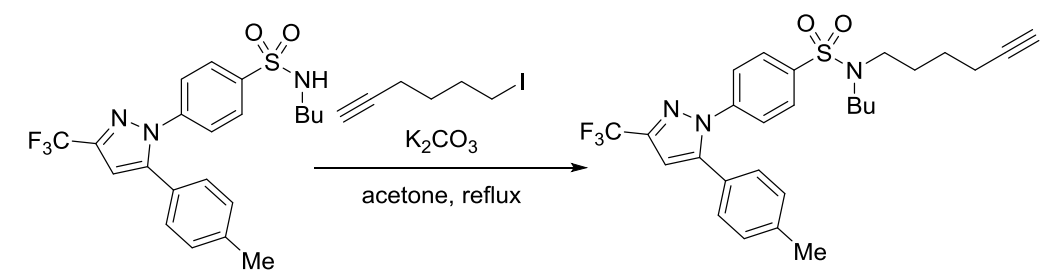

Under $\mathrm{N}_{2}$ atmosphere, to a solution ofcelecoxib derivative ${ }^{[5]}(2.19 \mathrm{~g}, 5.00 \mathrm{mmol}, 1.00$ equiv) in acetone $(10.0 \mathrm{~mL})$ were added $\mathrm{K}_{2} \mathrm{CO}_{3}(1.38 \mathrm{~g}, 10.0 \mathrm{mmol}, 2.00$ equiv) and 6-iodohex-1-yne (2.08 $\mathrm{g}, 10.0 \mathrm{mmol}, 2.00$ equiv) at room temperature. The reaction mixture was then refluxed for $12 \mathrm{~h}$. After cooling to room temperature, the reaction mixture was filtered through a pad of Celite and concentrated in vacuo. The residue was purified by chromatography on silica gel, eluting with hexanes/EtOAc 8:1 (v/v) to afford $1.34 \mathrm{~g}$ celecoxib derivative (1II) as a white solid (52\% yield). $\mathrm{R}_{f}=0.31$ (hexanes/EtOAc 10:1 (v/v)). NMR Spectroscopy: ${ }^{1} \mathrm{H}$ NMR $\left(400 \mathrm{MHz}, \mathrm{CDCl}_{3}\right) \delta 7.78(\mathrm{~d}$, $J=8.7 \mathrm{~Hz}, 2 \mathrm{H}), 7.45$ (d, $J=8.7 \mathrm{~Hz}, 2 \mathrm{H}), 7.16(\mathrm{~d}, J=8.0 \mathrm{~Hz}, 2 \mathrm{H}), 7.09$ (d, $J=8.1 \mathrm{~Hz}, 2 \mathrm{H}), 6.73$ (s, 1H), $3.18-3.05$ (m, 4H), 2.37 (s, 3H), 2.20 (td, $J=6.8,2.6 \mathrm{~Hz}, 2 \mathrm{H}), 1.94$ (t, $J=2.6 \mathrm{~Hz}, 1 \mathrm{H})$, $1.69-1.59(\mathrm{~m}, 2 \mathrm{H}), 1.57-1.42(\mathrm{~m}, 4 \mathrm{H}), 1.33-1.23(\mathrm{~m}, 2 \mathrm{H}), 0.89(\mathrm{t}, J=7.3 \mathrm{~Hz}, 3 \mathrm{H}) .{ }^{13} \mathrm{C}$ NMR $\left(101 \mathrm{MHz}, \mathrm{CDCl}_{3}\right) \delta 145.3,144.1(\mathrm{q}, J=38.3 \mathrm{~Hz}), 142.3,139.9,139.7,129.8,128.8,128.1,125.8$, 125.6, $121.2(\mathrm{q}, J=269.1 \mathrm{~Hz}), 106.3,83.8,69.0,48.0,47.6,30.6,27.5,25.4,21.4,20.0,18.0$, 13.7. ${ }^{19} \mathrm{~F}$ NMR (376 MHz, $\left.\mathrm{CDCl}_{3}\right) \delta-62.92$ (s, 3F). Mass Spectrometry: HRMS-ESI (m/z): Calcd for $\mathrm{C}_{27} \mathrm{H}_{31} \mathrm{~F}_{3} \mathrm{~N}_{3} \mathrm{O}_{2} \mathrm{~S}[\mathrm{M}+\mathrm{H}]^{+}$, 518.2089. Found, 518.2080. 


\section{Podophyllotoxin derivative (1 $\mathrm{mm})$}

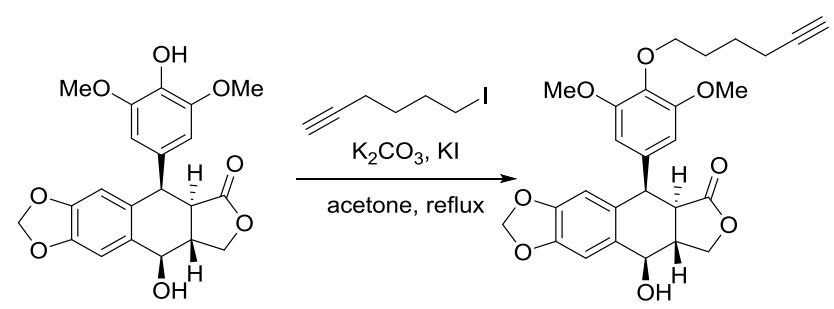

Under $\mathrm{N}_{2}$ atmosphere, to a solution of 4'-deMethylpodophyllotoxin $(2.00 \mathrm{~g}, 5.00 \mathrm{mmol}, 1.00$ equiv) in acetone ( $50.0 \mathrm{~mL}$ ) were added $\mathrm{K}_{2} \mathrm{CO}_{3}(1.38 \mathrm{~g}, 10.0 \mathrm{mmol}, 2.00$ equiv), $\mathrm{KI}$ ( $830 \mathrm{mg}, 5.00$ mmol, 1.00 equiv) and 6-iodohex-1-yne ( $1.54 \mathrm{~g}, 7.50 \mathrm{mmol}, 1.50$ equiv) at room temperature. The reaction mixture was then refluxed for $10 \mathrm{~h}$. After cooling to room temperature, the reaction mixture was filtered through a pad of Celite and concentrated in vacuo. The residue was purified by chromatography on silica gel, eluting with hexanes/EtOAc $1: 1(\mathrm{v} / \mathrm{v})$ to afford $2.31 \mathrm{~g}$ podophyllotoxin derivative (1 $\mathbf{m m}$ ') as a white solid (96\% yield).

$\mathrm{R}_{f}=0.30$ (hexanes/EtOAc 1:1 (v/v)). NMR Spectroscopy: ${ }^{1} \mathrm{H}$ NMR $\left(400 \mathrm{MHz}, \mathrm{CDCl}_{3}\right) \delta 6.98(\mathrm{~s}$, $1 \mathrm{H}), 6.56(\mathrm{~s}, 1 \mathrm{H}), 6.31(\mathrm{~s}, 2 \mathrm{H}), 5.93(\mathrm{~d}, J=12.1 \mathrm{~Hz}, 2 \mathrm{H}), 4.78-4.72(\mathrm{~m}, 1 \mathrm{H}), 4.39$ (d, $J=2.8 \mathrm{~Hz}$, $1 \mathrm{H}), 4.33-4.23(\mathrm{~m}, 2 \mathrm{H}), 3.93$ (t, $J=6.2 \mathrm{~Hz}, 2 \mathrm{H}), 3.72(\mathrm{~s}, 6 \mathrm{H}), 3.40(\mathrm{dd}, J=10.6,3.1 \mathrm{~Hz}, 1 \mathrm{H})$, $3.16-3.05(\mathrm{~m}, 1 \mathrm{H}), 2.77(\mathrm{~s}, 1 \mathrm{H}), 2.24(\mathrm{td}, J=6.9,2.3 \mathrm{~Hz}, 2 \mathrm{H}), 1.96-1.91(\mathrm{~m}, 1 \mathrm{H}), 1.86-1.77$ (m, 2H), $1.76-1.64(\mathrm{~m}, 2 \mathrm{H}) .{ }^{13} \mathrm{C}$ NMR $\left(101 \mathrm{MHz}, \mathrm{CDCl}_{3}\right) \delta$ 179.4, 153.6, 147.4, 147.1, 137.5, 136.0, 131.5, 130.0, 109.7, 106.4, 105.0, 101.2, 84.6, 72.7, 68.5, 68.3, 67.6, 56.3, 45.3, 44.5, 39.5, 29.1, 24.9, 18.1. Mass Spectrometry: HRMS-ESI (m/z): Calcd for $\mathrm{C}_{27} \mathrm{H}_{32} \mathrm{NO}_{8}\left[\mathrm{M}+\mathrm{NH}_{4}\right]^{+}$, 498.2122. Found, 498.2122.

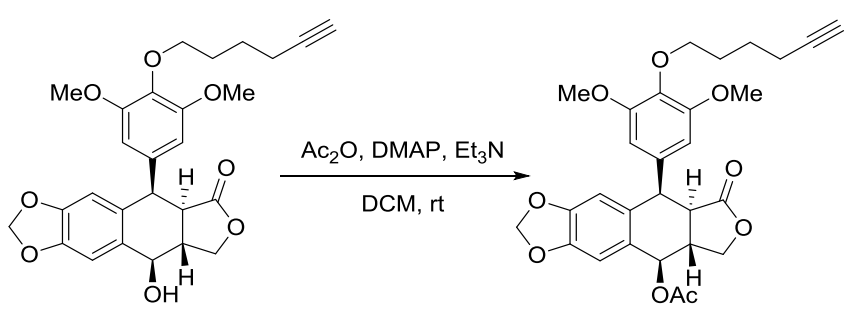

To a solution of podophyllotoxin derivative (1 mm') (960 mg, $2.00 \mathrm{mmol}, 1.00$ equiv), DMAP (4-dimethylaminepyridine) (24.4 mg, $0.200 \mathrm{mmol}, 0.100$ equiv) and $\mathrm{Et}_{3} \mathrm{~N}(303 \mathrm{mg}, 3.00 \mathrm{mmol}$, 1.50 equiv) in $\mathrm{CH}_{2} \mathrm{Cl}_{2}(20.0 \mathrm{~mL})$ at $0{ }^{\circ} \mathrm{C}$ were added dropwise $\mathrm{Ac}_{2} \mathrm{O}(255 \mathrm{mg}, 2.50 \mathrm{mmol}, 1.25$ equiv). The reaction mixture was stirred at room temperature for $10 \mathrm{~h}$ before quenched with $\mathrm{H}_{2} \mathrm{O}$ $(20.0 \mathrm{~mL})$ and extracted 3 times with $\mathrm{CH}_{2} \mathrm{Cl}_{2}(20.0 \mathrm{~mL})$. The combined organic layer was dried over $\mathrm{MgSO}_{4}$. The filtrate was concentrated in vacuo and the residue was purified by chromatography on silica gel, eluting with hexanes/EtOAc $2: 1(\mathrm{v} / \mathrm{v})$ to afford $948 \mathrm{mg}$ podophyllotoxin derivative (11I) as a colorless liquid (91\% yield).

$\mathrm{R}_{f}=0.41$ (hexanes/EtOAc 1:1 (v/v)). NMR Spectroscopy: ${ }^{1} \mathrm{H}$ NMR $\left(400 \mathrm{MHz}, \mathrm{CDCl}_{3}\right) \delta 6.83(\mathrm{~s}$, 1H), 6.49 (s, 1H), 6.39 (s, 2H), 5.98 (d, $J=4.5 \mathrm{~Hz}, 1 \mathrm{H}), 5.93$ (dd, $J=11.2,1.3 \mathrm{~Hz}, 2 \mathrm{H}), 4.40-$ $4.33(\mathrm{~m}, 2 \mathrm{H}), 4.23(\mathrm{dd}, J=9.8,3.0 \mathrm{~Hz}, 1 \mathrm{H}), 3.96(\mathrm{t}, J=6.3 \mathrm{~Hz}, 2 \mathrm{H}), 3.78(\mathrm{~s}, 6 \mathrm{H}), 3.38(\mathrm{dd}, J=$ $10.5,4.2 \mathrm{~Hz}, 1 \mathrm{H}), 3.25-3.17$ (m, 1H), 2.26 (td, $J=7.0,2.6 \mathrm{~Hz}, 2 \mathrm{H}), 2.10$ (s, 3H), 1.93 (t, $J=2.6$ $\mathrm{Hz}, 1 \mathrm{H}), 1.89-1.80(\mathrm{~m}, 2 \mathrm{H}), 1.78-1.70(\mathrm{~m}, 2 \mathrm{H}) .{ }^{13} \mathrm{C} \mathrm{NMR}\left(101 \mathrm{MHz}, \mathrm{CDCl}_{3}\right) \delta 178.4,170.3$, $153.8,148.2$, 146.9, 137.4, 136.2, 131.6, 127.2, 109.6, 107.3, 105.4, 101.4, 84.6, 72.6, 70.8, 68.4, 
68.3, 56.3, 45.2, 44.6, 38.1, 29.2, 25.0, 21.0, 18.1. Mass Spectrometry: HRMS-ESI (m/z): Calcd for $\mathrm{C}_{27} \mathrm{H}_{27} \mathrm{O}_{7}[\mathrm{M}+\mathrm{H}]^{+}$, 463.1757. Found, 463.1747 .

\section{Mycophenolic acid derivative (1nn)}

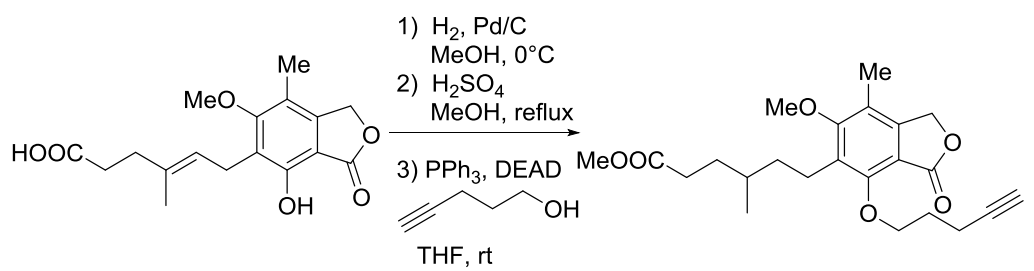

The suspension of mycophenolic acid (1.61 g, $5.00 \mathrm{mmol}, 1.00$ equiv) and $\mathrm{Pd} / \mathrm{C}$ (10\% wt) (400 $\mathrm{mg})$ in $\mathrm{MeOH}(200 \mathrm{~mL})$ was stired at $25^{\circ} \mathrm{C}$ for $9 \mathrm{~h}$ under $\mathrm{H}_{2}$ atmosphere. Then the reaction mixture was filtered through a pad of Celite and concentrated in vacuo to get the crude product.

Then to a solution of this crude product in methanol $(10 \mathrm{~mL})$ was added dropwise concentrated $\mathrm{H}_{2} \mathrm{SO}_{4}(1 \mathrm{~mL})$ under stirring at room temperature and heated under reflux overnight. After completion of the reaction, about $80 \%$ methanol was distilled off. The reaction mixture was diluted with water $(50 \mathrm{~mL})$ and extracted with $\mathrm{CH}_{2} \mathrm{Cl}_{2}(50 \mathrm{~mL})$ twice and the combined organic layer was washed with aqueous sodium bicarbonate $(10 \%, 50 \mathrm{~mL})$ solution followed by water. The organic layer was dried over anhydrous $\mathrm{Na}_{2} \mathrm{SO}_{4}$, and the solvent was removed by reduce pressure to afford crude mycophenolic acid derivative.

To a solution of crude mycophenolic acid derivative in dry THF $(10 \mathrm{~mL})$ under argon, $\mathrm{Ph}_{3} \mathrm{P}(1.57$ $\mathrm{g}, 6.00 \mathrm{mmol}, 1.20$ equiv) and pent-4-yn-1-ol (504 mg, $6.00 \mathrm{mmol}, 1.20$ equiv) were added. The mixture was cooled to $0{ }^{\circ} \mathrm{C}$ and DEAD ( $1.09 \mathrm{~g}, 6.25 \mathrm{mmol}, 1.25$ equiv) was added dropwise. The solution was allowed to stired at room temperature for $18 \mathrm{~h}$. The solvent was evaporated and the residue was taken up in $\mathrm{CH}_{2} \mathrm{Cl}_{2}(30 \mathrm{~mL})$. The resulting suspension was filtrated and washed with $\mathrm{CH}_{2} \mathrm{Cl}_{2}$. The filtrate was concentrated in vacuo and the residue was purified by column chromatography, eluting with hexanes/EtOAc 4:1 (v/v) to afford $1.30 \mathrm{~g}$ mycophenolic acid derivative (1 $\mathbf{m m})$ as a colorless liquid (65\% total yield over 3 steps).

$\mathrm{R}_{f}=0.16$ (hexanes/EtOAc 4:1 (v/v)). NMR Spectroscopy: ${ }^{1} \mathrm{H}$ NMR $\left(400 \mathrm{MHz}, \mathrm{CDCl}_{3}\right) \delta 5.11(\mathrm{~s}$, $2 \mathrm{H}), 4.30(\mathrm{t}, J=6.2 \mathrm{~Hz}, 2 \mathrm{H}), 3.79(\mathrm{~s}, 3 \mathrm{H}), 3.67$ (s, 3H), $2.73-2.60(\mathrm{~m}, 2 \mathrm{H}), 2.46(\mathrm{td}, J=7.1,2.6$ $\mathrm{Hz}, 2 \mathrm{H}), 2.40-2.30(\mathrm{~m}, 2 \mathrm{H}), 2.17(\mathrm{~s}, 3 \mathrm{H}), 2.11-2.03(\mathrm{~m}, 2 \mathrm{H}), 1.96(\mathrm{t}, J=2.6 \mathrm{~Hz}, 1 \mathrm{H}), 1.82-$ $1.71(\mathrm{~m}, 1 \mathrm{H}), 1.57-1.47(\mathrm{~m}, 3 \mathrm{H}), 1.42-1.32(\mathrm{~m}, 1 \mathrm{H}), 0.99(\mathrm{~d}, J=6.1 \mathrm{~Hz}, 3 \mathrm{H}) .{ }^{13} \mathrm{C}$ NMR $(101$ $\left.\mathrm{MHz}, \mathrm{CDCl}_{3}\right) \delta 174.6,169.2,163.0,155.7,146.6,130.5,120.1,112.8,100.1,83.9,73.9,68.9$, 68.4, 61.2, 51.6, 37.5, 33.1, 32.0, 31.8, 29.4, 22.3, 19.3, 15.3, 11.7. Mass Spectrometry: HRMS-ESI (m/z): Calcd for $\mathrm{C}_{23} \mathrm{H}_{31} \mathrm{O}_{6}[\mathrm{M}+\mathrm{H}]^{+}$, 403.2121. Found, 403.2112.

\section{Artesunate derivative (100)}

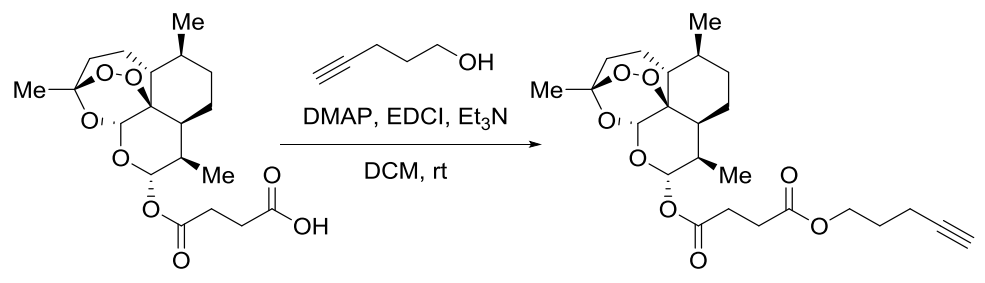


To a solution of Artesunate (1.92 g, $5.00 \mathrm{mmol}, 1.00$ equiv), DMAP (4-dimethylaminepyridine) (122 mg, $1.00 \mathrm{mmol}, 0.200$ equiv) and $\mathrm{Et}_{3} \mathrm{~N}\left(1.51 \mathrm{~g}, 15.0 \mathrm{mmol}, 3.00\right.$ equiv) in $\mathrm{CH}_{2} \mathrm{Cl}_{2}(20.0 \mathrm{~mL})$ at room temperature were added EDCI (1-ethyl-(3-(3-dimethylamino)propyl)-carbodiimide hydrochloride) (1.91 g, $10.0 \mathrm{mmol}, 2.00$ equiv) and pent-4-yn-1-ol (504 mg, $6.00 \mathrm{mmol}, 1.20$ equiv). The reaction mixture was stirred at room temperature for $6 \mathrm{~h}$ before quenched with $\mathrm{H}_{2} \mathrm{O}$ $(30.0 \mathrm{~mL})$ and extracted 3 times with $\mathrm{CH}_{2} \mathrm{Cl}_{2}(30.0 \mathrm{~mL})$. The combined organic layer was dried over $\mathrm{MgSO}_{4}$. The filtrate was concentrated in vacuo and the residue was purified by chromatography on silica gel, eluting with hexanes/EtOAc 4:1 (v/v) to afford $2.01 \mathrm{~g}$ Artesunate derivative (100) as a colorless liquid ( $89 \%$ yield).

$\mathrm{R}_{f}=0.23$ (hexanes/EtOAc 4:1 (v/v)). NMR Spectroscopy: ${ }^{1} \mathrm{H}$ NMR $\left(400 \mathrm{MHz}, \mathrm{CDCl}_{3}\right) \delta 5.74(\mathrm{~d}$, $J=9.8 \mathrm{~Hz}, 1 \mathrm{H}), 5.39(\mathrm{~s}, 1 \mathrm{H}), 4.15(\mathrm{t}, \mathrm{J}=6.3 \mathrm{~Hz}, 2 \mathrm{H}), 2.72-2.66(\mathrm{~m}, 2 \mathrm{H}), 2.66-2.46(\mathrm{~m}, 3 \mathrm{H})$, $2.33(\mathrm{td}, J=14.0,3.8 \mathrm{~Hz}, 1 \mathrm{H}), 2.24(\mathrm{td}, J=7.0,2.4 \mathrm{~Hz}, 2 \mathrm{H}), 2.03-1.99(\mathrm{~m}, 1 \mathrm{H}), 1.94$ (t, $J=2.4$ $\mathrm{Hz}, 1 \mathrm{H}), 1.89-1.77(\mathrm{~m}, 3 \mathrm{H}), 1.77-1.64(\mathrm{~m}, 2 \mathrm{H}), 1.58(\mathrm{dt}, J=13.7,4.2 \mathrm{~Hz}, 1 \mathrm{H}), 1.50-1.40(\mathrm{~m}$, $1 \mathrm{H}), 1.38(\mathrm{~s}, 3 \mathrm{H}), 1.36-1.19(\mathrm{~m}, 3 \mathrm{H}), 1.03-0.94(\mathrm{~m}, 1 \mathrm{H}), 0.92(\mathrm{~d}, J=5.9 \mathrm{~Hz}, 3 \mathrm{H}), 0.81(\mathrm{~d}, J=$ 7.1 Hz, 3H). ${ }^{13} \mathrm{C}$ NMR $\left(101 \mathrm{MHz}, \mathrm{CDCl}_{3}\right) \delta 172.0,171.1,104.4,92.2,91.5,83.0,80.1,69.1,63.3$, $51.5,45.2,37.3,36.2,34.1,31.8,29.2,28.8,27.5,26.0,24.6,22.0,20.2,15.2,12.1$. Mass Spectrometry: HRMS-ESI (m/z): Calcd for $\mathrm{C}_{24} \mathrm{H}_{34} \mathrm{NaO}_{8}[\mathrm{M}+\mathrm{Na}]^{+}$, 473.2146. Found, 473.2141.

\section{Ezetimibe derivative (1pp)}

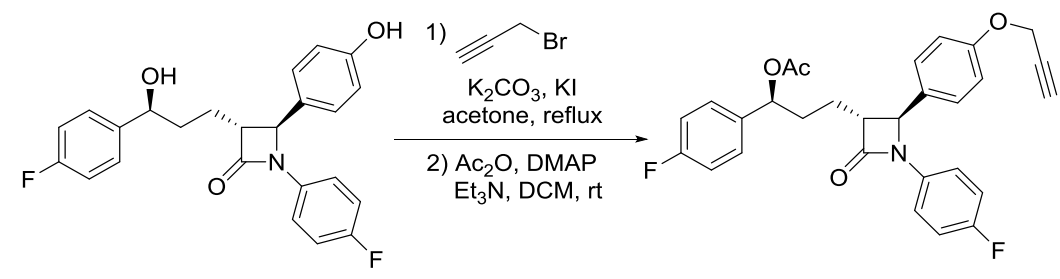

Under $\mathrm{N}_{2}$ atmosphere, to a solution of Ezetimibe ( $4.09 \mathrm{~g}, 10.0 \mathrm{mmol}, 1.00$ equiv) in acetone (50.0 $\mathrm{mL}$ ) were added $\mathrm{K}_{2} \mathrm{CO}_{3}(2.76 \mathrm{~g}, 20.0 \mathrm{mmol}, 2.00$ equiv), $\mathrm{KI}$ (1.66 g, $10.0 \mathrm{mmol}, 1.00$ equiv) and 3-bromoprop-1-yne ( $3.00 \mathrm{~g}, 20.0 \mathrm{mmol}, 2.00$ equiv) at room temperature. The reaction mixture was then refluxed for $12 \mathrm{~h}$. After cooling to room temperature, the reaction mixture was filteredthrough a pad of Celite and concentrated in vacuo.

To a solution of this residue, DMAP (4-dimethylaminepyridine) (122 $\mathrm{mg}, 1.00 \mathrm{mmol}, 0.200$ equiv) and $\mathrm{Et}_{3} \mathrm{~N}\left(1.21 \mathrm{~g}, 12.0 \mathrm{mmol}, 1.20\right.$ equiv) in $\mathrm{CH}_{2} \mathrm{Cl}_{2}(50.0 \mathrm{~mL})$ at $0{ }^{\circ} \mathrm{C}$ were added dropwise $\mathrm{Ac}_{2} \mathrm{O}$ (1.11 g, $11.0 \mathrm{mmol}, 1.10$ equiv). The reaction mixture was stirred at room temperature for $6 \mathrm{~h}$ before quenched with $\mathrm{H}_{2} \mathrm{O}(30.0 \mathrm{~mL})$ and extracted 3 times with $\mathrm{CH}_{2} \mathrm{Cl}_{2}(30.0$ $\mathrm{mL}$ ). The combined organic layer was dried over $\mathrm{MgSO}_{4}$. The filtrate was concentrated in vacuo and the residue was purified by chromatography on silica gel, eluting with hexanes/EtOAc 4:1 (v/v) to afford $3.50 \mathrm{~g}$ Ezetimibe derivative (10o) as a colorless liquid (73\% total yield over 2 steps).

$\mathrm{R}_{f}=0.19$ (hexanes/EtOAc 4:1 (v/v)). NMR Spectroscopy: ${ }^{1} \mathrm{H}$ NMR $\left(400 \mathrm{MHz}, \mathrm{CDCl}_{3}\right) \delta 7.31-$ $7.21(\mathrm{~m}, 6 \mathrm{H}), 7.07-6.98(\mathrm{~m}, 4 \mathrm{H}), 6.97-6.91(\mathrm{~m}, 2 \mathrm{H}), 5.72(\mathrm{t}, J=6.7 \mathrm{~Hz}, 1 \mathrm{H}), 4.71(\mathrm{~d}, J=2.4$ $\mathrm{Hz}, 2 \mathrm{H}), 4.58(\mathrm{~d}, J=2.2 \mathrm{~Hz}, 1 \mathrm{H}), 3.08(\mathrm{td}, J=7.7,2.2 \mathrm{~Hz}, 1 \mathrm{H}), 2.56(\mathrm{t}, J=2.3 \mathrm{~Hz}, 1 \mathrm{H}), 2.10-$ $2.01(\mathrm{~m}, 5 \mathrm{H}), 1.92-1.82(\mathrm{~m}, 2 \mathrm{H}) .{ }^{13} \mathrm{C}$ NMR $\left(101 \mathrm{MHz}, \mathrm{CDCl}_{3}\right) \delta 170.3,167.1,162.6(\mathrm{~d}, J=$ $246.9 \mathrm{~Hz}), 159.1$ (d, $J=243.6 \mathrm{~Hz}), 158.0,135.9$ (d, $J=3.1 \mathrm{~Hz}), 134.0$ (d, $J=2.7 \mathrm{~Hz}), 130.4$, 
$128.4(\mathrm{~d}, J=8.3 \mathrm{~Hz}), 127.3,118.5(\mathrm{~d}, J=7.9 \mathrm{~Hz}), 116.0(\mathrm{~d}, J=22.8 \mathrm{~Hz}), 115.8,115.7(\mathrm{~d}, J=21.5$ $\mathrm{Hz}), 78.4,76.0,75.0,64.0,60.2,56.0,33.7,25.0,21.3 .{ }^{19} \mathrm{~F} \mathrm{NMR}\left(376 \mathrm{MHz}, \mathrm{CDCl}_{3}\right) \delta-113.76-$ $-113.84(\mathrm{~m}, 1 \mathrm{~F}),-117.96--118.03(\mathrm{~m}, 1 \mathrm{~F})$. Mass Spectrometry: HRMS-ESI $(\mathrm{m} / \mathrm{z})$ : Calcd for $\mathrm{C}_{29} \mathrm{H}_{25} \mathrm{~F}_{2} \mathrm{NNaO}_{4}[\mathrm{M}+\mathrm{Na}]^{+}$, 512.1649. Found, 512.1641.

\section{(1S)-(-)-camphanic acid derivative (1qq)}

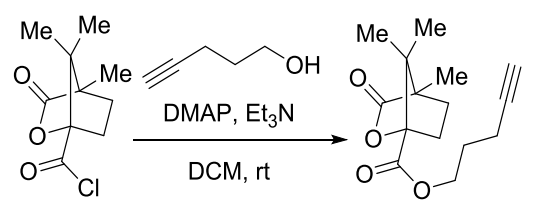

To a solution of (1S)-(-)-camphanic acid chloride (1.08 g, $5.00 \mathrm{mmol}, 1.00$ equiv), DMAP (4-dimethylaminepyridine) (122 mg, $1.00 \mathrm{mmol}, 0.200$ equiv) and $\mathrm{Et}_{3} \mathrm{~N}$ (757 mg, $7.50 \mathrm{mmol}, 1.50$ equiv) in $\mathrm{CH}_{2} \mathrm{Cl}_{2}(20.0 \mathrm{~mL})$ at $0{ }^{\circ} \mathrm{C}$ were added dropwise pent-4-yn-1-ol $(504 \mathrm{mg}, 6.00 \mathrm{mmol}$, 1.20 equiv).The reaction mixture was stirred at room temperature for $4 \mathrm{~h}$ before quenched with $\mathrm{H}_{2} \mathrm{O}(30.0 \mathrm{~mL})$ and extracted 3 times with $\mathrm{CH}_{2} \mathrm{Cl}_{2}(30.0 \mathrm{~mL})$. The combined organic layer was dried over $\mathrm{MgSO}_{4}$. The filtrate was concentrated in vacuo and the residue was purified by chromatography on silica gel, eluting with hexanes/EtOAc 10:1 (v/v) to afford $634 \mathrm{mg}$ (1S)-(-)-camphanic acid derivative (1qq) as a colorless liquid (48\% yield).

$\mathrm{R}_{f}=0.15$ (hexanes/EtOAc 10:1 (v/v)). NMR Spectroscopy: ${ }^{1} \mathrm{H}$ NMR (400 MHz, $\left.\mathrm{CDCl}_{3}\right) \delta 4.41-$ $4.25(\mathrm{~m}, 2 \mathrm{H}), 2.46-2.37(\mathrm{~m}, 1 \mathrm{H}), 2.30(\mathrm{td}, J=7.0,2.6 \mathrm{~Hz}, 2 \mathrm{H}), 2.06-1.98(\mathrm{~m}, 1 \mathrm{H}), 1.97(\mathrm{t}, J=$ $2.7 \mathrm{~Hz}, 1 \mathrm{H}), 1.95-1.86(\mathrm{~m}, 3 \mathrm{H}), 1.72-1.63(\mathrm{~m}, 1 \mathrm{H}), 1.10(\mathrm{~s}, 3 \mathrm{H}), 1.04(\mathrm{~s}, 3 \mathrm{H}), 0.95(\mathrm{~s}, 3 \mathrm{H}) .{ }^{13} \mathrm{C}$ $\operatorname{NMR}\left(101 \mathrm{MHz}, \mathrm{CDCl}_{3}\right) \delta 178.3,167.6,91.2,82.6,69.5,64.1,54.9,54.3,30.7,29.0,27.4,16.8$, 15.2, 9.8. Mass Spectrometry: HRMS-ESI (m/z): Calcd for $\mathrm{C}_{15} \mathrm{H}_{24} \mathrm{NO}_{4}\left[\mathrm{M}+\mathrm{NH}_{4}\right]^{+}, 282.1700$. Found, 282.1704.

\section{Febuxostat derivative (1rr)}

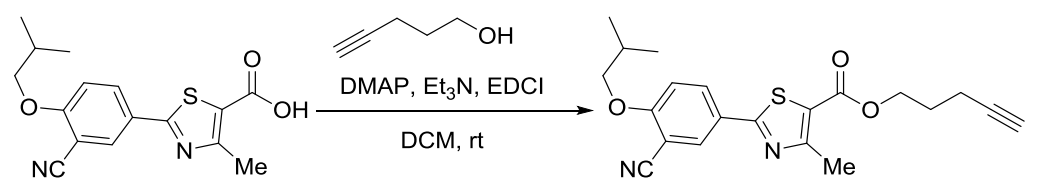

To a solution of Febuxostat (1.58 g, $5.00 \mathrm{mmol}, 1.00$ equiv), DMAP (4-dimethylaminepyridine) (122 mg, $1.00 \mathrm{mmol}, 0.200$ equiv) and $\mathrm{Et}_{3} \mathrm{~N}$ (1.51 g, $15.0 \mathrm{mmol}, 3.00$ equiv) in $\mathrm{CH}_{2} \mathrm{Cl}_{2}(20.0 \mathrm{~mL})$ at room temperature were added EDCI (1-ethyl-(3-(3-dimethylamino)propyl)-carbodiimide hydrochloride) (1.91 g, $10.0 \mathrm{mmol}, 2.00$ equiv) and pent-4-yn-1-ol (504 mg, $6.00 \mathrm{mmol}, 1.20$ equiv). The reaction mixture was stirred at room temperature for $6 \mathrm{~h}$ before quenched with $\mathrm{H}_{2} \mathrm{O}$ (30.0 mL) and extracted 3 times with $\mathrm{CH}_{2} \mathrm{Cl}_{2}(30.0 \mathrm{~mL})$. The combined organic layer was dried over $\mathrm{MgSO}_{4}$. The filtrate was concentrated in vacuo and the residue was purified by chromatography on silica gel, eluting with hexanes/EtOAc 10:1 (v/v) to afford 1.32 g Febuxostat derivative (1rr) as a white solid (69\% yield).

$\mathrm{R}_{f}=0.15$ (hexanes/EtOAc 10:1 (v/v)). NMR Spectroscopy: ${ }^{1} \mathrm{H}$ NMR $\left(400 \mathrm{MHz}, \mathrm{CDCl}_{3}\right) \delta 8.16(\mathrm{~d}$, $J=2.3 \mathrm{~Hz}, 1 \mathrm{H}), 8.08(\mathrm{dd}, J=8.8,2.3 \mathrm{~Hz}, 1 \mathrm{H}), 7.00(\mathrm{~d}, J=8.9 \mathrm{~Hz}, 1 \mathrm{H}), 4.40(\mathrm{t}, J=6.2 \mathrm{~Hz}, 2 \mathrm{H})$, $3.89(\mathrm{~d}, J=6.5 \mathrm{~Hz}, 2 \mathrm{H}), 2.75(\mathrm{~s}, 3 \mathrm{H}), 2.37(\mathrm{td}, J=7.0,2.6 \mathrm{~Hz}, 2 \mathrm{H}), 2.25-2.14(\mathrm{~m}, 1 \mathrm{H}), 2.02-$ 
$1.94(\mathrm{~m}, 3 \mathrm{H}), 1.08(\mathrm{~d}, J=6.7 \mathrm{~Hz}, 6 \mathrm{H}) .{ }^{13} \mathrm{C}$ NMR $\left(101 \mathrm{MHz}, \mathrm{CDCl}_{3}\right) \delta 167.4,162.6,162.0,161.4$, 132.7, 132.2, 126.1, 121.7, 115.5, 112.7, 103.1, 82.9, 75.8, 69.4, 63.9, 28.3, 27.7, 19.2, 17.6, 15.4. Mass Spectrometry: HRMS-ESI (m/z): Calcd for $\mathrm{C}_{21} \mathrm{H}_{23} \mathrm{~N}_{2} \mathrm{O}_{3} \mathrm{~S}[\mathrm{M}+\mathrm{H}]^{+}$, 383.1429. Found, 383.1420 .

\section{L-phenylalanine derivative (1ss)}

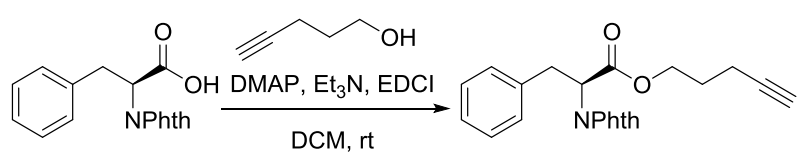

To a solution of $N$-phthaloyl-L-phenylalanine (1.48 g, $5.00 \mathrm{mmol}, 1.00$ equiv), DMAP (4-dimethylaminepyridine) (122 mg, $1.00 \mathrm{mmol}, 0.200$ equiv) and $\mathrm{Et}_{3} \mathrm{~N}(1.51 \mathrm{~g}, 15.0 \mathrm{mmol}, 3.00$ equiv) in $\mathrm{CH}_{2} \mathrm{Cl}_{2}(20.0 \mathrm{~mL})$ at room temperature were added EDCI (1-ethyl-(3-(3-dimethylamino)propyl)-carbodiimide hydrochloride) (1.91 g, $10.0 \mathrm{mmol}, 2.00$ equiv) and pent-4-yn-1-ol (504 mg, $6.00 \mathrm{mmol}, 1.20$ equiv). The reaction mixture was stirred at room temperature for $6 \mathrm{~h}$ before quenched with $\mathrm{H}_{2} \mathrm{O}(30.0 \mathrm{~mL})$ and extracted 3 times with $\mathrm{CH}_{2} \mathrm{Cl}_{2}$ $(30.0 \mathrm{~mL})$. The combined organic layer was dried over $\mathrm{MgSO}_{4}$. The filtrate was concentrated in vacuo and the residue was purified by chromatography on silica gel, eluting with hexanes/EtOAc 10:1 (v/v) to afford $1.23 \mathrm{~g}$ Febuxostat derivative (1 $\mathbf{s s})$ as a white solid (68\% yield).

$\mathrm{R}_{f}=0.15$ (hexanes/EtOAc 10:1 (v/v)). NMR Spectroscopy: ${ }^{1} \mathrm{H}$ NMR $\left(400 \mathrm{MHz}, \mathrm{CDCl}_{3}\right) \delta 7.81-$ $7.74(\mathrm{~m}, 2 \mathrm{H}), 7.71-7.64(\mathrm{~m}, 2 \mathrm{H}), 7.21-7.09(\mathrm{~m}, 5 \mathrm{H}), 5.16(\mathrm{dd}, J=11.2,5.3 \mathrm{~Hz}, 1 \mathrm{H}), 4.36-$ $4.24(\mathrm{~m}, 2 \mathrm{H}), 3.64-3.48(\mathrm{~m}, 2 \mathrm{H}), 2.21(\mathrm{td}, J=7.0,2.6 \mathrm{~Hz}, 1 \mathrm{H}), 1.94(\mathrm{t}, J=2.6 \mathrm{~Hz}, 1 \mathrm{H}), 1.89-$ $1.80(\mathrm{~m}, 2 \mathrm{H}) .{ }^{13} \mathrm{C}$ NMR $\left(101 \mathrm{MHz}, \mathrm{CDCl}_{3}\right) \delta 168.9,167.6,136.8,134.2,131.6,128.9,128.7$, 127.0, 123.6, 82.9, 69.3, 64.6, 53.4, 34.8, 27.4, 15.2. Mass Spectrometry: HRMS-ESI (m/z): Calcd for $\mathrm{C}_{22} \mathrm{H}_{20} \mathrm{NO}_{4}[\mathrm{M}+\mathrm{H}]^{+}, 362.1387$. Found, 362.1394.

\section{Lithocholic acid derivative (1tt)}

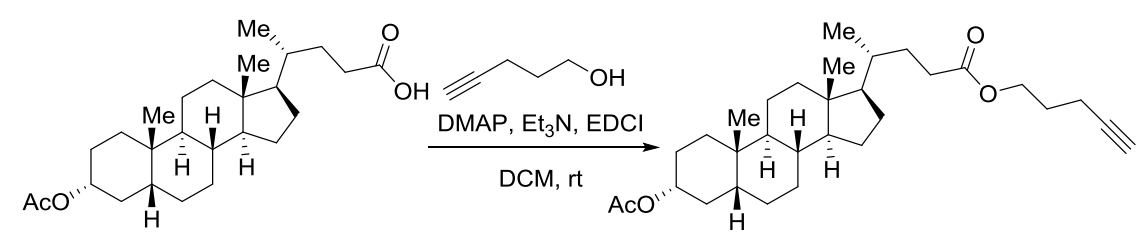

To a solution of lithocholic acid derivative ${ }^{[6]}$ (2.09 g, $5.00 \mathrm{mmol}, 1.00$ equiv), DMAP (4-dimethylaminepyridine) (122 mg, $1.00 \mathrm{mmol}, 0.200$ equiv) and $\mathrm{Et}_{3} \mathrm{~N}(1.51 \mathrm{~g}, 15.0 \mathrm{mmol}, 3.00$ equiv) in $\mathrm{CH}_{2} \mathrm{Cl}_{2}(20.0 \mathrm{~mL})$ at room temperature were added EDCI (1-ethyl-(3-(3-dimethylamino)propyl)-carbodiimide hydrochloride) (1.91 g, $10.0 \mathrm{mmol}, 2.00$ equiv) and pent-4-yn-1-ol (504 mg, $6.00 \mathrm{mmol}, 1.20$ equiv). The reaction mixture was stirred at room temperature for $6 \mathrm{~h}$ before quenched with $\mathrm{H}_{2} \mathrm{O}(30.0 \mathrm{~mL})$ and extracted 3 times with $\mathrm{CH}_{2} \mathrm{Cl}_{2}$ $(30.0 \mathrm{~mL})$. The combined organic layer was dried over $\mathrm{MgSO}_{4}$. The filtrate was concentrated in vacuo and the residue was purified by chromatography on silica gel, eluting with hexanes/EtOAc 10:1 (v/v) to afford $1.83 \mathrm{~g}$ lithocholic acid derivative (1tt) as a white solid (78\% yield).

$\mathrm{R}_{f}=0.38$ (hexanes/EtOAc 10:1 (v/v)). NMR Spectroscopy: ${ }^{1} \mathrm{H}$ NMR $\left(400 \mathrm{MHz}, \mathrm{CDCl}_{3}\right) \delta 4.77-$ $4.65(\mathrm{~m}, 1 \mathrm{H}), 4.16(\mathrm{t}, J=6.3 \mathrm{~Hz}, 2 \mathrm{H}), 2.39-2.15(\mathrm{~m}, 4 \mathrm{H}), 2.02(\mathrm{~s}, 3 \mathrm{H}), 1.99-1.91(\mathrm{~m}, 2 \mathrm{H}), 1.90$ 
$-1.72(\mathrm{~m}, 7 \mathrm{H}), 1.60-1.48(\mathrm{~m}, 2 \mathrm{H}), 1.48-1.18(\mathrm{~m}, 11 \mathrm{H}), 1.18-0.96(\mathrm{~m}, 6 \mathrm{H}), 0.95-0.85(\mathrm{~m}$, $6 \mathrm{H}), 0.63(\mathrm{~s}, 3 \mathrm{H}) .{ }^{13} \mathrm{C} \mathrm{NMR}\left(101 \mathrm{MHz}, \mathrm{CDCl}_{3}\right) \delta 174.4,170.8,83.2,74.5,69.1,62.9,56.6,56.1$, 42.9, 42.0, 40.5, 40.3, 35.9, 35.5, 35.2, 34.7, 32.4, 31.4, 31.1, 28.3, 27.7, 27.2, 26.8, 26.4, 24.3, 23.5, 21.6, 21.0, 18.4, 15.4, 12.2. Mass Spectrometry: HRMS-ESI (m/z): Calcd for $\mathrm{C}_{31} \mathrm{H}_{52} \mathrm{NO}_{4}[\mathrm{M}$ $\left.+\mathrm{NH}_{4}\right]^{+}$, 502.3891. Found, 502.3896 .

\section{Arbutin derivative (1uu)}

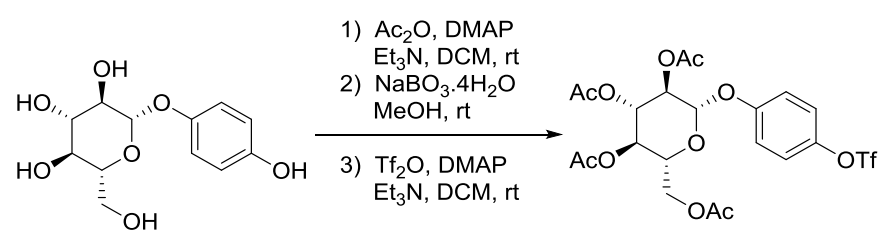

To a solution of Arbutin ( $2.72 \mathrm{~g}, 10.0 \mathrm{mmol}, 1.00$ equiv), DMAP (4-dimethylaminepyridine) (122 $\mathrm{mg}, 1.00 \mathrm{mmol}, 0.100$ equiv) and $\mathrm{Et}_{3} \mathrm{~N}\left(8.08 \mathrm{~g}, 80.0 \mathrm{mmol}, 8.00\right.$ equiv) in $\mathrm{CH}_{2} \mathrm{Cl}_{2}(50.0 \mathrm{~mL})$ at $0{ }^{\circ} \mathrm{C}$ were added dropwise $\mathrm{Ac}_{2} \mathrm{O}(6.06 \mathrm{~g}, 60.0 \mathrm{mmol}, 6.00$ equiv). The reaction mixture was stirred at room temperature for $10 \mathrm{~h}$ before quenched with $\mathrm{H}_{2} \mathrm{O}(50.0 \mathrm{~mL})$ and extracted 3 times with $\mathrm{CH}_{2} \mathrm{Cl}_{2}(50.0 \mathrm{~mL})$. The combined organic layer was dried over $\mathrm{MgSO}_{4}$. The filtrate was concentrated in vacuo. Then to a solution of this crude product in $\mathrm{MeOH}(50.0 \mathrm{~mL})$ at $0{ }^{\circ} \mathrm{C}$ were added $\mathrm{NaBO}_{3} .4 \mathrm{H}_{2} \mathrm{O}$ ( $3.08 \mathrm{~g}, 20.0 \mathrm{mmol}, 2.00$ equiv). The reaction mixture was stirred at room temperature. The reaction mixture was monitored by TLC. When the reaction is complete, filtered through a pad of Celite and concentrated in vacuo.

To a solution of this residue, DMAP (4-dimethylaminepyridine) (122 mg, $1.00 \mathrm{mmol}, 0.100$ equiv) and $\mathrm{Et}_{3} \mathrm{~N}\left(1.21 \mathrm{~g}, 12.0 \mathrm{mmol}, 1.20\right.$ equiv) in $\mathrm{CH}_{2} \mathrm{Cl}_{2}(20.0 \mathrm{~mL})$ at $0{ }^{\circ} \mathrm{C}$ were added dropwise $\mathrm{Tf}_{2} \mathrm{O}(3.38 \mathrm{~g}, 12.0 \mathrm{mmol}, 1.20$ equiv). The reaction mixture was stirred at room temperature for $1 \mathrm{~h}$ before quenched with $\mathrm{H}_{2} \mathrm{O}(20.0 \mathrm{~mL})$ and extracted 3 times with $\mathrm{CH}_{2} \mathrm{Cl}_{2}(20.0$ $\mathrm{mL}$ ). The combined organic layer was dried over $\mathrm{MgSO}_{4}$. The filtrate was concentrated in vacuo and the residue was purified by chromatography on silica gel, eluting with hexanes/EtOAc 2:1 (v/v) to afford $2.17 \mathrm{~g}$ Arbutin derivative (1 $\left.\mathbf{u u}^{\prime}\right)$ as a white solid (38\% total yield over 3 steps).

$\mathrm{R}_{f}=0.66$ (hexanes/EtOAc 1:1 (v/v)). NMR Spectroscopy: ${ }^{1} \mathrm{H}$ NMR $\left(400 \mathrm{MHz}, \mathrm{CDCl}_{3}\right) \delta 7.23-$ $7.17(\mathrm{~m}, 2 \mathrm{H}), 7.06-7.01(\mathrm{~m}, 2 \mathrm{H}), 5.32-5.24(\mathrm{~m}, 2 \mathrm{H}), 5.16$ (t, $J=9.4 \mathrm{~Hz}, 1 \mathrm{H}), 5.08$ (d, $J=7.3$ $\mathrm{Hz}, 1 \mathrm{H}), 4.28(\mathrm{dd}, J=12.3,5.4 \mathrm{~Hz}, 1 \mathrm{H}), 4.16(\mathrm{dd}, J=12.3,2.2 \mathrm{~Hz}, 1 \mathrm{H}), 3.92-3.81(\mathrm{~m}, 1 \mathrm{H}), 2.06$ (s, 6H), 2.04 (s, 3H), 2.03 (s, 3H). ${ }^{13} \mathrm{C} \mathrm{NMR}\left(101 \mathrm{MHz}, \mathrm{CDCl}_{3}\right) \delta$ 170.6, 170.3, 169.5, 169.4, 156.2, 144.9, 122.7, 118.8 (q, $J=320.8 \mathrm{~Hz}), 118.5,99.0,72.6,72.3,71.1,68.2,61.9,20.8,20.7$, 20.7. ${ }^{19} \mathrm{~F}$ NMR $\left(376 \mathrm{MHz}, \mathrm{CDCl}_{3}\right) \delta-72.73$ (s, 3F). Mass Spectrometry: HRMS-ESI (m/z): Calcd for $\mathrm{C}_{21} \mathrm{H}_{27} \mathrm{~F}_{3} \mathrm{NO}_{13} \mathrm{~S}\left[\mathrm{M}+\mathrm{NH}_{4}\right]^{+}, 590.1150$. Found, 590.1155.

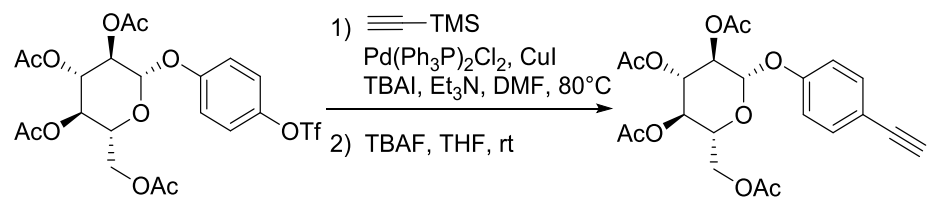

In an oven dried flask degas DMF $(5.00 \mathrm{~mL})$ by gently bubbling $\mathrm{N}_{2}$ for $2 \mathrm{~h}$, add then Arbutin derivative (1 uu') (572 mg, $1.00 \mathrm{mmol}, 1.00$ equiv), TBAI (1.11 g, $3.00 \mathrm{mmol}, 3.00$ equiv), CuI 
(57.0 mg, 0.3 equiv, 0.300 equiv), $\mathrm{Et}_{3} \mathrm{~N}(2.00 \mathrm{~mL}), \mathrm{Pd}\left(\mathrm{Ph}_{3} \mathrm{P}_{2}\right)_{2} \mathrm{Cl}_{2}(70.2 \mathrm{mg}, 0.100 \mathrm{mmol}, 0.100$ equiv) and trimethylsilylacetylene (296 $\mathrm{mg}, 3.00 \mathrm{mmol}, 3.00$ equiv) and heat the resulting mixture at $80{ }^{\circ} \mathrm{C}$ under $\mathrm{N}_{2}$ atmosphere while stirring overnight. Concentrate the mixture in vacuo, and then filter through a short pad of celite eluting with EtOAc, and then removed the solvent in vacuo. 1M TBAF in THF $(5.00 \mathrm{~mL}, 5.00$ equiv) was added slowly to a solution of this residue in dry THF $(10.0 \mathrm{~mL})$ cooled at $0{ }^{\circ} \mathrm{C}$. The resulting solution was stirred at $0{ }^{\circ} \mathrm{C}$ for 30 mins. The reaction was quenched by addition of $\mathrm{NH}_{4} \mathrm{Cl}(\mathrm{aq})$ and the mixture extracted with $\mathrm{CH}_{2} \mathrm{Cl}_{2} 3$ times $(30 \mathrm{~mL})$. The organic layer was washed with brine, dried over $\mathrm{MgSO}_{4}$, and evaporated under vaccum, the residue was purified by chromatography on silica gel, eluting with hexanes/EtOAc 2:1 (v/v) to afford $398 \mathrm{mg}$ Arbutin derivative (1 $\mathbf{u u})$ as a white solid (89\% total yield over 2 steps).

$\mathrm{R}_{f}=0.61$ (hexanes/EtOAc 1:1 (v/v)). NMR Spectroscopy: ${ }^{1} \mathrm{H}$ NMR $\left(400 \mathrm{MHz}, \mathrm{CDCl}_{3}\right) \delta 7.42(\mathrm{~d}$, $J=8.7 \mathrm{~Hz}, 1 \mathrm{H}), 6.92(\mathrm{~d}, J=8.8 \mathrm{~Hz}, 2 \mathrm{H}), 5.32-5.24(\mathrm{~m}, 2 \mathrm{H}), 5.20-5.12(\mathrm{~m}, 1 \mathrm{H}), 5.09(\mathrm{~d}, J=$ $7.4 \mathrm{~Hz}, 1 \mathrm{H}), 4.27(\mathrm{dd}, J=12.3,5.4 \mathrm{~Hz}, 1 \mathrm{H}), 4.15(\mathrm{dd}, J=12.3,2.3 \mathrm{~Hz}, 1 \mathrm{H}), 3.91-3.81(\mathrm{~m}, 1 \mathrm{H})$, $3.03(\mathrm{~s}, 1 \mathrm{H}), 2.06(\mathrm{~s}, 3 \mathrm{H}), 2.05(\mathrm{~s}, 3 \mathrm{H}), 2.04(\mathrm{~s}, 3 \mathrm{H}), 2.03(\mathrm{~s}, 3 \mathrm{H}) .{ }^{13} \mathrm{C} \mathrm{NMR}\left(101 \mathrm{MHz}, \mathrm{CDCl}_{3}\right) \delta$ 170.7, 170.3, 169.5, 169.4, 157.0, 133.7, 117.5, 116.9, 98.8, 83.1, 72.7, 72.2, 71.2, 68.3, 62.0, 20.8, 20.7. Mass Spectrometry: HRMS-ESI (m/z): Calcd for $\mathrm{C}_{22} \mathrm{H}_{24} \mathrm{NaO}_{10}[\mathrm{M}+\mathrm{Na}]^{+}, 471.1267$. Found, 471.1257 .

\section{$\delta$-D-tocopherol derivative (1ww')}

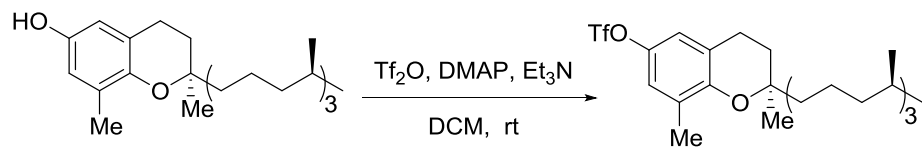

To a solution of $\delta$-D-tocopherol $(2.01 \mathrm{~g}, \quad 5.00 \mathrm{mmol}, \quad 1.00$ equiv), DMAP (4-dimethylaminepyridine) (122 mg, $1.00 \mathrm{mmol}, 0.200$ equiv) and $\mathrm{Et}_{3} \mathrm{~N}(1.21 \mathrm{~g}, 12.0 \mathrm{mmol}, 2.40$ equiv) in $\mathrm{CH}_{2} \mathrm{Cl}_{2}(20.0 \mathrm{~mL})$ at $0{ }^{\circ} \mathrm{C}$ were added dropwise $\mathrm{Tf}_{2} \mathrm{O}(1.70 \mathrm{~g}, 6.00 \mathrm{mmol}, 1.20$ equiv). The reaction mixture was stirred at room temperature for $1 \mathrm{~h}$ before quenched with $\mathrm{H}_{2} \mathrm{O}(20.0 \mathrm{~mL})$ and extracted 3 times with $\mathrm{CH}_{2} \mathrm{Cl}_{2}(30.0 \mathrm{~mL})$. The combined organic layer was dried over $\mathrm{MgSO}_{4}$. The filtrate was concentrated in vacuo and the residue was purified by chromatography on silica gel, eluting with hexanes/EtOAc 30:1 (v/v) to afford $2.59 \mathrm{~g} \delta$-D-tocopherol derivative (1ww') as a colorless liquid (97\% yield).

$\mathrm{R}_{f}=0.75$ (hexanes/EtOAc 40:1 (v/v)). NMR Spectroscopy: ${ }^{1} \mathrm{H}$ NMR (400 MHz, $\left.\mathrm{CDCl}_{3}\right) \delta 6.85(\mathrm{~d}$, $J=2.8 \mathrm{~Hz}, 1 \mathrm{H}), 6.81(\mathrm{~d}, J=2.9 \mathrm{~Hz}, 1 \mathrm{H}), 2.83-2.67(\mathrm{~m}, 2 \mathrm{H}), 2.17(\mathrm{~s}, 3 \mathrm{H}), 1.87-1.71(\mathrm{~m}, 2 \mathrm{H})$, $1.62-1.02(\mathrm{~m}, 24 \mathrm{H}), 0.91-0.81(\mathrm{~m}, 12 \mathrm{H}) .{ }^{13} \mathrm{C} \mathrm{NMR}\left(101 \mathrm{MHz}, \mathrm{CDCl}_{3}\right) \delta 151.8,141.6,128.5$, 121.8, 120.9, 119.2, 118.9 (q, $J=320.9 \mathrm{~Hz}), 77.0,40.3,39.5,37.6,37.5,37.5,37.4,33.0,32.8$, $30.8,28.1,25.0,24.6,24.3,22.9,22.8,22.6,21.1,19.9,19.8,16.3$. Mass Spectrometry: HRMS-ESI (m/z): Calcd for $\mathrm{C}_{29} \mathrm{H}_{47} \mathrm{O}[\mathrm{M}+\mathrm{H}]^{+}$, 411.3621. Found, 411.3622.

\section{$\delta$-D-tocopherol derivative (1ww)}

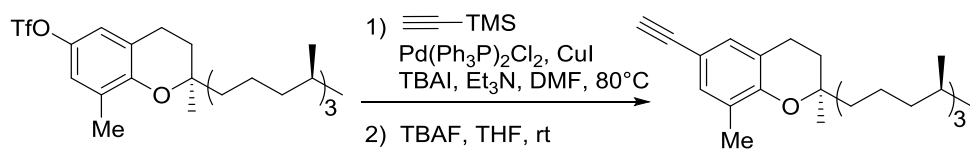

In an oven dried flask degas DMF $(15.0 \mathrm{~mL})$ by gently bubbling $\mathrm{N}_{2}$ for $2 \mathrm{~h}$, then add 
$\delta$-D-tocopherol derivative (1ww') (1.60 g, $3.00 \mathrm{mmol}, 1.00$ equiv), TBAI (3.33 g, $9.00 \mathrm{mmol}, 3.00$ equiv), $\mathrm{CuI}$ (171 mg, $0.900 \mathrm{mmol}, 0.300$ equiv), $\mathrm{Et}_{3} \mathrm{~N}(6.00 \mathrm{~mL}), \mathrm{Pd}\left(\mathrm{Ph}_{3} \mathrm{P}\right)_{2} \mathrm{Cl}_{2}(210 \mathrm{mg}, 0.300$ mmol, 0.100 equiv) and trimethylsilylacetylene $(888 \mathrm{mg}, 9.00 \mathrm{mmol}, 3.00$ equiv) and heat the resulting mixture at $80{ }^{\circ} \mathrm{C}$ under $\mathrm{N}_{2}$ atmosphere while stirring overnight. Concentrate the mixture in vacuo, and then filter through a short pad of celite eluting with EtOAc, and then removed the solvent in vacuo.

1M TBAF in THF $(15.0 \mathrm{~mL}, 5.00$ equiv) was added slowly to a solution of this residue in dry THF $(20.0 \mathrm{~mL})$ cooled at $0{ }^{\circ} \mathrm{C}$. The resulting solution was stirred at $0{ }^{\circ} \mathrm{C}$ for 30 mins. The reaction was quenched by addition of $\mathrm{NH}_{4} \mathrm{Cl}$ (aq) and the mixture extracted with $\mathrm{CH}_{2} \mathrm{Cl}_{2} 3$ times $(30 \mathrm{~mL})$. The organic layer was washed with $\mathrm{H}_{2} \mathrm{O}$ and brine, dried over $\mathrm{MgSO}_{4}$, and evaporated under vaccum, the residue was purified by chromatography on silica gel, eluting with hexanes/EtOAc 30:1 (v/v) to afford $996 \mathrm{mg} \delta$-D-tocopherol derivative (1ww) as a colorless liquid (81\% total yield over 2 steps)

$\mathrm{R}_{f}=0.75$ (hexanes/EtOAc 40:1 (v/v)). NMR Spectroscopy: ${ }^{1} \mathrm{H}$ NMR (400 MHz, $\left.\mathrm{CDCl}_{3}\right) \delta 7.11(\mathrm{~s}$, 1H), $7.09(\mathrm{~s}, 1 \mathrm{H}), 2.94(\mathrm{~s}, 1 \mathrm{H}), 2.72(\mathrm{t}, J=6.3 \mathrm{~Hz}, 2 \mathrm{H}), 2.14(\mathrm{~s}, 3 \mathrm{H}), 1.87-1.70(\mathrm{~m}, 2 \mathrm{H}), 1.64-$ $1.50(\mathrm{~m}, 3 \mathrm{H}), 1.48-1.01(\mathrm{~m}, 21 \mathrm{H}), 0.92-0.82(\mathrm{~m}, 12 \mathrm{H}) .{ }^{13} \mathrm{C} \mathrm{NMR}\left(101 \mathrm{MHz}, \mathrm{CDCl}_{3}\right) \delta 153.1$, 132.2, 131.2, 126.6, 120.6, 112.1, 84.6, 76.8, 74.9, 40.2, 39.5, 37.6, 37.5, 37.4, 32.9, 32.8, 31.1, 28.1, 25.0, 24.6, 24.4, 22.9, 22.8, 22.2, 21.1, 19.9, 19.8, 16.0. Mass Spectrometry: HRMS-ESI $(\mathrm{m} / \mathrm{z})$ : Calcd for $\mathrm{C}_{29} \mathrm{H}_{47} \mathrm{O}[\mathrm{M}+\mathrm{H}]^{+}$, 411.3621. Found, 411.3622 .

\section{1,1-Dibromo-2-(trifluoromethoxy)dodec-1-ene (3a)}

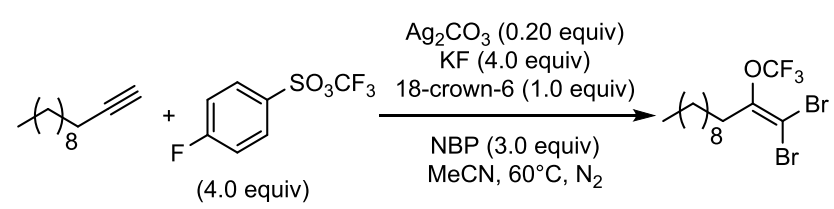

In a glove box, to a $15.0 \mathrm{~mL}$ sealed tube were added in sequence 1-dodecyne (1a) $(83 \mathrm{mg}, 0.500$ mmol, 1.00 equiv), KF (116 mg, $2.00 \mathrm{mmol}, 4.00$ equiv), 18-crown-6 (132 mg, $0.500 \mathrm{mmol}, 1.00$ equiv), $\mathrm{Ag}_{2} \mathrm{CO}_{3}$ (27.5 mg, $0.100 \mathrm{mmol}, 0.200$ equiv), NBP ( $N$-Bromophthalimide) ( $339 \mathrm{mg}, 1.50$ mmol, 3.00 equiv) and $1.00 \mathrm{~mL} \mathrm{MeCN}$. The mixture was stirring at room temperature for 5 mins, then added TFMS ( $489 \mathrm{mg}, 2.00 \mathrm{mmol}, 4.00$ equiv). After stirring for $4.0 \mathrm{~h}$ at $60{ }^{\circ} \mathrm{C}$, the reaction mixture was concentrated in vacuo. The residue was then added $10 \mathrm{~mL} \mathrm{CH}_{2} \mathrm{Cl}_{2}$ and filtered. The organic layer was concentrated in vacuo. The residue was purified by preparative HPLC ( 9 $\mathrm{mL} / \mathrm{min}$, detector $\mathrm{UV} \lambda \max 210 \mathrm{~nm}, \mathrm{MeCN} / \mathrm{H}_{2} \mathrm{O}=90: 10(0 \mathrm{~min}), \mathrm{MeCN} / \mathrm{H}_{2} \mathrm{O}=100: 0$ (10 min), $\left.\mathrm{MeCN} / \mathrm{H}_{2} \mathrm{O}=100: 0(40 \mathrm{~min})\right)$ to afford $163 \mathrm{mg}$ 1,1-dibromo-2-(trifluoromethoxy)dodec-1-ene (3a) (29.5 $\mathrm{min}$ ) as a colorless liquid ( $80 \%$ yield).

$\mathrm{R}_{f}=0.98$ (hexanes). NMR Spectroscopy: ${ }^{1} \mathrm{H}$ NMR $\left(400 \mathrm{MHz}, \mathrm{CDCl}_{3}\right) \delta 2.55(\mathrm{t}, J=7.5 \mathrm{~Hz}, 2 \mathrm{H})$, $1.63-1.53(\mathrm{~m}, 2 \mathrm{H}), 1.37-1.20(\mathrm{~m}, 14 \mathrm{H}), 0.89(\mathrm{t}, J=5.9 \mathrm{~Hz}, 3 \mathrm{H}) .{ }^{13} \mathrm{C} \mathrm{NMR}\left(101 \mathrm{MHz}, \mathrm{CDCl}_{3}\right)$ $\delta 149.5,119.9$ (q, $J=260.9 \mathrm{~Hz}), 87.8,32.1,32.1,29.7,29.6,29.5,29.3,28.9,26.0,22.9,14.3 .{ }^{19} \mathrm{~F}$ NMR $\left(376 \mathrm{MHz}, \mathrm{CDCl}_{3}\right) \delta-55.81(\mathrm{~s}, 3 \mathrm{~F})$. Mass Spectrometry: HRMS-EI $(\mathrm{m} / \mathrm{z})$ : Calcd for $\mathrm{C}_{13} \mathrm{H}_{21} \mathrm{Br}_{2} \mathrm{~F}_{3} \mathrm{O}[\mathrm{M}]^{+}$, 407.9911. Found, 407.9910. 


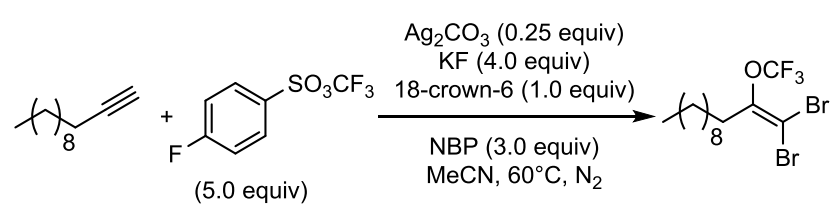

In a glove box, to a $15.0 \mathrm{~mL}$ sealed tube were added in sequence 1-dodecyne (1a) ( $83 \mathrm{mg}, 0.500$ mmol, 1.00 equiv), KF (116 mg, $2.00 \mathrm{mmol}, 4.00$ equiv), 18-crown-6 (132 mg, $0.500 \mathrm{mmol}, 1.00$ equiv), $\mathrm{Ag}_{2} \mathrm{CO}_{3}$ ( $34.4 \mathrm{mg}, 0.125 \mathrm{mmol}, 0.250$ equiv), NBP ( $N$-Bromophthalimide) ( $339 \mathrm{mg}, 1.50$ mmol, 3.00 equiv) and $1.00 \mathrm{~mL} \mathrm{MeCN}$. The mixture was stirring at room temperature for 5 mins, then added TFMS (612 mg, $2.50 \mathrm{mmol}, 5.00$ equiv). After stirring for $4.0 \mathrm{~h}$ at $60{ }^{\circ} \mathrm{C}$, the reaction mixture was concentrated in vacuo. The residue was then added $10 \mathrm{~mL} \mathrm{CH}_{2} \mathrm{Cl}_{2}$ and filtered. The organic layer was concentrated in vacuo. The residue was purified by preparative HPLC ( 9 $\mathrm{mL} / \mathrm{min}$, detector UV $\lambda \max 210 \mathrm{~nm}, \mathrm{MeCN} / \mathrm{H}_{2} \mathrm{O}=90: 10(0 \mathrm{~min}), \mathrm{MeCN} / \mathrm{H}_{2} \mathrm{O}=100: 0$ (10 min), $\left.\mathrm{MeCN} / \mathrm{H}_{2} \mathrm{O}=100: 0(40 \mathrm{~min})\right)$ to afford $180 \mathrm{mg}$ 1,1-dibromo-2-(trifluoromethoxy)dodec-1-ene (3a) (29.5 $\mathrm{min})$ as a colorless liquid ( $88 \%$ yield).

\section{6,6-Dibromo-5-(trifluoromethoxy)hex-5-en-1-yl 3-methoxybenzoate (3b)}

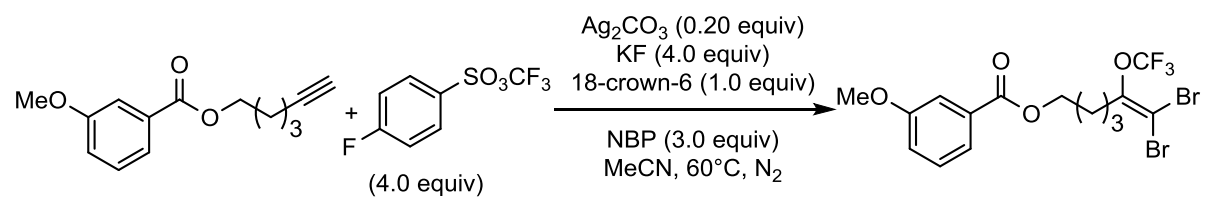

In a glove box, to a $15.0 \mathrm{~mL}$ sealed tube were added in sequence hex-5-yn-1-yl 3-methoxybenzoate (1b) (116 mg, $0.500 \mathrm{mmol}, 1.00$ equiv), KF (116 mg, $2.00 \mathrm{mmol}, 4.00$ equiv), 18-crown-6 (132 mg, 0.500 mmol, 1.00 equiv), $\mathrm{Ag}_{2} \mathrm{CO}_{3}$ (27.5 mg, $0.100 \mathrm{mmol}, 0.200$ equiv), NBP ( $N$-Bromophthalimide) (339 mg, $1.50 \mathrm{mmol}, 3.00$ equiv) and $1.00 \mathrm{~mL} \mathrm{MeCN}$. The mixture was stirring at room temperature for $5 \mathrm{mins}$, then added TFMS (489 mg, $2.00 \mathrm{mmol}, 4.00$ equiv). After stirring for $4.0 \mathrm{~h}$ at $60{ }^{\circ} \mathrm{C}$, the reaction mixture was concentrated in vacuo. The residue was then added $10 \mathrm{~mL} \mathrm{CH} \mathrm{Cl}_{2}$ and filtered. The organic layer was concentrated in vacuo. The residue was purified by preparative TLC, eluting with hexanes/EtOAc 50:1 (v/v) to afford $176 \mathrm{mg}$ 6,6-dibromo-5-(trifluoromethoxy)hex-5-en-1-yl 3-methoxybenzoate (3b) as a colorless liquid (74\% yield).

$\mathrm{R}_{f}=0.11$ (hexanes/EtOAc 40:1 (v/v)). NMR Spectroscopy: ${ }^{1} \mathrm{H}$ NMR $\left(400 \mathrm{MHz}, \mathrm{CDCl}_{3}\right) \delta 7.63(\mathrm{~d}$, $J=7.7 \mathrm{~Hz}, 1 \mathrm{H}), 7.59-7.52(\mathrm{~m}, 1 \mathrm{H}), 7.34$ (t, $J=7.9 \mathrm{~Hz}, 1 \mathrm{H}), 7.10$ (dd, $J=7.6,2.5 \mathrm{~Hz}, 1 \mathrm{H}), 4.34$ (t, $J=6.1 \mathrm{~Hz}, 2 \mathrm{H}), 3.85(\mathrm{~s}, 3 \mathrm{H}), 2.65(\mathrm{t}, J=7.1 \mathrm{~Hz}, 2 \mathrm{H}), 1.92-1.71(\mathrm{~m}, 4 \mathrm{H}) .{ }^{13} \mathrm{C}$ NMR $(101$ $\left.\mathrm{MHz}, \mathrm{CDCl}_{3}\right) \delta 166.5,159.7,148.7,131.6,129.5,122.0,119.9$ (q, $\left.J=261.0 \mathrm{~Hz}\right), 119.5,114.2$, 88.6, 64.3, 55.5, 31.7, 27.9, 22.6. ${ }^{19} \mathrm{~F}$ NMR (376 $\left.\mathrm{MHz}, \mathrm{CDCl}_{3}\right) \delta-56.29$ (s, 3F). Mass Spectrometry: HRMS-ESI (m/z): Calcd for $\mathrm{C}_{15} \mathrm{H}_{16} \mathrm{Br}_{2} \mathrm{~F}_{3} \mathrm{O}_{4}[\mathrm{M}+\mathrm{H}]^{+}$, 474.9362. Found, 474.9358.

\section{5,5-Dibromo-4-(trifluoromethoxy)pent-4-en-1-yl 4-phenoxybenzoate (3c)}

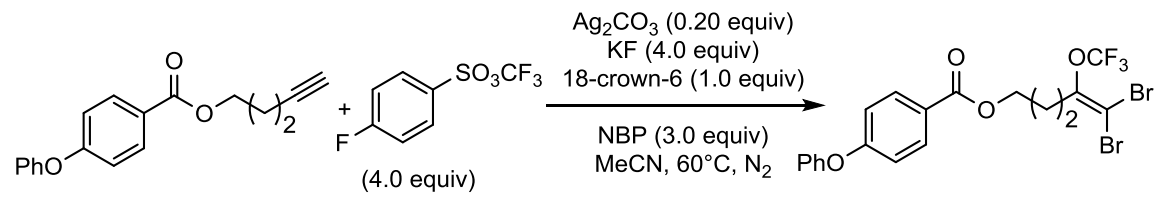

In a glove box, to a $15.0 \mathrm{~mL}$ sealed tube were added in sequence pent-4-yn-1-yl 4-phenoxybenzoate (1c) (140 mg, $0.500 \mathrm{mmol}, 1.00$ equiv), KF (116 mg, $2.00 \mathrm{mmol}, 4.00$ equiv), 
18-crown-6 (132 mg, $0.500 \mathrm{mmol}, 1.00$ equiv), $\mathrm{Ag}_{2} \mathrm{CO}_{3}$ (27.5 mg, $0.100 \mathrm{mmol}, 0.200$ equiv), NBP ( $N$-Bromophthalimide) (339 mg, $1.50 \mathrm{mmol}, 3.00$ equiv) and $1.00 \mathrm{~mL} \mathrm{MeCN}$. The mixture was stirring at room temperature for $5 \mathrm{mins}$, then added TFMS (489 $\mathrm{mg}, 2.00 \mathrm{mmol}, 4.00$ equiv). After stirring for $4.0 \mathrm{~h}$ at $60{ }^{\circ} \mathrm{C}$, the reaction mixture was concentrated in vacuo. The residue was then added $10 \mathrm{~mL} \mathrm{CH} \mathrm{Cl}_{2}$ and filtered. The organic layer was concentrated in vacuo. The residue was purified by preparative TLC, eluting with hexanes/EtOAc 50:1 (v/v) to afford $220 \mathrm{mg}$ 5,5-dibromo-4-(trifluoromethoxy)pent-4-en-1-yl 4-phenoxybenzoate (3c) as a colorless liquid (82\% yield).

$\mathrm{R}_{f}=0.15$ (hexanes/EtOAc 40:1 (v/v)). NMR Spectroscopy: ${ }^{1} \mathrm{H}$ NMR (400 MHz, $\left.\mathrm{CDCl}_{3}\right) \delta 8.02(\mathrm{~d}$, $J=8.7 \mathrm{~Hz}, 2 \mathrm{H}), 7.39$ (t, $J=7.2 \mathrm{~Hz}, 2 \mathrm{H}), 7.19(\mathrm{t}, J=6.9 \mathrm{~Hz}, 1 \mathrm{H}), 7.07(\mathrm{~d}, J=7.9 \mathrm{~Hz}, 2 \mathrm{H}), 7.00(\mathrm{~d}$, $J=8.6 \mathrm{~Hz}, 2 \mathrm{H}), 4.34(\mathrm{t}, J=5.9 \mathrm{~Hz}, 2 \mathrm{H}), 2.78(\mathrm{t}, J=7.4 \mathrm{~Hz}, 2 \mathrm{H}), 2.18-1.97(\mathrm{~m}, 2 \mathrm{H}) .{ }^{13} \mathrm{C} \mathrm{NMR}$ $\left(101 \mathrm{MHz}, \mathrm{CDCl}_{3}\right) \delta 165.9,162.0,155.6,148.1,131.7,130.1,124.6,124.3,120.2,119.8$ (q, $J=$ 261.0 Hz), 117.3, 89.0, 63.3, 29.1, 25.2. ${ }^{19} \mathrm{~F}$ NMR (376 MHz, $\left.\mathrm{CDCl}_{3}\right) \delta-56.70$ (s, 3F). Mass Spectrometry: HRMS-ESI (m/z): Calcd for $\mathrm{C}_{19} \mathrm{H}_{16} \mathrm{Br}_{2} \mathrm{~F}_{3} \mathrm{O}_{4}[\mathrm{M}+\mathrm{H}]^{+}$, 522.9362. Found, 522.9362 .

\section{6,6-Dibromo-5-(trifluoromethoxy)hex-5-en-1-yl [1,1'-biphenyl]-4-carboxylate (3d)}

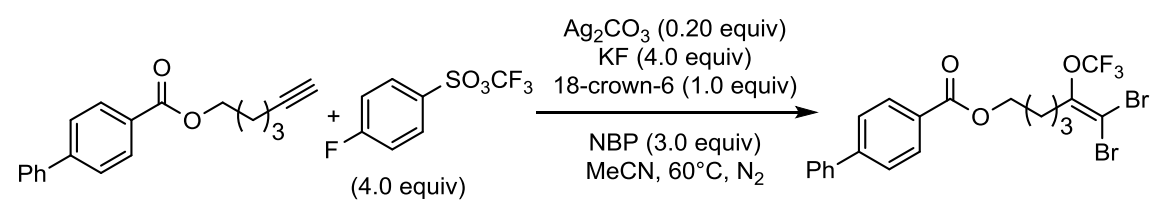

In a glove box, to a $15.0 \mathrm{~mL}$ sealed tube were added in sequence hex-5-yn-1-yl [1,1'-biphenyl]-4-carboxylate (1d) (139 mg, $0.500 \mathrm{mmol}, 1.00$ equiv), KF (116 mg, $2.00 \mathrm{mmol}$, 4.00 equiv), 18-crown-6 (132 mg, $0.500 \mathrm{mmol}, 1.00$ equiv), $\mathrm{Ag}_{2} \mathrm{CO}_{3}$ (27.5 mg, $0.100 \mathrm{mmol}, 0.200$ equiv), NBP ( $N$-Bromophthalimide) $(339 \mathrm{mg}, 1.50 \mathrm{mmol}, 3.00$ equiv) and $1.00 \mathrm{~mL} \mathrm{MeCN}$. The mixture was stirring at room temperature for $5 \mathrm{mins}$, then added TFMS (489 mg, $2.00 \mathrm{mmol}, 4.00$ equiv). After stirring for $4.0 \mathrm{~h}$ at $60{ }^{\circ} \mathrm{C}$, the reaction mixture was concentrated in vacuo. The residue was then added $10 \mathrm{~mL} \mathrm{CH} \mathrm{Cl}_{2}$ and filtered. The organic layer was concentrated in vacuo. The residue was purified by preparative TLC, eluting with hexanes/EtOAc 40:1 (v/v) to afford 214 mg 6,6-dibromo-5-(trifluoromethoxy)hex-5-en-1-yl [1,1'-biphenyl]-4-carboxylate (3d) as a colorless liquid ( $82 \%$ yield).

$\mathrm{R}_{f}=0.11$ (hexanes/EtOAc 40:1 (v/v)). NMR Spectroscopy: ${ }^{1} \mathrm{H}$ NMR $\left(400 \mathrm{MHz}, \mathrm{CDCl}_{3}\right) \delta 8.13(\mathrm{~d}$, $J=8.3 \mathrm{~Hz}, 2 \mathrm{H}), 7.68(\mathrm{~d}, J=8.3 \mathrm{~Hz}, 2 \mathrm{H}), 7.64(\mathrm{~d}, J=7.3 \mathrm{~Hz}, 2 \mathrm{H}), 7.48(\mathrm{t}, J=7.5 \mathrm{~Hz}, 2 \mathrm{H}), 7.41(\mathrm{t}$, $J=7.3 \mathrm{~Hz}, 1 \mathrm{H}), 4.39(\mathrm{t}, J=6.0 \mathrm{~Hz}, 2 \mathrm{H}), 2.69(\mathrm{t}, J=7.0 \mathrm{~Hz}, 2 \mathrm{H}), 1.91-1.76(\mathrm{~m}, 4 \mathrm{H}) .{ }^{13} \mathrm{C} \mathrm{NMR}$ $\left(101 \mathrm{MHz}, \mathrm{CDCl}_{3}\right) \delta 166.5,148.7,145.8,140.1,130.2,129.0,128.3,127.4,127.2,119.9$ (q, $J=$ 261.2 Hz), 88.6, 64.2, 31.7, 27.9, 22.6. ${ }^{19} \mathrm{~F}$ NMR (376 MHz, $\left.\mathrm{CDCl}_{3}\right) \delta-56.20$ (s, 3F). Mass Spectrometry: HRMS-ESI (m/z): Calcd for $\mathrm{C}_{20} \mathrm{H}_{18} \mathrm{Br}_{2} \mathrm{~F}_{3} \mathrm{O}_{3}[\mathrm{M}+\mathrm{H}]^{+}$, 520.9569. Found, 520.9567.

\section{6,6-Dibromo-5-(trifluoromethoxy)hex-5-en-1-yl 3-methylbenzoate (3e)}

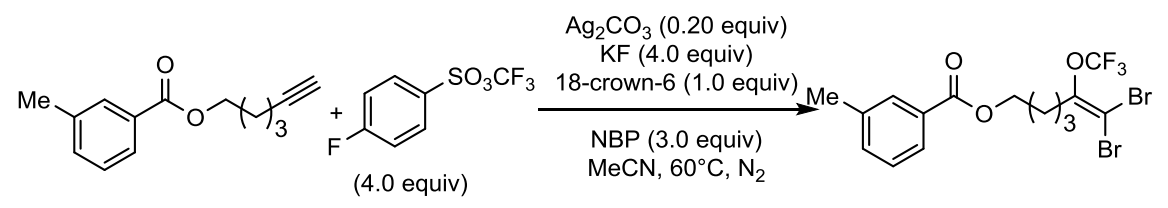

In a glove box, to a $15.0 \mathrm{~mL}$ sealed tube were added in sequence hex-5-yn-1-yl 3-methylbenzoate (1e) (108 mg, $0.500 \mathrm{mmol}, 1.00$ equiv), KF (116 mg, $2.00 \mathrm{mmol}, 4.00$ equiv), 18-crown-6 (132 
mg, 0.500 mmol, 1.00 equiv), $\mathrm{Ag}_{2} \mathrm{CO}_{3}(27.5 \mathrm{mg}, 0.100 \mathrm{mmol}, 0.200$ equiv), NBP ( $N$-Bromophthalimide) (339 mg, $1.50 \mathrm{mmol}, 3.00$ equiv) and $1.00 \mathrm{~mL} \mathrm{MeCN}$. The mixture was stirring at room temperature for 5 mins, then added TFMS (489 mg, $2.00 \mathrm{mmol}, 4.00$ equiv). After stirring for $4.0 \mathrm{~h}$ at $60{ }^{\circ} \mathrm{C}$, the reaction mixture was concentrated in vacuo. The residue was then added $10 \mathrm{~mL} \mathrm{CH}_{2} \mathrm{Cl}_{2}$ and filtered. The organic layer was concentrated in vacuo. The residue was purified by preparative TLC, eluting with hexanes/EtOAc 40:1 (v/v) to afford $184 \mathrm{mg}$ 6,6-dibromo-5-(trifluoromethoxy)hex-5-en-1-yl 3-methylbenzoate (3e) as a colorless liquid (80\% yield).

$\mathrm{R}_{f}=0.18$ (hexanes/EtOAc 40:1 (v/v)). NMR Spectroscopy: ${ }^{1} \mathrm{H}$ NMR $\left(400 \mathrm{MHz}, \mathrm{CDCl}_{3}\right) \delta 7.88-$ $7.80(\mathrm{~m}, 2 \mathrm{H}), 7.40-7.29(\mathrm{~m}, 2 \mathrm{H}), 4.34(\mathrm{t}, J=5.8 \mathrm{~Hz}, 2 \mathrm{H}), 2.66(\mathrm{t}, J=6.9 \mathrm{~Hz}, 2 \mathrm{H}), 2.41(\mathrm{~s}, 3 \mathrm{H})$, $1.89-1.71(\mathrm{~m}, 4 \mathrm{H}) .{ }^{13} \mathrm{C} \mathrm{NMR}\left(101 \mathrm{MHz}, \mathrm{CDCl}_{3}\right) \delta 166.8,148.7,138.3,133.8,130.3,130.2$, 128.4, 126.8, 119.9 (q, $J=260.9 \mathrm{~Hz}), 88.6,64.2,31.7,27.9,22.6,21.4 .{ }^{19} \mathrm{~F}$ NMR $(376 \mathrm{MHz}$, $\left.\mathrm{CDCl}_{3}\right) \delta-56.35$ (s, 3F). Mass Spectrometry: HRMS-ESI (m/z): Calcd for $\mathrm{C}_{15} \mathrm{H}_{16} \mathrm{Br}_{2} \mathrm{~F}_{3} \mathrm{O}_{3}[\mathrm{M}+$ $\mathrm{H}]^{+}, 458.9413$. Found, 458.9408 .

\section{6,6-Dibromo-5-(trifluoromethoxy)hex-5-en-1-yl 4-(tert-butyl)benzoate (3f)}

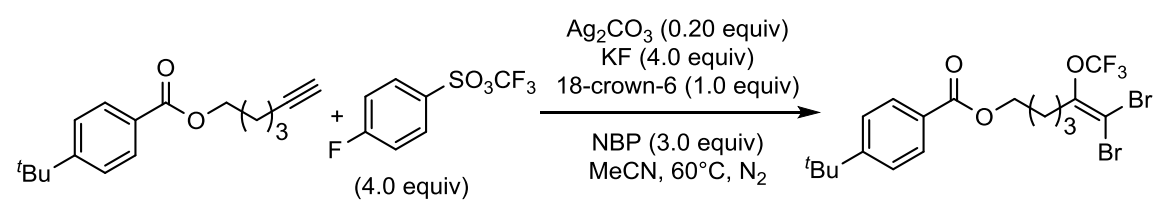

In a glove box, to a $15.0 \mathrm{~mL}$ sealed tube were added in sequence hex-5-yn-1-yl 4-(tert-butyl)benzoate (1f) (129 mg, $0.500 \mathrm{mmol}, 1.00$ equiv), KF (116 mg, $2.00 \mathrm{mmol}, 4.00$ equiv), 18-crown-6 (132 mg, $0.500 \mathrm{mmol}, 1.00$ equiv), $\mathrm{Ag}_{2} \mathrm{CO}_{3}$ (27.5 mg, $0.100 \mathrm{mmol}, 0.200$ equiv), NBP ( $N$-Bromophthalimide) $(339 \mathrm{mg}, 1.50 \mathrm{mmol}, 3.00$ equiv) and $1.00 \mathrm{~mL} \mathrm{MeCN}$. The mixture was stirring at room temperature for $5 \mathrm{mins}$, then added TFMS (489 mg, $2.00 \mathrm{mmol}, 4.00$ equiv). After stirring for $4.0 \mathrm{~h}$ at $60{ }^{\circ} \mathrm{C}$, the reaction mixture was concentrated in vacuo. The residue was then added $10 \mathrm{~mL} \mathrm{CH}_{2} \mathrm{Cl}_{2}$ and filtered. The organic layer was concentrated in vacuo. The residue was purified by preparative TLC, eluting with hexanes/EtOAc 40:1 (v/v) to afford 206 mg 6,6-dibromo-5-(trifluoromethoxy)hex-5-en-1-yl 4-(tert-butyl)benzoate (3f) as a colorless liquid (82\% yield).

$\mathrm{R}_{f}=0.18$ (hexanes/EtOAc 40:1 (v/v)). NMR Spectroscopy: ${ }^{1} \mathrm{H}$ NMR $\left(400 \mathrm{MHz}, \mathrm{CDCl}_{3}\right) \delta 8.01-$ $7.92(\mathrm{~m}, 2 \mathrm{H}), 7.52-7.40(\mathrm{~m}, 2 \mathrm{H}), 4.34(\mathrm{t}, J=6.1 \mathrm{~Hz}, 2 \mathrm{H}), 2.66(\mathrm{t}, J=7.1 \mathrm{~Hz}, 2 \mathrm{H}), 1.88-1.71$ $(\mathrm{m}, 4 \mathrm{H}), 1.34(\mathrm{~s}, 9 \mathrm{H}) .{ }^{13} \mathrm{C}$ NMR $\left(101 \mathrm{MHz}, \mathrm{CDCl}_{3}\right) \delta 166.7,156.8,148.7,129.6,127.6,125.5$, 119.9 (q, $J=261.2 \mathrm{~Hz}), 88.6,64.0,35.2,31.7,31.2,28.0,22.6 .{ }^{19} \mathrm{~F} \mathrm{NMR}\left(376 \mathrm{MHz}, \mathrm{CDCl}_{3}\right) \delta$ $-56.20(\mathrm{~s}, 3 \mathrm{~F})$. Mass Spectrometry: HRMS-ESI (m/z): Calcd for $\mathrm{C}_{18} \mathrm{H}_{22} \mathrm{Br}_{2} \mathrm{~F}_{3} \mathrm{O}_{3}[\mathrm{M}+\mathrm{H}]^{+}$, 500.9882. Found, 500.9878.

\section{6,6-Dibromo-5-(trifluoromethoxy)hex-5-en-1-yl benzoate (3g)}

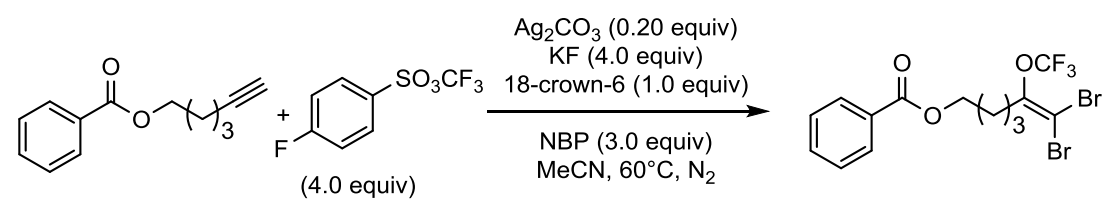

In a glove box, to a $15.0 \mathrm{~mL}$ sealed tube were added in sequence hex-5-yn-1-yl benzoate (1g) (101 $\mathrm{mg}, 0.500 \mathrm{mmol}, 1.00$ equiv), KF (116 mg, $2.00 \mathrm{mmol}, 4.00$ equiv), 18-crown-6 (132 mg, 0.500 
mmol, 1.00 equiv), $\mathrm{Ag}_{2} \mathrm{CO}_{3}$ ( $27.5 \mathrm{mg}, 0.100 \mathrm{mmol}, 0.200$ equiv), NBP ( $N$-Bromophthalimide) (339 mg, $1.50 \mathrm{mmol}, 3.00$ equiv) and $1.00 \mathrm{~mL} \mathrm{MeCN}$. The mixture was stirring at room temperature for $5 \mathrm{mins}$, then added TFMS ( $489 \mathrm{mg}, 2.00 \mathrm{mmol}, 4.00$ equiv). After stirring for 4.0 $\mathrm{h}$ at $60^{\circ} \mathrm{C}$, the reaction mixture was concentrated in vacuo. The residue was then added $10 \mathrm{~mL}$ $\mathrm{CH}_{2} \mathrm{Cl}_{2}$ and filtered. The organic layer was concentrated in vacuo. The residue was purified by preparative TLC, eluting with hexanes/EtOAc 40:1 (v/v) to afford $178 \mathrm{mg}$ 6,6-dibromo-5-(trifluoromethoxy)hex-5-en-1-yl benzoate (3g) as a colorless liquid (80\% yield). $\mathrm{R}_{f}=0.18$ (hexanes/EtOAc 40:1 (v/v)). NMR Spectroscopy: ${ }^{1} \mathrm{H}$ NMR $\left(400 \mathrm{MHz}, \mathrm{CDCl}_{3}\right) \delta 8.04(\mathrm{~d}$, $J=7.6 \mathrm{~Hz}, 2 \mathrm{H}), 7.56(\mathrm{t}, J=7.4 \mathrm{~Hz}, 1 \mathrm{H}), 7.45(\mathrm{t}, J=7.5 \mathrm{~Hz}, 2 \mathrm{H}), 4.35(\mathrm{t}, J=5.9 \mathrm{~Hz}, 2 \mathrm{H}), 2.66$ (t, $J=7.0 \mathrm{~Hz}, 2 \mathrm{H}), 1.89-1.71(\mathrm{~m}, 4 \mathrm{H}) .{ }^{13} \mathrm{C} \mathrm{NMR}\left(101 \mathrm{MHz}, \mathrm{CDCl}_{3}\right) \delta 166.7,148.7,133.1,130.4$, 129.7, 128.5, 119.9 (q, $J=261.0 \mathrm{~Hz}), 88.6,64.3,31.7,27.9,22.6 .{ }^{19} \mathrm{~F} \mathrm{NMR}\left(376 \mathrm{MHz}, \mathrm{CDCl}_{3}\right) \delta$ -56.14 (s, 3F). Mass Spectrometry: HRMS-EI (m/z): Calcd for $\mathrm{C}_{14} \mathrm{H}_{13} \mathrm{Br}_{2} \mathrm{~F}_{3} \mathrm{O}_{3}[\mathrm{M}]^{+}, 443.9184$. Found, 443.9179.

6,6-Dibromo-5-(trifluoromethoxy)hex-5-en-1-yl 4-fluorobenzoate (3h)

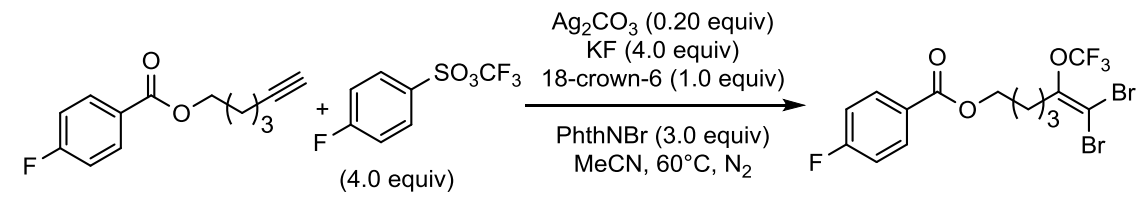

In a glove box, to a $15.0 \mathrm{~mL}$ sealed tube were added in sequence hex-5-yn-1-yl 4-fluorobenzoate (1h) (110 mg, $0.500 \mathrm{mmol}, 1.00$ equiv), KF (116 mg, $2.00 \mathrm{mmol}, 4.00$ equiv), 18-crown-6 (132 $\mathrm{mg}, \quad 0.500 \mathrm{mmol}, 1.00$ equiv), $\mathrm{Ag}_{2} \mathrm{CO}_{3}(27.5 \mathrm{mg}, 0.100 \mathrm{mmol}, 0.200$ equiv), NBP ( $N$-Bromophthalimide) (339 mg, $1.50 \mathrm{mmol}, 3.00$ equiv) and $1.00 \mathrm{~mL} \mathrm{MeCN}$. The mixture was stirring at room temperature for 5 mins, then added TFMS (489 mg, $2.00 \mathrm{mmol}, 4.00$ equiv). After stirring for $4.0 \mathrm{~h}$ at $60{ }^{\circ} \mathrm{C}$, the reaction mixture was concentrated in vacuo. The residue was then added $10 \mathrm{~mL} \mathrm{CH}_{2} \mathrm{Cl}_{2}$ and filtered. The organic layer was concentrated in vacuo. The residue was purified by preparative TLC, eluting with hexanes/EtOAc 40:1 (v/v) to afford $183 \mathrm{mg}$ 6,6-dibromo-5-(trifluoromethoxy)hex-5-en-1-yl 4-fluorobenzoate (3h) as a colorless liquid (78\% yield).

$\mathrm{R}_{f}=0.18$ (hexanes/EtOAc 40:1 (v/v)). NMR Spectroscopy: ${ }^{1} \mathrm{H}$ NMR $\left(400 \mathrm{MHz}, \mathrm{CDCl}_{3}\right) \delta 8.13-$ $7.96(\mathrm{~m}, 2 \mathrm{H}), 7.18-7.06(\mathrm{~m}, 2 \mathrm{H}), 4.34(\mathrm{t}, J=6.2 \mathrm{~Hz}, 2 \mathrm{H}), 2.66(\mathrm{t}, J=7.1 \mathrm{~Hz}, 2 \mathrm{H}), 1.89-1.71$ (m, 4H). ${ }^{13} \mathrm{C}$ NMR $\left(101 \mathrm{MHz}, \mathrm{CDCl}_{3}\right) \delta 165.9(\mathrm{~d}, J=254.0 \mathrm{~Hz}), 165.7,148.7,132.2(\mathrm{~d}, J=9.3$ $\mathrm{Hz}), 126.6$ (d, $J=2.8 \mathrm{~Hz}), 119.9$ (q, $J=261.0 \mathrm{~Hz}), 115.7$ (d, $J=22.0 \mathrm{~Hz}), 88.7,64.4,31.7,27.9$, 22.6. ${ }^{19} \mathrm{~F} \mathrm{NMR}\left(376 \mathrm{MHz}, \mathrm{CDCl}_{3}\right) \delta-56.21(\mathrm{~s}, 3 \mathrm{~F}),-105.86--105.76(\mathrm{~m}, 1 \mathrm{~F})$. Mass Spectrometry: HRMS-ESI ( $\mathrm{m} / \mathrm{z}$ ): Calcd for $\mathrm{C}_{14} \mathrm{H}_{13} \mathrm{Br}_{2} \mathrm{~F}_{4} \mathrm{O}_{3}[\mathrm{M}+\mathrm{H}]^{+}$, 462.9162. Found, 462.9143.

\section{6,6-Dibromo-5-(trifluoromethoxy)hex-5-en-1-yl 4-chlorobenzoate (3i)}

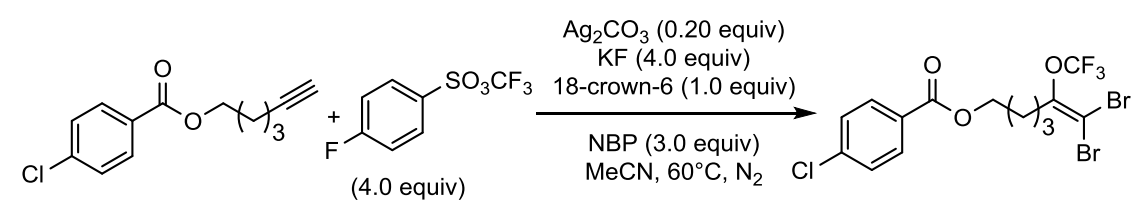

In a glove box, to a $15.0 \mathrm{~mL}$ sealed tube were added in sequence hex-5-yn-1-yl 4-chlorobenzoate (1i) (118 mg, $0.500 \mathrm{mmol}, 1.00$ equiv), KF (116 mg, $2.00 \mathrm{mmol}, 4.00$ equiv), 18-crown-6 (132 mg, 0.500 mmol, 1.00 equiv), $\quad \mathrm{Ag}_{2} \mathrm{CO}_{3} \quad(27.5 \mathrm{mg}, \quad 0.100 \mathrm{mmol}, 0.200$ equiv), NBP 
( $N$-Bromophthalimide) (339 mg, $1.50 \mathrm{mmol}, 3.00$ equiv) and $1.00 \mathrm{~mL} \mathrm{MeCN}$. The mixture was stirring at room temperature for $5 \mathrm{mins}$, then added TFMS ( $489 \mathrm{mg}, 2.00 \mathrm{mmol}, 4.00$ equiv). After stirring for $4.0 \mathrm{~h}$ at $60{ }^{\circ} \mathrm{C}$, the reaction mixture was concentrated in vacuo. The residue was then added $10 \mathrm{~mL} \mathrm{CH}_{2} \mathrm{Cl}_{2}$ and filtered. The organic layer was concentrated in vacuo. The residue was purified by preparative TLC, eluting with hexanes/EtOAc 40:1 (v/v) to afford $194 \mathrm{mg}$ 6,6-dibromo-5-(trifluoromethoxy)hex-5-en-1-yl 4-chlorobenzoate (3i) as a colorless liquid (81\% yield).

$\mathrm{R}_{f}=0.20$ (hexanes/EtOAc 40:1 (v/v)). NMR Spectroscopy: ${ }^{1} \mathrm{H}$ NMR $\left(400 \mathrm{MHz}, \mathrm{CDCl}_{3}\right) \delta 7.96(\mathrm{~d}$, $J=8.5 \mathrm{~Hz}, 2 \mathrm{H}), 7.41(\mathrm{~d}, J=8.5 \mathrm{~Hz}, 2 \mathrm{H}), 4.34(\mathrm{t}, J=6.1 \mathrm{~Hz}, 2 \mathrm{H}), 2.65(\mathrm{t}, J=7.1 \mathrm{~Hz}, 2 \mathrm{H}), 1.90-$ $1.70(\mathrm{~m}, 4 \mathrm{H}) .{ }^{13} \mathrm{C}$ NMR $\left(101 \mathrm{MHz}, \mathrm{CDCl}_{3}\right) \delta 165.8,148.6,139.6,131.1,128.9,128.8,119.9$ (q, $J$ $=261.1 \mathrm{~Hz}$ ), 88.7, 64.5, 31.7, 27.8, 22.5. ${ }^{19} \mathrm{~F}$ NMR (376 MHz, $\left.\mathrm{CDCl}_{3}\right) \delta-56.60$ (s, 3F). Mass Spectrometry: HRMS-ESI (m/z): Calcd for $\mathrm{C}_{14} \mathrm{H}_{13} \mathrm{Br}_{2} \mathrm{ClF}_{3} \mathrm{O}_{3}[\mathrm{M}+\mathrm{H}]^{+}$, 478.8867. Found, 478.8861 .

\section{5,5-Dibromo-4-(trifluoromethoxy)pent-4-en-1-yl 4-bromobenzoate (3j)}

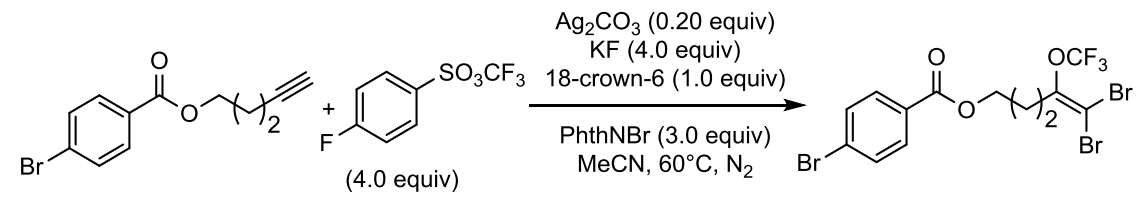

In a glove box, to a $15.0 \mathrm{~mL}$ sealed tube were added in sequence pent-4-yn-1-yl 4-bromobenzoate (1j) (133 mg, $0.500 \mathrm{mmol}, 1.00$ equiv), KF (116 mg, $2.00 \mathrm{mmol}, 4.00$ equiv), 18-crown-6 (132 mg, 0.500 mmol, 1.00 equiv), $\mathrm{Ag}_{2} \mathrm{CO}_{3}(27.5 \mathrm{mg}, 0.100 \mathrm{mmol}, 0.200$ equiv), NBP ( $N$-Bromophthalimide) (339 mg, $1.50 \mathrm{mmol}, 3.00$ equiv) and $1.00 \mathrm{~mL} \mathrm{MeCN}$. The mixture was stirring at room temperature for $5 \mathrm{mins}$, then added TFMS ( $489 \mathrm{mg}, 2.00 \mathrm{mmol}, 4.00$ equiv). After stirring for $4.0 \mathrm{~h}$ at $60{ }^{\circ} \mathrm{C}$, the reaction mixture was concentrated in vacuo. The residue was then added $10 \mathrm{~mL} \mathrm{CH}_{2} \mathrm{Cl}_{2}$ and filtered. The organic layer was concentrated in vacuo. The residue was purified by preparative TLC, eluting with hexanes/EtOAc 50:1 (v/v) to afford $204 \mathrm{mg}$ 5,5-dibromo-4-(trifluoromethoxy)pent-4-en-1-yl 4-bromobenzoate (3j) as a colorless liquid ( $80 \%$ yield).

$\mathrm{R}_{f}=0.30$ (hexanes/EtOAc 40:1 (v/v)). NMR Spectroscopy: ${ }^{1} \mathrm{H}$ NMR (400 MHz, $\left.\mathrm{CDCl}_{3}\right) \delta 7.89(\mathrm{~d}$, $J=8.5 \mathrm{~Hz}, 2 \mathrm{H}), 7.58(\mathrm{~d}, J=8.5 \mathrm{~Hz}, 2 \mathrm{H}), 4.34(\mathrm{t}, J=6.1 \mathrm{~Hz}, 2 \mathrm{H}), 2.76(\mathrm{t}, J=7.4 \mathrm{~Hz}, 2 \mathrm{H}), 2.11-$ $2.03(\mathrm{~m}, 2 \mathrm{H}) .{ }^{13} \mathrm{C}$ NMR $\left(101 \mathrm{MHz}, \mathrm{CDCl}_{3}\right) \delta 165.8,148.0,131.9,131.2,129.0,128.3,119.9$ (q, $J$ $=261.1 \mathrm{~Hz}$ ), 89.1, 63.8, 29.2, 25.2. ${ }^{19} \mathrm{~F}$ NMR $\left(376 \mathrm{MHz}, \mathrm{CDCl}_{3}\right) \delta-56.02$ (s, 3F). Mass Spectrometry: HRMS-ESI (m/z): Calcd for $\mathrm{C}_{13} \mathrm{H}_{11} \mathrm{Br}_{3} \mathrm{~F}_{3} \mathrm{O}_{3}[\mathrm{M}+\mathrm{H}]^{+}$, 508.8205. Found, 508.8203.

\section{6,6-Dibromo-5-(trifluoromethoxy)hex-5-en-1-yl 4-iodobenzoate (3k)}

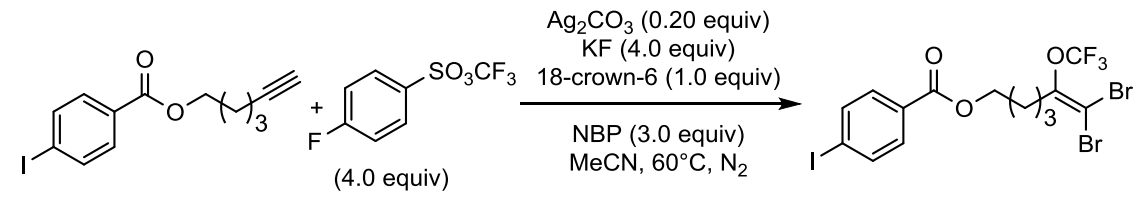

In a glove box, to a $15.0 \mathrm{~mL}$ sealed tube were added in sequence hex-5-yn-1-yl 4-iodobenzoate (1k) (164 mg, $0.500 \mathrm{mmol}, 1.00$ equiv), KF (116 mg, $2.00 \mathrm{mmol}, 4.00$ equiv), 18-crown-6 (132 mg, 0.500 mmol, 1.00 equiv), $\mathrm{Ag}_{2} \mathrm{CO}_{3}(27.5 \mathrm{mg}, 0.100 \mathrm{mmol}, 0.200$ equiv), NBP ( $N$-Bromophthalimide) (339 mg, $1.50 \mathrm{mmol}, 3.00$ equiv) and $1.00 \mathrm{~mL} \mathrm{MeCN}$. The mixture was 
stirring at room temperature for $5 \mathrm{mins}$, then added TFMS ( $489 \mathrm{mg}, 2.00 \mathrm{mmol}, 4.00$ equiv). After stirring for $4.0 \mathrm{~h}$ at $60{ }^{\circ} \mathrm{C}$, the reaction mixture was concentrated in vacuo. The residue was then added $10 \mathrm{~mL} \mathrm{CH}_{2} \mathrm{Cl}_{2}$ and filtered. The organic layer was concentrated in vacuo. The residue was purified by preparative TLC, eluting with hexanes/EtOAc 40:1 (v/v) to afford $235 \mathrm{mg}$ 6,6-dibromo-5-(trifluoromethoxy)hex-5-en-1-yl 4-iodobenzoate (3k) as a colorless liquid (82\% yield).

$\mathrm{R}_{f}=0.27$ (hexanes/EtOAc 40:1 (v/v)). NMR Spectroscopy: ${ }^{1} \mathrm{H}$ NMR $\left(400 \mathrm{MHz}, \mathrm{CDCl}_{3}\right) \delta 7.80(\mathrm{~d}$, $J=8.3 \mathrm{~Hz}, 2 \mathrm{H}), 7.73(\mathrm{~d}, J=8.4 \mathrm{~Hz}, 2 \mathrm{H}), 4.33(\mathrm{t}, J=6.1 \mathrm{~Hz}, 2 \mathrm{H}), 2.65(\mathrm{t}, J=7.1 \mathrm{~Hz}, 2 \mathrm{H}), 1.87-$ $1.71(\mathrm{~m}, 4 \mathrm{H}) .{ }^{13} \mathrm{C}$ NMR $\left(101 \mathrm{MHz}, \mathrm{CDCl}_{3}\right) \delta 166.1,148.6,137.9,131.1,129.8,119.9$ (q, $J=$ $260.9 \mathrm{~Hz}$ ), 100.9, 88.7, 64.5, 31.7, 27.9, 22.6. ${ }^{19} \mathrm{~F}$ NMR (376 MHz, $\left.\mathrm{CDCl}_{3}\right) \delta-56.23$ (s, 3F). Mass Spectrometry: HRMS-EI (m/z): Calcd for $\mathrm{C}_{14} \mathrm{H}_{12} \mathrm{Br}_{2} \mathrm{~F}_{3} \mathrm{IO}_{3}[\mathrm{M}]^{+}$, 569.8150. Found, 569.8159 .

\section{6,6-Dibromo-5-(trifluoromethoxy)hex-5-en-1-yl 4-(trifluoromethyl)benzoate (3I)}

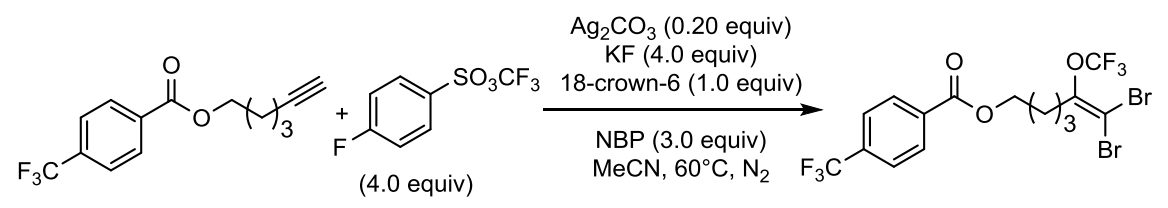

In a glove box, to a $15.0 \mathrm{~mL}$ sealed tube were added in sequence hex-5-yn-1-yl 4-(trifluoromethyl)benzoate (11) (135 mg, $0.500 \mathrm{mmol}, 1.00$ equiv), KF (116 mg, $2.00 \mathrm{mmol}, 4.00$ equiv), 18-crown-6 (132 mg, $0.500 \mathrm{mmol}, 1.00$ equiv), $\mathrm{Ag}_{2} \mathrm{CO}_{3}$ (27.5 mg, $0.100 \mathrm{mmol}, 0.200$ equiv), NBP ( $N$-Bromophthalimide) $(339 \mathrm{mg}, 1.50 \mathrm{mmol}, 3.00$ equiv) and $1.00 \mathrm{~mL} \mathrm{MeCN}$. The mixture was stirring at room temperature for $5 \mathrm{mins}$, then added TFMS (489 mg, $2.00 \mathrm{mmol}, 4.00$ equiv). After stirring for $4.0 \mathrm{~h}$ at $60{ }^{\circ} \mathrm{C}$, the reaction mixture was concentrated in vacuo. The residue was then added $10 \mathrm{~mL} \mathrm{CH} \mathrm{Cl}_{2}$ and filtered. The organic layer was concentrated in vacuo. The residue was purified by preparative TLC, eluting with hexanes/EtOAc 50:1 (v/v) to afford 203 mg 6,6-dibromo-5-(trifluoromethoxy)hex-5-en-1-yl 4-(trifluoromethyl)benzoate (3I) as a colorless liquid (79\% yield).

$\mathrm{R}_{f}=0.20$ (hexanes/EtOAc 40:1 (v/v)). NMR Spectroscopy: ${ }^{1} \mathrm{H}$ NMR $\left(400 \mathrm{MHz}, \mathrm{CDCl}_{3}\right) \delta 8.15(\mathrm{~d}$, $J=8.1 \mathrm{~Hz}, 2 \mathrm{H}), 7.71(\mathrm{~d}, J=8.2 \mathrm{~Hz}, 2 \mathrm{H}), 4.38(\mathrm{t}, J=6.2 \mathrm{~Hz}, 2 \mathrm{H}), 2.66(\mathrm{t}, J=7.2 \mathrm{~Hz}, 2 \mathrm{H}), 1.90-$ $1.73(\mathrm{~m}, 4 \mathrm{H}) .{ }^{13} \mathrm{C}$ NMR $\left(101 \mathrm{MHz}, \mathrm{CDCl}_{3}\right) \delta 165.4,148.6,134.6(\mathrm{q}, J=32.5 \mathrm{~Hz}), 133.6,130.1$, $125.6(\mathrm{q}, J=3.5 \mathrm{~Hz}), 123.8$ (q, $J=272.7 \mathrm{~Hz}), 119.9$ (q, $J=261.2 \mathrm{~Hz}), 88.7,64.8,31.7,27.9,22.6$. ${ }^{19} \mathrm{~F}$ NMR $\left(376 \mathrm{MHz}, \mathrm{CDCl}_{3}\right) \delta-56.63(\mathrm{~s}, 3 \mathrm{~F}),-62.62(\mathrm{~s}, 3 \mathrm{~F})$. Mass Spectrometry: HRMS-EI (m/z): Calcd for $\mathrm{C}_{15} \mathrm{H}_{12} \mathrm{Br}_{2} \mathrm{~F}_{6} \mathrm{O}_{3}[\mathrm{M}]^{+}$, 511.9057. Found, 511.9046 .

\section{5,5-Dibromo-4-(trifluoromethoxy)hex-5-en-1-yl 4-(trifluoromethoxy)benzoate (3m)}

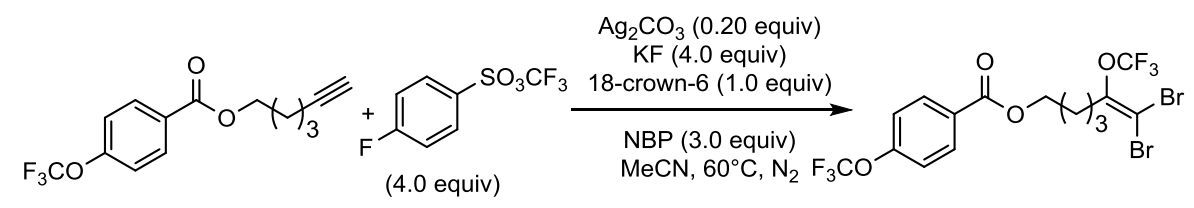

In a glove box, to a $15.0 \mathrm{~mL}$ sealed tube were added in sequence hex-5-yn-1-yl 4-(trifluoromethoxy)benzoate (1m) (143 mg, $0.500 \mathrm{mmol}, 1.00$ equiv), KF (116 mg, $2.00 \mathrm{mmol}$, 4.00 equiv), 18-crown-6 (132 mg, $0.500 \mathrm{mmol}, 1.00$ equiv), $\mathrm{Ag}_{2} \mathrm{CO}_{3}$ (27.5 mg, $0.100 \mathrm{mmol}, 0.200$ equiv), NBP ( $N$-Bromophthalimide) (339 mg, $1.50 \mathrm{mmol}, 3.00$ equiv) and $1.00 \mathrm{~mL} \mathrm{MeCN}$. The 
mixture was stirring at room temperature for 5 mins, then added TFMS ( $489 \mathrm{mg}, 2.00 \mathrm{mmol}, 4.00$ equiv). After stirring for $4.0 \mathrm{~h}$ at $60{ }^{\circ} \mathrm{C}$, the reaction mixture was concentrated in vacuo. The residue was then added $10 \mathrm{~mL} \mathrm{CH} \mathrm{Cl}_{2}$ and filtered. The organic layer was concentrated in vacuo. The residue was purified by preparative TLC, eluting with hexanes/EtOAc 40:1 (v/v) to afford 207mg 5,5-dibromo-4-(trifluoromethoxy)hex-5-en-1-yl 4-(trifluoromethoxy)benzoate (3m) as a colorless liquid ( $78 \%$ yield).

$\mathrm{R}_{f}=0.20$ (hexanes/EtOAc 40:1 (v/v)). NMR Spectroscopy: ${ }^{1} \mathrm{H}$ NMR $\left(400 \mathrm{MHz}, \mathrm{CDCl}_{3}\right) \delta 8.09(\mathrm{~d}$, $J=8.6 \mathrm{~Hz}, 2 \mathrm{H}), 7.27(\mathrm{~d}, J=8.2 \mathrm{~Hz}, 2 \mathrm{H}), 4.36(\mathrm{t}, J=5.9 \mathrm{~Hz}, 2 \mathrm{H}), 2.66(\mathrm{t}, J=7.0 \mathrm{~Hz}, 2 \mathrm{H}), 1.89-$ $1.73(\mathrm{~m}, 4 \mathrm{H}) .{ }^{13} \mathrm{C}$ NMR $\left(101 \mathrm{MHz}, \mathrm{CDCl}_{3}\right) \delta 165.4,152.8,148.7,131.7,128.8,120.5(\mathrm{q}, J=$ $258.8 \mathrm{~Hz}), 120.4,120.0$ (q, $J=261.0 \mathrm{~Hz}), 88.7,64.6,31.7,27.9,22.6 .{ }^{19} \mathrm{~F}$ NMR $(376 \mathrm{MHz}$, $\left.\mathrm{CDCl}_{3}\right) \delta-56.19$ (s, 3F), -57.12 (s, 3F). Mass Spectrometry: HRMS-ESI (m/z): Calcd for $\mathrm{C}_{15} \mathrm{H}_{13} \mathrm{Br}_{2} \mathrm{~F}_{6} \mathrm{O}_{4}[\mathrm{M}+\mathrm{H}]^{+}$, 528.9079. Found, 528.9085.

\section{5,5-Dibromo-4-(trifluoromethoxy)pent-4-en-1-yl 4-benzoylbenzoate (3n)}

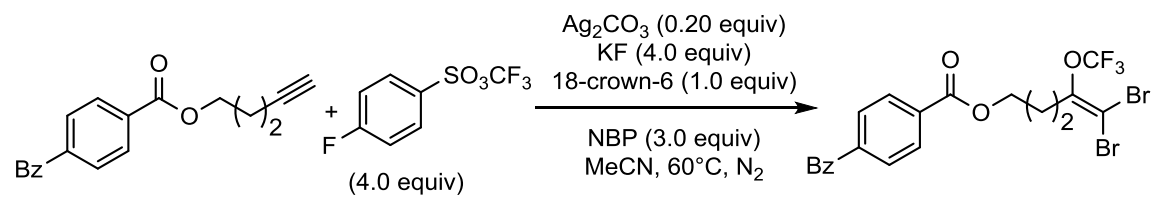

In a glove box, to a $15.0 \mathrm{~mL}$ sealed tube were added in sequence pent-4-yn-1-yl 4-benzoylbenzoate (1n) (146 mg, $0.500 \mathrm{mmol}, 1.00$ equiv), KF (116 mg, $2.00 \mathrm{mmol}, 4.00$ equiv), 18-crown-6 (132 mg, $0.500 \mathrm{mmol}, 1.00$ equiv), $\mathrm{Ag}_{2} \mathrm{CO}_{3}$ (27.5 mg, $0.100 \mathrm{mmol}, 0.200$ equiv), NBP ( $N$-Bromophthalimide) (339 mg, $1.50 \mathrm{mmol}, 3.00$ equiv) and $1.00 \mathrm{~mL} \mathrm{MeCN}$. The mixture was stirring at room temperature for 5 mins, then added TFMS ( $489 \mathrm{mg}, 2.00 \mathrm{mmol}, 4.00$ equiv). After stirring for $4.0 \mathrm{~h}$ at $60{ }^{\circ} \mathrm{C}$, the reaction mixture was concentrated in vacuo. The residue was then added $10 \mathrm{~mL} \mathrm{CH}_{2} \mathrm{Cl}_{2}$ and filtered. The organic layer was concentrated in vacuo. The residue was purified by preparative TLC, eluting with hexanes/EtOAc 20:1 (v/v) to afford $215 \mathrm{mg}$ 5,5-dibromo-4-(trifluoromethoxy)pent-4-en-1-yl 4-benzoylbenzoate (3n) as a white solid (78\% yield).

$\mathrm{R}_{f}=0.25$ (hexanes/EtOAc 20:1 (v/v)). NMR Spectroscopy: ${ }^{1} \mathrm{H}$ NMR (400 MHz, $\left.\mathrm{CDCl}_{3}\right) \delta 8.14(\mathrm{~d}$, $J=8.3 \mathrm{~Hz}, 2 \mathrm{H}), 7.84(\mathrm{~d}, J=8.3 \mathrm{~Hz}, 2 \mathrm{H}), 7.80(\mathrm{~d}, J=8.3 \mathrm{~Hz}, 2 \mathrm{H}), 7.61(\mathrm{t}, J=7.4 \mathrm{~Hz}, 1 \mathrm{H}), 7.53-$ $7.46(\mathrm{~m}, 2 \mathrm{H}), 4.40(\mathrm{t}, J=6.1 \mathrm{~Hz}, 2 \mathrm{H}), 2.79(\mathrm{t}, J=7.4 \mathrm{~Hz}, 2 \mathrm{H}), 2.16-2.04(\mathrm{~m}, 2 \mathrm{H}) .{ }^{13} \mathrm{C} \mathrm{NMR}$ $\left(101 \mathrm{MHz}, \mathrm{CDCl}_{3}\right) \delta 196.1,165.7,148.0,141.6,137.0,133.1,133.1,130.2,129.9,129.6,128.6$, 119.9 (q, $J=261.3 \mathrm{~Hz}), 89.2,64.0,29.2,25.2 .{ }^{19} \mathrm{~F} \mathrm{NMR}\left(376 \mathrm{MHz}, \mathrm{CDCl}_{3}\right) \delta-56.50$ (s, 3F). Mass Spectrometry: HRMS-ESI (m/z): Calcd for $\mathrm{C}_{20} \mathrm{H}_{16} \mathrm{Br}_{2} \mathrm{~F}_{3} \mathrm{O}_{4}[\mathrm{M}+\mathrm{H}]^{+}$, 534.9362. Found, 534.9358.

\section{6,6-Dibromo-5-(trifluoromethoxy)hex-5-en-1-yl 4-cyanobenzoate (3o)}

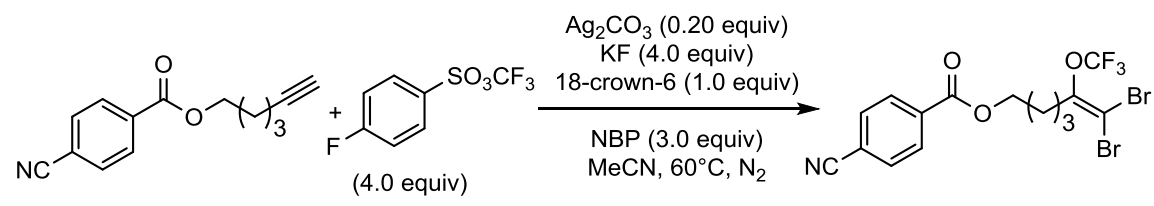

In a glove box, to a $15.0 \mathrm{~mL}$ sealed tube were added in sequence hex-5-yn-1-yl 4-cyanobenzoate (1o) (114 mg, $0.500 \mathrm{mmol}, 1.00$ equiv), KF (116 mg, $2.00 \mathrm{mmol}, 4.00$ equiv), 18-crown-6 (132 $\mathrm{mg}, \quad 0.500$ mmol, 1.00 equiv), $\mathrm{Ag}_{2} \mathrm{CO}_{3}(27.5 \mathrm{mg}, 0.100 \mathrm{mmol}, 0.200$ equiv), NBP ( $N$-Bromophthalimide) (339 mg, $1.50 \mathrm{mmol}, 3.00$ equiv) and $1.00 \mathrm{~mL} \mathrm{MeCN}$. The mixture was 
stirring at room temperature for $5 \mathrm{mins}$, then added TFMS ( $489 \mathrm{mg}, 2.00 \mathrm{mmol}, 4.00$ equiv). After stirring for $4.0 \mathrm{~h}$ at $60{ }^{\circ} \mathrm{C}$, the reaction mixture was concentrated in vacuo. The residue was then added $10 \mathrm{~mL} \mathrm{CH}_{2} \mathrm{Cl}_{2}$ and filtered. The organic layer was concentrated in vacuo. The residue was purified by preparative TLC, eluting with hexanes/EtOAc 10:1 (v/v) to afford $186 \mathrm{mg}$ 6,6-dibromo-5-(trifluoromethoxy)hex-5-en-1-yl 4-cyanobenzoate (30) as a colorless liquid (79\% yield).

$\mathrm{R}_{f}=0.08$ (hexanes/EtOAc 20:1 (v/v)). NMR Spectroscopy: ${ }^{1} \mathrm{H}$ NMR $\left(400 \mathrm{MHz}, \mathrm{CDCl}_{3}\right) \delta 8.12(\mathrm{~d}$, $J=8.0 \mathrm{~Hz}, 2 \mathrm{H}), 7.74(\mathrm{~d}, J=8.1 \mathrm{~Hz}, 2 \mathrm{H}), 4.37(\mathrm{t}, J=6.2 \mathrm{~Hz}, 2 \mathrm{H}), 2.65(\mathrm{t}, J=7.2 \mathrm{~Hz}, 2 \mathrm{H}), 1.91-$ $1.70(\mathrm{~m}, 4 \mathrm{H}) .{ }^{13} \mathrm{C}$ NMR $\left(101 \mathrm{MHz}, \mathrm{CDCl}_{3}\right) \delta 165.0,148.5,134.1,132.3,130.2,119.9$ (q, $J=$ $261.1 \mathrm{~Hz}), 118.0,116.6,88.7,65.0,31.6,27.7,22.5 .{ }^{19} \mathrm{~F} \mathrm{NMR}\left(376 \mathrm{MHz}, \mathrm{CDCl}_{3}\right) \delta-55.67(\mathrm{~s}, 3 \mathrm{~F})$. Mass Spectrometry: HRMS-EI (m/z): Calcd for $\mathrm{C}_{15} \mathrm{H}_{12} \mathrm{Br}_{2} \mathrm{~F}_{3} \mathrm{NO}_{3}[\mathrm{M}]^{+}$, 468.9136. Found, 468.9134 .

\section{6,6-Dibromo-5-(trifluoromethoxy)hex-5-en-1-yl 4-nitrobenzoate (3p)}

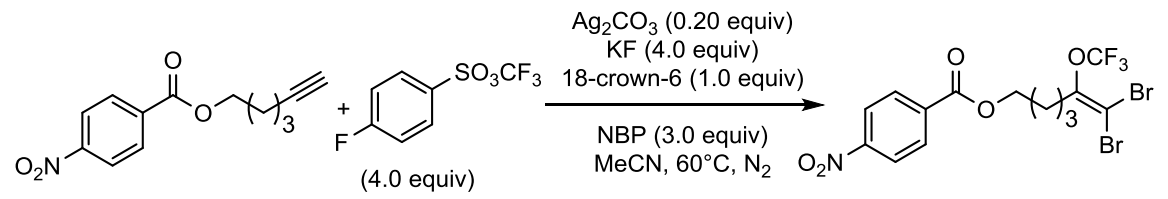

In a glove box, to a $15.0 \mathrm{~mL}$ sealed tube were added in sequence hex-5-yn-1-yl 4-nitrobenzoate (1p) (124 mg, $0.500 \mathrm{mmol}, 1.00$ equiv), KF (116 mg, $2.00 \mathrm{mmol}, 4.00$ equiv), 18-crown-6 (132 $\mathrm{mg}, 0.500 \mathrm{mmol}, 1.00$ equiv), $\mathrm{Ag}_{2} \mathrm{CO}_{3}(27.5 \mathrm{mg}, 0.100 \mathrm{mmol}, 0.200$ equiv), NBP ( $N$-Bromophthalimide) (339 mg, $1.50 \mathrm{mmol}, 3.00$ equiv) and $1.00 \mathrm{~mL} \mathrm{MeCN}$. The mixture was stirring at room temperature for $5 \mathrm{mins}$, then added TFMS (489 mg, $2.00 \mathrm{mmol}, 4.00$ equiv). After stirring for $4.0 \mathrm{~h}$ at $60{ }^{\circ} \mathrm{C}$, the reaction mixture was concentrated in vacuo. The residue was then added $10 \mathrm{~mL} \mathrm{CH}_{2} \mathrm{Cl}_{2}$ and filtered. The organic layer was concentrated in vacuo. The residue was purified by preparative TLC, eluting with hexanes/EtOAc 30:1 (v/v) to afford $199 \mathrm{mg}$ 6,6-dibromo-5-(trifluoromethoxy)hex-5-en-1-yl 4-nitrobenzoate (3p) as a yellow liquid (81\% yield).

$\mathrm{R}_{f}=0.30$ (hexanes/EtOAc 20:1 (v/v)). NMR Spectroscopy: ${ }^{1} \mathrm{H}$ NMR $\left(400 \mathrm{MHz}, \mathrm{CDCl}_{3}\right) \delta 8.28(\mathrm{~d}$, $J=8.8 \mathrm{~Hz}, 2 \mathrm{H}), 8.20$ (d, $J=8.8 \mathrm{~Hz}, 2 \mathrm{H}), 4.40(\mathrm{t}, J=6.2 \mathrm{~Hz}, 2 \mathrm{H}), 2.66(\mathrm{t}, J=7.3 \mathrm{~Hz}, 2 \mathrm{H}), 1.90-$ $1.72(\mathrm{~m}, 4 \mathrm{H}) .{ }^{13} \mathrm{C}$ NMR $\left(101 \mathrm{MHz}, \mathrm{CDCl}_{3}\right) \delta 164.7,150.7,148.5$ (q, $\left.J=1.2 \mathrm{~Hz}\right), 135.7,130.8$, 123.7, 119.9 (q, $J=261.1 \mathrm{~Hz}), 88.8,65.2,31.6,27.8,22.5 .{ }^{19} \mathrm{~F} \mathrm{NMR}\left(376 \mathrm{MHz}, \mathrm{CDCl}_{3}\right) \delta-56.49$ (s, 3F). Mass Spectrometry: HRMS-EI (m/z): Calcd for $\mathrm{C}_{14} \mathrm{H}_{12} \mathrm{Br}_{2} \mathrm{~F}_{3} \mathrm{NO}_{5}[\mathrm{M}]^{+}, 488.9034$. Found, 488.9042 .

\section{6,6-Dibromo-5-(trifluoromethoxy)hex-5-en-1-yl 4-(methylsulfonyl)benzoate (3q)}

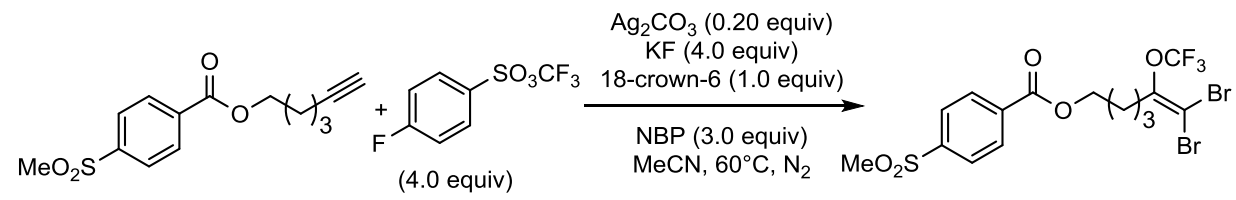

In a glove box, to a $15.0 \mathrm{~mL}$ sealed tube were added in sequence hex-5-yn-1-yl 4-(methylsulfonyl)benzoate (1q) (140 mg, $0.500 \mathrm{mmol}, 1.00$ equiv), KF (116 mg, $2.00 \mathrm{mmol}, 4.00$ equiv), 18-crown-6 (132 mg, $0.500 \mathrm{mmol}, 1.00$ equiv), $\mathrm{Ag}_{2} \mathrm{CO}_{3}$ (27.5 mg, $0.100 \mathrm{mmol}, 0.200$ equiv), NBP ( $N$-Bromophthalimide) $(339 \mathrm{mg}, 1.50 \mathrm{mmol}, 3.00$ equiv) and $1.00 \mathrm{~mL} \mathrm{MeCN}$. The 
mixture was stirring at room temperature for 5 mins, then added TFMS (489 mg, $2.00 \mathrm{mmol}, 4.00$ equiv). After stirring for $4.0 \mathrm{~h}$ at $60{ }^{\circ} \mathrm{C}$, the reaction mixture was concentrated in vacuo. The residue was then added $10 \mathrm{~mL} \mathrm{CH}_{2} \mathrm{Cl}_{2}$ and filtered. The organic layer was concentrated in vacuo. The residue was purified by preparative TLC, eluting with hexanes/EtOAc 8:1 (v/v) to afford 210 mg 6,6-dibromo-5-(trifluoromethoxy)hex-5-en-1-yl 4-(methylsulfonyl)benzoate (3q) as a colorless liquid ( $80 \%$ yield).

$\mathrm{R}_{f}=0.05$ (hexanes/EtOAc 8:1 (v/v)). NMR Spectroscopy: ${ }^{1} \mathrm{H}$ NMR $\left(400 \mathrm{MHz}, \mathrm{CDCl}_{3}\right) \delta 8.18(\mathrm{~d}$, $J=8.4 \mathrm{~Hz}, 2 \mathrm{H}), 7.99$ (d, $J=8.4 \mathrm{~Hz}, 2 \mathrm{H}), 4.36$ (t, $J=6.2 \mathrm{~Hz}, 2 \mathrm{H}), 3.05$ (s, 3H), 2.62 (t, $J=7.2 \mathrm{~Hz}$, 2H), $1.90-1.65(\mathrm{~m}, 4 \mathrm{H}) .{ }^{13} \mathrm{C}$ NMR $\left(101 \mathrm{MHz}, \mathrm{CDCl}_{3}\right) \delta 164.9,148.4(\mathrm{q}, J=1.2 \mathrm{~Hz}), 144.3$, $134.9,130.5,127.5,119.8$ (q, $J=261.0 \mathrm{~Hz}), 88.6,65.0,44.3,31.5,27.6,22.4 .{ }^{19} \mathrm{~F}$ NMR $(376$ $\left.\mathrm{MHz}, \mathrm{CDCl}_{3}\right) \delta-56.56(\mathrm{~s}, 3 \mathrm{~F})$. Mass Spectrometry: HRMS-ESI (m/z): Calcd for $\mathrm{C}_{15} \mathrm{H}_{16} \mathrm{Br}_{2} \mathrm{~F}_{3} \mathrm{O}_{5} \mathrm{~S}$ $[\mathrm{M}+\mathrm{H}]^{+}$, 522.9032. Found, 522.9028.

\section{4-((3,3-Dibromo-2-(trifluoromethoxy)allyl)oxy)benzaldehyde (3r)}

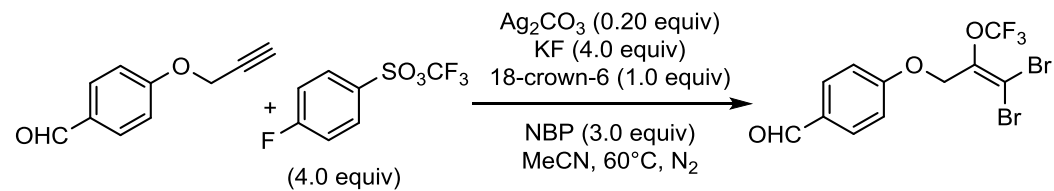

In a glove box, to a $15.0 \mathrm{~mL}$ sealed tube were added in sequence 4-(prop-2-yn-1-yloxy)benzaldehyde (1r) ${ }^{[7]}$ ( $80 \mathrm{mg}, 0.500 \mathrm{mmol}, 1.00$ equiv), KF (116 mg, 2.00 mmol, 4.00 equiv), 18 -crown-6 (132 mg, $0.500 \mathrm{mmol}, 1.00$ equiv), $\mathrm{Ag}_{2} \mathrm{CO}_{3}(27.5 \mathrm{mg}, 0.100 \mathrm{mmol}$, 0.200 equiv), NBP ( $N$-Bromophthalimide) $(339 \mathrm{mg}, 1.50 \mathrm{mmol}, 3.00$ equiv) and $1.00 \mathrm{~mL} \mathrm{MeCN}$. The mixture was stirring at room temperature for $5 \mathrm{mins}$, then added TFMS (489 mg, $2.00 \mathrm{mmol}$, 4.00 equiv). After stirring for $4.0 \mathrm{~h}$ at $60^{\circ} \mathrm{C}$, the reaction mixture was concentrated in vacuo. The residue was then added $10 \mathrm{~mL} \mathrm{CH}_{2} \mathrm{Cl}_{2}$ and filtered. The organic layer was concentrated in vacuo. The residue was purified by preparative TLC, eluting with hexanes/EtOAc30:1 (v/v) to afford 127 mg 4-((3,3-dibromo-2-(trifluoromethoxy)allyl)oxy)benzaldehyde (3r) as a colorless liquid (63\% yield).

$\mathrm{R}_{f}=0.10$ (hexanes/EtOAc 20:1 (v/v)). NMR Spectroscopy: ${ }^{1} \mathrm{H}$ NMR $\left(400 \mathrm{MHz}, \mathrm{CDCl}_{3}\right) \delta 9.90(\mathrm{~s}$, $1 \mathrm{H}), 7.86(\mathrm{~d}, J=8.6 \mathrm{~Hz}, 2 \mathrm{H}), 7.03(\mathrm{~d}, J=8.5 \mathrm{~Hz}, 2 \mathrm{H}), 4.92(\mathrm{~s}, 2 \mathrm{H}) .{ }^{13} \mathrm{C} \mathrm{NMR}\left(101 \mathrm{MHz}, \mathrm{CDCl}_{3}\right)$ $\delta 190.8,162.5,143.2,143.2,132.1,131.0,119.9(\mathrm{q}, J=262.3 \mathrm{~Hz}), 115.1,95.7,65.3 .{ }^{19} \mathrm{~F}$ NMR $\left(376 \mathrm{MHz}, \mathrm{CDCl}_{3}\right) \delta-56.12(\mathrm{~s}, 3 \mathrm{~F})$. Mass Spectrometry: HRMS-ESI $(\mathrm{m} / \mathrm{z})$ : Calcd for $\mathrm{C}_{11} \mathrm{H}_{8} \mathrm{Br}_{2} \mathrm{~F}_{3} \mathrm{O}_{3}[\mathrm{M}+\mathrm{H}]^{+}$, 402.8787. Found, 402.8782 .

\section{4-((3,3-Dibromo-2-(trifluoromethoxy)allyl)oxy)benzoic acid (3s)}

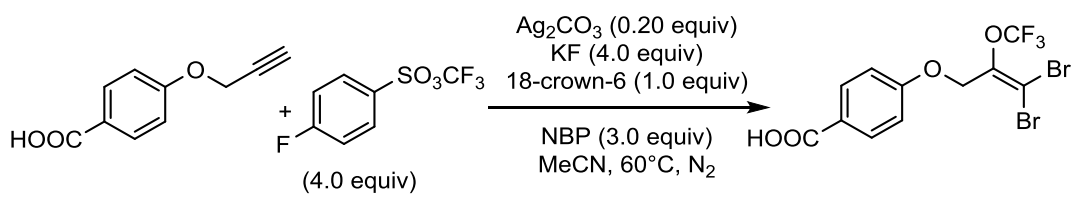

In a glove box, to a $15.0 \mathrm{~mL}$ sealed tube were added in sequence 4-(prop-2-yn-1-yloxy)benzoic acid (1s) ( $88 \mathrm{mg}, 0.500 \mathrm{mmol}, 1.00$ equiv), KF (116 mg, $2.00 \mathrm{mmol}, 4.00$ equiv), 18-crown-6 (132 $\mathrm{mg}, \quad 0.500 \mathrm{mmol}, 1.00$ equiv), $\mathrm{Ag}_{2} \mathrm{CO}_{3}(27.5 \mathrm{mg}, 0.100 \mathrm{mmol}, 0.200$ equiv), NBP ( $N$-Bromophthalimide) (339 mg, $1.50 \mathrm{mmol}, 3.00$ equiv) and $1.00 \mathrm{~mL} \mathrm{MeCN}$. The mixture was stirring at room temperature for $5 \mathrm{mins}$, then added TFMS ( $489 \mathrm{mg}, 2.00 \mathrm{mmol}, 4.00$ equiv). After 
stirring for $4.0 \mathrm{~h}$ at $60{ }^{\circ} \mathrm{C}$, the reaction mixture was concentrated in vacuo. The residue was then added $10 \mathrm{~mL} \mathrm{CH}_{2} \mathrm{Cl}_{2}$ and filtered. The organic layer was concentrated in vacuo. The residue was purified by preparative TLC, eluting with hexanes/EtOAc 1:1 (v/v) to afford $78 \mathrm{mg}$ 4-((3,3-dibromo-2-(trifluoromethoxy)allyl)oxy)benzoic acid (3s) as a white solid (37\% yield). $\mathrm{R}_{f}=0.78$ (hexanes/EtOAc 1:1 (v/v)). NMR Spectroscopy: ${ }^{1} \mathrm{H}$ NMR $\left(400 \mathrm{MHz}, \mathrm{CDCl}_{3}\right) \delta 8.10(\mathrm{~d}$, $J=8.6 \mathrm{~Hz}, 2 \mathrm{H}), 6.98(\mathrm{~d}, J=8.6 \mathrm{~Hz}, 2 \mathrm{H}), 4.92(\mathrm{~s}, 2 \mathrm{H}) .{ }^{13} \mathrm{C} \mathrm{NMR}\left(101 \mathrm{MHz}, \mathrm{CDCl}_{3}\right) \delta 162.1$, 143.4, 132.6, 123.8, 123.1, 119.9 (q, $J=262.4 \mathrm{~Hz}), 116.0,114.6,95.6,65.3 .{ }^{19} \mathrm{~F}$ NMR (376 MHz, $\left.\mathrm{CDCl}_{3}\right) \delta-56.01$ (s, 3F). Mass Spectrometry: HRMS-ESI (m/z): Calcd for $\mathrm{C}_{11} \mathrm{H}_{6} \mathrm{Br}_{2} \mathrm{~F}_{3} \mathrm{O}_{4}[\mathrm{M}-\mathrm{H}]^{-}$, 416.8590. Found, 416.8589 .

\section{4-Chlorobutyl 4-((3,3-dibromo-2-(trifluoromethoxy)allyl)oxy)benzoate (3t)}

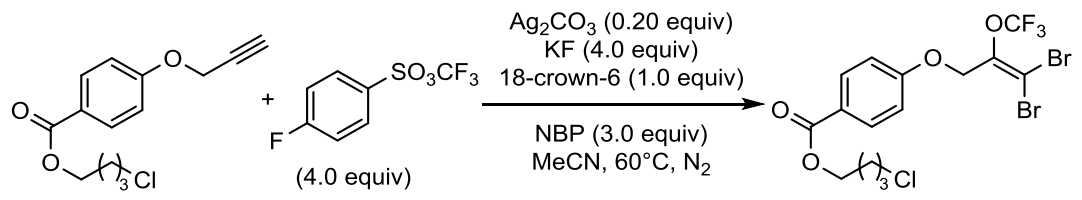

In a glove box, to a $15.0 \mathrm{~mL}$ sealed tube were added in sequence 4-chlorobutyl 4-(prop-2-yn-1-yloxy)benzoate (1t) $(133 \mathrm{mg}, 0.500 \mathrm{mmol}, 1.00$ equiv), KF (116 mg, $2.00 \mathrm{mmol}$, 4.00 equiv), 18-crown-6 (132 mg, $0.500 \mathrm{mmol}, 1.00$ equiv), $\mathrm{Ag}_{2} \mathrm{CO}_{3}$ (27.5 mg, $0.100 \mathrm{mmol}, 0.200$ equiv), NBP ( $N$-Bromophthalimide) $(339 \mathrm{mg}, 1.50 \mathrm{mmol}, 3.00$ equiv) and $1.00 \mathrm{~mL} \mathrm{MeCN}$. The mixture was stirring at room temperature for $5 \mathrm{mins}$, then added TFMS (489 mg, $2.00 \mathrm{mmol}, 4.00$ equiv). After stirring for $4.0 \mathrm{~h}$ at $60^{\circ} \mathrm{C}$, the reaction mixture was concentrated in vacuo. The residue was then added $10 \mathrm{~mL} \mathrm{CH} \mathrm{Cl}_{2}$ and filtered. The organic layer was concentrated in vacuo. The residue was purified by preparative TLC, eluting with hexanes/EtOAc 50:1 (v/v) to afford 204 mg 4-chlorobutyl 4-((3,3-dibromo-2-(trifluoromethoxy)allyl)oxy)benzoate (3t) as a colorless liquid ( $80 \%$ yield).

$\mathrm{R}_{f}=0.08$ (hexanes/EtOAc 40:1 (v/v)). NMR Spectroscopy: ${ }^{1} \mathrm{H}$ NMR $\left(400 \mathrm{MHz}, \mathrm{CDCl}_{3}\right) \delta 8.00(\mathrm{~d}$, $J=8.8 \mathrm{~Hz}, 2 \mathrm{H}), 6.94(\mathrm{~d}, J=8.8 \mathrm{~Hz}, 2 \mathrm{H}), 4.89$ (s, 2H), $4.37-4.27$ (m, 2H), 3.59 (t, $J=5.8 \mathrm{~Hz}$, 2H), $1.99-1.82(\mathrm{~m}, 4 \mathrm{H}) .{ }^{13} \mathrm{C} \mathrm{NMR}\left(101 \mathrm{MHz}, \mathrm{CDCl}_{3}\right) \delta 166.0,161.4,143.4,131.7,124.0,119.8$ $(\mathrm{q}, J=262.1 \mathrm{~Hz}), 114.5,95.4,65.2,64.0,44.6,29.3,26.2 .{ }^{19} \mathrm{~F} \mathrm{NMR}\left(376 \mathrm{MHz}, \mathrm{CDCl}_{3}\right) \delta-56.66$ (s, 3F). Mass Spectrometry: HRMS-ESI (m/z): Calcd for $\mathrm{C}_{15} \mathrm{H}_{15} \mathrm{Br}_{2} \mathrm{ClF}_{3} \mathrm{O}_{4}[\mathrm{M}+\mathrm{H}]^{+}, 508.8972$. Found, 508.8967.

\section{Oxiran-2-ylmethyl 4-((3,3-dibromo-2-(trifluoromethoxy)allyl)oxy)benzoate (3u)}

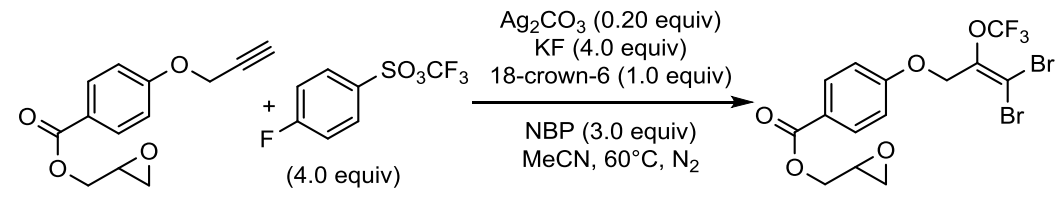

In a glove box, to a $15.0 \mathrm{~mL}$ sealed tube were added in sequence oxiran-2-ylmethyl 4-(prop-2-yn-1-yloxy)benzoate (1u) (116 mg, $0.500 \mathrm{mmol}, 1.00$ equiv), KF (116 mg, $2.00 \mathrm{mmol}$, 4.00 equiv), 18-crown-6 (132 mg, $0.500 \mathrm{mmol}, 1.00$ equiv), $\mathrm{Ag}_{2} \mathrm{CO}_{3}$ (27.5 mg, $0.100 \mathrm{mmol}, 0.200$ equiv), NBP ( $N$-Bromophthalimide) $(339 \mathrm{mg}, 1.50 \mathrm{mmol}, 3.00$ equiv) and $1.00 \mathrm{~mL} \mathrm{MeCN}$. The mixture was stirring at room temperature for 5 mins, then added TFMS (489 mg, $2.00 \mathrm{mmol}, 4.00$ equiv). After stirring for $4.0 \mathrm{~h}$ at $60{ }^{\circ} \mathrm{C}$, the reaction mixture was concentrated in vacuo. The residue was then added $10 \mathrm{~mL} \mathrm{CH}_{2} \mathrm{Cl}_{2}$ and filtered. The organic layer was concentrated in vacuo. 
The residue was purified by preparative TLC, eluting with hexanes/EtOAc 10:1 (v/v) to afford 171 mg oxiran-2-ylmethyl 4-((3,3-dibromo-2-(trifluoromethoxy)allyl)oxy)benzoate (3u) as a colorless liquid (72\% yield).

$\mathrm{R}_{f}=0.07$ (hexanes/EtOAc 20:1 (v/v)). NMR Spectroscopy: ${ }^{1} \mathrm{H}$ NMR (400 MHz, $\left.\mathrm{CDCl}_{3}\right) \delta 8.04(\mathrm{~d}$, $J=8.7 \mathrm{~Hz}, 2 \mathrm{H}), 6.95(\mathrm{~d}, J=8.7 \mathrm{~Hz}, 2 \mathrm{H}), 4.90(\mathrm{~s}, 2 \mathrm{H}), 4.63(\mathrm{dd}, J=12.3,2.7 \mathrm{~Hz}, 1 \mathrm{H}), 4.13(\mathrm{dd}, J$ $=12.3,6.3 \mathrm{~Hz}, 1 \mathrm{H}), 3.36-3.28(\mathrm{~m}, 1 \mathrm{H}), 2.88(\mathrm{t}, J=4.4 \mathrm{~Hz}, 1 \mathrm{H}), 2.74-2.69(\mathrm{~m}, 1 \mathrm{H}) .{ }^{13} \mathrm{C} \mathrm{NMR}$ $\left(101 \mathrm{MHz}, \mathrm{CDCl}_{3}\right) \delta 165.8,161.6,143.4,132.0,123.4,119.9$ (q, $\left.J=262.3 \mathrm{~Hz}\right), 114.6,95.5,65.4$, 65.3, 49.6, 44.8. ${ }^{19} \mathrm{~F}$ NMR $\left(376 \mathrm{MHz}, \mathrm{CDCl}_{3}\right) \delta-56.57$ (s, 3F). Mass Spectrometry: HRMS-ESI (m/z): Calcd for $\mathrm{C}_{14} \mathrm{H}_{12} \mathrm{Br}_{2} \mathrm{~F}_{3} \mathrm{O}_{5}[\mathrm{M}+\mathrm{H}]^{+}$, 474.8998. Found, 474.8994.

\section{2-(Trimethylsilyl)ethyl 4-((3,3-dibromo-2-(trifluoromethoxy)allyl)oxy)benzoate (3v)}

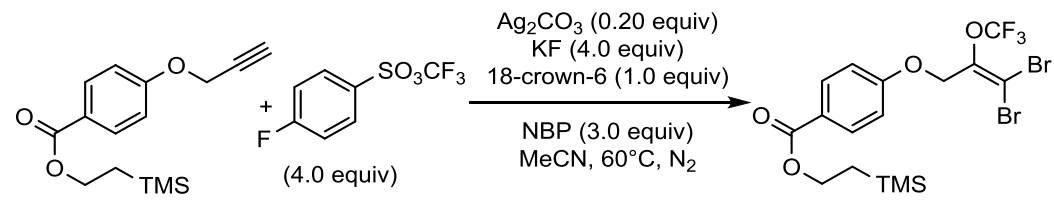

In a glove box, to a $15.0 \mathrm{~mL}$ sealed tube were added in sequence 2-(trimethylsilyl)ethyl 4-(prop-2-yn-1-yloxy)benzoate (1v) (138 mg, $0.500 \mathrm{mmol}, 1.00$ equiv), KF (116 mg, $2.00 \mathrm{mmol}$, 4.00 equiv), 18-crown-6 (132 mg, $0.500 \mathrm{mmol}, 1.00$ equiv), $\mathrm{Ag}_{2} \mathrm{CO}_{3}$ (27.5 mg, $0.100 \mathrm{mmol}, 0.200$ equiv), NBP ( $N$-Bromophthalimide) $(339 \mathrm{mg}, 1.50 \mathrm{mmol}, 3.00$ equiv) and $1.00 \mathrm{~mL} \mathrm{MeCN}$. The mixture was stirring at room temperature for $5 \mathrm{mins}$, then added TFMS (489 $\mathrm{mg}, 2.00 \mathrm{mmol}, 4.00$ equiv). After stirring for $4.0 \mathrm{~h}$ at $60{ }^{\circ} \mathrm{C}$, the reaction mixture was concentrated in vacuo. The residue was then added $10 \mathrm{~mL} \mathrm{CH} \mathrm{Cl}_{2}$ and filtered. The organic layer was concentrated in vacuo. The residue was purified by preparative TLC, eluting with hexanes/EtOAc 40:1 (v/v) to afford 177 mg 2-(trimethylsilyl)ethyl 4-((3,3-dibromo-2-(trifluoromethoxy)allyl)oxy)benzoate (3v) as a colorless liquid (68\% yield).

$\mathrm{R}_{f}=0.18$ (hexanes/EtOAc 40:1 (v/v)). NMR Spectroscopy: ${ }^{1} \mathrm{H}$ NMR $\left(400 \mathrm{MHz}, \mathrm{CDCl}_{3}\right) \delta 8.01(\mathrm{~d}$, $J=8.9 \mathrm{~Hz}, 2 \mathrm{H}), 6.94(\mathrm{~d}, J=8.9 \mathrm{~Hz}, 2 \mathrm{H}), 4.89(\mathrm{~s}, 2 \mathrm{H}), 4.43-4.36(\mathrm{~m}, 2 \mathrm{H}), 1.15-1.08(\mathrm{~m}, 2 \mathrm{H})$, 0.08 (s, 9H). ${ }^{13} \mathrm{C} \mathrm{NMR}\left(101 \mathrm{MHz}, \mathrm{CDCl}_{3}\right) \delta 166.3,161.3,143.6,131.7,124.6,119.9$ (q, $J=262.3$ $\mathrm{Hz}$ ), 114.5, 95.4, 65.3, 63.2, 17.6, -1.3. ${ }^{19} \mathrm{~F} \mathrm{NMR}\left(376 \mathrm{MHz}, \mathrm{CDCl}_{3}\right) \delta-56.54$ (s, 3F). Mass Spectrometry: HRMS-ESI (m/z): Calcd for $\mathrm{C}_{16} \mathrm{H}_{19} \mathrm{Br}_{2} \mathrm{~F}_{3} \mathrm{NaO}_{4} \mathrm{Si}[\mathrm{M}+\mathrm{Na}]^{+}$, 540.9264. Found, 540.9262 .

\section{2-(6,6-Dibromo-5-(trifluoromethoxy)hex-5-en-1-yl)isoindoline-1,3-dione (3w)}

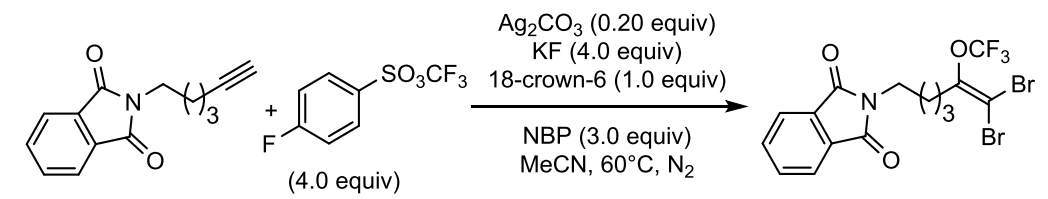

In a glove box, to a $15.0 \mathrm{~mL}$ sealed tube were added in sequence 2-(hex-5-yn-1-yl)isoindoline-1,3-dione (1w) ${ }^{[8]}$ (114 mg, $0.500 \mathrm{mmol}, 1.00$ equiv), KF (116 mg, $2.00 \mathrm{mmol}, 4.00$ equiv), 18-crown-6 (132 mg, $0.500 \mathrm{mmol}, 1.00$ equiv), $\mathrm{Ag}_{2} \mathrm{CO}_{3}$ (27.5 mg, 0.100 mmol, 0.200 equiv), NBP ( $N$-Bromophthalimide) (339 mg, $1.50 \mathrm{mmol}, 3.00$ equiv) and $1.00 \mathrm{~mL}$ $\mathrm{MeCN}$. The mixture was stirring at room temperature for $5 \mathrm{mins}$, then added TFMS ( $489 \mathrm{mg}, 2.00$ mmol, 4.00 equiv). After stirring for $4.0 \mathrm{~h}$ at $60{ }^{\circ} \mathrm{C}$, the reaction mixture was concentrated in vacuo. The residue was then added $10 \mathrm{~mL} \mathrm{CH}_{2} \mathrm{Cl}_{2}$ and filtered. The organic layer was concentrated 
in vacuo. The residue was purified by preparative TLC, eluting with hexanes/EtOAc 10:1 (v/v) to afford $141 \mathrm{mg}$ 2-(6,6-dibromo-5-(trifluoromethoxy)hex-5-en-1-yl)isoindoline-1,3-dione (3w) as a colorless liquid ( $60 \%$ yield).

$\mathrm{R}_{f}=0.11$ (hexanes/EtOAc 20:1 (v/v)). NMR Spectroscopy: ${ }^{1} \mathrm{H}$ NMR $\left(400 \mathrm{MHz}, \mathrm{CDCl}_{3}\right) \delta 7.82-$ $7.74(\mathrm{~m}, 2 \mathrm{H}), 7.70-7.63(\mathrm{~m}, 2 \mathrm{H}), 3.66(\mathrm{t}, J=6.9 \mathrm{~Hz}, 2 \mathrm{H}), 2.57(\mathrm{t}, J=7.2 \mathrm{~Hz}, 2 \mathrm{H}), 1.74-1.64$ (m, 2H), $1.64-1.54(\mathrm{~m}, 2 \mathrm{H}) .{ }^{13} \mathrm{C}$ NMR $\left(101 \mathrm{MHz}, \mathrm{CDCl}_{3}\right) \delta 168.3,148.5,134.0,132.1,123.2$, 119.8 (q, $J=261.1 \mathrm{~Hz}), 88.5,37.4,31.5,27.7,23.2 .{ }^{19} \mathrm{~F} \mathrm{NMR}\left(376 \mathrm{MHz}, \mathrm{CDCl}_{3}\right) \delta-56.22(\mathrm{~s}, 3 \mathrm{~F})$. Mass Spectrometry: HRMS-ESI (m/z): Calcd for $\mathrm{C}_{15} \mathrm{H}_{13} \mathrm{Br}_{2} \mathrm{~F}_{3} \mathrm{NO}_{3}[\mathrm{M}+\mathrm{H}]^{+}$, 469.9209. Found, 469.9205 .

\section{$N$-(6,6-dibromo-5-(trifluoromethoxy)hex-5-en-1-yl) Saccharin (3x)}

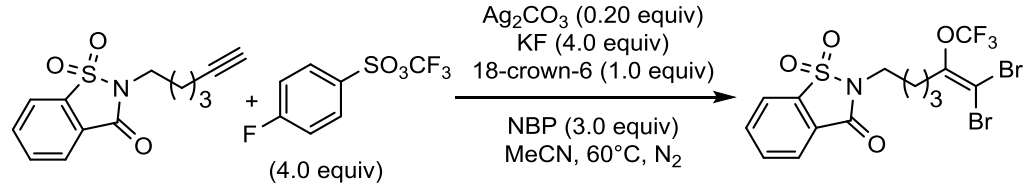

In a glove box, to a $15.0 \mathrm{~mL}$ sealed tube were added in sequence $N$-(hex-5-yn-1-yl)Saccharin (1x) (132 mg, $0.500 \mathrm{mmol}, 1.00$ equiv), KF (116 mg, $2.00 \mathrm{mmol}, 4.00$ equiv), 18-crown-6 (132 mg, 0.500 mmol, 1.00 equiv), $\mathrm{Ag}_{2} \mathrm{CO}_{3} \quad(27.5 \mathrm{mg}, 0.100 \mathrm{mmol}, 0.200$ equiv), NBP ( $N$-Bromophthalimide) (339 mg, $1.50 \mathrm{mmol}, 3.00$ equiv) and $1.00 \mathrm{~mL} \mathrm{MeCN}$. The mixture was stirring at room temperature for $5 \mathrm{mins}$, then added TFMS ( $489 \mathrm{mg}, 2.00 \mathrm{mmol}, 4.00$ equiv). After stirring for $4.0 \mathrm{~h}$ at $60{ }^{\circ} \mathrm{C}$, the reaction mixture was concentrated in vacuo. The residue was then added $10 \mathrm{~mL} \mathrm{CH} \mathrm{Cl}_{2}$ and filtered. The organic layer was concentrated in vacuo. The residue was purified by preparative TLC, eluting with hexanes/EtOAc 10:1 (v/v) to afford $183 \mathrm{mg}$ $N$-(6,6-dibromo-5-(trifluoromethoxy)hex-5-en-1-yl) Saccharin (3x) as a colorless liquid (72\% yield).

$\mathrm{R}_{f}=0.06$ (hexanes/EtOAc 20:1 (v/v)). NMR Spectroscopy: ${ }^{1} \mathrm{H}$ NMR $\left(400 \mathrm{MHz}, \mathrm{CDCl}_{3}\right) \delta 8.03(\mathrm{~d}$, $J=6.9 \mathrm{~Hz}, 1 \mathrm{H}), 7.93-7.88(\mathrm{~m}, 1 \mathrm{H}), 7.87-7.77(\mathrm{~m}, 2 \mathrm{H}), 3.78(\mathrm{t}, J=7.2 \mathrm{~Hz}, 2 \mathrm{H}), 2.62(\mathrm{t}, J=7.5$ $\mathrm{Hz}, 2 \mathrm{H}), 1.93-1.83(\mathrm{~m}, 2 \mathrm{H}), 1.75-1.65(\mathrm{~m}, 2 \mathrm{H}) .{ }^{13} \mathrm{C}$ NMR $\left(101 \mathrm{MHz}, \mathrm{CDCl}_{3}\right) \delta 159.0,148.4$, 137.7, 134.9, 134.4, 127.3, 125.2, 121.0, 119.8 (q, $J=261.2 \mathrm{~Hz}$ ), 88.7, 38.8, 31.5, 27.5, 23.1. ${ }^{19} \mathrm{~F}$ NMR $\left(376 \mathrm{MHz}, \mathrm{CDCl}_{3}\right) \delta-56.14(\mathrm{~s}, 3 \mathrm{~F})$. Mass Spectrometry: HRMS-ESI $(\mathrm{m} / \mathrm{z})$ : Calcd for $\mathrm{C}_{14} \mathrm{H}_{13} \mathrm{Br}_{2} \mathrm{~F}_{3} \mathrm{NO}_{4} \mathrm{~S}[\mathrm{M}+\mathrm{H}]^{+}$, 505.8879. Found, 505.8873.

\section{$N$-butyl- $N$-(6,6-dibromo-5-(trifluoromethoxy)hex-5-en-1-yl)benzenesulfonamide (3y)}

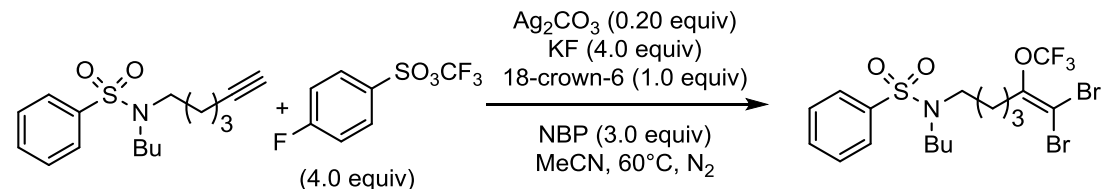

In a glove box, to a $15.0 \mathrm{~mL}$ sealed tube were added in sequence $N$-butyl- $N$-(hex-5-yn-1-yl)benzenesulfonamide (1y) (147 mg, $0.500 \mathrm{mmol}, 1.00$ equiv), KF (116 $\mathrm{mg}, 2.00 \mathrm{mmol}, 4.00$ equiv), 18-crown-6 (132 mg, $0.500 \mathrm{mmol}, 1.00$ equiv), $\mathrm{Ag}_{2} \mathrm{CO}_{3}$ (27.5 mg, $0.100 \mathrm{mmol}, 0.200$ equiv), NBP ( $N$-Bromophthalimide) (339 mg, $1.50 \mathrm{mmol}, 3.00$ equiv) and 1.00 $\mathrm{mL} \mathrm{MeCN}$. The mixture was stirring at room temperature for 5 mins, then added TFMS (489 mg, $2.00 \mathrm{mmol}, 4.00$ equiv). After stirring for $4.0 \mathrm{~h}$ at $60^{\circ} \mathrm{C}$, the reaction mixture was concentrated in vасио. The residue was then added $10 \mathrm{~mL} \mathrm{CH}_{2} \mathrm{Cl}_{2}$ and filtered. The organic layer was concentrated 
in vacuo. The residue was purified by preparative TLC, eluting with hexanes/EtOAc 12:1 (v/v) to afford $218 \mathrm{mg} N$-butyl- $N$-(6,6-dibromo-5-(trifluoromethoxy)hex-5-en-1-yl)benzenesulfonamide $(\mathbf{3 y})$ as a colorless liquid ( $81 \%$ yield).

$\mathrm{R}_{f}=0.15$ (hexanes/EtOAc 20:1 (v/v)). NMR Spectroscopy: ${ }^{1} \mathrm{H}$ NMR $\left(400 \mathrm{MHz}, \mathrm{CDCl}_{3}\right) \delta 7.78(\mathrm{~d}$, $J=7.4 \mathrm{~Hz}, 2 \mathrm{H}), 7.58-7.51(\mathrm{~m}, 1 \mathrm{H}), 7.48(\mathrm{t}, J=7.4 \mathrm{~Hz}, 2 \mathrm{H}), 3.16-3.02(\mathrm{~m}, 4 \mathrm{H}), 2.60-2.50(\mathrm{~m}$, 2H), $1.64-1.41(\mathrm{~m}, 6 \mathrm{H}), 1.32-1.22(\mathrm{~m}, 2 \mathrm{H}), 0.87(\mathrm{t}, J=7.3 \mathrm{~Hz}, 3 \mathrm{H}) .{ }^{13} \mathrm{C}$ NMR $(101 \mathrm{MHz}$, $\left.\mathrm{CDCl}_{3}\right) \delta 148.6,139.9,132.5,129.1,127.1,119.8(\mathrm{q}, J=260.8 \mathrm{~Hz}), 88.5,48.3,47.9,31.6,30.9$, 27.8, 23.1, 20.0, 13.7. ${ }^{19} \mathrm{~F}$ NMR (376 $\mathrm{MHz}, \mathrm{CDCl}_{3}$ ) $\delta-56.12$ (s, 3F). Mass Spectrometry: HRMS-ESI (m/z): Calcd for $\mathrm{C}_{17} \mathrm{H}_{23} \mathrm{Br}_{2} \mathrm{~F}_{3} \mathrm{NO}_{3} \mathrm{~S}[\mathrm{M}+\mathrm{H}]^{+}$, 535.9712. Found, 535.9708.

\section{6,6-Dibromo-5-(trifluoromethoxy)hex-5-en-1-yl 1-naphthoate (3z)}

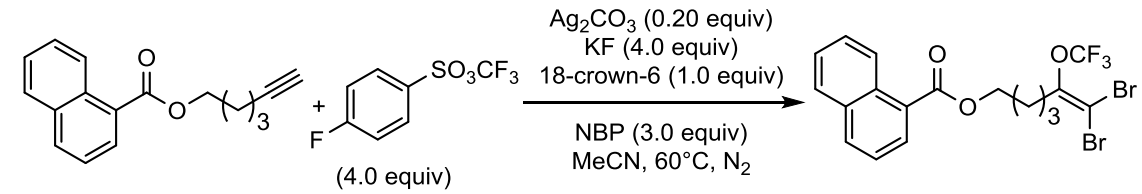

In a glove box, to a $15.0 \mathrm{~mL}$ sealed tube were added in sequence hex-5-yn-1-yl 1-naphthoate (1z) (126 mg, $0.500 \mathrm{mmol}, 1.00$ equiv), KF (116 mg, $2.00 \mathrm{mmol}, 4.00$ equiv), 18-crown-6 (132 mg, 0.500 mmol, 1.00 equiv), $\mathrm{Ag}_{2} \mathrm{CO}_{3} \quad(27.5 \mathrm{mg}, 0.100 \mathrm{mmol}, 0.200$ equiv), NBP ( $N$-Bromophthalimide) $(339 \mathrm{mg}, 1.50 \mathrm{mmol}, 3.00$ equiv) and $1.00 \mathrm{~mL} \mathrm{MeCN}$. The mixture was stirring at room temperature for $5 \mathrm{mins}$, then added TFMS ( $489 \mathrm{mg}, 2.00 \mathrm{mmol}, 4.00$ equiv). After stirring for $4.0 \mathrm{~h}$ at $60{ }^{\circ} \mathrm{C}$, the reaction mixture was concentrated in vacuo. The residue was then added $10 \mathrm{~mL} \mathrm{CH} \mathrm{Cl}_{2}$ and filtered. The organic layer was concentrated in vacuo. The residue was purified by preparative TLC, eluting with hexanes/EtOAc 50:1 (v/v) to afford $201 \mathrm{mg}$ 6,6-dibromo-5-(trifluoromethoxy)hex-5-en-1-yl 1-naphthoate (3z) as a colorless liquid (81\% yield).

$\mathrm{R}_{f}=0.30$ (hexanes/EtOAc 40:1 (v/v)). NMR Spectroscopy: ${ }^{1} \mathrm{H}$ NMR (400 MHz, $\left.\mathrm{CDCl}_{3}\right) \delta 8.94(\mathrm{~d}$, $J=8.7 \mathrm{~Hz}, 1 \mathrm{H}), 8.20(\mathrm{~d}, J=7.2 \mathrm{~Hz}, 1 \mathrm{H}), 8.03(\mathrm{~d}, J=8.2 \mathrm{~Hz}, 1 \mathrm{H}), 7.90(\mathrm{~d}, J=8.1 \mathrm{~Hz}, 1 \mathrm{H}), 7.64(\mathrm{t}$, $J=7.7 \mathrm{~Hz}, 1 \mathrm{H}), 7.58-7.48(\mathrm{~m}, 2 \mathrm{H}), 4.46(\mathrm{t}, J=5.9 \mathrm{~Hz}, 2 \mathrm{H}), 2.69(\mathrm{t}, J=7.1 \mathrm{~Hz}, 2 \mathrm{H}), 1.94-1.77$ (m, 4H). ${ }^{13} \mathrm{C}$ NMR $\left(101 \mathrm{MHz}, \mathrm{CDCl}_{3}\right) \delta 167.6,148.7,134.0,133.5,131.5,130.3,128.7,127.9$, $127.3,126.3,125.9,124.6,119.9(\mathrm{q}, J=260.8 \mathrm{~Hz}), 88.6,64.3,31.8,28.0,22.7 .{ }^{19} \mathrm{~F}$ NMR $(376$ $\left.\mathrm{MHz}, \mathrm{CDCl}_{3}\right) \delta-56.23$ (s, 3F). Mass Spectrometry: HRMS-ESI (m/z): Calcd for $\mathrm{C}_{18} \mathrm{H}_{16} \mathrm{Br}_{2} \mathrm{~F}_{3} \mathrm{O}_{3}$ $[\mathrm{M}+\mathrm{H}]^{+}$, 494.9413. Found, 494.9409.

\section{6,6-Dibromo-5-(trifluoromethoxy)hex-5-en-1-yl benzofuran-2-carboxylate (3aa)}

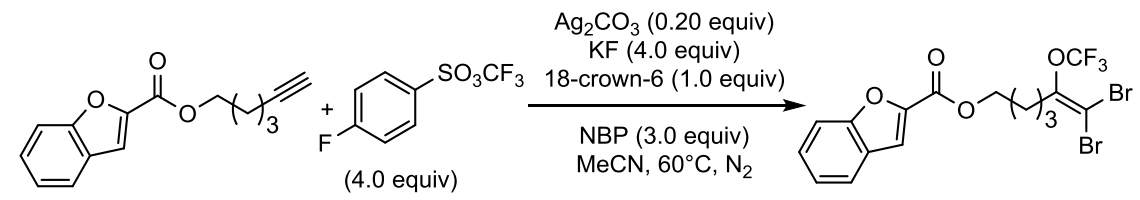

In a glove box, to a $15.0 \mathrm{~mL}$ sealed tube were added in sequence hex-5-yn-1-yl benzofuran-2-carboxylate (1aa) (121 mg, $0.500 \mathrm{mmol}, 1.00$ equiv), KF (116 mg, $2.00 \mathrm{mmol}, 4.00$ equiv), 18-crown-6 (132 mg, $0.500 \mathrm{mmol}, 1.00$ equiv), $\mathrm{Ag}_{2} \mathrm{CO}_{3}$ (27.5 mg, $0.100 \mathrm{mmol}, 0.200$ equiv), NBP ( $N$-Bromophthalimide) $(339 \mathrm{mg}, 1.50 \mathrm{mmol}, 3.00$ equiv) and $1.00 \mathrm{~mL} \mathrm{MeCN}$. The mixture was stirring at room temperature for $5 \mathrm{mins}$, then added TFMS (489 mg, $2.00 \mathrm{mmol}, 4.00$ equiv). After stirring for $4.0 \mathrm{~h}$ at $60{ }^{\circ} \mathrm{C}$, the reaction mixture was concentrated in vacuo. The 
residue was then added $10 \mathrm{~mL} \mathrm{CH}_{2} \mathrm{Cl}_{2}$ and filtered. The organic layer was concentrated in vacuo. The residue was purified by preparative TLC, eluting with hexanes/EtOAc 30:1 (v/v) to afford 170 mg 6,6-dibromo-5-(trifluoromethoxy)hex-5-en-1-yl benzofuran-2-carboxylate (3aa) as a colorless liquid (70\% yield).

$\mathrm{R}_{f}=0.11$ (hexanes/EtOAc 40:1 (v/v)). NMR Spectroscopy: ${ }^{1} \mathrm{H}$ NMR $\left(400 \mathrm{MHz}, \mathrm{CDCl}_{3}\right) \delta 7.67(\mathrm{~d}$, $J=7.8 \mathrm{~Hz}, 1 \mathrm{H}), 7.58(\mathrm{dd}, J=8.4,0.7 \mathrm{~Hz}, 1 \mathrm{H}), 7.52(\mathrm{~d}, J=0.9 \mathrm{~Hz}, 1 \mathrm{H}), 7.47-7.41(\mathrm{~m}, 1 \mathrm{H}), 7.33$ $-7.27(\mathrm{~m}, 1 \mathrm{H}), 4.40(\mathrm{t}, J=6.3 \mathrm{~Hz}, 2 \mathrm{H}), 2.66(\mathrm{t}, J=7.2 \mathrm{~Hz}, 2 \mathrm{H}), 1.90-1.70(\mathrm{~m}, 4 \mathrm{H}) .{ }^{13} \mathrm{C}$ NMR $\left(101 \mathrm{MHz}, \mathrm{CDCl}_{3}\right) \delta 159.6,155.8,148.6(\mathrm{q}, J=1.1 \mathrm{~Hz}), 145.5,127.8,127.0,123.9,122.9,119.9$ (q, $J=261.1 \mathrm{~Hz}), 114.0,112.4,88.7,64.7,31.6,31.6,27.8,22.4 .{ }^{19} \mathrm{~F} \mathrm{NMR}\left(376 \mathrm{MHz}, \mathrm{CDCl}_{3}\right) \delta$ -56.22 (s, 3F). Mass Spectrometry: HRMS-ESI (m/z): Calcd for $\mathrm{C}_{16} \mathrm{H}_{13} \mathrm{Br}_{2} \mathrm{~F}_{3} \mathrm{NaO}_{4}[\mathrm{M}+\mathrm{Na}]^{+}$, 506.9025. Found, 506.9020.

\section{6,6-Dibromo-5-(trifluoromethoxy)hex-5-en-1-yl 5-bromothiophene-2-carboxylate (3bb)}

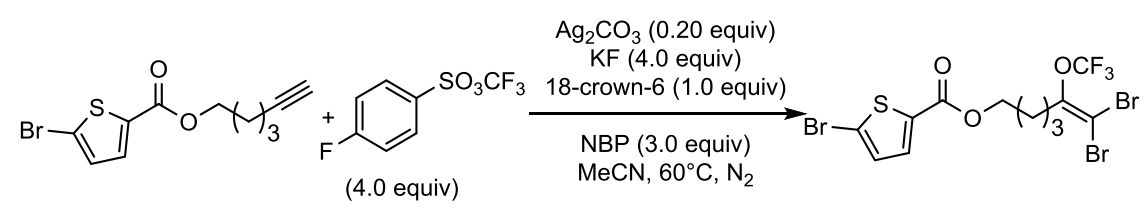

In a glove box, to a $15.0 \mathrm{~mL}$ sealed tube were added in sequence hex-5-yn-1-yl 5-bromothiophene-2-carboxylate (1bb) (143 mg, $0.500 \mathrm{mmol}, 1.00$ equiv), KF (116 mg, 2.00 mmol, 4.00 equiv), 18 -crown-6 (132 mg, $0.500 \mathrm{mmol}, 1.00$ equiv), $\mathrm{Ag}_{2} \mathrm{CO}_{3}(27.5 \mathrm{mg}, 0.100 \mathrm{mmol}$, 0.200 equiv), NBP ( $N$-Bromophthalimide) (339 mg, $1.50 \mathrm{mmol}, 3.00$ equiv) and $1.00 \mathrm{~mL} \mathrm{MeCN}$. The mixture was stirring at room temperature for $5 \mathrm{mins}$, then added TFMS (489 $\mathrm{mg}, 2.00 \mathrm{mmol}$, 4.00 equiv). After stirring for $4.0 \mathrm{~h}$ at $60^{\circ} \mathrm{C}$, the reaction mixture was concentrated in vacuo. The residue was then added $10 \mathrm{~mL} \mathrm{CH} \mathrm{Cl}_{2}$ and filtered. The organic layer was concentrated in vacuo. The residue was purified by preparative TLC, eluting with hexanes/EtOAc 40:1 (v/v) to afford 202 mg 6,6-dibromo-5-(trifluoromethoxy)hex-5-en-1-yl 5-bromothiophene-2-carboxylate (3bb) as a colorless liquid ( $76 \%$ yield).

$\mathrm{R}_{f}=0.30$ (hexanes/EtOAc 40:1 (v/v)). NMR Spectroscopy: ${ }^{1} \mathrm{H}$ NMR (400 MHz, $\left.\mathrm{CDCl}_{3}\right) \delta 7.53(\mathrm{~d}$, $J=3.6 \mathrm{~Hz}, 1 \mathrm{H}), 7.06(\mathrm{~d}, J=3.6 \mathrm{~Hz}, 1 \mathrm{H}), 4.29(\mathrm{t}, J=5.6 \mathrm{~Hz}, 2 \mathrm{H}), 2.63(\mathrm{t}, J=6.6 \mathrm{~Hz}, 2 \mathrm{H}), 1.90-$ $1.58(\mathrm{~m}, 4 \mathrm{H}) .{ }^{13} \mathrm{C} \mathrm{NMR}\left(101 \mathrm{MHz}, \mathrm{CDCl}_{3}\right) \delta 161.1,148.6(\mathrm{q}, J=1.1 \mathrm{~Hz}), 134.9,133.8,131.0$, 120.4, 119.9 (q, $J=261.1 \mathrm{~Hz}), 88.7,64.6,31.6,27.8,22.4 .{ }^{19} \mathrm{~F} \mathrm{NMR}\left(376 \mathrm{MHz}, \mathrm{CDCl}_{3}\right) \delta-56.31$ (s, 3F). Mass Spectrometry: HRMS-ESI (m/z): Calcd for $\mathrm{C}_{12} \mathrm{H}_{11} \mathrm{~B}_{\mathrm{r} 3} \mathrm{~F}_{3} \mathrm{O}_{3} \mathrm{~S}[\mathrm{M}+\mathrm{H}]^{+}, 528.7926$. Found, 528.7920.

\section{6,6-Dibromo-5-(trifluoromethoxy)hex-5-en-1-yl quinoline-2-carboxylate (3cc)}

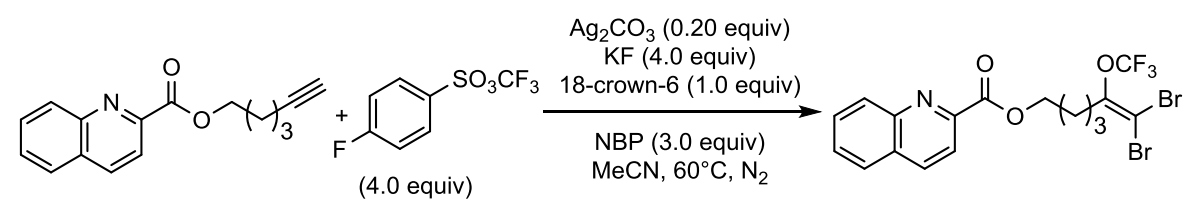

In a glove box, to a $15.0 \mathrm{~mL}$ sealed tube were added in sequence hex-5-yn-1-yl quinoline-2-carboxylate (1cc) (126 mg, $0.500 \mathrm{mmol}, 1.00$ equiv), KF (116 mg, $2.00 \mathrm{mmol}, 4.00$ equiv), 18-crown-6 (132 mg, $0.500 \mathrm{mmol}, 1.00$ equiv), $\mathrm{Ag}_{2} \mathrm{CO}_{3}$ (27.5 mg, $0.100 \mathrm{mmol}, 0.200$ equiv), NBP ( $N$-Bromophthalimide) $(339 \mathrm{mg}, 1.50 \mathrm{mmol}, 3.00$ equiv) and $1.00 \mathrm{~mL} \mathrm{MeCN}$. The mixture was stirring at room temperature for 5 mins, then added TFMS (489 mg, $2.00 \mathrm{mmol}, 4.00$ 
equiv). After stirring for $4.0 \mathrm{~h}$ at $60^{\circ} \mathrm{C}$, the reaction mixture was concentrated in vacuo. The residue was then added $10 \mathrm{~mL} \mathrm{CH}_{2} \mathrm{Cl}_{2}$ and filtered. The organic layer was concentrated in vacuo. The residue was purified by preparative TLC, eluting with hexanes/EtOAc/Et $3 \mathrm{~N}$ 15:1:0.02 (v/v) to afford $191 \mathrm{mg}$ 6,6-dibromo-5-(trifluoromethoxy)hex-5-en-1-yl quinoline-2-carboxylate (3cc) as a colorless liquid ( $77 \%$ yield).

$\mathrm{R}_{f}=0.19$ (hexanes/EtOAc 8:1 (v/v)). NMR Spectroscopy: ${ }^{1} \mathrm{H}$ NMR $\left(400 \mathrm{MHz}, \mathrm{CDCl}_{3}\right) \delta 8.31-$ $8.15(\mathrm{~m}, 2 \mathrm{H}), 8.09$ (d, $J=8.3 \mathrm{~Hz}, 1 \mathrm{H}), 7.79(\mathrm{~d}, J=8.1 \mathrm{~Hz}, 1 \mathrm{H}), 7.71$ (t, $J=7.6 \mathrm{~Hz}, 1 \mathrm{H}), 7.56(\mathrm{t}, J$ $=7.4 \mathrm{~Hz}, 1 \mathrm{H}), 4.45(\mathrm{t}, J=6.3 \mathrm{~Hz}, 2 \mathrm{H}), 2.62(\mathrm{t}, J=7.3 \mathrm{~Hz}, 2 \mathrm{H}), 1.94-1.80(\mathrm{~m}, 2 \mathrm{H}), 1.80-1.63$ (m, 2H). ${ }^{13} \mathrm{C}$ NMR $\left(101 \mathrm{MHz}, \mathrm{CDCl}_{3}\right) \delta 165.3,148.6,148.0,147.7,137.2,130.8,130.2,129.3$, 128.6, 127.5, 120.9, 119.8 (q, $J=261.1 \mathrm{~Hz}), 88.5,65.2,31.6,27.7,22.4 .{ }^{19} \mathrm{~F}$ NMR (376 MHz, $\mathrm{CDCl}_{3}$ ) $\delta-56.09$ (s, 3F). Mass Spectrometry: HRMS-ESI (m/z): Calcd for $\mathrm{C}_{17} \mathrm{H}_{15} \mathrm{Br}_{2} \mathrm{~F}_{3} \mathrm{NO}_{3}[\mathrm{M}+$ $\mathrm{H}]^{+}, 495.9365$. Found, 495.9362.

6,6-Dibromo-5-(trifluoromethoxy)hex-5-en-1-yl 6-fluoropicolinate (3dd)

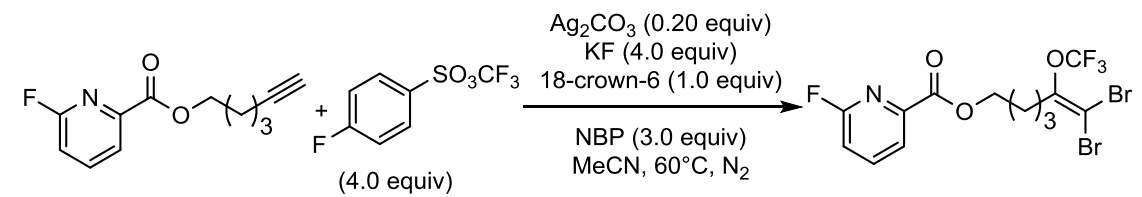

In a glove box, to a $15.0 \mathrm{~mL}$ sealed tube were added in sequence hex-5-yn-1-yl 6-fluoropicolinate (1dd) (110 mg, $0.500 \mathrm{mmol}, 1.00$ equiv), KF (116 mg, $2.00 \mathrm{mmol}, 4.00$ equiv), 18-crown-6 (132 $\mathrm{mg}, 0.500 \mathrm{mmol}, 1.00$ equiv), $\mathrm{Ag}_{2} \mathrm{CO}_{3}(27.5 \mathrm{mg}, 0.100 \mathrm{mmol}, 0.200$ equiv), NBP ( $N$-Bromophthalimide) (339 mg, $1.50 \mathrm{mmol}, 3.00$ equiv) and $1.00 \mathrm{~mL} \mathrm{MeCN}$. The mixture was stirring at room temperature for $5 \mathrm{mins}$, then added TFMS ( $489 \mathrm{mg}, 2.00 \mathrm{mmol}, 4.00$ equiv). After stirring for $4.0 \mathrm{~h}$ at $60{ }^{\circ} \mathrm{C}$, the reaction mixture was concentrated in vacuo. The residue was then added $10 \mathrm{~mL} \mathrm{CH}_{2} \mathrm{Cl}_{2}$ and filtered. The organic layer was concentrated in vacuo. The residue was purified by preparative TLC, eluting with hexanes/EtOAc/Et ${ }_{3} \mathrm{~N}$ 15:1:0.02 (v/v) to afford $172 \mathrm{mg}$ 6,6-dibromo-5-(trifluoromethoxy)hex-5-en-1-yl 6-fluoropicolinate (3dd) as a colorless liquid (74\% yield).

$\mathrm{R}_{f}=0.22$ (hexanes/EtOAc 8:1 (v/v)). NMR Spectroscopy: ${ }^{1} \mathrm{H}$ NMR (400 MHz, $\left.\mathrm{CDCl}_{3}\right) \delta 8.04-$ $7.88(\mathrm{~m}, 2 \mathrm{H}), 7.13(\mathrm{~d}, J=7.9 \mathrm{~Hz}, 1 \mathrm{H}), 4.39$ (t, $J=6.4 \mathrm{~Hz}, 2 \mathrm{H}), 2.62(\mathrm{t}, J=7.3 \mathrm{~Hz}, 2 \mathrm{H}), 1.89-$ $1.78(\mathrm{~m}, 2 \mathrm{H}), 1.77-1.66(\mathrm{~m}, 2 \mathrm{H}) .{ }^{13} \mathrm{C} \mathrm{NMR}\left(101 \mathrm{MHz}, \mathrm{CDCl}_{3}\right) \delta 163.8,162.9(\mathrm{~d}, J=242.8 \mathrm{~Hz})$, 148.4, 146.3 (d, $J=12.3 \mathrm{~Hz}), 142.1$ (d, $J=7.5 \mathrm{~Hz}), 122.7$ (d, $J=3.5 \mathrm{~Hz}), 119.7$ (q, $J=261.3 \mathrm{~Hz})$, $113.9(\mathrm{~d}, J=37.0 \mathrm{~Hz}), 88.5,65.3,31.5,27.6,22.3 .{ }^{19} \mathrm{~F} \mathrm{NMR}\left(376 \mathrm{MHz}, \mathrm{CDCl}_{3}\right) \delta-56.16(\mathrm{~s}, 3 \mathrm{~F})$, -64.70 (s, 1F). Mass Spectrometry: HRMS-ESI (m/z): Calcd for $\mathrm{C}_{13} \mathrm{H}_{12} \mathrm{Br}_{2} \mathrm{~F}_{4} \mathrm{NO}_{3}[\mathrm{M}+\mathrm{H}]^{+}$, 463.9115. Found, 463.9110.

\section{1-(2,2-Dibromo-1-(trifluoromethoxy)vinyl)-4-methoxybenzene (3ee)}

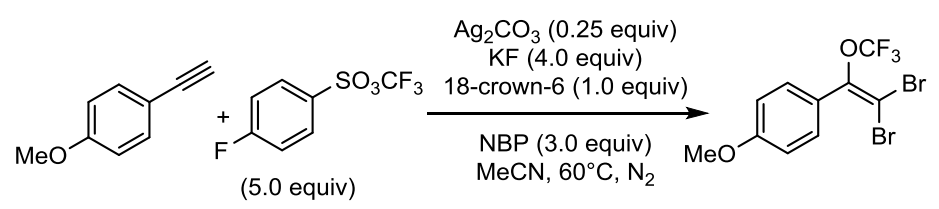

In a glove box, to a $15.0 \mathrm{~mL}$ sealed tube were added in sequence 1-ethynyl-4-methoxybenzene (1ee) (66 mg, $0.500 \mathrm{mmol}, 1.00$ equiv), KF (116 mg, $2.00 \mathrm{mmol}, 4.00$ equiv), 18-crown-6 (132 mg, 0.500 mmol, 1.00 equiv), $\mathrm{Ag}_{2} \mathrm{CO}_{3}$ (34.4 mg, $0.125 \mathrm{mmol}, 0.250$ equiv), NBP 
( $N$-Bromophthalimide) (339 mg, $1.50 \mathrm{mmol}, 3.00$ equiv) and $1.00 \mathrm{~mL} \mathrm{MeCN}$. The mixture was stirring at room temperature for $5 \mathrm{mins}$, then added TFMS ( $612 \mathrm{mg}, 2.50 \mathrm{mmol}, 5.00$ equiv). After stirring for $4.0 \mathrm{~h}$ at $60{ }^{\circ} \mathrm{C}$, the reaction mixture was concentrated in vacuo. The residue was then added $10 \mathrm{~mL} \mathrm{CH}_{2} \mathrm{Cl}_{2}$ and filtered. The organic layer was concentrated in vacuo. The residue was purified by preparative TLC, eluting with hexanes/EtOAc 50:1 (v/v) to afford $158 \mathrm{mg}$ 1-(2,2-dibromo-1-(trifluoromethoxy)vinyl)-4-methoxybenzene (3ee) as a colorless liquid (84\% yield).

$\mathrm{R}_{f}=0.40$ (hexanes/EtOAc 40:1 (v/v)). NMR Spectroscopy: ${ }^{1} \mathrm{H}$ NMR $\left(400 \mathrm{MHz}, \mathrm{CDCl}_{3}\right) \delta 7.37(\mathrm{~d}$, $J=8.7 \mathrm{~Hz}, 2 \mathrm{H}), 6.83(\mathrm{~d}, J=8.6 \mathrm{~Hz}, 2 \mathrm{H}), 3.74(\mathrm{~s}, 3 \mathrm{H}) .{ }^{13} \mathrm{C} \mathrm{NMR}\left(101 \mathrm{MHz}, \mathrm{CDCl}_{3}\right) \delta 161.1$, $146.5(\mathrm{q}, J=1.5 \mathrm{~Hz}), 131.0,123.6,120.1$ (q, $J=261.9 \mathrm{~Hz}), 113.9,88.1,55.4 .{ }^{19} \mathrm{~F}$ NMR $(376 \mathrm{MHz}$, $\left.\mathrm{CDCl}_{3}\right) \delta-55.67$ (s, 3F). Mass Spectrometry: HRMS-EI (m/z): Calcd for $\mathrm{C}_{10} \mathrm{H}_{7} \mathrm{Br}_{2} \mathrm{~F}_{3} \mathrm{O}_{2}[\mathrm{M}]^{+}$, 373.8765. Found, 373.8759 .

Gram scale synthesis of 1-(2,2-dibromo-1-(trifluoromethoxy)vinyl)-4-methoxybenzene (3ee)

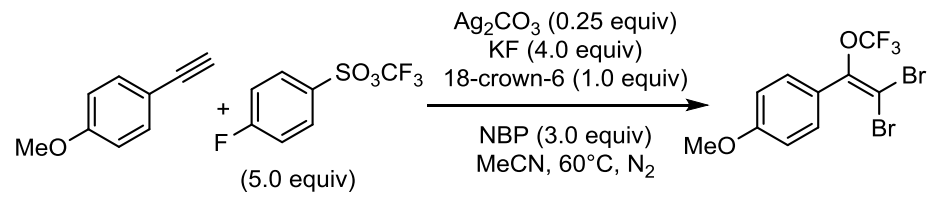

In a glove box, to a $150 \mathrm{~mL}$ sealed tube were added in sequence 1-ethynyl-4-methoxybenzene (1ee) (1.32 g, $10.0 \mathrm{mmol}, 1.00$ equiv), KF (2.32 g, $40.0 \mathrm{mmol}, 4.00$ equiv), 18-crown-6 (2.64 g, $10.0 \mathrm{mmol}, 1.00$ equiv), $\mathrm{Ag}_{2} \mathrm{CO}_{3}$ ( $688 \mathrm{mg}, 2.50 \mathrm{mmol}, 0.250$ equiv), NBP ( $N$-Bromophthalimide) $(6.78 \mathrm{~g}, 30.0 \mathrm{mmol}, 3.00$ equiv) and $20.0 \mathrm{~mL} \mathrm{MeCN}$. The mixture was stirring at room temperature for 5 mins, then added TFMS (12.2 g, $50.0 \mathrm{mmol}, 5.00$ equiv). After stirring for $4.0 \mathrm{~h}$ at $60{ }^{\circ} \mathrm{C}$, the reaction mixture was concentrated in vacuo. The residue was then added $100 \mathrm{~mL}$ $\mathrm{CH}_{2} \mathrm{Cl}_{2}$ and filtered. The organic layer was concentrated in vacuo. The residue was purified by chromatography on silica gel, eluting with hexanes/EtOAc $50: 1(\mathrm{v} / \mathrm{v})$ to afford $3.12 \mathrm{~g}$ 1-(2,2-dibromo-1-(trifluoromethoxy)vinyl)-4-methoxybenzene (3ee) as a colorless liquid (83\% yield).

\section{4-(2,2-Dibromo-1-(trifluoromethoxy)vinyl)-1,1'-biphenyl (3ff)}

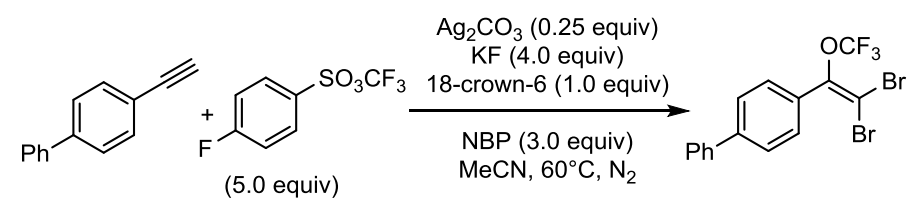

In a glove box, to a $15.0 \mathrm{~mL}$ sealed tube were added in sequence 4-ethynyl-1,1'-biphenyl (1ff) (89 $\mathrm{mg}, 0.500 \mathrm{mmol}, 1.00$ equiv), KF (116 mg, $2.00 \mathrm{mmol}, 4.00$ equiv), 18-crown-6 (132 mg, 0.500 mmol, 1.00 equiv), $\mathrm{Ag}_{2} \mathrm{CO}_{3}$ ( $34.4 \mathrm{mg}, 0.125 \mathrm{mmol}, 0.250$ equiv), NBP ( $N$-Bromophthalimide) (339 mg, $1.50 \mathrm{mmol}, 3.00$ equiv) and $1.00 \mathrm{~mL} \mathrm{MeCN}$. The mixture was stirring at room temperature for $5 \mathrm{mins}$, then added TFMS ( $612 \mathrm{mg}, 2.50 \mathrm{mmol}$, 5.00 equiv). After stirring for 4.0 $\mathrm{h}$ at $60{ }^{\circ} \mathrm{C}$, the reaction mixture was concentrated in vacuo. The residue was then added $10 \mathrm{~mL}$ $\mathrm{CH}_{2} \mathrm{Cl}_{2}$ and filtered. The organic layer was concentrated in vacuo. The residue was purified by preparative TLC, eluting with hexanes/EtOAc 100:1 (v/v) to afford $150 \mathrm{mg}$ 4-(2,2-dibromo-1-(trifluoromethoxy)vinyl)-1,1'-biphenyl (3ff) as a colorless liquid (71\% yield). 
$\mathrm{R}_{f}=0.63$ (hexanes/EtOAc 40:1 (v/v)). NMR Spectroscopy: ${ }^{1} \mathrm{H}$ NMR $\left(400 \mathrm{MHz}, \mathrm{CDCl}_{3}\right) \delta 7.78-$ $7.62(\mathrm{~m}, 6 \mathrm{H}), 7.57-7.48(\mathrm{~m}, 2 \mathrm{H}), 7.48-7.40(\mathrm{~m}, 1 \mathrm{H}) .{ }^{13} \mathrm{C} \mathrm{NMR}\left(101 \mathrm{MHz}, \mathrm{CDCl}_{3}\right) \delta 146.3$, 143.2, 134.0, 130.4, 129.8, 129.1, 128.2, 127.3, 127.1, 120.2 (q, $J=262.3 \mathrm{~Hz}), 89.4 .{ }^{19} \mathrm{~F}$ NMR $\left(376 \mathrm{MHz}, \mathrm{CDCl}_{3}\right) \delta-56.77$ (s, 3F). Mass Spectrometry: HRMS-EI (m/z): Calcd for $\mathrm{C}_{15} \mathrm{H}_{9} \mathrm{Br}_{2} \mathrm{~F}_{3} \mathrm{O}$ $[\mathrm{M}]^{+}, 419.8972$. Found, 419.8967 .

\section{1-(Tert-butyl)-4-(2,2-dibromo-1-(trifluoromethoxy)vinyl) benzene (3gg)}

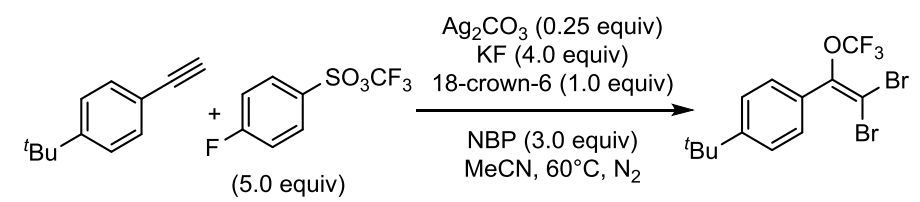

In a glove box, to a $15.0 \mathrm{~mL}$ sealed tube were added in sequence 1-(tert-butyl)-4-ethynylbenzene (19g) (79 mg, $0.500 \mathrm{mmol}, 1.00$ equiv), KF (116 mg, $2.00 \mathrm{mmol}, 4.00$ equiv), 18-crown-6 (132 $\mathrm{mg}, \quad 0.500$ mmol, 1.00 equiv), $\mathrm{Ag}_{2} \mathrm{CO}_{3}$ (34.4 mg, $0.125 \mathrm{mmol}, 0.250$ equiv), NBP ( $N$-Bromophthalimide) (339 mg, $1.50 \mathrm{mmol}, 3.00$ equiv) and $1.00 \mathrm{~mL} \mathrm{MeCN}$. The mixture was stirring at room temperature for $5 \mathrm{mins}$, then added TFMS (612 mg, $2.50 \mathrm{mmol}, 5.00$ equiv). After stirring for $4.0 \mathrm{~h}$ at $60{ }^{\circ} \mathrm{C}$, the reaction mixture was concentrated in vacuo. The residue was then added $10 \mathrm{~mL} \mathrm{CH}_{2} \mathrm{Cl}_{2}$ and filtered. The organic layer was concentrated in vacuo. The residue was purified by preparative TLC, eluting with hexanes/EtOAc 100:1 (v/v) to afford $127 \mathrm{mg}$ 1-(tert-butyl)-4-(2,2-dibromo-1-(trifluoromethoxy)vinyl) benzene (3gg) as a colorless liquid (63\% yield).

$\mathrm{R}_{f}=0.46$ (hexanes/EtOAc 40:1 (v/v)). NMR Spectroscopy: ${ }^{1} \mathrm{H}$ NMR (400 MHz, $\left.\mathrm{CDCl}_{3}\right) \delta 7.50-$ $7.45(\mathrm{~m}, 2 \mathrm{H}), 7.45-7.40(\mathrm{~m}, 2 \mathrm{H}), 1.35(\mathrm{~s}, 9 \mathrm{H}) .{ }^{13} \mathrm{C} \mathrm{NMR}\left(101 \mathrm{MHz}, \mathrm{CDCl}_{3}\right) \delta 153.8,146.7$, 129.1, 128.6, 125.4, 120.1 (q, $J=262.0 \mathrm{~Hz}), 88.7,35.6,31.3 .{ }^{19} \mathrm{~F} \mathrm{NMR}\left(376 \mathrm{MHz}, \mathrm{CDCl}_{3}\right) \delta$ -56.41 (s, 3F). Mass Spectrometry: HRMS-EI (m/z): Calcd for $\mathrm{C}_{13} \mathrm{H}_{13} \mathrm{Br}_{2} \mathrm{~F}_{3} \mathrm{O}[\mathrm{M}]^{+}, 399.9285$. Found, 399.9280 .

\section{(2,2-Dibromo-1-(trifluoromethoxy)vinyl)benzene (3hh)}

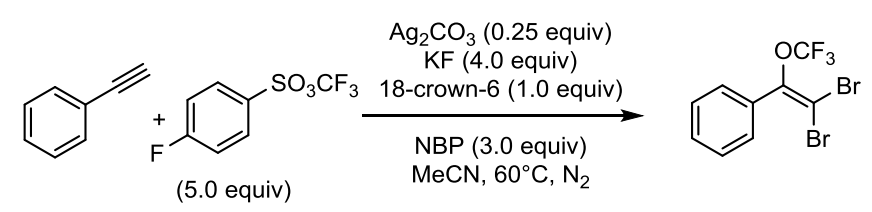

In a glove box, to a $15.0 \mathrm{~mL}$ sealed tube were added in sequence ethynylbenzene (1hh) $(51 \mathrm{mg}$, $0.500 \mathrm{mmol}, 1.00$ equiv), KF (116 mg, $2.00 \mathrm{mmol}, 4.00$ equiv), 18 -crown-6 (132 mg, $0.500 \mathrm{mmol}$, 1.00 equiv), $\mathrm{Ag}_{2} \mathrm{CO}_{3}$ (34.4 mg, $0.125 \mathrm{mmol}, 0.250$ equiv), NBP (N-Bromophthalimide) (339 mg, $1.50 \mathrm{mmol}, 3.00$ equiv) and $1.00 \mathrm{~mL} \mathrm{MeCN}$. The mixture was stirring at room temperature for 5 mins, then added TFMS (612 mg, $2.50 \mathrm{mmol}, 5.00$ equiv). After stirring for $4.0 \mathrm{~h}$ at $60{ }^{\circ} \mathrm{C}$, the reaction mixture was concentrated in vacuo. The residue was then added $10 \mathrm{~mL} \mathrm{CH}_{2} \mathrm{Cl}_{2}$ and filtered. The organic layer was concentrated in vacuo. The residue was purified by preparative TLC, eluting with hexanes/EtOAc 100:1 (v/v) to afford $107 \quad \mathrm{mg}$ (2,2-dibromo-1-(trifluoromethoxy)vinyl)benzene ( $\mathbf{3 h h}$ ) as a colorless liquid (62\% yield).

$\mathrm{R}_{f}=0.43$ (hexanes/EtOAc 40:1 (v/v)). NMR Spectroscopy: ${ }^{1} \mathrm{H}$ NMR $\left(400 \mathrm{MHz}, \mathrm{CDCl}_{3}\right) \delta 7.57-$ $7.48(\mathrm{~m}, 2 \mathrm{H}), 7.46-7.38(\mathrm{~m}, 3 \mathrm{H}) .{ }^{13} \mathrm{C}$ NMR $\left(101 \mathrm{MHz}, \mathrm{CDCl}_{3}\right) \delta 146.6$ (q, $\left.J=1.8 \mathrm{~Hz}\right), 131.7$, 
130.5, 129.4, 128.6, 120.1 (q, $J=262.2 \mathrm{~Hz}), 89.3 .{ }^{19} \mathrm{~F} \mathrm{NMR}\left(376 \mathrm{MHz}, \mathrm{CDCl}_{3}\right) \delta-56.44(\mathrm{~s}, 3 \mathrm{~F})$. Mass Spectrometry: HRMS-EI (m/z): Calcd for $\mathrm{C}_{9} \mathrm{H}_{5} \mathrm{Br}_{2} \mathrm{~F}_{3} \mathrm{O}[\mathrm{M}]^{+}$, 343.8659. Found, 343.8650 .

\section{1-Chloro-4-(2,2-dibromo-1-(trifluoromethoxy)vinyl)benzene (3ii)}

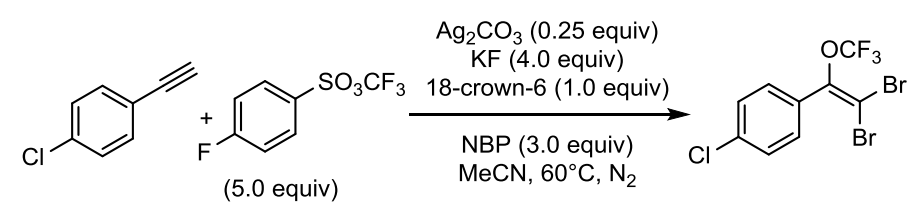

In a glove box, to a $15.0 \mathrm{~mL}$ sealed tube were added in sequence 1-chloro-4-ethynylbenzene (1ii) (68 mg, $0.500 \mathrm{mmol}, 1.00$ equiv), KF (116 mg, $2.00 \mathrm{mmol}, 4.00$ equiv), 18-crown-6 (132 mg, 0.500 mmol, 1.00 equiv), $\mathrm{Ag}_{2} \mathrm{CO}_{3} \quad(34.4 \mathrm{mg}, 0.125 \mathrm{mmol}, 0.250$ equiv), NBP ( $N$-Bromophthalimide) (339 mg, $1.50 \mathrm{mmol}, 3.00$ equiv) and $1.00 \mathrm{~mL} \mathrm{MeCN}$. The mixture was stirring at room temperature for $5 \mathrm{mins}$, then added TFMS (612 $\mathrm{mg}, 2.50 \mathrm{mmol}, 5.00$ equiv). After stirring for $4.0 \mathrm{~h}$ at $60{ }^{\circ} \mathrm{C}$, the reaction mixture was concentrated in vacuo. The residue was then added $10 \mathrm{~mL} \mathrm{CH} \mathrm{Cl}_{2}$ and filtered. The organic layer was concentrated in vacuo. The residue was purified by preparative TLC, eluting with hexanes/EtOAc 50:1 (v/v) to afford $99 \mathrm{mg}$ 1-chloro-4-(2,2-dibromo-1-(trifluoromethoxy)vinyl) benzene (3ii) as a colorless liquid (52\% yield).

$\mathrm{R}_{f}=0.45$ (hexanes/EtOAc 40:1 (v/v)). NMR Spectroscopy: ${ }^{1} \mathrm{H}$ NMR $\left(400 \mathrm{MHz}, \mathrm{CDCl}_{3}\right) \delta 7.48(\mathrm{~d}$, $J=8.5 \mathrm{~Hz}, 2 \mathrm{H}), 7.40(\mathrm{~d}, J=8.5 \mathrm{~Hz}, 2 \mathrm{H}) .{ }^{13} \mathrm{C} \mathrm{NMR}\left(101 \mathrm{MHz}, \mathrm{CDCl}_{3}\right) \delta 145.4,136.7,130.7$, 130.2, 129.0, 120.1 (q, $J=262.4 \mathrm{~Hz}), 90.1 .{ }^{19} \mathrm{~F} \mathrm{NMR}\left(376 \mathrm{MHz}, \mathrm{CDCl}_{3}\right) \delta-56.65$ (s, 3F). Mass Spectrometry: HRMS-EI (m/z): Calcd for $\mathrm{C}_{9} \mathrm{H}_{4} \mathrm{Br}_{2} \mathrm{ClF}_{3} \mathrm{O}[\mathrm{M}]^{+}, 377.8270$. Found, 377.8261.

\section{1-(2,2-Dibromo-1-(trifluoromethoxy)vinyl)-4-nitrobenzene (3jj)}

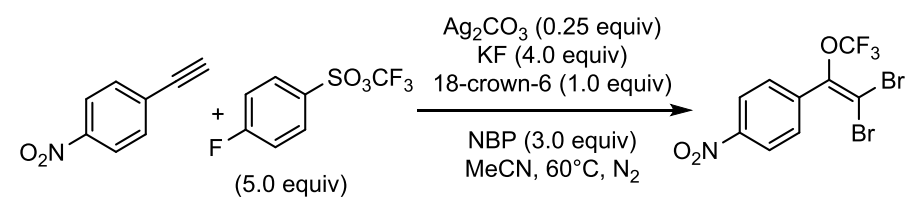

In a glove box, to a $15.0 \mathrm{~mL}$ sealed tube were added in sequence 1-ethynyl-4-nitrobenzene (1 $\mathbf{j} \mathbf{j})$ (74 mg, $0.500 \mathrm{mmol}, 1.00$ equiv), KF (116 mg, $2.00 \mathrm{mmol}, 4.00$ equiv), 18-crown-6 (132 mg, 0.500 mmol, 1.00 equiv), $\quad \mathrm{Ag}_{2} \mathrm{CO}_{3} \quad(34.4 \mathrm{mg}, 0.125 \mathrm{mmol}, 0.250$ equiv), NBP ( $N$-Bromophthalimide) (339 mg, $1.50 \mathrm{mmol}, 3.00$ equiv) and $1.00 \mathrm{~mL} \mathrm{MeCN}$. The mixture was stirring at room temperature for $5 \mathrm{mins}$, then added TFMS (612 $\mathrm{mg}, 2.50 \mathrm{mmol}, 5.00$ equiv). After stirring for $4.0 \mathrm{~h}$ at $60^{\circ} \mathrm{C}$, the reaction mixture was concentrated in vacuo. The residue was then added $10 \mathrm{~mL} \mathrm{CH}_{2} \mathrm{Cl}_{2}$ and filtered. The organic layer was concentrated in vacuo. The residue was purified by preparative TLC, eluting with hexanes/EtOAc 50:1 (v/v) to afford $90 \mathrm{mg}$ 1-(2,2-dibromo-1-(trifluoromethoxy)vinyl)-4-nitrobenzene (3jj) as a yellow liquid (46\% yield). $\mathrm{R}_{f}=0.30$ (hexanes/EtOAc 20:1 (v/v)). NMR Spectroscopy: ${ }^{1} \mathrm{H}$ NMR $\left(400 \mathrm{MHz}, \mathrm{CDCl}_{3}\right) \delta 8.28(\mathrm{~d}$, $J=8.8 \mathrm{~Hz}, 2 \mathrm{H}), 7.75(\mathrm{~d}, J=8.8 \mathrm{~Hz}, 2 \mathrm{H}) .{ }^{13} \mathrm{C}$ NMR $\left(101 \mathrm{MHz}, \mathrm{CDCl}_{3}\right) \delta 148.6,144.2$ (q, $J=1.9$ $\mathrm{Hz}), 138.0,130.5,123.8,120.0$ (q, $J=263.2 \mathrm{~Hz}), 92.6 .{ }^{19} \mathrm{~F} \mathrm{NMR}\left(376 \mathrm{MHz}, \mathrm{CDCl}_{3}\right) \delta-56.78(\mathrm{~s}$, 3F). Mass Spectrometry: HRMS-EI (m/z): Calcd for $\mathrm{C}_{9} \mathrm{H}_{4} \mathrm{Br}_{2} \mathrm{~F}_{3} \mathrm{NO}_{3}[\mathrm{M}]^{+}, 388.8510$. Found, 388.8503 . 


\section{(1-Bromo-2-(trifluoromethoxy)ethene-1,2-diyl)dibenzene $(Z)+(E)(3 \mathbf{k k})$}

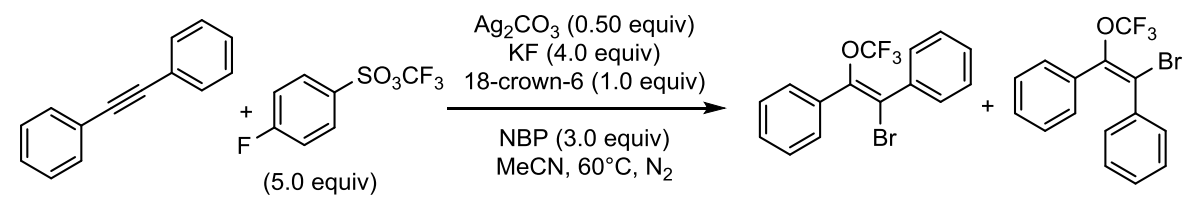

In a glove box, to a $15.0 \mathrm{~mL}$ sealed tube were added in sequence 1,2-diphenylethyne (1 kk) $(89 \mathrm{mg}$, $0.500 \mathrm{mmol}, 1.00$ equiv), KF (116 mg, $2.00 \mathrm{mmol}, 4.00$ equiv), 18 -crown-6 (132 mg, $0.500 \mathrm{mmol}$, 1.00 equiv), $\mathrm{Ag}_{2} \mathrm{CO}_{3}$ (68.8 mg, $0.250 \mathrm{mmol}, 0.500$ equiv), NBP ( $N$-Bromophthalimide) (339 mg, $1.50 \mathrm{mmol}, 3.00$ equiv) and $1.00 \mathrm{~mL} \mathrm{MeCN}$. The mixture was stirring at room temperature for 5 mins, then added TFMS (612 mg, $2.50 \mathrm{mmol}, 5.00$ equiv). After stirring for $4.0 \mathrm{~h}$ at $60{ }^{\circ} \mathrm{C}$, the reaction mixture was concentrated in vacuo. The residue was then added $10 \mathrm{~mL} \mathrm{CH}_{2} \mathrm{Cl}_{2}$ and filtered. The organic layer was concentrated in vacuo. The residue was purified by preparative TLC, eluting with hexanes/EtOAc 100:1 (v/v) to afford $94 \quad \mathrm{mg}$ (1-bromo-2-(trifluoromethoxy)ethene-1,2-diyl)dibenzene $(Z)+(E)(\mathbf{3 k k})$ as a white solid (total $55 \%$ yield).

$\mathrm{R}_{f}=0.24$ (hexanes/EtOAc 40:1 (v/v)). NMR Spectroscopy: ${ }^{1} \mathrm{H}$ NMR $\left(400 \mathrm{MHz}, \mathrm{CDCl}_{3}\right) \delta 7.63$ (dd, $J=6.3,2.8 \mathrm{~Hz}, 2 \mathrm{H}), 7.54$ (d, $J=7.3 \mathrm{~Hz}, 2 \mathrm{H}), 7.48-7.37$ (m, 5H), 7.33 (d, $J=7.3 \mathrm{~Hz}, 1 \mathrm{H}), 7.26-$ $7.13(\mathrm{~m}, 3 \mathrm{H}) .{ }^{13} \mathrm{C} \mathrm{NMR}\left(101 \mathrm{MHz}, \mathrm{CDCl}_{3}\right) \delta 144.8,144.8,142.9,137.2,136.7,133.5,132.4$, $130.2,130.0,129.9,129.5,129.5,129.2,129.0,128.5,128.4,128.3,128.3,120.8$ (q, $J=260.8$ $\mathrm{Hz}), 120.2(\mathrm{q}, J=260.4 \mathrm{~Hz}), 117.9,116.7 .{ }^{19} \mathrm{~F} \mathrm{NMR}\left(376 \mathrm{MHz}, \mathrm{CDCl}_{3}\right) \delta-55.04(\mathrm{~s}, 3 \mathrm{~F}),-55.29(\mathrm{~s}$ 10F). Mass Spectrometry: HRMS-EI (m/z): Calcd for $\mathrm{C}_{15} \mathrm{H}_{10} \mathrm{BrF}_{3} \mathrm{O}[\mathrm{M}]^{+}$, 341.9867. Found, 341.9859 .

\section{Celecoxib derivative (3II)}

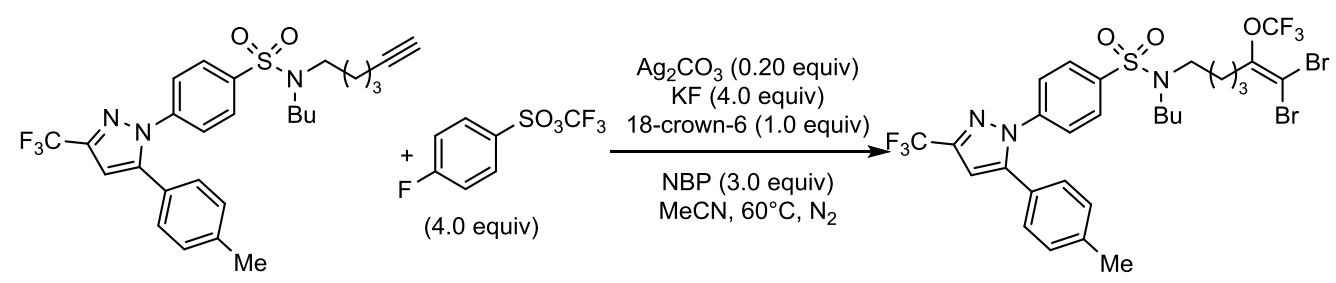

In a glove box, to a $15.0 \mathrm{~mL}$ sealed tube were added in sequence $N$-butyl- $N$-(hex-5-yn-1-yl)Celecoxib (11l) (258 mg, 0.500 mmol, 1.00 equiv), KF (116 mg, 2.00 mmol, 4.00 equiv), 18-crown-6 (132 mg, $0.500 \mathrm{mmol}, 1.00$ equiv), $\mathrm{Ag}_{2} \mathrm{CO}_{3}$ (27.5 mg, $0.100 \mathrm{mmol}$, 0.200 equiv), NBP ( $N$-Bromophthalimide) (339 mg, $1.50 \mathrm{mmol}, 3.00$ equiv) and $1.00 \mathrm{~mL} \mathrm{MeCN}$. The mixture was stirring at room temperature for $5 \mathrm{mins}$, then added TFMS (489 $\mathrm{mg}, 2.00 \mathrm{mmol}$, 4.00 equiv). After stirring for $4.0 \mathrm{~h}$ at $60^{\circ} \mathrm{C}$, the reaction mixture was concentrated in vacuo. The residue was then added $10 \mathrm{~mL} \mathrm{CH} \mathrm{Cl}_{2}$ and filtered. The organic layer was concentrated in vacuo. The residue was purified by preparative TLC, eluting with hexanes/EtOAc10:1 (v/v) to afford 304 mg Celecoxib derivative (3II) as a white solid ( $80 \%$ yield).

$\mathrm{R}_{f}=0.36$ (hexanes/EtOAc 10:1 (v/v)). NMR Spectroscopy: ${ }^{1} \mathrm{H}$ NMR $\left(400 \mathrm{MHz}, \mathrm{CDCl}_{3}\right) \delta 7.80(\mathrm{~d}$, $J=8.4 \mathrm{~Hz}, 2 \mathrm{H}), 7.48(\mathrm{~d}, J=8.4 \mathrm{~Hz}, 2 \mathrm{H}), 7.17(\mathrm{~d}, J=8.0 \mathrm{~Hz}, 2 \mathrm{H}), 7.11(\mathrm{~d}, J=8.0 \mathrm{~Hz}, 2 \mathrm{H}), 6.75$ 
(s, 1H), $3.22-3.06(\mathrm{~m}, 4 \mathrm{H}), 2.60(\mathrm{t}, J=6.0 \mathrm{~Hz}, 2 \mathrm{H}), 2.37(\mathrm{~s}, 3 \mathrm{H}), 1.70-1.55(\mathrm{~m}, 4 \mathrm{H}), 1.50(\mathrm{dt}, J$ $=15.1,7.6 \mathrm{~Hz}, 2 \mathrm{H}), 1.28(\mathrm{dt}, J=14.5,7.3 \mathrm{~Hz}, 3 \mathrm{H}), 0.90(\mathrm{t}, J=7.3 \mathrm{~Hz}, 3 \mathrm{H}) .{ }^{13} \mathrm{C}$ NMR $(101 \mathrm{MHz}$, $\left.\mathrm{CDCl}_{3}\right) \delta 148.5,145.2,144.0(\mathrm{q}, J=38.5 \mathrm{~Hz}), 142.3,139.8,139.4,129.7,128.7,128.0,125.7$, 125.5, 121.1 (q, $J=269.2 \mathrm{~Hz}), 119.7$ (q, $J=261.0 \mathrm{~Hz}), 106.2,88.5,48.3,47.8,31.4,30.7,27.7$, 22.9, 21.2, 19.9, 13.6. ${ }^{19} \mathrm{~F}$ NMR (376 MHz, $\left.\mathrm{CDCl}_{3}\right) \delta-56.12$ (s, 3F), -62.37 (s, 3F). Mass Spectrometry: HRMS-ESI (m/z): Calcd for $\mathrm{C}_{28} \mathrm{H}_{30} \mathrm{Br}_{2} \mathrm{~F}_{6} \mathrm{~N}_{3} \mathrm{O}_{3} \mathrm{~S}[\mathrm{M}+\mathrm{H}]^{+}$, 760.0273. Found, 760.0270 .

\section{Epipodophyllotoxin derivative (3mm)}

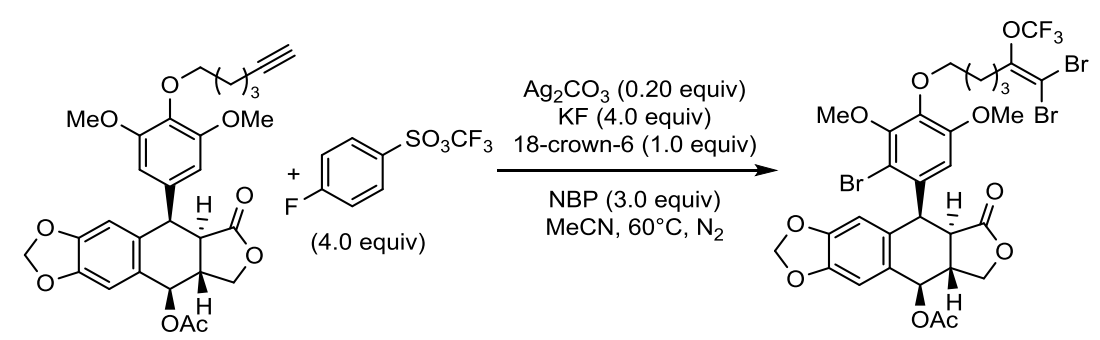

In a glove box, to a $15.0 \mathrm{~mL}$ sealed tube were added in sequence 4'-O-(hex-5-yn-1-yl)Acetyl epipodophyllotoxin (1mm) (261 mg, $0.500 \mathrm{mmol}, 1.00$ equiv), KF (116 mg, $2.00 \mathrm{mmol}, 4.00$ equiv), 18-crown-6 (132 mg, $0.500 \mathrm{mmol}, 1.00$ equiv), $\mathrm{Ag}_{2} \mathrm{CO}_{3}(27.5 \mathrm{mg}, 0.100 \mathrm{mmol}, 0.200$ equiv), NBP ( $N$-Bromophthalimide) $(339 \mathrm{mg}, 1.50 \mathrm{mmol}, 3.00$ equiv) and $1.00 \mathrm{~mL} \mathrm{MeCN}$. The mixture was stirring at room temperature for $5 \mathrm{mins}$, then added TFMS (489 mg, $2.00 \mathrm{mmol}, 4.00$ equiv). After stirring for $4.0 \mathrm{~h}$ at $60{ }^{\circ} \mathrm{C}$, the reaction mixture was concentrated in vacuo. The residue was then added $10 \mathrm{~mL} \mathrm{CH}_{2} \mathrm{Cl}_{2}$ and filtered. The organic layer was concentrated in vacuo. The residue was purified by preparative TLC, eluting with hexanes/EtOAc 3:1 (v/v) to afford 143 $\mathrm{mg}$ epipodophyllotoxin derivative (3 $\mathbf{m m})$ as a white solid ( $34 \%$ yield).

$\mathrm{R}_{f}=0.65$ (hexanes/EtOAc 1:1 (v/v)). NMR Spectroscopy: ${ }^{1} \mathrm{H}$ NMR $\left(400 \mathrm{MHz}, \mathrm{CDCl}_{3}\right) \delta 6.86(\mathrm{~s}$, 1H), $6.71(\mathrm{~s}, 1 \mathrm{H}), 6.27(\mathrm{~s}, 1 \mathrm{H}), 6.06(\mathrm{~d}, J=3.5 \mathrm{~Hz}, 1 \mathrm{H}), 5.92(\mathrm{dd}, J=13.5,1.3 \mathrm{~Hz}, 2 \mathrm{H}), 4.56$ (d, $J$ $=5.0 \mathrm{~Hz}, 1 \mathrm{H}), 4.47(\mathrm{dd}, J=9.8,7.2 \mathrm{~Hz}, 1 \mathrm{H}), 4.36(\mathrm{dd}, J=9.8,1.5 \mathrm{~Hz}, 1 \mathrm{H}), 4.04(\mathrm{t}, J=5.0 \mathrm{~Hz}$, $2 \mathrm{H}), 3.88(\mathrm{~s}, 3 \mathrm{H}), 3.83(\mathrm{~s}, 3 \mathrm{H}), 3.46(\mathrm{dd}, J=10.1,5.2 \mathrm{~Hz}, 1 \mathrm{H}), 3.14-3.04(\mathrm{~m}, 1 \mathrm{H}), 2.66(\mathrm{t}, J=$ $6.7 \mathrm{~Hz}, 2 \mathrm{H}), 2.06(\mathrm{~s}, 3 \mathrm{H}), 1.89-1.79(\mathrm{~m}, 4 \mathrm{H}) .{ }^{13} \mathrm{C} \mathrm{NMR}\left(101 \mathrm{MHz}, \mathrm{CDCl}_{3}\right) \delta 178.5,170.6,153.1$, 152.1, 149.1, 148.8, 146.5, 141.7, 136.2, 131.6, 126.4, 119.9 (q, $J=260.9 \mathrm{~Hz}), 110.5,108.7$, $108.3,101.4,88.3,73.2,72.0,68.9,61.2,56.3,45.5,42.5,38.7,31.9,29.4,22.7,21.0 .{ }^{19} \mathrm{~F}$ NMR $\left(376 \mathrm{MHz}, \mathrm{CDCl}_{3}\right) \delta-56.03$ (s, 3F). Mass Spectrometry: HRMS-ESI $(\mathrm{m} / \mathrm{z})$ : Calcd for $\mathrm{C}_{30} \mathrm{H}_{28} \mathrm{Br}_{3} \mathrm{~F}_{3} \mathrm{NaO}_{10}[\mathrm{M}+\mathrm{Na}]^{+}$, 864.9077. Found, 864.9078.

\section{Mycophenolic acid derivative (3nn)}

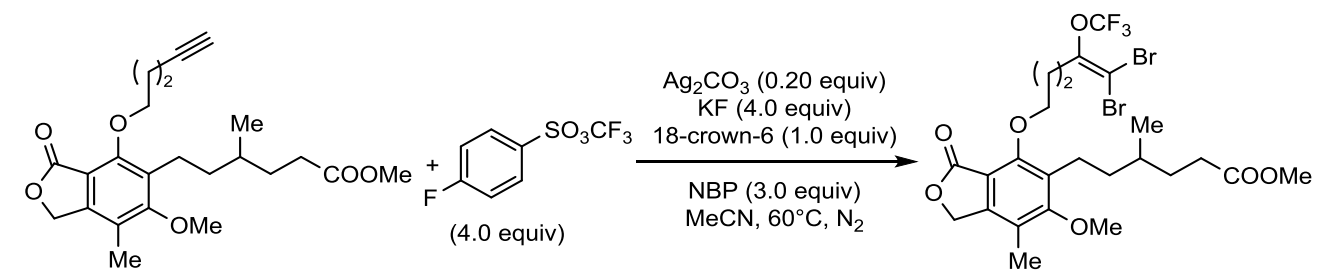

In a glove box, to a $15.0 \mathrm{~mL}$ sealed tube were added in sequence mycophenolic acid derivative 
(1nn) (201 mg, $0.500 \mathrm{mmol}, 1.00$ equiv), KF (116 mg, $2.00 \mathrm{mmol}, 4.00$ equiv), 18-crown-6 (132 $\mathrm{mg}, \quad 0.500$ mmol, 1.00 equiv), $\mathrm{Ag}_{2} \mathrm{CO}_{3}(27.5 \mathrm{mg}, 0.100 \mathrm{mmol}, 0.200$ equiv), NBP ( $N$-Bromophthalimide) (339 mg, $1.50 \mathrm{mmol}, 3.00$ equiv) and $1.00 \mathrm{~mL} \mathrm{MeCN}$. The mixture was stirring at room temperature for 5 mins, then added TFMS (489 mg, $2.00 \mathrm{mmol}, 4.00$ equiv). After stirring for $4.0 \mathrm{~h}$ at $60{ }^{\circ} \mathrm{C}$, the reaction mixture was concentrated in vacuo. The residue was then added $10 \mathrm{~mL} \mathrm{CH}_{2} \mathrm{Cl}_{2}$ and filtered. The organic layer was concentrated in vacuo. The residue was purified by preparative TLC, eluting with hexanes/EtOAc 3:1 (v/v) to afford $107 \mathrm{mg}$ mycophenolic acid derivative (3nn) as a colorless liquid (33\% yield).

$\mathrm{R}_{f}=0.25$ (hexanes/EtOAc 4:1 (v/v)). NMR Spectroscopy: ${ }^{1} \mathrm{H}$ NMR $\left(400 \mathrm{MHz}, \mathrm{CDCl}_{3}\right) \delta 5.12(\mathrm{~s}$, $2 \mathrm{H}), 4.26(\mathrm{t}, J=6.1 \mathrm{~Hz}, 2 \mathrm{H}), 3.79(\mathrm{~s}, 3 \mathrm{H}), 3.66(\mathrm{~s}, 3 \mathrm{H}), 2.90-2.81(\mathrm{~m}, 2 \mathrm{H}), 2.71-2.57(\mathrm{~m}, 2 \mathrm{H})$, $2.40-2.25(\mathrm{~m}, 2 \mathrm{H}), 2.17(\mathrm{~s}, 3 \mathrm{H}), 2.14-2.06(\mathrm{~m}, 2 \mathrm{H}), 1.80-1.70(\mathrm{~m}, 1 \mathrm{H}), 1.58-1.44(\mathrm{~m}, 3 \mathrm{H})$, $1.41-1.29(\mathrm{~m}, 1 \mathrm{H}), 0.98(\mathrm{~d}, J=6.1 \mathrm{~Hz}, 2 \mathrm{H}) .{ }^{13} \mathrm{C} \mathrm{NMR}\left(101 \mathrm{MHz}, \mathrm{CDCl}_{3}\right) \delta 174.5,169.2,163.0$, $155.4,148.8,146.7,130.3,120.1,119.9$ (q, $J=260.7 \mathrm{~Hz}), 112.6,88.5,74.0,68.4,61.2,51.6,37.5$, 33.0, 31.9, 31.7, 29.3, 26.9, 22.3, 19.3, 11.7. $\left.{ }^{19} \mathrm{~F} \mathrm{NMR} \mathrm{(376} \mathrm{MHz,} \mathrm{CDCl}_{3}\right) \delta-56.07$ (s, 3F). Mass Spectrometry: HRMS-ESI (m/z): Calcd for $\mathrm{C}_{24} \mathrm{H}_{30} \mathrm{Br}_{2} \mathrm{~F}_{3} \mathrm{O}_{7}[\mathrm{M}+\mathrm{H}]^{+}$, 645.0305. Found, 645.0308.

\section{Artesunatederivative (30o)}

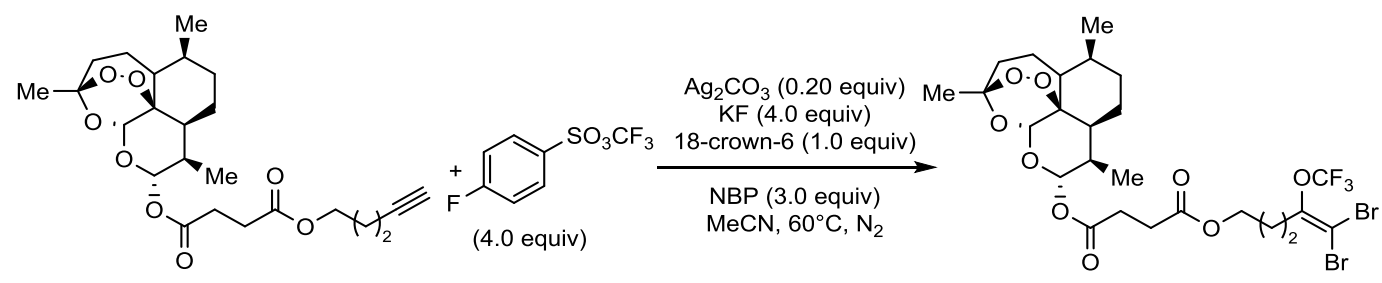

In a glove box, to a $15.0 \mathrm{~mL}$ sealed tube were added in sequence artesunate derivative (10o) $(225$ $\mathrm{mg}, 0.500 \mathrm{mmol}, 1.00$ equiv), KF (116 mg, $2.00 \mathrm{mmol}, 4.00$ equiv), 18-crown-6 (132 mg, 0.500 mmol, 1.00 equiv), $\mathrm{Ag}_{2} \mathrm{CO}_{3}$ (27.5 mg, $0.100 \mathrm{mmol}, 0.200$ equiv), NBP ( $N$-Bromophthalimide) (339 mg, $1.50 \mathrm{mmol}, 3.00$ equiv) and $1.00 \mathrm{~mL} \mathrm{MeCN}$. The mixture was stirring at room temperature for $5 \mathrm{mins}$, then added TFMS ( $489 \mathrm{mg}, 2.00 \mathrm{mmol}, 4.00$ equiv). After stirring for 4.0 $\mathrm{h}$ at $60^{\circ} \mathrm{C}$, the reaction mixture was concentrated in vacuo. The residue was then added $10 \mathrm{~mL}$ $\mathrm{CH}_{2} \mathrm{Cl}_{2}$ and filtered. The organic layer was concentrated in vacuo. The residue was purified by preparative HPLC $\left(9 \mathrm{~mL} / \mathrm{min}\right.$, detector $\mathrm{UV} \lambda \max 210 \mathrm{~nm}, \mathrm{MeCN} / \mathrm{H}_{2} \mathrm{O}=70: 30(0 \mathrm{~min})$, $\mathrm{MeCN} / \mathrm{H}_{2} \mathrm{O}=100: 0(30 \mathrm{~min}), \mathrm{MeCN} / \mathrm{H}_{2} \mathrm{O}=100: 0(40 \mathrm{~min})$ ) to afford $246 \mathrm{mg}$ artesunate derivative (3oo) (30.4 $\mathrm{min}$ ) as a colorless liquid (71\% yield).

$\mathrm{R}_{f}=0.38$ (hexanes/EtOAc 4:1 (v/v)). NMR Spectroscopy: ${ }^{1} \mathrm{H}$ NMR $\left(400 \mathrm{MHz}, \mathrm{CDCl}_{3}\right) \delta 5.76(\mathrm{~d}$, $J=9.8 \mathrm{~Hz}, 1 \mathrm{H}), 5.40(\mathrm{~s}, 1 \mathrm{H}), 4.09(\mathrm{t}, J=5.6 \mathrm{~Hz}, 2 \mathrm{H}), 2.76-2.46(\mathrm{~m}, 7 \mathrm{H}), 2.34(\mathrm{td}, J=14.0,3.8$ $\mathrm{Hz}, 1 \mathrm{H}), 2.04-1.96(\mathrm{~m}, 1 \mathrm{H}), 1.94-1.81(\mathrm{~m}, 3 \mathrm{H}), 1.79-1.65(\mathrm{~m}, 2 \mathrm{H}), 1.59(\mathrm{dt}, J=13.7,4.3 \mathrm{~Hz}$, 1H), $1.46(\mathrm{dd}, J=20.5,9.2 \mathrm{~Hz}, 1 \mathrm{H}), 1.39(\mathrm{~s}, 3 \mathrm{H}), 1.37-1.19(\mathrm{~m}, 3 \mathrm{H}), 1.05-0.96(\mathrm{~m}, 1 \mathrm{H}), 0.93$ $(\mathrm{d}, J=5.9 \mathrm{~Hz}, 3 \mathrm{H}), 0.82$ (d, $J=7.1 \mathrm{~Hz}, 3 \mathrm{H}) .{ }^{13} \mathrm{C} \mathrm{NMR}\left(101 \mathrm{MHz}, \mathrm{CDCl}_{3}\right) \delta 172.0,171.1,148.0$, $119.8(\mathrm{q}, J=261.0 \mathrm{~Hz}), 104.5,92.2,91.6,88.9,80.2,63.2,51.6,45.3,37.3,36.3,34.1,31.8,29.2$, 28.9, 26.0, 24.9, 24.6, 22.0, 20.3, 12.1. ${ }^{19} \mathrm{~F}$ NMR (376 MHz, $\left.\mathrm{CDCl}_{3}\right) \delta-56.17$ (s, 3F). Mass Spectrometry: HRMS-ESI (m/z): Calcd for $\mathrm{C}_{25} \mathrm{H}_{33} \mathrm{Br}_{2} \mathrm{~F}_{3} \mathrm{NaO}_{9}[\mathrm{M}+\mathrm{Na}]^{+}$, 715.0336. Found, 715.0336 . 


\section{Ezetimibe derivative (3pp)}

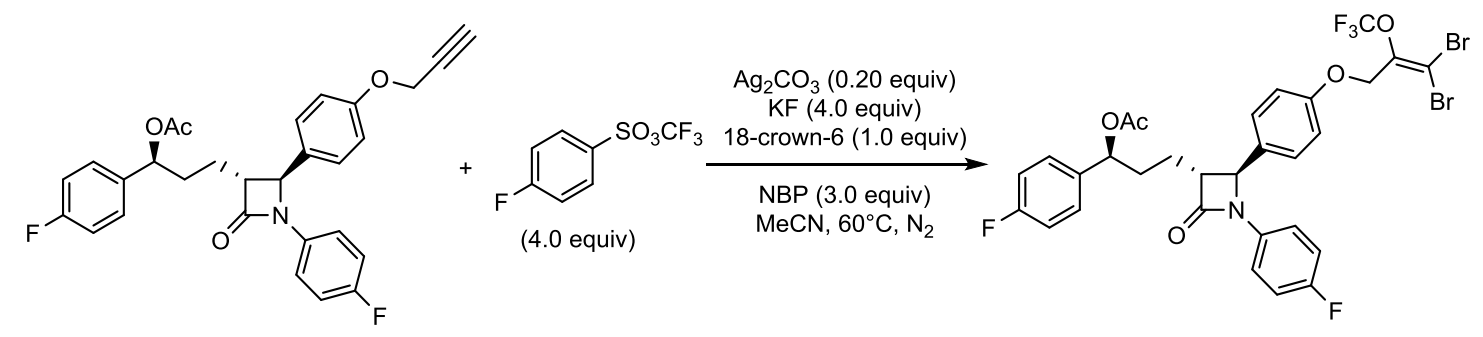

In a glove box, to a $15.0 \mathrm{~mL}$ sealed tube were added in sequence ezetimibe derivative (100) $(245$ $\mathrm{mg}, 0.500 \mathrm{mmol}, 1.00$ equiv), KF (116 mg, $2.00 \mathrm{mmol}, 4.00$ equiv), 18 -crown-6 (132 mg, 0.500 mmol, 1.00 equiv), $\mathrm{Ag}_{2} \mathrm{CO}_{3}$ (27.5 mg, $0.100 \mathrm{mmol}, 0.200$ equiv), NBP ( $N$-Bromophthalimide) (339 mg, $1.50 \mathrm{mmol}, 3.00$ equiv) and $1.00 \mathrm{~mL} \mathrm{MeCN}$. The mixture was stirring at room temperature for $5 \mathrm{mins}$, then added TFMS ( $489 \mathrm{mg}, 2.00 \mathrm{mmol}, 4.00$ equiv). After stirring for 4.0 $\mathrm{h}$ at $60{ }^{\circ} \mathrm{C}$, the reaction mixture was concentrated in vacuo. The residue was then added $10 \mathrm{~mL}$ $\mathrm{CH}_{2} \mathrm{Cl}_{2}$ and filtered. The organic layer was concentrated in vacuo. The residue was purified by preparative TLC, eluting with hexanes/EtOAc 8:1 (v/v) to afford $208 \mathrm{mg}$ ezetimibe derivative (3pp) as a white solid (57\% yield).

$\mathrm{R}_{f}=0.34$ (hexanes/EtOAc 4:1 (v/v)). NMR Spectroscopy: ${ }^{1} \mathrm{H}$ NMR $\left(400 \mathrm{MHz}, \mathrm{CDCl}_{3}\right) \delta 7.36-$ $7.17(\mathrm{~m}, 6 \mathrm{H}), 6.99$ (t, $J=7.9 \mathrm{~Hz}, 2 \mathrm{H}), 6.96-6.82(\mathrm{~m}, 4 \mathrm{H}), 5.71(\mathrm{t}, J=6.1 \mathrm{~Hz}, 1 \mathrm{H}), 4.84(\mathrm{~s}, 2 \mathrm{H})$, $4.59(\mathrm{~s}, 1 \mathrm{H}), 3.06(\mathrm{t}, J=7.4 \mathrm{~Hz}, 1 \mathrm{H}), 2.10-2.00(\mathrm{~m}, 5 \mathrm{H}), 1.92-1.77(\mathrm{~m}, 2 \mathrm{H}) .{ }^{13} \mathrm{C}$ NMR $(101$ $\left.\mathrm{MHz}, \mathrm{CDCl}_{3}\right) \delta 170.1,166.9,162.3(\mathrm{~d}, J=246.5 \mathrm{~Hz}), 158.9(\mathrm{~d}, J=243.3 \mathrm{~Hz}), 157.9,143.7,135.8$ (d, $J=3.2 \mathrm{~Hz}), 133.8$ (d, $J=2.6 \mathrm{~Hz}), 130.8,128.2(\mathrm{~d}, J=8.2 \mathrm{~Hz}), 127.3,119.7$ (q, $J=262.1 \mathrm{~Hz})$, $118.3(\mathrm{~d}, J=7.8 \mathrm{~Hz}), 115.7$ (d, $J=23.9 \mathrm{~Hz}), 115.6,115.4(\mathrm{~d}, J=21.5 \mathrm{~Hz}), 95.0,74.7,65.2,60.6$, 60.1, 33.6, 24.8, 21.0. ${ }^{19} \mathrm{~F}$ NMR (376 MHz, $\left.\mathrm{CDCl}_{3}\right) \delta-56.41$ (s, 3F), -113.67--113.74 (m, 1F), $-117.79--117.86$ (m. 1F). Mass Spectrometry: HRMS-ESI (m/z): Calcd for $\mathrm{C}_{30} \mathrm{H}_{24} \mathrm{Br}_{2} \mathrm{~F}_{5} \mathrm{NNaO}_{5}$ $[\mathrm{M}+\mathrm{Na}]^{+}$, 753.9834. Found, 753.9831 .

\section{(1S)-(-)-camphanic acid derivative(3qq)}

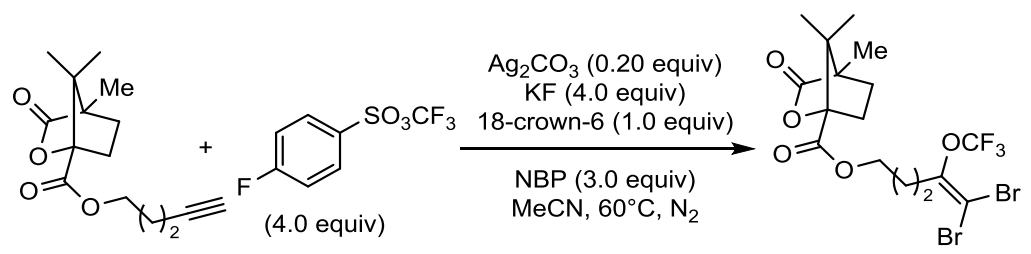

In a glove box, to a $15.0 \mathrm{~mL}$ sealed tube were added in sequence (1S)-(-)-camphanic acid derivative (1qq) (132 mg, $0.500 \mathrm{mmol}, 1.00$ equiv), $\mathrm{KF}$ (116 mg, $2.00 \mathrm{mmol}, 4.00$ equiv), 18-crown-6 (132 mg, 0.500 mmol, 1.00 equiv), $\mathrm{Ag}_{2} \mathrm{CO}_{3}$ (27.5 mg, 0.100 mmol, 0.200 equiv), NBP ( $N$-Bromophthalimide) (339 mg, $1.50 \mathrm{mmol}, 3.00$ equiv) and $1.00 \mathrm{~mL} \mathrm{MeCN}$. The mixture was stirring at room temperature for 5 mins, then added TFMS ( $489 \mathrm{mg}, 2.00 \mathrm{mmol}, 4.00$ equiv). After stirring for $4.0 \mathrm{~h}$ at $60{ }^{\circ} \mathrm{C}$, the reaction mixture was concentrated in vacuo. The residue was then added $10 \mathrm{~mL} \mathrm{CH} \mathrm{Cl}_{2}$ and filtered. The organic layer was concentrated in vacuo. The residue was purified by preparative HPLC $\left(9 \mathrm{~mL} / \mathrm{min}\right.$, detector UV $\lambda \max 210 \mathrm{~nm}, \mathrm{MeCN} / \mathrm{H}_{2} \mathrm{O}=50: 50(0$ 
$\min ), \mathrm{MeCN} / \mathrm{H}_{2} \mathrm{O}=70: 30(20 \mathrm{~min}), \mathrm{MeCN} / \mathrm{H}_{2} \mathrm{O}=90: 10$ (30 min), $\mathrm{MeCN} / \mathrm{H}_{2} \mathrm{O}=100: 0$ (40 min)) to afford $178 \mathrm{mg}(1 \mathrm{~S})$-(-)-camphanic acid derivative (3qq) (17.1 $\mathrm{min})$ as a colorless liquid (70\% yield).

$\mathrm{R}_{f}=0.14$ (hexanes/EtOAc 10:1 (v/v)). NMR Spectroscopy: ${ }^{1} \mathrm{H}$ NMR (400 MHz, $\left.\mathrm{CDCl}_{3}\right) \delta 4.27-$ $4.16(\mathrm{~m}, 2 \mathrm{H}), 2.65(\mathrm{t}, J=7.6 \mathrm{~Hz}, 2 \mathrm{H}), 2.44-2.32(\mathrm{~m}, 1 \mathrm{H}), 2.03-1.84(\mathrm{~m}, 4 \mathrm{H}), 1.69-1.59(\mathrm{~m}$, 1H), 1.07 (s, 3H), $1.02(\mathrm{~s}, 3 \mathrm{H}), 0.92(\mathrm{~s}, 3 \mathrm{H}) .{ }^{13} \mathrm{C} \mathrm{NMR}\left(101 \mathrm{MHz}, \mathrm{CDCl}_{3}\right) \delta 178.0,167.4,147.6(\mathrm{q}$, $J=1.0 \mathrm{~Hz}), 119.8$ (q, $J=261.3 \mathrm{~Hz}), 91.0,89.2,64.0,54.8,54.2,30.7,28.9,28.9,25.0,16.8,16.8$, 9.7, 9.7. ${ }^{19} \mathrm{~F}$ NMR $\left(376 \mathrm{MHz}, \mathrm{CDCl}_{3}\right) \delta-56.22(\mathrm{~s}, 3 \mathrm{~F})$. Mass Spectrometry: HRMS-ESI (m/z): Calcd for $\mathrm{C}_{16} \mathrm{H}_{20} \mathrm{Br}_{2} \mathrm{~F}_{3} \mathrm{O}_{5}[\mathrm{M}+\mathrm{H}]^{+}$, 506.9624. Found, 506.9619.

\section{Febuxostate derivative (3rr)}

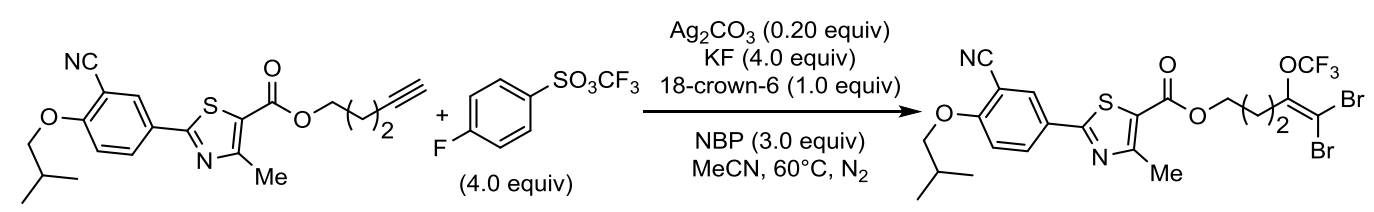

In a glove box, to a $15.0 \mathrm{~mL}$ sealed tube were added in sequence febuxostate derivative (1 $\mathbf{r r})$ (191 $\mathrm{mg}, 0.500 \mathrm{mmol}, 1.00$ equiv), KF (116 mg, $2.00 \mathrm{mmol}, 4.00$ equiv), 18-crown-6 (132 mg, 0.500 mmol, 1.00 equiv), $\mathrm{Ag}_{2} \mathrm{CO}_{3}$ (27.5 mg, $0.100 \mathrm{mmol}, 0.200$ equiv), NBP ( $N$-Bromophthalimide) (339 mg, $1.50 \mathrm{mmol}, 3.00$ equiv) and $1.00 \mathrm{~mL} \mathrm{MeCN}$. The mixture was stirring at room temperature for $5 \mathrm{mins}$, then added TFMS ( $489 \mathrm{mg}, 2.00 \mathrm{mmol}, 4.00$ equiv). After stirring for 4.0 $\mathrm{h}$ at $60^{\circ} \mathrm{C}$, the reaction mixture was concentrated in vacuo. The residue was then added $10 \mathrm{~mL}$ $\mathrm{CH}_{2} \mathrm{Cl}_{2}$ and filtered. The organic layer was concentrated in vacuo. The residue was purified by preparative TLC, eluting with hexanes/EtOAc 10:1 (v/v) to afford $210 \mathrm{mg}$ febuxostate derivative (3rr) as a white solid (67\% yield).

$\mathrm{R}_{f}=0.14$ (hexanes/EtOAc 10:1 (v/v)). NMR Spectroscopy: ${ }^{1} \mathrm{H}$ NMR $\left(400 \mathrm{MHz}, \mathrm{CDCl}_{3}\right) \delta 8.11(\mathrm{~d}$, $J=1.7 \mathrm{~Hz}, 1 \mathrm{H}), 8.03(\mathrm{dd}, J=8.8,1.7 \mathrm{~Hz}, 1 \mathrm{H}), 6.98(\mathrm{~d}, J=8.9 \mathrm{~Hz}, 1 \mathrm{H}), 4.30(\mathrm{t}, J=6.0 \mathrm{~Hz}, 2 \mathrm{H})$, $3.87(\mathrm{~d}, J=6.5 \mathrm{~Hz}, 2 \mathrm{H}), 2.84-2.66(\mathrm{~m}, 5 \mathrm{H}), 2.23-2.11(\mathrm{~m}, 1 \mathrm{H}), 2.10-1.99(\mathrm{~m}, 2 \mathrm{H}), 1.06(\mathrm{~d}, J$ $=6.7 \mathrm{~Hz}, 6 \mathrm{H}) .{ }^{13} \mathrm{C} \mathrm{NMR}\left(101 \mathrm{MHz}, \mathrm{CDCl}_{3}\right) \delta 167.4,162.5,161.8,161.5,147.8(\mathrm{q}, J=1.1 \mathrm{~Hz})$, 132.6, 132.0, 125.9, 121.3, 119.8 (q, $J=261.2 \mathrm{~Hz}$ ), 115.4, 112.7, 102.9, 89.2, 75.7, 63.7, 29.0, 28.2, 25.0, 19.1, 17.5. ${ }^{19} \mathrm{~F}$ NMR (376 $\mathrm{MHz}, \mathrm{CDCl}_{3}$ ) $\delta-56.10$ (s, 3F). Mass Spectrometry: HRMS-ESI ( $\mathrm{m} / \mathrm{z}$ ): Calcd for $\mathrm{C}_{22} \mathrm{H}_{22} \mathrm{Br}_{2} \mathrm{~F}_{3} \mathrm{~N}_{2} \mathrm{O}_{4} \mathrm{~S}[\mathrm{M}+\mathrm{H}]^{+}$, 624.9614. Found, 624.9611.

\section{L-phenylalanine derivative (3ss)}

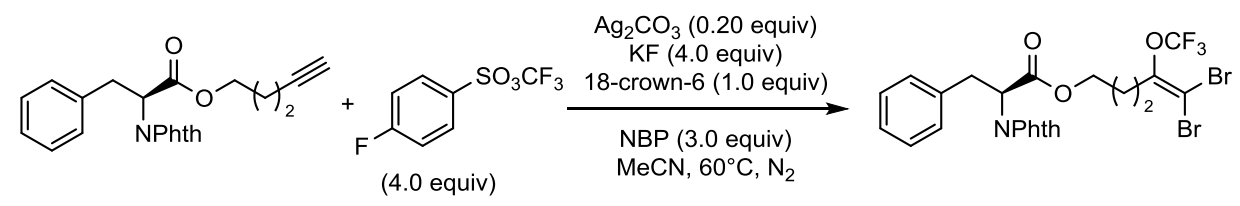

In a glove box, to a $15.0 \mathrm{~mL}$ sealed tube were added in sequence L-phenylalanine derivative (1ss) (180 mg, $0.500 \mathrm{mmol}, 1.00$ equiv), KF (116 mg, $2.00 \mathrm{mmol}, 4.00$ equiv), 18-crown-6 (132 mg, 0.500 mmol, 1.00 equiv), $\mathrm{Ag}_{2} \mathrm{CO}_{3}(27.5 \mathrm{mg}, \quad 0.100 \mathrm{mmol}, \quad 0.200$ equiv $), \quad \mathrm{NBP}$ ( $N$-Bromophthalimide) (339 mg, $1.50 \mathrm{mmol}, 3.00$ equiv) and $1.00 \mathrm{~mL} \mathrm{MeCN}$. The mixture was stirring at room temperature for $5 \mathrm{mins}$, then added TFMS (489 mg, $2.00 \mathrm{mmol}, 4.00$ equiv). After 
stirring for $4.0 \mathrm{~h}$ at $60{ }^{\circ} \mathrm{C}$, the reaction mixture was concentrated in vacuo. The residue was then added $10 \mathrm{~mL} \mathrm{CH}_{2} \mathrm{Cl}_{2}$ and filtered. The organic layer was concentrated in vacuo. The residue was purified by preparative TLC, eluting with hexanes/EtOAc 10:1 (v/v) to afford $220 \mathrm{mg}$ L-phenylalanine derivative (3ss) as a colorless liquid (73\% yield).

$\mathrm{R}_{f}=0.18$ (hexanes/EtOAc 10:1 (v/v)). NMR Spectroscopy: ${ }^{1} \mathrm{H}$ NMR $\left(400 \mathrm{MHz}, \mathrm{CDCl}_{3}\right) \delta 7.81-$ $7.74(\mathrm{~m}, 2 \mathrm{H}), 7.73-7.65(\mathrm{~m}, 2 \mathrm{H}), 7.22-7.09(\mathrm{~m}, 5 \mathrm{H}), 5.16(\mathrm{dd}, J=11.3,5.2 \mathrm{~Hz}, 1 \mathrm{H}), 4.31-$ $4.15(\mathrm{~m}, 2 \mathrm{H}), 3.57$ (ddd, $J=25.6,14.3,8.4 \mathrm{~Hz}, 2 \mathrm{H}), 2.64-2.53(\mathrm{~m}, 2 \mathrm{H}), 1.97-1.85(\mathrm{~m}, 2 \mathrm{H}) .{ }^{13} \mathrm{C}$ NMR $\left(101 \mathrm{MHz}, \mathrm{CDCl}_{3}\right) \delta 168.9,167.5,147.8,136.7,134.3,131.6,128.9,128.7,127.0,123.6$, $119.8(\mathrm{q}, J=261.6 \mathrm{~Hz}), 89.1,64.5,53.4,34.8,28.9,25.0 .{ }^{19} \mathrm{~F} \mathrm{NMR}\left(376 \mathrm{MHz}, \mathrm{CDCl}_{3}\right) \delta-56.17(\mathrm{~s}$, 3F). Mass Spectrometry: HRMS-ESI (m/z): Calcd for $\mathrm{C}_{23} \mathrm{H}_{19} \mathrm{Br}_{2} \mathrm{~F}_{3} \mathrm{NO}_{5}[\mathrm{M}+\mathrm{H}]^{+}, 603.9577$. Found, 603.9572.

\section{Lithocholic acid derivative (3tt)}

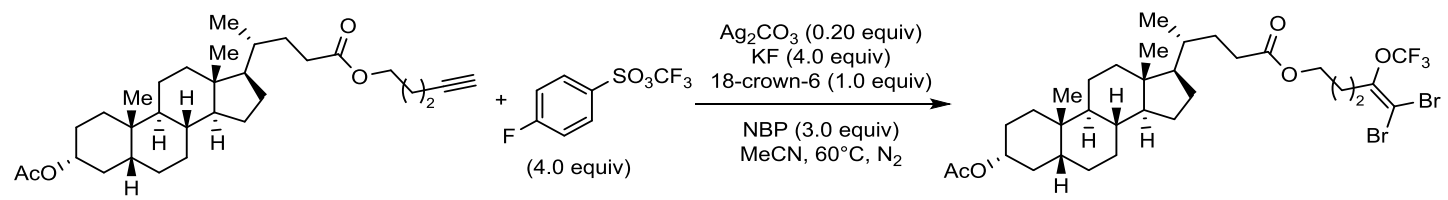

In a glove box, to a $15.0 \mathrm{~mL}$ sealed tube were added in sequence lithocholic acid derivative (1tt) ( $242 \mathrm{mg}, 0.500 \mathrm{mmol}, 1.00$ equiv), $\mathrm{KF}$ (116 mg, $2.00 \mathrm{mmol}, 4.00$ equiv), 18-crown-6 (132 mg, 0.500 mmol, 1.00 equiv), $\mathrm{Ag}_{2} \mathrm{CO}_{3} \quad(27.5 \mathrm{mg}, 0.100 \mathrm{mmol}, 0.200$ equiv), NBP ( $N$-Bromophthalimide) (339 mg, $1.50 \mathrm{mmol}, 3.00$ equiv) and $1.00 \mathrm{~mL} \mathrm{MeCN}$. The mixture was stirring at room temperature for $5 \mathrm{mins}$, then added TFMS ( $489 \mathrm{mg}, 2.00 \mathrm{mmol}, 4.00$ equiv). After stirring for $4.0 \mathrm{~h}$ at $60{ }^{\circ} \mathrm{C}$, the reaction mixture was concentrated in vacuo. The residue was then added $10 \mathrm{~mL} \mathrm{CH}_{2} \mathrm{Cl}_{2}$ and filtered. The organic layer was concentrated in vacuo. The residue was purified by preparative HPLC $\left(9 \mathrm{~mL} / \mathrm{min}\right.$, detector $\mathrm{UV} \lambda \max 210 \mathrm{~nm}, \mathrm{MeCN} / \mathrm{H}_{2} \mathrm{O}=70: 30(0 \mathrm{~min})$, $\left.\mathrm{MeCN} / \mathrm{H}_{2} \mathrm{O}=90: 10(30 \mathrm{~min}), \mathrm{MeCN} / \mathrm{H}_{2} \mathrm{O}=100: 0(80 \mathrm{~min})\right)$ to afford $258 \mathrm{mg}$ lithocholic acid derivative (3tt) (77.05 $\mathrm{min}$ ) as a colorless liquid (71\% yield).

$\mathrm{R}_{f}=0.35$ (hexanes/EtOAc 10:1 (v/v)). NMR Spectroscopy: ${ }^{1} \mathrm{H}$ NMR $\left(400 \mathrm{MHz}, \mathrm{CDCl}_{3}\right) \delta 4.77-$ $4.63(\mathrm{~m}, 1 \mathrm{H}), 4.08(\mathrm{t}, J=6.1 \mathrm{~Hz}, 2 \mathrm{H}), 2.66(\mathrm{t}, J=7.5 \mathrm{~Hz}, 2 \mathrm{H}), 2.38-2.30(\mathrm{~m}, 1 \mathrm{H}), 2.24-2.16$ $(\mathrm{m}, 1 \mathrm{H}), 2.01(\mathrm{~s}, 3 \mathrm{H}), 1.99-1.88(\mathrm{~m}, 3 \mathrm{H}), 1.88-1.75(\mathrm{~m}, 5 \mathrm{H}), 1.69-1.63(\mathrm{~m}, 1 \mathrm{H}), 1.60-1.50$ (m, 2H), $1.46-1.34(\mathrm{~m}, 7 \mathrm{H}), 1.30-1.20(\mathrm{~m}, 4 \mathrm{H}), 1.15-0.95(\mathrm{~m}, 6 \mathrm{H}), 0.94-0.86(\mathrm{~m}, 6 \mathrm{H}), 0.63$ (s, 3H). ${ }^{13} \mathrm{C}$ NMR $\left(101 \mathrm{MHz}, \mathrm{CDCl}_{3}\right) \delta 174.3,170.8,148.2,119.9$ (q, $\left.J=261.0 \mathrm{~Hz}\right), 88.9,74.5$, 62.8, 56.6, 56.1, 42.7, 42.0, 40.5, 40.3, 35.9, 35.5, 35.2, 34.7, 32.4, 31.3, 31.1, 29.1, 28.3, 27.1, 26.8, 26.4, 25.1, 24.3, 23.4, 21.6, 21.0, 18.4, 12.2. $\left.{ }^{19} \mathrm{~F} \mathrm{NMR} \mathrm{(376} \mathrm{MHz,} \mathrm{CDCl}_{3}\right) \delta-56.16(\mathrm{~s}, 3 \mathrm{~F})$. Mass Spectrometry: HRMS-ESI (m/z): Calcd for $\mathrm{C}_{32} \mathrm{H}_{47} \mathrm{Br}_{2} \mathrm{~F}_{3} \mathrm{NaO}_{5}[\mathrm{M}+\mathrm{Na}]^{+}, 749.1635$. Found, 749.1633.

\section{Arbutin derivative (3uu)}

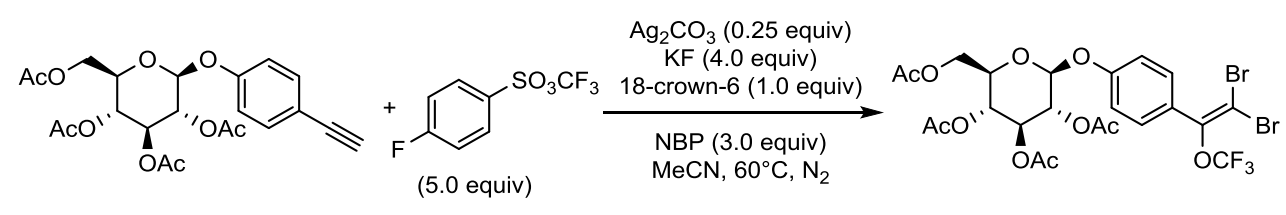


In a glove box, to a $15.0 \mathrm{~mL}$ sealed tube were added in sequence arbutin derivative (1uu) $(224 \mathrm{mg}$, $0.500 \mathrm{mmol}, 1.00$ equiv), KF (116 mg, $2.00 \mathrm{mmol}, 4.00$ equiv), 18 -crown-6 (132 mg, $0.500 \mathrm{mmol}$, 1.00 equiv), $\mathrm{Ag}_{2} \mathrm{CO}_{3}$ (34.4 mg, $0.125 \mathrm{mmol}, 0.250$ equiv), NBP ( $N$-Bromophthalimide) ( $339 \mathrm{mg}$, $1.50 \mathrm{mmol}, 3.00$ equiv) and $1.00 \mathrm{~mL} \mathrm{MeCN}$. The mixture was stirring at room temperature for 5 mins, then added TFMS (612 mg, $2.50 \mathrm{mmol}, 5.00$ equiv). After stirring for $4.0 \mathrm{~h}$ at $60{ }^{\circ} \mathrm{C}$, the reaction mixture was concentrated in vacuo. The residue was then added $10 \mathrm{~mL} \mathrm{CH}_{2} \mathrm{Cl}_{2}$ and filtered. The organic layer was concentrated in vacuo. The residue was purified by preparative TLC, eluting with hexanes/EtOAc 1:1 (v/v) to afford $305 \mathrm{mg}$ arbutin derivative (3uu) as a white solid ( $88 \%$ yield).

$\mathrm{R}_{f}=0.74$ (hexanes/EtOAc 1:1 (v/v)). NMR Spectroscopy: ${ }^{1} \mathrm{H}$ NMR $\left(400 \mathrm{MHz}, \mathrm{CDCl}_{3}\right) \delta 7.45(\mathrm{~d}$, $J=8.4 \mathrm{~Hz}, 2 \mathrm{H}), 6.99(\mathrm{~d}, J=8.5 \mathrm{~Hz}, 2 \mathrm{H}), 5.34-5.23(\mathrm{~m}, 2 \mathrm{H}), 5.20-5.10(\mathrm{~m}, 2 \mathrm{H}), 4.28(\mathrm{dd}, J=$ $12.3,5.4 \mathrm{~Hz}, 1 \mathrm{H}), 4.16(\mathrm{~d}, J=12.1 \mathrm{~Hz}, 1 \mathrm{H}), 3.94-3.84(\mathrm{~m}, 1 \mathrm{H}), 2.14-1.94(\mathrm{~m}, 12 \mathrm{H}) .{ }^{13} \mathrm{C} \mathrm{NMR}$ $\left(101 \mathrm{MHz}, \mathrm{CDCl}_{3}\right) \delta 170.6,170.3,169.5,169.3,157.9,145.9$ (q, $\left.J=1.7 \mathrm{~Hz}\right), 131.0,126.3,120.0$ $(\mathrm{q}, J=262.1 \mathrm{~Hz}), 116.6,98.4,89.0,72.7,72.3,71.2,68.3,62.0,20.7,20.7,20.7,20.6 .{ }^{19} \mathrm{~F}$ NMR $\left(376 \mathrm{MHz}, \mathrm{CDCl}_{3}\right) \delta-56.68(\mathrm{~s}, 3 \mathrm{~F})$. Mass Spectrometry: HRMS-ESI $(\mathrm{m} / \mathrm{z})$ : Calcd for $\mathrm{C}_{23} \mathrm{H}_{23} \mathrm{Br}_{2} \mathrm{~F}_{3} \mathrm{NaO}_{11}[\mathrm{M}+\mathrm{Na}]^{+}, 712.9451$. Found, 712.9447 .

\section{Estronederivative (3vv)}

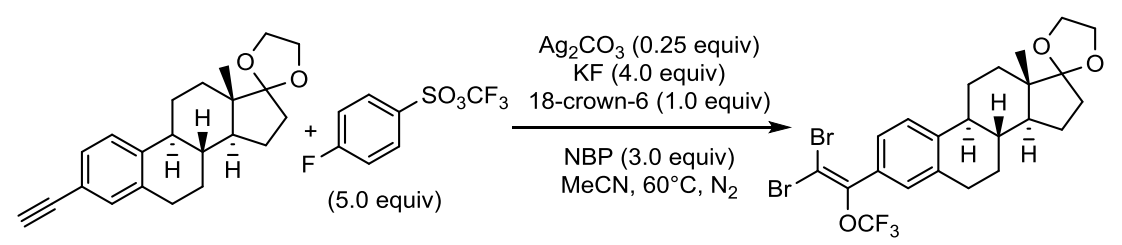

In a glove box, to a $15.0 \mathrm{~mL}$ sealed tube were added in sequenceestrone derivative $(\mathbf{1 v v})^{[9]}(161$ $\mathrm{mg}, 0.500 \mathrm{mmol}, 1.00$ equiv), $\mathrm{KF}$ (116 mg, $2.00 \mathrm{mmol}, 4.00$ equiv), 18-crown-6 (132 mg, 0.500 mmol, 1.00 equiv), $\mathrm{Ag}_{2} \mathrm{CO}_{3}$ (34.4 mg, $0.125 \mathrm{mmol}, 0.250$ equiv), NBP ( $N$-Bromophthalimide) (339 mg, $1.50 \mathrm{mmol}, 3.00$ equiv) and $1.00 \mathrm{~mL} \mathrm{MeCN}$. The mixture was stirring at room temperature for $5 \mathrm{mins}$, then added TFMS ( $612 \mathrm{mg}, 2.50 \mathrm{mmol}$, 5.00 equiv). After stirring for 4.0 $\mathrm{h}$ at $60^{\circ} \mathrm{C}$, the reaction mixture was concentrated in vacuo. The residue was then added $10 \mathrm{~mL}$ $\mathrm{CH}_{2} \mathrm{Cl}_{2}$ and filtered. The organic layer was concentrated in vacuo. The residue was purified by preparative TLC, eluting with hexanes/EtOAc 40:1 (v/v) to afford $161 \mathrm{mg}$ estrone derivative (3vv) as a white solid ( $57 \%$ yield).

$\mathrm{R}_{f}=0.38$ (hexanes/EtOAc 10:1 (v/v)). NMR Spectroscopy: ${ }^{1} \mathrm{H}$ NMR (400 MHz, $\left.\mathrm{CDCl}_{3}\right) \delta 7.36-$ $7.28(\mathrm{~m}, 2 \mathrm{H}), 7.25(\mathrm{~s}, 1 \mathrm{H}), 4.12-3.85(\mathrm{~m}, 4 \mathrm{H}), 2.94-2.87$ (m, 2H), $2.42-2.26(\mathrm{~m}, 2 \mathrm{H}), 2.12-$ $2.02(\mathrm{~m}, 1 \mathrm{H}), 2.00-1.75(\mathrm{~m}, 4 \mathrm{H}), 1.74-1.37(\mathrm{~m}, 6 \mathrm{H}), 0.92(\mathrm{~s}, 3 \mathrm{H}) .{ }^{13} \mathrm{C} \mathrm{NMR}\left(101 \mathrm{MHz}, \mathrm{CDCl}_{3}\right)$ $\delta 146.8,143.2,137.1,129.6,128.7,126.5,125.4,120.1$ (q, $J=262.0 \mathrm{~Hz}), 119.5,88.5,65.4,64.7$, 49.6, 46.2, 44.3, 38.6, 34.3, 30.8, 29.5, 26.7, 25.8, 22.5, 14.5. ${ }^{19} \mathrm{~F}$ NMR $\left(376 \mathrm{MHz}, \mathrm{CDCl}_{3}\right) \delta$ -55.59 (s, 3F). Mass Spectrometry: HRMS-ESI (m/z): Calcd for $\mathrm{C}_{23} \mathrm{H}_{26} \mathrm{Br}_{2} \mathrm{~F}_{3} \mathrm{O}_{3}[\mathrm{M}+\mathrm{H}]^{+}$, 565.0195. Found, 565.0191. 


\section{$\delta$-D-tocopherolderivative (3ww)}

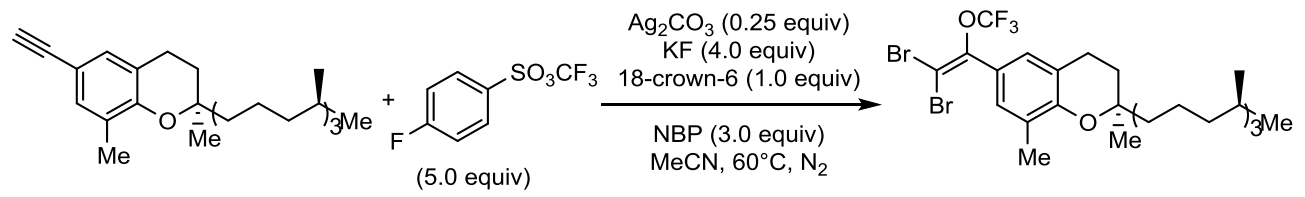

In a glove box, to a $15.0 \mathrm{~mL}$ sealed tube were added in sequence $\delta$-D-tocopherol derivative (1ww) (205 mg, $0.500 \mathrm{mmol}, 1.00$ equiv), KF (116 mg, $2.00 \mathrm{mmol}, 4.00$ equiv), 18 -crown-6 (132 mg, 0.500 mmol, 1.00 equiv), $\mathrm{Ag}_{2} \mathrm{CO}_{3} \quad(34.4 \mathrm{mg}, 0.125 \mathrm{mmol}, 0.250$ equiv), NBP ( $N$-Bromophthalimide) (339 mg, $1.50 \mathrm{mmol}, 3.00$ equiv) and $1.00 \mathrm{~mL} \mathrm{MeCN}$. The mixture was stirring at room temperature for $5 \mathrm{mins}$, then added TFMS (612 $\mathrm{mg}, 2.50 \mathrm{mmol}, 5.00$ equiv). After stirring for $4.0 \mathrm{~h}$ at $60{ }^{\circ} \mathrm{C}$, the reaction mixture was concentrated in vacuo. The residue was then added $10 \mathrm{~mL} \mathrm{CH}_{2} \mathrm{Cl}_{2}$ and filtered. The organic layer was concentrated in vacuo. The residue was purified by preparative TLC, eluting with hexanes/EtOAc 100:1 (v/v) to afford $213 \mathrm{mg}$ $\delta$-D-tocopherol derivative (3ww) as a colorless liquid (65\% yield).

$\mathrm{R}_{f}=0.79$ (hexanes/EtOAc 40:1 (v/v)). NMR Spectroscopy: ${ }^{1} \mathrm{H}$ NMR $\left(400 \mathrm{MHz}, \mathrm{CDCl}_{3}\right) \delta 7.10(\mathrm{~s}$, $1 \mathrm{H}), 7.07(\mathrm{~s}, 1 \mathrm{H}), 2.83-2.68(\mathrm{~m}, 2 \mathrm{H}), 2.16(\mathrm{~s}, 3 \mathrm{H}), 1.87-1.73(\mathrm{~m}, 2 \mathrm{H}), 1.64-1.57(\mathrm{~m}, 2 \mathrm{H})$, $1.55-1.49(\mathrm{~m}, 1 \mathrm{H}), 1.46-1.19(\mathrm{~m}, 15 \mathrm{H}), 1.17-1.02(\mathrm{~m}, 6 \mathrm{H}), 0.91-0.80(\mathrm{~m}, 12 \mathrm{H}) .{ }^{13} \mathrm{C}$ NMR $\left(101 \mathrm{MHz}, \mathrm{CDCl}_{3}\right) \delta 154.1,147.2,129.3,128.4,126.4,121.5,120.3,120.1$ (q, $\left.J=261.7 \mathrm{~Hz}\right), 87.0$, 77.1, 40.6, 39.6, 37.6, 37.6, 37.5, 33.0, 32.8, 31.0, 28.2, 25.0, 24.6, 24.4, 22.9, 22.8, 22.3, 21.1, 19.9, 19.8, 16.2. ${ }^{19} \mathrm{~F}$ NMR $\left(376 \mathrm{MHz}, \mathrm{CDCl}_{3}\right) \delta-55.90$ (s, 3F). Mass Spectrometry: HRMS-ESI $(\mathrm{m} / \mathrm{z})$ : Calcd for $\mathrm{C}_{30} \mathrm{H}_{46} \mathrm{Br}_{2} \mathrm{~F}_{3} \mathrm{O}_{2}[\mathrm{M}+\mathrm{H}]^{+}$, 653.1811. Found, 653.1811.

\section{(E)-6-bromo-7-(4-methoxyphenyl)-7-(trifluoromethoxy)hept-6-en-4-yn-1-ol (4ee)}

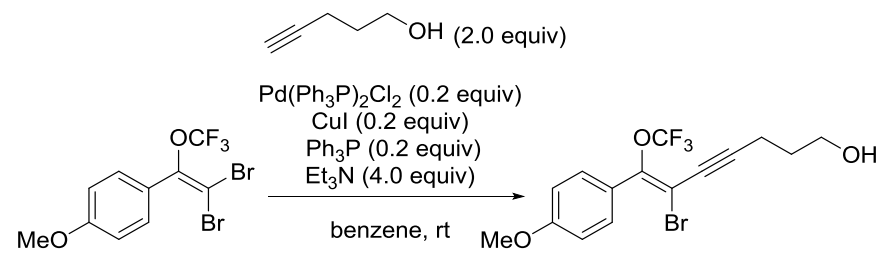

In a glove box, to a $15.0 \mathrm{~mL}$ sealed tube were added in sequence 1-(2,2-dibromo-1-(trifluoromethoxy)vinyl)-4-methoxybenzene (3ee) (188 mg, $0.500 \mathrm{mmol}, 1.00$ equiv), $\mathrm{Pd}\left(\mathrm{Ph}_{3} \mathrm{P}\right)_{2} \mathrm{Cl}_{2}$ (70.2 mg, $0.100 \mathrm{mmol}, 0.200$ equiv), $\mathrm{Ph}_{3} \mathrm{P}$ (26.2 mg, $0.100 \mathrm{mmol}, 0.200$ equiv), $\mathrm{CuI}$ (19 mg, $0.100 \mathrm{mmol}, 0.200$ equiv), $\mathrm{Et}_{3} \mathrm{~N}$ (202 mg, $2.00 \mathrm{mmol}, 4.00$ equiv), pent-4-yn-1-ol ( $84 \mathrm{mg}, 1.00 \mathrm{mmol}, 2.00$ equiv) and $5.00 \mathrm{~mL}$ benzene. After stirring for $12.0 \mathrm{~h}$ at $25{ }^{\circ} \mathrm{C}$, the reaction mixture was added $10 \mathrm{~mL} \mathrm{CH}_{2} \mathrm{Cl}_{2}$ and filtered. The organic layer was concentrated in vacuo. The residue was purified by preparative TLC, eluting with hexanes/EtOAc $\begin{array}{lllll}4: 1 & (\mathrm{v} / \mathrm{v}) & \text { to } & \text { afford } & 76\end{array}$ (E)-6-bromo-7-(4-methoxyphenyl)-7-(trifluoromethoxy)hept-6-en-4-yn-1-ol (4ee) as a colorless liquid (40\% yield).

$\mathrm{R}_{f}=0.06$ (hexanes/EtOAc 4:1 (v/v)). NMR Spectroscopy: ${ }^{1} \mathrm{H}$ NMR (400 MHz, $\left.\mathrm{CDCl}_{3}\right) \delta 7.73-$ $7.47(\mathrm{~m}, 2 \mathrm{H}), 7.07-6.71(\mathrm{~m}, 2 \mathrm{H}), 3.84(\mathrm{~s}, 3 \mathrm{H}), 3.66(\mathrm{t}, J=6.1 \mathrm{~Hz}, 2 \mathrm{H}), 2.51(\mathrm{t}, J=7.0 \mathrm{~Hz}, 2 \mathrm{H})$, $1.81-1.71(\mathrm{~m}, 2 \mathrm{H}), 1.64-1.56(\mathrm{br} \mathrm{m}, 1 \mathrm{H}) .{ }^{13} \mathrm{C}$ NMR $\left(101 \mathrm{MHz}, \mathrm{CDCl}_{3}\right) \delta 161.0,149.9(\mathrm{q}, J=$ $1.8 \mathrm{~Hz}), 130.1,124.1,120.3$ (q, $J=261.5 \mathrm{~Hz}), 113.7,98.9,94.8,61.4,55.5,30.8,16.4 .{ }^{19} \mathrm{~F}$ NMR $\left(376 \mathrm{MHz}, \mathrm{CDCl}_{3}\right) \delta-55.27(\mathrm{~s}, 3 \mathrm{~F})$. Mass Spectrometry: HRMS-ESI $(\mathrm{m} / \mathrm{z})$ : Calcd for $\mathrm{C}_{15} \mathrm{H}_{14} \mathrm{BrF}_{3} \mathrm{O}_{3}[\mathrm{M}]^{+}$, 378.0078. Found, 378.0070. 
(E)-1-(2-bromo-2-phenyl-1-(trifluoromethoxy)vinyl)-4-methoxybenzene (5ee)

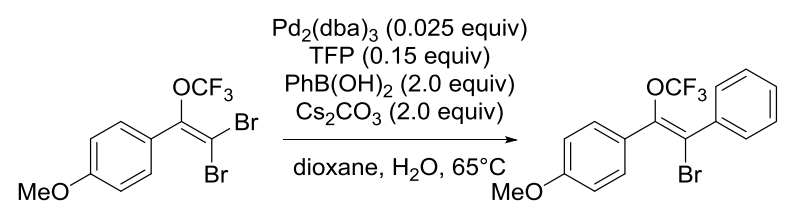

In a glove box, to a $15.0 \mathrm{~mL}$ sealed tube were added in sequence 1-(2,2-dibromo-1-(trifluoromethoxy)vinyl)-4-methoxybenzene (3ee) (188 mg, $0.500 \mathrm{mmol}, 1.00$ equiv), $\mathrm{Pd}_{2}(\mathrm{dba})_{3}(11.4 \mathrm{mg}, 0.0125 \mathrm{mmol}, 0.025$ equiv), TFP (17.4 mg, $0.075 \mathrm{mmol}, 0.150$ equiv), $\mathrm{PhB}(\mathrm{OH})_{2}$ (128 mg, $1.00 \mathrm{mmol}, 2.00$ equiv), $\mathrm{Cs}_{2} \mathrm{CO}_{3}$ (326 mg, $1.00 \mathrm{mmol}, 2.00$ equiv), $0.35 \mathrm{~mL}$ $\mathrm{H}_{2} \mathrm{O}$ and $2.00 \mathrm{~mL}$ 1,4-Dioxane. After stirring for $12.0 \mathrm{~h}$ at $65^{\circ} \mathrm{C}$, the reaction mixture was added $10 \mathrm{~mL} \mathrm{CH}_{2} \mathrm{Cl}_{2}$ and filtered. The organic layer was concentrated in vacuo. The residue was purified by preparative TLC, eluting with hexanes/EtOAc 100:1 (v/v) to afford $112 \mathrm{mg}$ (E)-1-(2-bromo-2-phenyl-1-(trifluoromethoxy)vinyl)-4-methoxybenzene (6ee) as a white solid (60\% yield).

$\mathrm{R}_{f}=0.16$ (hexanes). NMR Spectroscopy: ${ }^{1} \mathrm{H}$ NMR $\left(400 \mathrm{MHz}, \mathrm{CDCl}_{3}\right) \delta 7.26-7.19(\mathrm{~m}, 5 \mathrm{H}), 7.15$ $-7.10(\mathrm{~m}, 2 \mathrm{H}), 6.74-6.68(\mathrm{~m}, 2 \mathrm{H}), 3.75(\mathrm{~s}, 3 \mathrm{H}) .{ }^{13} \mathrm{C} \mathrm{NMR}\left(101 \mathrm{MHz}, \mathrm{CDCl}_{3}\right) \delta 160.3,144.7$ (q, $J=1.5 \mathrm{~Hz}), 137.5,131.0,130.3,128.8,128.5,124.5,120.8$ (q, $J=260.7 \mathrm{~Hz}), 115.1,113.7,55.3$.

${ }^{19} \mathrm{~F}$ NMR (376 MHz, $\left.\mathrm{CDCl}_{3}\right) \delta-54.96$ (s, 3F). Mass Spectrometry: HRMS-ESI (m/z): Calcd for $\mathrm{C}_{16} \mathrm{H}_{12} \mathrm{BrF}_{3} \mathrm{O}_{2}[\mathrm{M}]^{+}, 371.9973$. Found, 371.9963.

(4-Fluorophenyl)(2-(4-methoxyphenyl)-2-(trifluoromethoxy)vinyl)sulfane (E-6ee)+(Z-6ee)

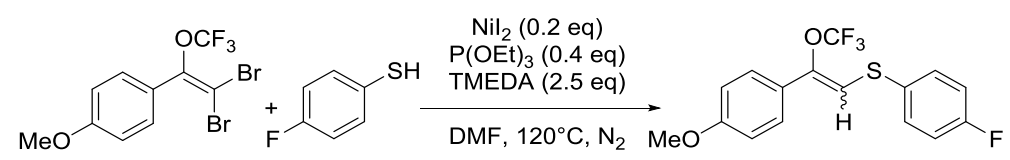

In a glove box, to a $15.0 \mathrm{~mL}$ sealed tube were added in sequence 1-(2,2-dibromo-1-(trifluoromethoxy)vinyl)-4-methoxybenzene (3ee) (188 mg, $0.500 \mathrm{mmol}, 1.00$ equiv), 4-fluorothiophenol (320 mg, $2.50 \mathrm{mmol}, 5.00$ equiv), $\mathrm{NiI}_{2}$ (31.2 mg, $0.100 \mathrm{mmol}, 0.200$ equiv), $\mathrm{P}(\mathrm{OEt})_{3}(33.2 \mathrm{mg}, 0.200 \mathrm{mmol}, 0.400$ equiv), TMEDA (290 mg, $2.50 \mathrm{mmol}, 5.00$ equiv) and $4.00 \mathrm{~mL}$ DMF. After stirring for $12.0 \mathrm{~h}$ at $120^{\circ} \mathrm{C}$, the reaction mixture was concentrated in vacuo. The residue was then added $10 \mathrm{~mL} \mathrm{CH}_{2} \mathrm{Cl}_{2}$ and filtered through a pad of silica gel. The organic layer was concentrated in vacuo. The residue was purified by preparative TLC, eluting $\begin{array}{lllllll}\text { with hexanes/EtOAc } & 40: 1 \quad(\mathrm{v} / \mathrm{v}) \quad \text { to } & \text { afford } & 46 & \mathrm{mg}\end{array}$ (E)-(4-fluorophenyl)(2-(4-methoxyphenyl)-2-(trifluoromethoxy)vinyl)sulfane (E-6ee) as a $\begin{array}{llllll}\text { colorless liquid } \quad(27 \% & \text { yield }), \quad \text { and } & \text { afford } & 103 & \mathrm{mg}\end{array}$ (Z)-(4-fluorophenyl)(2-(4-methoxyphenyl)-2-(trifluoromethoxy)vinyl)sulfane $\quad$ (Z-6ee) as a colorless liquid ( $60 \%$ yield).

(Z-6ee) $\mathrm{R}_{f}=0.10$ (hexanes). NMR Spectroscopy: ${ }^{1} \mathrm{H}$ NMR $\left(400 \mathrm{MHz}, \mathrm{CDCl}_{3}\right) \delta 7.49-7.36(\mathrm{~m}$, 4H), $7.06(\mathrm{t}, J=8.5 \mathrm{~Hz}, 2 \mathrm{H}), 6.90(\mathrm{~d}, J=8.7 \mathrm{~Hz}, 2 \mathrm{H}), 6.34(\mathrm{~s}, 1 \mathrm{H}), 3.83(\mathrm{~s}, 3 \mathrm{H}) .{ }^{13} \mathrm{C}$ NMR $(101$ $\left.\mathrm{MHz}, \mathrm{CDCl}_{3}\right) \delta 162.6(\mathrm{~d}, J=248.1 \mathrm{~Hz}), 160.4,144.1,132.9(\mathrm{~d}, J=8.3 \mathrm{~Hz}), 129.7(\mathrm{~d}, J=3.2 \mathrm{~Hz})$, 126.7, 126.4, $121.0(\mathrm{q}, J=260.1 \mathrm{~Hz}), 116.7,116.6(\mathrm{~d}, J=22.1 \mathrm{~Hz}), 114.2,55.4 .{ }^{19} \mathrm{~F}$ NMR $(376$ $\left.\mathrm{MHz}, \mathrm{CDCl}_{3}\right) \delta-56.72(\mathrm{~s}, 3 \mathrm{~F}),-114.33--114.40(\mathrm{~m}, 1 \mathrm{~F})$. Mass Spectrometry: HRMS-ESI (m/z): Calcd for $\mathrm{C}_{16} \mathrm{H}_{12} \mathrm{~F}_{4} \mathrm{O}_{2} \mathrm{~S}[\mathrm{M}]^{+}$, 344.0494. Found, 344.0487. 
$\left(\boldsymbol{E}\right.$-6ee) $\mathrm{R}_{f}=0.13$ (hexanes). NMR Spectroscopy: ${ }^{1} \mathrm{H}$ NMR $\left(400 \mathrm{MHz}, \mathrm{CDCl}_{3}\right) \delta 7.59-7.52(\mathrm{~m}$, $2 \mathrm{H}), 7.43-7.35(\mathrm{~m}, 2 \mathrm{H}), 7.10-7.02(\mathrm{~m}, 2 \mathrm{H}), 7.00-6.92(\mathrm{~m}, 2 \mathrm{H}), 6.39(\mathrm{~s}, 1 \mathrm{H}), 3.85(\mathrm{~s}, 3 \mathrm{H}) .{ }^{13} \mathrm{C}$ NMR $\left(101 \mathrm{MHz}, \mathrm{CDCl}_{3}\right) \delta 162.5(\mathrm{~d}, J=248.1 \mathrm{~Hz}), 160.5,143.6,132.2(\mathrm{~d}, J=8.3 \mathrm{~Hz}), 130.3(\mathrm{~d}$, $J=3.3 \mathrm{~Hz}), 129.5,124.9,120.9(\mathrm{q}, J=257.9 \mathrm{~Hz}), 116.6(\mathrm{~d}, J=22.2 \mathrm{~Hz}), 116.2,113.8,55.4 .{ }^{19} \mathrm{~F}$ NMR $\left(376 \mathrm{MHz}, \mathrm{CDCl}_{3}\right) \delta-57.00(\mathrm{~s}, 3 \mathrm{~F}),-113.50--113.57(\mathrm{~m}, 1 \mathrm{~F})$. Mass Spectrometry: HRMS-ESI (m/z): Calcd for $\mathrm{C}_{16} \mathrm{H}_{12} \mathrm{~F}_{4} \mathrm{O}_{2} \mathrm{~S}[\mathrm{M}]^{+}$, 344.0494. Found, 344.0486.

\section{(E)-1-(2-bromo-1-(trifluoromethoxy)vinyl)-4-methoxybenzene (7ee)}

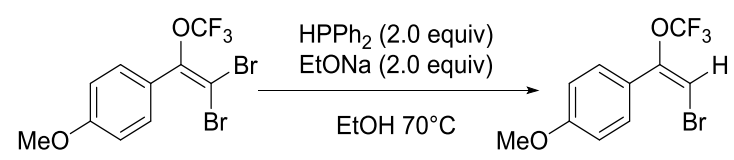

In a glove box, to a $15.0 \mathrm{~mL}$ sealed tube were added in sequence 1-(2,2-dibromo-1-(trifluoromethoxy)vinyl)-4-methoxybenzene (3ee) (188 mg, $0.500 \mathrm{mmol}, 1.00$ equiv), $\mathrm{HPPh}_{2}$ (186 mg, $1.00 \mathrm{mmol}, 2.00$ equiv), EtONa (68 mg, $1.00 \mathrm{mmol}, 2.00$ equiv) and 1.00 $\mathrm{mL}$ EtOH. After stirring for $12.0 \mathrm{~h}$ at $70{ }^{\circ} \mathrm{C}$, the reaction mixture was concentrated in vacuo. The residue was then added $10 \mathrm{~mL} \mathrm{CH} \mathrm{Cl}_{2}$ and filtered. The organic layer was concentrated in vacuo. The residue was purified by preparative TLC, eluting with hexanes/EtOAc 100:1 (v/v) to afford $107 \mathrm{mg}$ (E)-1-(2-bromo-1-(trifluoromethoxy)vinyl)-4-methoxybenzene (7ee) as a colorless liquid (72\% yield).

$\mathrm{R}_{f}=0.18$ (hexanes). NMR Spectroscopy: ${ }^{1} \mathrm{H}$ NMR $\left(400 \mathrm{MHz}, \mathrm{CDCl}_{3}\right) \delta 7.48-7.32(\mathrm{~m}, 2 \mathrm{H}), 7.00$ $-6.82(\mathrm{~m}, 2 \mathrm{H}), 6.31(\mathrm{~s}, 1 \mathrm{H}), 3.83(\mathrm{~s}, 3 \mathrm{H}) .{ }^{13} \mathrm{C} \mathrm{NMR}\left(101 \mathrm{MHz}, \mathrm{CDCl}_{3}\right) \delta 161.1,149.5$ (q, $J=1.4$ $\mathrm{Hz}), 127.6,125.4,120.4(\mathrm{q}, J=260.7 \mathrm{~Hz}), 114.3,97.2,55.4 .{ }^{19} \mathrm{~F}$ NMR $\left(376 \mathrm{MHz}, \mathrm{CDCl}_{3}\right) \delta$ -56.30 (s, 3F). Mass Spectrometry: HRMS-ESI (m/z): Calcd for $\mathrm{C}_{10} \mathrm{H}_{8} \mathrm{BrF}_{3} \mathrm{O}_{2}[\mathrm{M}]^{+}, 295.9660$. Found, 295.9652.

\section{(E)-1-bromo-2-(trifluoromethoxy)dodec-1-ene (E-7a)}

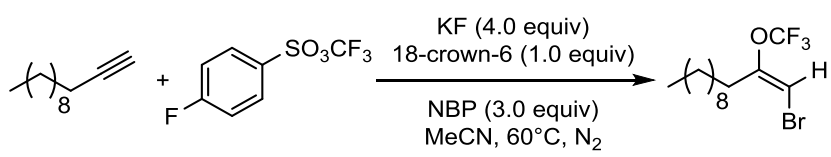

In a glove box, to a $15 \mathrm{~mL}$ sealed tube were added in sequence 1-dodecyne (1a) ( $83 \mathrm{mg}, 0.500$ mmol, 1.00 equiv), $1.00 \mathrm{~mL}$ MeCN, KF (116 mg, $2.00 \mathrm{mmol}, 4.00$ equiv), 18-crown-6 (132 mg, $0.50 \mathrm{mmol}, 1.00$ equiv), NBP ( $N$-Bromophthalimide) $(339 \mathrm{mg}, 1.50 \mathrm{mmol}, 3.00$ equiv). The mixture was stirring at room temperature for $5 \mathrm{mins}$, then added TFMS (489 mg, $2.00 \mathrm{mmol}, 4.00$ equiv). After stirring for $4.0 \mathrm{~h}$ at $60^{\circ} \mathrm{C}$, the reaction mixture was concentrated in vacuo. The residue was then added $10 \mathrm{~mL} \mathrm{CH} \mathrm{Cl}_{2}$ and filtered. The organic layer was concentrated in vacuo. The residue was purified by preparative HPLC $(9 \mathrm{~mL} / \mathrm{min}$, detector UV $\lambda \max 210 \mathrm{~nm}$, $\mathrm{MeCN} / \mathrm{H}_{2} \mathrm{O}=90: 10(0 \mathrm{~min}), \mathrm{MeCN} / \mathrm{H}_{2} \mathrm{O}=100: 0(10 \mathrm{~min}), \mathrm{MeCN} / \mathrm{H}_{2} \mathrm{O}=100: 0(40 \mathrm{~min})$ ) to afford $48 \mathrm{mg}(E)$-1-bromo-2-(trifluoromethoxy)dodec-1-ene $(\boldsymbol{E}-\mathbf{7 a})(31.0 \mathrm{~min})$ as a colorless liquid (29\% yield).

$\mathrm{R}_{f}=0.81$ (hexanes). NMR Spectroscopy: ${ }^{1} \mathrm{H}$ NMR $\left(400 \mathrm{MHz}, \mathrm{CDCl}_{3}\right) \delta 6.17$ (s, 1H), 2.45 (t, $J=$ $7.6 \mathrm{~Hz}, 2 \mathrm{H}), 1.62-1.49(\mathrm{~m}, 2 \mathrm{H}), 1.40-1.20(\mathrm{~m}, 14 \mathrm{H}), 0.89(\mathrm{t}, J=6.8 \mathrm{~Hz}, 3 \mathrm{H}) .{ }^{13} \mathrm{C}$ NMR $(101$ $\left.\mathrm{MHz}, \mathrm{CDCl}_{3}\right) \delta 151.6,120.2(\mathrm{q}, J=258.3 \mathrm{~Hz}), 97.5,32.1,30.9,29.7,29.6,29.5,29.4,28.9,25.8$, 22.9, 14.3. ${ }^{19} \mathrm{~F}$ NMR $\left(376 \mathrm{MHz}, \mathrm{CDCl}_{3}\right) \delta-57.21$ (s, 3F). Mass Spectrometry: HRMS-EI (m/z): 
Calcd for $\mathrm{C}_{13} \mathrm{H}_{22} \mathrm{BrF}_{3} \mathrm{O}[\mathrm{M}]^{+}, 330.0796$. Found, 330.0805.

(Z)-1-bromo-2-(trifluoromethoxy)dodec-1-ene (Z-7a)

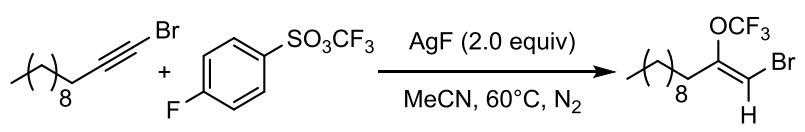

In a glove box, to a $15 \mathrm{~mL}$ sealed tube were added in sequence 1-bromododec-1-yne (1a') (122 $\mathrm{mg}, 0.500 \mathrm{mmol}, 1.00$ equiv), $1.00 \mathrm{~mL} \mathrm{MeCN}, \operatorname{AgF}(127 \mathrm{mg}, 1.00 \mathrm{mmol}, 2.00$ equiv). The mixture was stirring at room temperature for 5 mins, then added TFMS (489 mg, $2.00 \mathrm{mmol}, 4.00$ equiv). After stirring for $4.0 \mathrm{~h}$ at $60{ }^{\circ} \mathrm{C}$, the reaction mixture was concentrated in vacuo. The residue was then added $10 \mathrm{~mL} \mathrm{CH}_{2} \mathrm{Cl}_{2}$ and filtered. The organic layer was concentrated in vacuo. The residue was purified by preparative HPLC $(9 \mathrm{~mL} / \mathrm{min}$, detector UV $\lambda \max 210 \mathrm{~nm}$, $\mathrm{MeCN} / \mathrm{H}_{2} \mathrm{O}=90: 10$ (0 min), $\mathrm{MeCN} / \mathrm{H}_{2} \mathrm{O}=100: 0(10 \mathrm{~min}), \mathrm{MeCN} / \mathrm{H}_{2} \mathrm{O}=100: 0$ (40 min)) to afford $94 \mathrm{mg}(Z)$-1-bromo-2-(trifluoromethoxy)dodec-1-ene $(\boldsymbol{Z}-7 \mathbf{a})(24.5 \mathrm{~min})$ as a colorless liquid ( $57 \%$ yield).

$\mathrm{R}_{f}=0.90$ (hexanes). NMR Spectroscopy: ${ }^{1} \mathrm{H}$ NMR $\left(400 \mathrm{MHz}, \mathrm{CDCl}_{3}\right) \delta 5.84$ (s, 1H), 2.32 (t, $J=$ $7.5 \mathrm{~Hz}, 2 \mathrm{H}), 1.58-1.48(\mathrm{~m}, 2 \mathrm{H}), 1.39-1.20(\mathrm{~m}, 14 \mathrm{H}), 0.88(\mathrm{t}, J=6.8 \mathrm{~Hz}, 3 \mathrm{H}) .{ }^{13} \mathrm{C}$ NMR $(101$ $\left.\mathrm{MHz}, \mathrm{CDCl}_{3}\right) \delta 151.4,120.2(\mathrm{q}, J=259.4 \mathrm{~Hz}), 95.9,33.3,32.1,29.7,29.6,29.5,29.3,28.9,26.3$, 22.8, 14.2. ${ }^{19} \mathrm{~F}$ NMR $\left(376 \mathrm{MHz}, \mathrm{CDCl}_{3}\right) \delta-55.77$ (s, 3F). Mass Spectrometry: HRMS-EI (m/z): Calcd for $\mathrm{C}_{13} \mathrm{H}_{22} \mathrm{BrF}_{3} \mathrm{O}[\mathrm{M}]^{+}$, 330.0796. Found, 330.0796.

\section{Mechanism Studies}

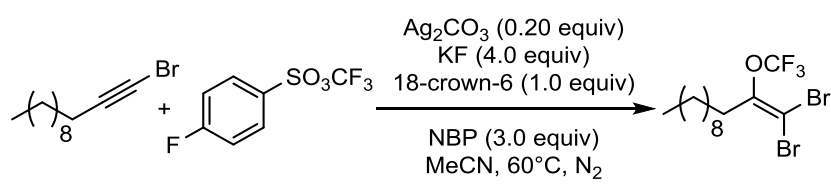

In a glove box, to a $2.0 \mathrm{~mL}$ sealed tube were added in sequence 1-bromododec-1-yne (1a') (12.2 $\mathrm{mg}, 0.0500 \mathrm{mmol}, 1.00$ equiv), $0.10 \mathrm{~mL} \mathrm{MeCN}, \mathrm{Ag}_{2} \mathrm{CO}_{3}$ (2.8 mg, $0.0100 \mathrm{mmol}, 0.200$ equiv), $\mathrm{KF}$ (11.6 mg, $0.200 \mathrm{mmol}, 4.00$ equiv), 18 -crown-6 (13.2 mg, $0.050 \mathrm{mmol}, 1.00$ equiv), NBP ( $N$-Bromophthalimide) $(33.9 \mathrm{mg}, 0.150 \mathrm{mmol}, 3.00$ equiv). The mixture was stirring at room temperature for $5 \mathrm{mins}$, then added TFMS ( $48.9 \mathrm{mg}, 0.200 \mathrm{mmol}, 4.00$ equiv). After stirring for $4.0 \mathrm{~h}$ at $60{ }^{\circ} \mathrm{C}$, trifluoromethylthiobenzene $(7 \mu \mathrm{L}, 0.0500 \mathrm{mmol})$ was added. The yield of 1,1-dibromo-2-(trifluoromethoxy)dodec-1-ene (3a) was determined by comparing the integration of the ${ }^{19}$ F NMR resonance of 1,1-dibromo-2-(trifluoromethoxy)dodec-1-ene (3a) (-55.81 ppm) with that of trifluoromethylthiobenzene (-42.80 ppm). $\left({ }^{19} \mathrm{~F}\right.$ NMR yield: $\left.82 \%\right)$.

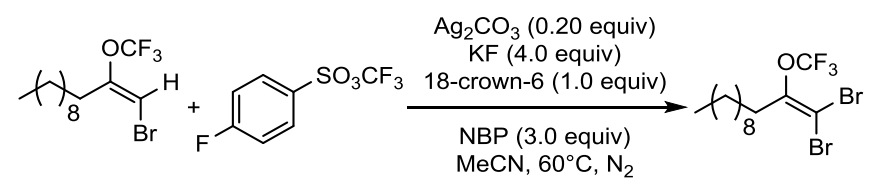


In a glove box, to a $2.0 \mathrm{~mL}$ sealed tube were added in sequence (E)-1-bromo-2-(trifluoromethoxy)dodec-1-ene (7a) (16.5 mg, $0.0500 \mathrm{mmol}, 1.00$ equiv), $0.10 \mathrm{~mL}$ $\mathrm{MeCN}, \mathrm{Ag}_{2} \mathrm{CO}_{3}$ (2.8 mg, $0.0100 \mathrm{mmol}, 0.200$ equiv), $\mathrm{KF}$ (11.6 mg, $0.200 \mathrm{mmol}, 4.00$ equiv), 18-crown-6 (13.2 mg, $0.050 \mathrm{mmol}, 1.00$ equiv), NBP ( $N$-Bromophthalimide) (33.9 mg, 0.150 mmol, 3.00 equiv). The mixture was stirring at room temperature for 5 mins, then added TFMS (48.9 mg, $0.200 \mathrm{mmol}, 4.00$ equiv). After stirring for $4.0 \mathrm{~h}$ at $60^{\circ} \mathrm{C}$, trifluoromethylthiobenzene ( 7 $\mu \mathrm{L}, 0.0500 \mathrm{mmol}$ ) was added. The yield of 1,1-dibromo-2-(trifluoromethoxy)dodec-1-ene (3a) was determined by comparing the integration of the ${ }^{19} \mathrm{~F}$ NMR resonance of 1,1-dibromo-2-(trifluoromethoxy)dodec-1-ene $\quad$ (3a) $\quad(-55.81 \quad \mathrm{ppm})$ with that of trifluoromethylthiobenzene $(-42.80 \mathrm{ppm}) .\left({ }^{19} \mathrm{~F}\right.$ NMR yield: $\left.0 \%\right)$.

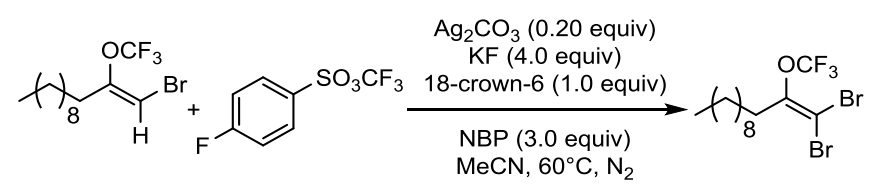

In a glove box, to a $2.0 \mathrm{~mL}$ sealed tube were added in sequence (Z)-1-bromo-2-(trifluoromethoxy)dodec-1-ene ( $Z$-7a) $(16.5 \mathrm{mg}, 0.0500 \mathrm{mmol}, 1.00$ equiv), 0.10 $\mathrm{mL} \mathrm{MeCN}, \mathrm{Ag}_{2} \mathrm{CO}_{3}$ (2.8 mg, $0.0100 \mathrm{mmol}, 0.200$ equiv), $\mathrm{KF}$ (11.6 mg, $0.200 \mathrm{mmol}, 4.00$ equiv), 18-crown-6 (13.2 mg, $0.050 \mathrm{mmol}, 1.00$ equiv), NBP ( $N$-Bromophthalimide) (33.9 mg, 0.150 mmol, 3.00 equiv). The mixture was stirring at room temperature for $5 \mathrm{mins}$, then added TFMS (48.9 mg, $0.200 \mathrm{mmol}, 4.00$ equiv). After stirring for $4.0 \mathrm{~h}$ at $60^{\circ} \mathrm{C}$, trifluoromethylthiobenzene ( 7 $\mu \mathrm{L}, 0.0500 \mathrm{mmol}$ ) was added. The yield of 1,1-dibromo-2-(trifluoromethoxy)dodec-1-ene (3a) was determined by comparing the integration of the ${ }^{19} \mathrm{~F}$ NMR resonance of 1,1-dibromo-2-(trifluoromethoxy)dodec-1-ene (3a) (-55.81 $\quad$ ppm) with that of trifluoromethylthiobenzene (-42.80 ppm). $\left({ }^{19} \mathrm{~F}\right.$ NMR yield: $\left.0 \%\right)$.

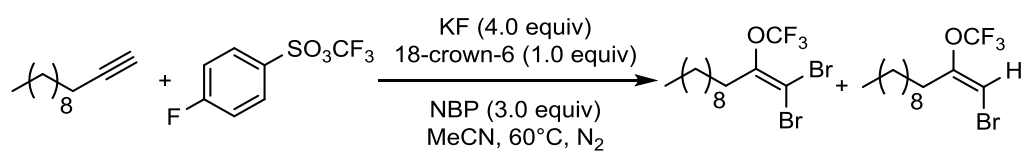

In a glove box, to a $2.0 \mathrm{~mL}$ sealed tube were added in sequence 1-dodecyne (1a) $(8.3 \mathrm{mg}, 0.0500$ mmol, 1.00 equiv), $0.10 \mathrm{~mL} \mathrm{MeCN}, \mathrm{KF}$ (11.6 mg, $0.200 \mathrm{mmol}, 4.00$ equiv), 18-crown-6 (13.2 mg, $0.050 \mathrm{mmol}, 1.00$ equiv), NBP ( $N$-Bromophthalimide) $(33.9 \mathrm{mg}, 0.150 \mathrm{mmol}, 3.00$ equiv). The mixture was stirring at room temperature for $5 \mathrm{mins}$, then added TFMS (48.9 $\mathrm{mg}, 0.200 \mathrm{mmol}$, 4.00 equiv). After stirring for $4.0 \mathrm{~h}$ at $60^{\circ} \mathrm{C}$, trifluoromethylthiobenzene $(7 \mu \mathrm{L}, 0.0500 \mathrm{mmol})$ was added. The yield of 1,1-dibromo-2-(trifluoromethoxy)dodec-1-ene (3a) was determined by comparing the integration of the ${ }^{19} \mathrm{~F}$ NMR resonance of 1,1-dibromo-2-(trifluoromethoxy)dodec-1-ene (3a) (-55.81 $\mathrm{ppm})$ with that of trifluoromethylthiobenzene $\quad(-42.80 \mathrm{ppm}) . \quad\left({ }^{19} \mathrm{~F} \quad \mathrm{NMR}\right.$ yield: $\left.33 \%\right)$. The yield of (E)-1-bromo-2-(trifluoromethoxy)dodec-1-ene (7a) was determined by comparing the integration of the ${ }^{19} \mathrm{~F}$ NMR resonance of (E)-1-bromo-2-(trifluoromethoxy)dodec-1-ene (E-7a) (-57.21 ppm) with that of trifluoromethylthiobenzene (-42.80 ppm). $\left({ }^{19} \mathrm{~F}\right.$ NMR yield: $\left.30 \%\right)$. 


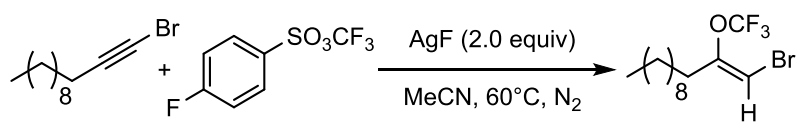

In a glove box, to a $2.0 \mathrm{~mL}$ sealed tube were added in sequence 1-bromododec-1-yne (1a') (12.2 $\mathrm{mg}, 0.0500 \mathrm{mmol}, 1.00$ equiv), $0.10 \mathrm{~mL} \mathrm{MeCN}, \mathrm{AgF}(12.7 \mathrm{mg}, 0.100 \mathrm{mmol}, 2.00$ equiv). The mixture was stirring at room temperature for $5 \mathrm{mins}$, then added TFMS (48.9 mg, $0.200 \mathrm{mmol}$, 4.00 equiv). After stirring for $4.0 \mathrm{~h}$ at $60^{\circ} \mathrm{C}$, trifluoromethylthiobenzene $(7 \mu \mathrm{L}, 0.0500 \mathrm{mmol})$ was added. The yield of (Z)-1-bromo-2-(trifluoromethoxy)dodec-1-ene (Z-7a) was determined by comparing the integration of the ${ }^{19} \mathrm{~F}$ NMR resonance of (Z)-1-bromo-2-(trifluoromethoxy)dodec-1-ene $\quad(\boldsymbol{Z}-7 \mathbf{a}) \quad(-55.77 \quad \mathrm{ppm})$ with that of trifluoromethylthiobenzene (-42.80 ppm). $\left({ }^{19} \mathrm{~F}\right.$ NMR yield: $\left.58 \%\right)$.

\section{Reference}

1. Guo, S.; Cong, F.; Guo, R; Wang, L; Tang, P. Asymmetric Silver-Catalysed Intermolecular Bromotrifluoromethoxylation of Alkenes with a New Trifluoromethoxylation Reagent. Nat. Chem. 2017, 9, 546.

2. Deng, C.; Fang, R.; Guan, Y.; Jiang, J.; Lin, C.; Wang, L. Sonication-Induced Self-assembly of Flexible Tris(ureidobenzyl)amine: From Dimeric Aggregates to Supramolecular Gels. Chem. Commun. 2012, 48, 7973.

3. Laha, J. K.; Sharma, S.; Dayal, N. Palladium-Catalyzed Regio- and Chemoselective Reactions of 2-Bromobenzyl Bromides: Expanding the Scope for the Synthesis of Biaryls Fused to a Seven-Membered Sultam. Eur. J. Org. Chem. 2015, 7885.

4. Trost, B. M.; Breder, A.; Kai, B. Atom-Economical Synthesis of Functionalized Cycloalkanes via Catalytic Redox Cycloisomerization of Propargyl Alcohols. Org. Lett. 2012, 14, 1708.

5. Yang, H.; Wang, F.; Jiang, X.; Zhou, Y.; Xu, X.; Tang, P. Silver-Promoted Oxidative Benzylic C-H Trifluoromethoxylation. Angew. Chem., Int. Ed. 2018, 57, 13266.

6. Mukherjee, S.; Garza-Sanchez, R. A.; Tlahuext-Aca, A.; Glorius, F. Alkynylation of Csp ${ }^{2}$ (O)-H Bonds Enabled by Photoredox-Mediated Hydrogen-Atom Transfer. Angew. Chem., Int. Ed. 2017, 56, 14723.

7. Meng, L.; Li, D.; Xiong, S.; Hu, X.; Wang, L.; Li, G. FRET-capable Supramolecular Polymers Based on a BODIPY-bridged Pillar[5] arene Dimer with BODIPY Guests for Mimicking the Light-harvesting System of Natural Photosynthesis. Chem. Commun. 2015, $51,4643$.

8. Upadhyay, N. S.; Jayakumar, J.; Cheng, C. Facile One-pot Synthesis of 2,3-Dihydro-1H-indolizinium Derivatives by Rhodium(III)-catalyzed Intramolecular Oxidative Annulation via C-H Activation: Application to Ficuseptine Synthesis. Chem. Commun. 2017, 53, 2491. 
9. Zhao, Q.; Hao, W.; Shi, H.; Xu, T.; Tu, X.; Jiang, B. Photocatalytic Annulation-Alkynyl Migration Strategy for Multiple Functionalization of Dual Unactivated Alkenes. Org. Lett. 2019, 21, 9784. 


\section{Spectrum Data}

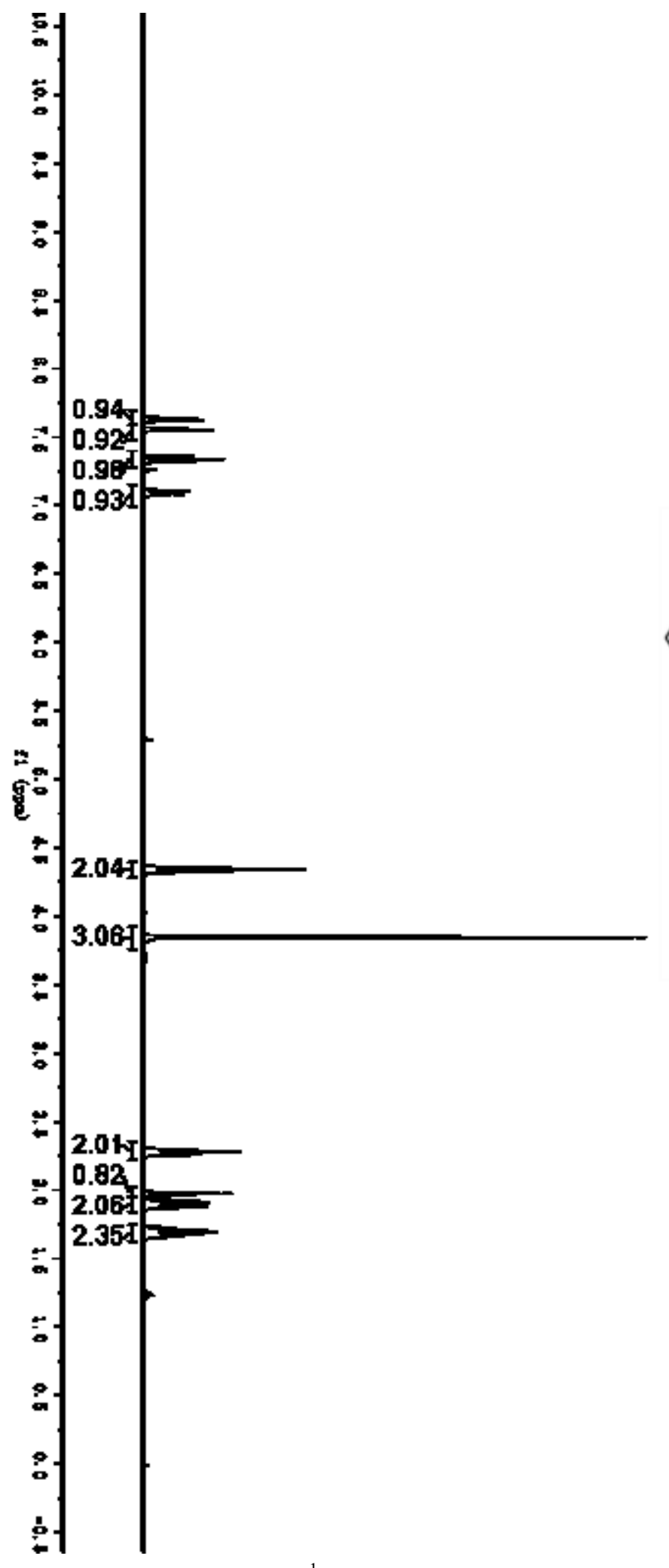

$\left\{\begin{array}{l}7.64 \\ 7.62 \\ 7.55 \\ 7.36 \\ 7.34 \\ 7.32 \\ 7.11 \\ 7.10 \\ 7.09 \\ 7.08\end{array}\right.$

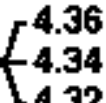

$-3.85$

2.30

2.29

2.28

2.28

2.26

2.26

1.98

$-1.97$

1.97

$-1.94$

1.92

1.90

1.88

1.87

1.73

1.71

1.69

1.67

1.66

${ }^{1} \mathrm{H}$ NMR spectrum (400 MHz, $\mathrm{CDCl}_{3}$ ) of $\mathbf{1 b}$ 


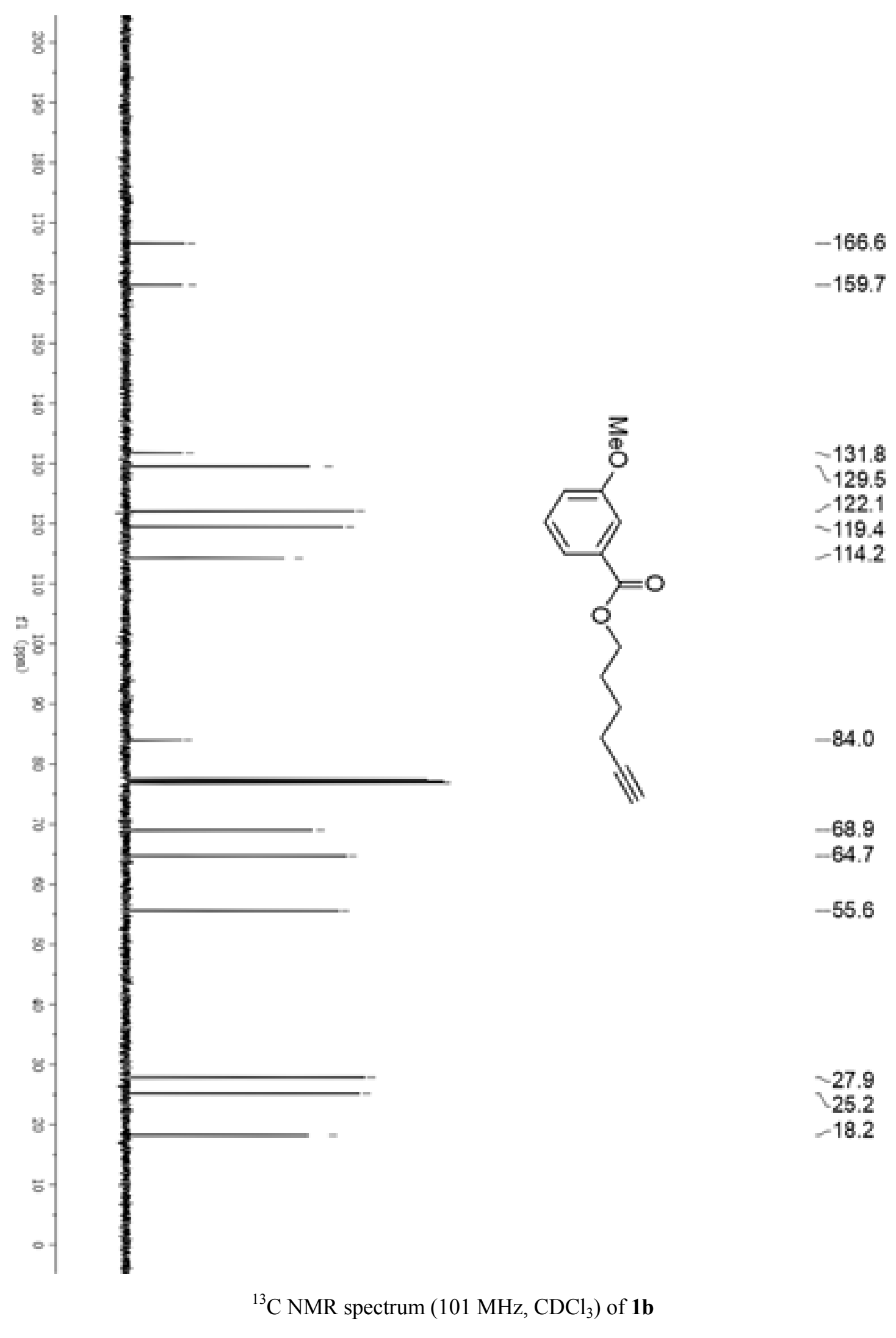




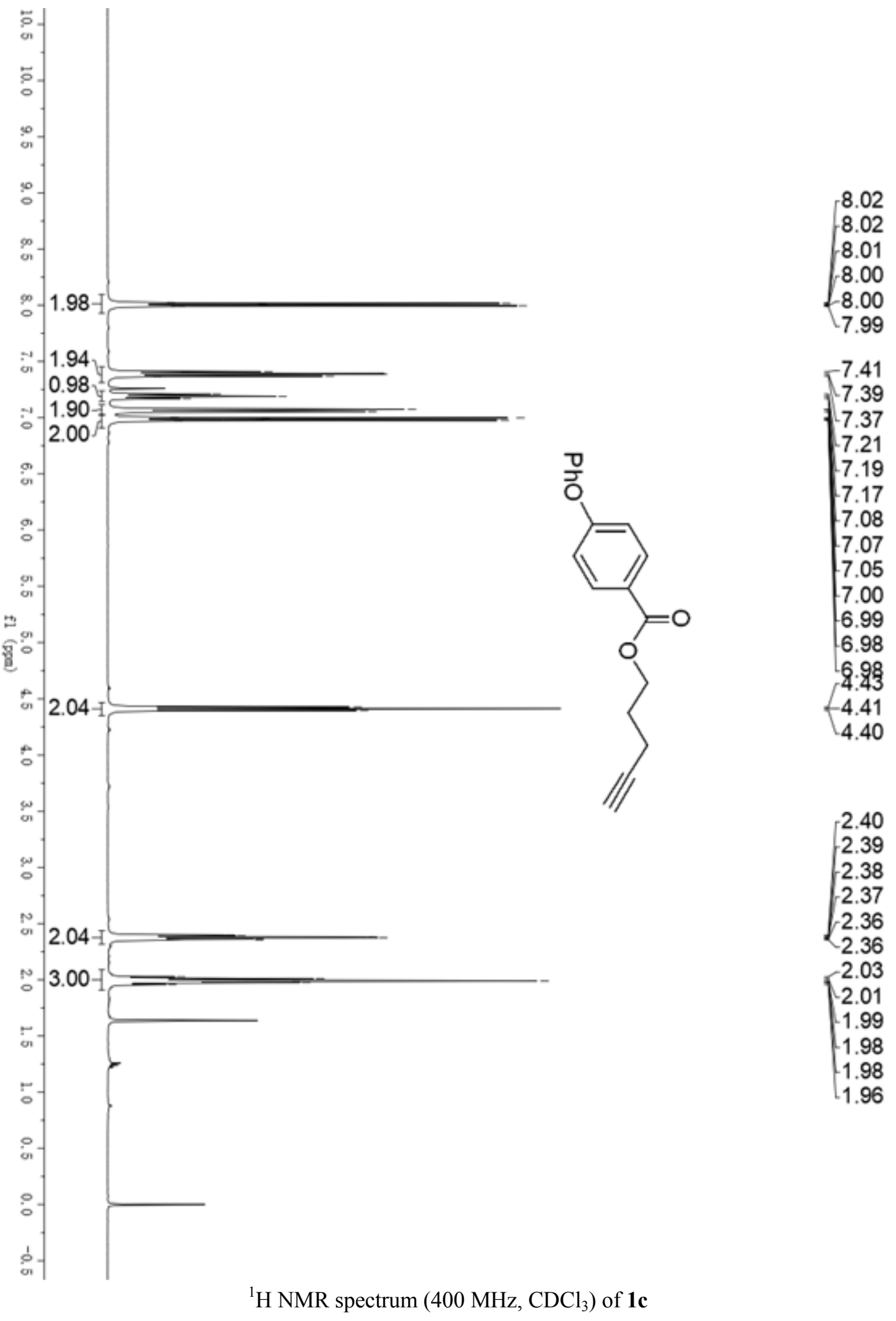




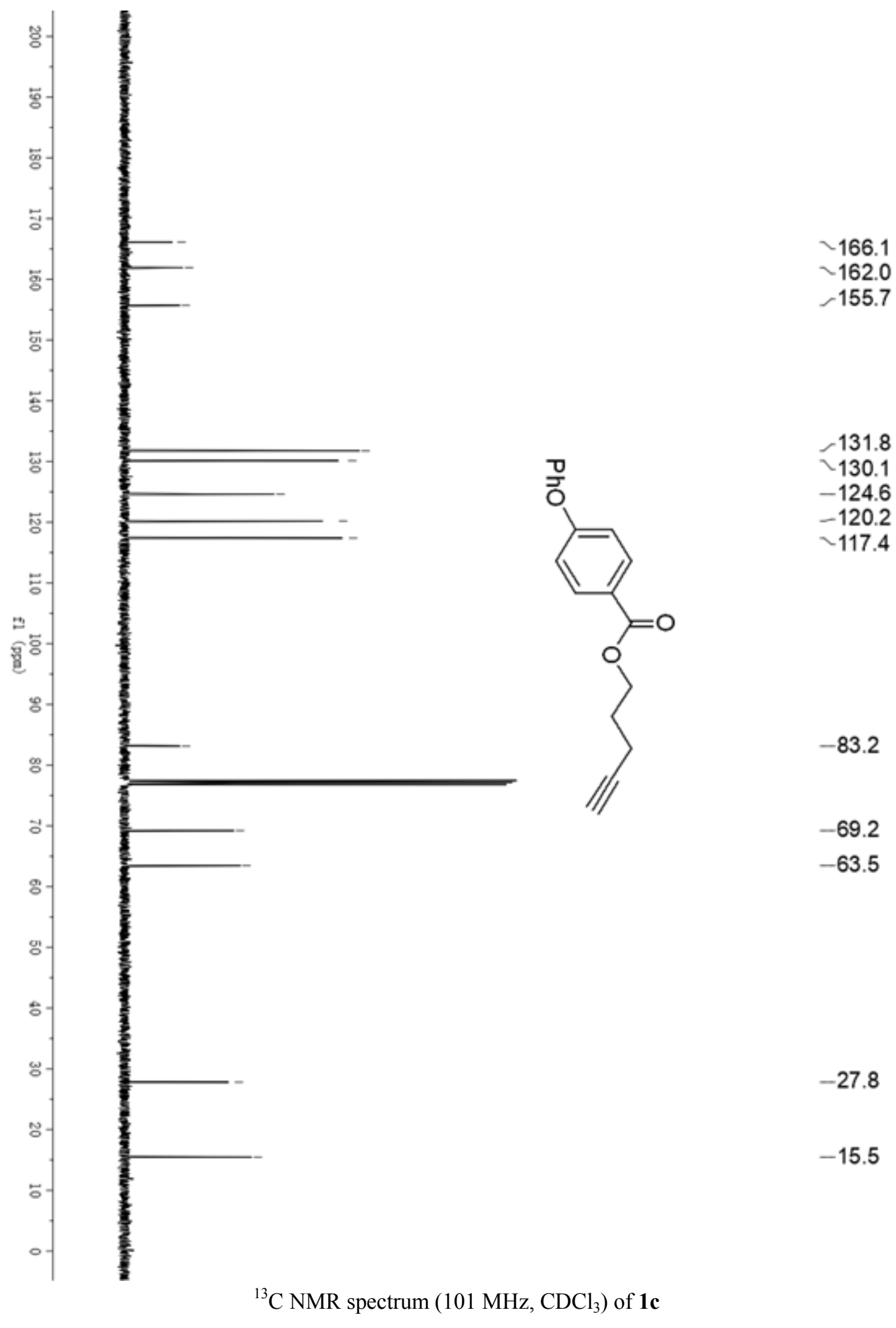




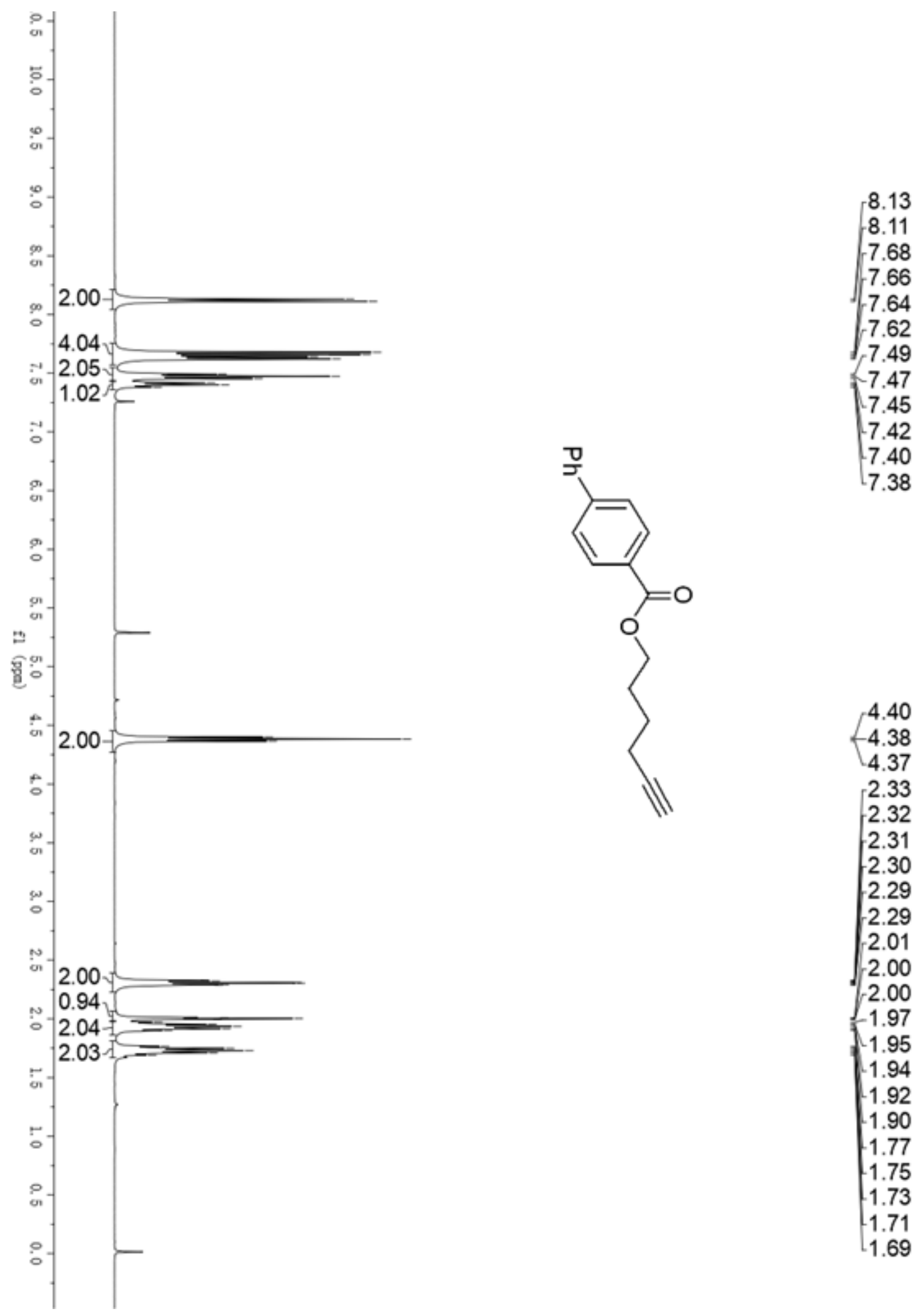

${ }^{1} \mathrm{H}$ NMR spectrum $\left(400 \mathrm{MHz}, \mathrm{CDCl}_{3}\right.$ ) of $\mathbf{1 d}$ 


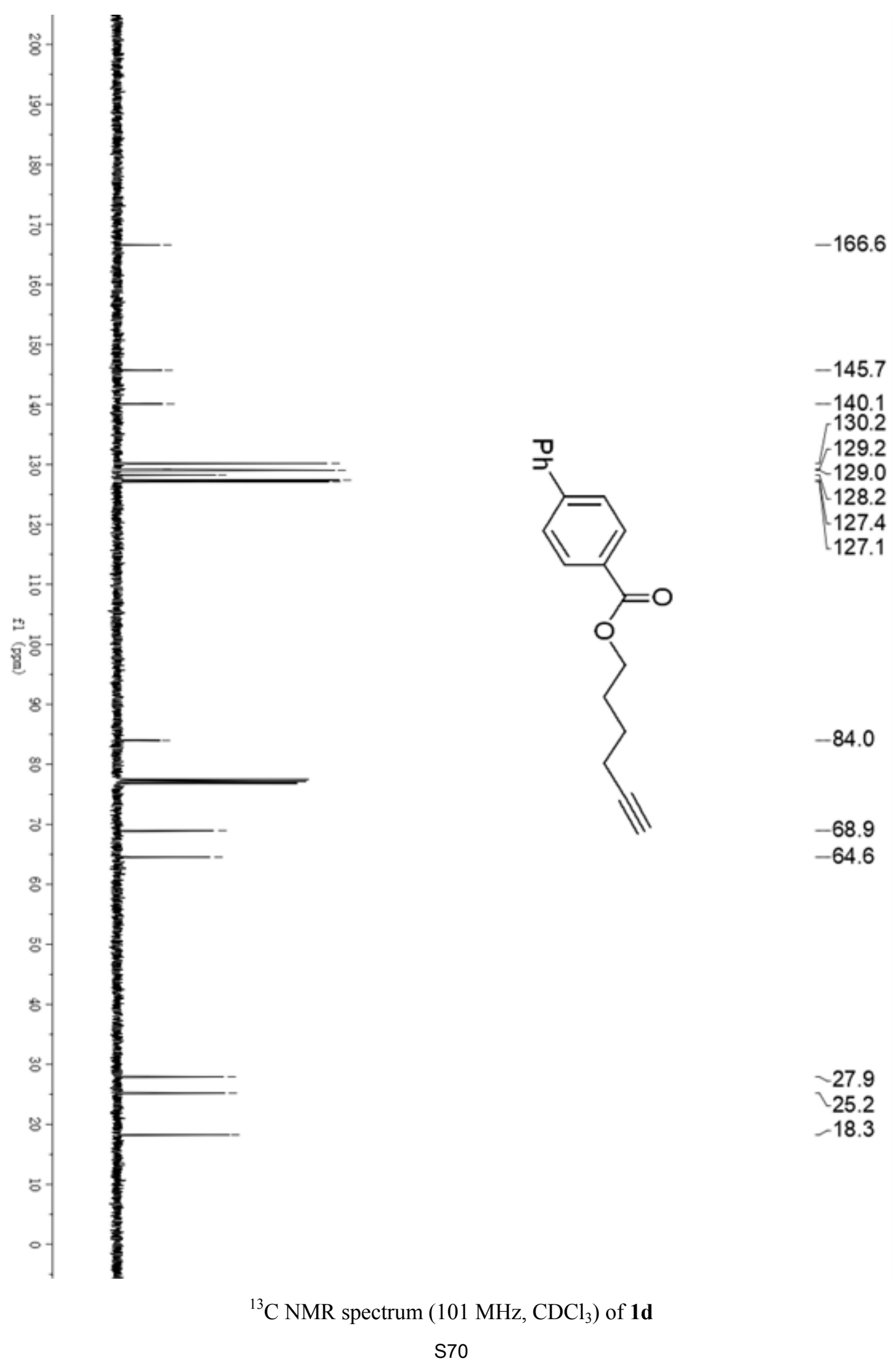




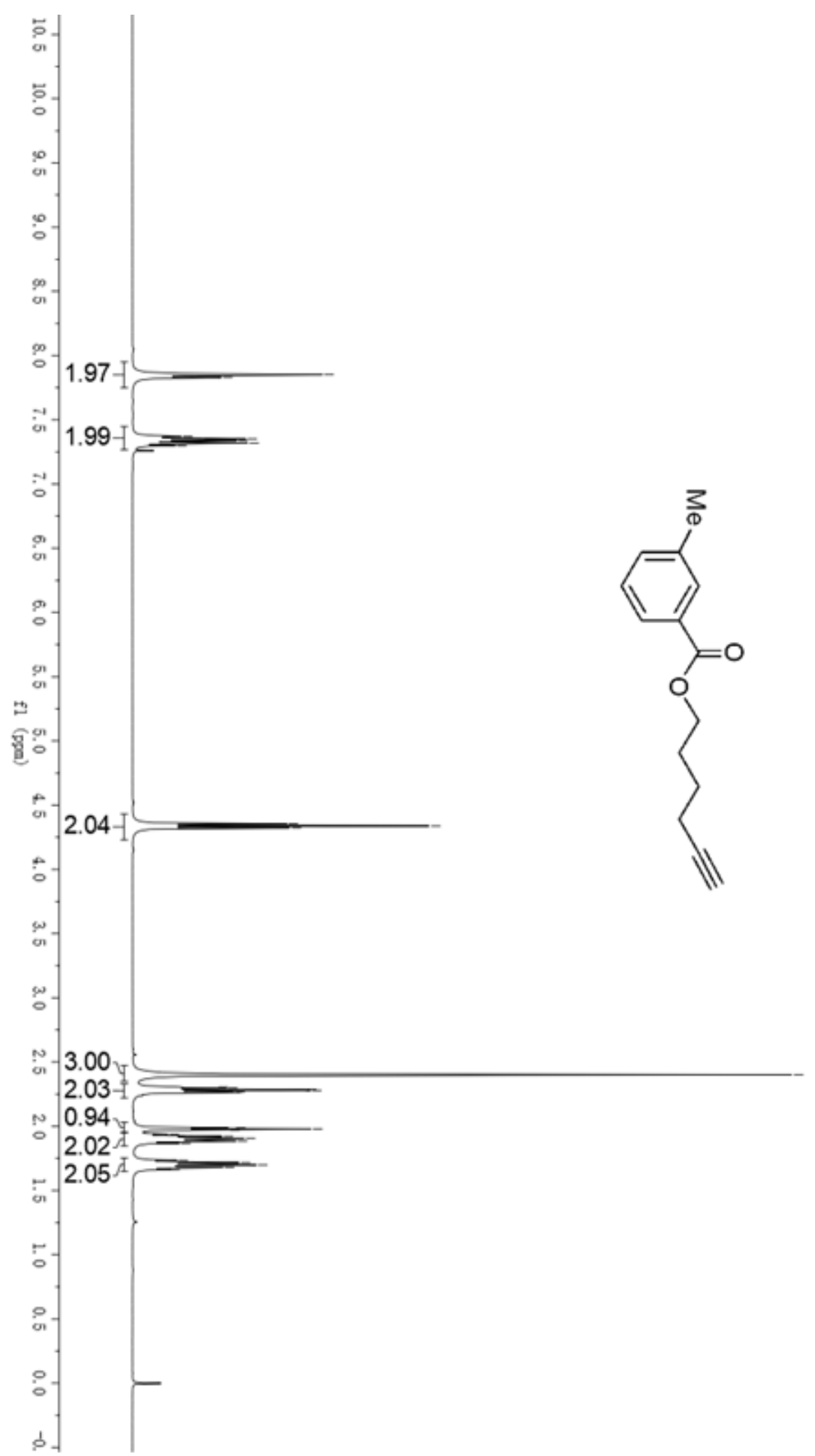

${ }^{1} \mathrm{H}$ NMR spectrum $\left(400 \mathrm{MHz}, \mathrm{CDCl}_{3}\right)$ of $\mathbf{1 e}$ 


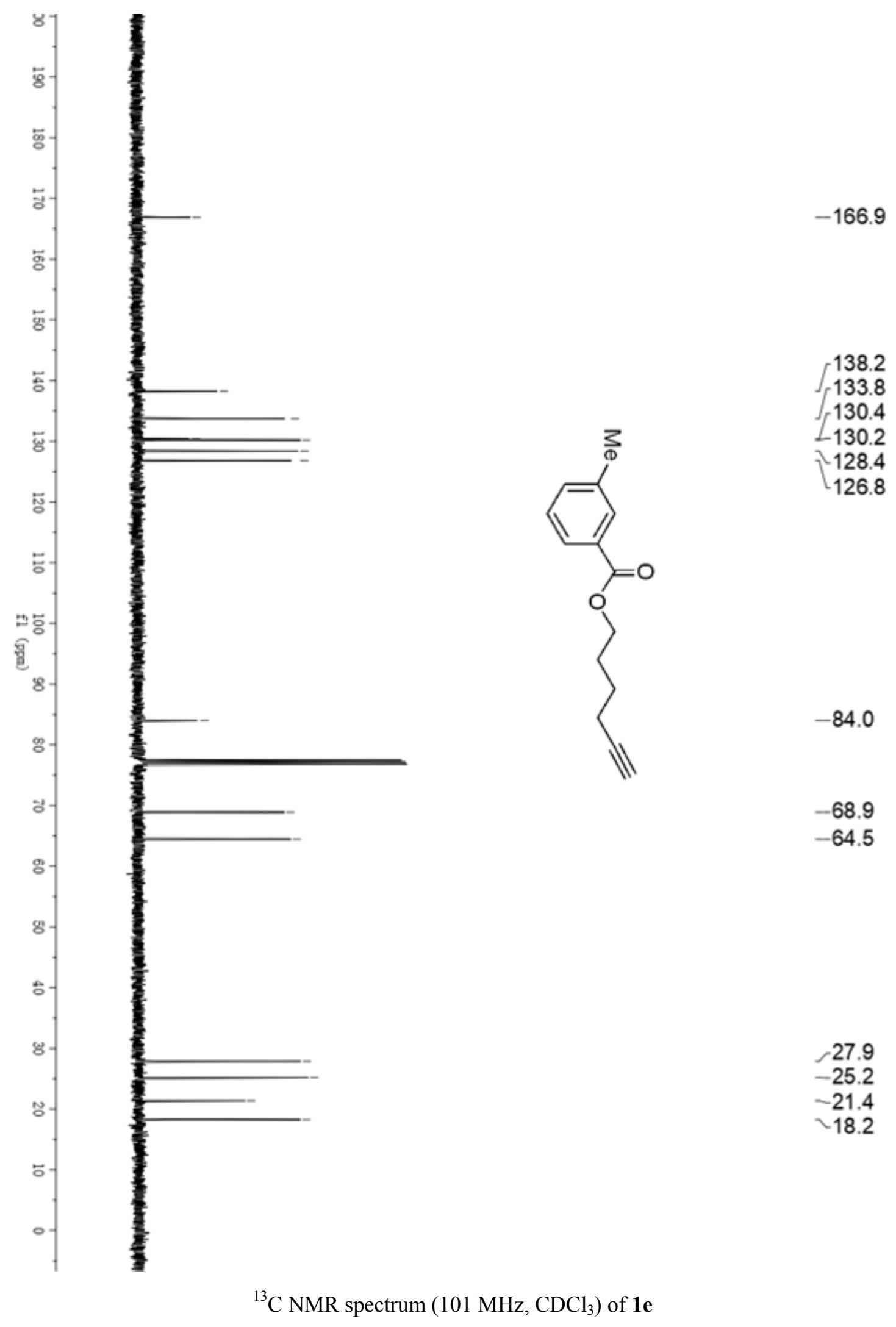




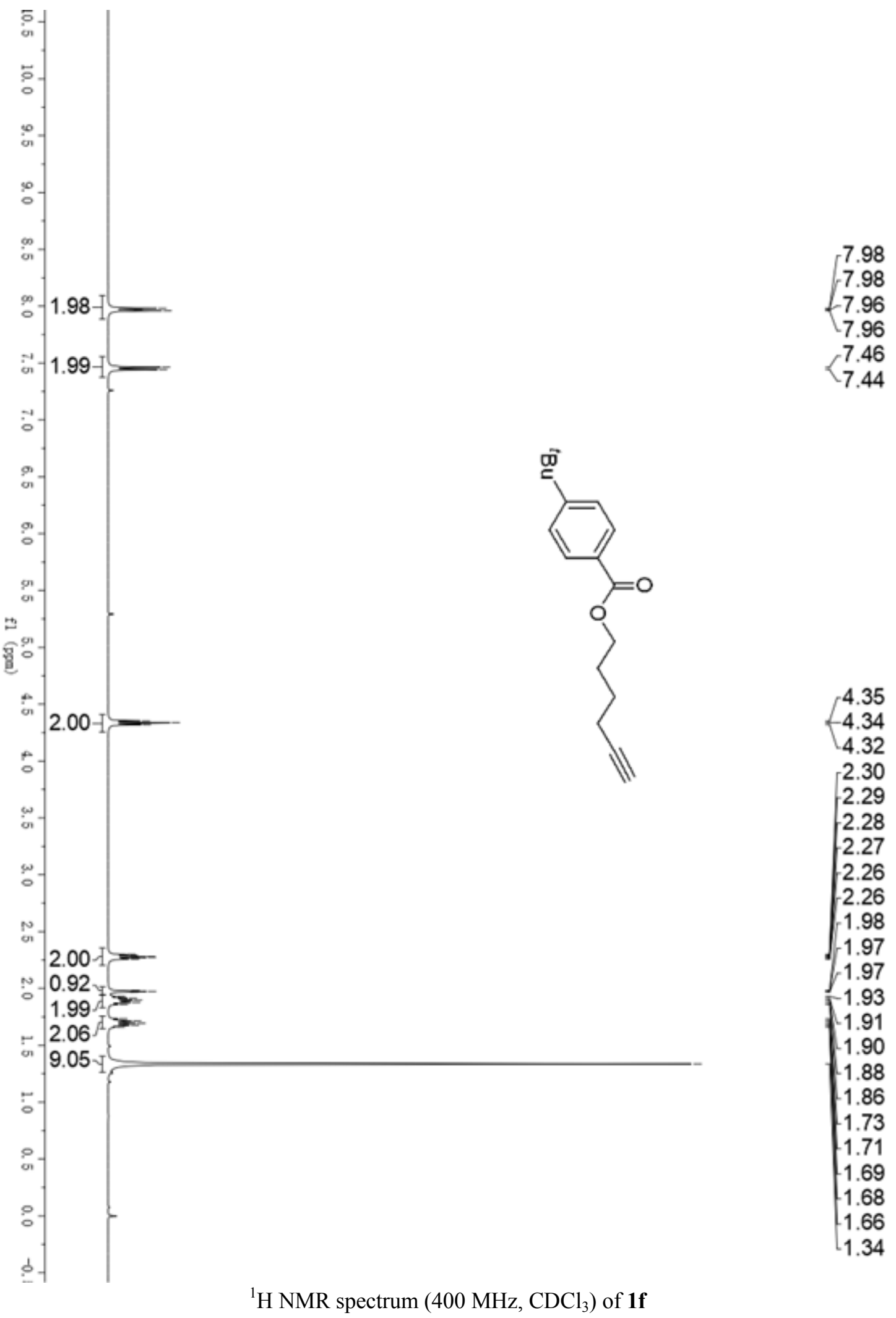




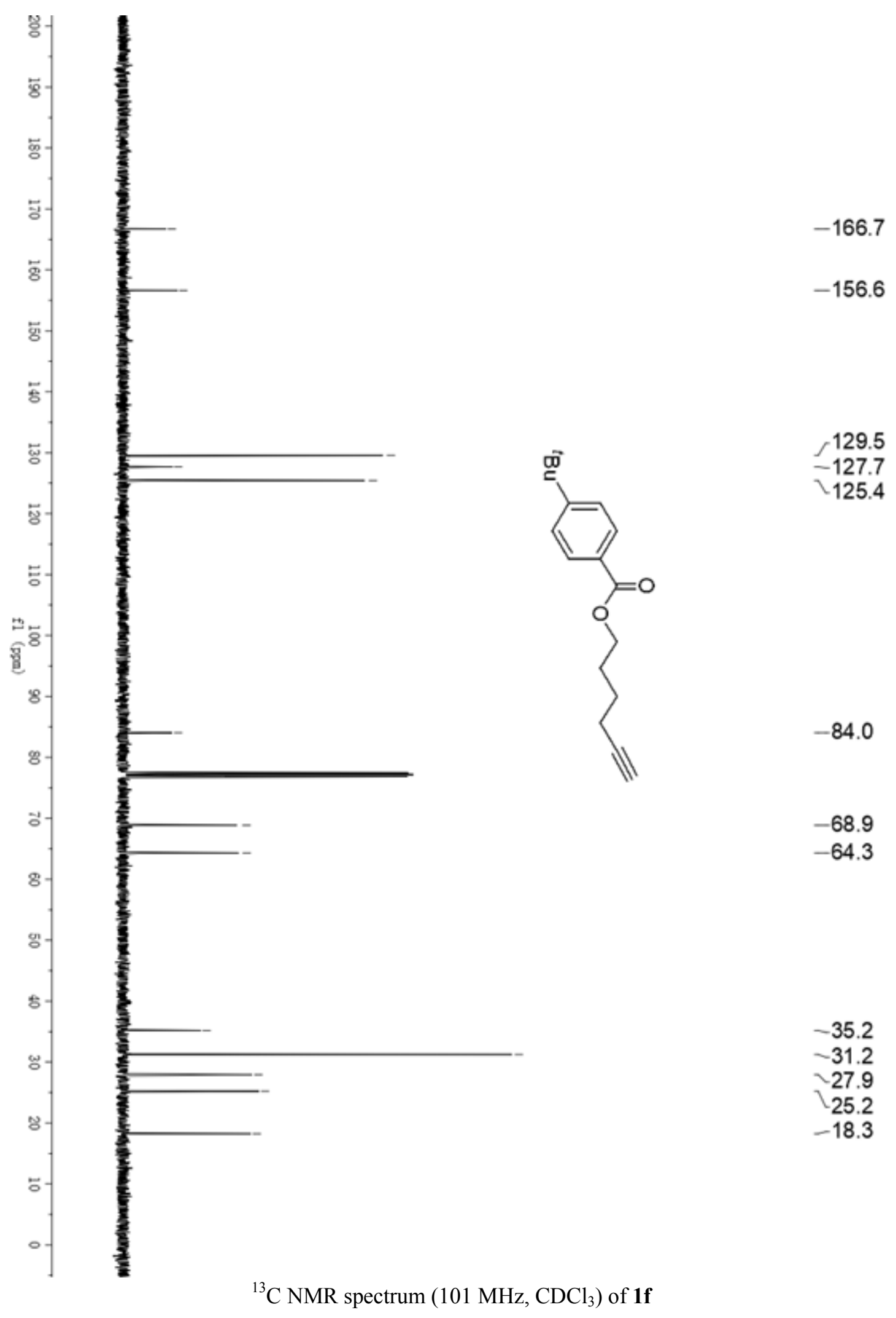




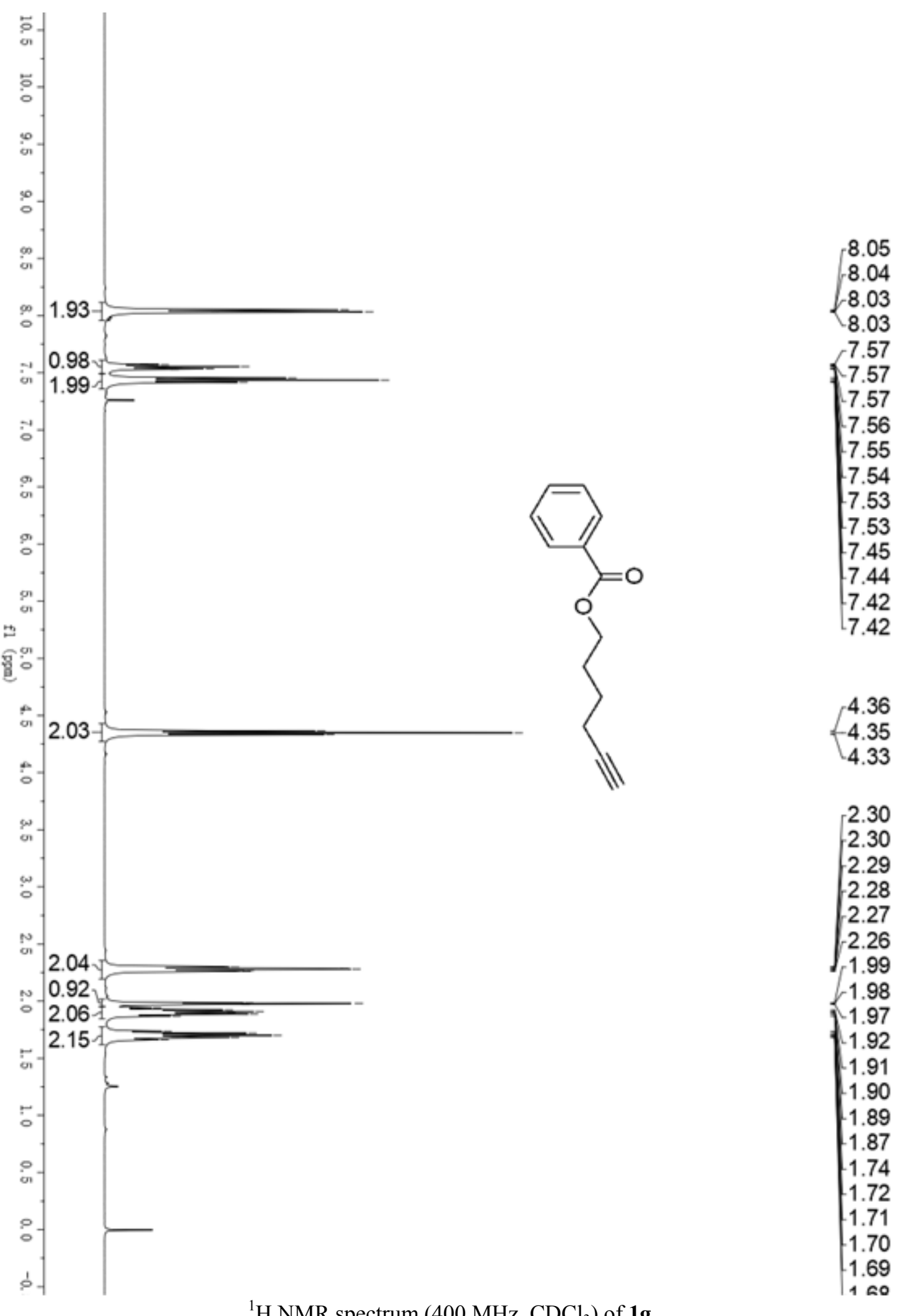

${ }^{1} \mathrm{H}$ NMR spectrum $\left(400 \mathrm{MHz}, \mathrm{CDCl}_{3}\right)$ of $\mathbf{1 g}$ 


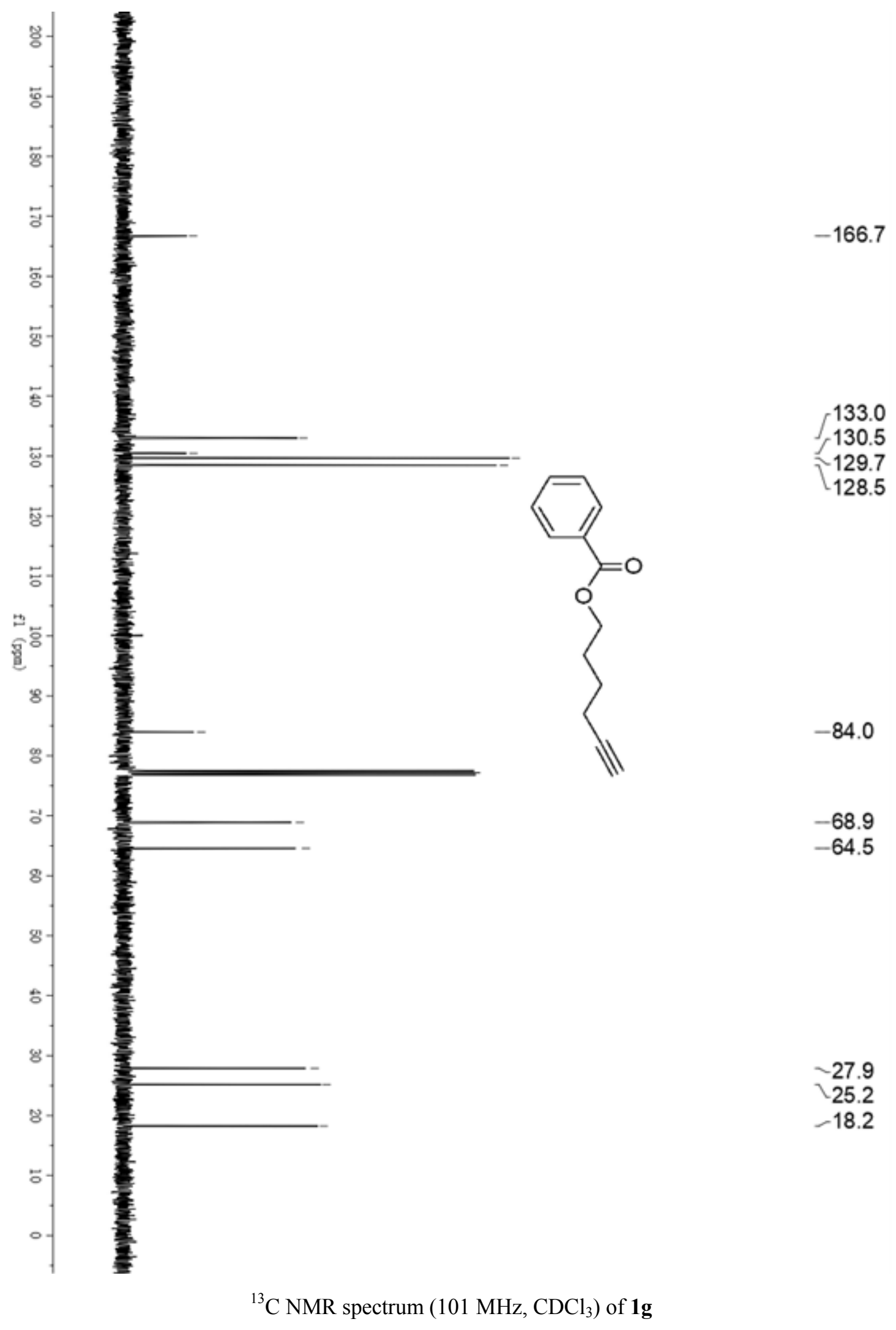



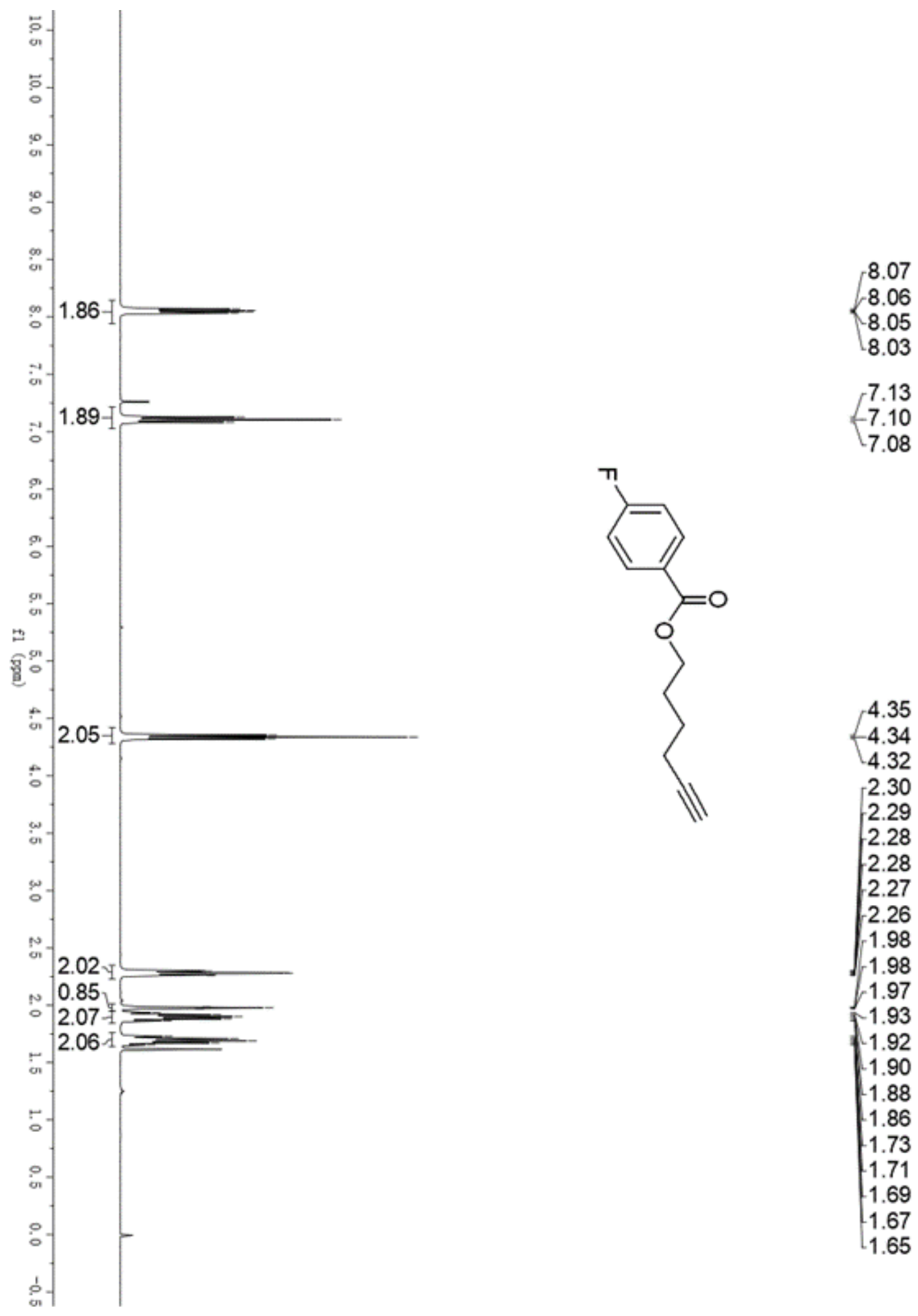

$\left\{\begin{array}{r}4.35 \\ 4.34 \\ 4.32\end{array}\right.$

2.30

2.29

2.28

$-2.28$

2.27

$-2.26$

1.98

1.98

1.97

1.93

1.92

$-1.90$

1.88

1.86

1.73

1.71

1.69

$-1.67$

1.65

${ }^{1} \mathrm{H}$ NMR spectrum $\left(400 \mathrm{MHz}, \mathrm{CDCl}_{3}\right)$ of $\mathbf{1 h}$ 


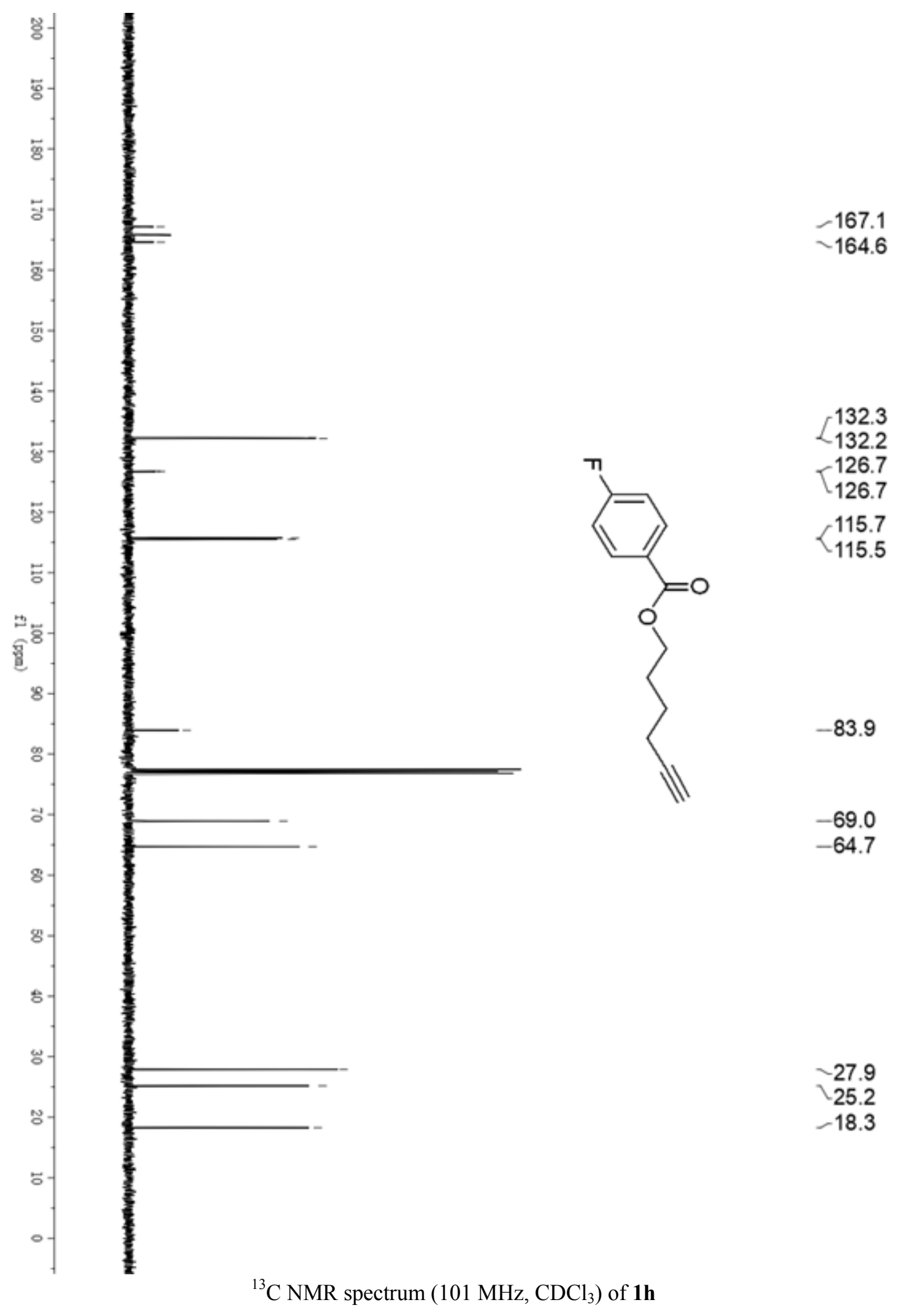




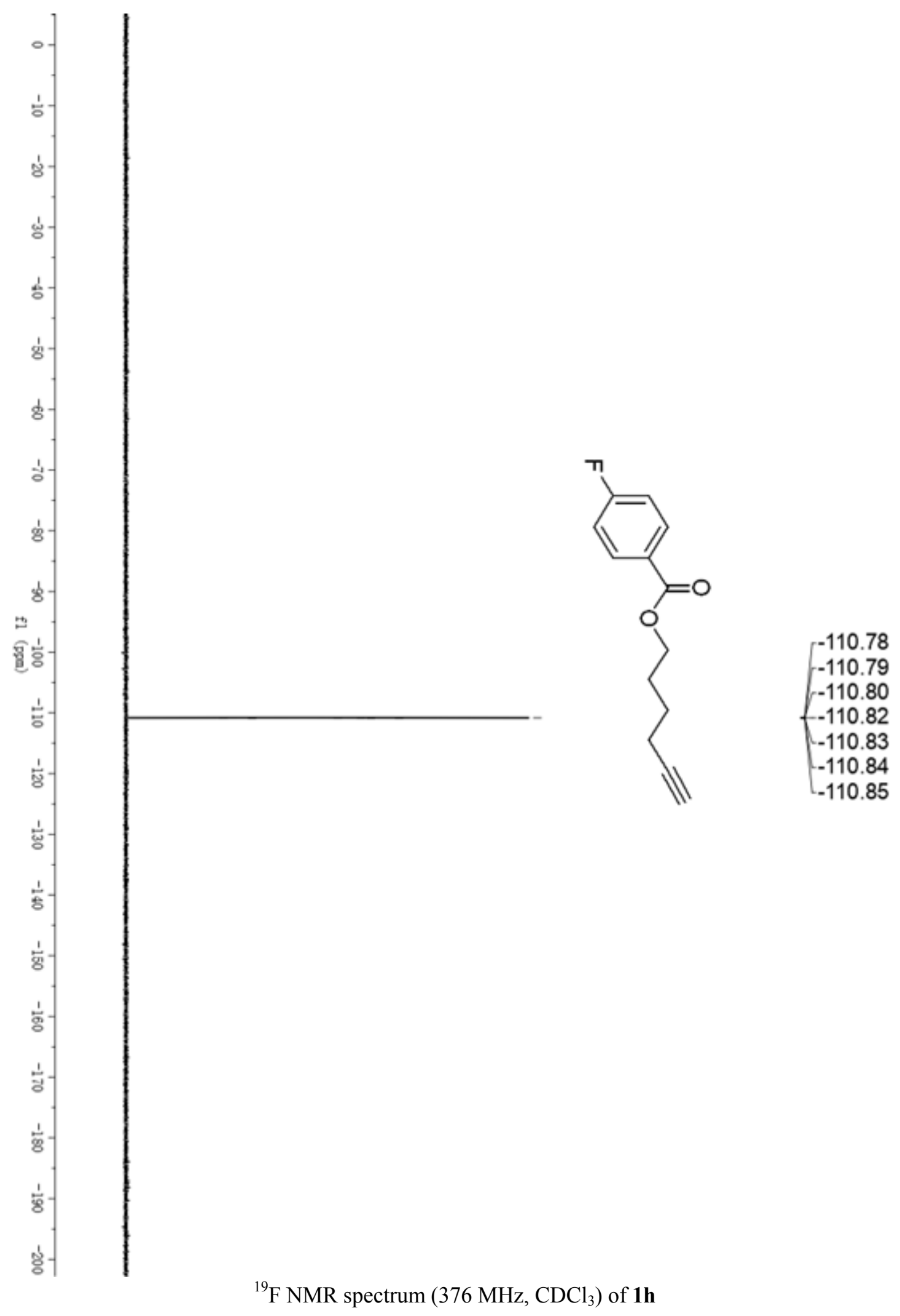




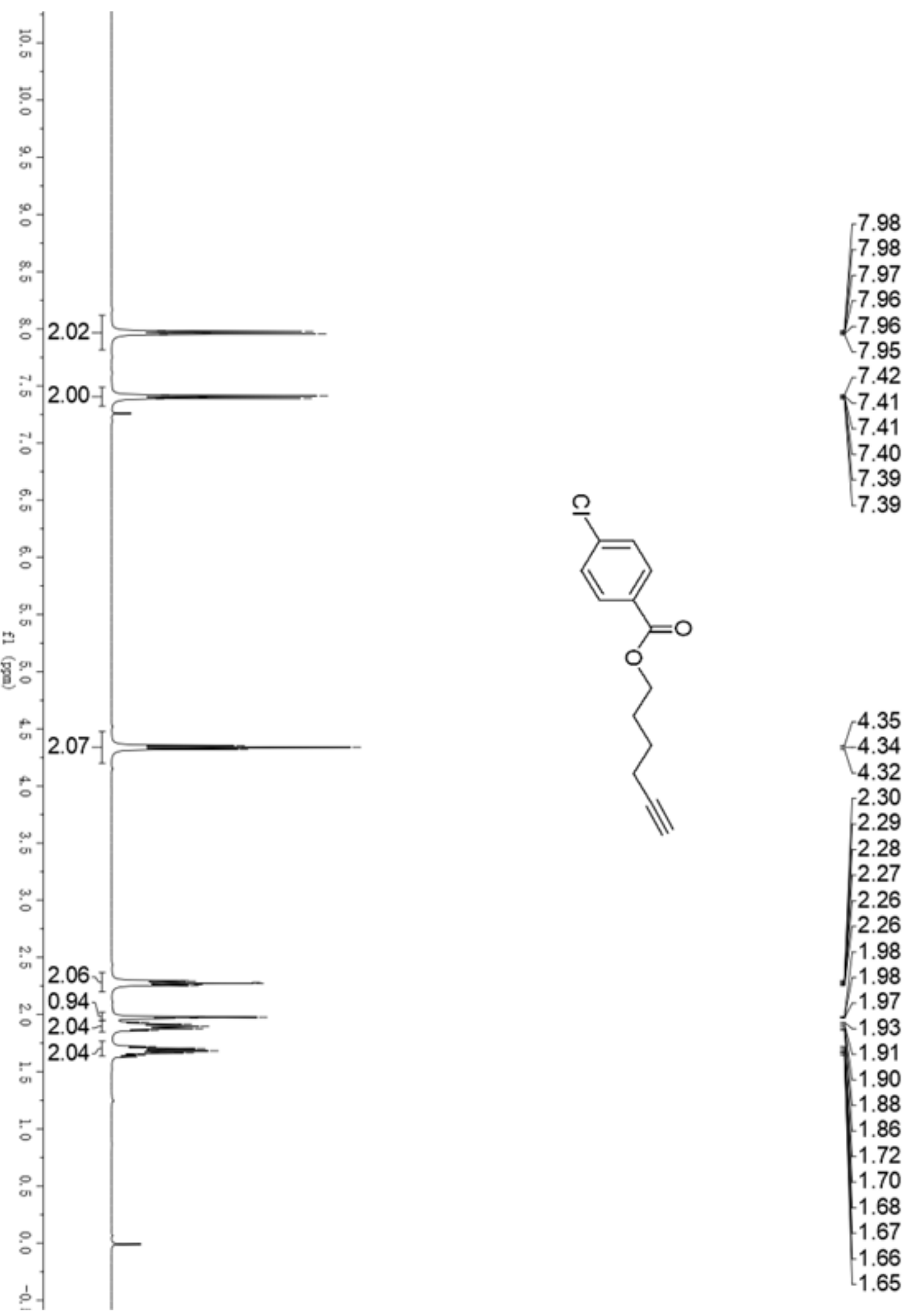

${ }^{1} \mathrm{H}$ NMR spectrum $\left(400 \mathrm{MHz}, \mathrm{CDCl}_{3}\right.$ ) of $\mathbf{1 i}$ 


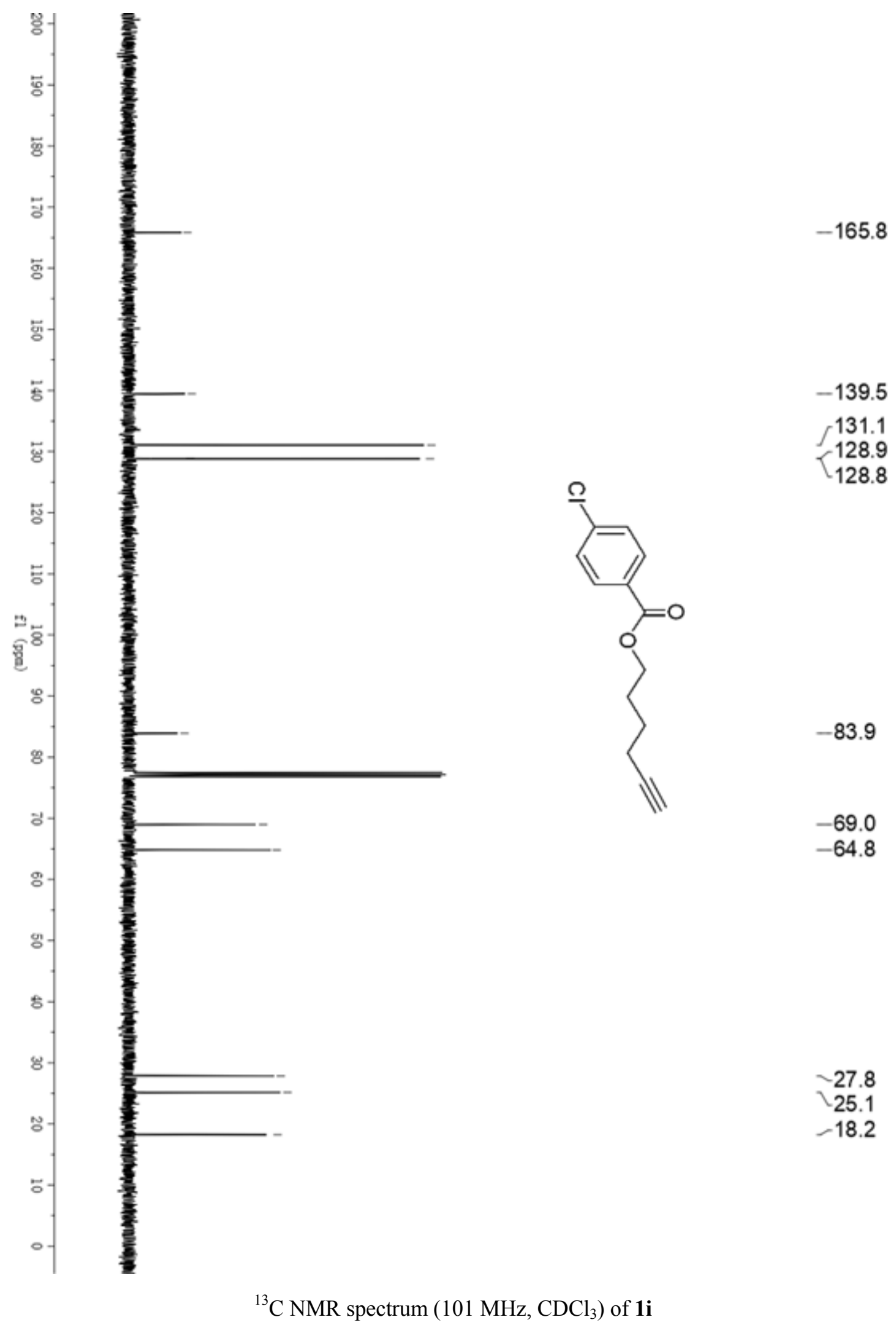




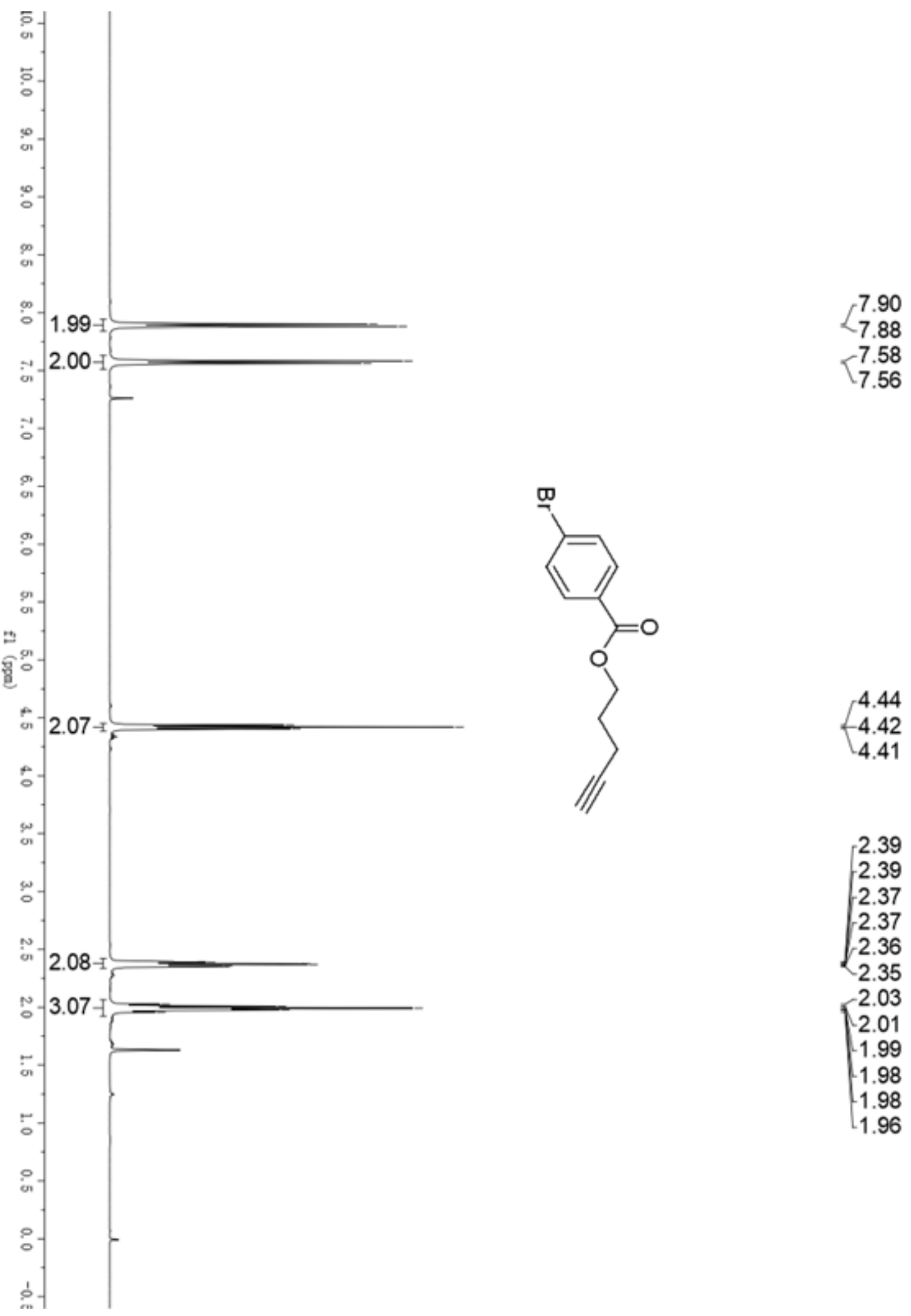

${ }^{1} \mathrm{H}$ NMR spectrum $\left(400 \mathrm{MHz}, \mathrm{CDCl}_{3}\right)$ of $\mathbf{1} \mathbf{j}$ 


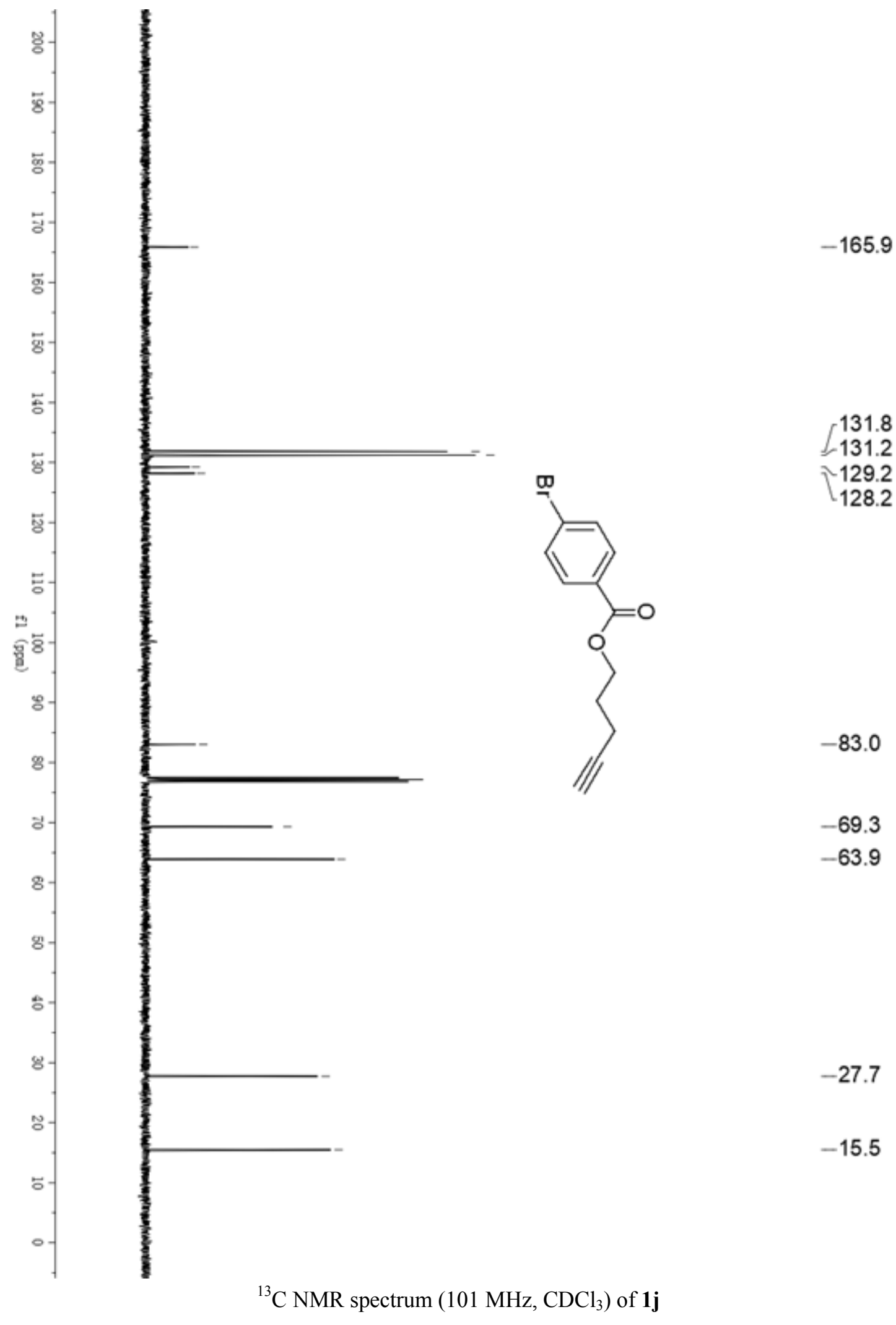




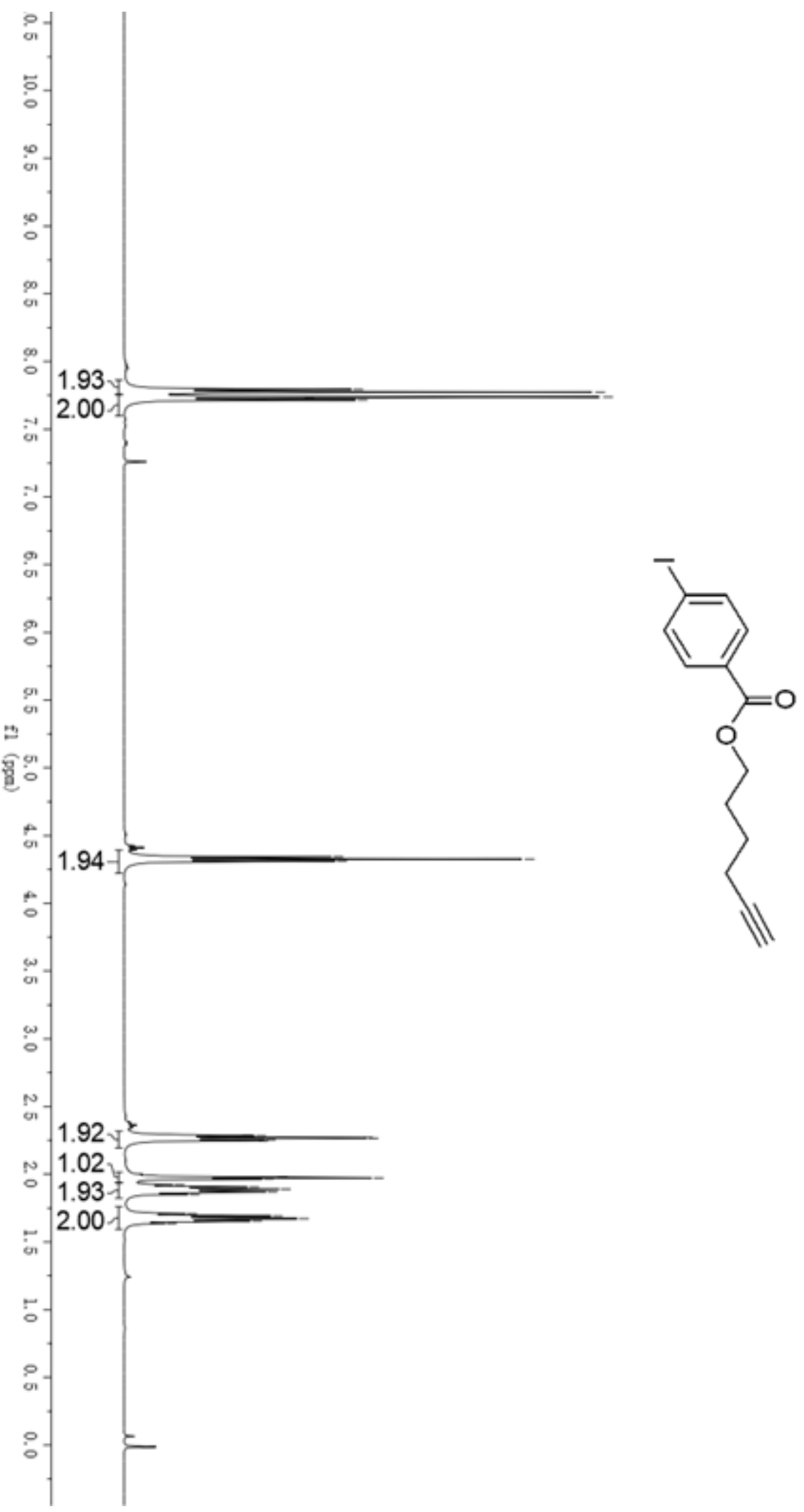

$\left\{\begin{array}{l}7.80 \\ 7.79 \\ 7.77 \\ 7.74 \\ 7.73 \\ 7.72 \\ 7.72\end{array}\right.$

${ }^{1} \mathrm{H}$ NMR spectrum $\left(400 \mathrm{MHz}, \mathrm{CDCl}_{3}\right)$ of $\mathbf{1 k}$ 


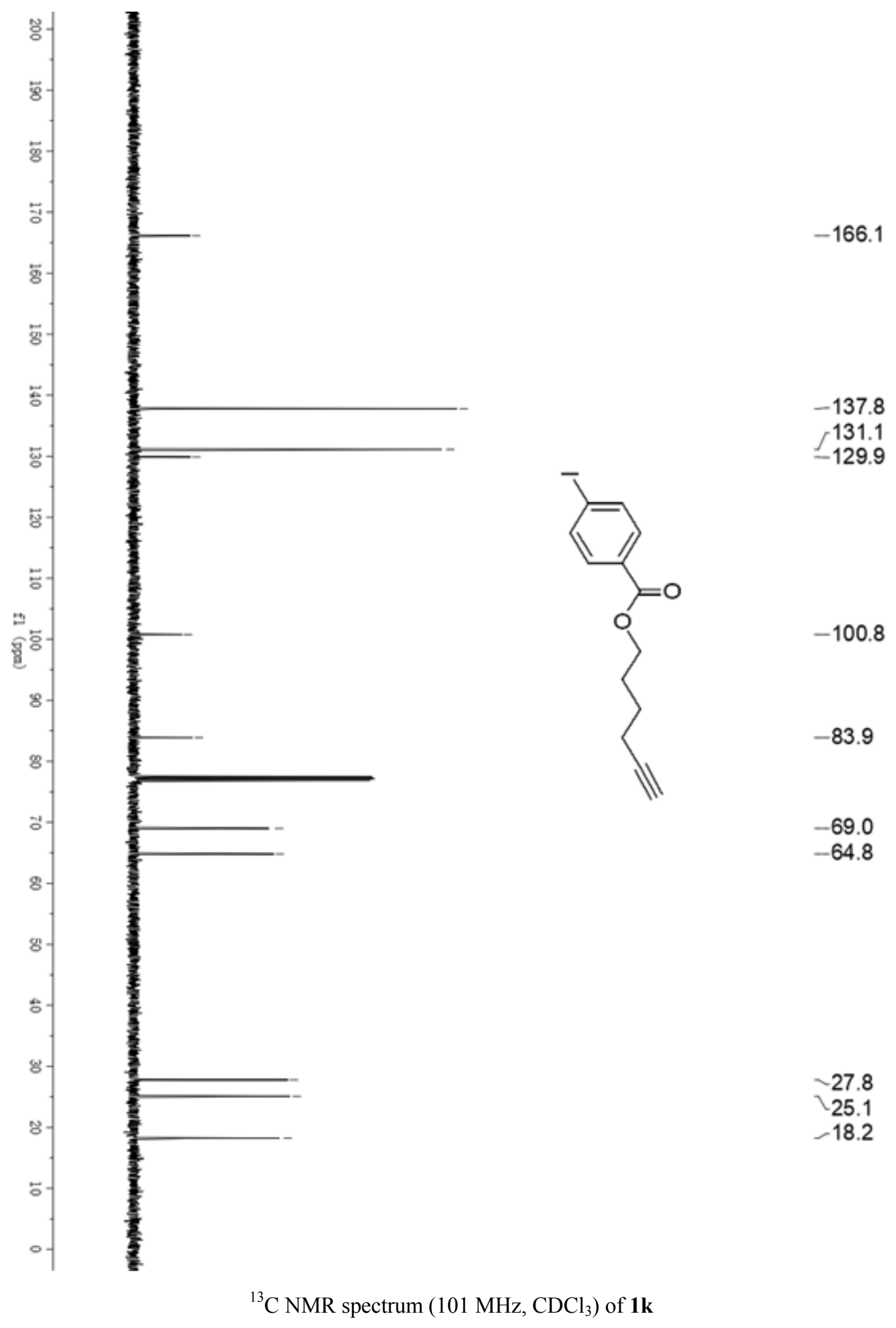




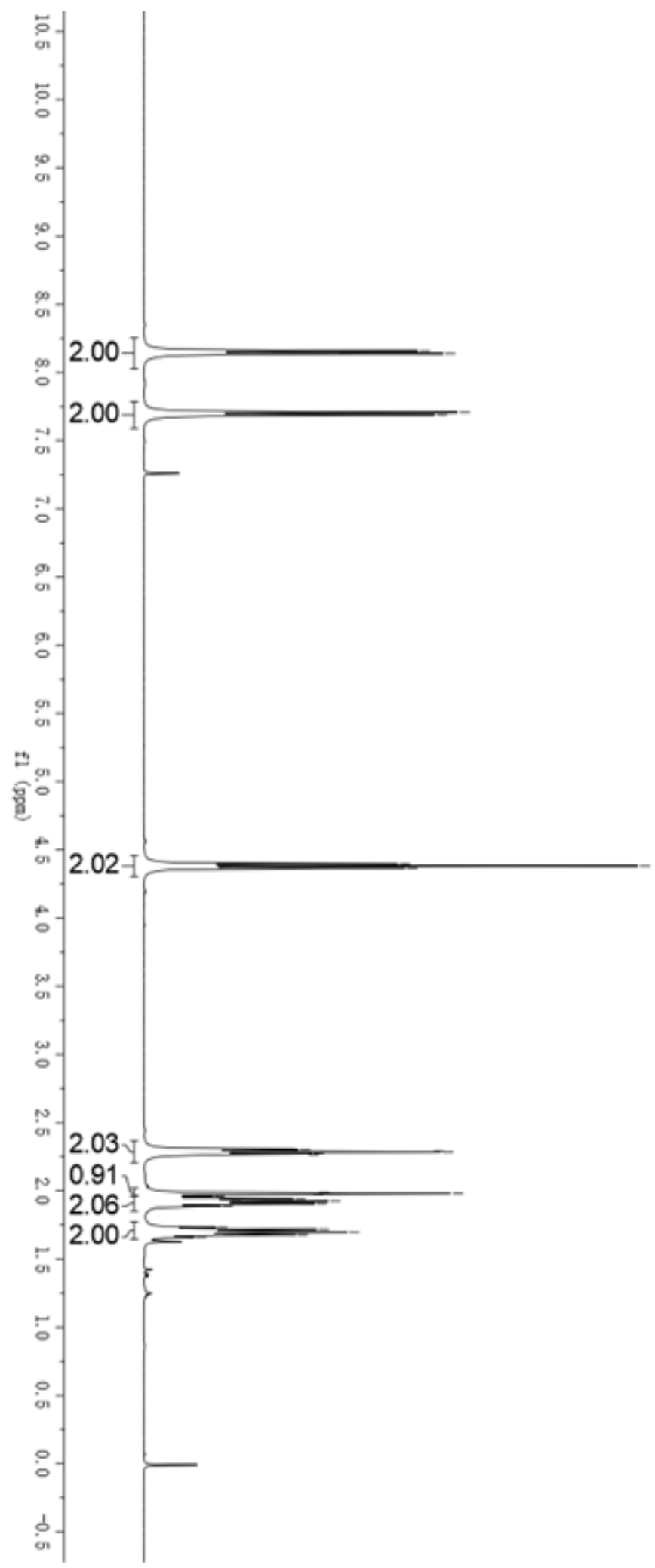

8.16

8.14

7.71

7.69
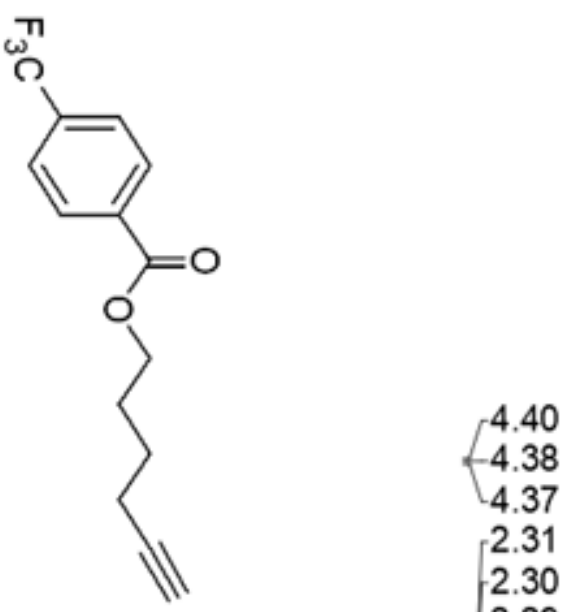

4.37

2.31

2.30

$-2.29$

2.28

$-2.27$

2.27

1.99

1.98

1.97

1.96

1.94

1.93

$-1.92$

$-1.90$

1.89

1.73

1.72

1.70

1.68

$-1.68$

1.66

${ }^{1} \mathrm{H}$ NMR spectrum $\left(400 \mathrm{MHz}, \mathrm{CDCl}_{3}\right)$ of $\mathbf{1 l}$ 


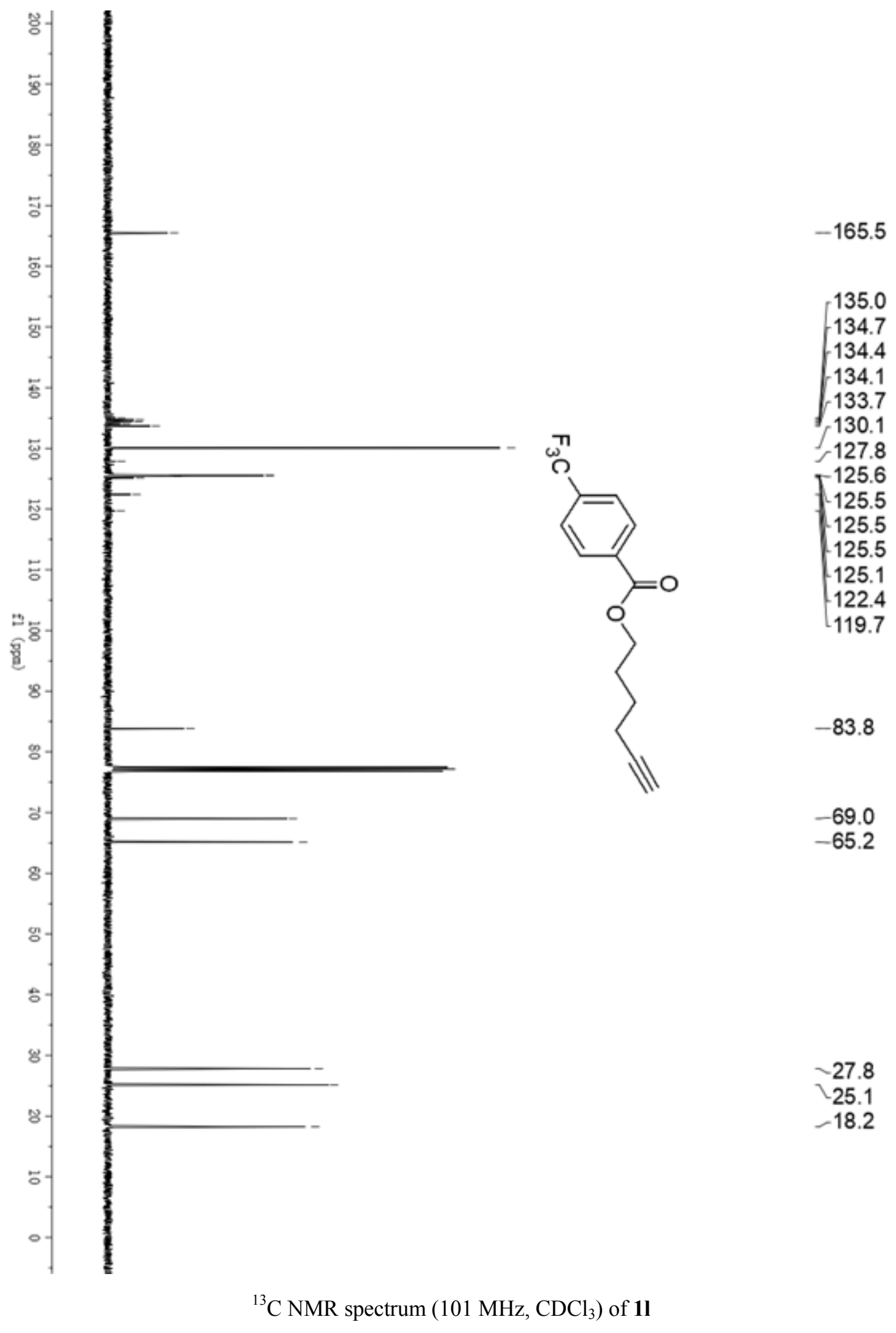




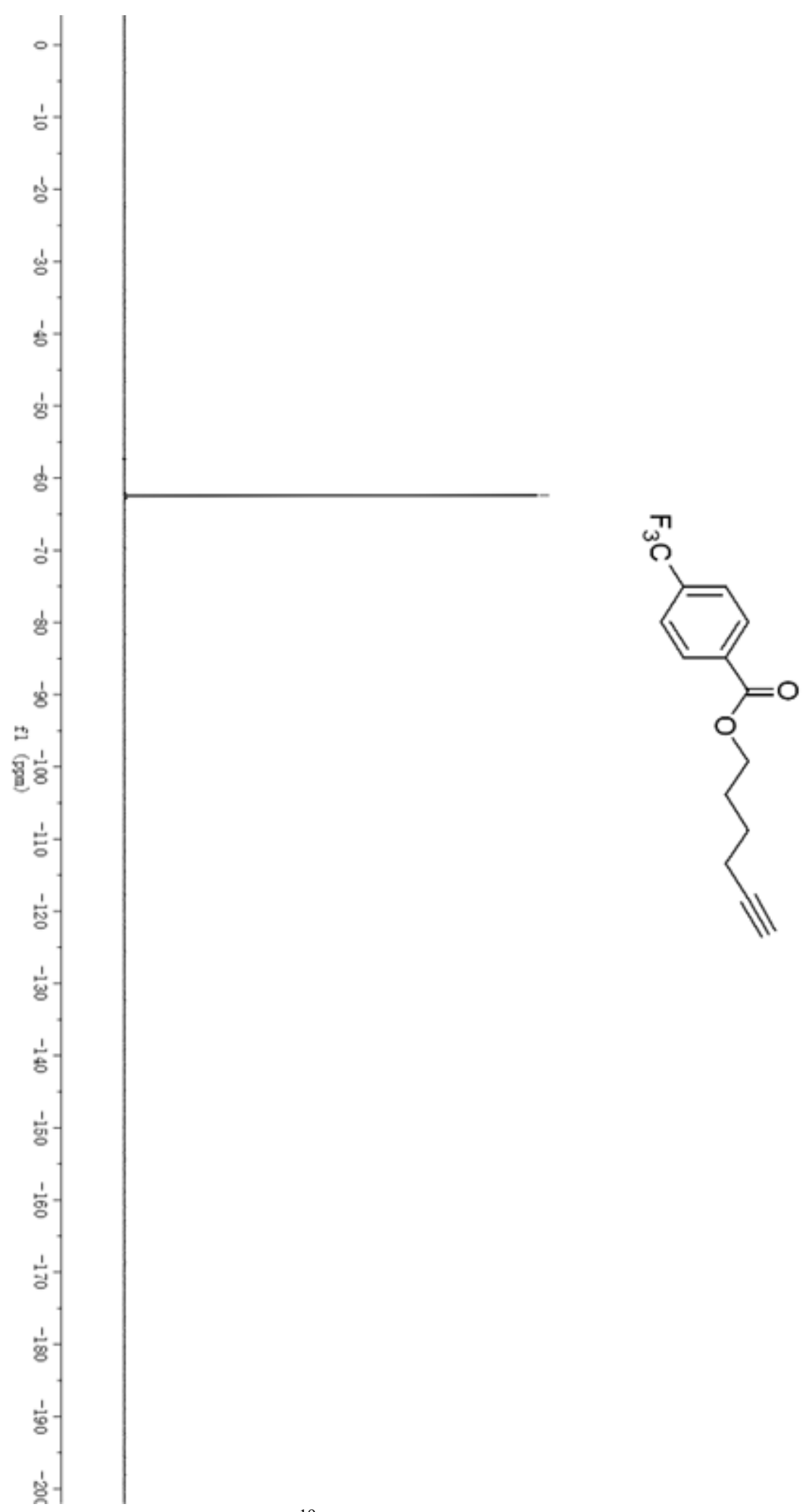

${ }^{19} \mathrm{~F}$ NMR spectrum $\left(376 \mathrm{MHz}, \mathrm{CDCl}_{3}\right)$ of $\mathbf{1 l}$ 


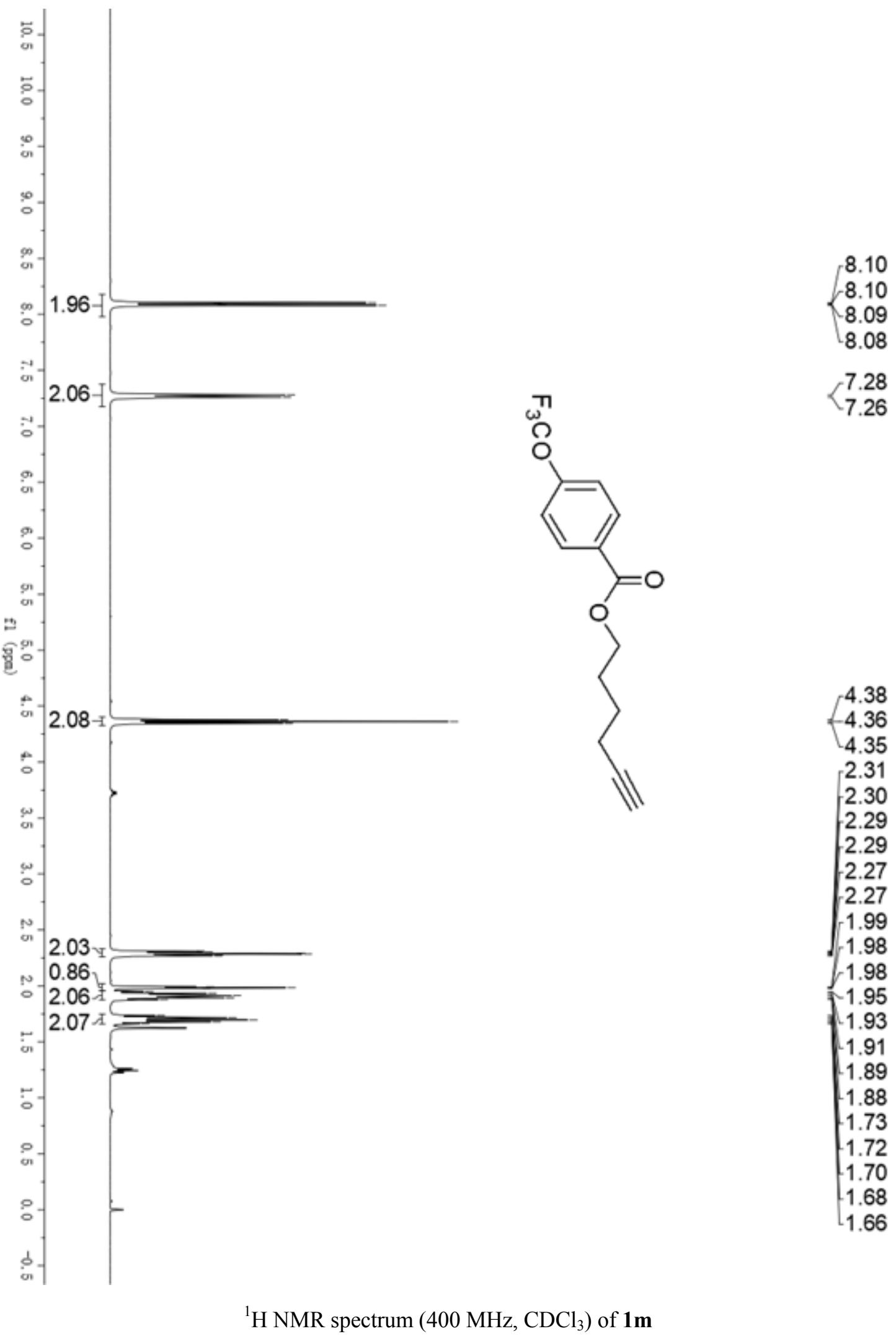




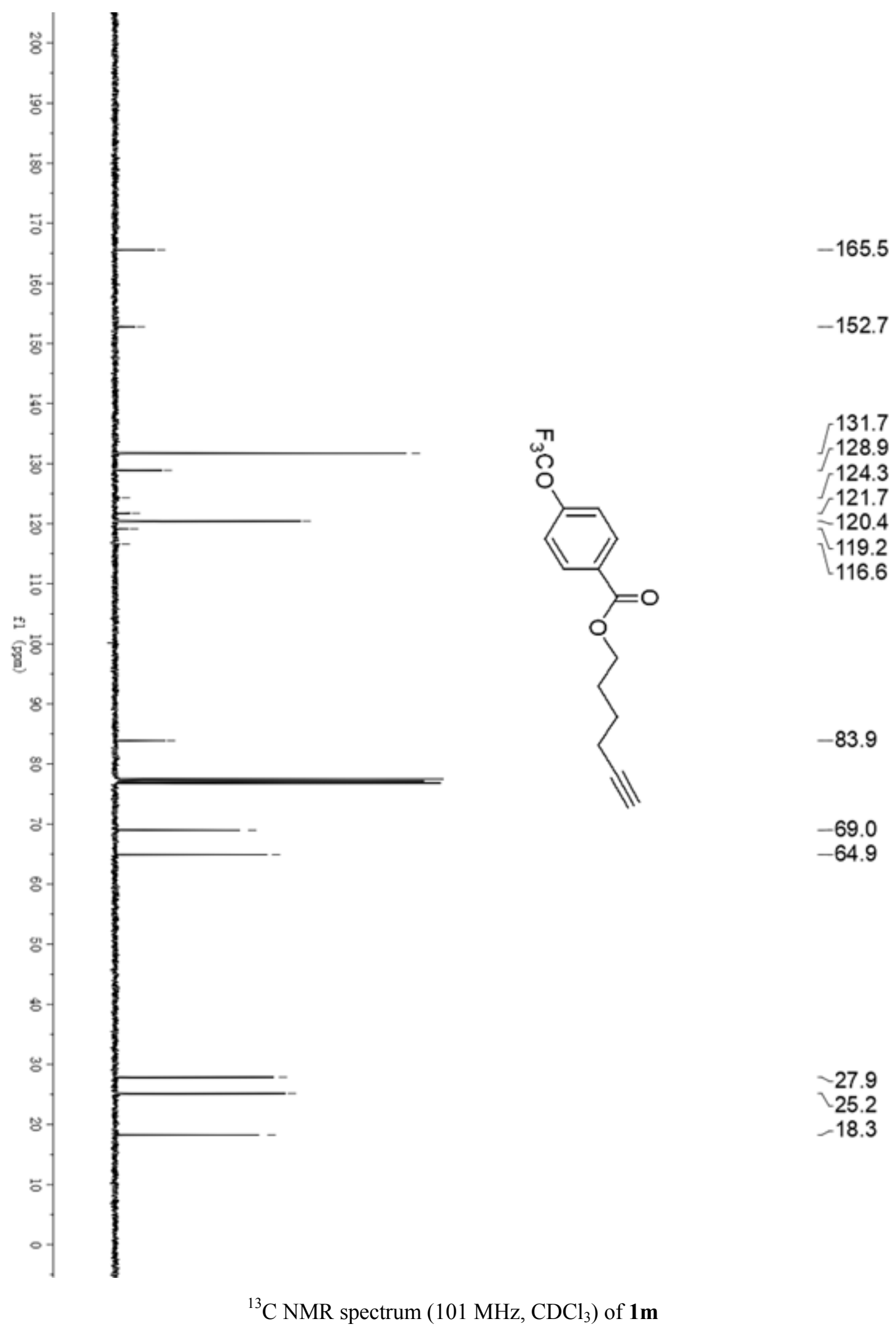




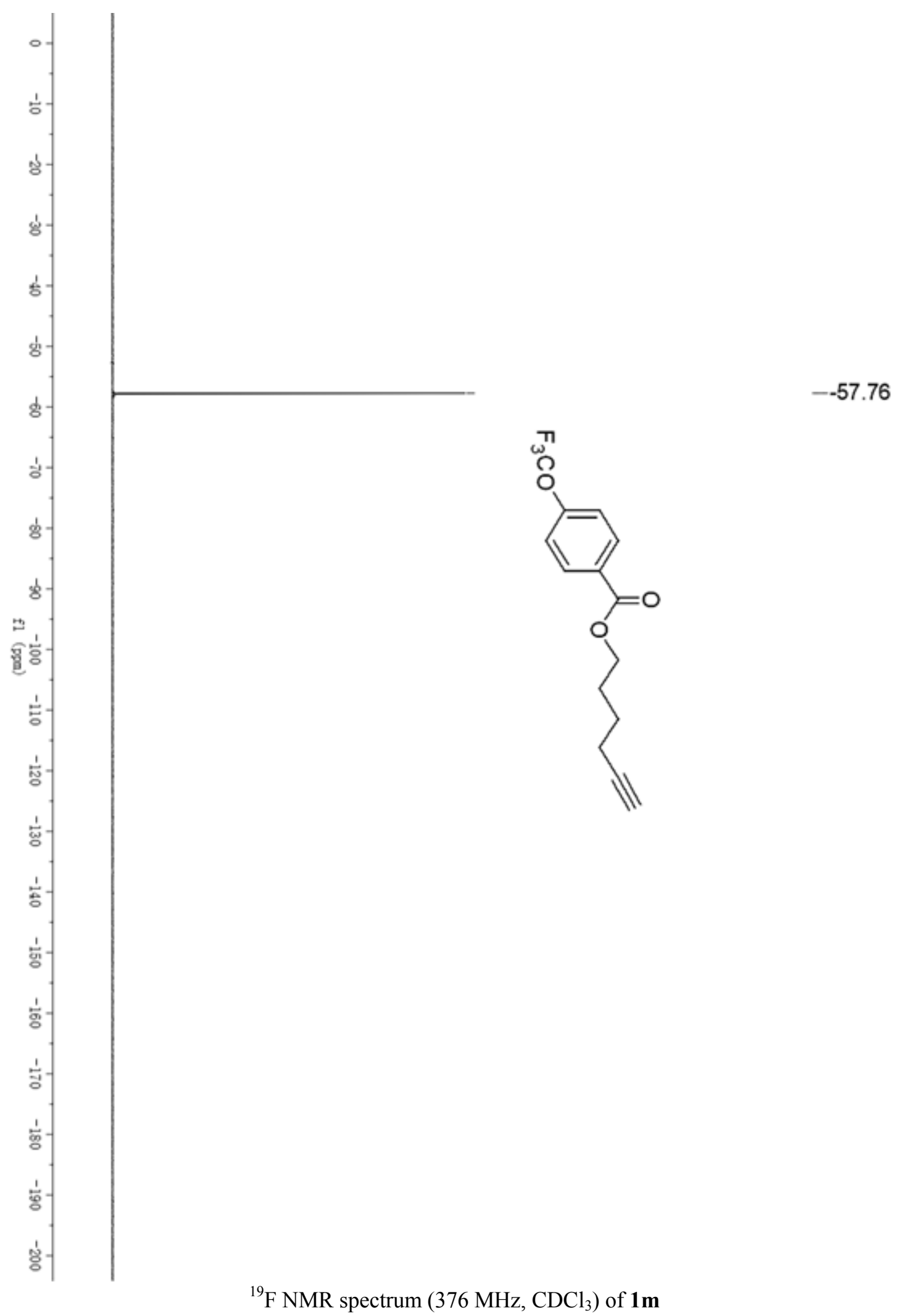




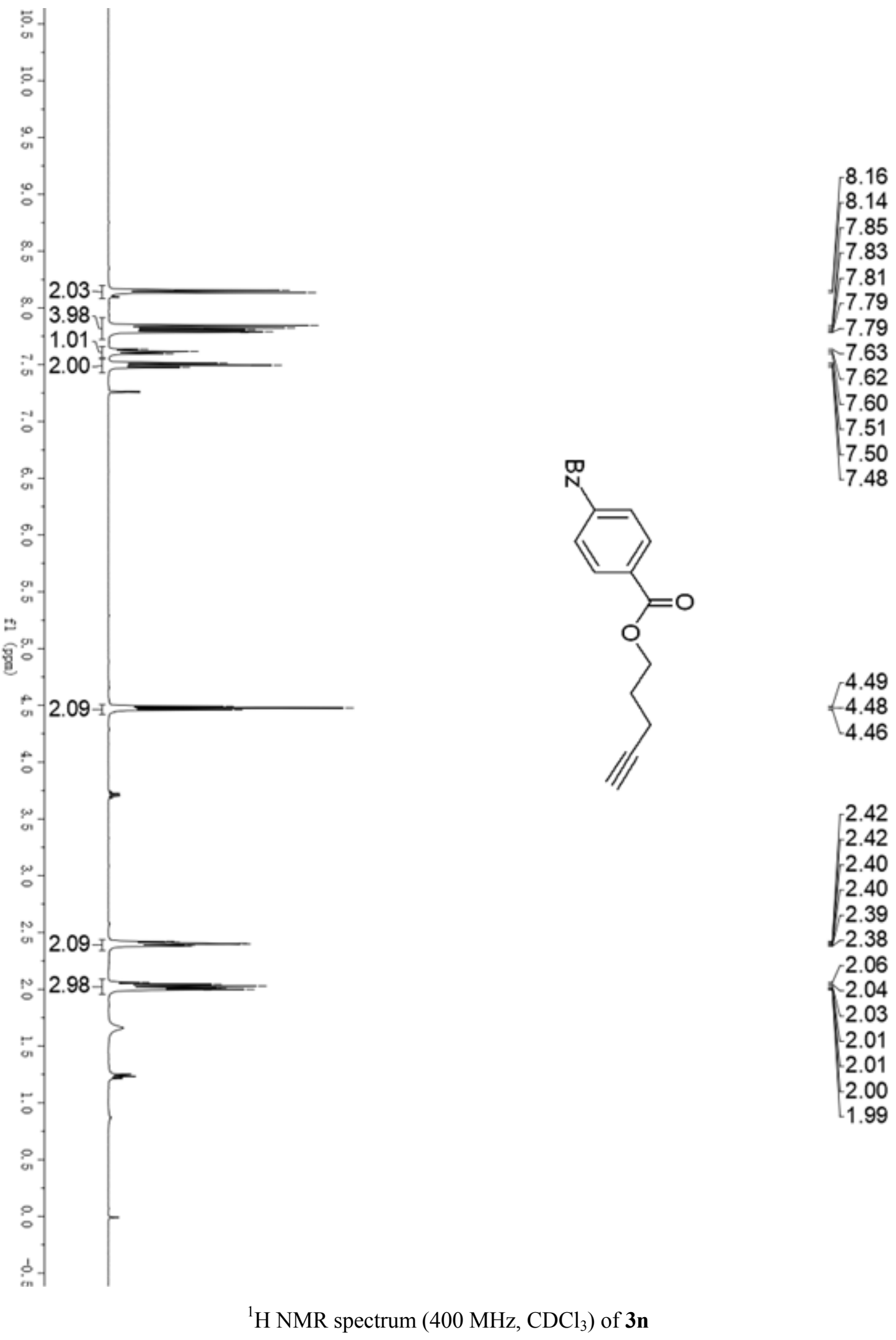




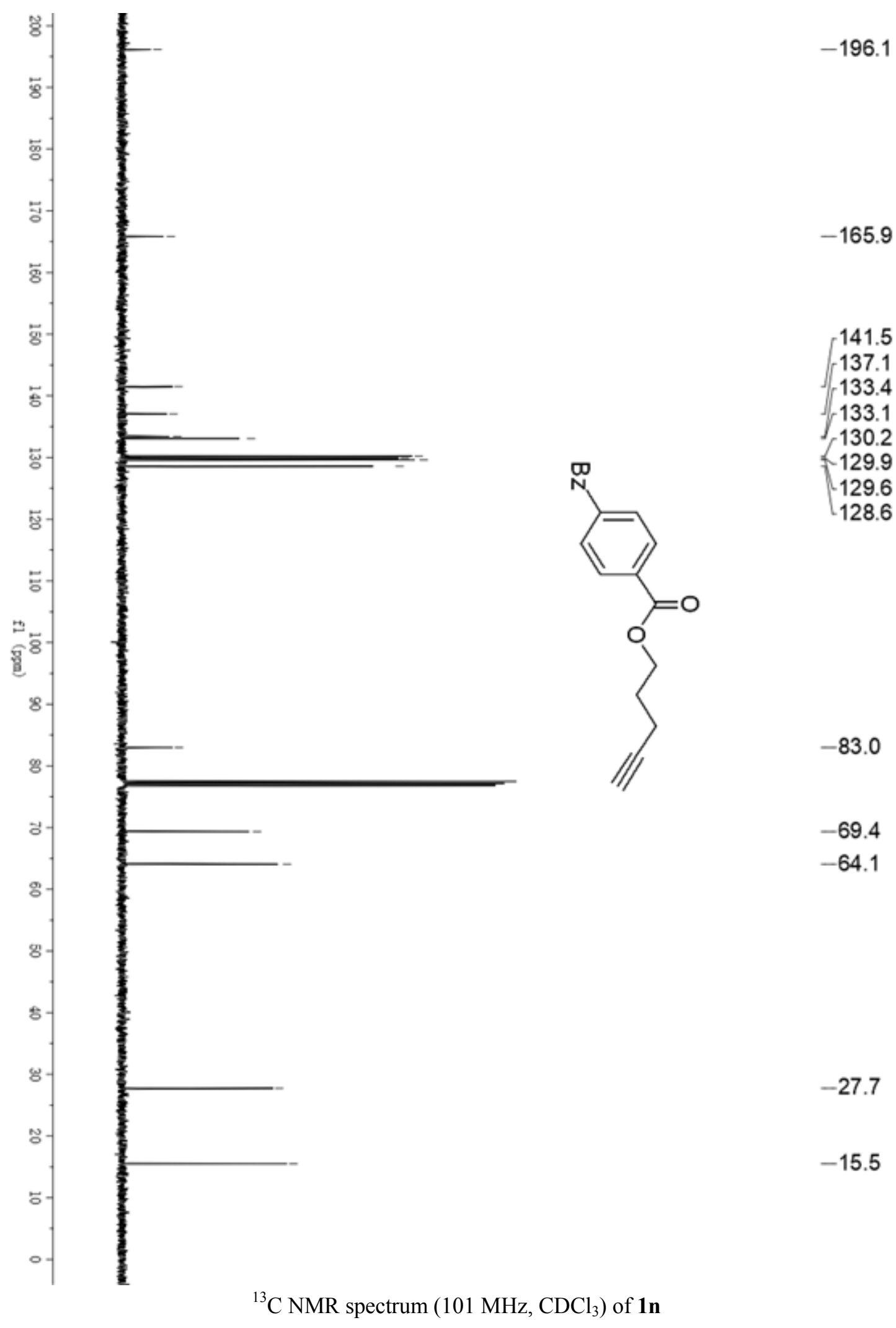




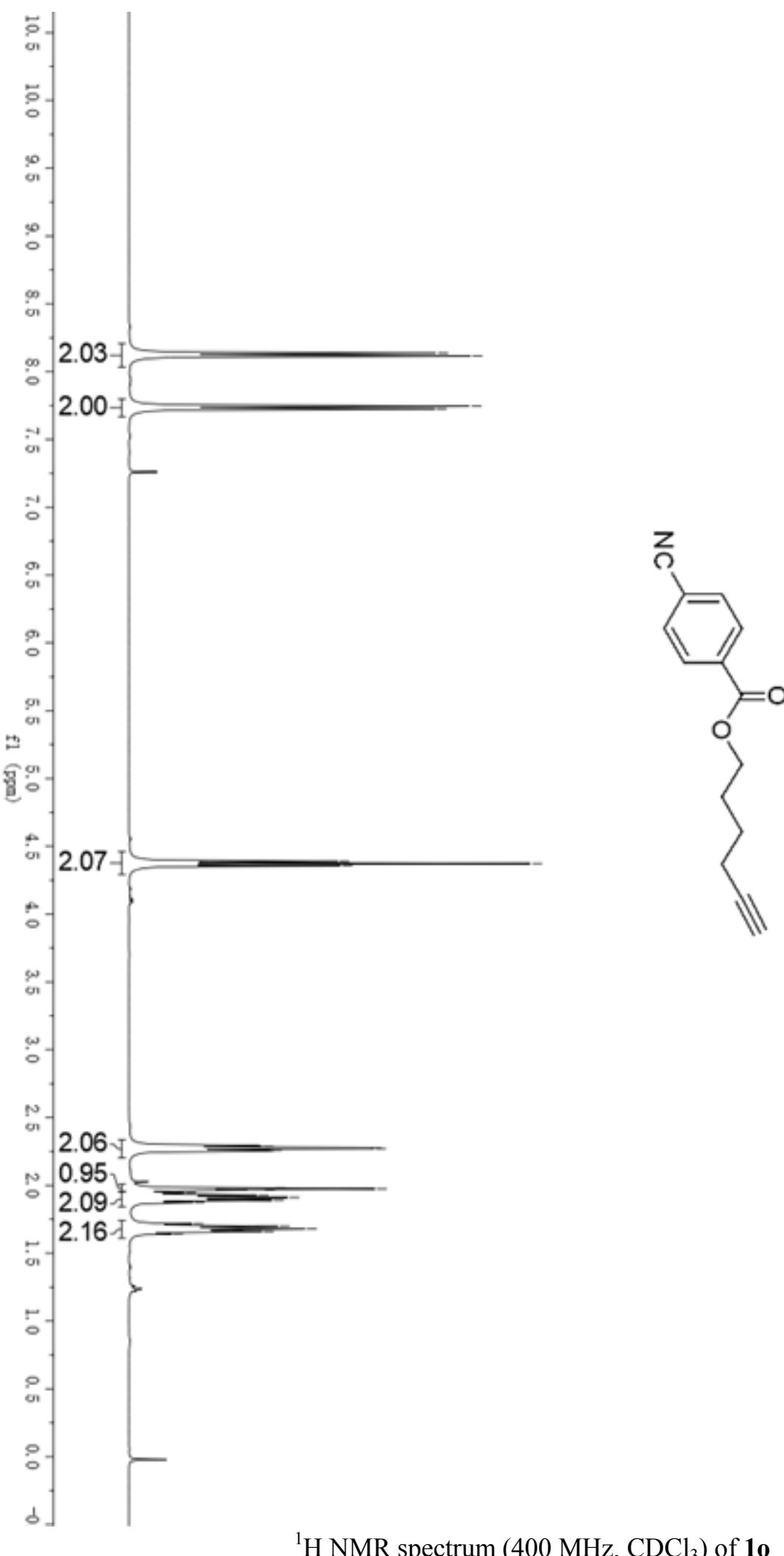

8.14

8.11

7.75

7.72

4.39

$-4.37$

4.36

2.29

2.29

2.28

2.27

$-2.26$

$-2.25$

1.98

1.97

1.97

$-1.94$

1.93

$-1.91$

$-1.89$

$-1.87$

1.72

$-1.70$

1.68

1.67

$-1.66$

1.64

${ }^{1} \mathrm{H}$ NMR spectrum $\left(400 \mathrm{MHz}, \mathrm{CDCl}_{3}\right.$ ) of $\mathbf{1 o}$ 


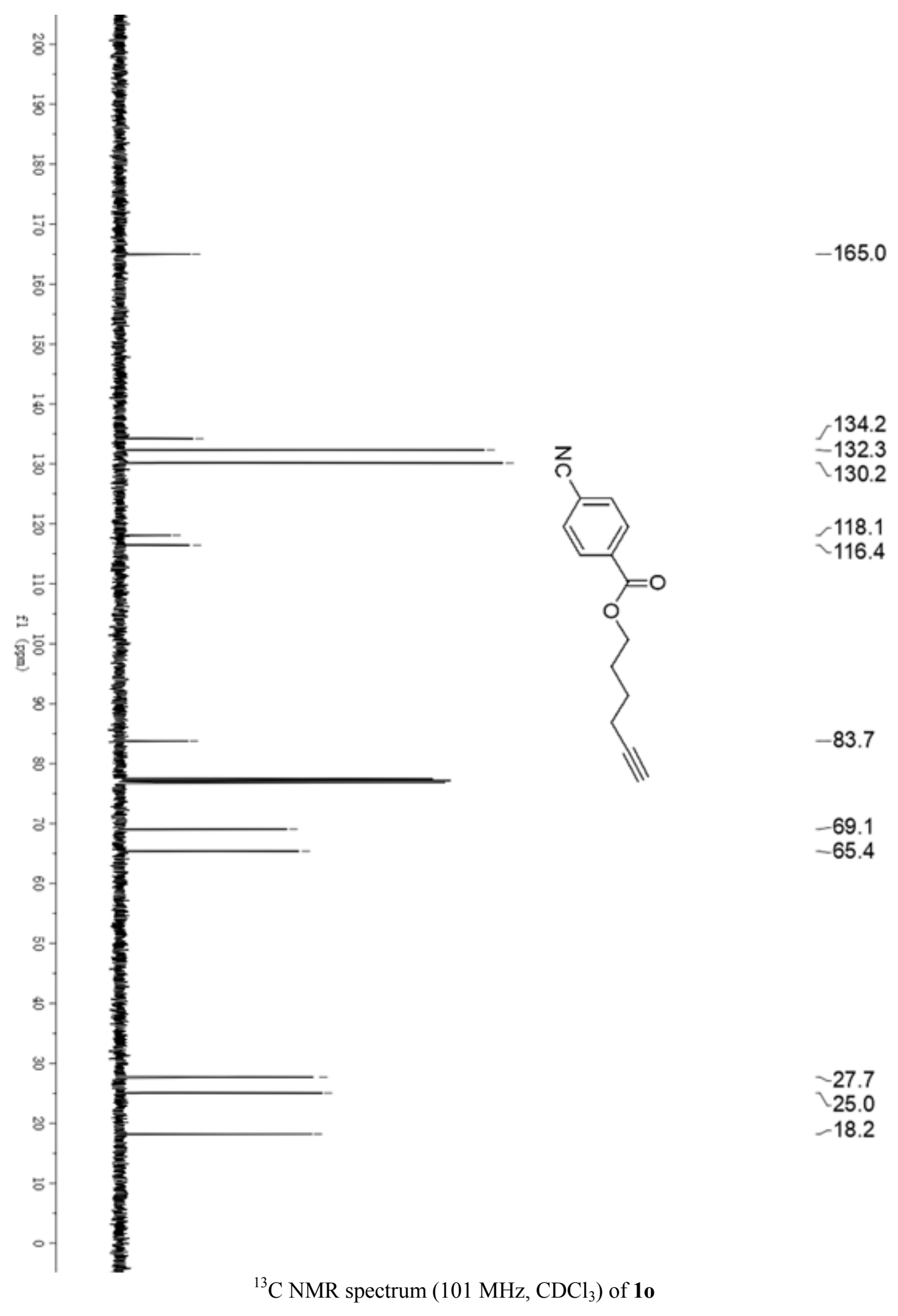




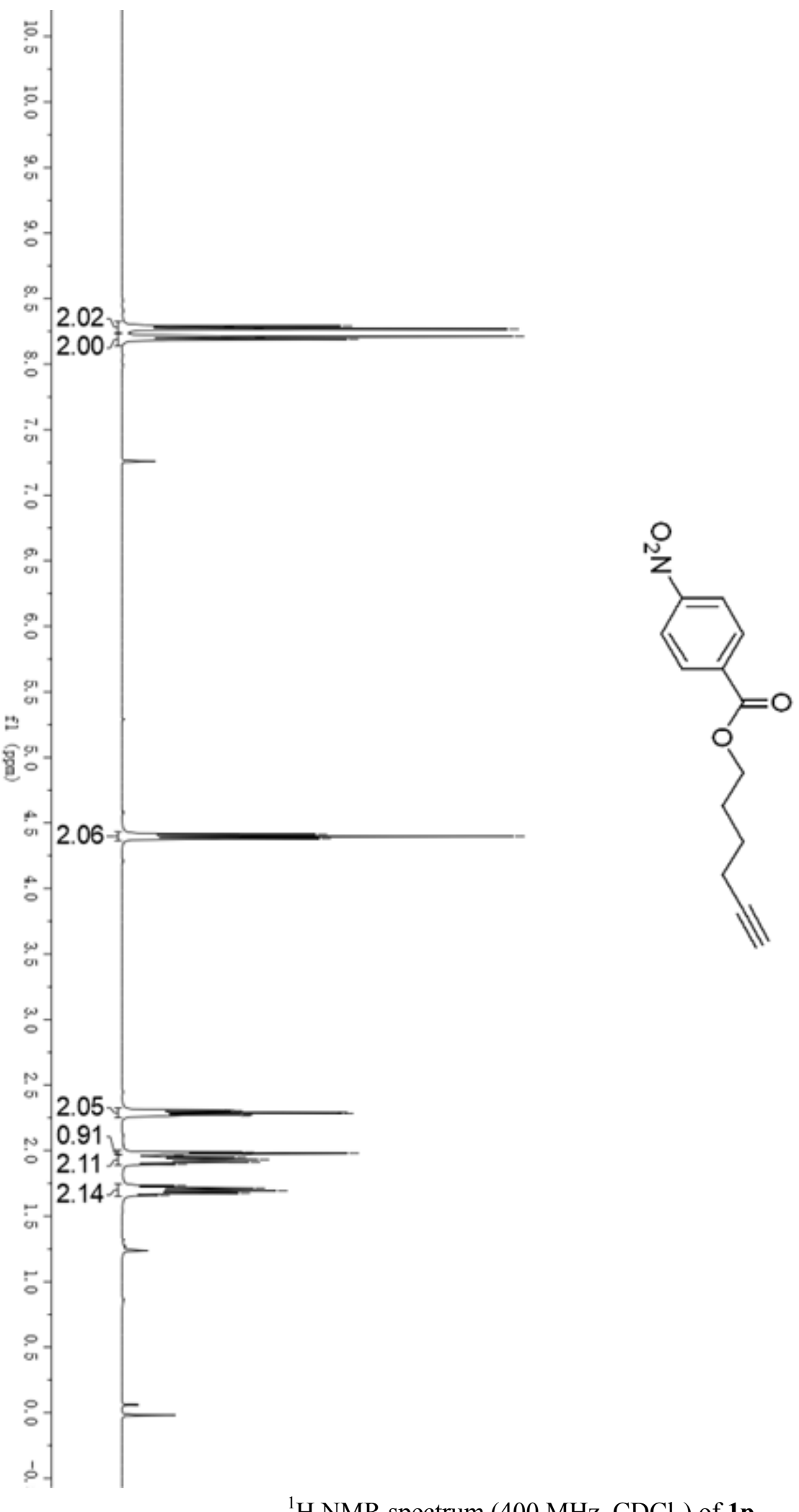

$\left\{\begin{array}{l}8.29 \\ 8.29 \\ 8.27 \\ 8.27 \\ 8.21 \\ 8.21 \\ 8.19 \\ 8.19\end{array}\right.$

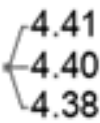

4.38

2.31

$-2.30$

2.29

$-2.28$

2.27

$-2.27$

1.99

1.98

1.97

$-1.97$

$-1.95$

1.93

$-1.91$

1.90

1.73

1.71

1.69

$-1.68$

$-1.68$

${ }^{1} \mathrm{H}$ NMR spectrum $\left(400 \mathrm{MHz}, \mathrm{CDCl}_{3}\right)$ of $\mathbf{1 p}$ 


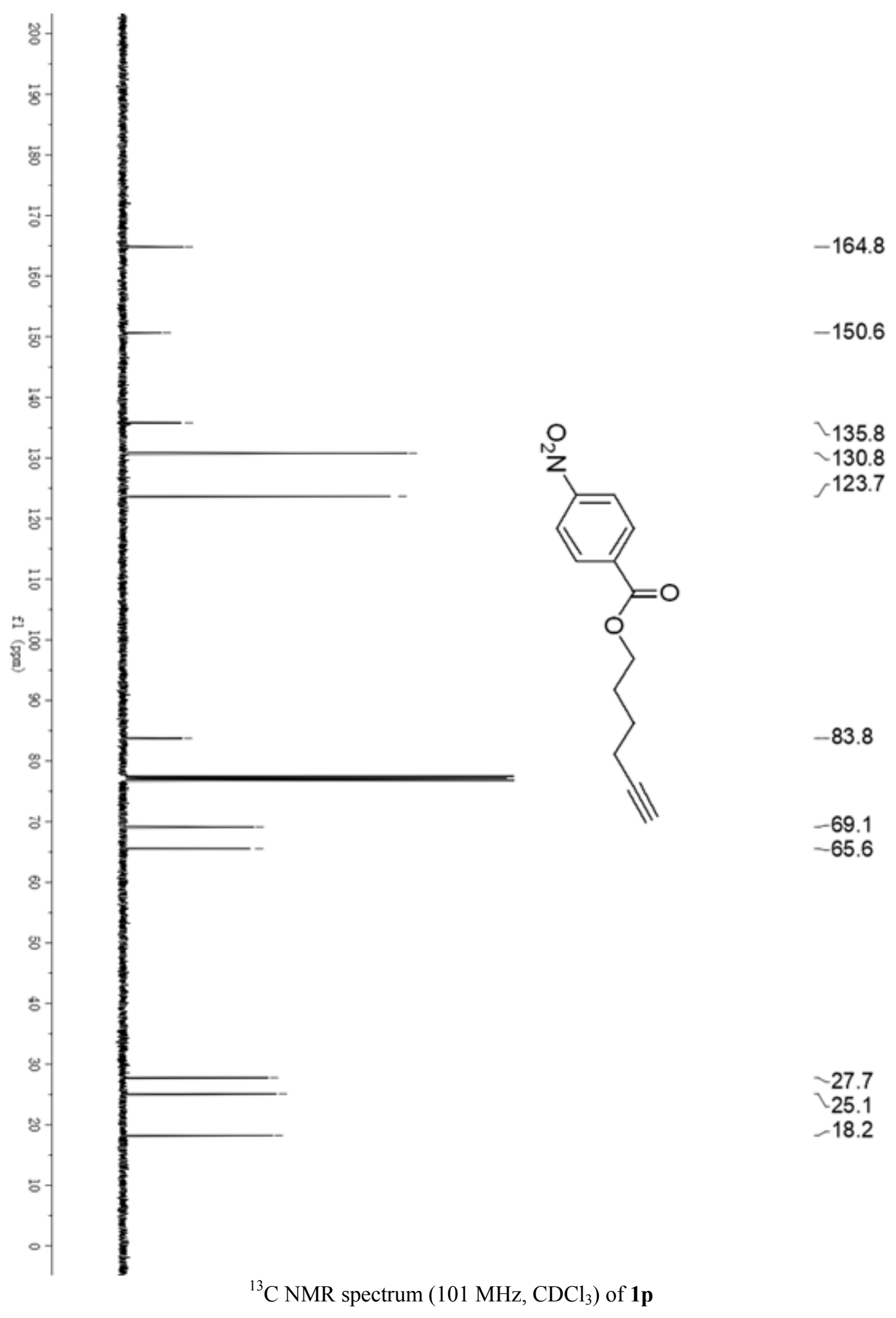



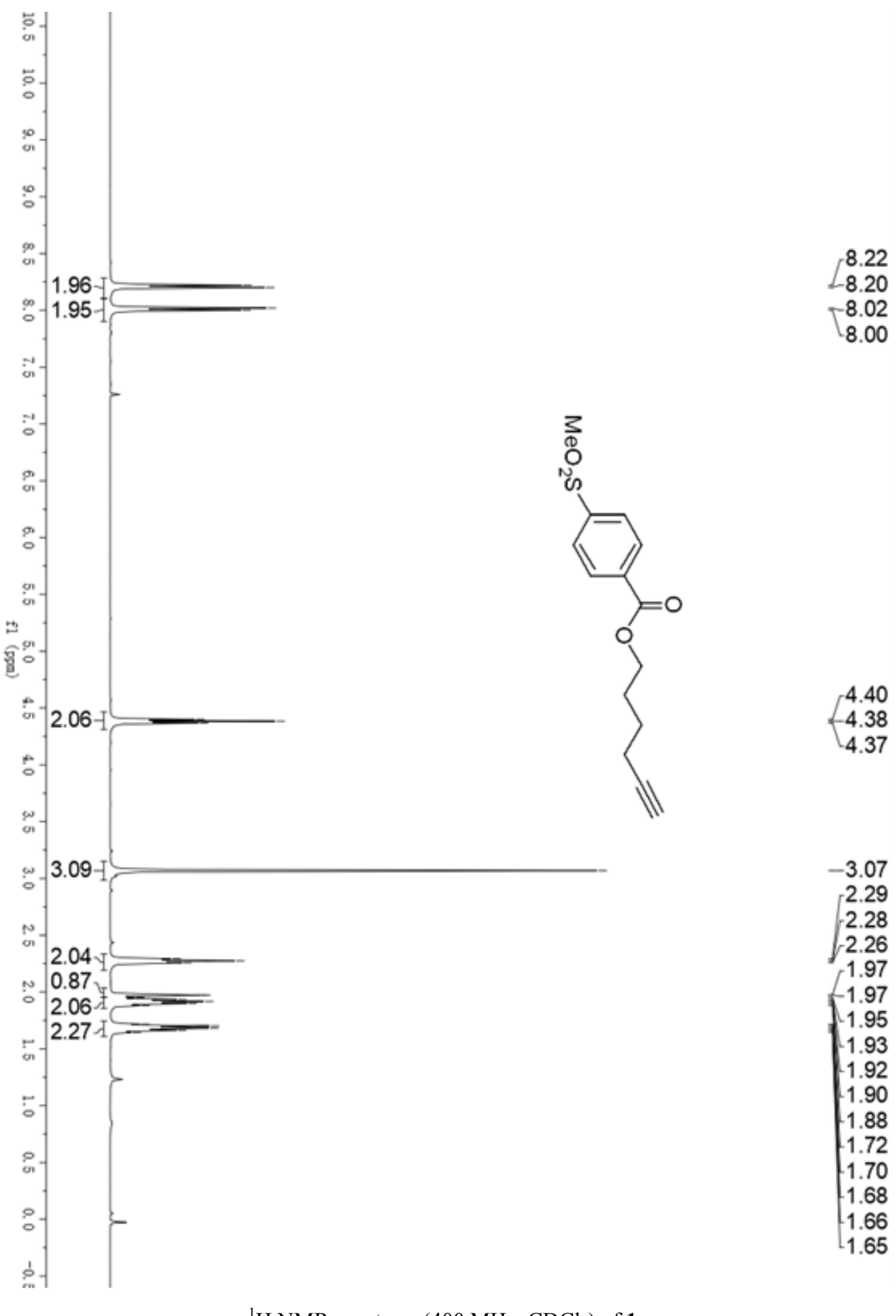

${ }^{1} \mathrm{H}$ NMR spectrum $\left(400 \mathrm{MHz}, \mathrm{CDCl}_{3}\right)$ of $\mathbf{1 q}$ 


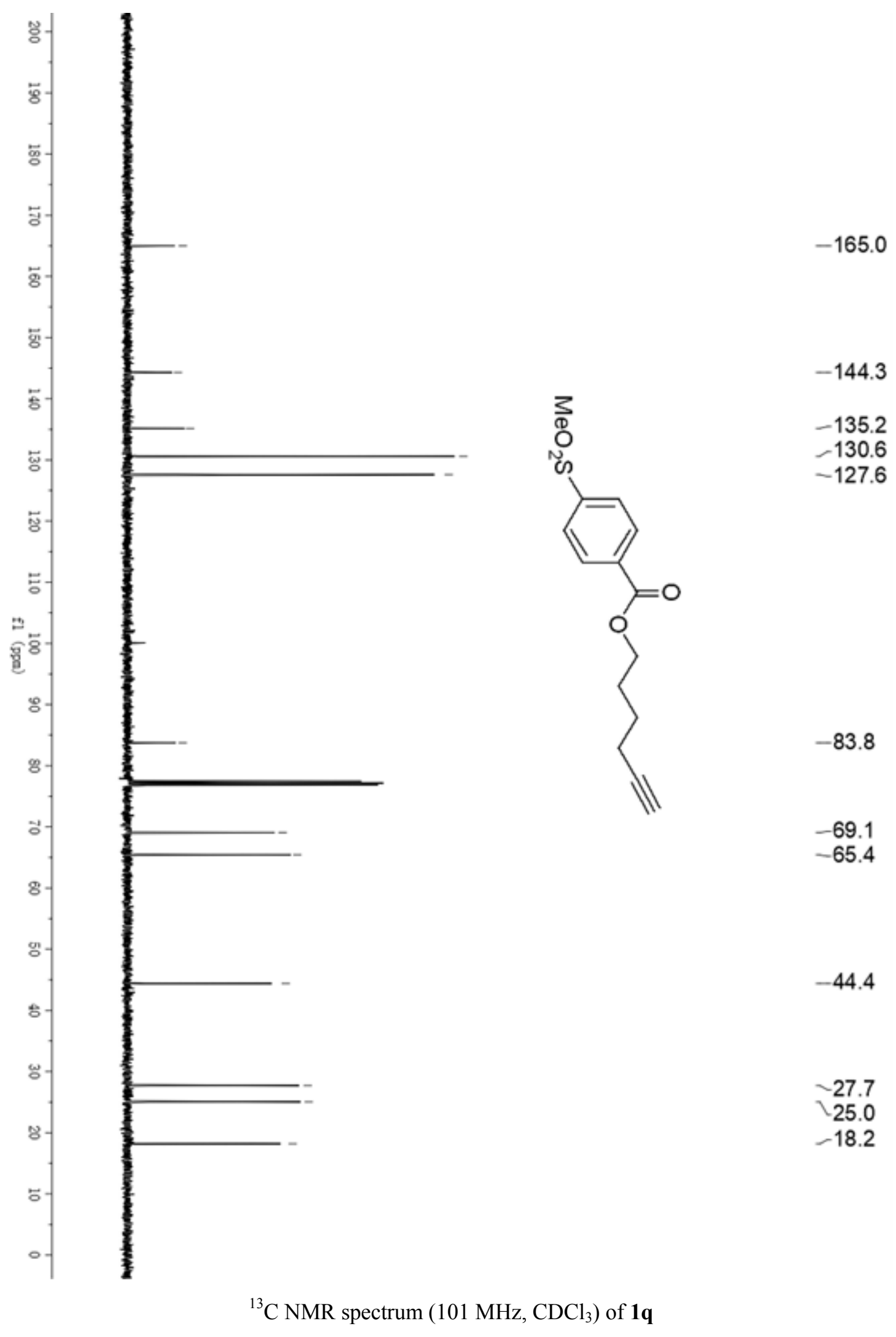




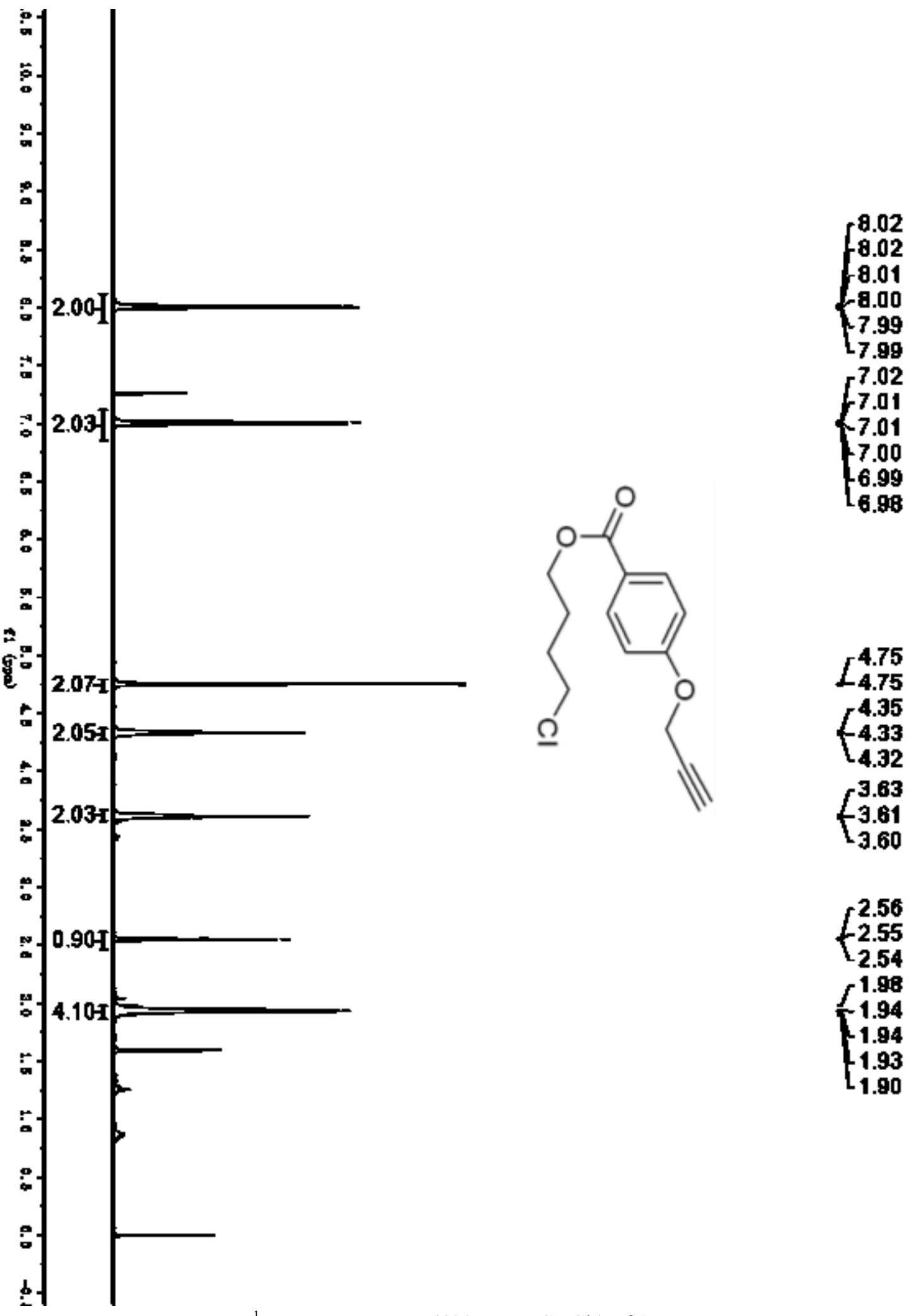

${ }^{1} \mathrm{H}$ NMR spectrum $\left(400 \mathrm{MHz}, \mathrm{CDCl}_{3}\right)$ of $\mathbf{1 t}$ 


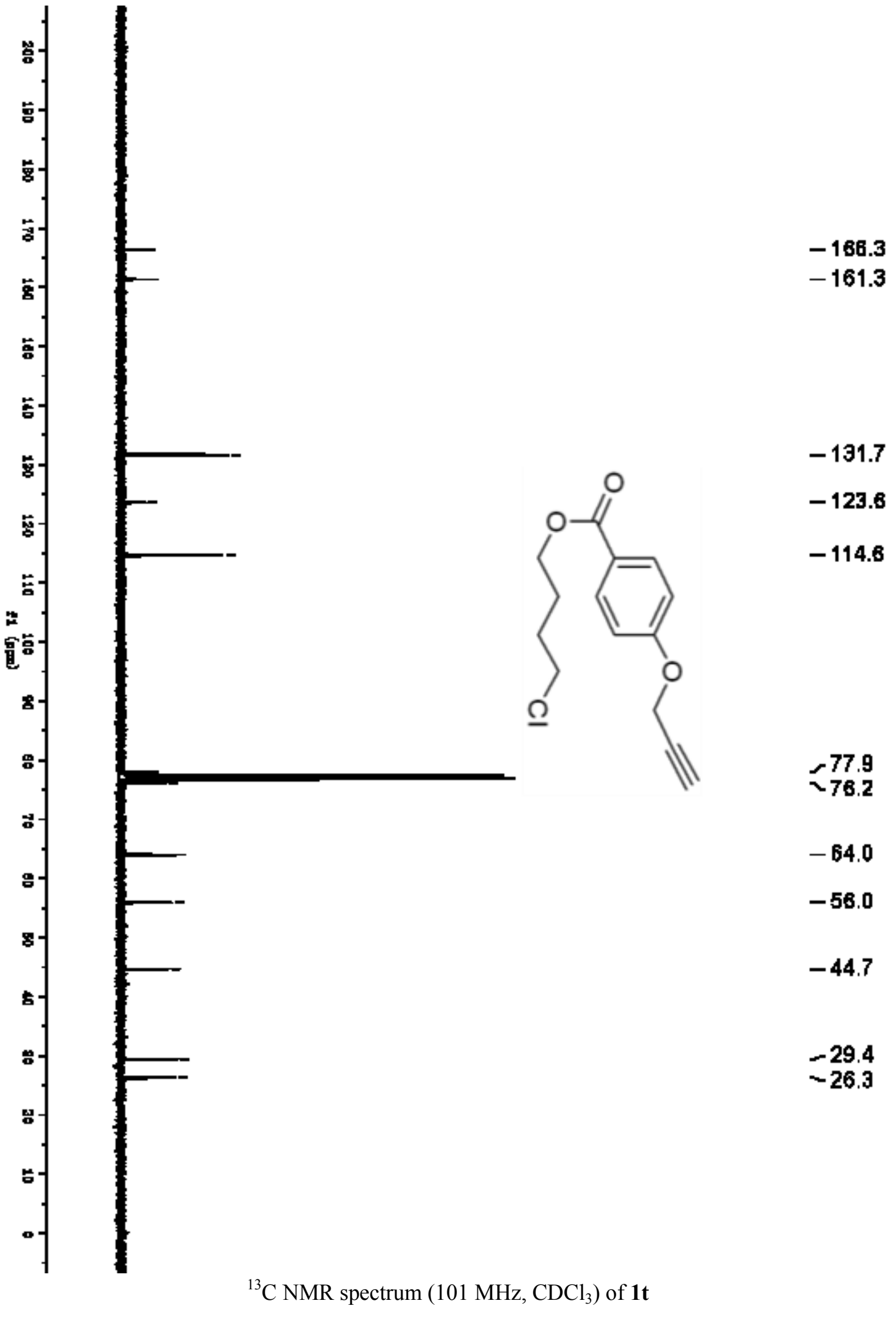




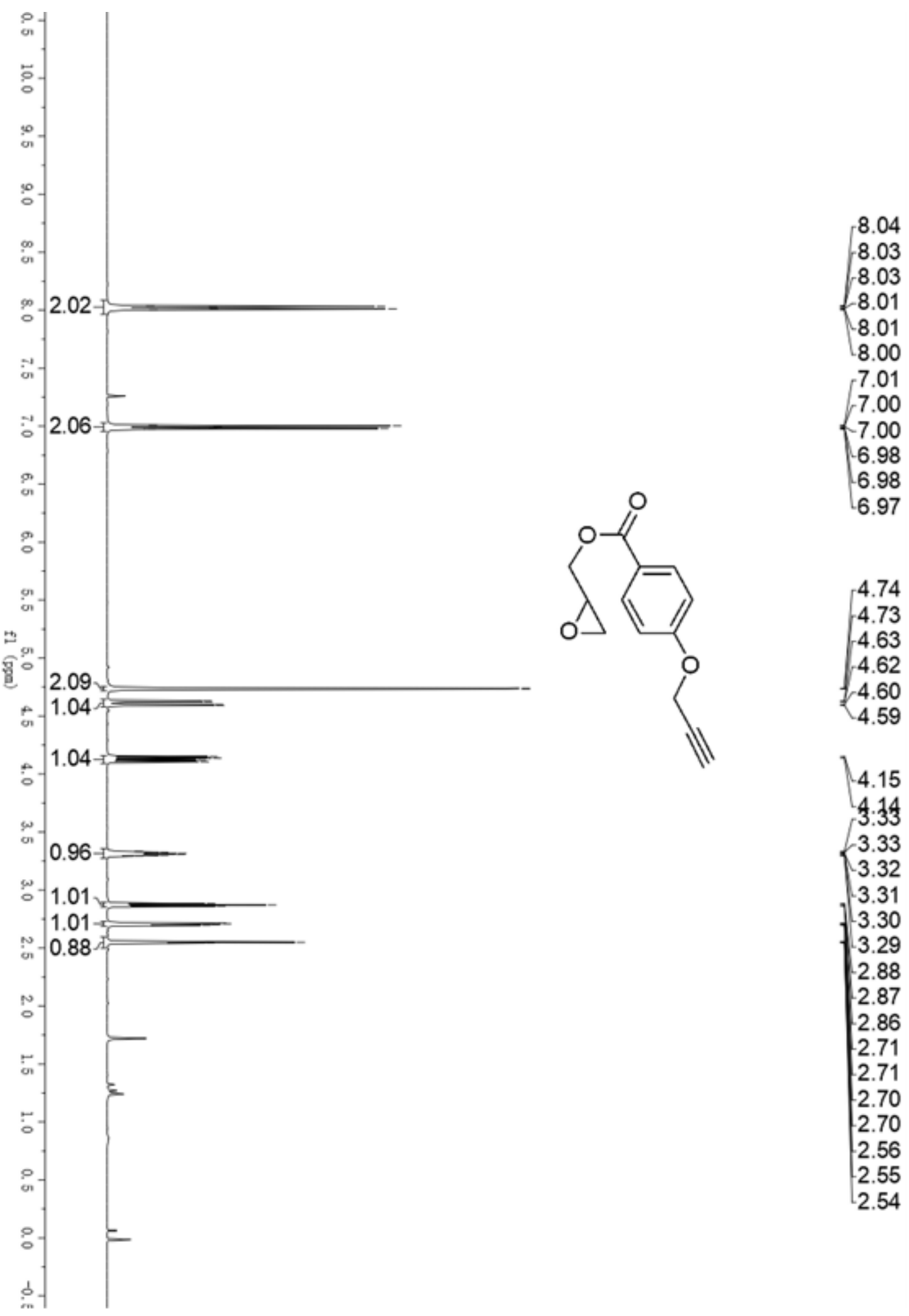

${ }^{1} \mathrm{H}$ NMR spectrum $\left(400 \mathrm{MHz}, \mathrm{CDCl}_{3}\right.$ ) of $\mathbf{1 u}$ 


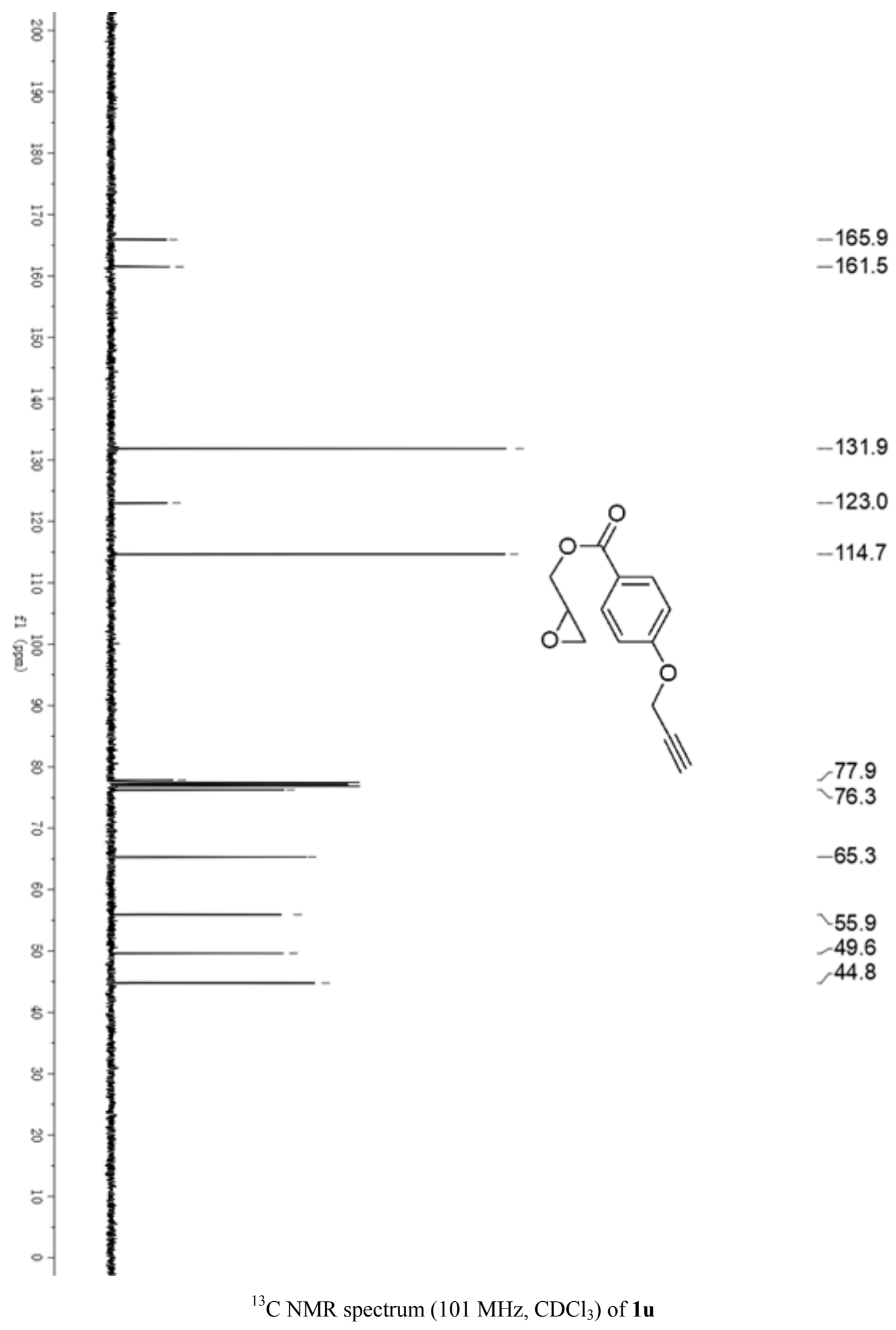




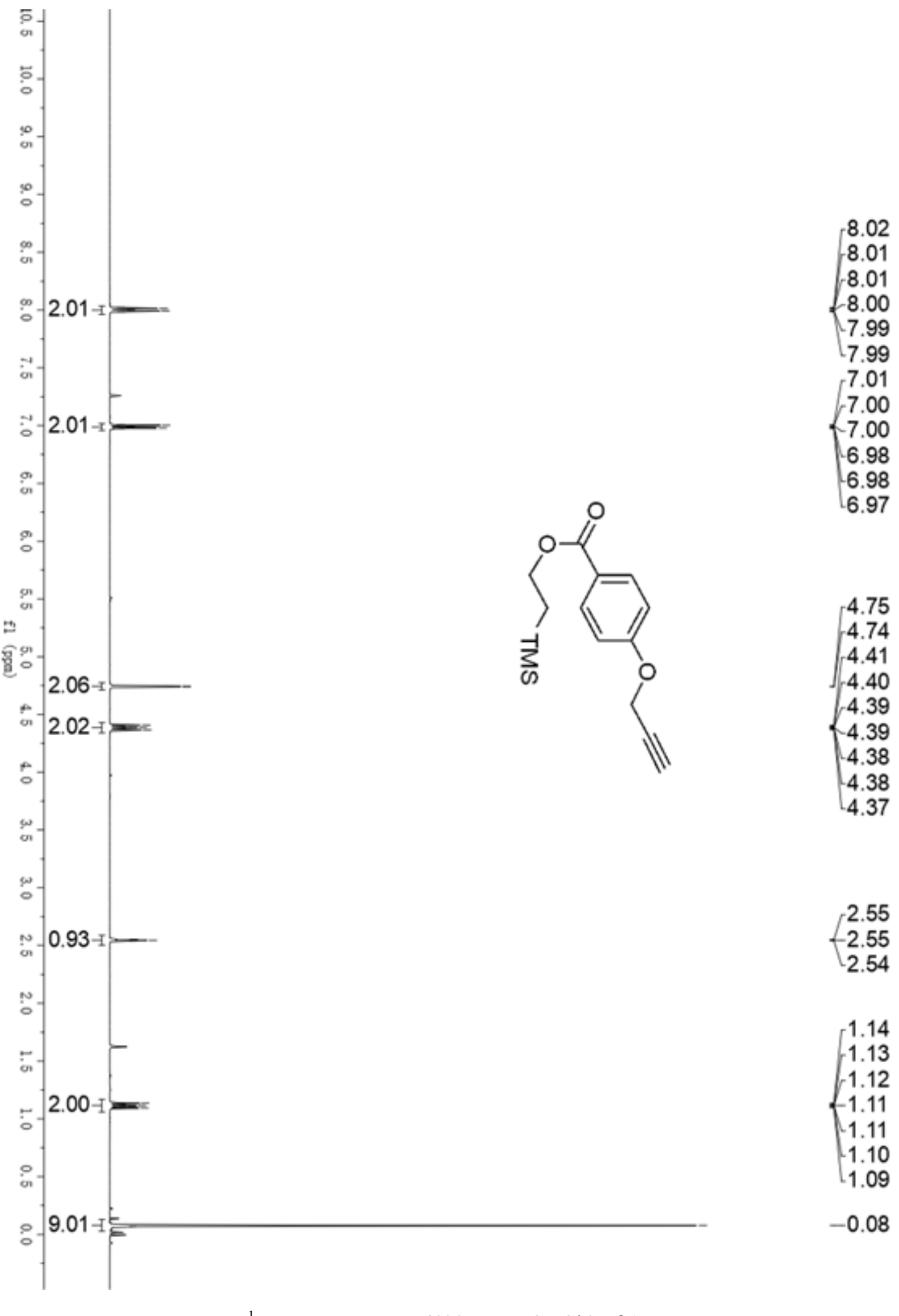

${ }^{1} \mathrm{H}$ NMR spectrum $\left(400 \mathrm{MHz}, \mathrm{CDCl}_{3}\right)$ of $\mathbf{1 v}$ 


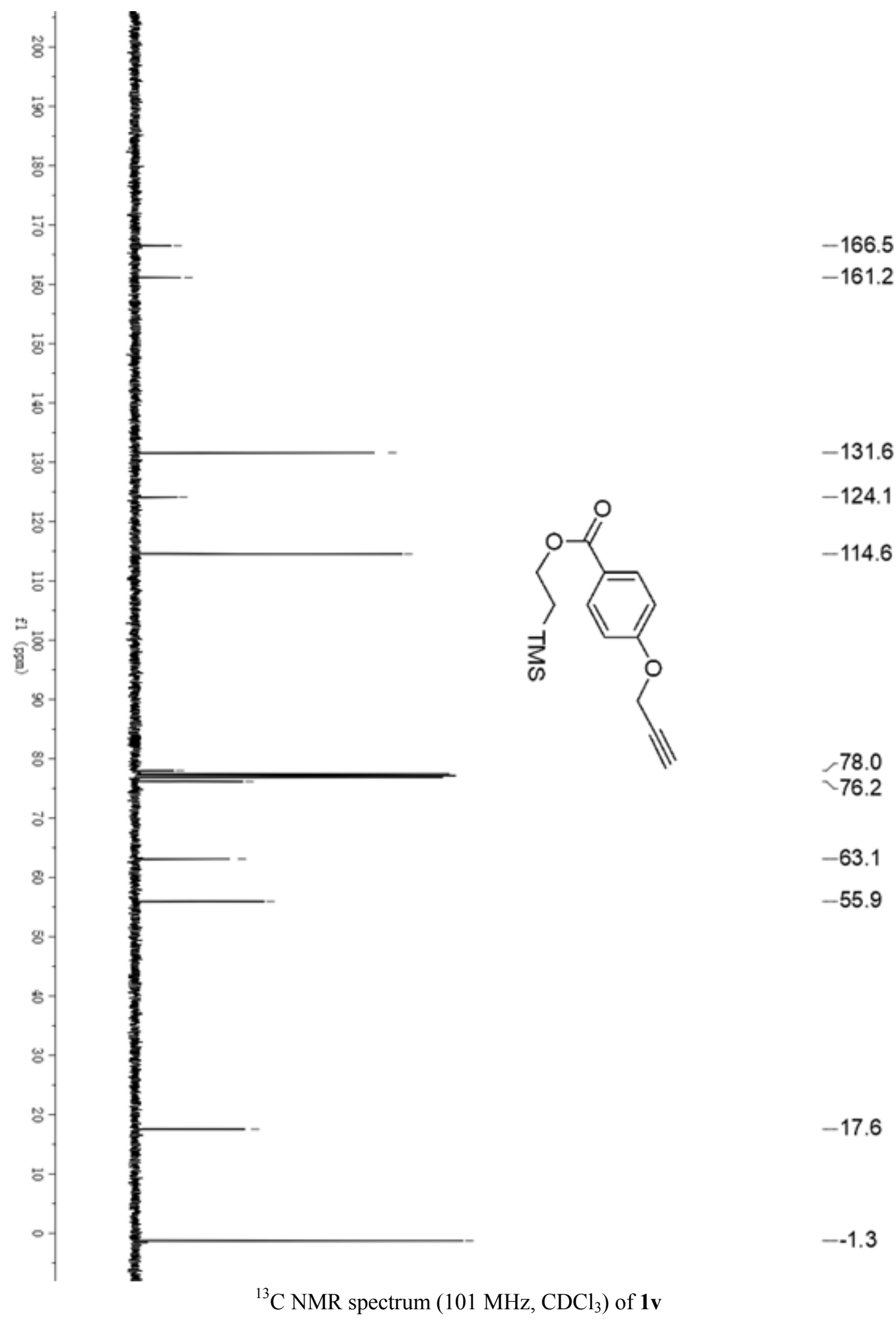




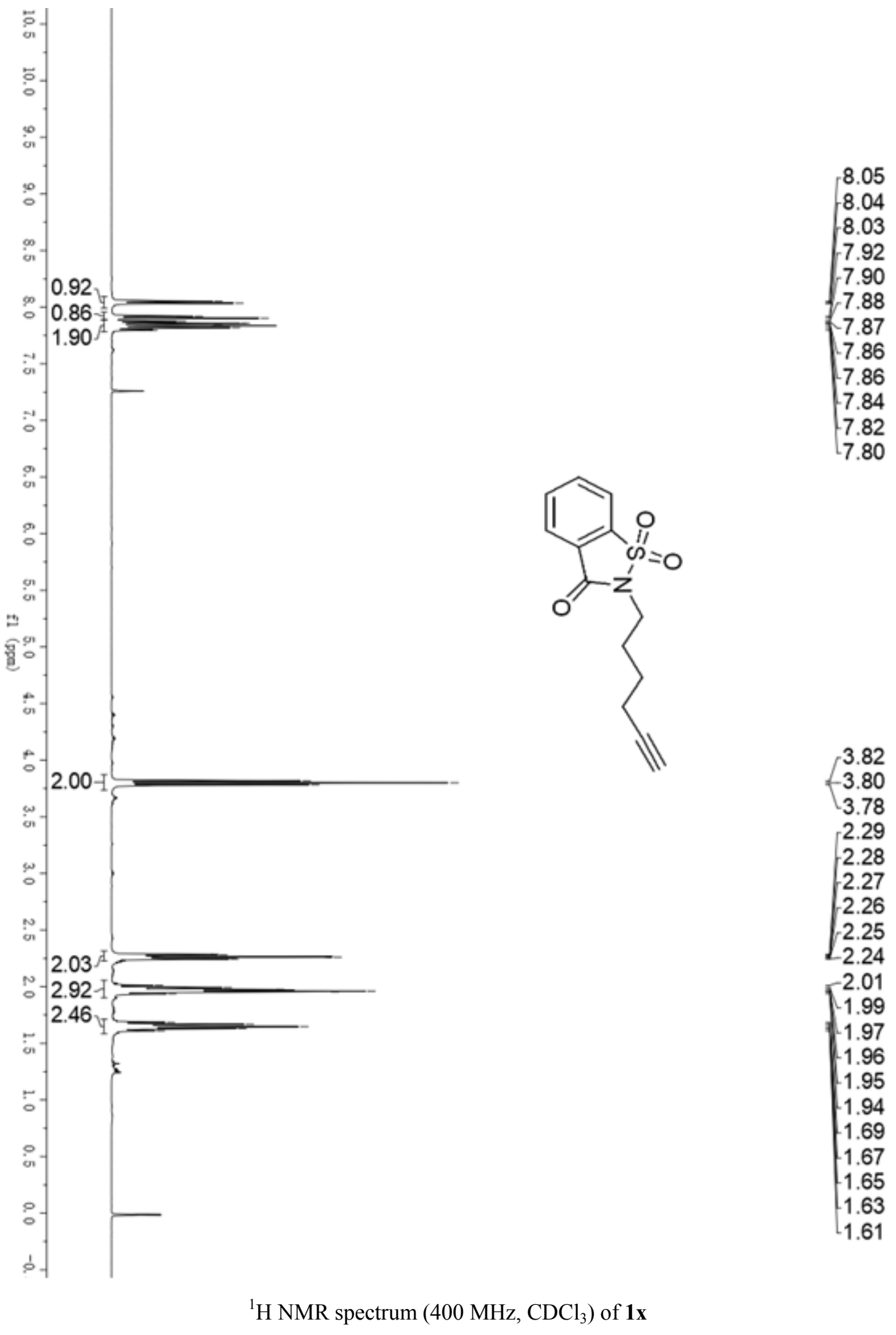




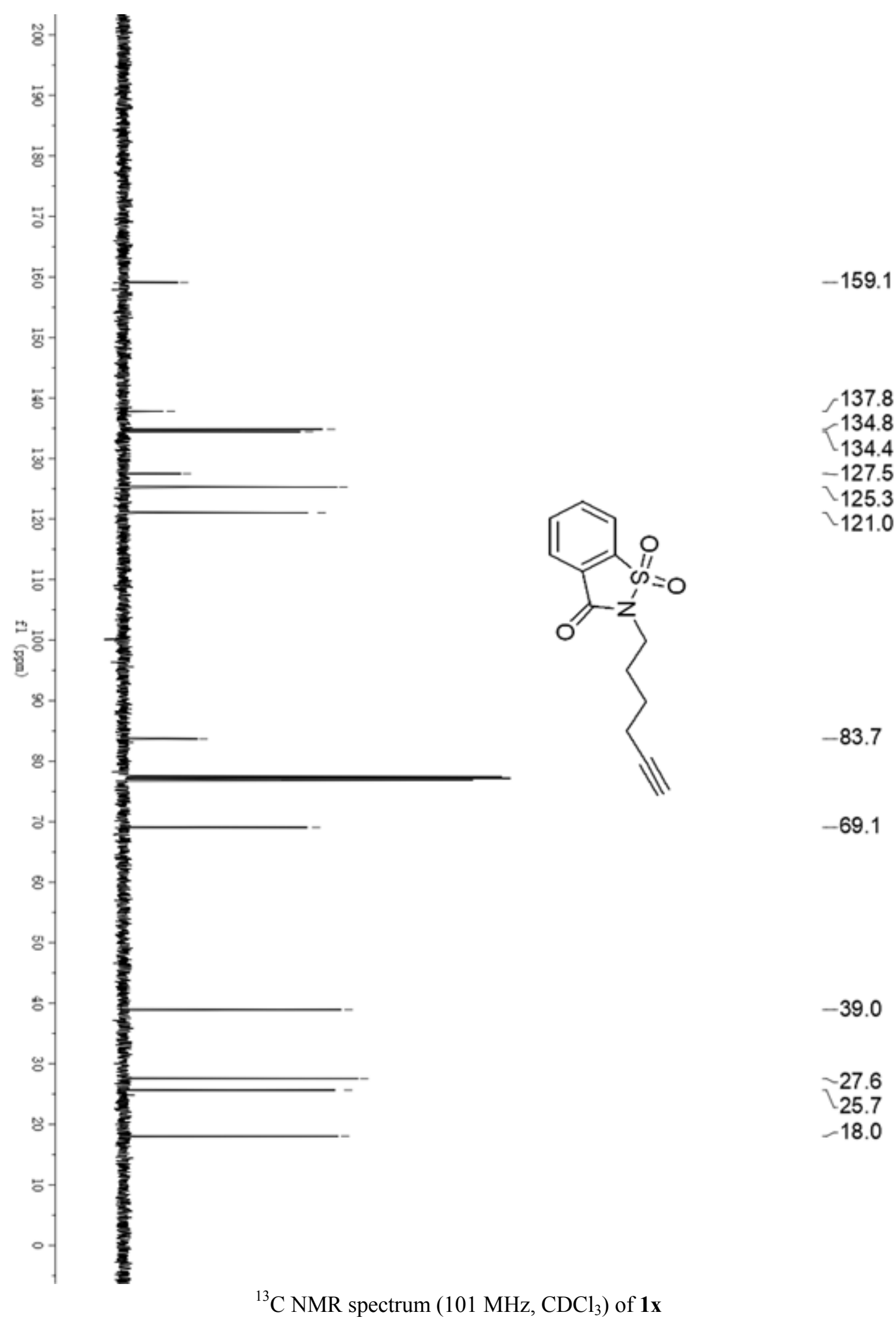




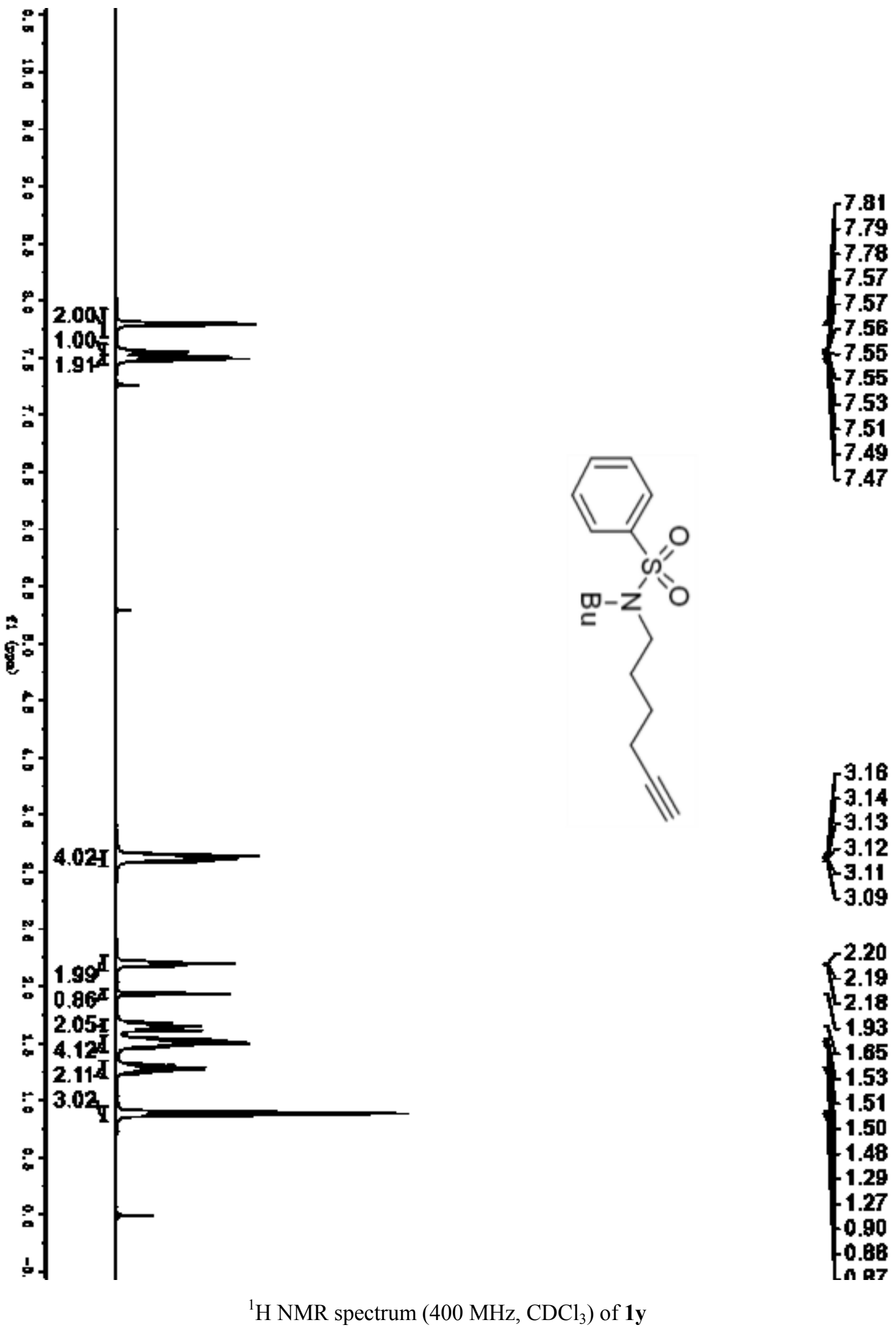




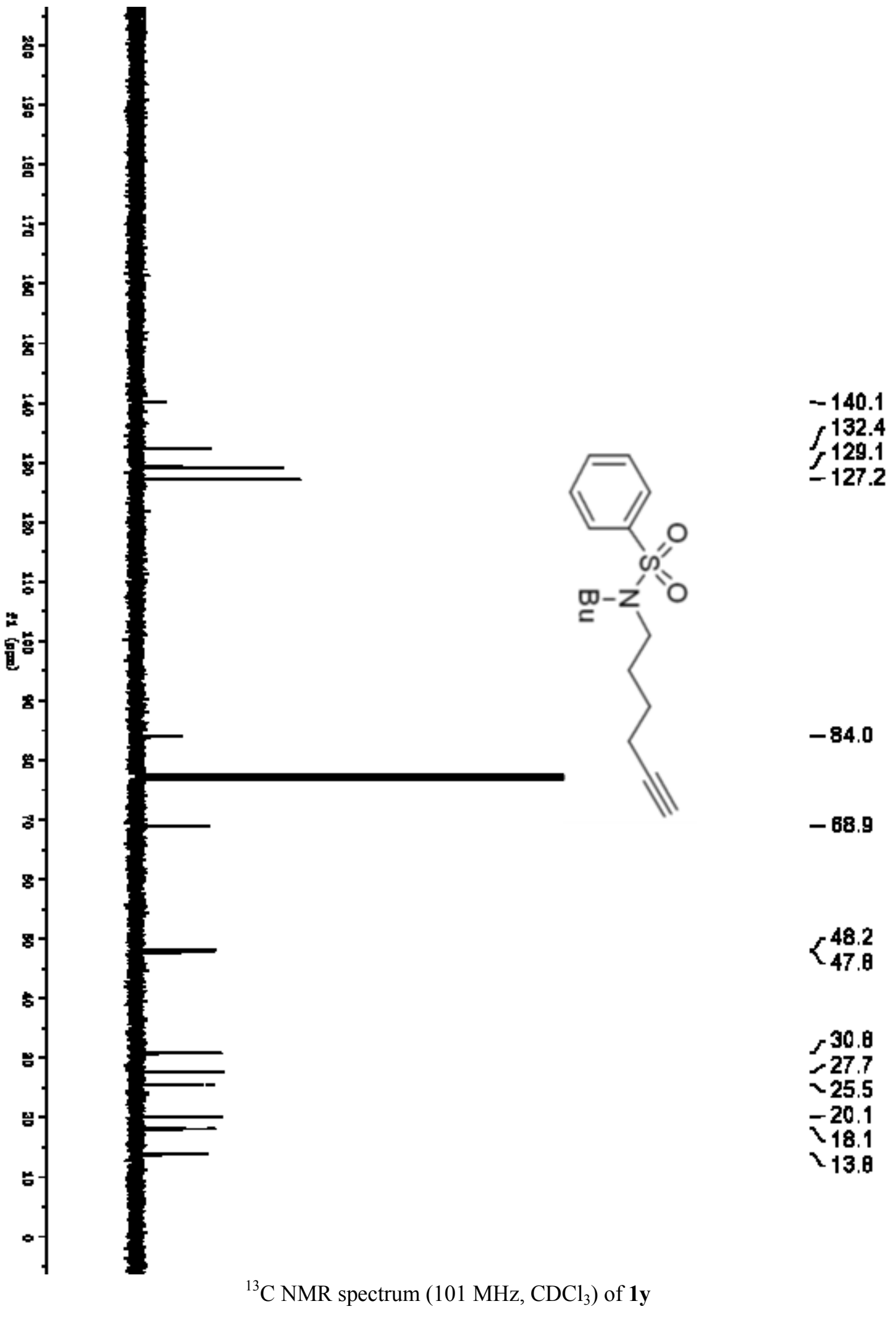




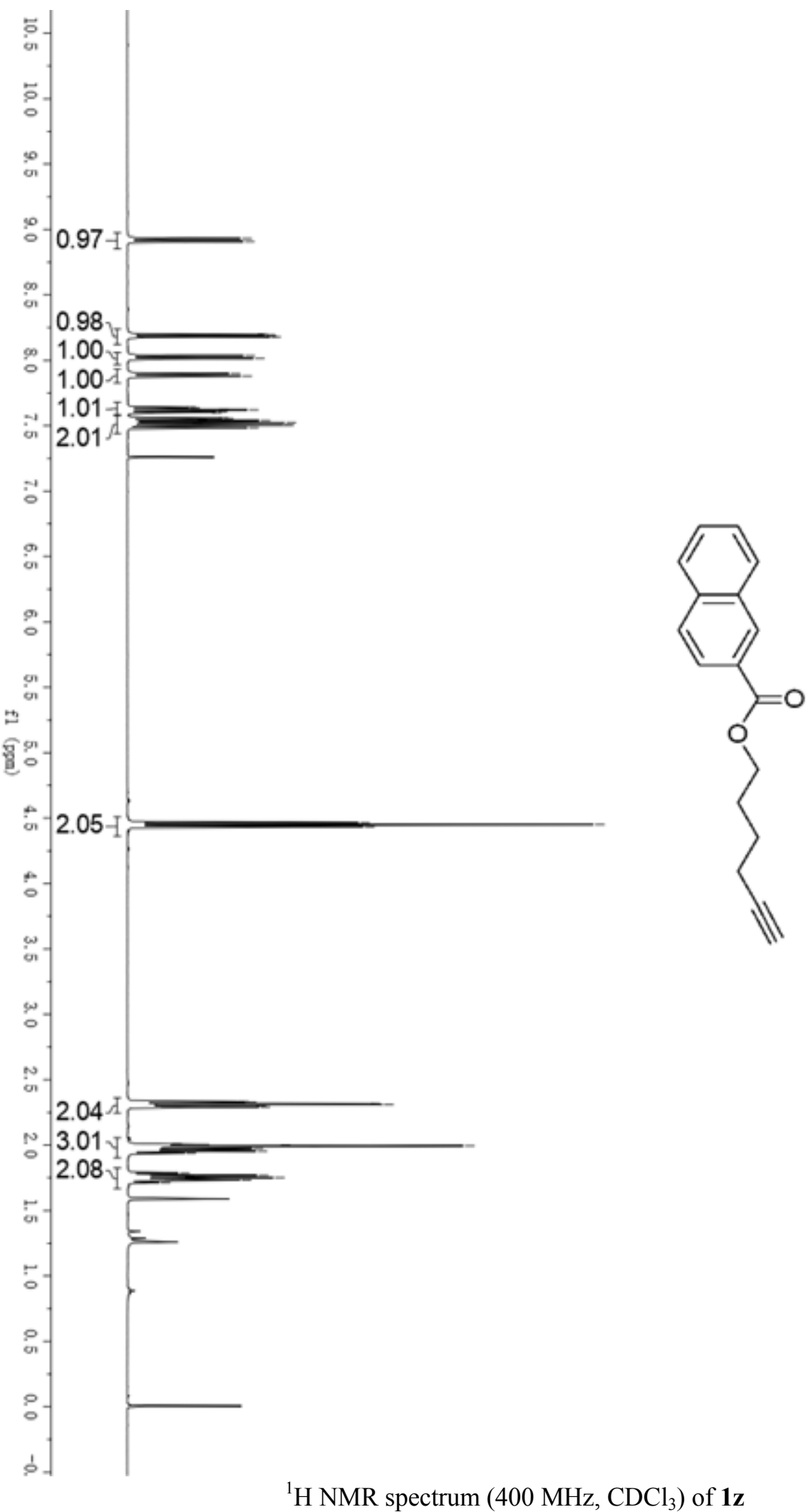

8.93

8.91

8.20

8.20

8.18

8.18

8.03

8.01

7.90

7.88

7.64

7.64

7.62

7.62

7.62

7.60

7.60

7.56

7.56

7.54

7.54

7.54

7.52

7.52

7.51

7.50

7.48

4.47

4.45

4.43

2.33

$-2.33$

2.32

2.31

2.30

$-2.29$

2.01

2.00

$-2.00$

$-1.99$

1.98

1.97

1.95

1.94

1.79

1.77

$-1.76$

$-1.75$

$-1.74$

${ }^{1} \mathrm{H}$ NMR spectrum $\left(400 \mathrm{MHz}, \mathrm{CDCl}_{3}\right.$ ) of $\mathbf{1 z}$

1.73 


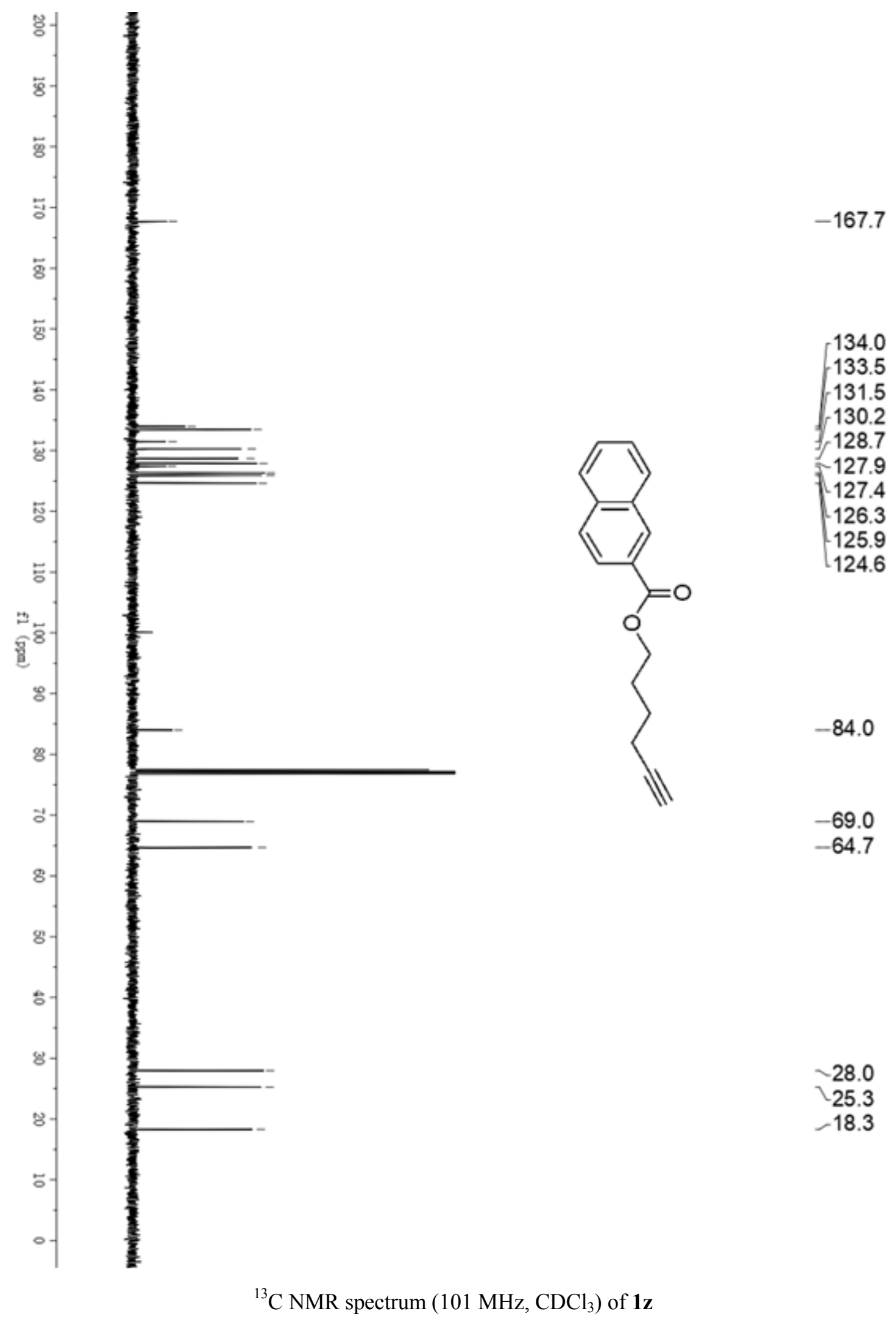




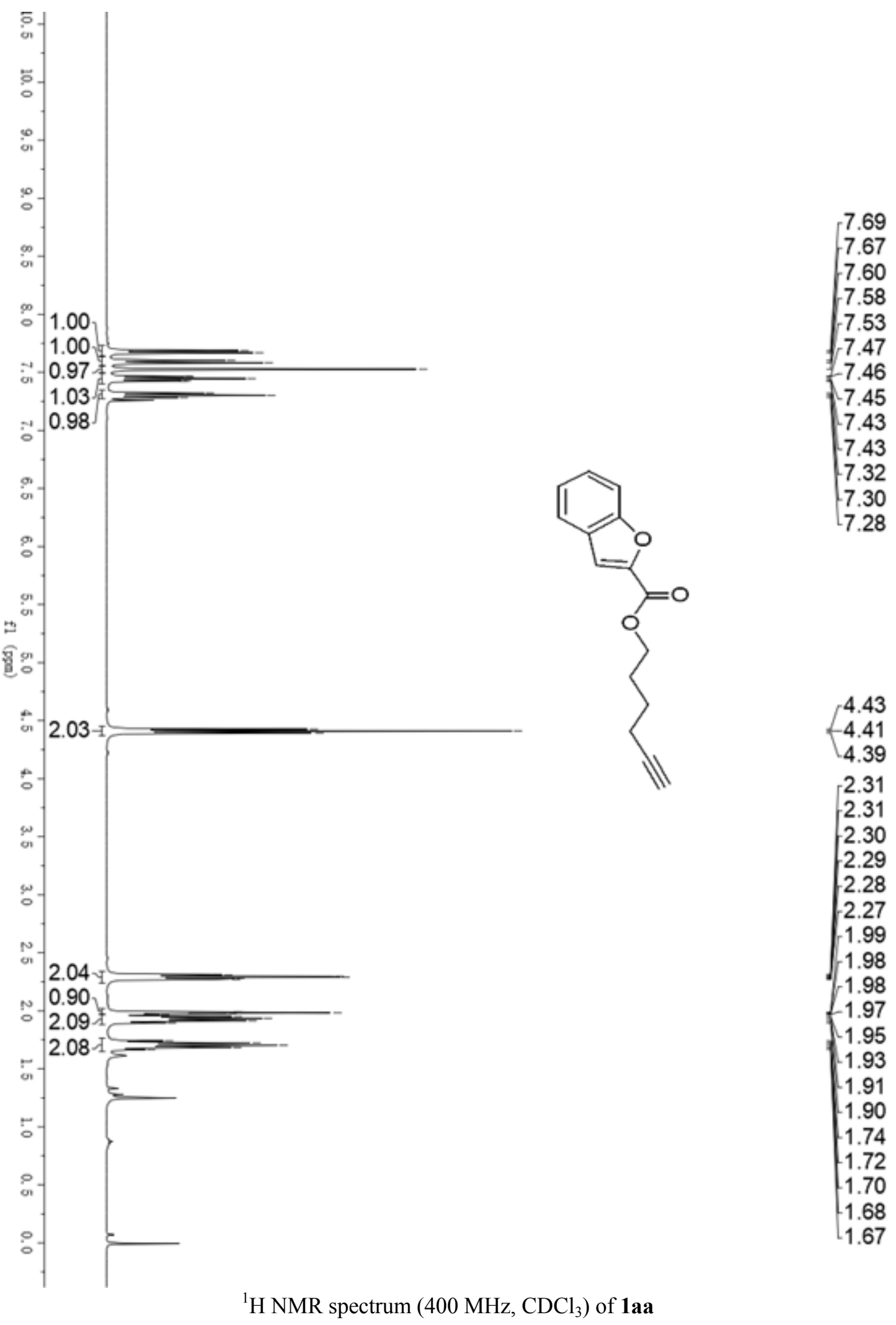




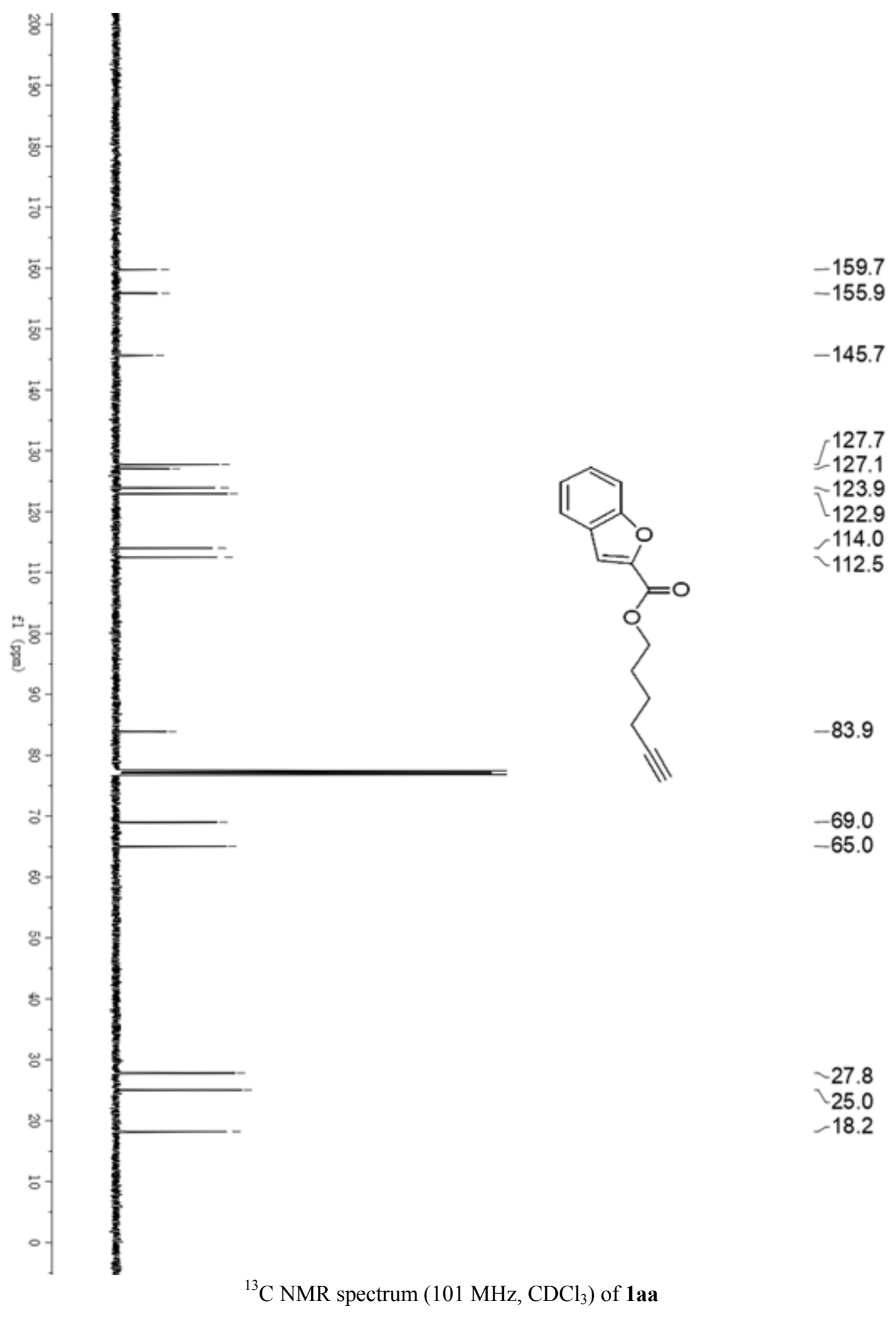



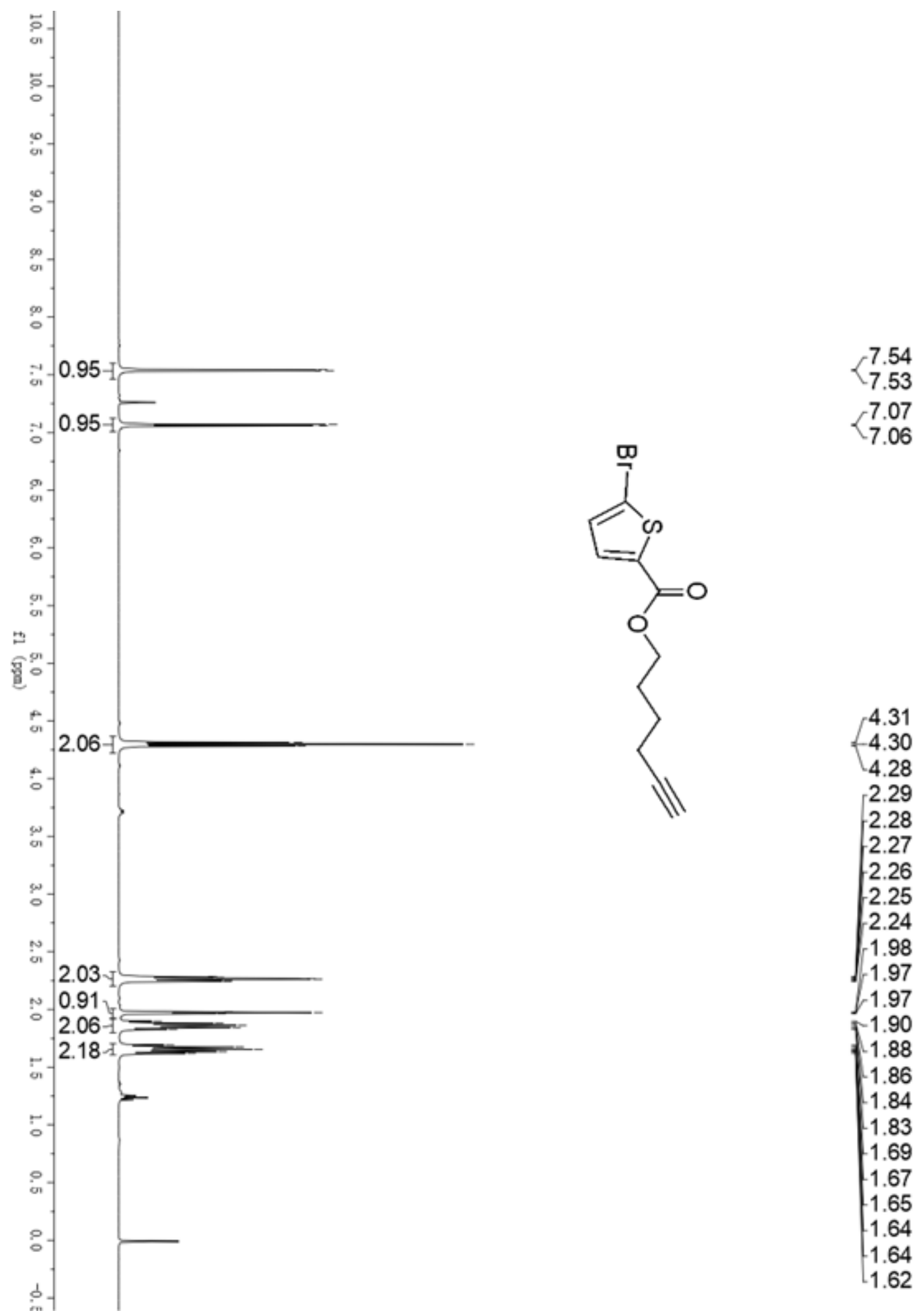

2.29

$-2.28$

2.27

2.26

$-2.25$

2.24

1.98

1.97

1.97

1.90

1.88

1.86

1.84

1.83

1.69

1.67

$-1.65$

1.64

1.64

1.62

${ }^{1} \mathrm{H}$ NMR spectrum $\left(400 \mathrm{MHz}, \mathrm{CDCl}_{3}\right.$ ) of $\mathbf{1 b b}$ 


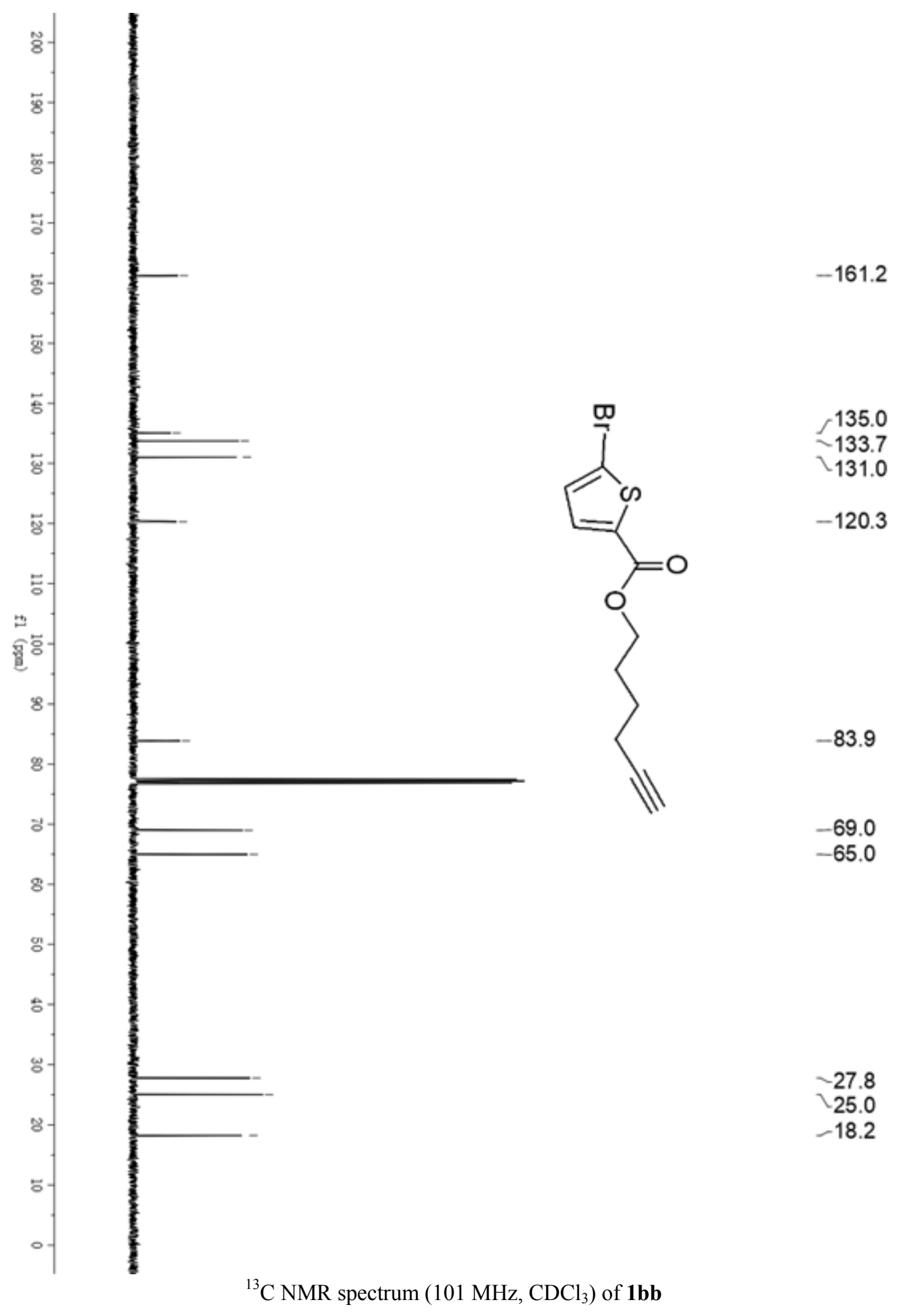




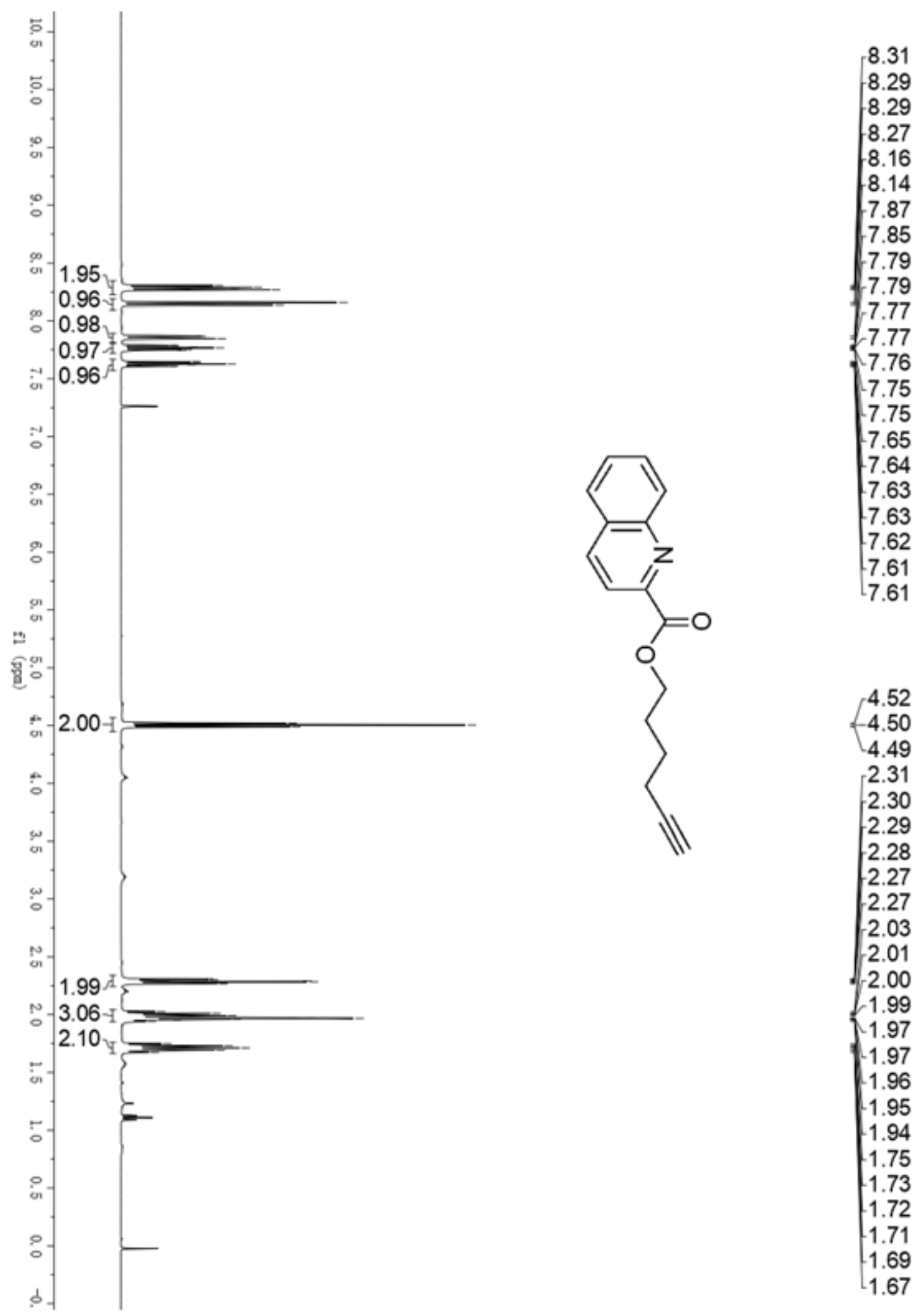

${ }^{1} \mathrm{H}$ NMR spectrum $\left(400 \mathrm{MHz}, \mathrm{CDCl}_{3}\right.$ ) of $\mathbf{1 c c}$ 


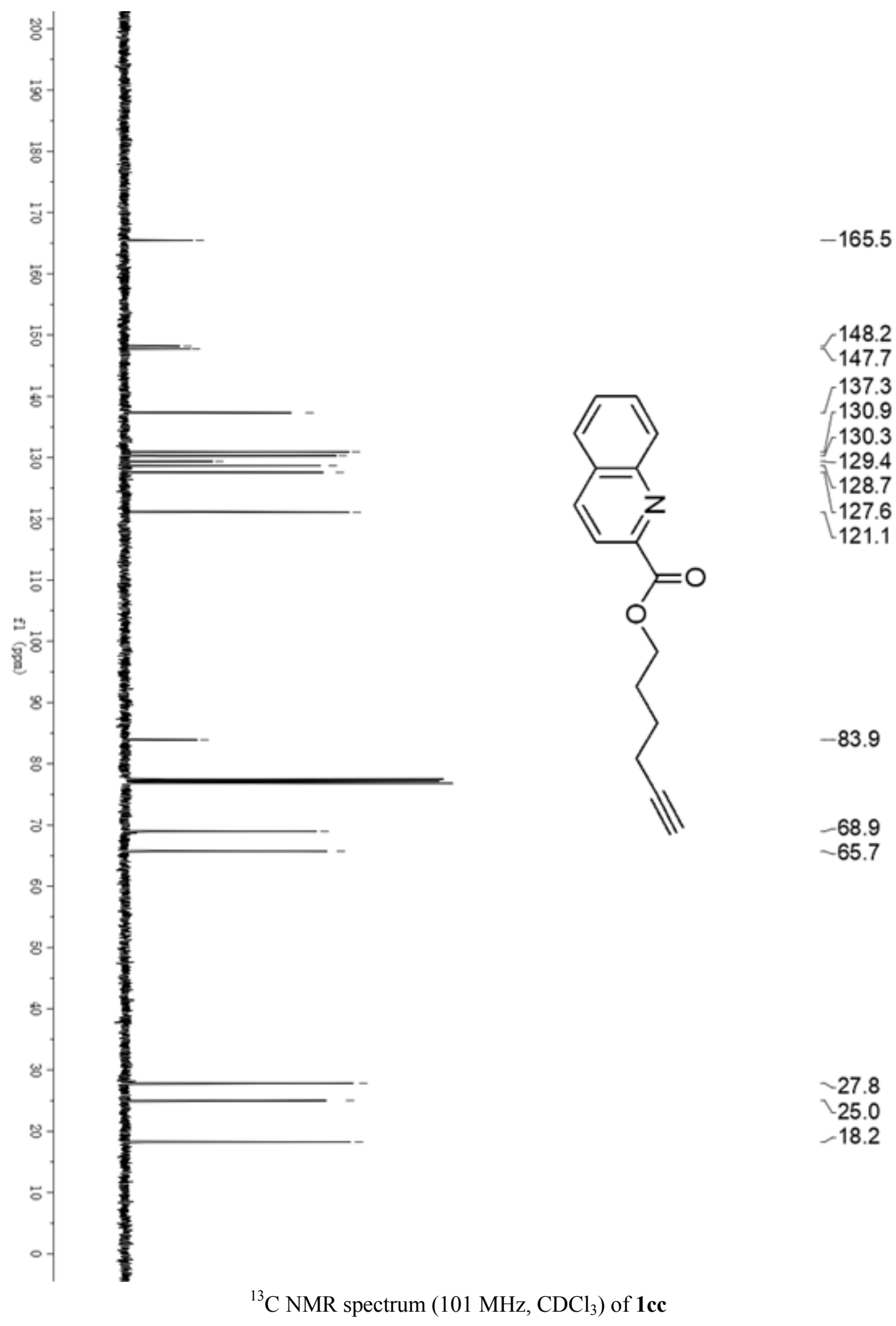




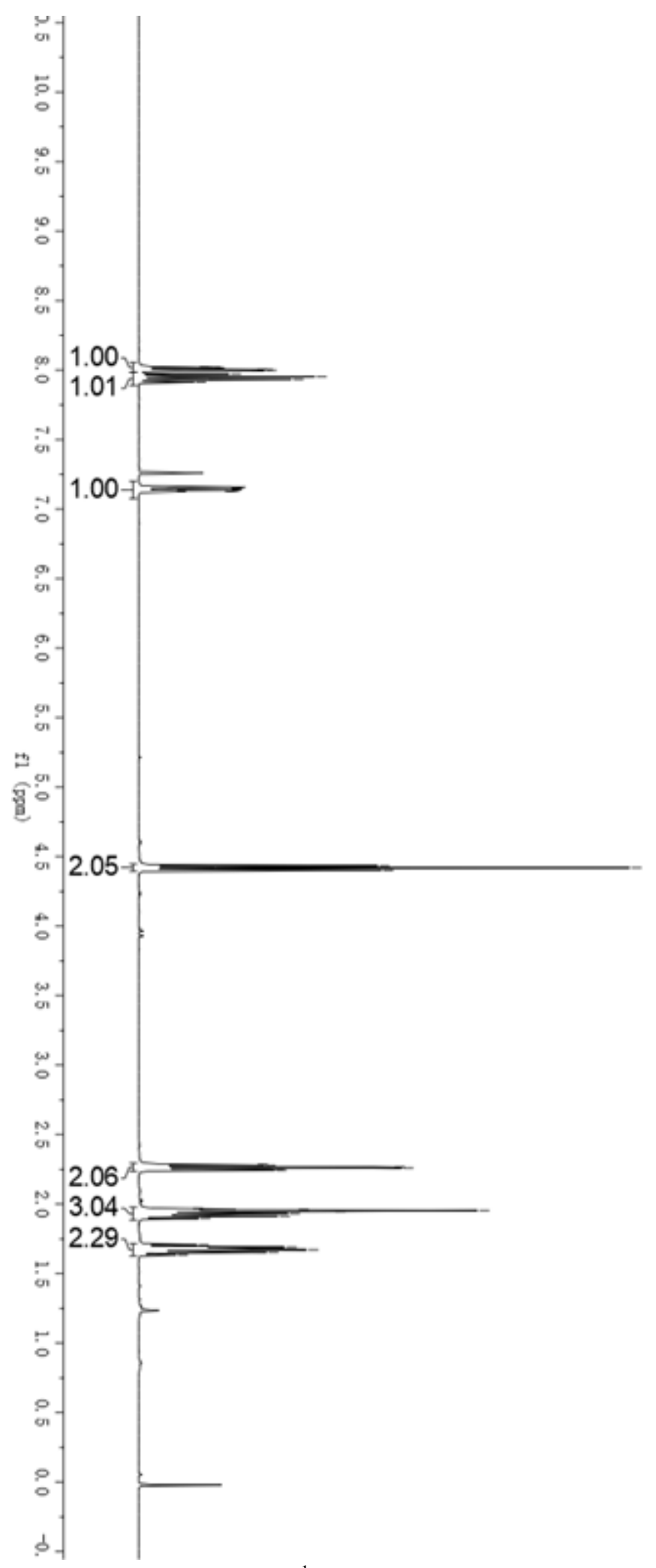

8.02

$-8.02$

8.02

8.02

8.00

8.00

8.00

8.00

7.97

7.95

7.93

7.91

7.16

7.16

7.15

$-7.15$

7.14

$-7.14$

7.13

7.13

4.44

$-4.42$

4.40

2.29

2.28

$-2.27$

2.26

$-2.25$

2.24

1.97

1.96

1.95

$-1.95$

1.93

1.91

$-1.90$

1.71

$-1.69$

1.68

$-1.68$

1.67

1.65

${ }^{1} \mathrm{H}$ NMR spectrum $\left(400 \mathrm{MHz}, \mathrm{CDCl}_{3}\right)$ of $\mathbf{1 d d}$ 


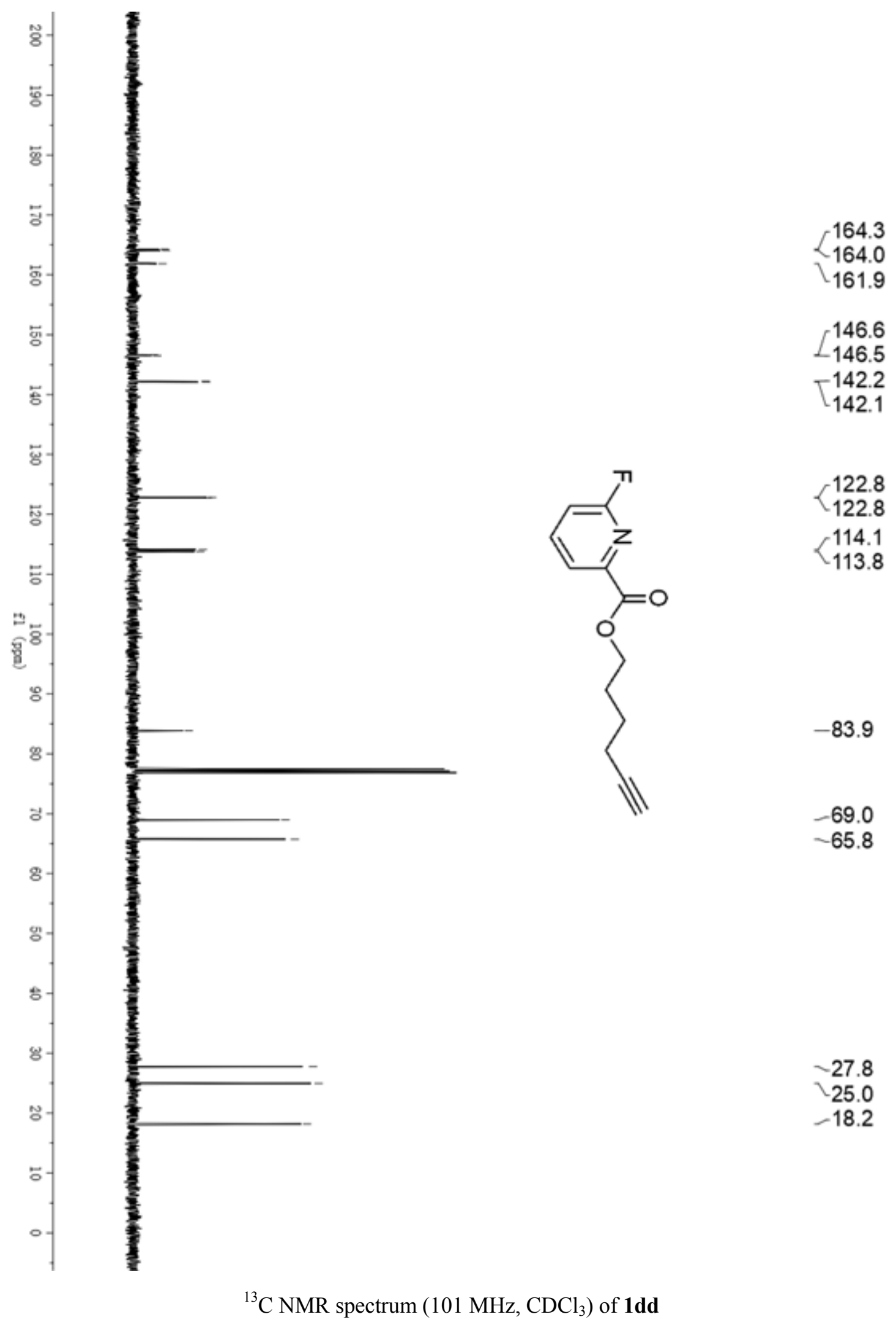




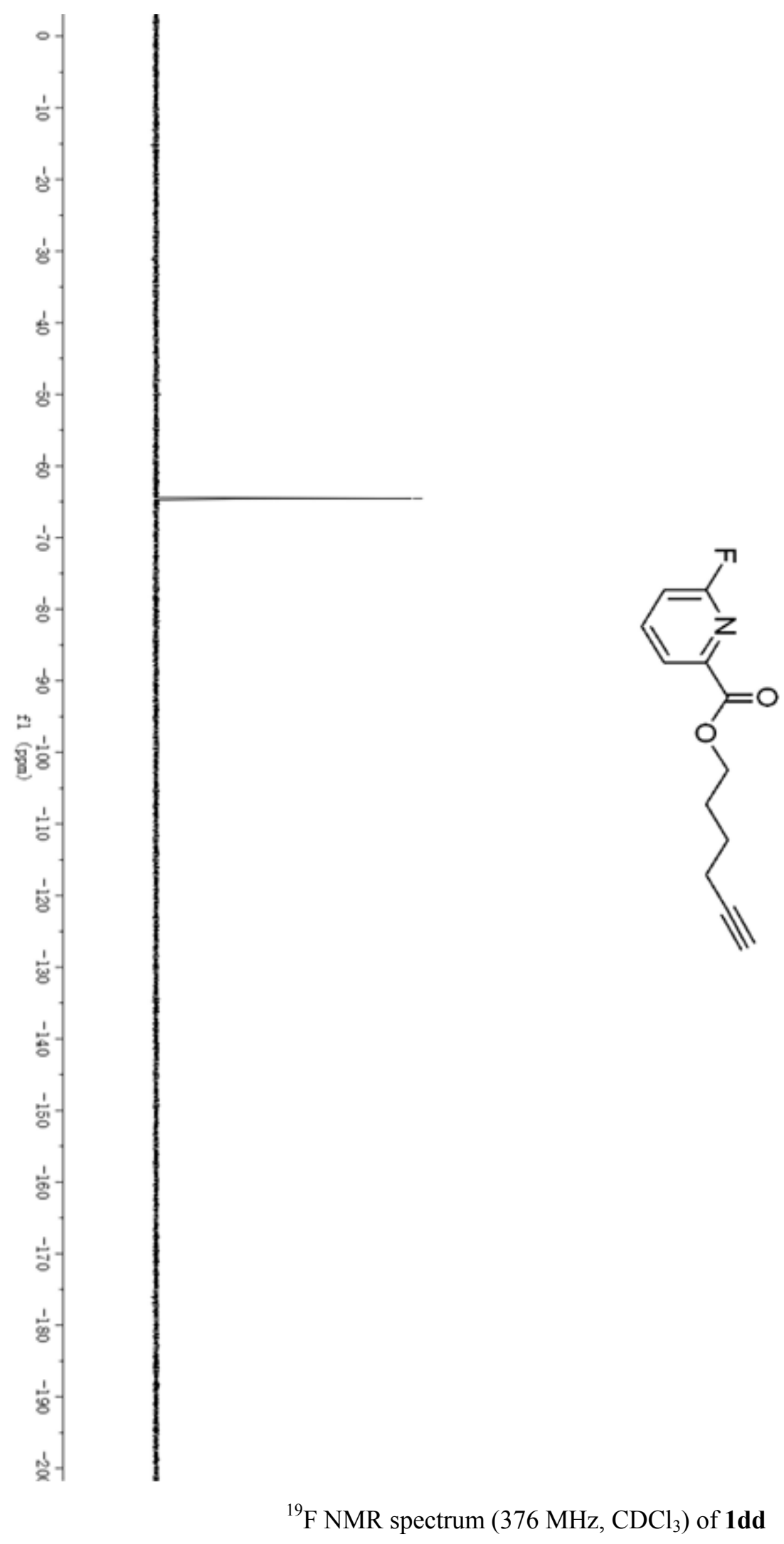

$--64.55$ 


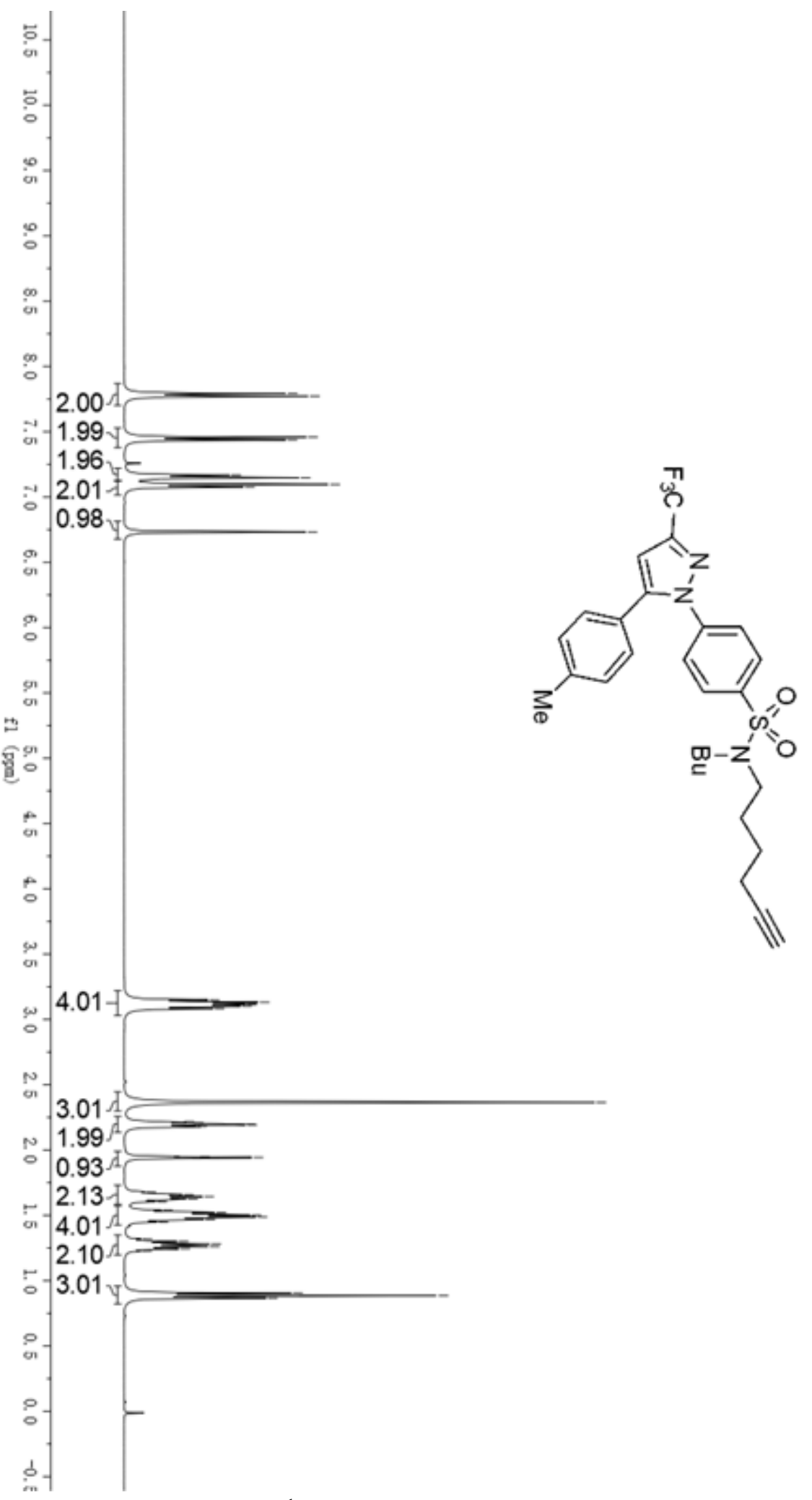

${ }^{1} \mathrm{H}$ NMR spectrum (400 MHz, $\mathrm{CDCl}_{3}$ ) of $\mathbf{1 I l}$ 


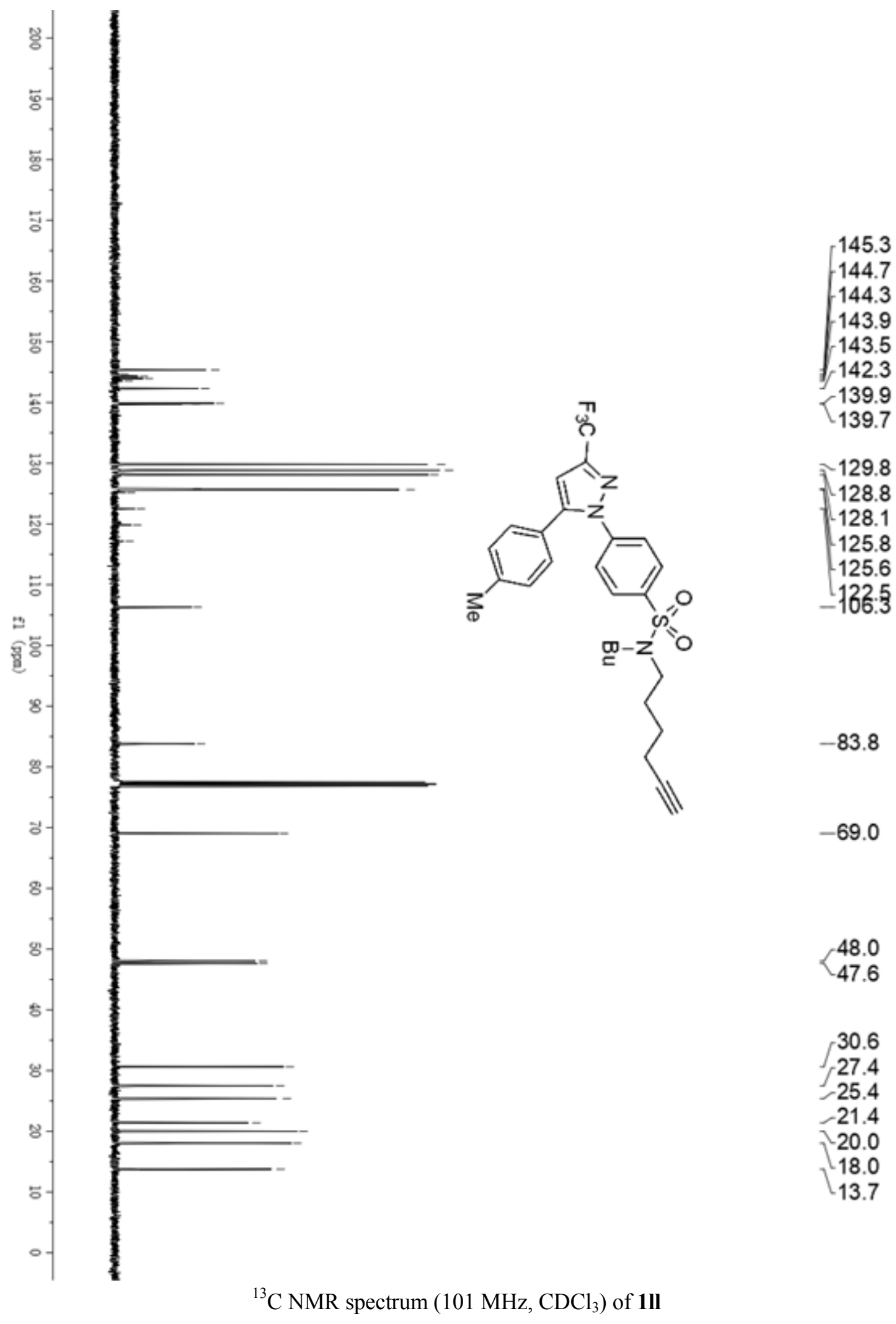




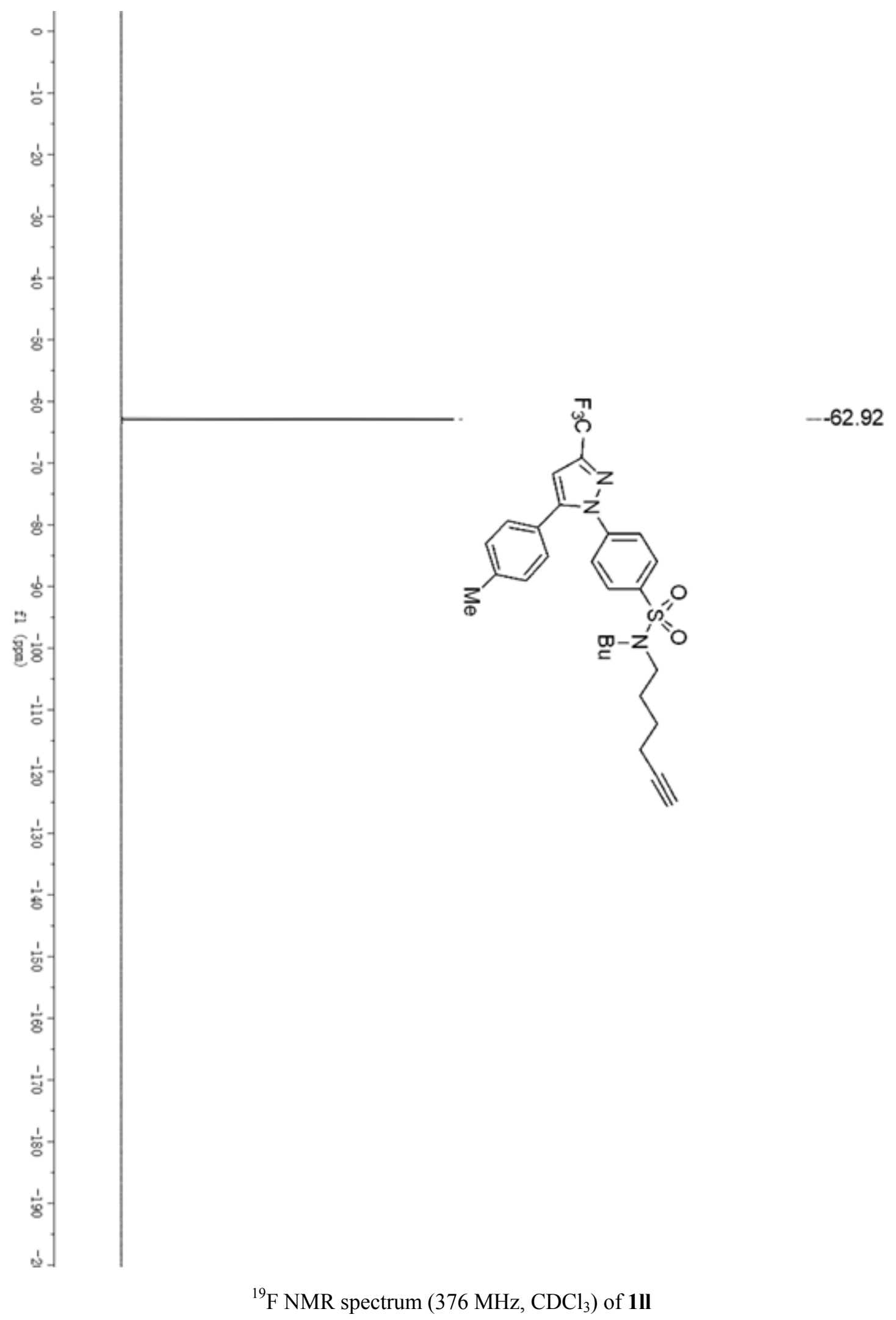




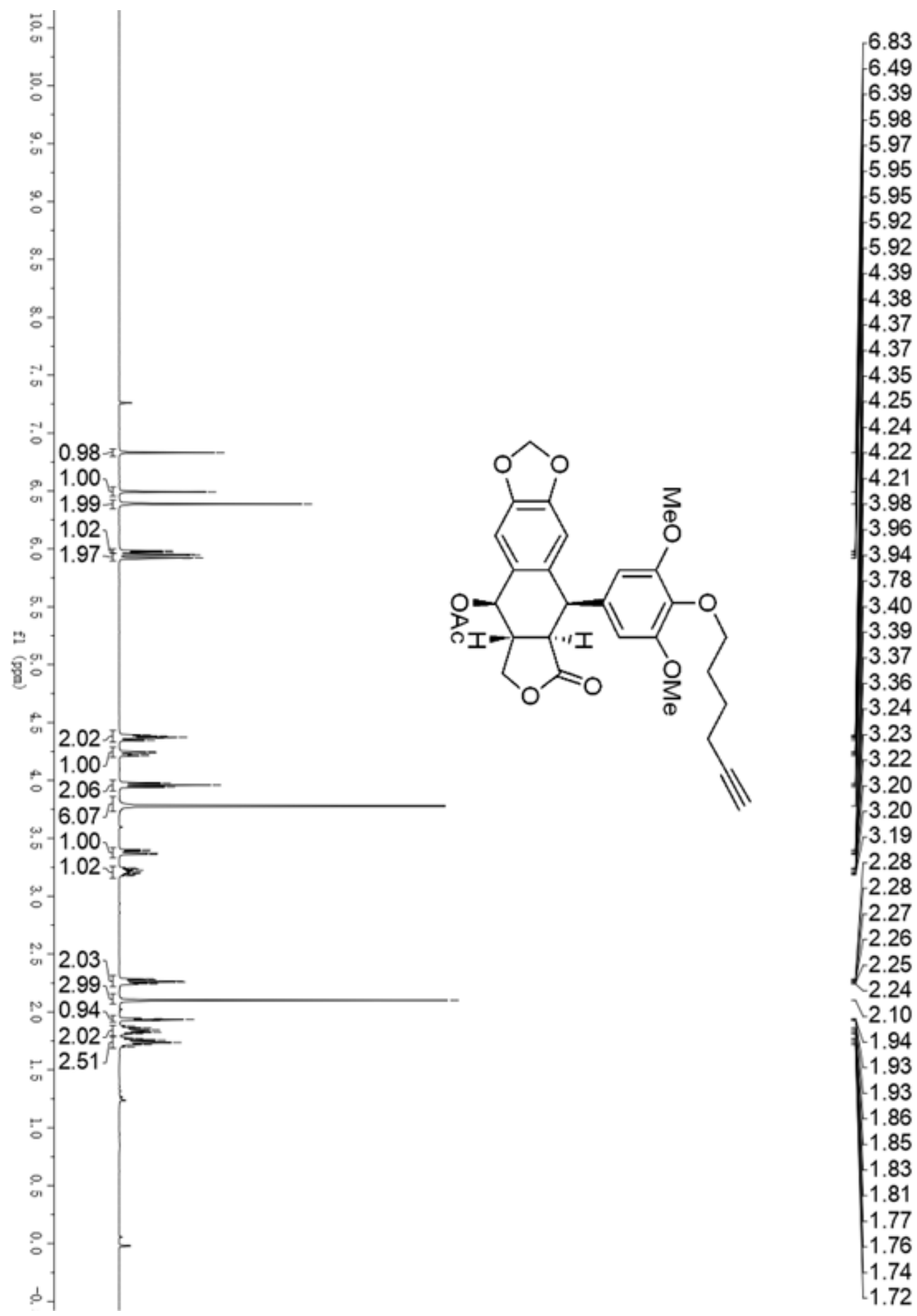

${ }^{1} \mathrm{H}$ NMR spectrum $\left(400 \mathrm{MHz}, \mathrm{CDCl}_{3}\right)$ of $\mathbf{1} \mathbf{m m}$ 


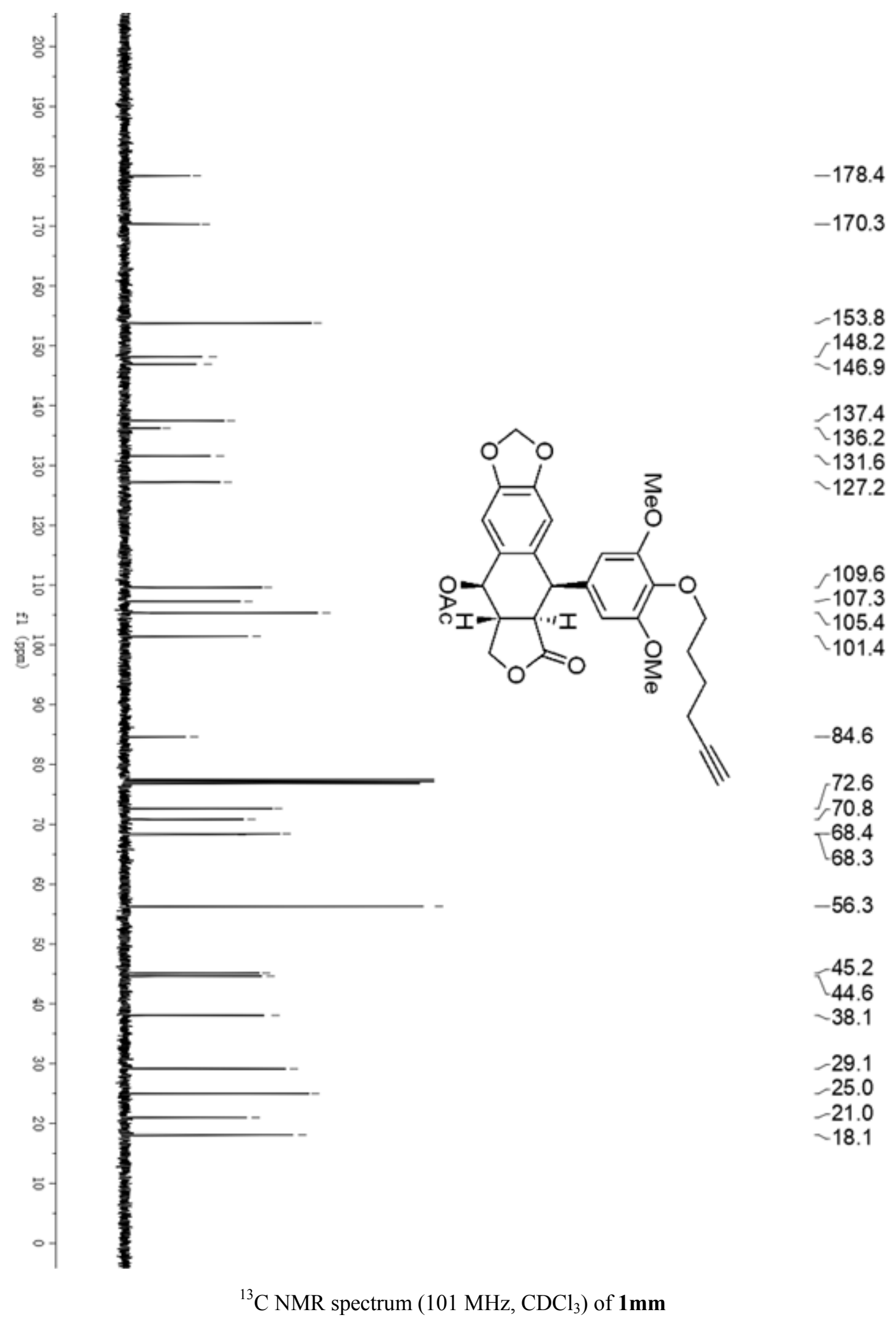




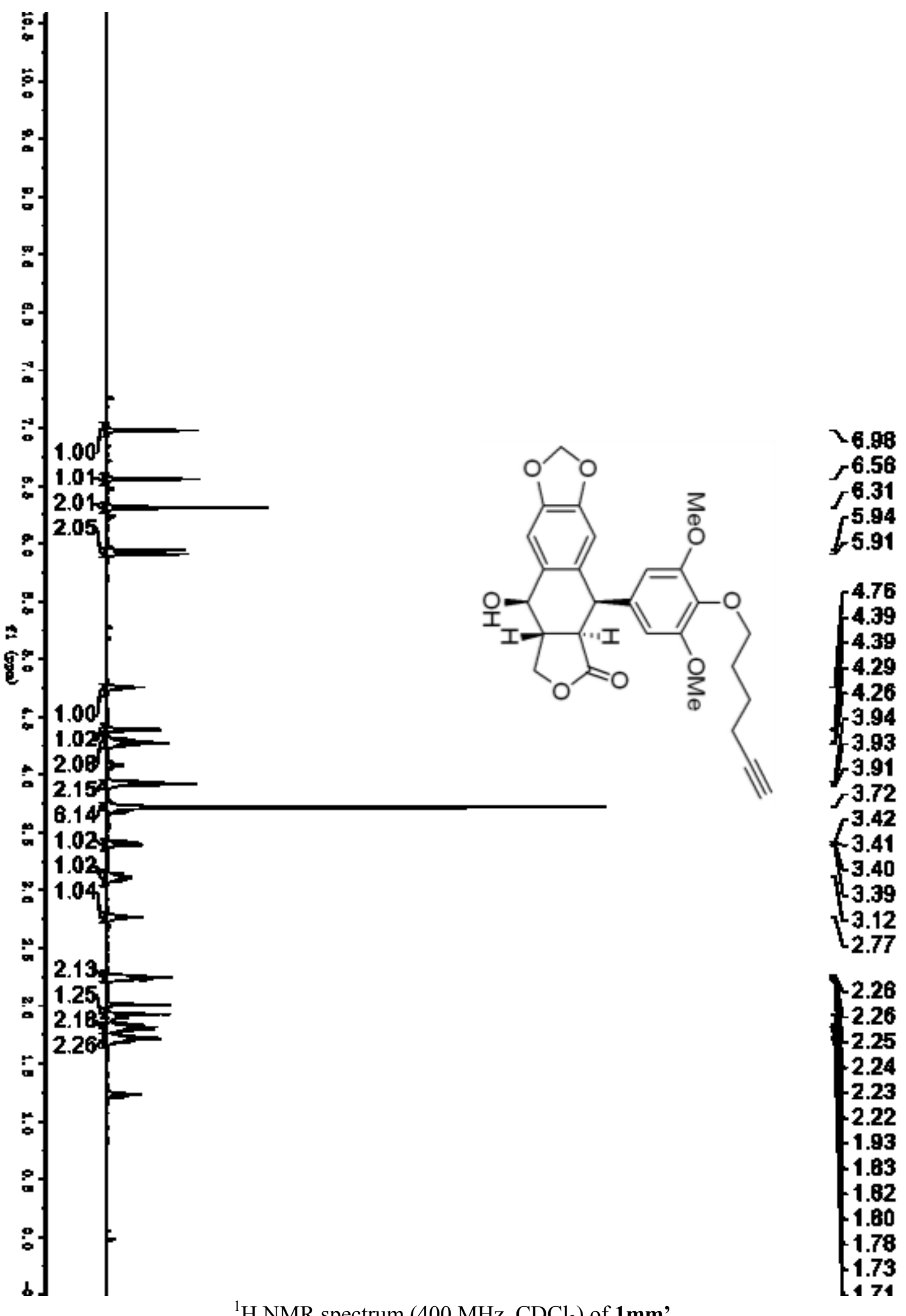

${ }^{1} \mathrm{H}$ NMR spectrum (400 MHz, $\left.\mathrm{CDCl}_{3}\right)$ of $\mathbf{1} \mathbf{m m}$ ' 


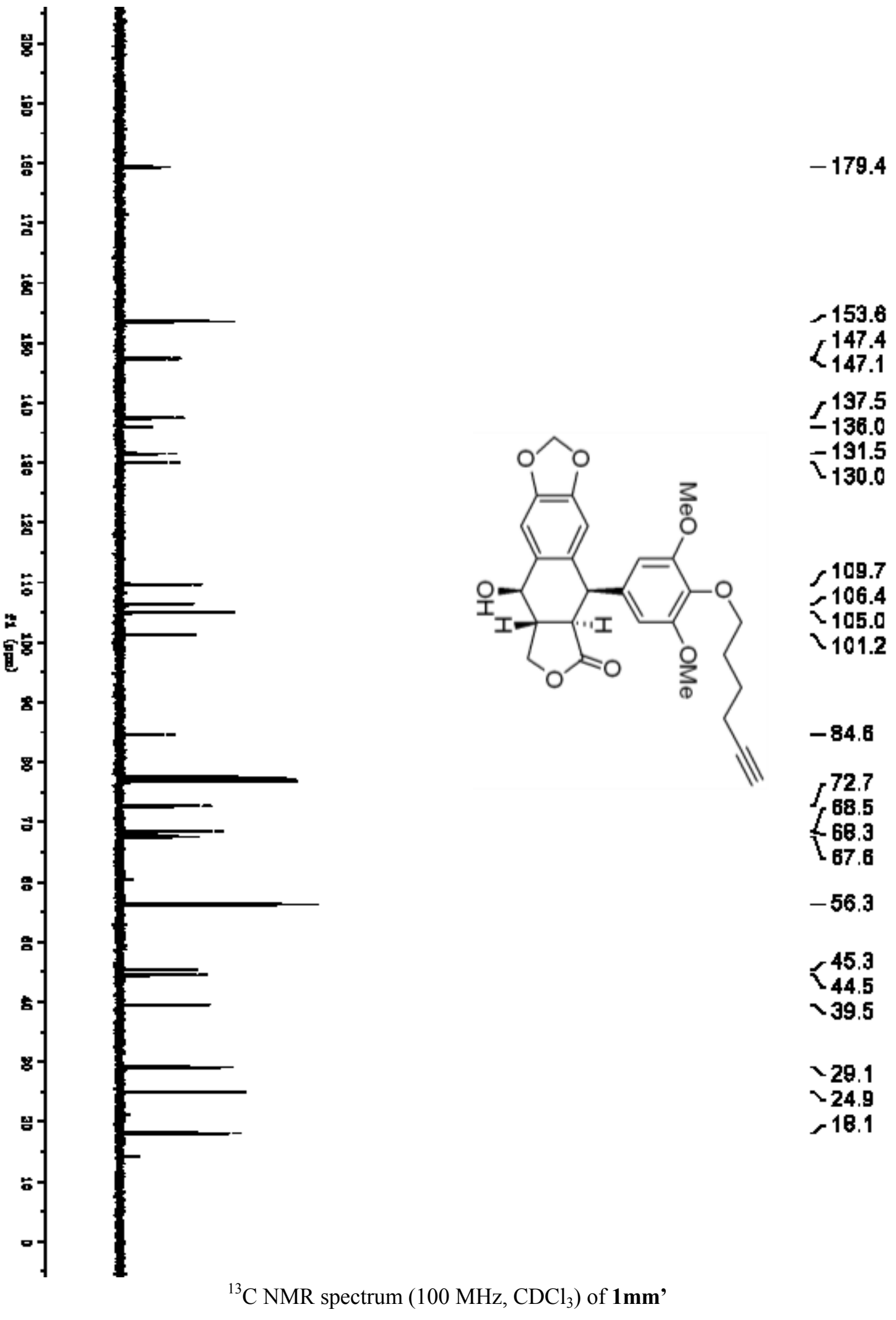




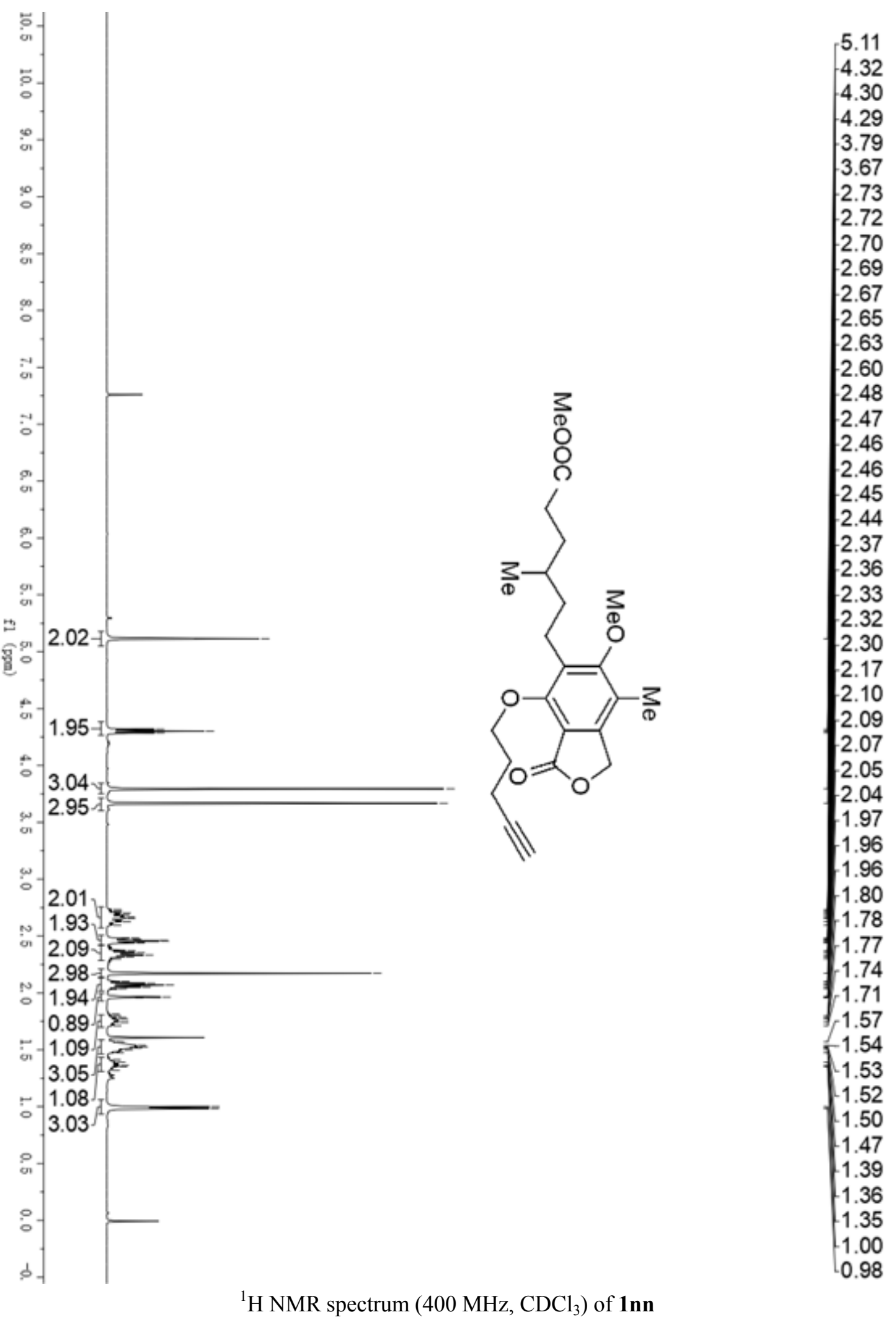




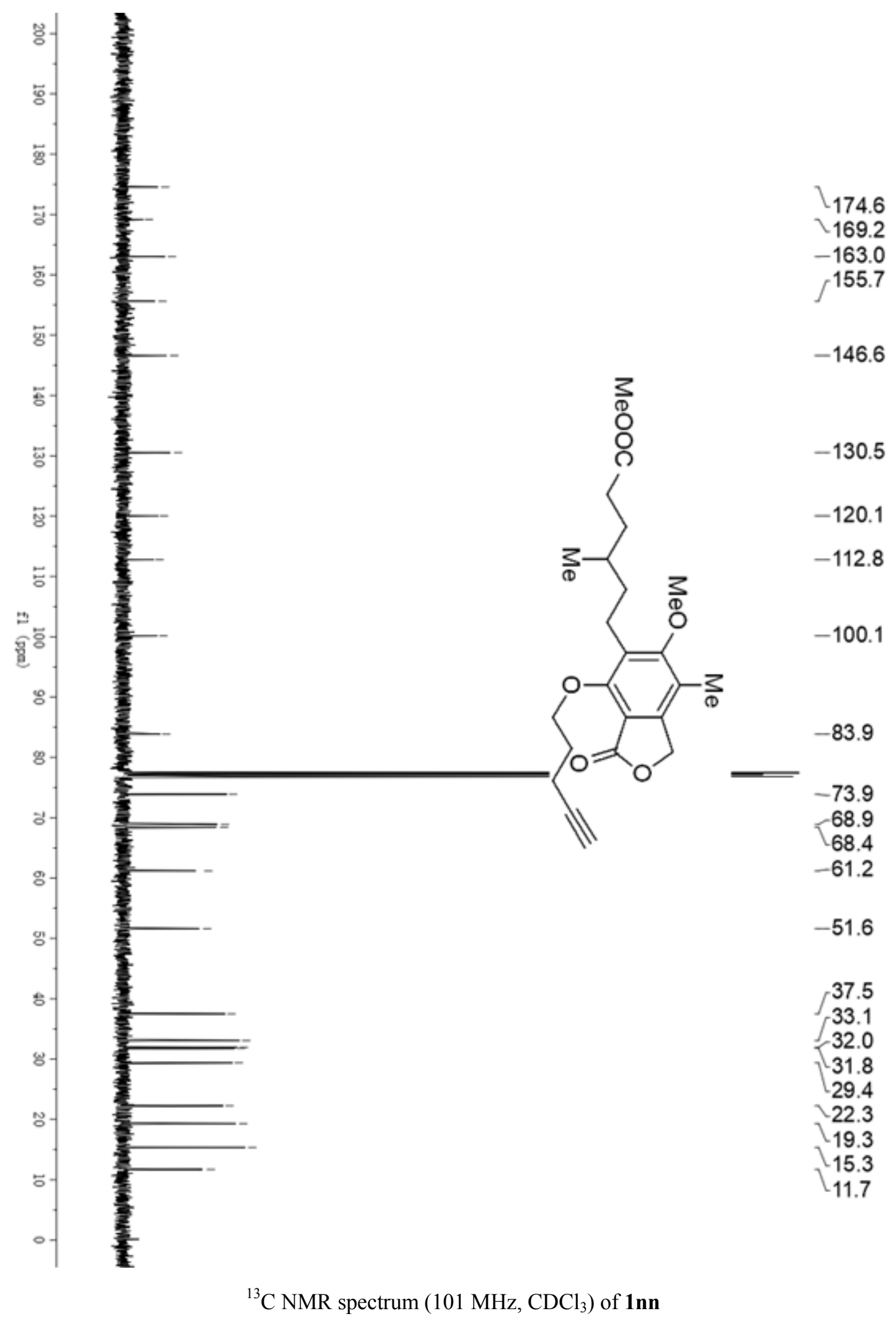



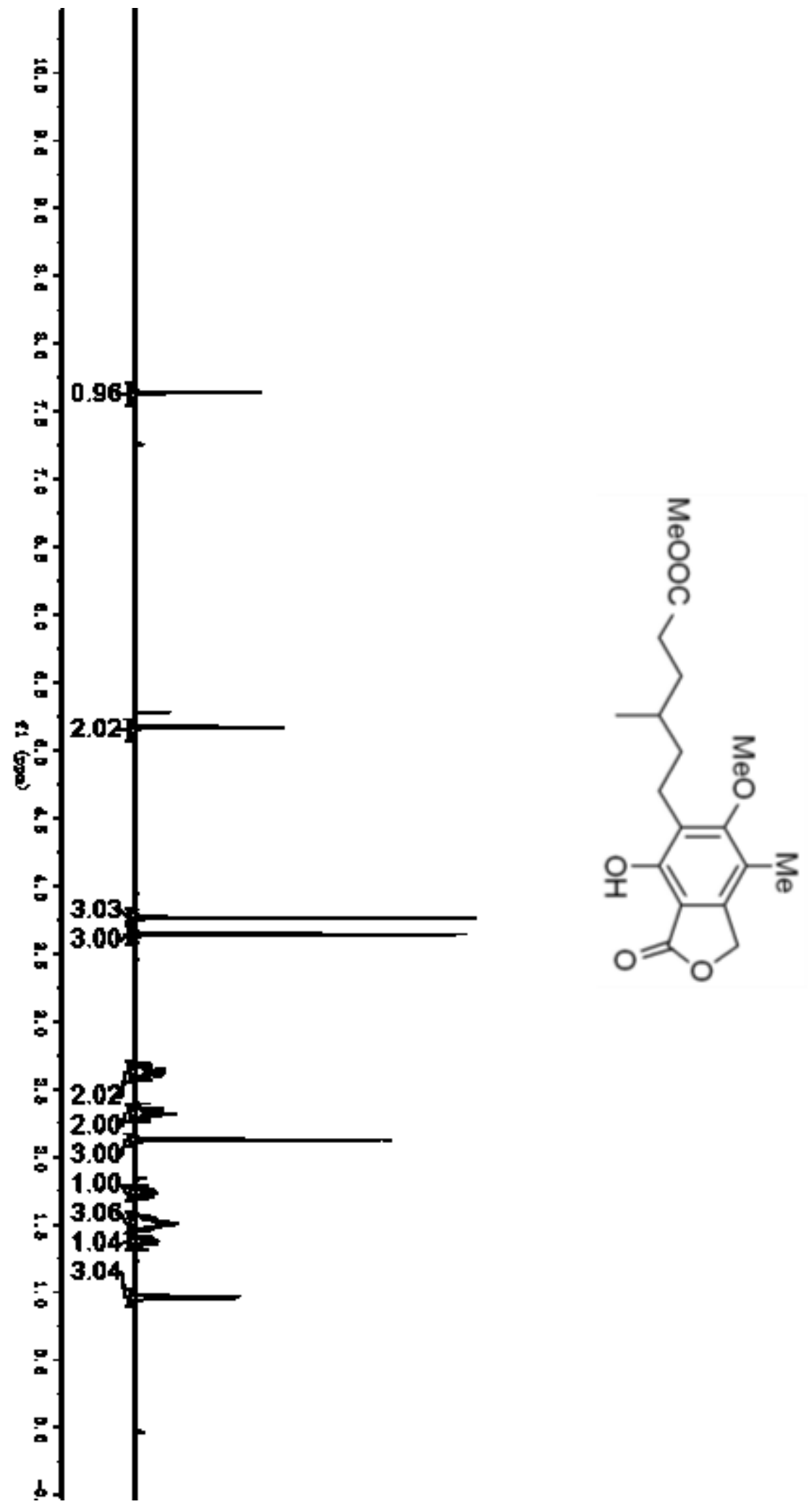

${ }^{1} \mathrm{H}$ NMR spectrum $\left(400 \mathrm{MHz}, \mathrm{CDCl}_{3}\right)$ of $\mathbf{1 n n}$ ' 


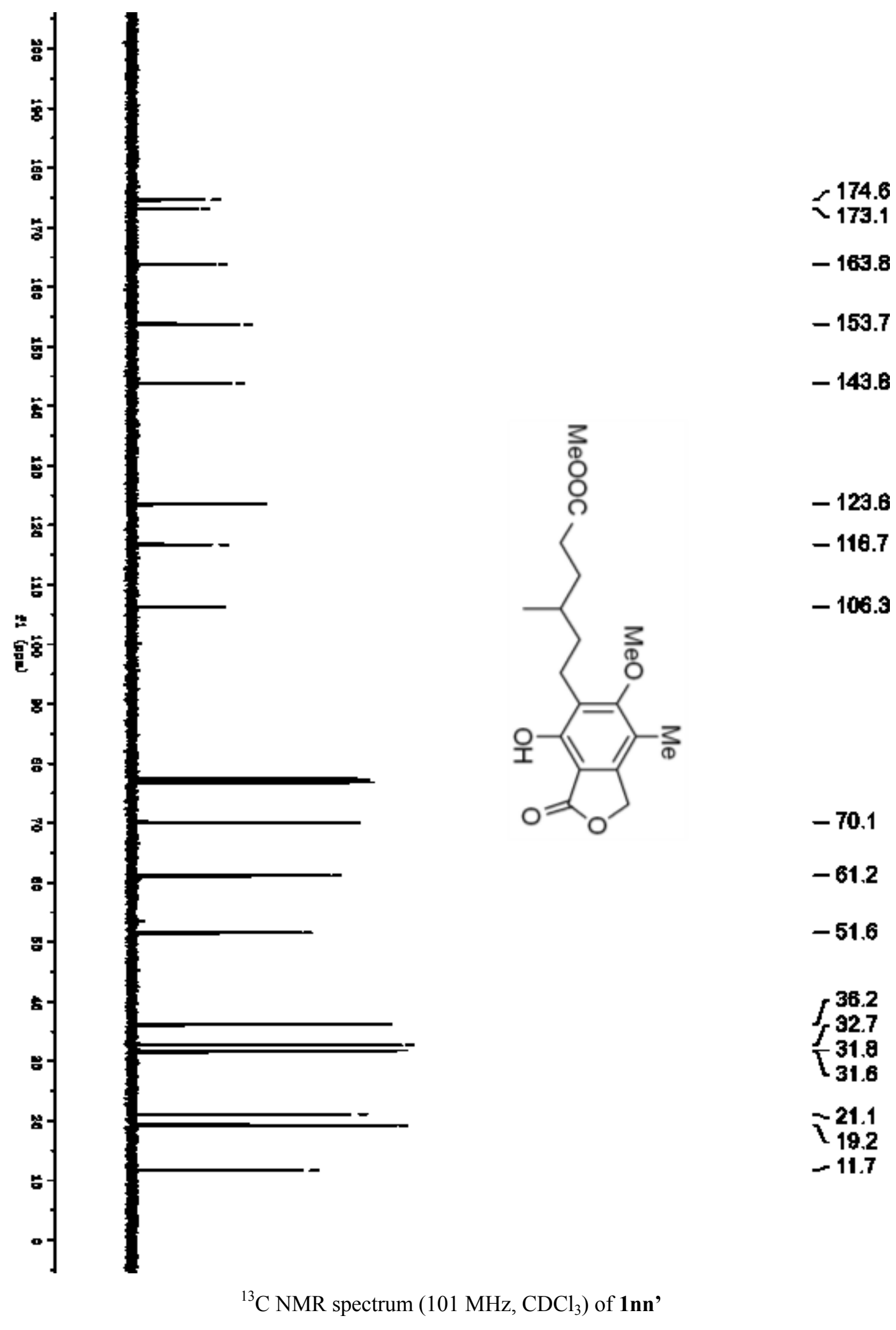




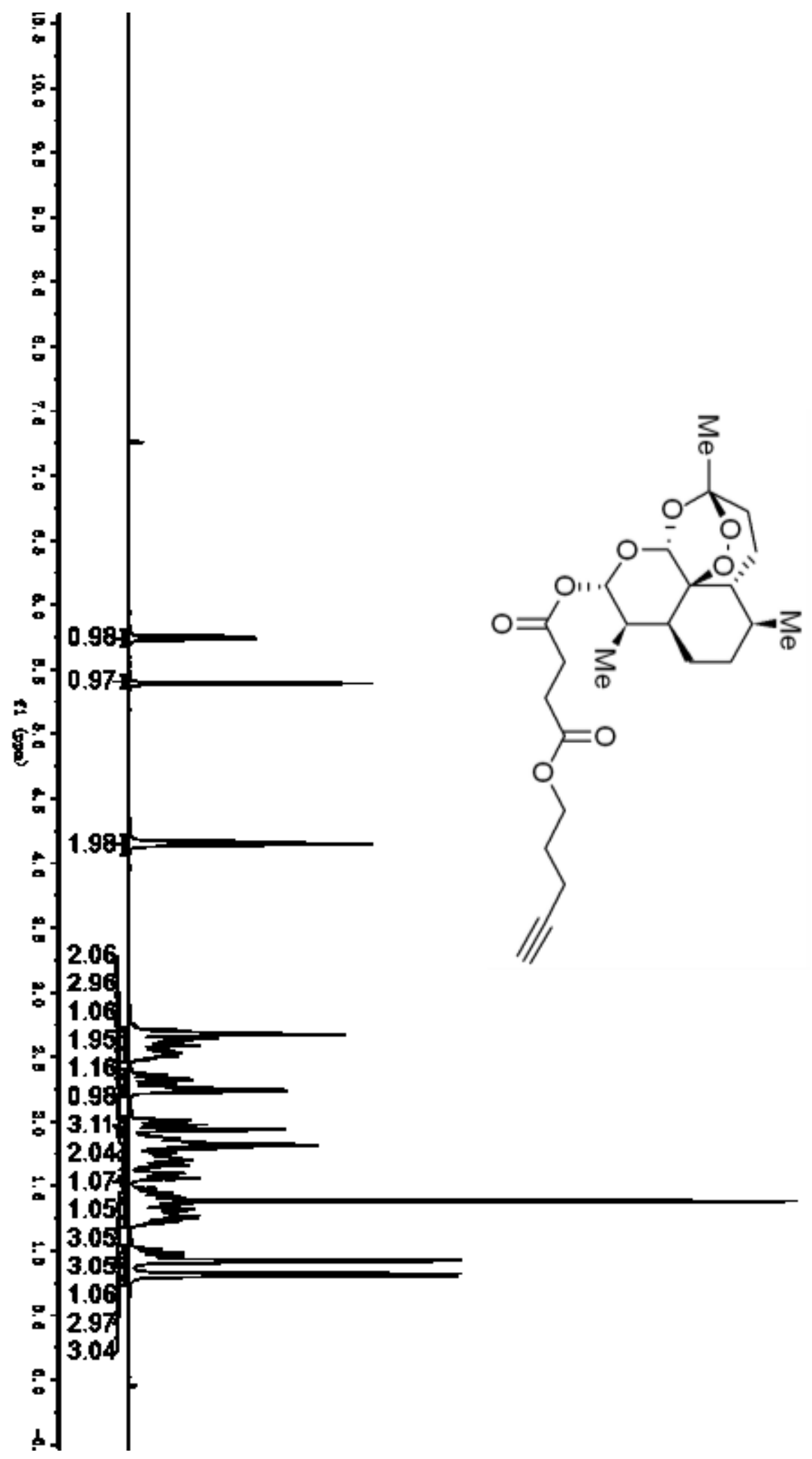

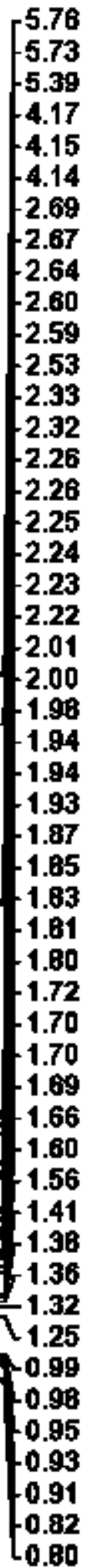

${ }^{1} \mathrm{H}$ NMR spectrum $\left(400 \mathrm{MHz}, \mathrm{CDCl}_{3}\right)$ of $\mathbf{1 0 o}$ 


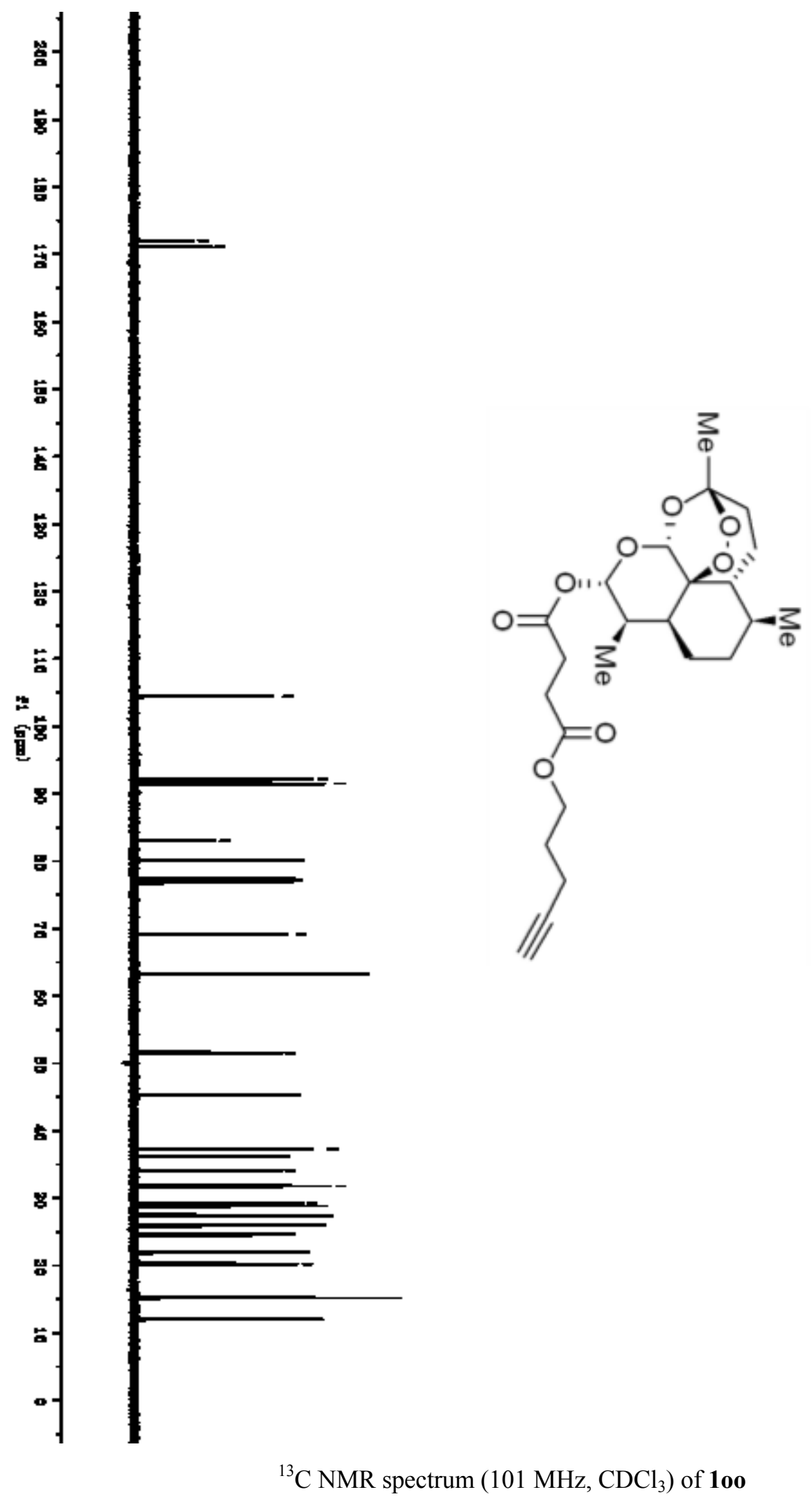

$-104.5$

92.2

91.5

$-83.0$

$-80.1$

$-69.1$

$-63.3$

51.5

45.2

37.3

36.2

34.1

31.8

29.2

28.8

27.5

26.0

24.6

22.0

20.2

15.2

12.1

${ }^{13} \mathrm{C}$ NMR spectrum $\left(101 \mathrm{MHz}, \mathrm{CDCl}_{3}\right)$ of $\mathbf{1 o o}$ 


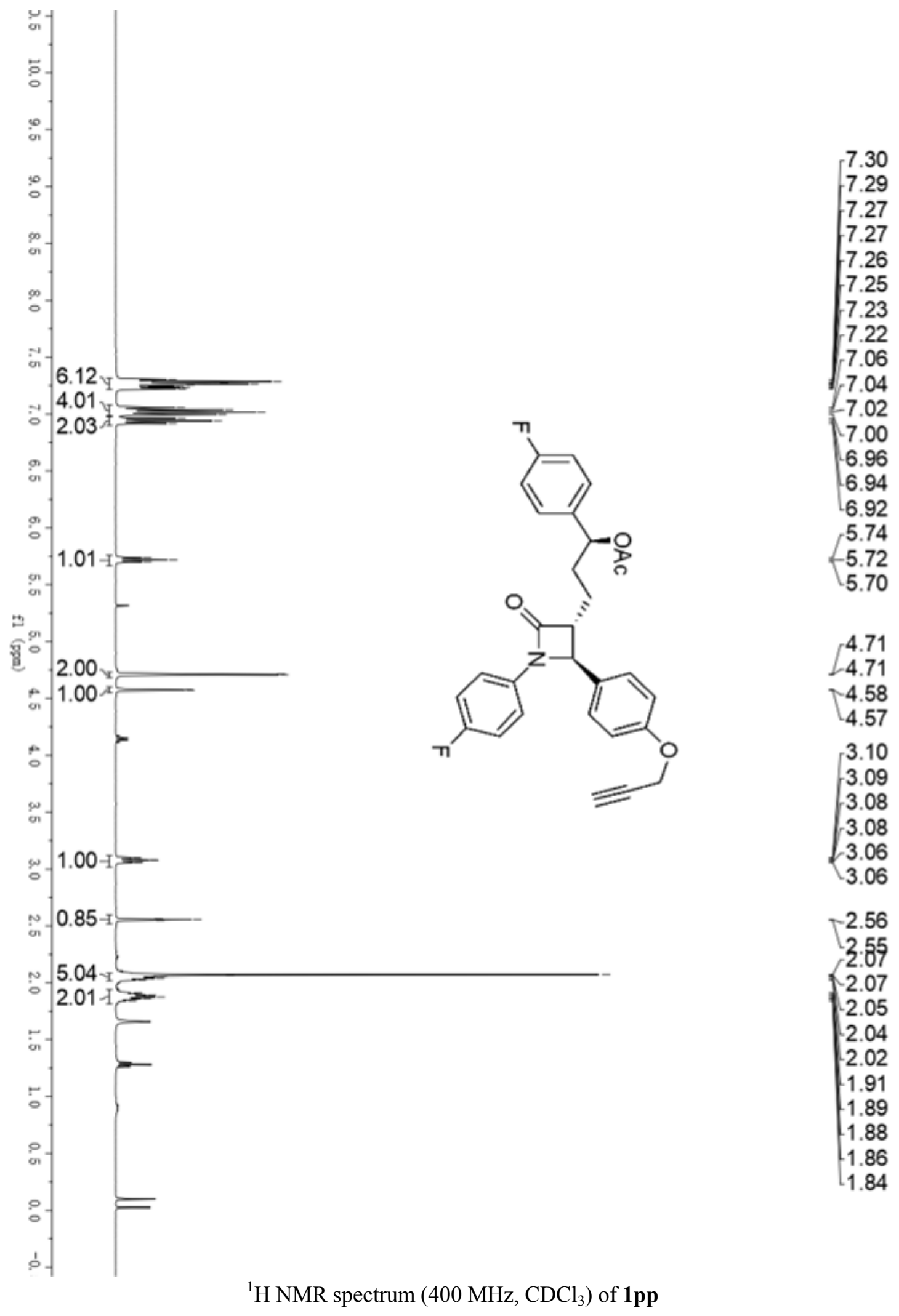




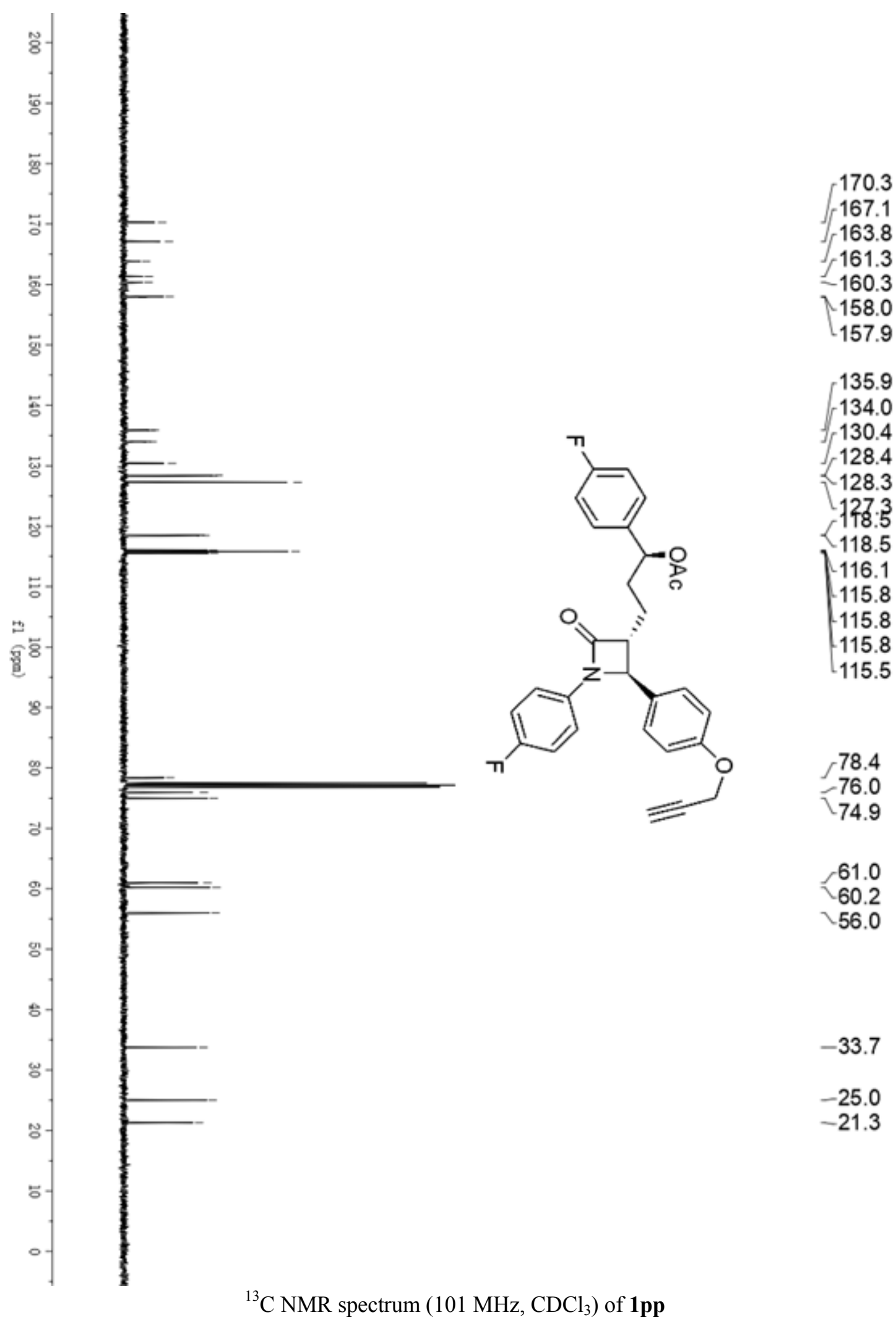




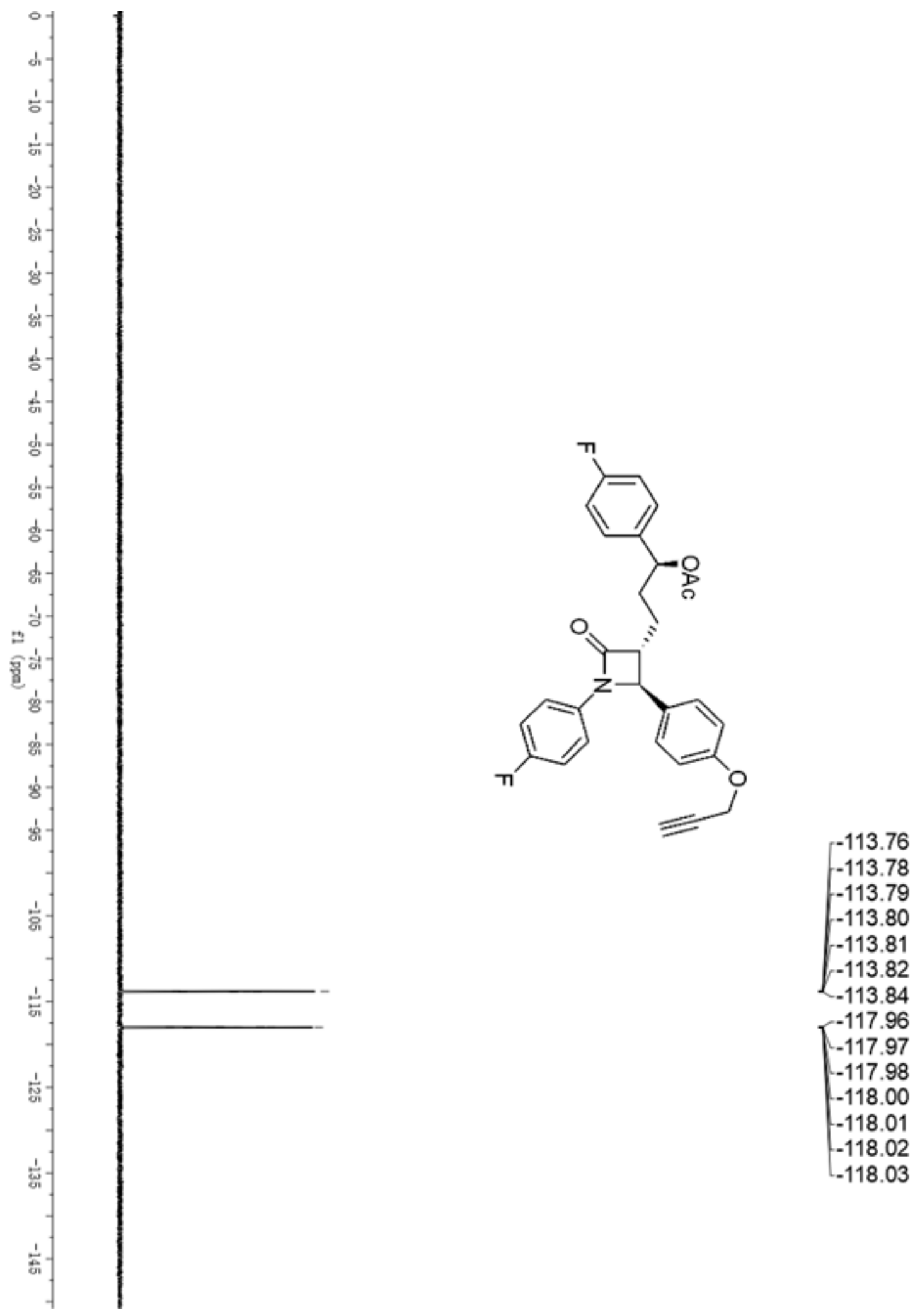

${ }^{19} \mathrm{~F}$ NMR spectrum $\left(376 \mathrm{MHz}, \mathrm{CDCl}_{3}\right.$ ) of $\mathbf{1 p p}$ 

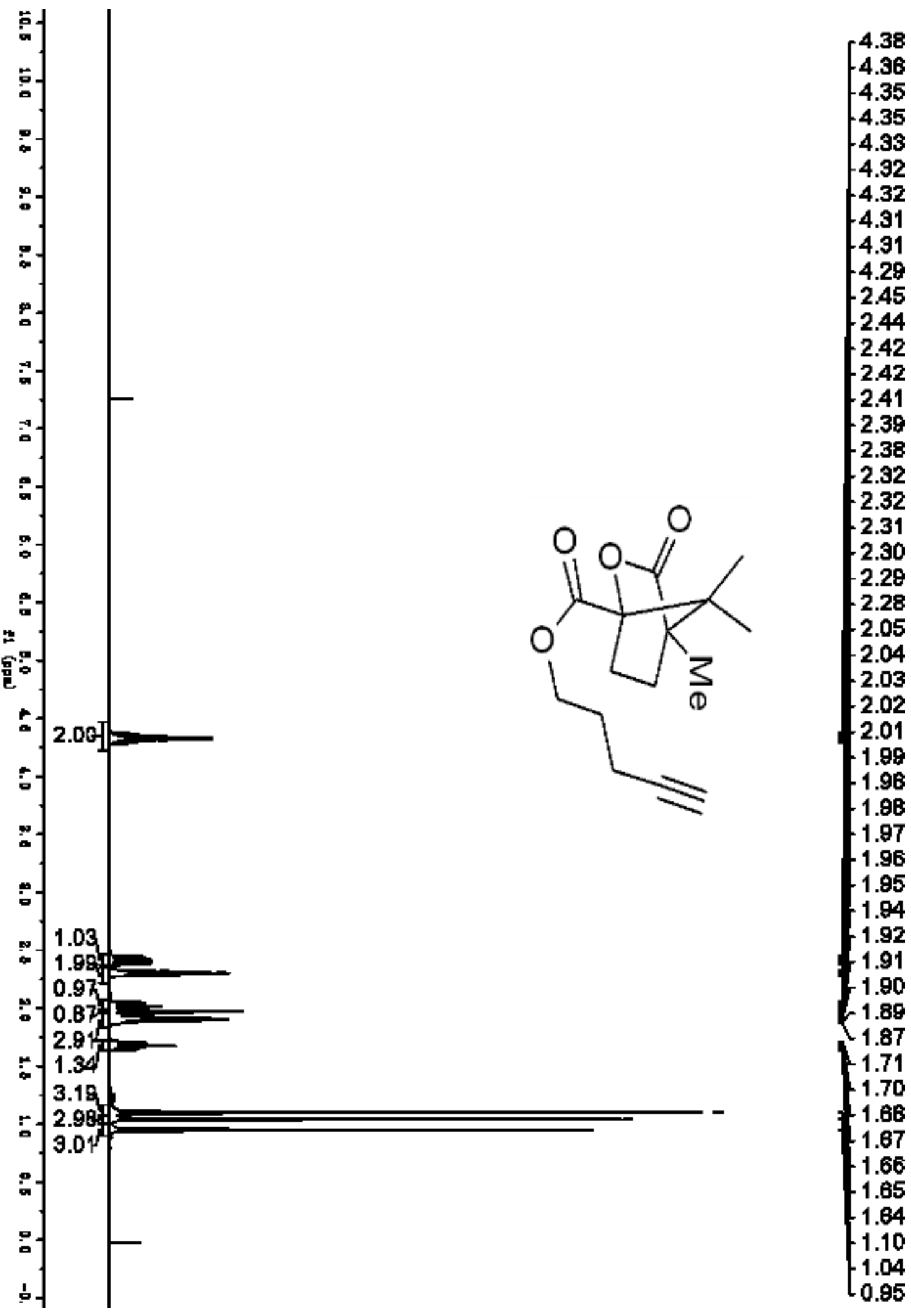

${ }^{1} \mathrm{H}$ NMR spectrum $\left(400 \mathrm{MHz}, \mathrm{CDCl}_{3}\right)$ of $\mathbf{1 q q}$ 


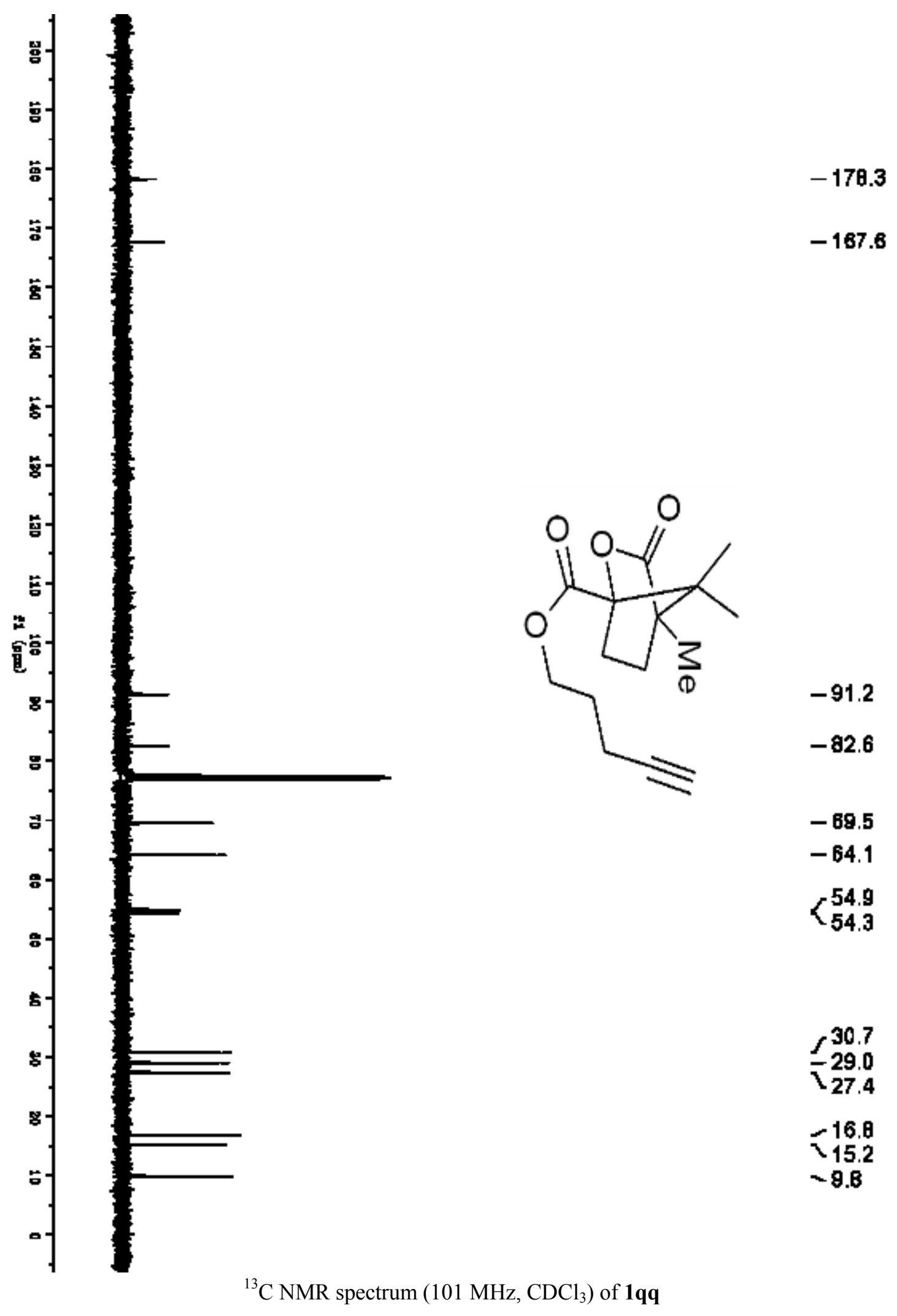




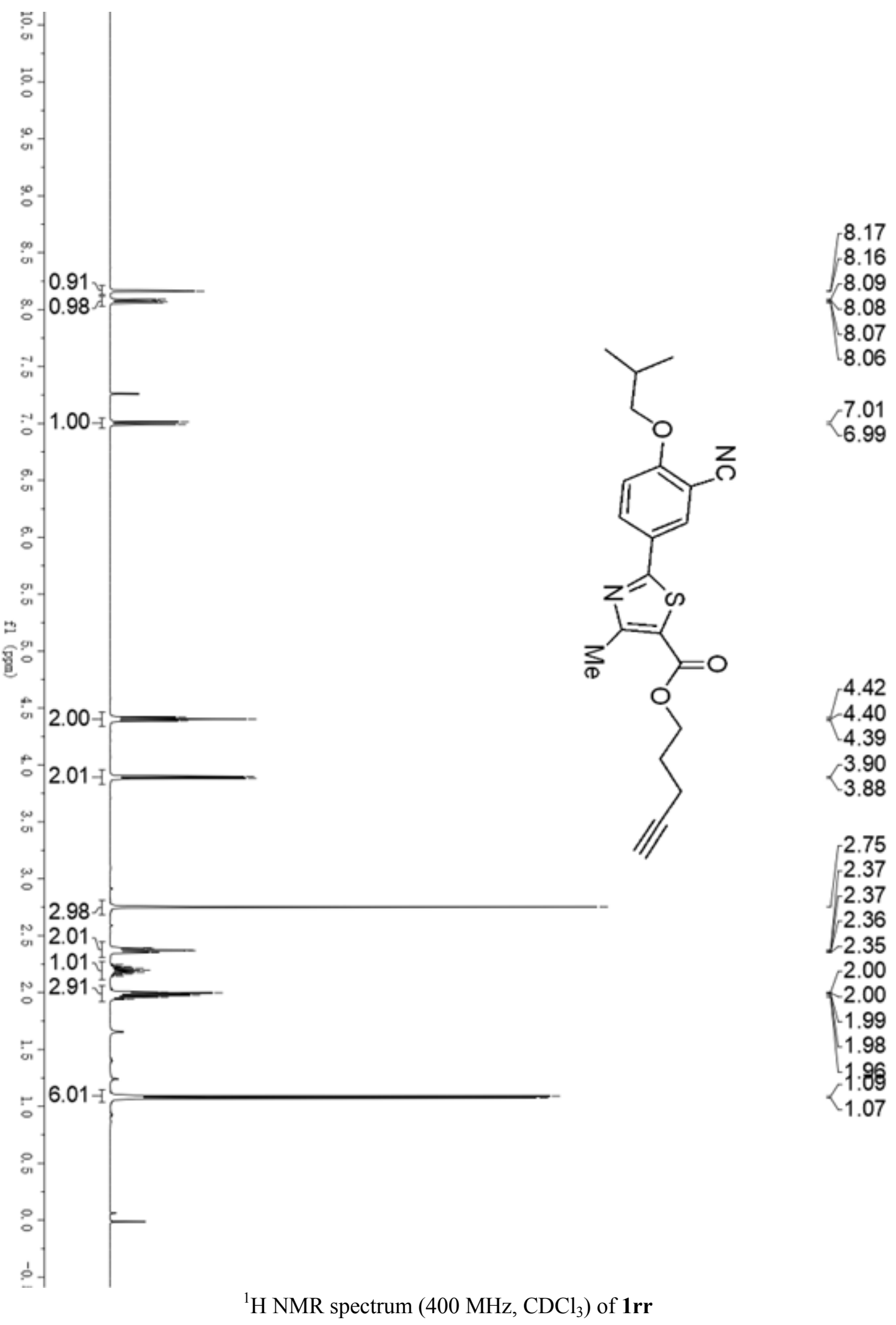




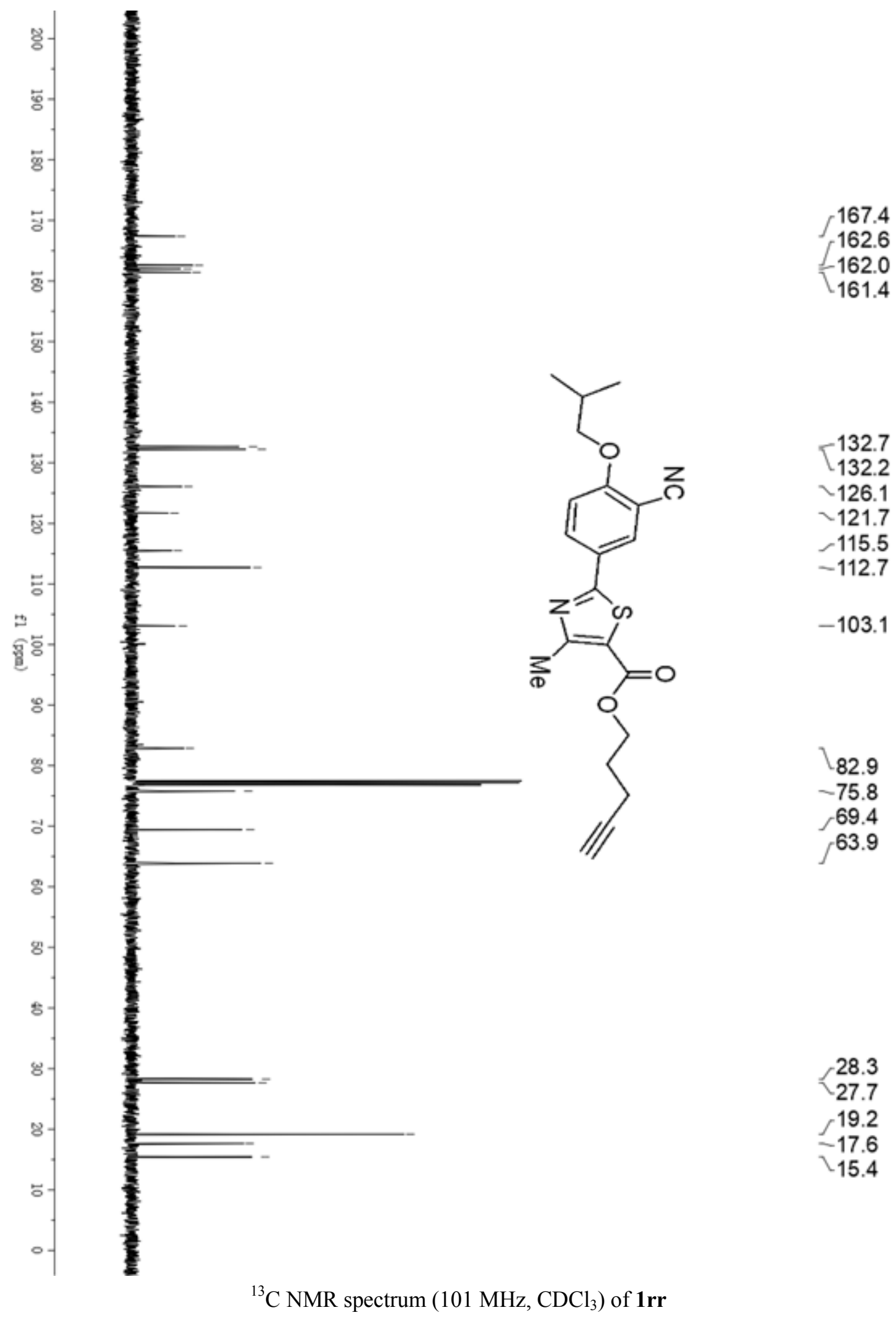




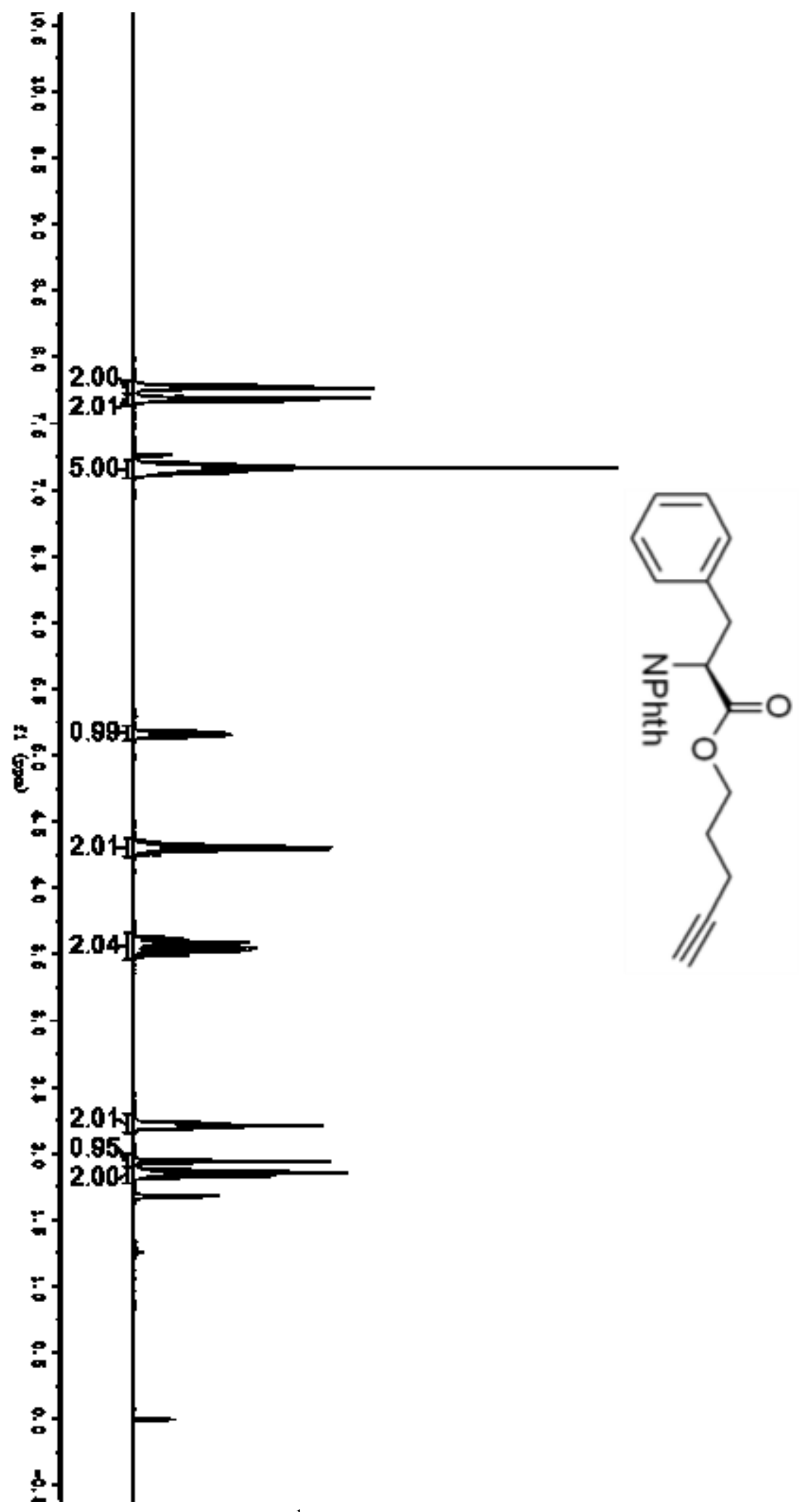

$-7.12$

7.11

5.18

5.17

$-5.15$

5.14

4.34

1. 4.33

4.31

$-4.30$

$-4.28$

$-4.27$

3.63

3.61

3.59

$-3.58$

$-3.55$

3.53

$-3.52$

3.49

2.23

2.23

$-2.21$

2.21

2.20

2.19

1.95

1.94

1.93

1.88

1.86

1.85

1.83

${ }^{1} \mathrm{H}$ NMR spectrum $\left(400 \mathrm{MHz}, \mathrm{CDCl}_{3}\right)$ of $\mathbf{1 s s}$

1.81 


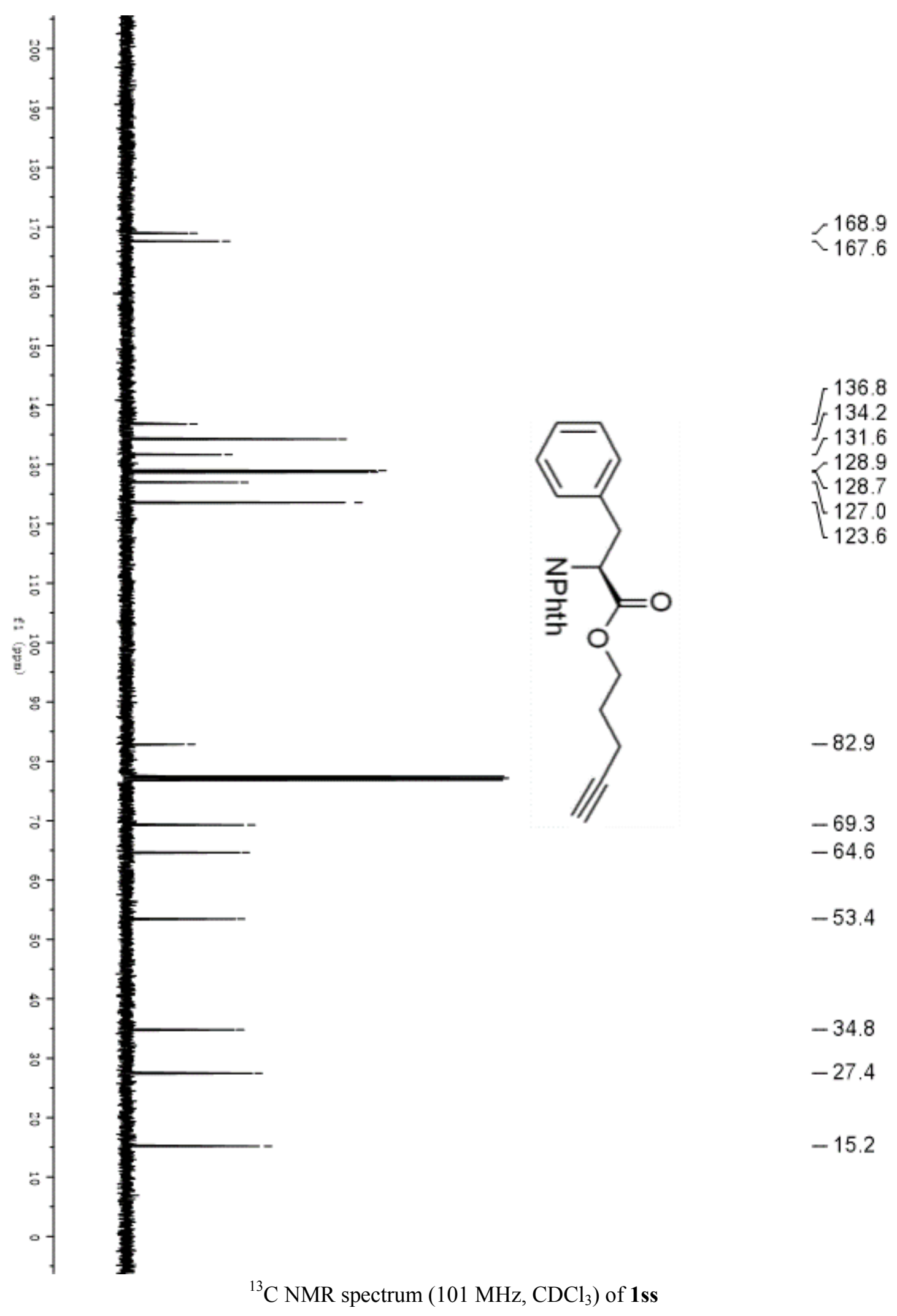




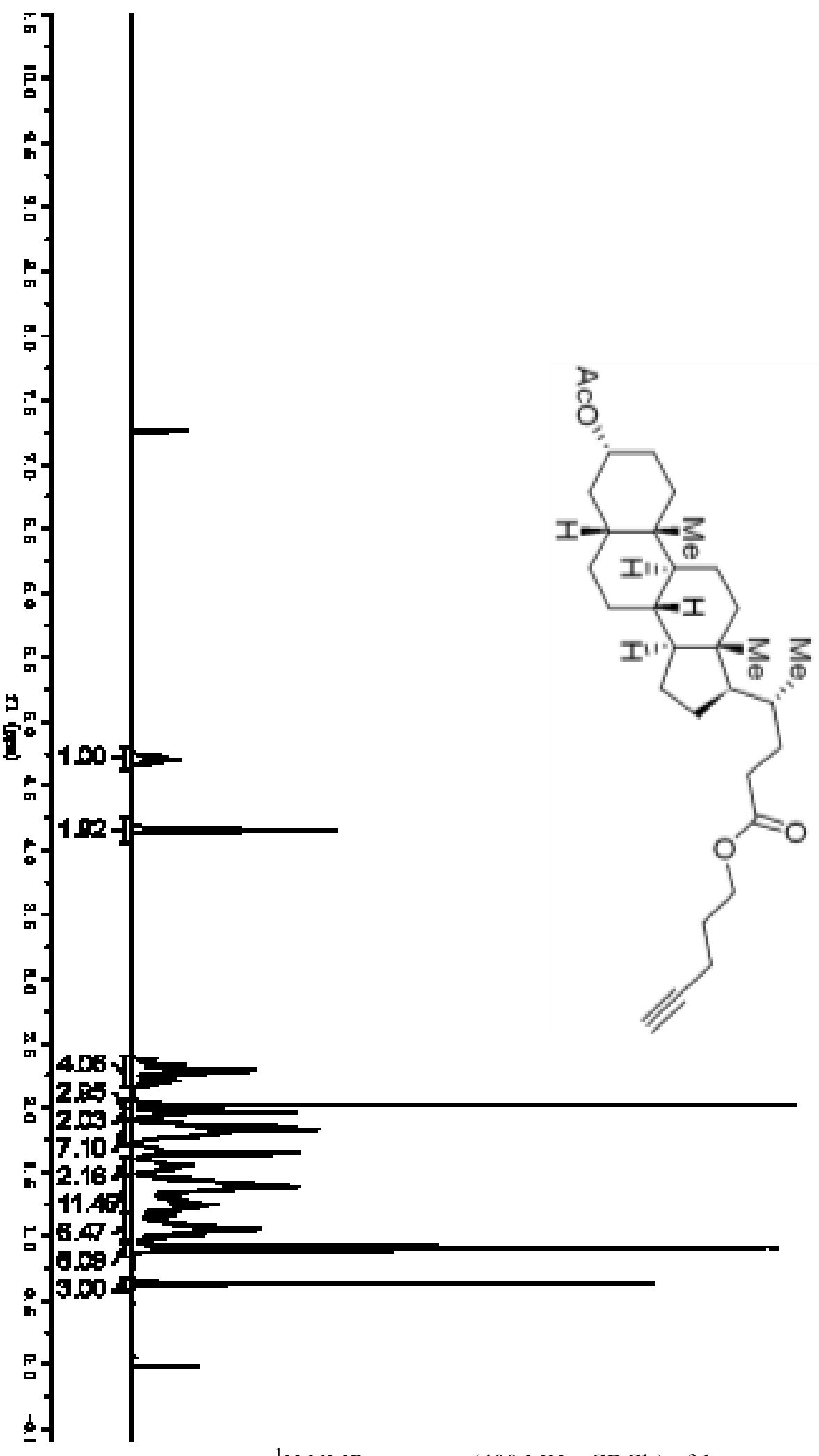

${ }^{1} \mathrm{H}$ NMR spectrum (400 MHz, $\mathrm{CDCl}_{3}$ ) of $\mathbf{1} \mathbf{t t}$ 


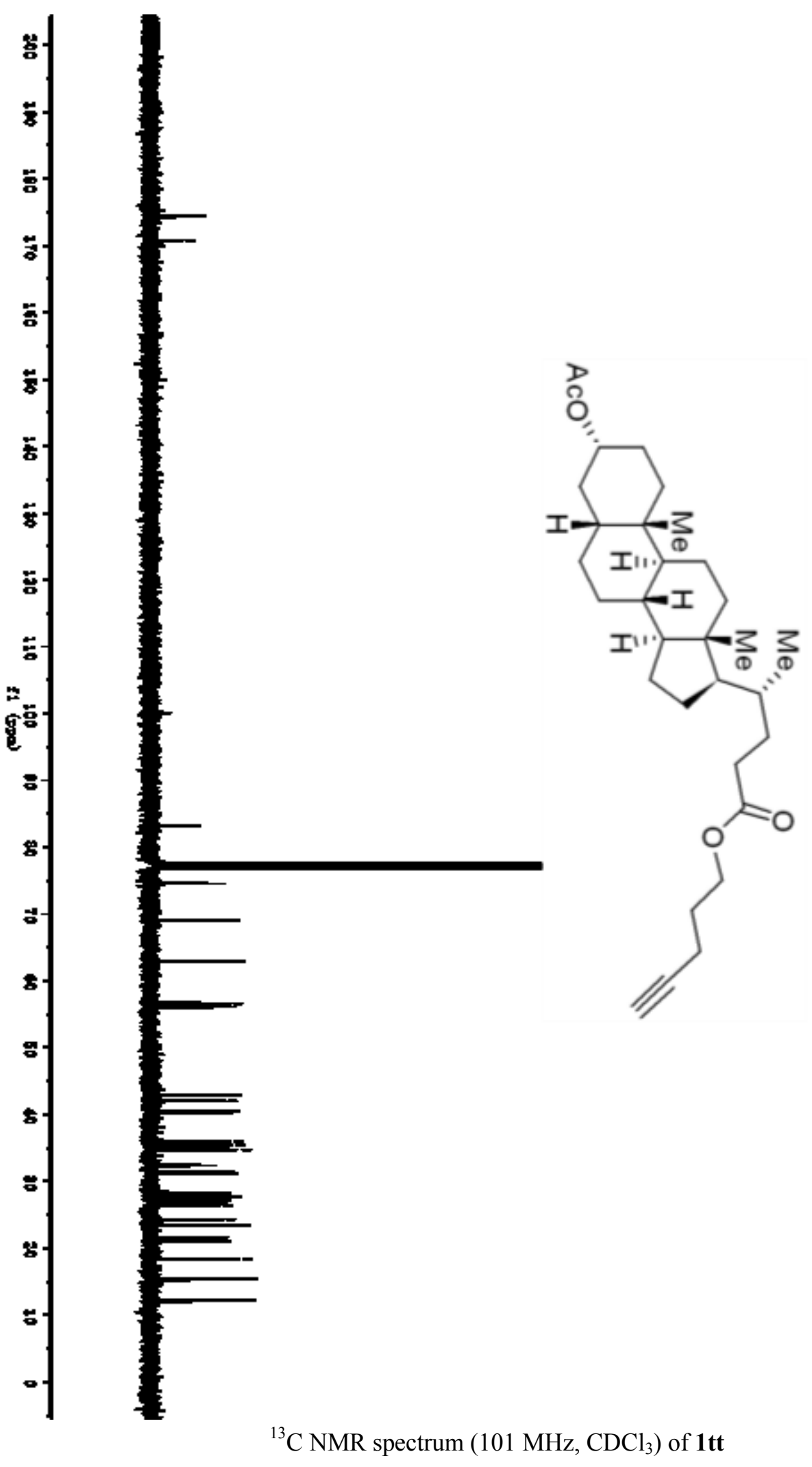

$-174.4$

$=170.8$

$-83.2$

$\checkmark 74.5$

$-69.1$

62.9

56.6

L56.1

42.9

442.0

$-40.5$

$-35.9$

135.5

34.7

31.4

31.1

27.7

$-24.3$

23.5

$-18.4$

$-15.4$

${ }^{13} \mathrm{C}$ NMR spectrum $\left(101 \mathrm{MHz}, \mathrm{CDCl}_{3}\right)$ of $\mathbf{1 t t}$ 


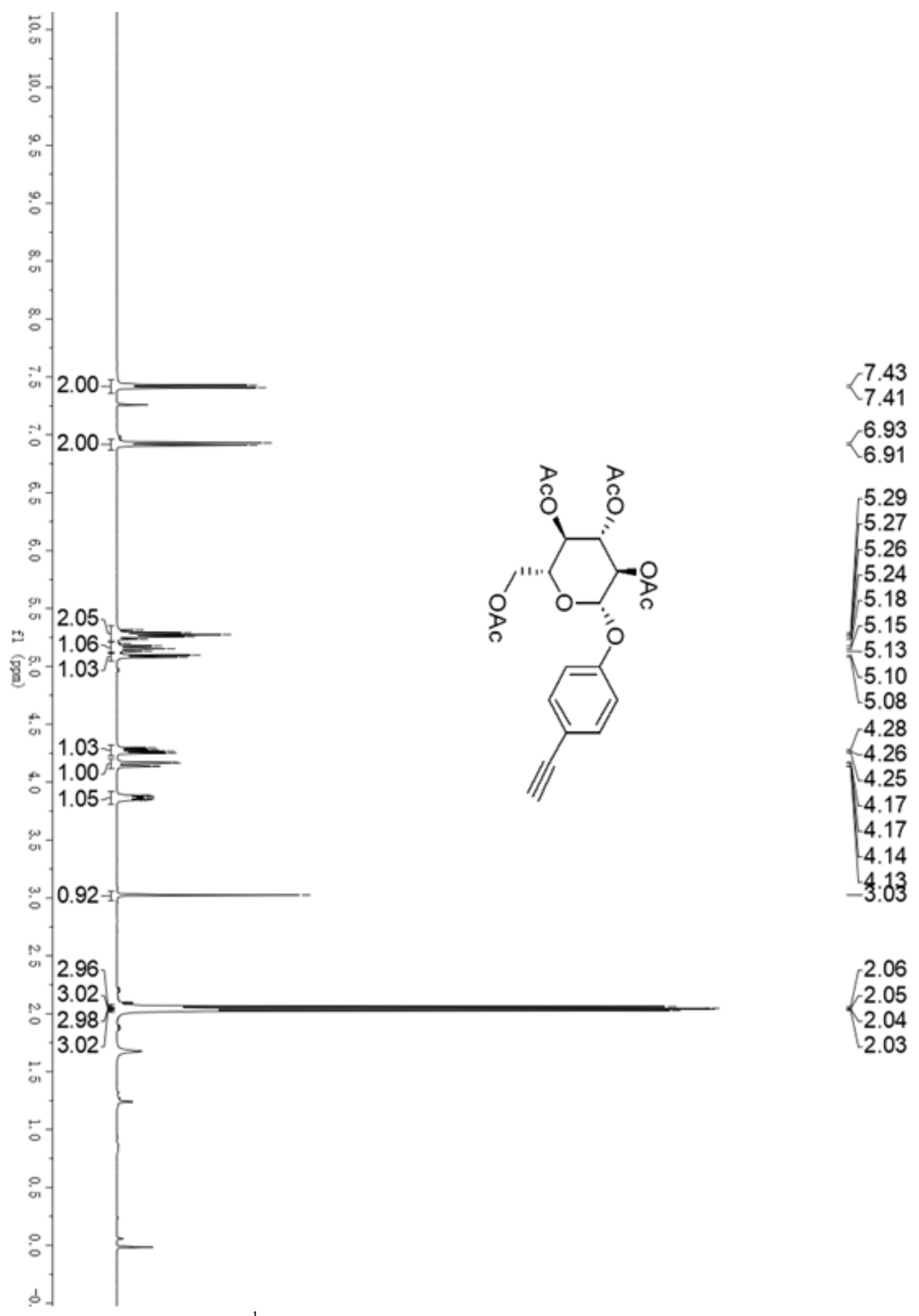

${ }^{1} \mathrm{H}$ NMR spectrum $\left(400 \mathrm{MHz}, \mathrm{CDCl}_{3}\right)$ of $\mathbf{1 u u}$ 


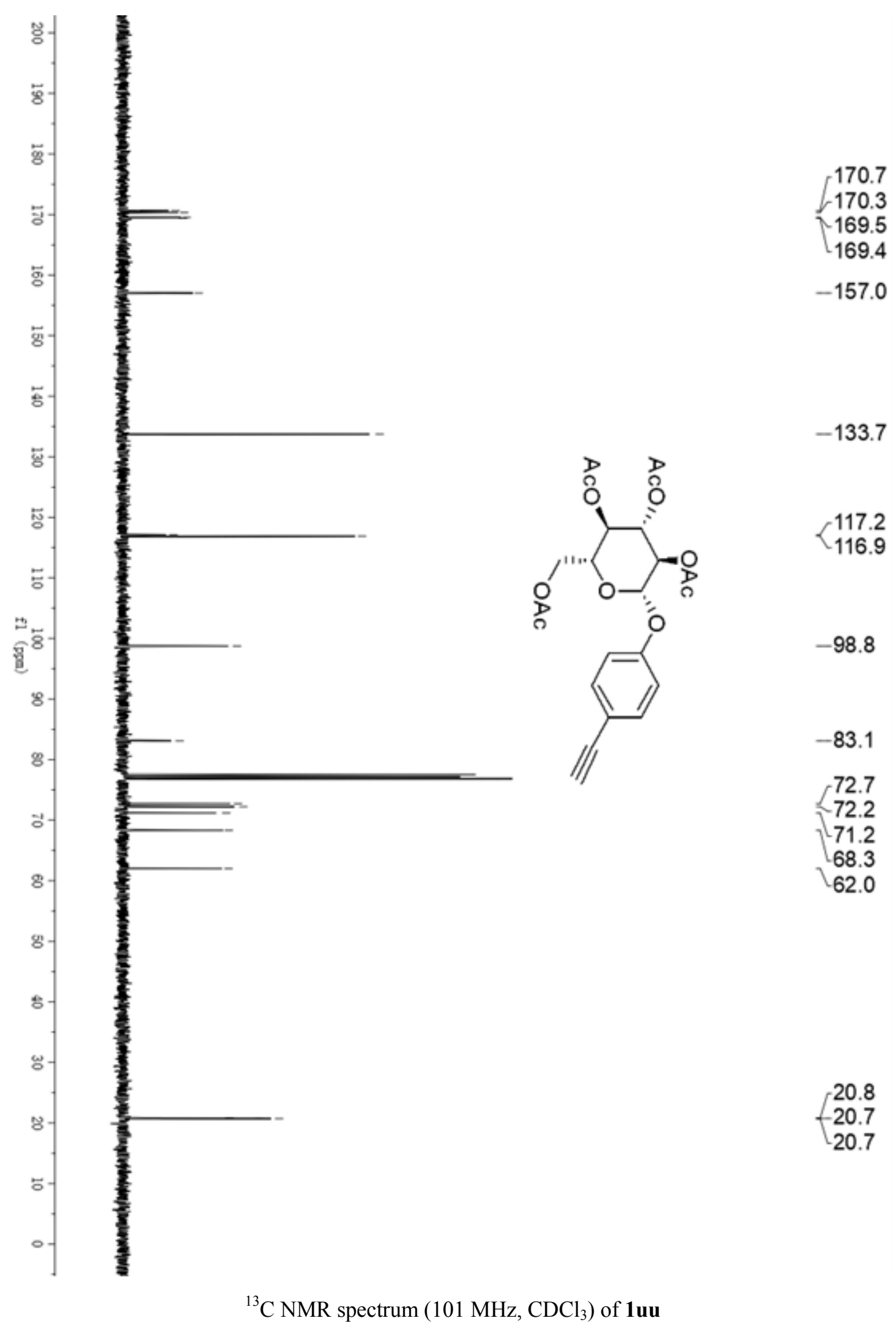




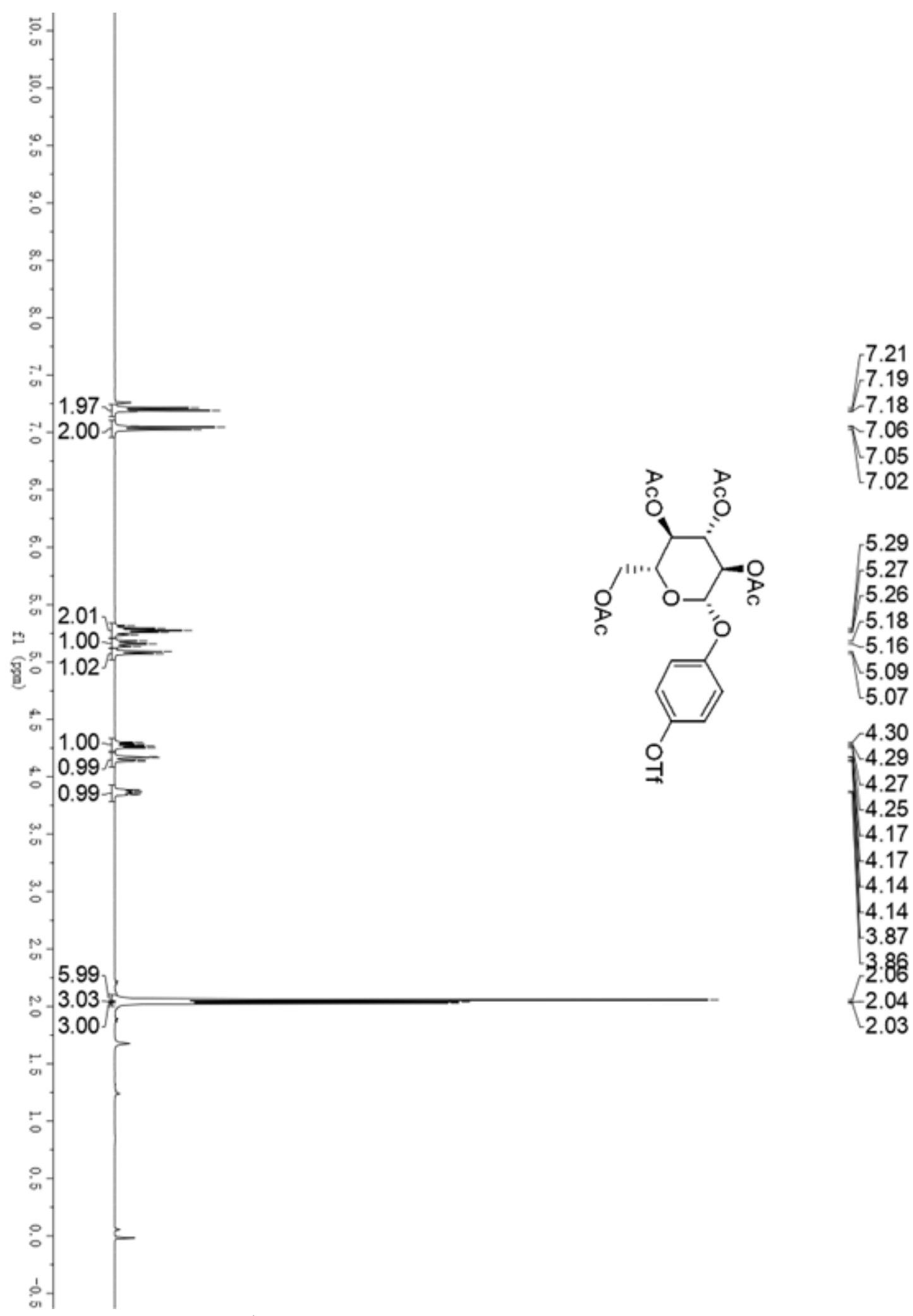

${ }^{1} \mathrm{H}$ NMR spectrum (400 MHz, $\left.\mathrm{CDCl}_{3}\right)$ of $1 \mathbf{u u}$ ' 


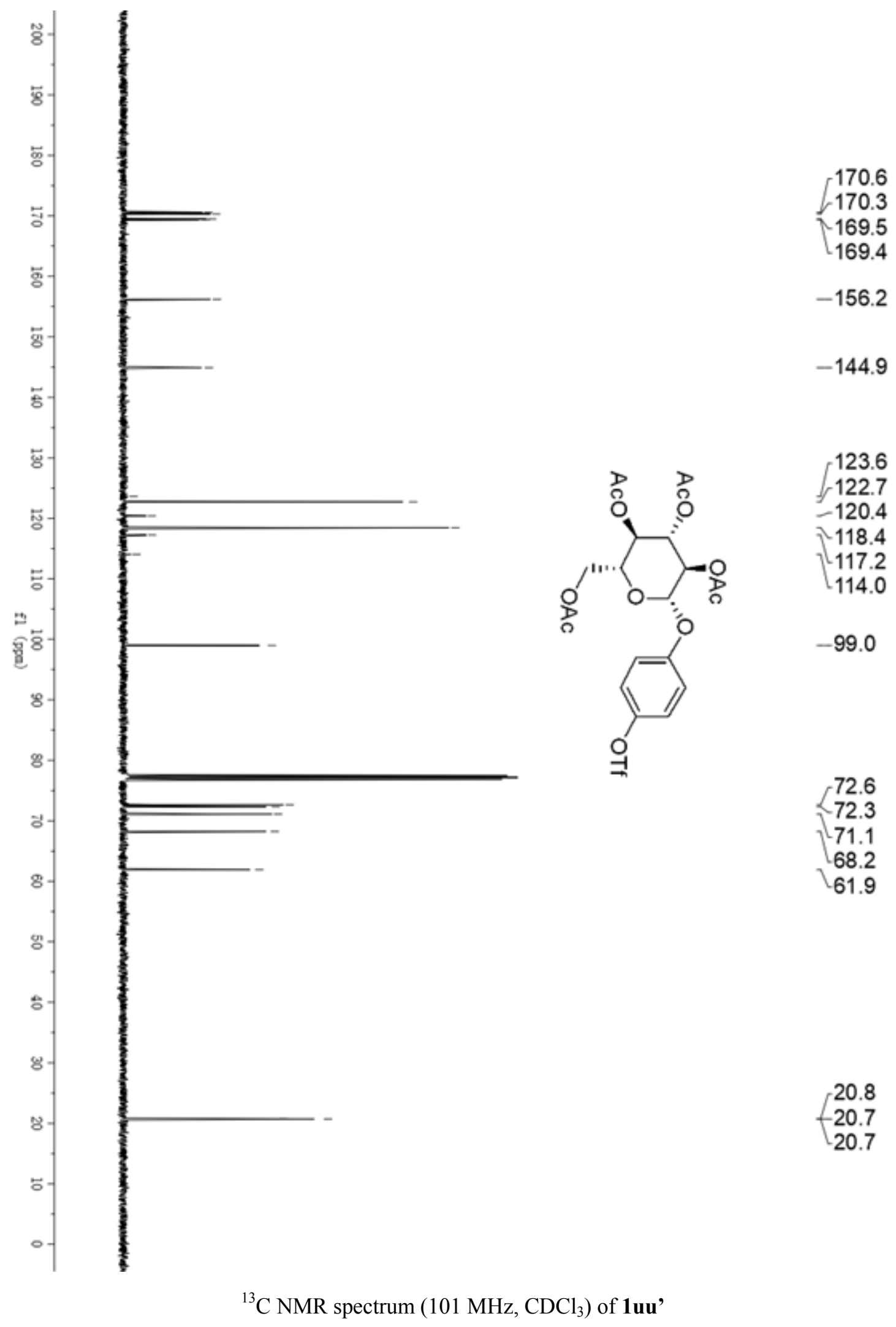




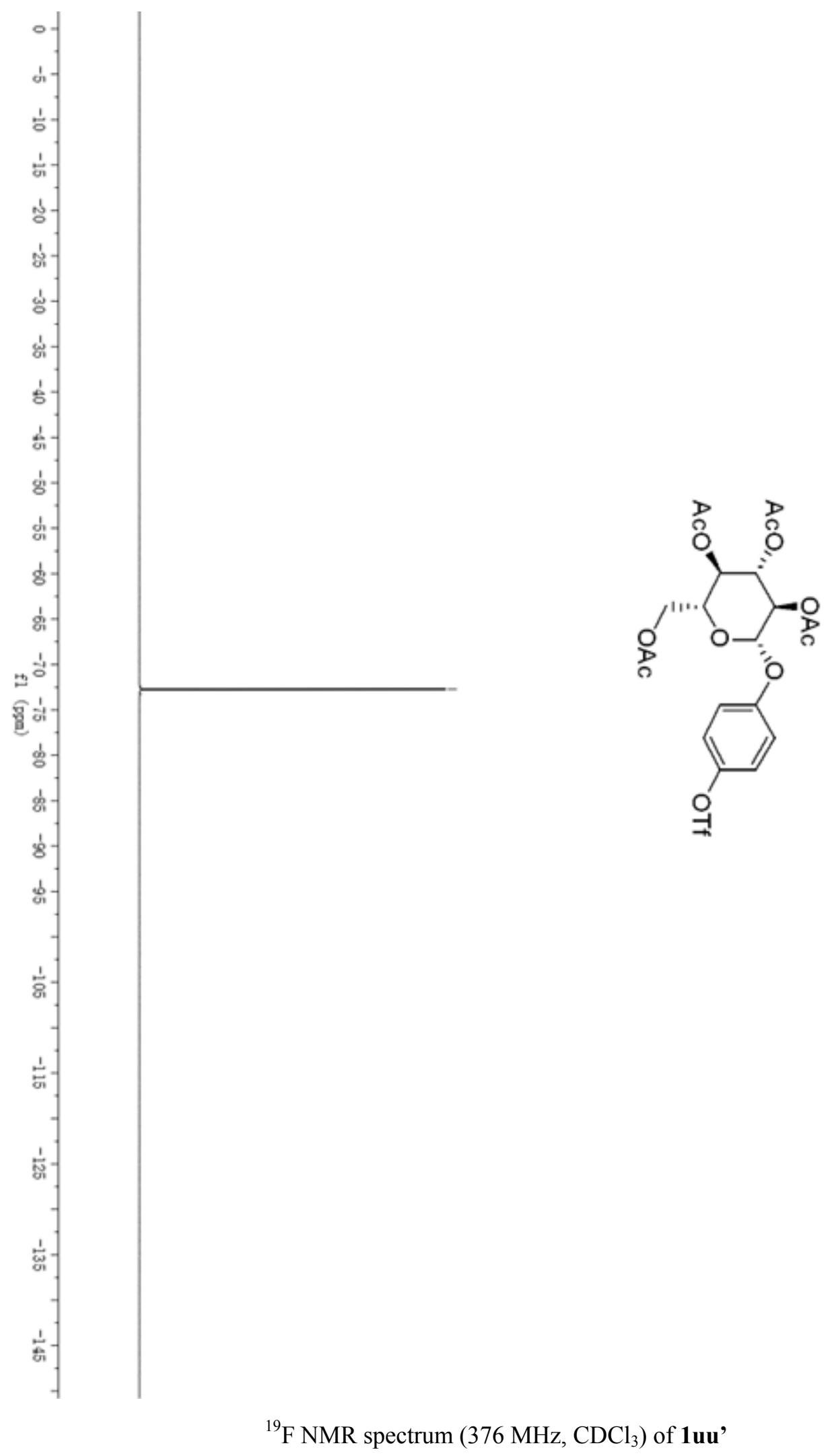




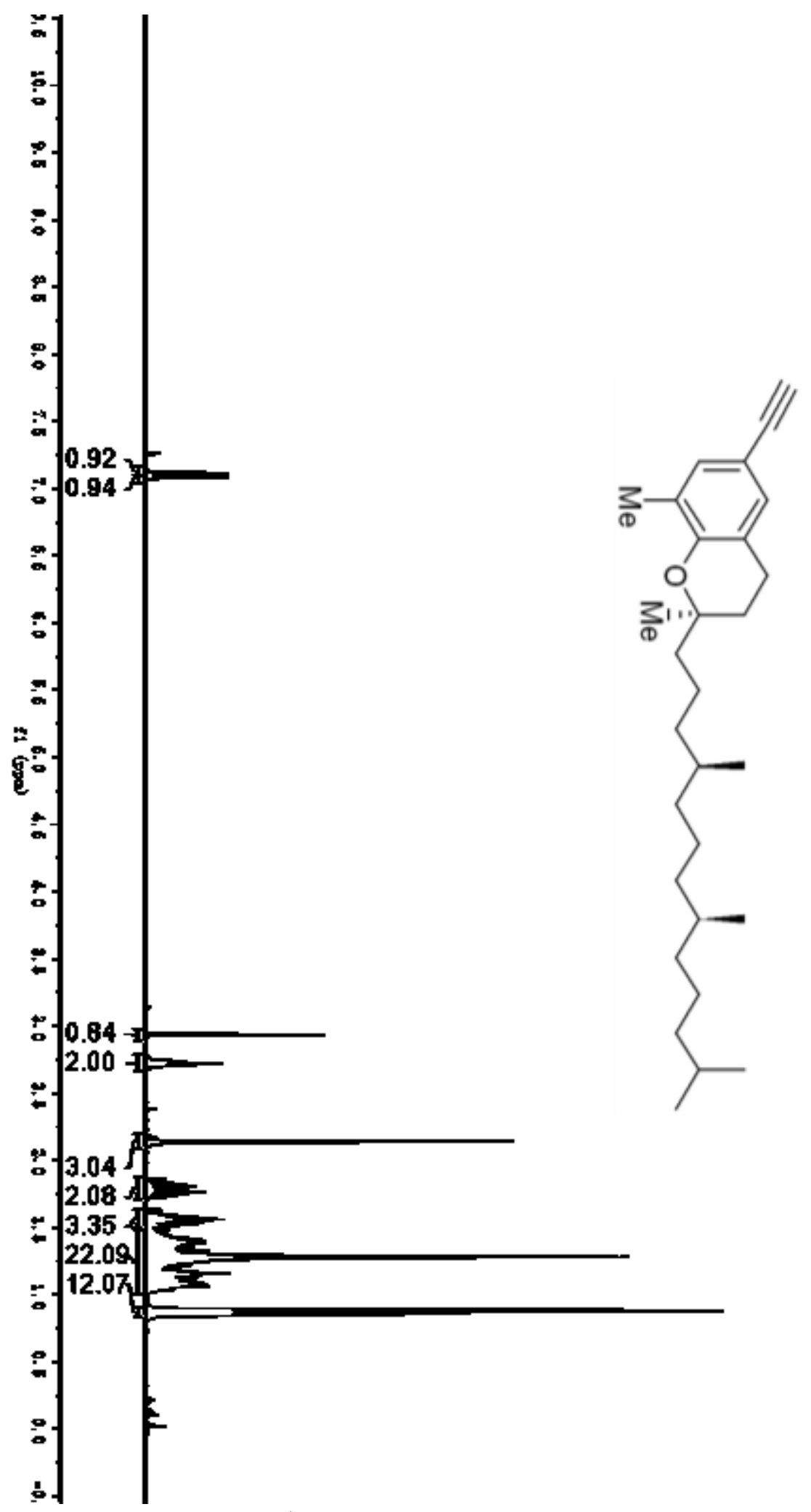

${ }^{1} \mathrm{H}$ NMR spectrum $\left(400 \mathrm{MHz}, \mathrm{CDCl}_{3}\right)$ of $\mathbf{1 w w}$

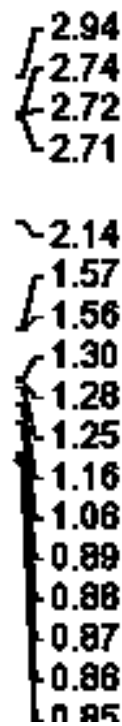




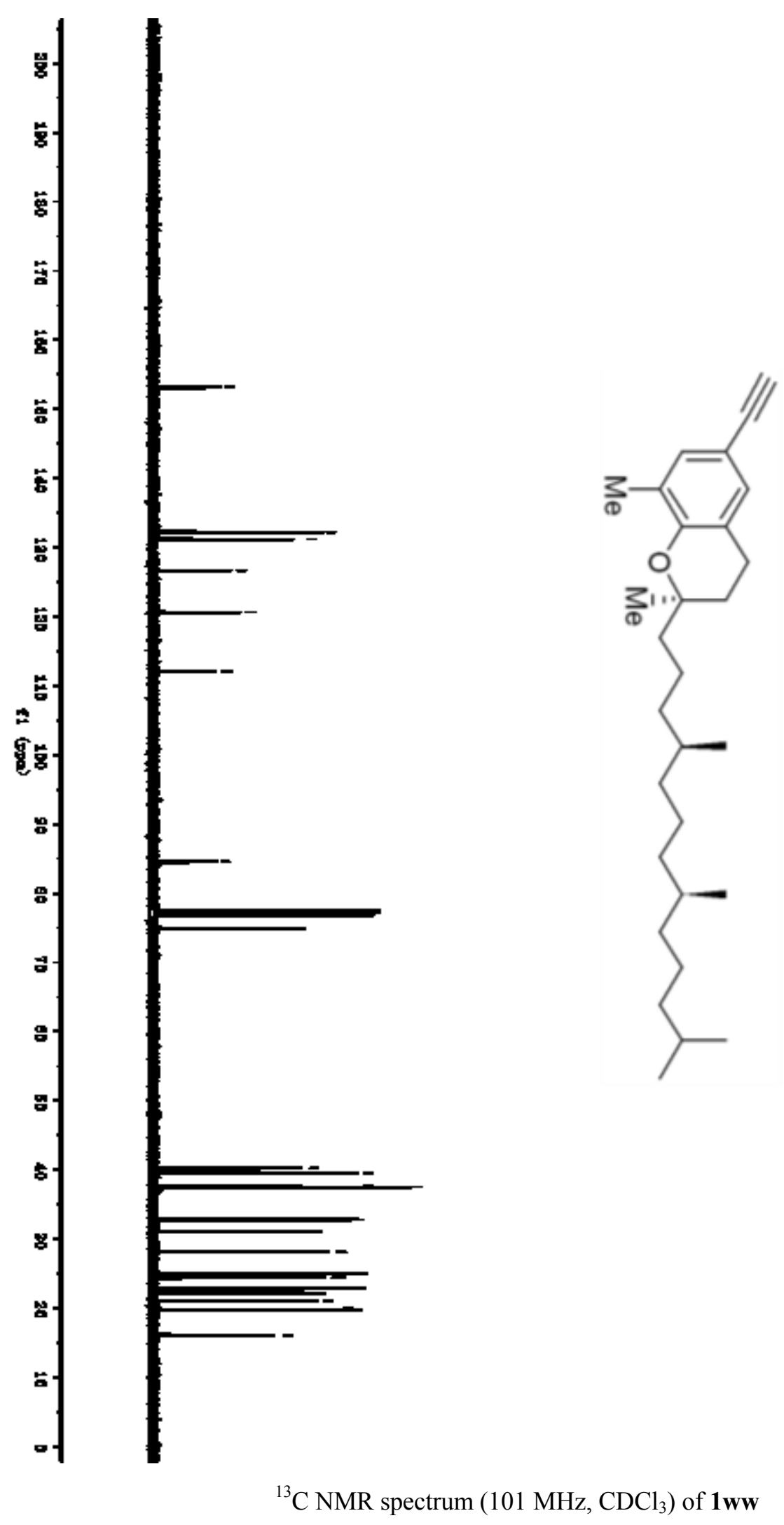

$\begin{array}{r}132.2 \\ 131.2 \\ \hdashline 128.6\end{array}$

$-120.6$

$-112.1$

-84.5
$J_{74.9}^{76.8}$

40.2

39.5

37.6

37.5

37.4

32.9

32.8

31.1

28.1

25.0

24.6

24.4

22.9

22.8

22.2

21.1

19.9

${ }^{13} \mathrm{C}$ NMR spectrum $\left(101 \mathrm{MHz}, \mathrm{CDCl}_{3}\right)$ of $\mathbf{1 w w}$ 


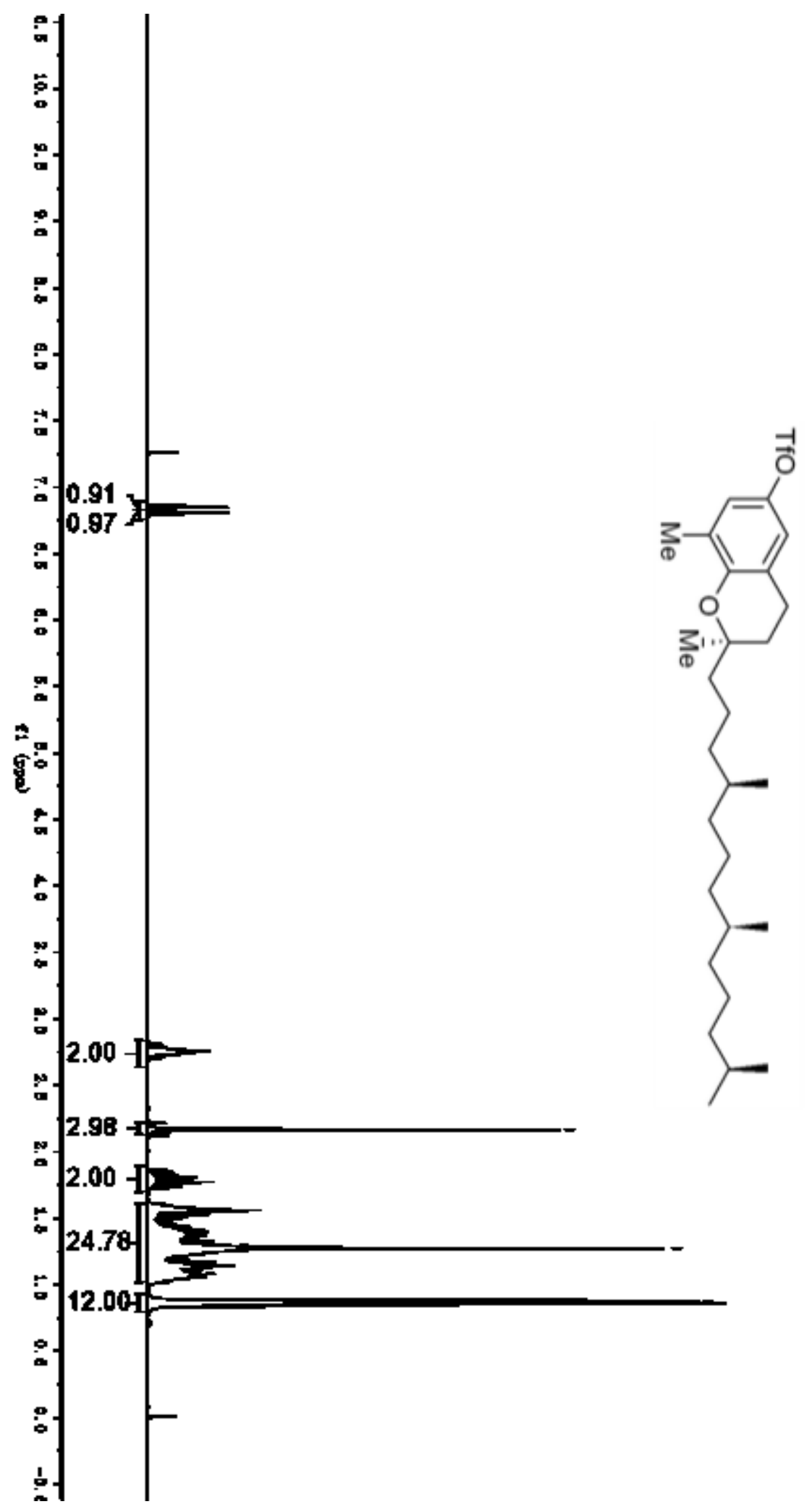

6.86

6.85

$-6.81$

6.80

2.82

2.80

2.78

$-2.76$

2.74

2.74

271

2.69

$\backslash 2.17$

1.56

1.29

$-1.28$

1.27

1.25

$-1.16$

$-1.15$

$-0.88$

$-0.87$

0.86

0.86

${ }^{1} \mathrm{H}$ NMR spectrum $\left(400 \mathrm{MHz}, \mathrm{CDCl}_{3}\right)$ of $\mathbf{1} \mathbf{w w}$ 


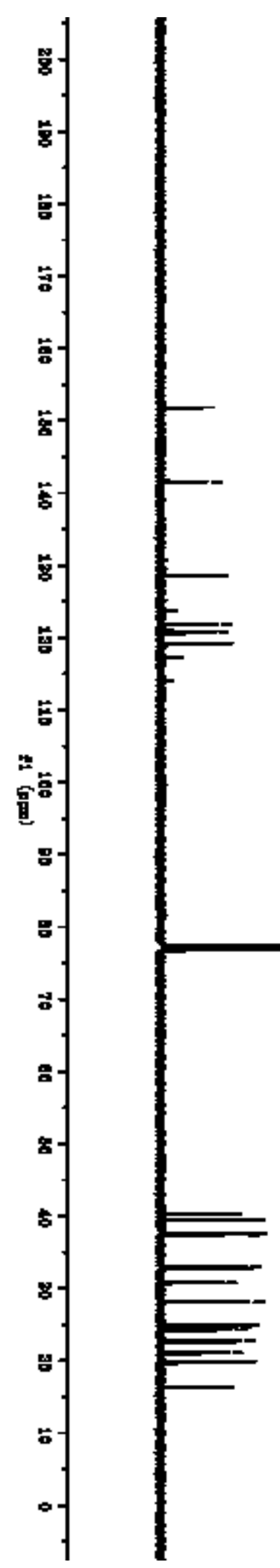

$-151.8$

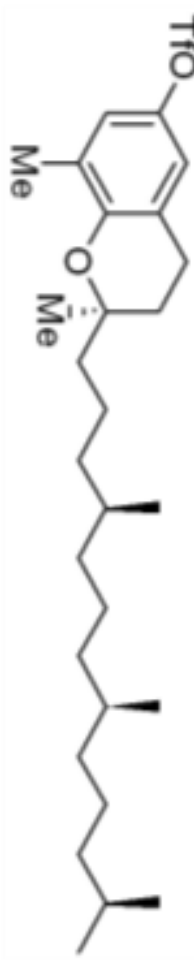

$-141.6$

128.5

123.7

121.8

120.8

120.5

119.2

117.3

114.0

$-77.0$

40.3

39.5

37.6

37.5

37.5

37.4

32.9

32.8

30.8

28.1

25.0

24.6

24.3

22.9

22.8

22.6

21.1

19.9

${ }^{13} \mathrm{C}$ NMR spectrum (101 MHz, $\mathrm{CDCl}_{3}$ ) of $\mathbf{1} \mathbf{w w}^{\mathbf{*}}$ 


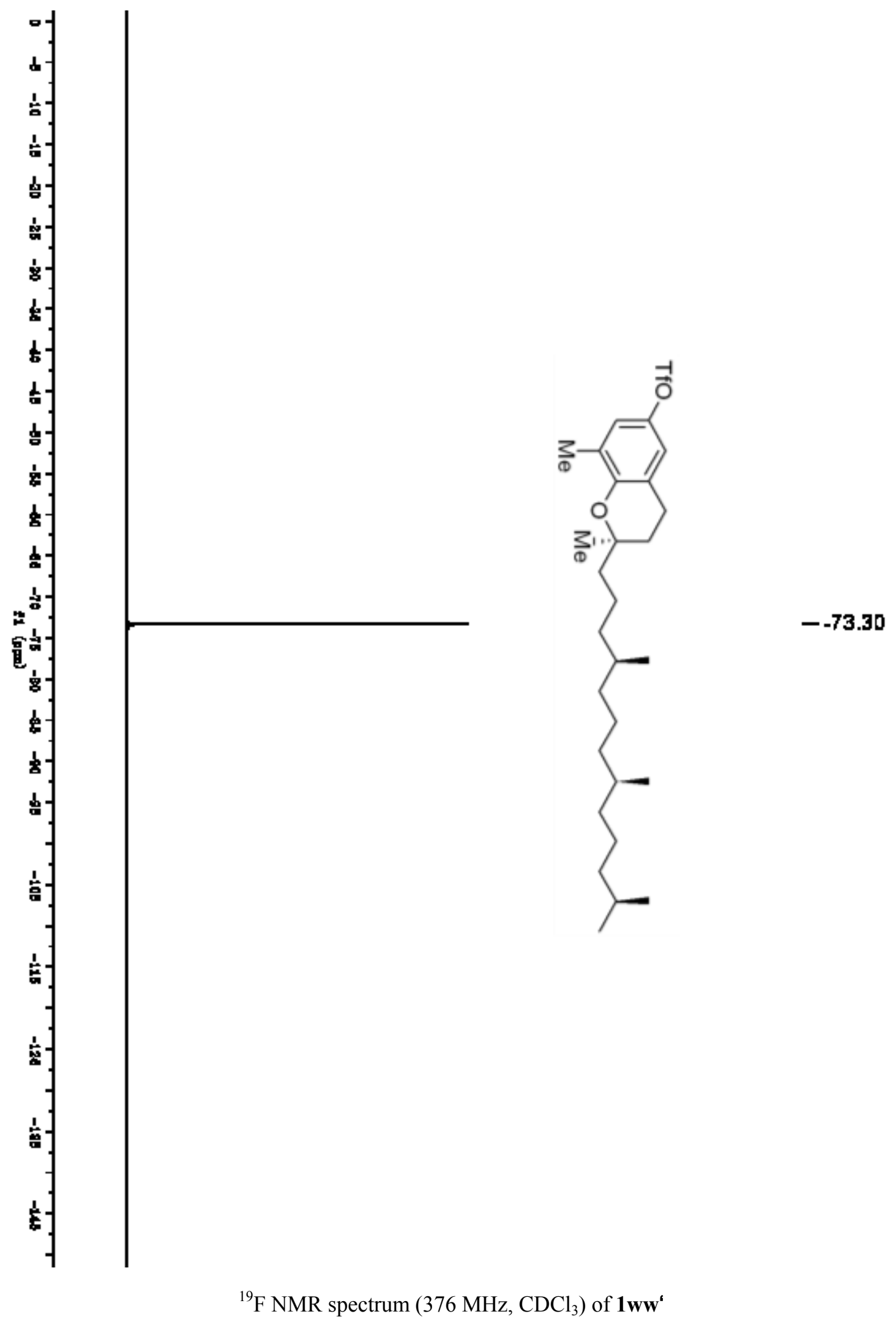




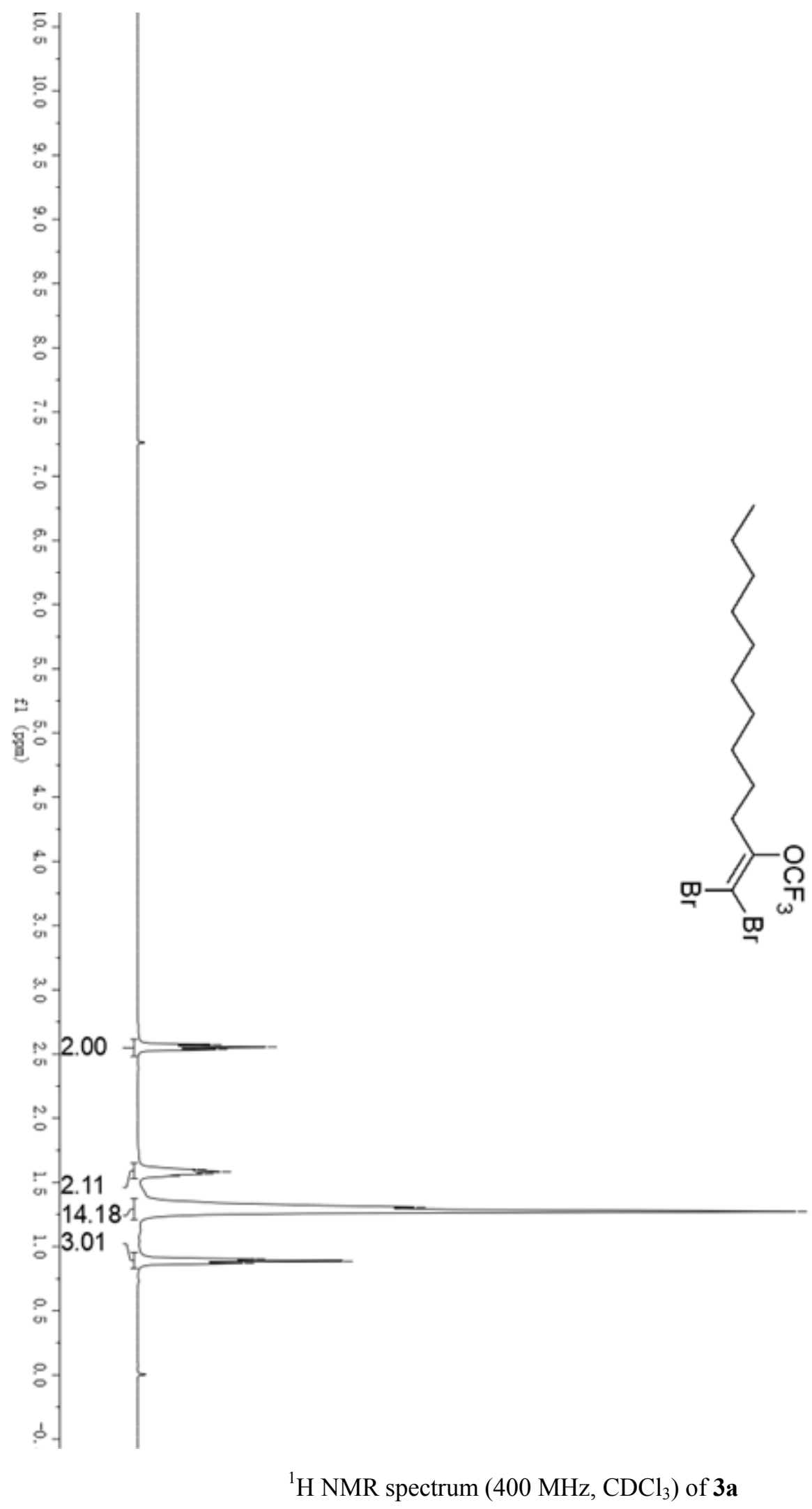

2.57

$-2.55$

2.54

1.60

1.58

$-1.57$

1.55

1.31

1.27

0.90

0.89

0.87 


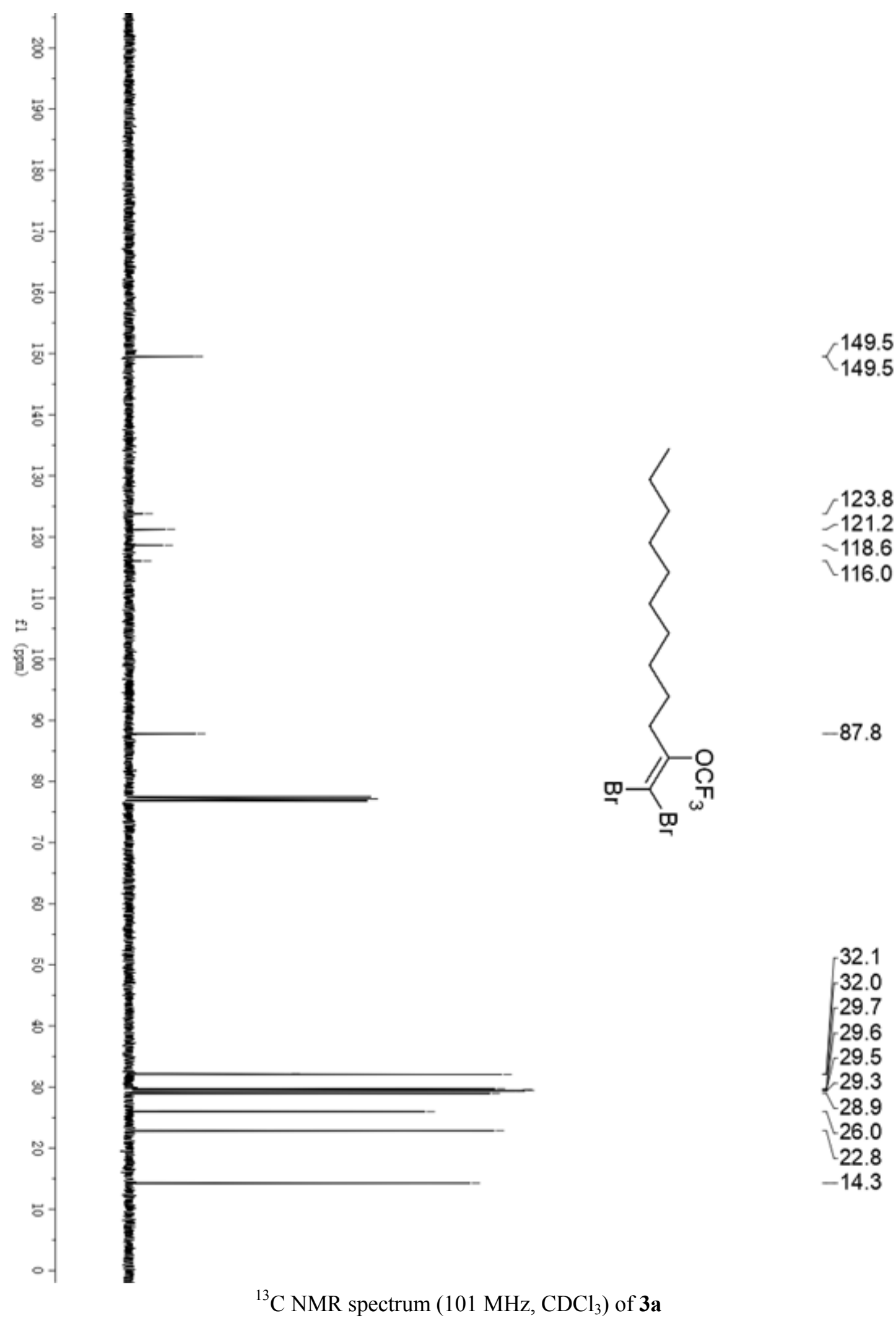




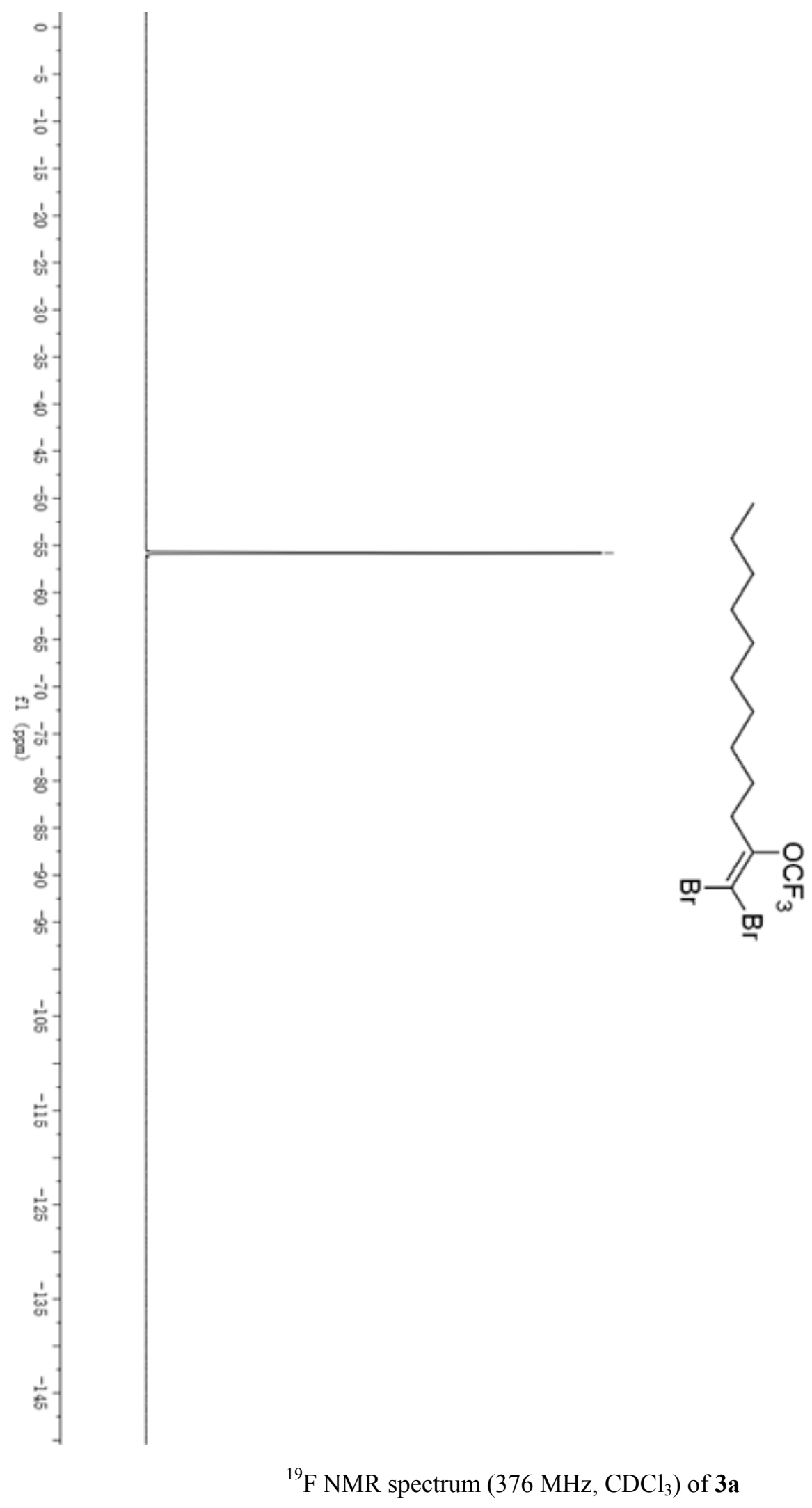




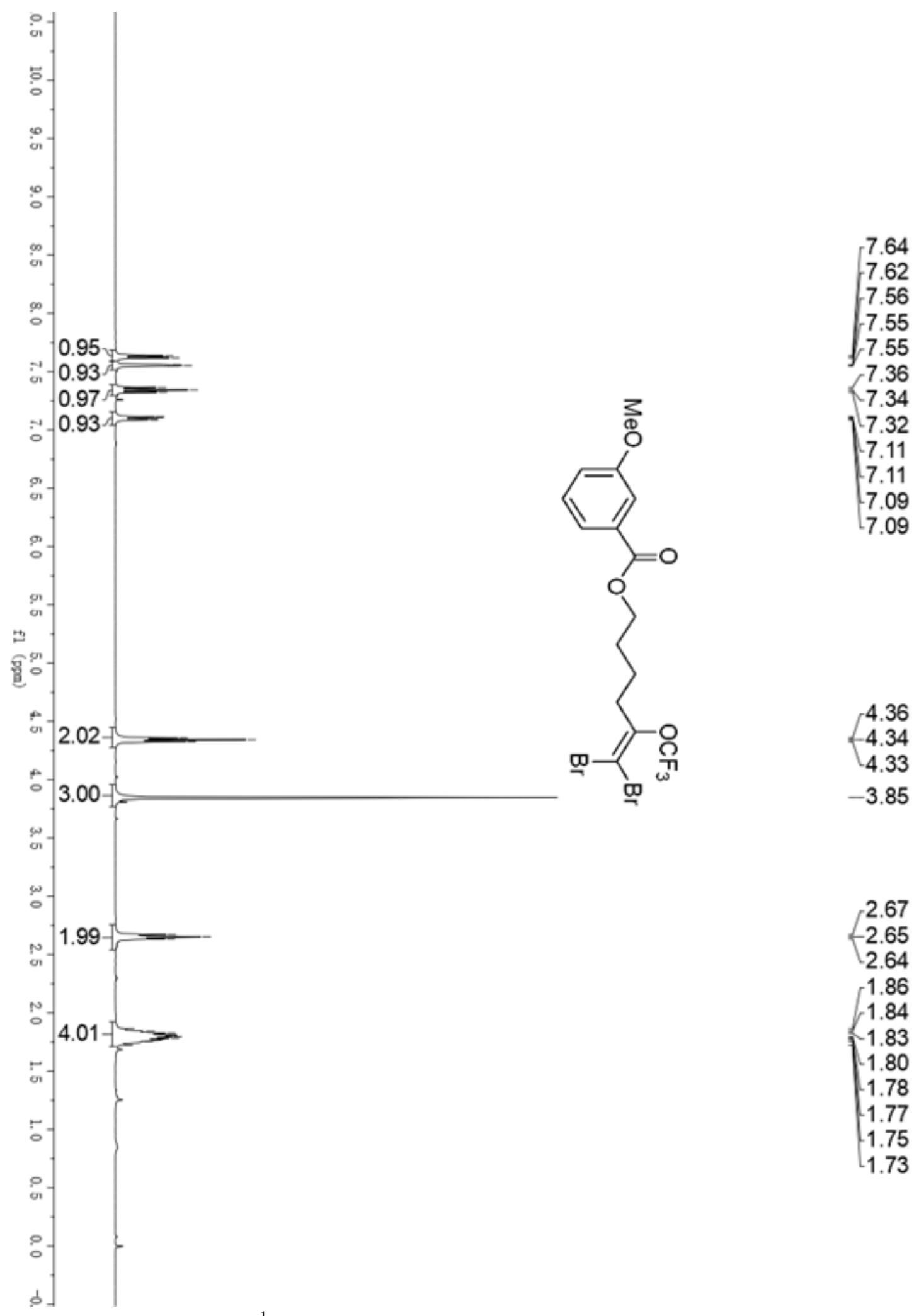

${ }^{1} \mathrm{H}$ NMR spectrum $\left(400 \mathrm{MHz}, \mathrm{CDCl}_{3}\right)$ of $\mathbf{3 b}$ 


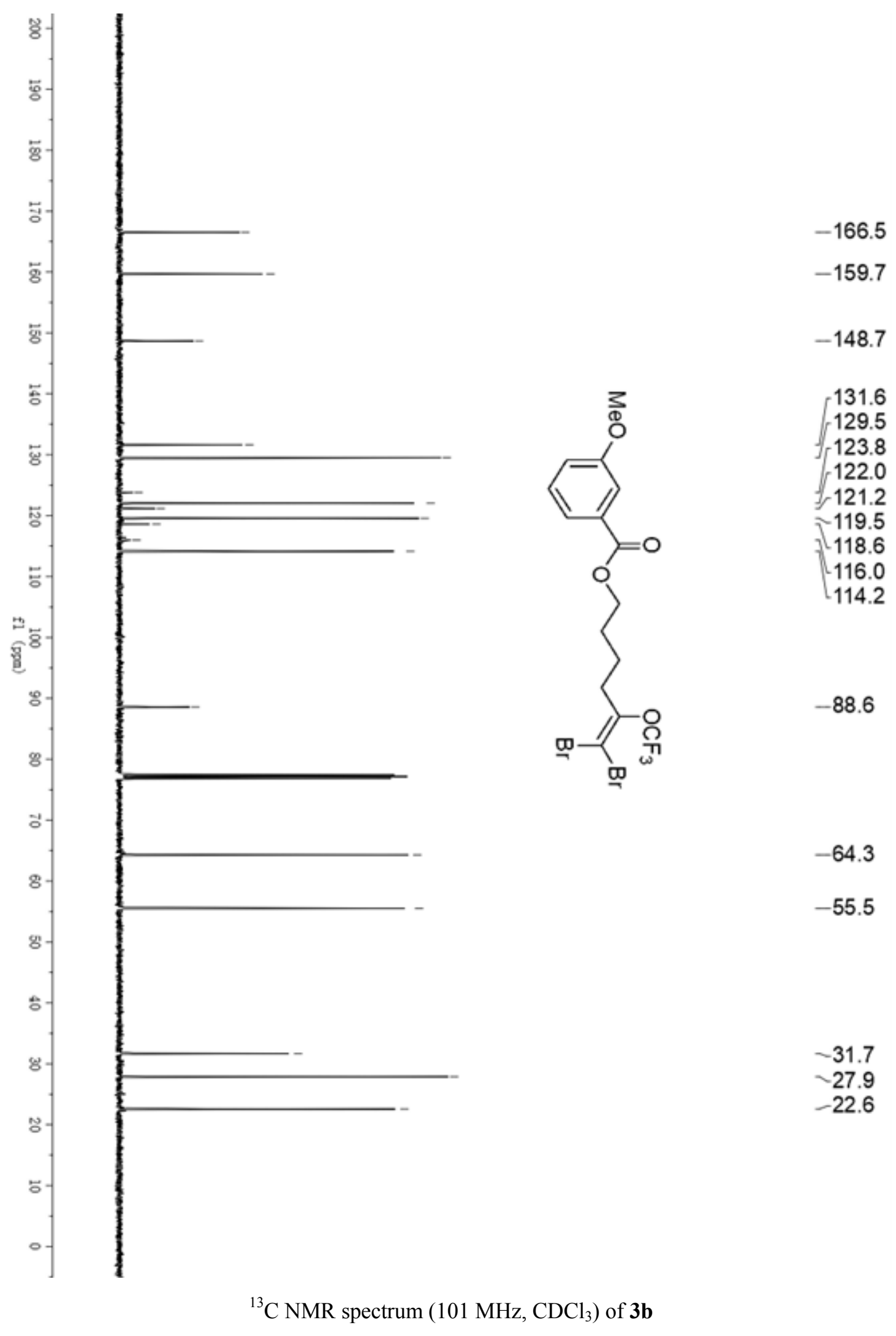




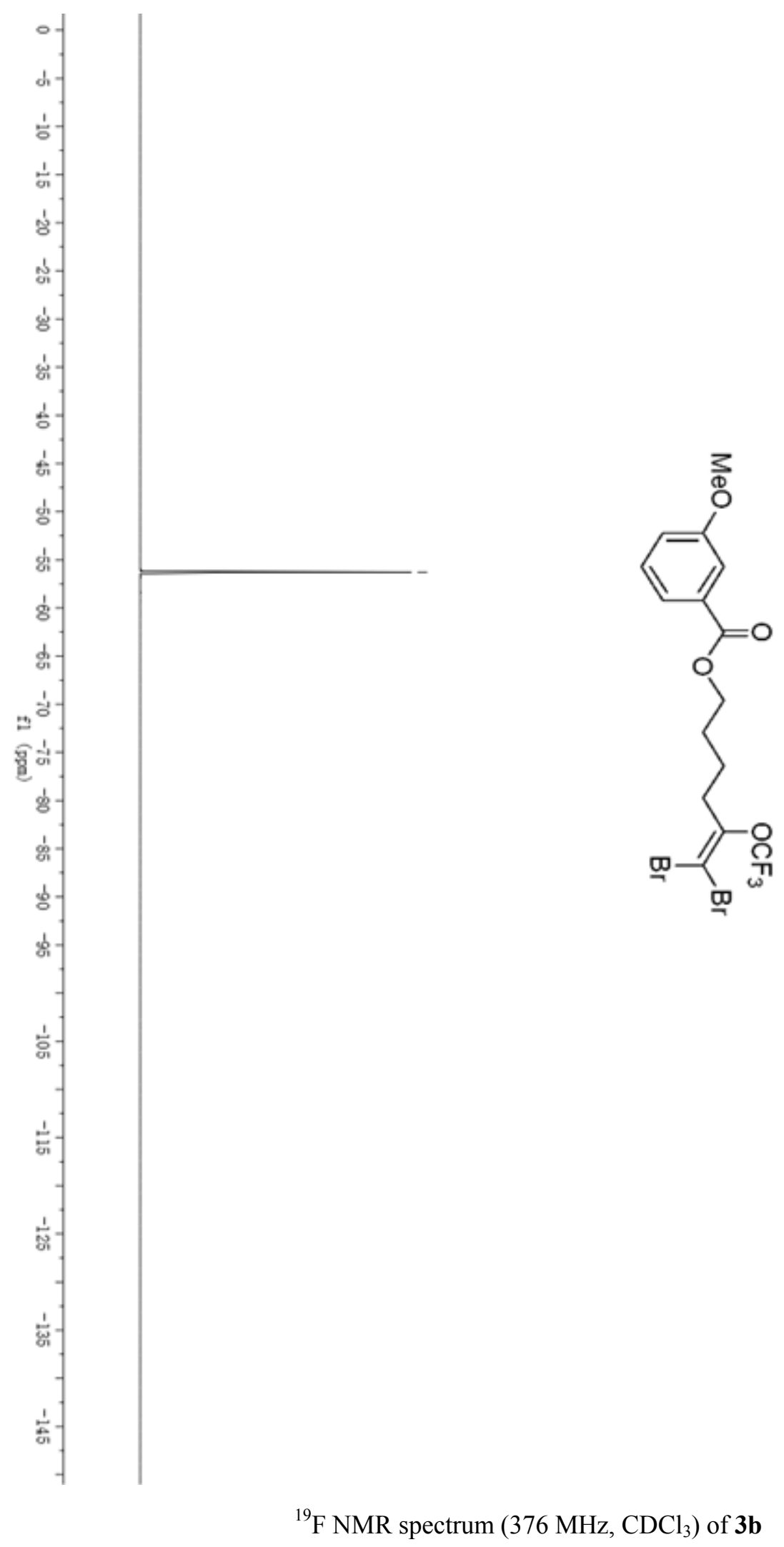




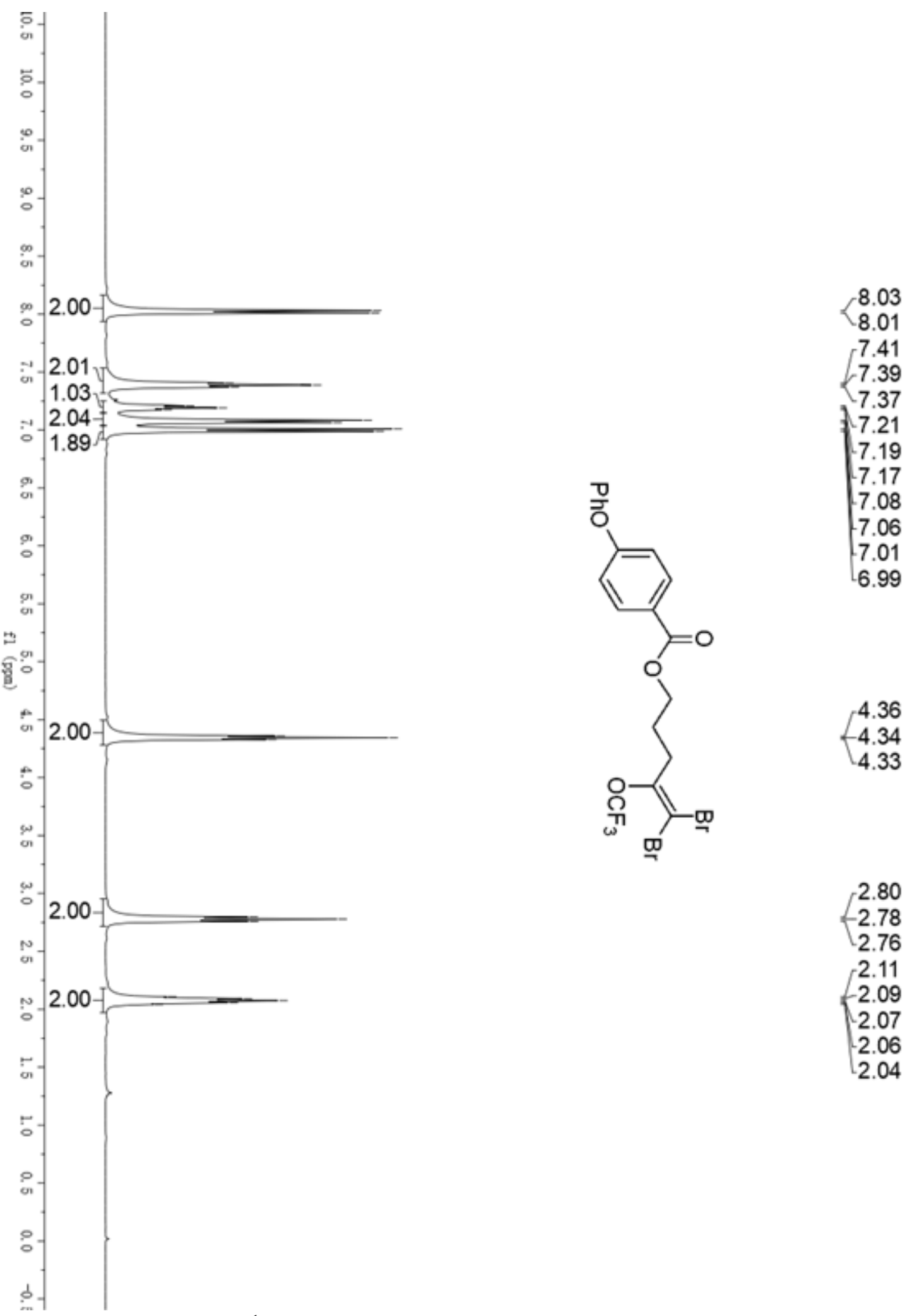

${ }^{1} \mathrm{H}$ NMR spectrum $\left(400 \mathrm{MHz}, \mathrm{CDCl}_{3}\right)$ of $\mathbf{3 c}$ 


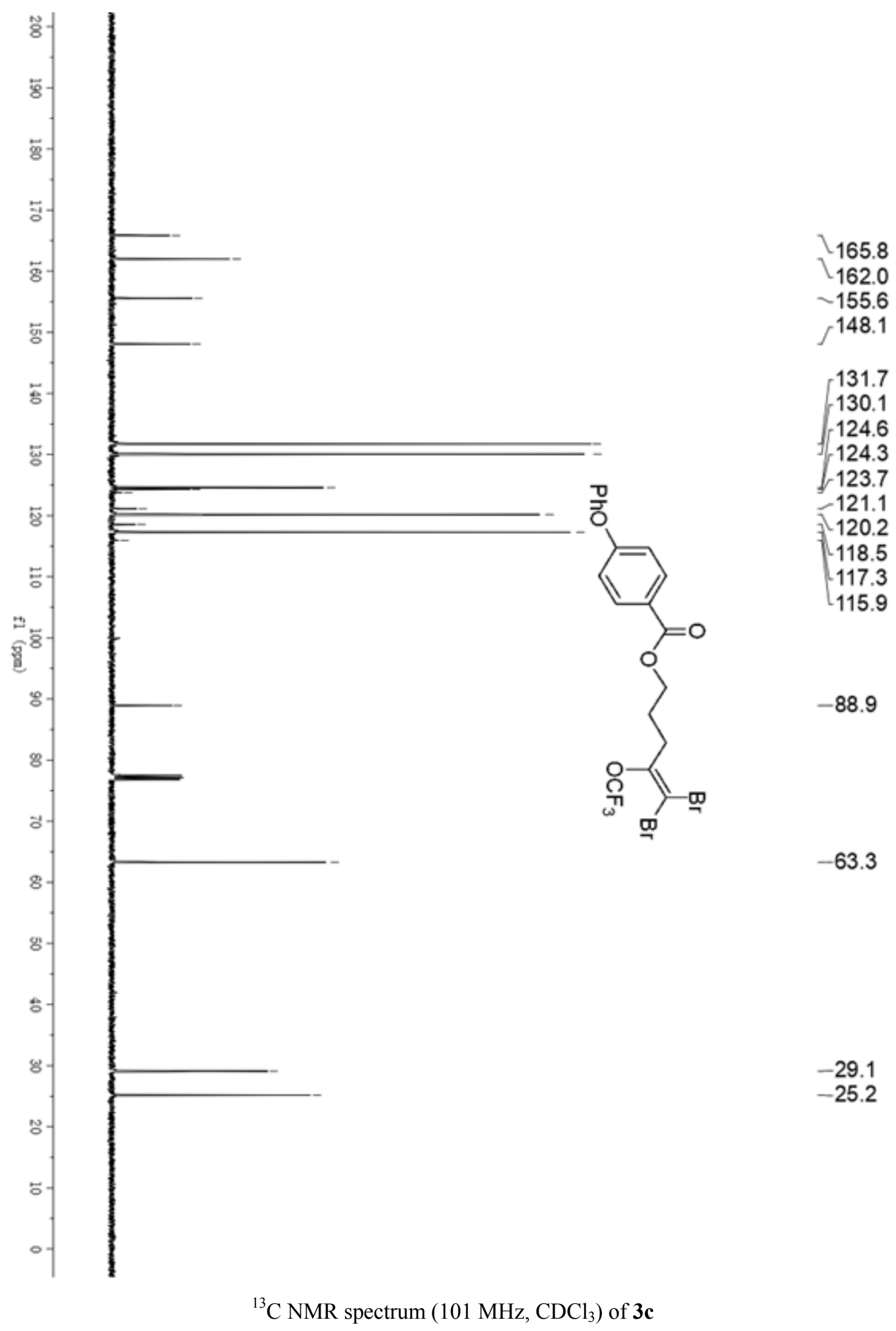




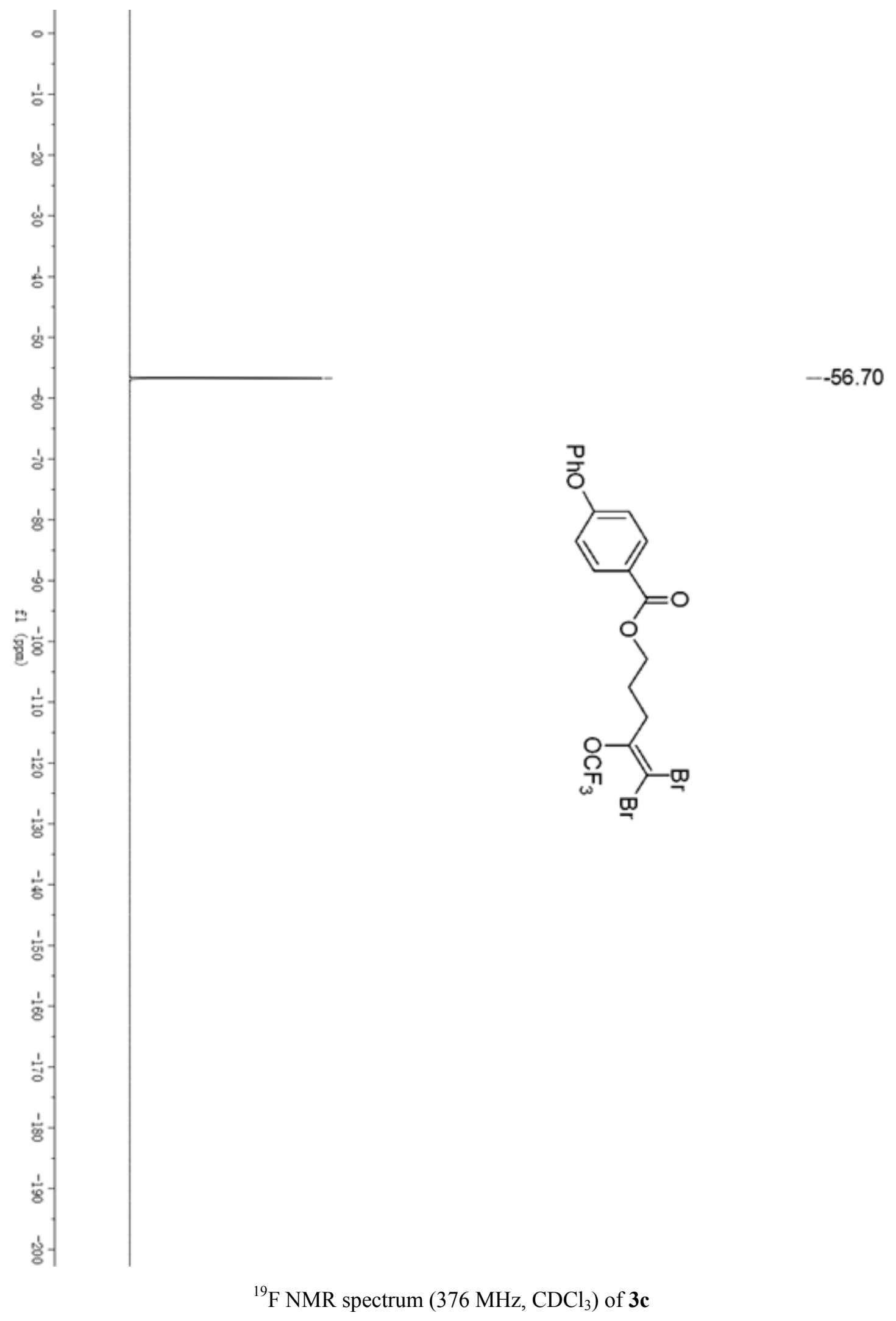




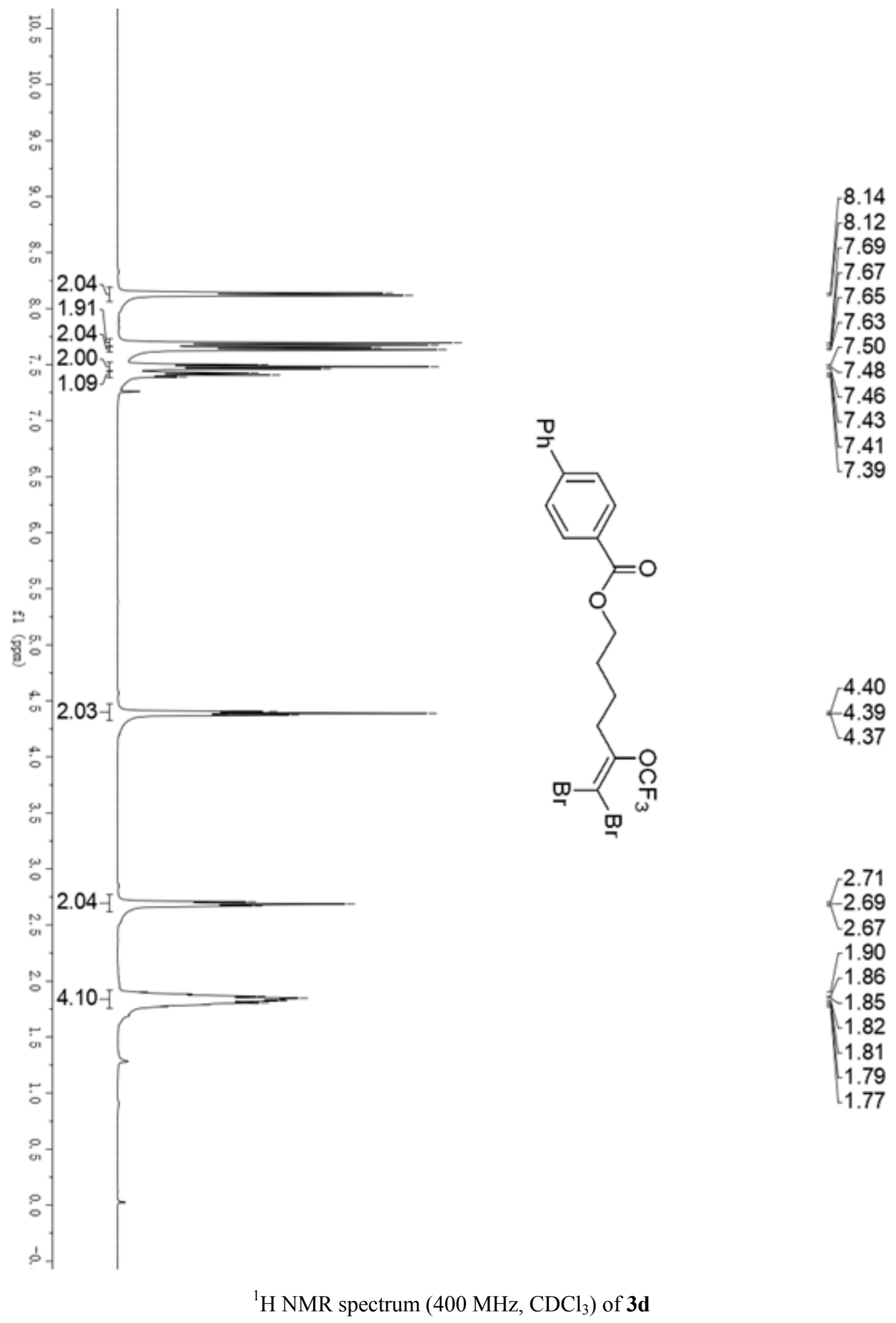




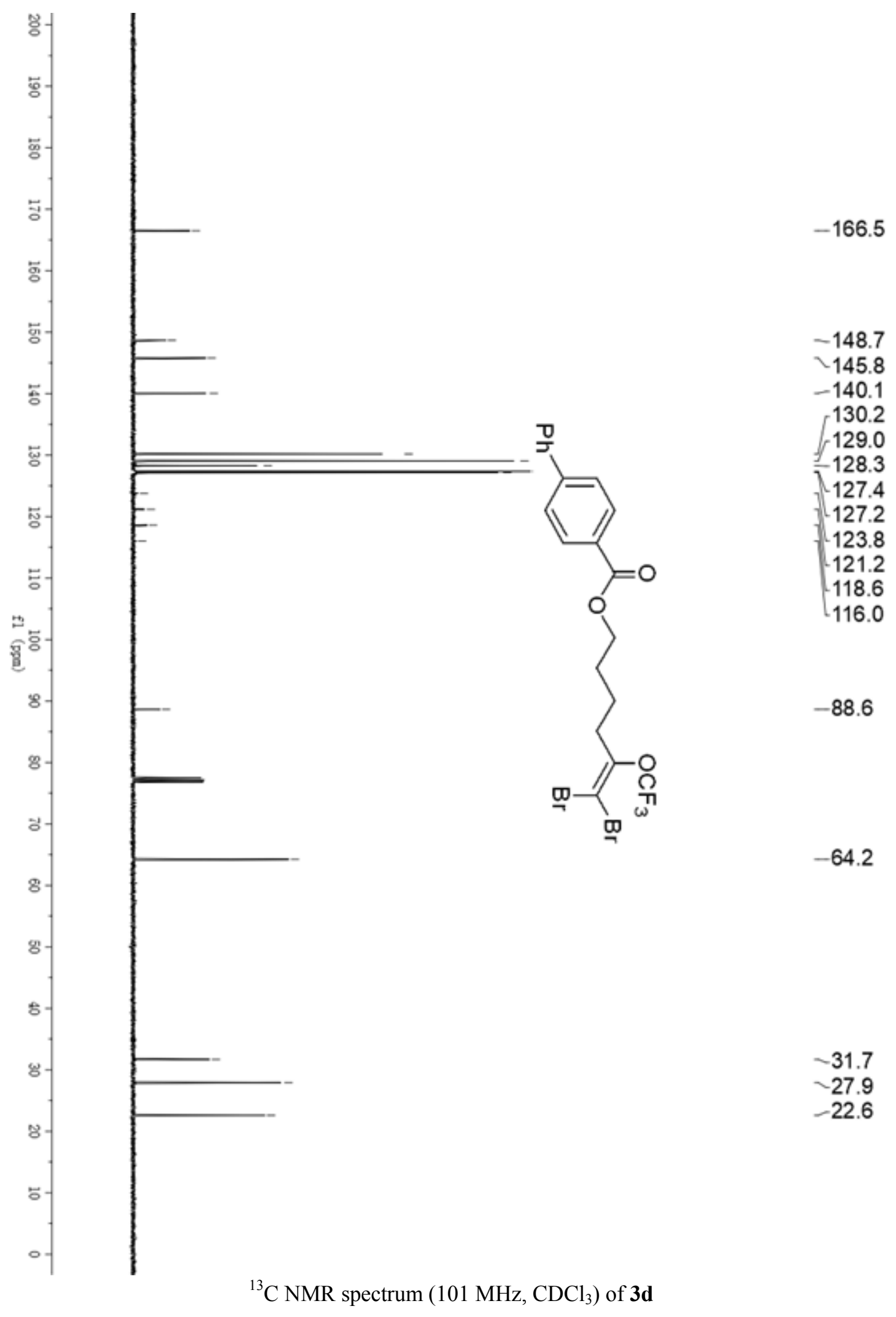




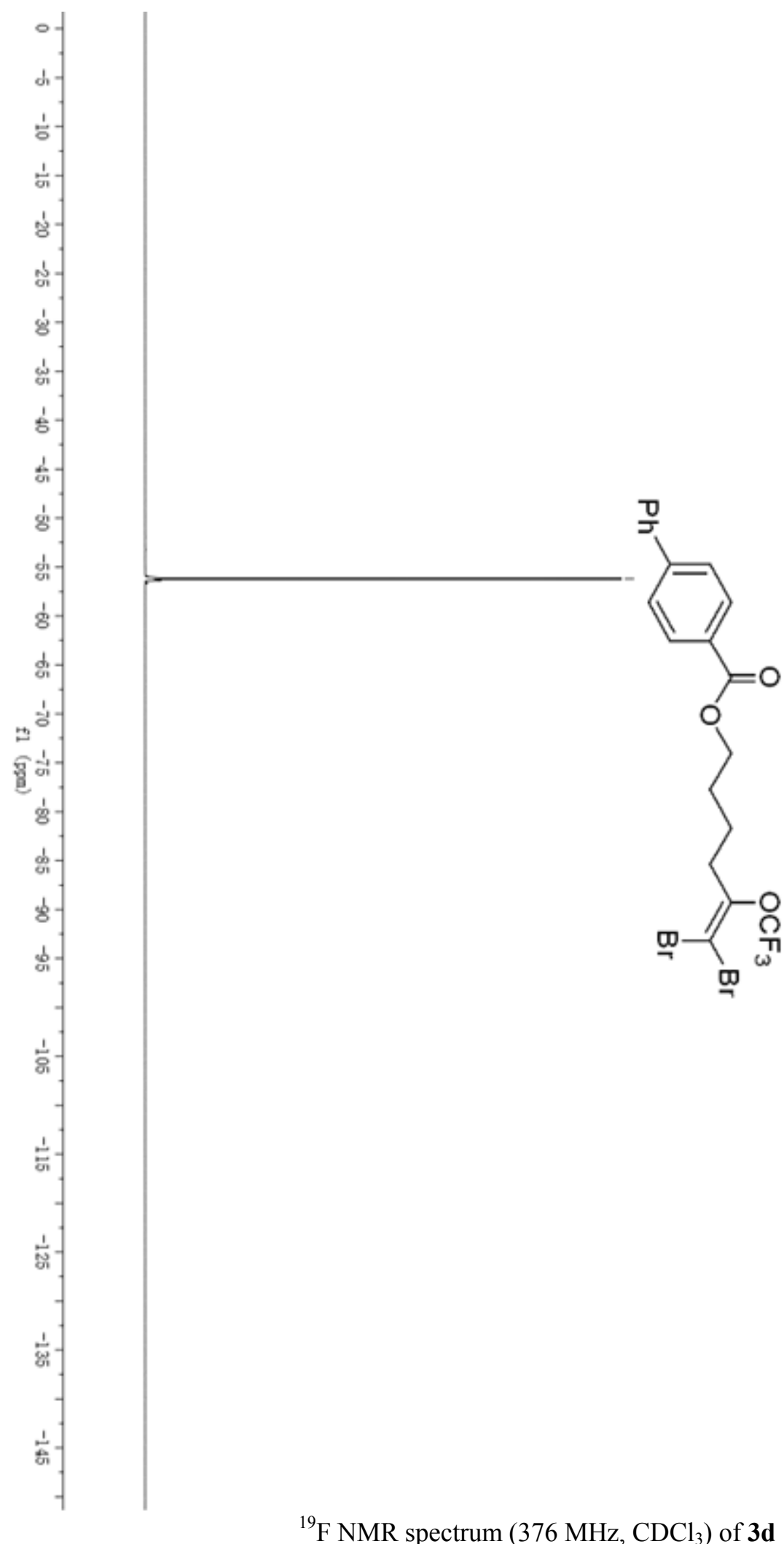

${ }^{19} \mathrm{~F} \mathrm{NMR} \mathrm{spectrum}\left(376 \mathrm{MHz}, \mathrm{CDCl}_{3}\right)$ of $\mathbf{3 d}$ 


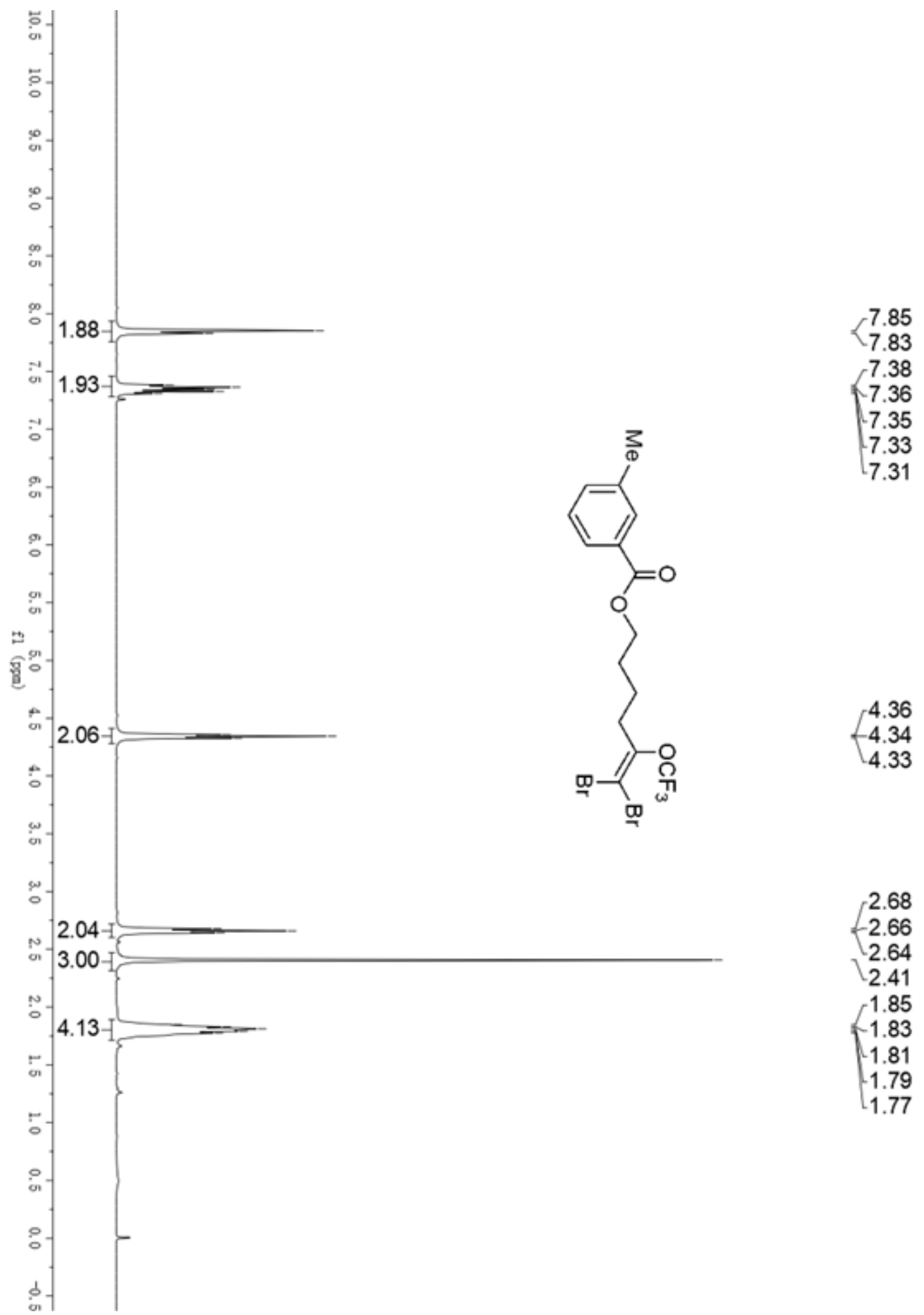

${ }^{1} \mathrm{H}$ NMR spectrum $\left(400 \mathrm{MHz}, \mathrm{CDCl}_{3}\right)$ of $\mathbf{3 e}$ 


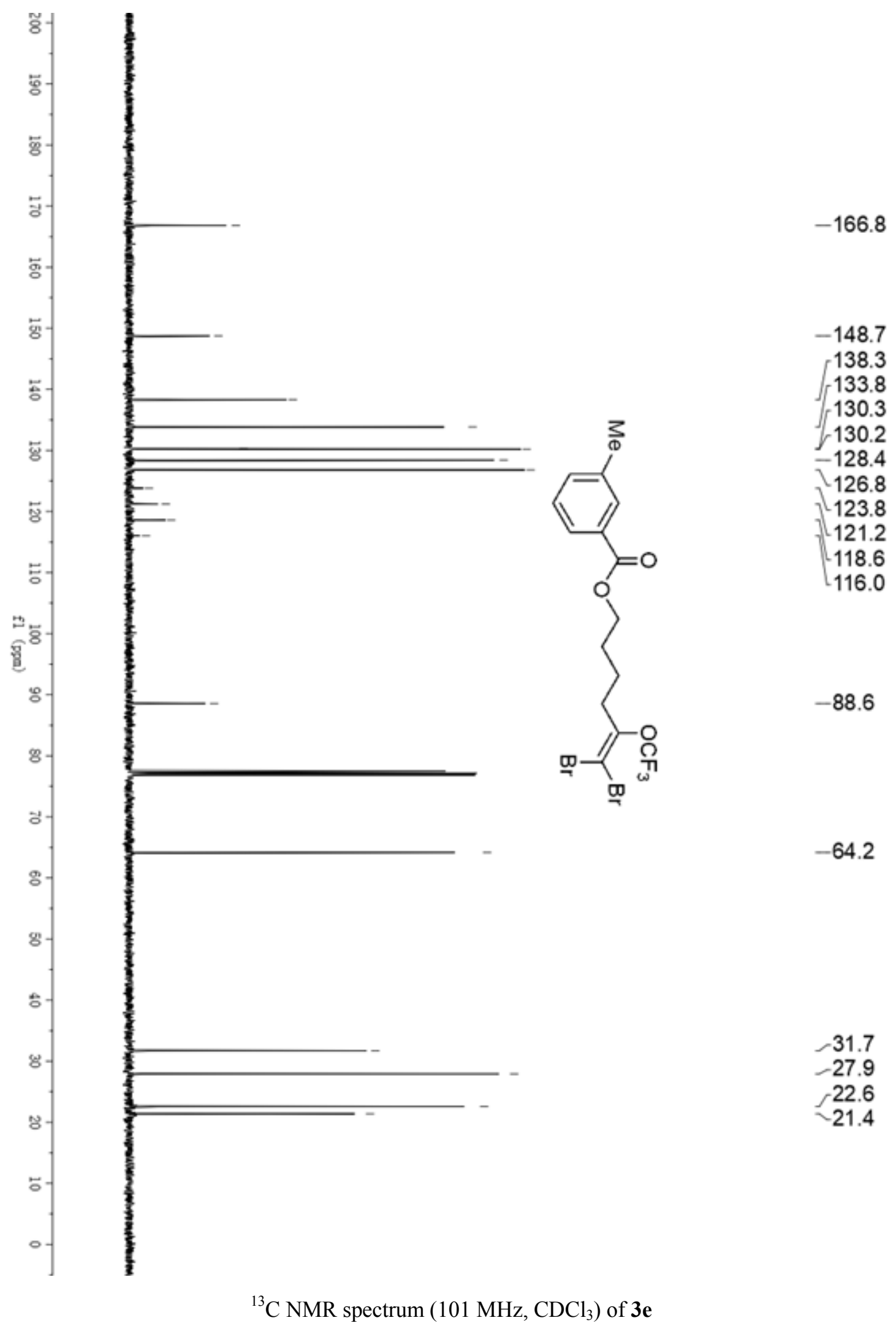




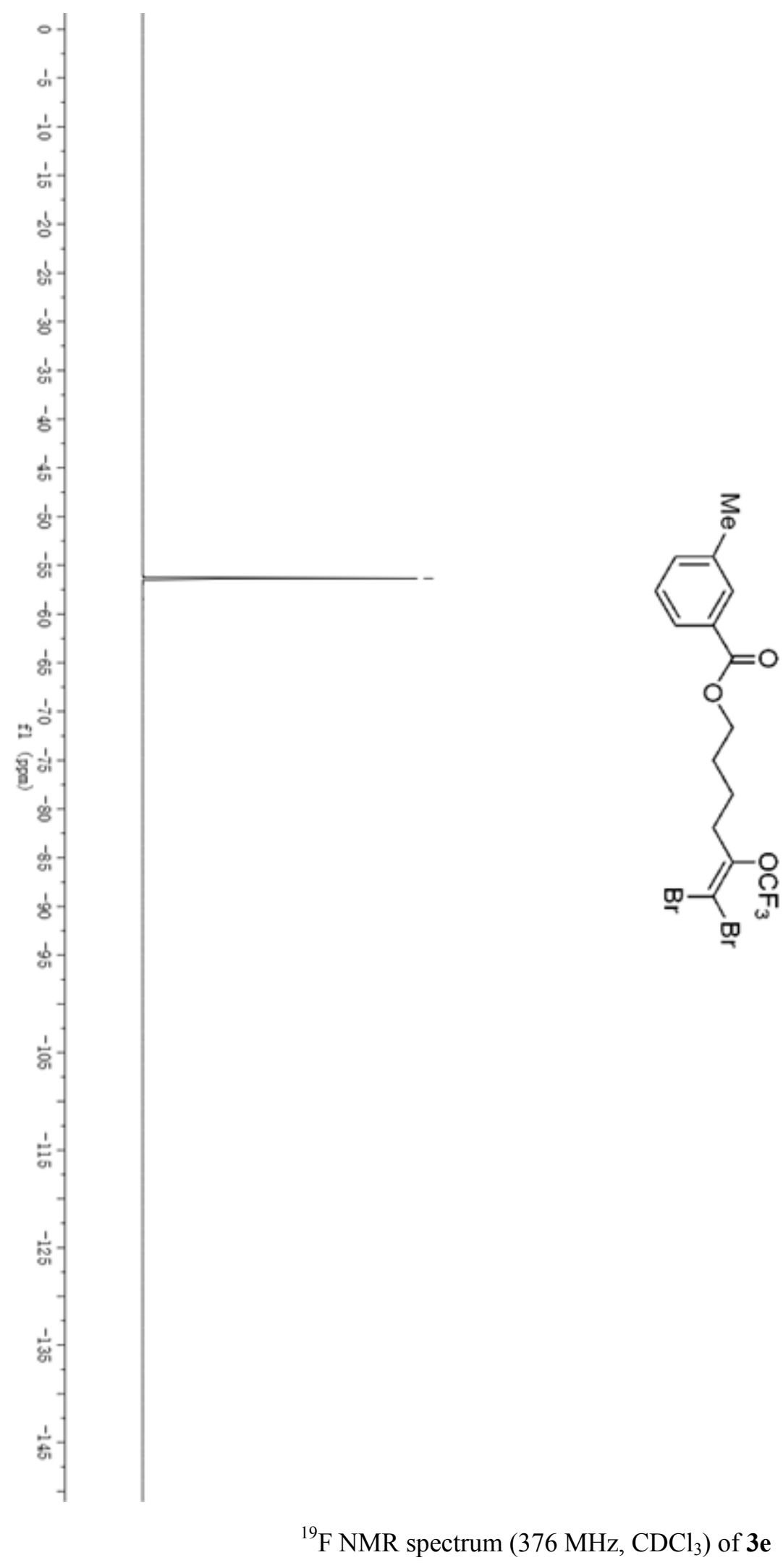




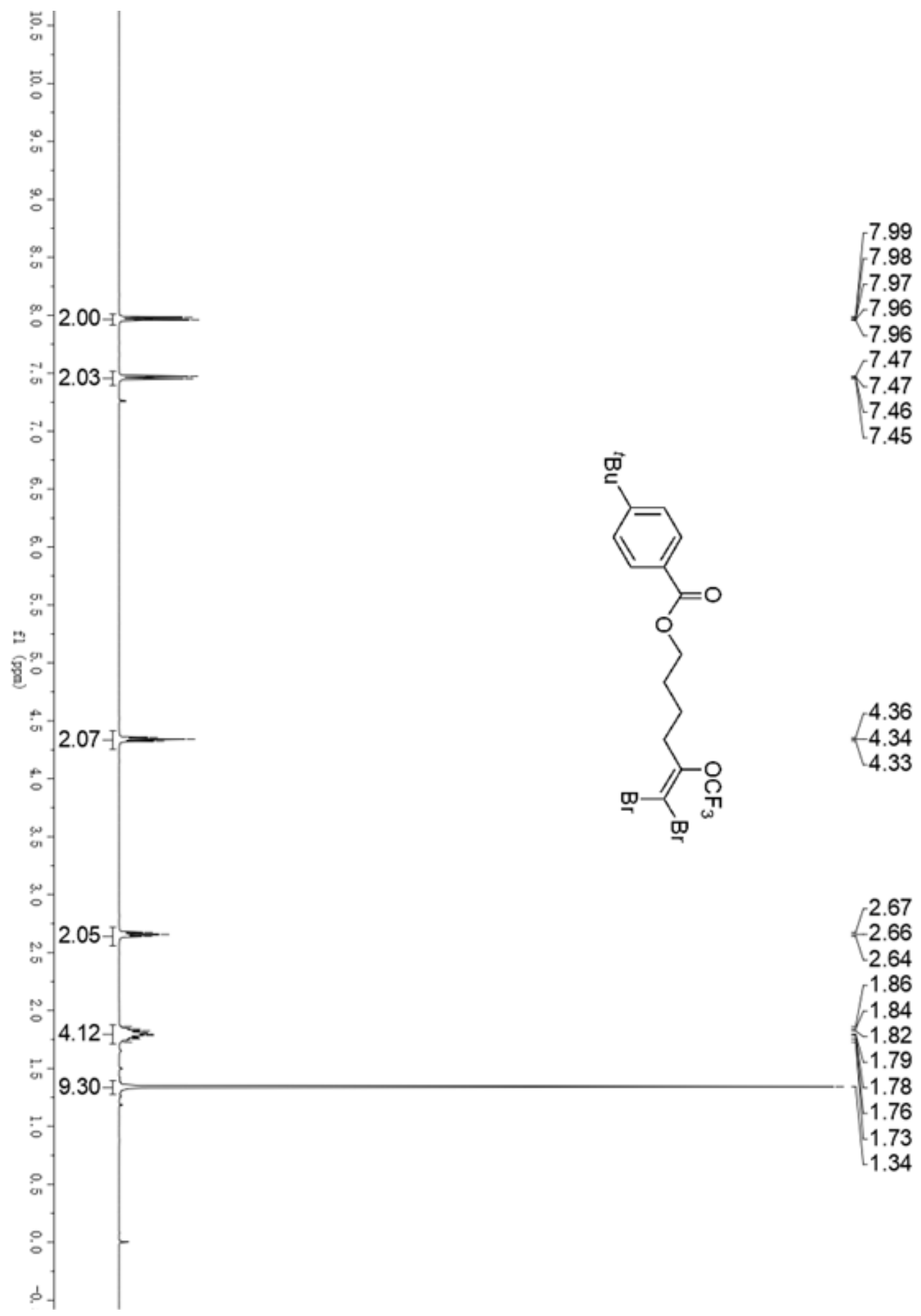

${ }^{1} \mathrm{H}$ NMR spectrum $\left(400 \mathrm{MHz}, \mathrm{CDCl}_{3}\right)$ of $\mathbf{3 f}$ 


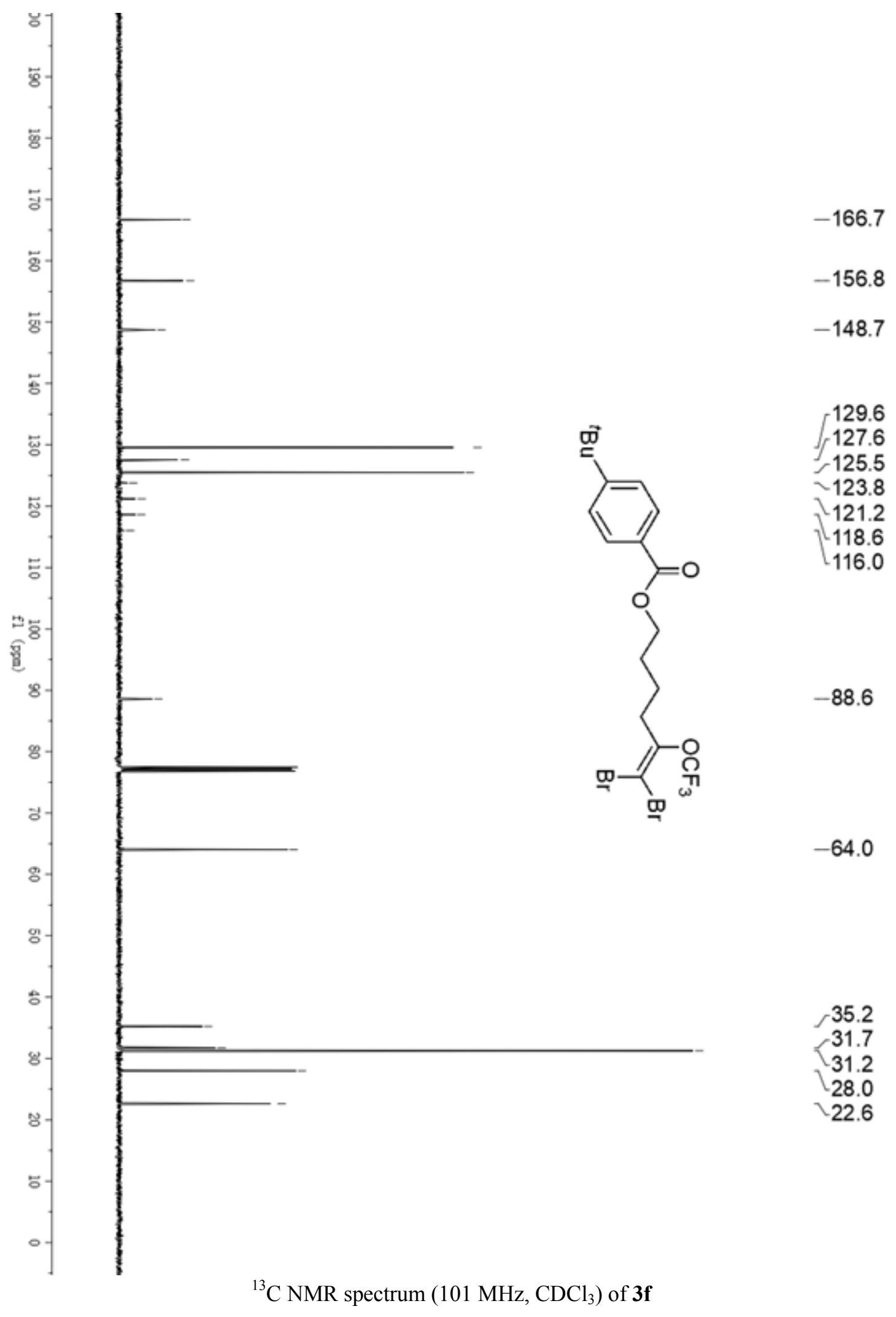




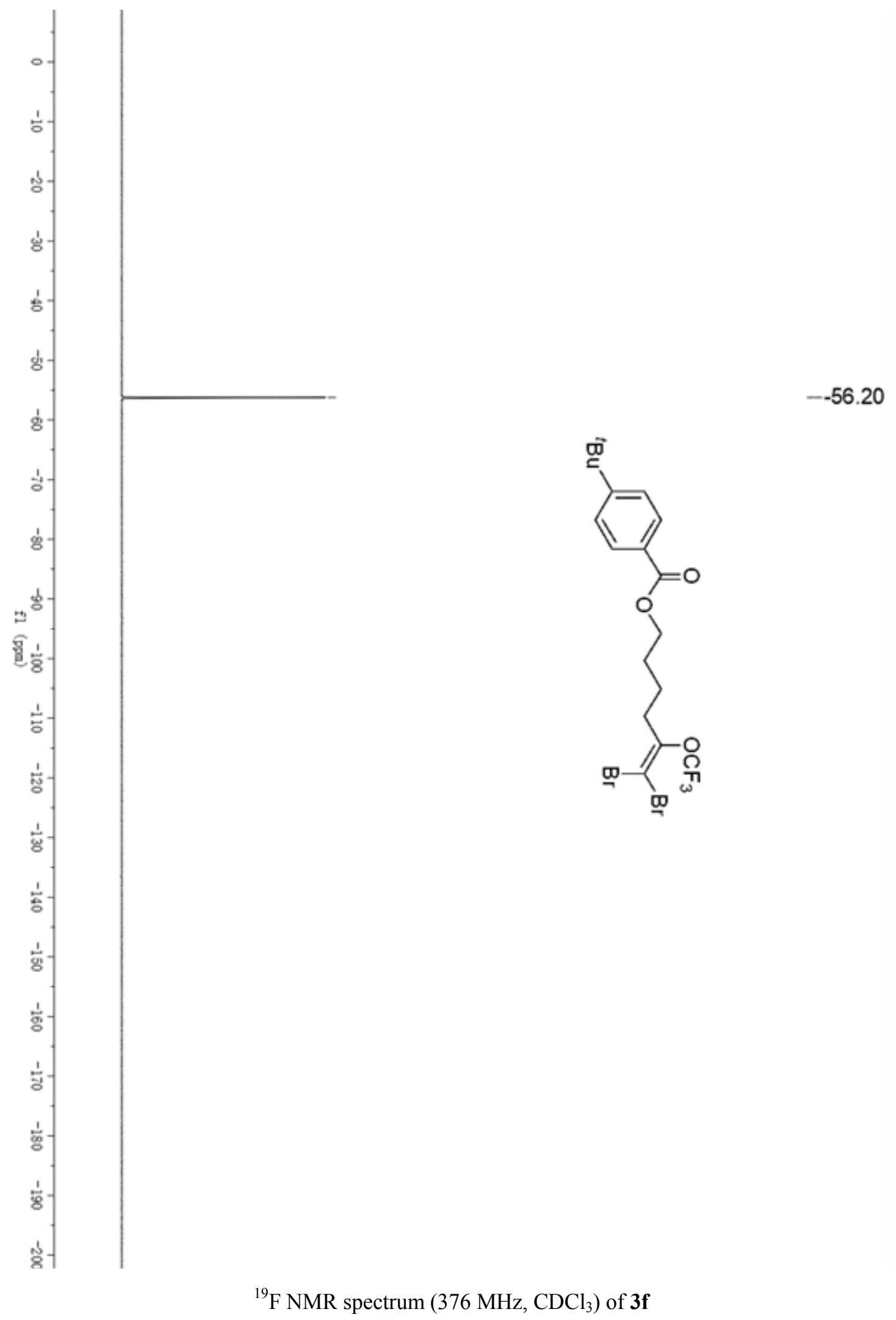




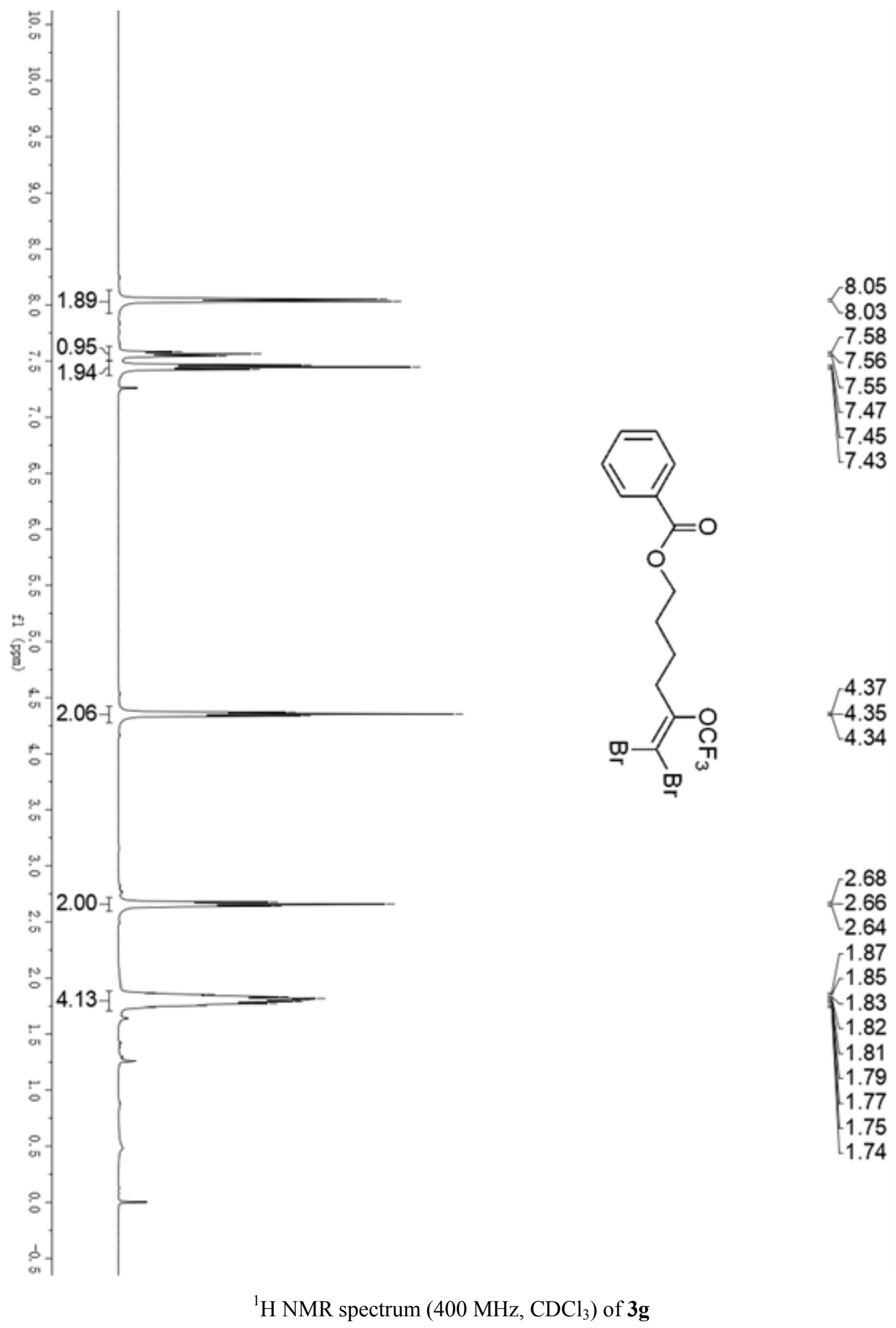




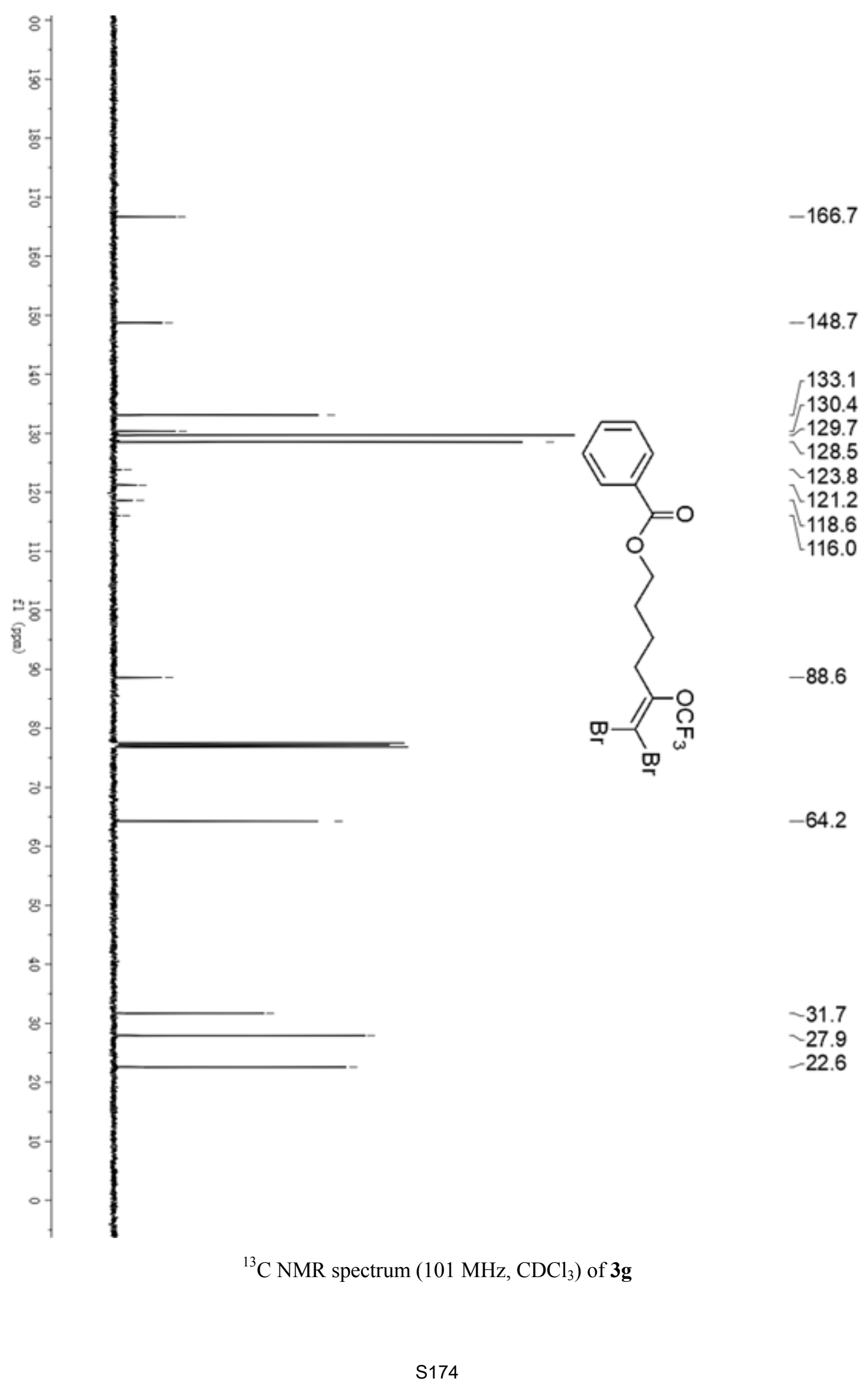




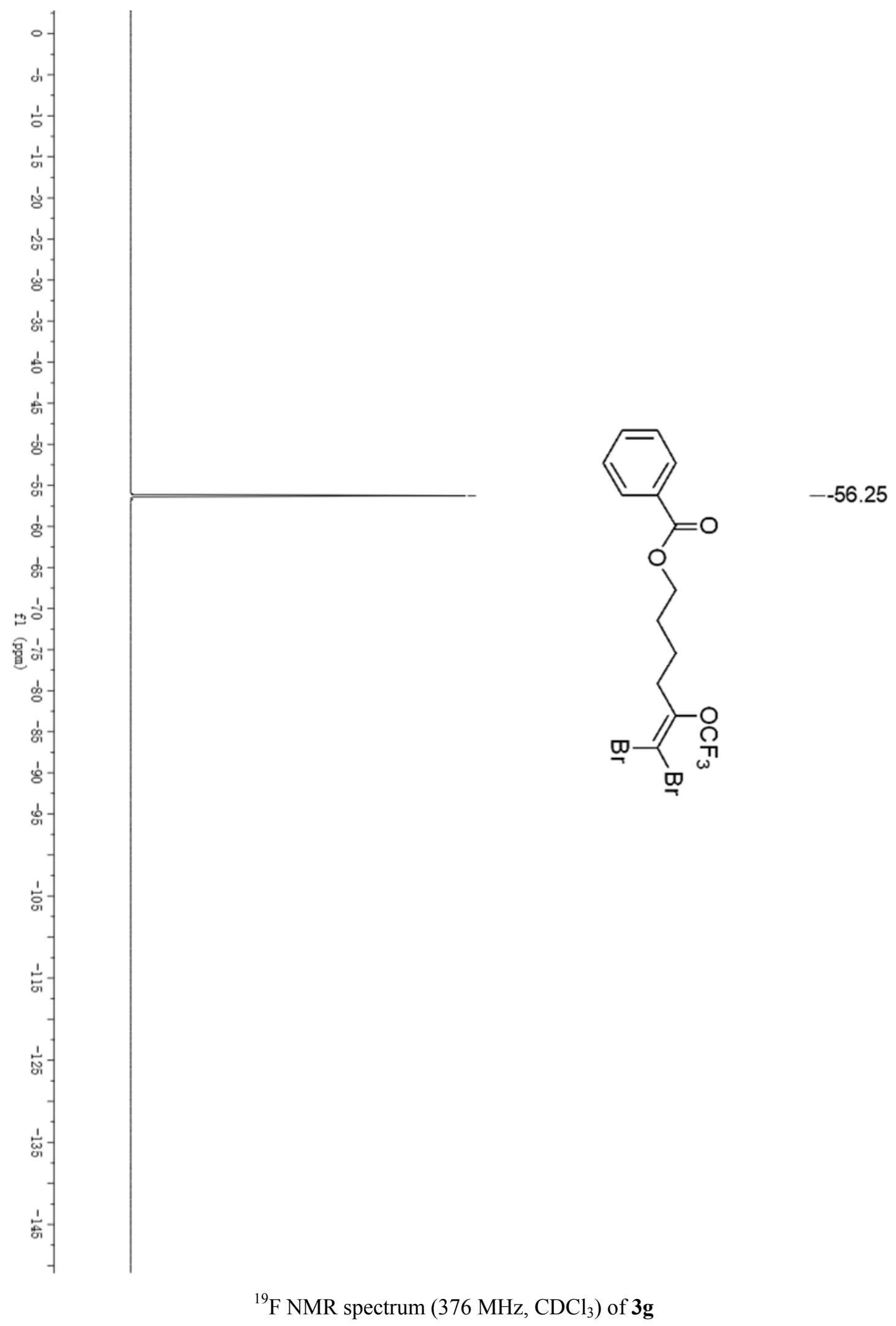




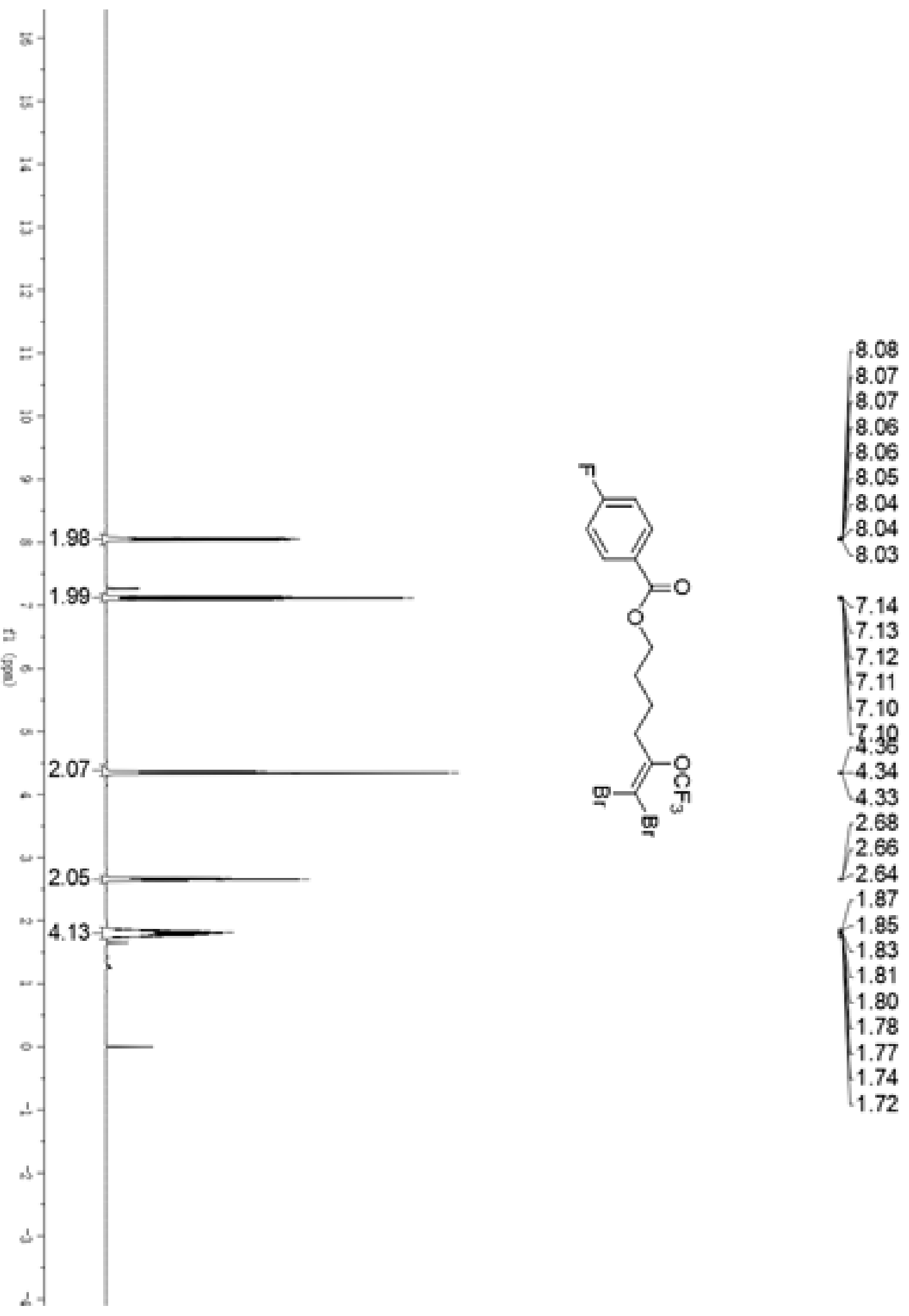

${ }^{1} \mathrm{H}$ NMR spectrum $\left(400 \mathrm{MHz}, \mathrm{CDCl}_{3}\right)$ of $\mathbf{3 h}$ 


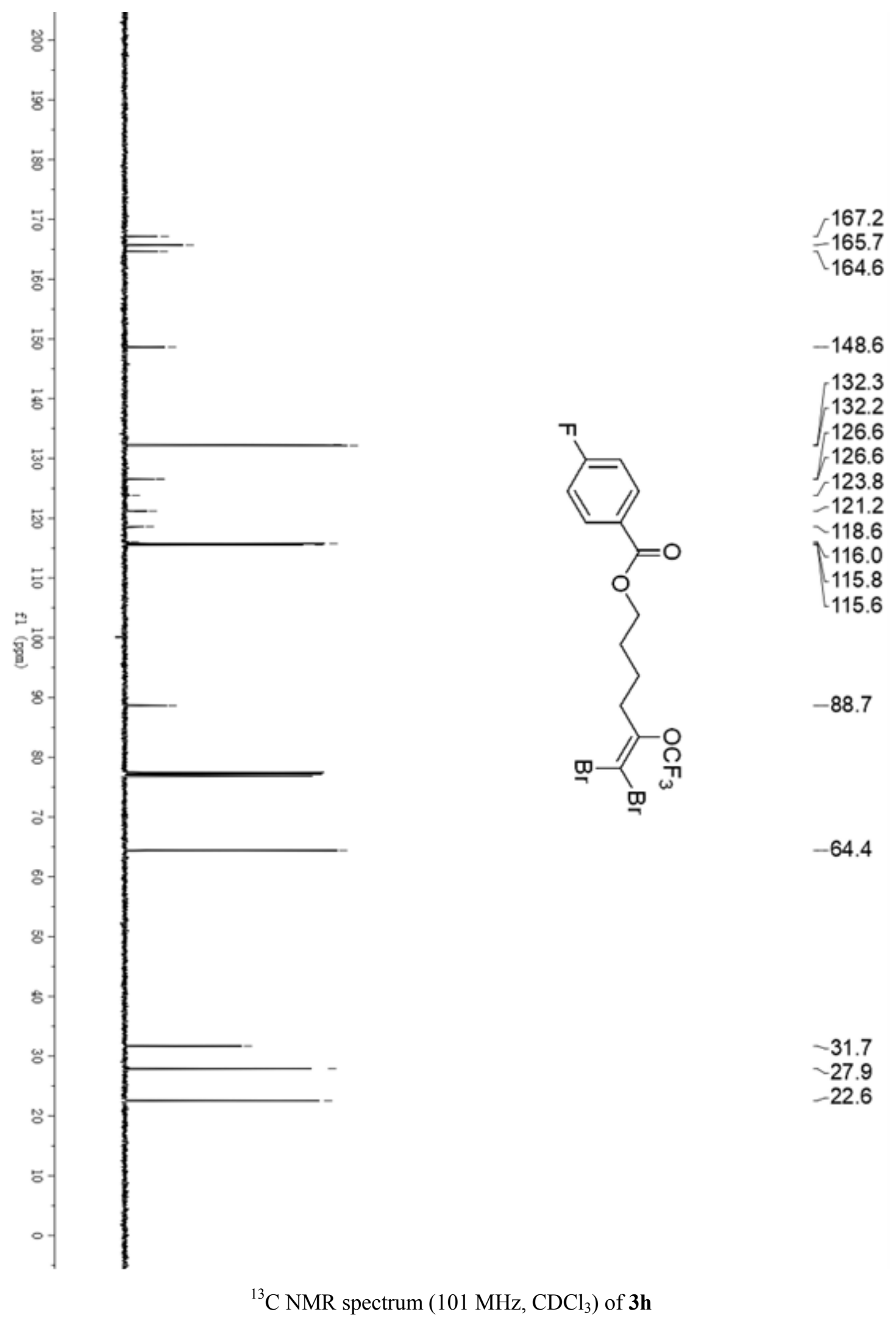




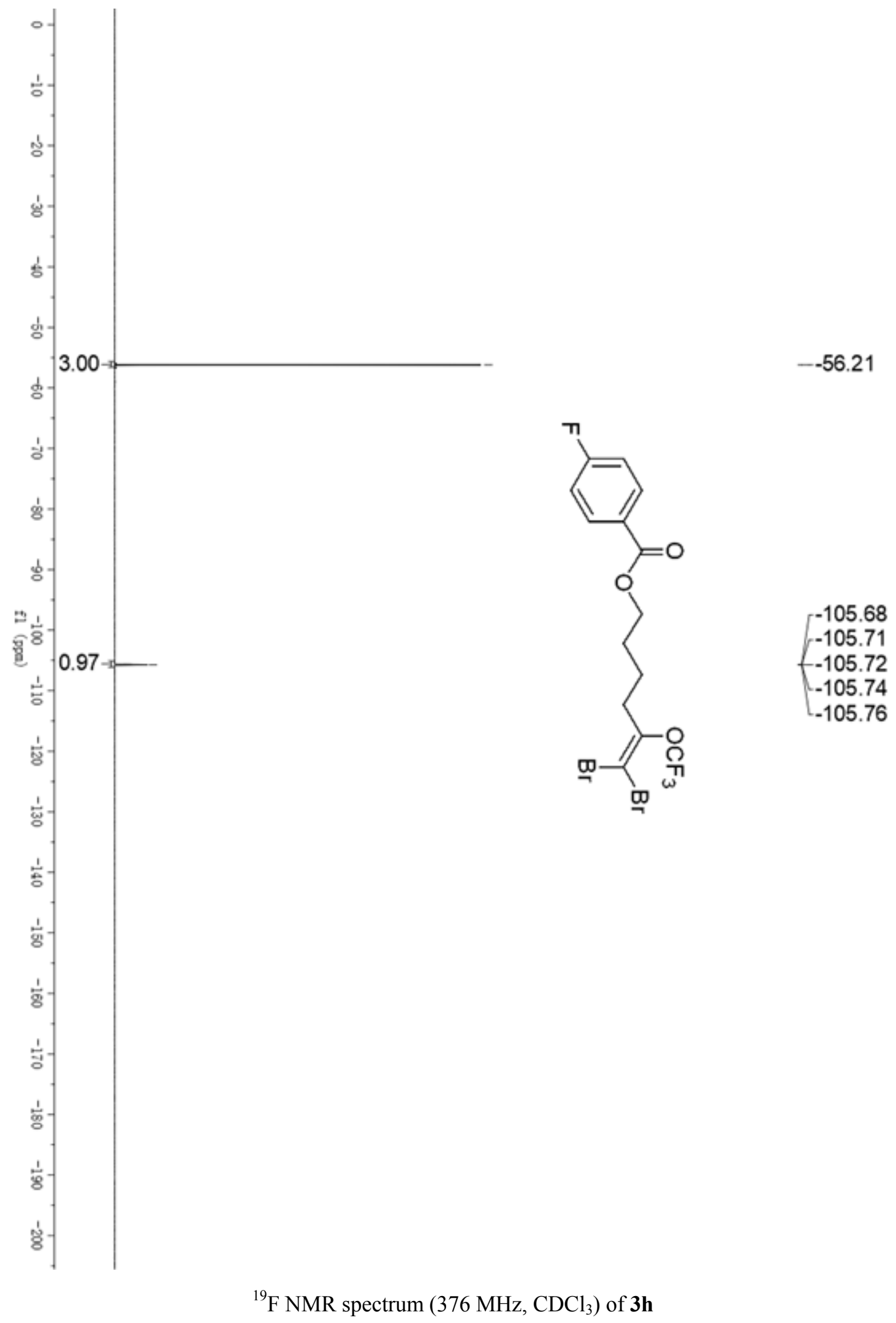




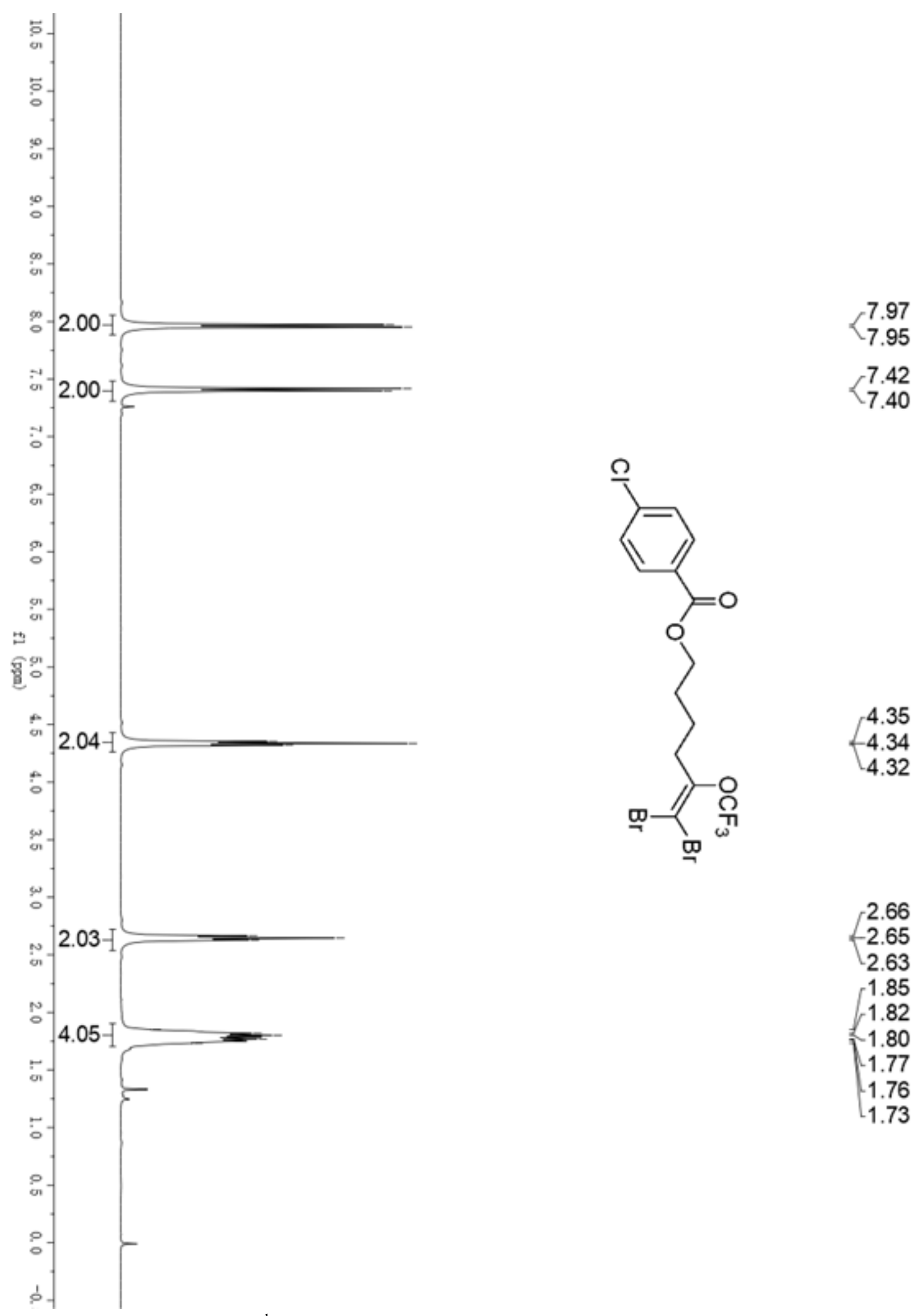

${ }^{1} \mathrm{H}$ NMR spectrum (400 MHz, $\mathrm{CDCl}_{3}$ ) of $\mathbf{3 i}$ 


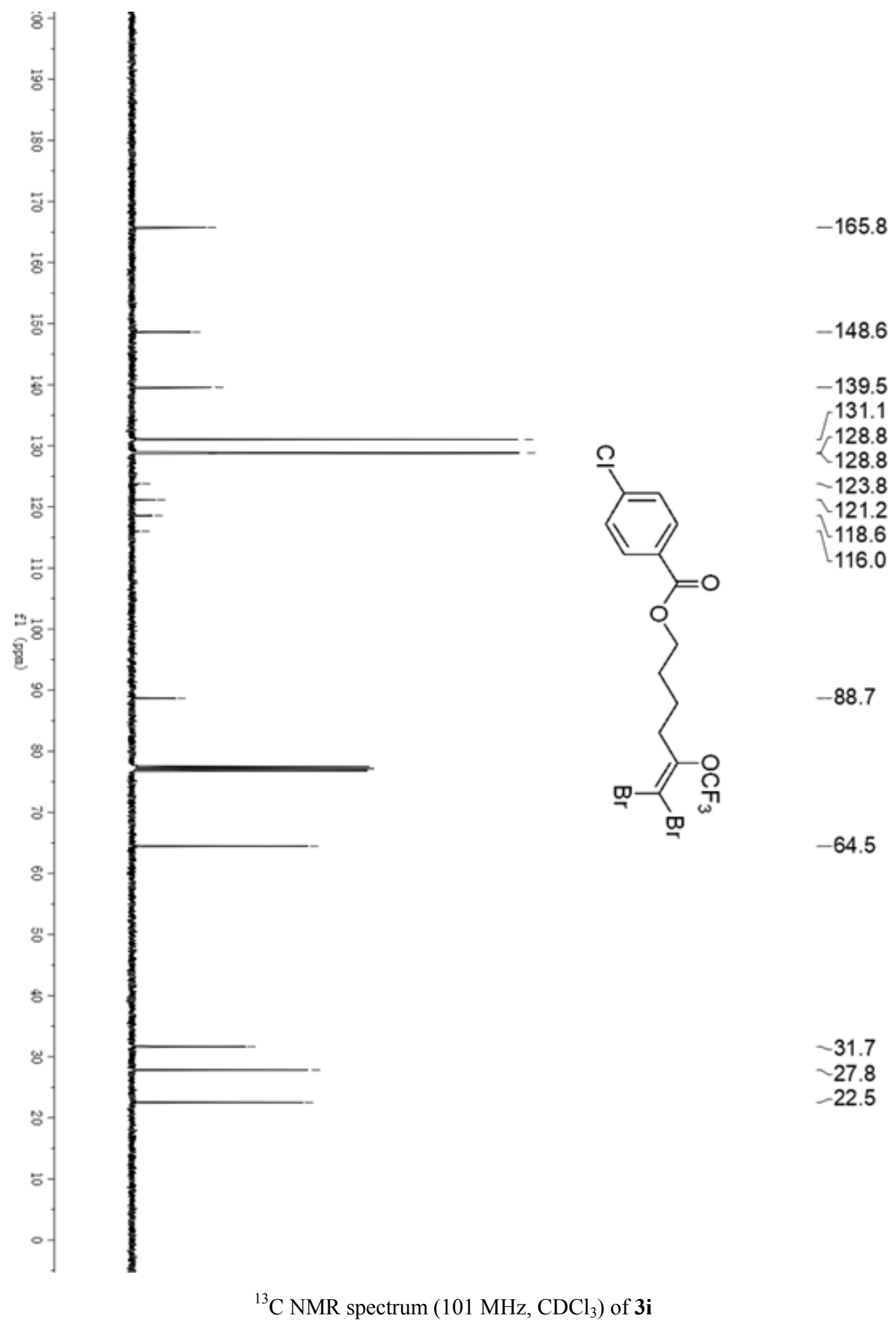




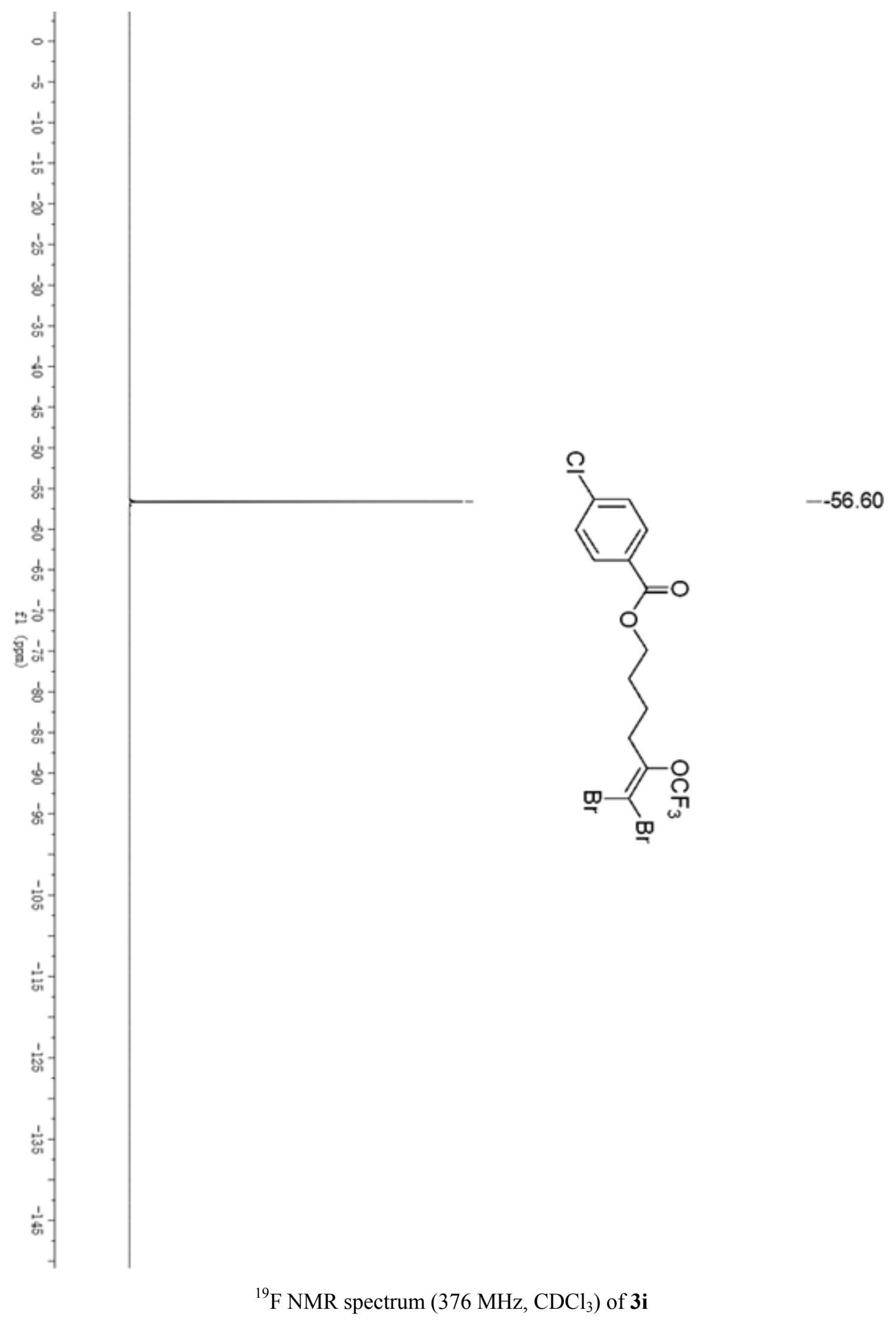




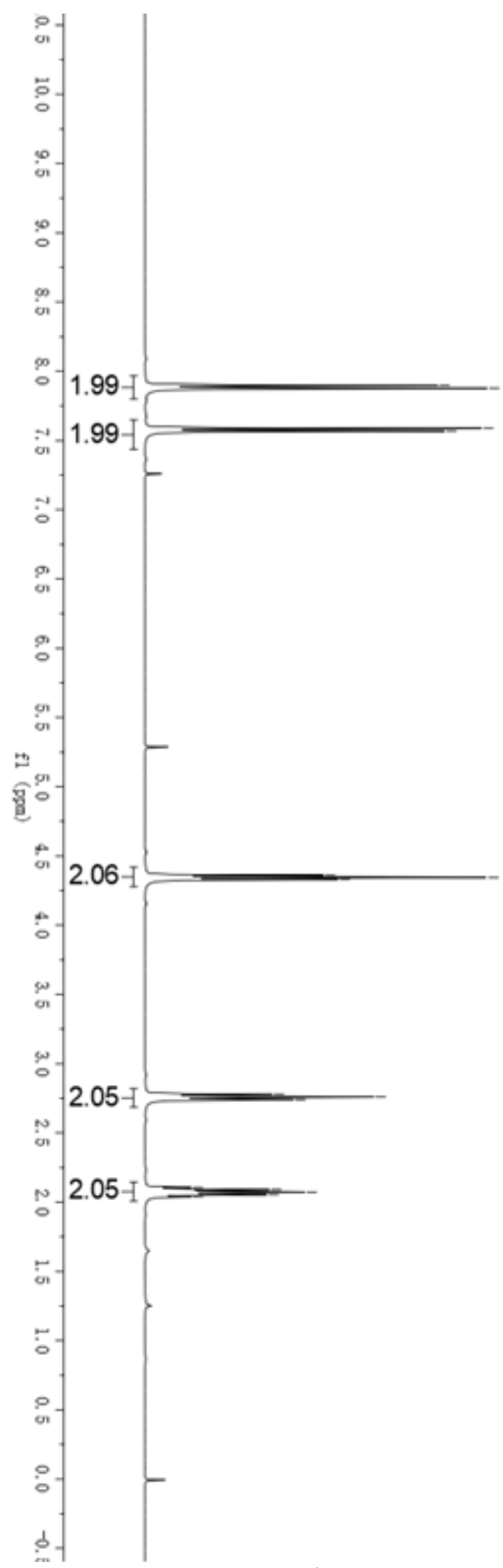

$\stackrel{\text { m }}{\longrightarrow}$<smiles>Cc1ccc(C(=O)O)cc1</smiles><smiles>CC(O)=C([O])CCCO</smiles>

4.36

$-4.35$

4.33

2.04

${ }^{1} \mathrm{H}$ NMR spectrum $\left(400 \mathrm{MHz}, \mathrm{CDCl}_{3}\right)$ of $\mathbf{3 j}$ 


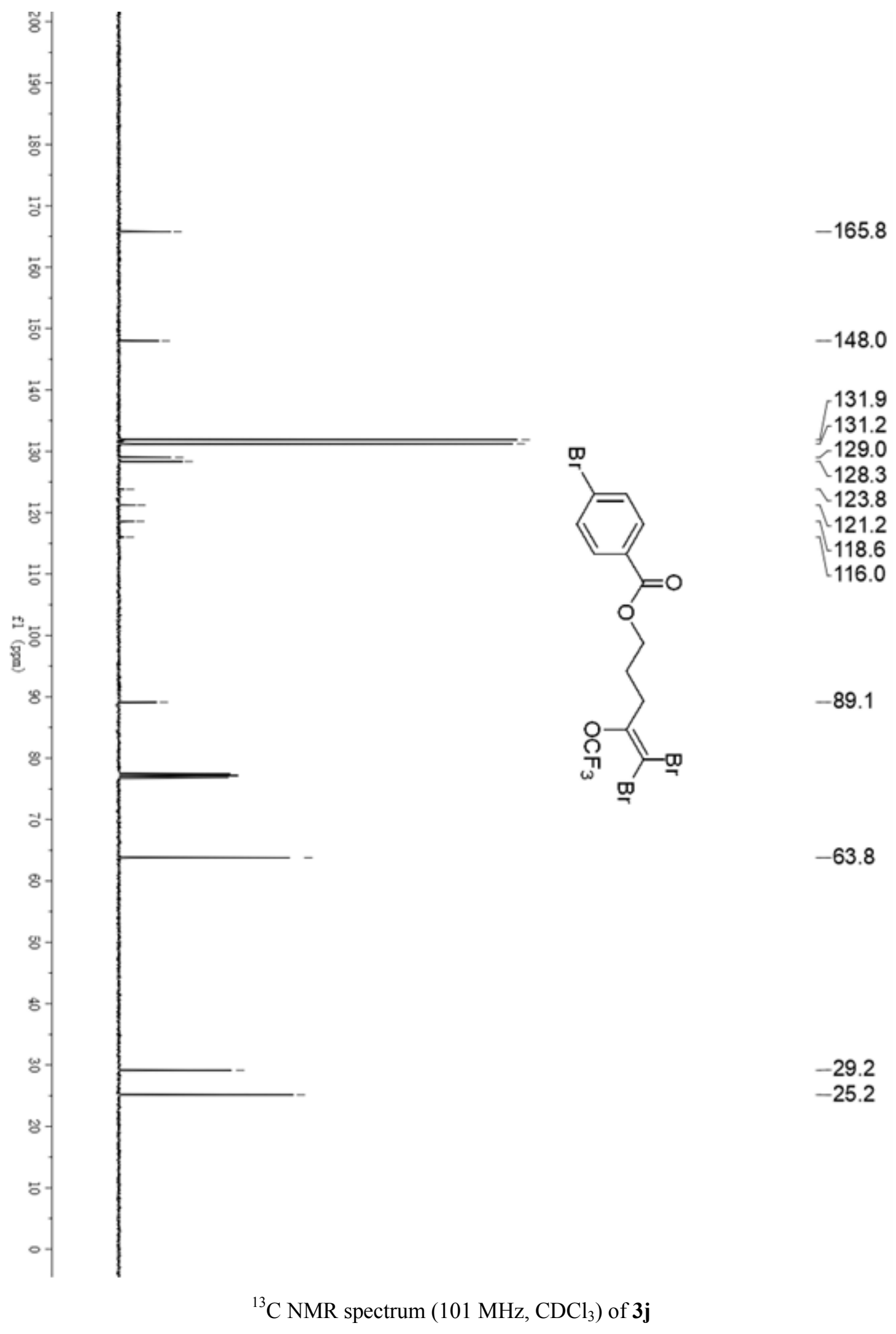




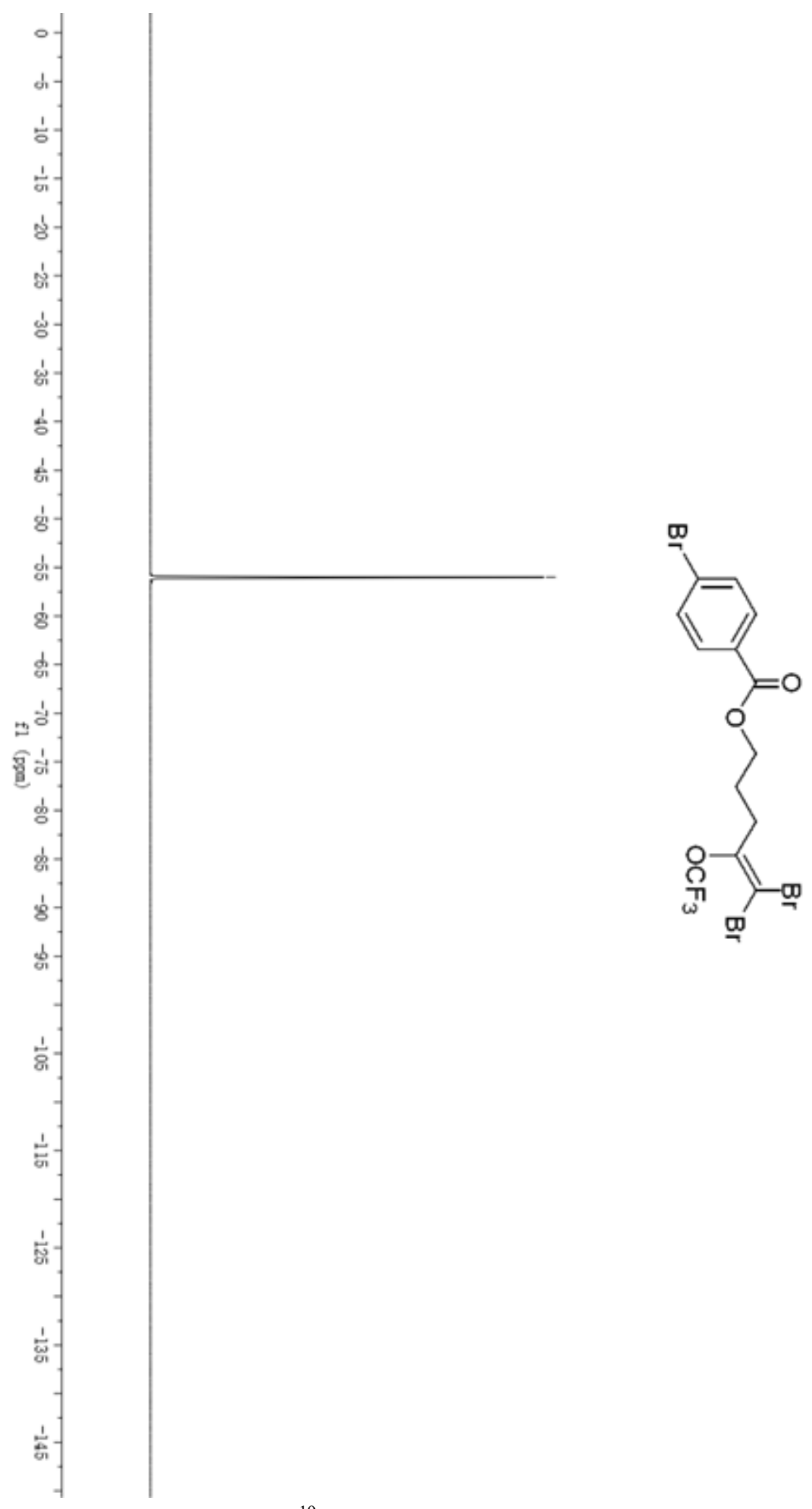

${ }^{19} \mathrm{~F}$ NMR spectrum $\left(376 \mathrm{MHz}, \mathrm{CDCl}_{3}\right)$ of $\mathbf{3 j}$ 

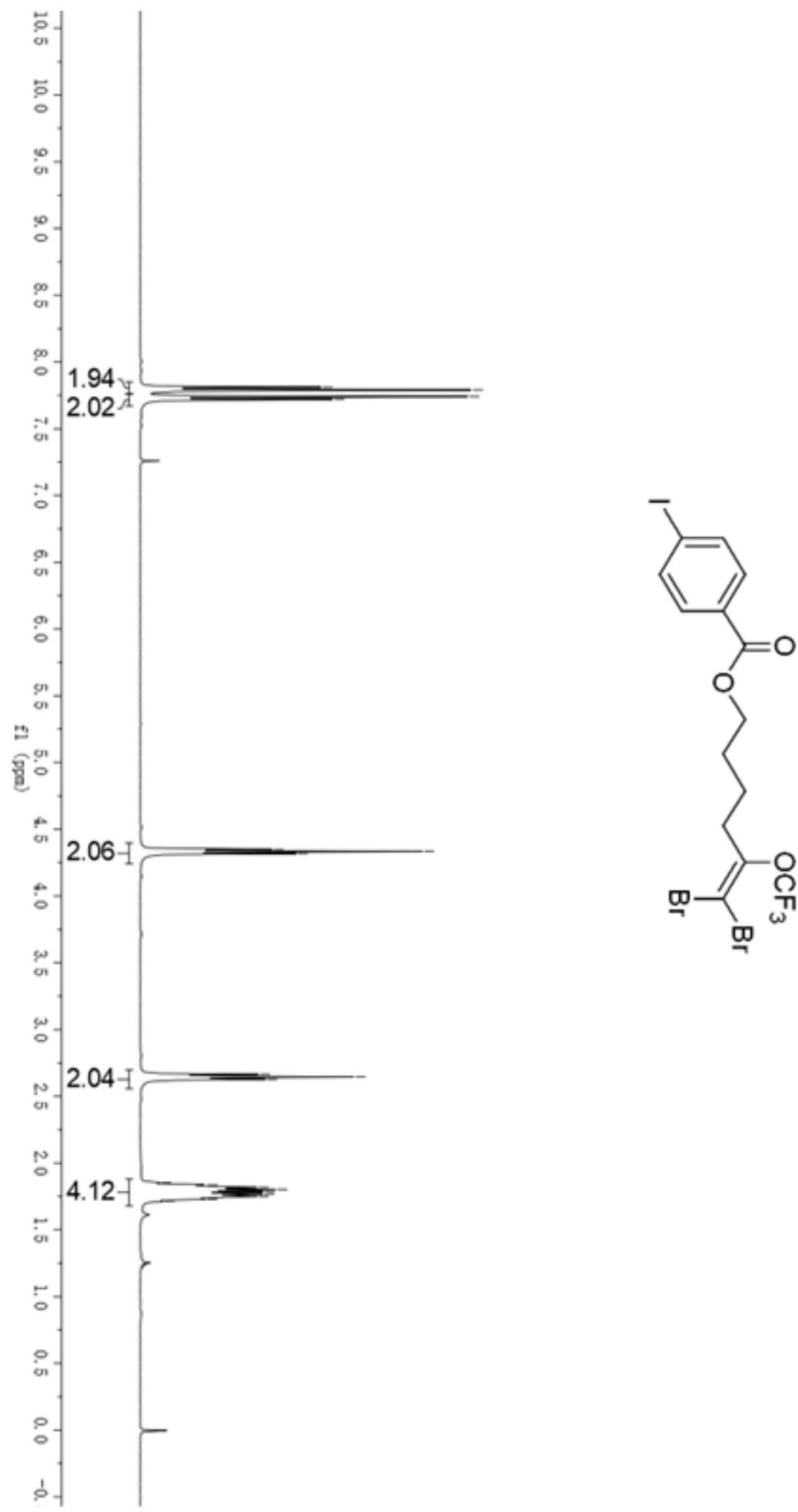

${ }^{1} \mathrm{H}$ NMR spectrum $\left(400 \mathrm{MHz}, \mathrm{CDCl}_{3}\right)$ of $\mathbf{3} \mathbf{k}$ 


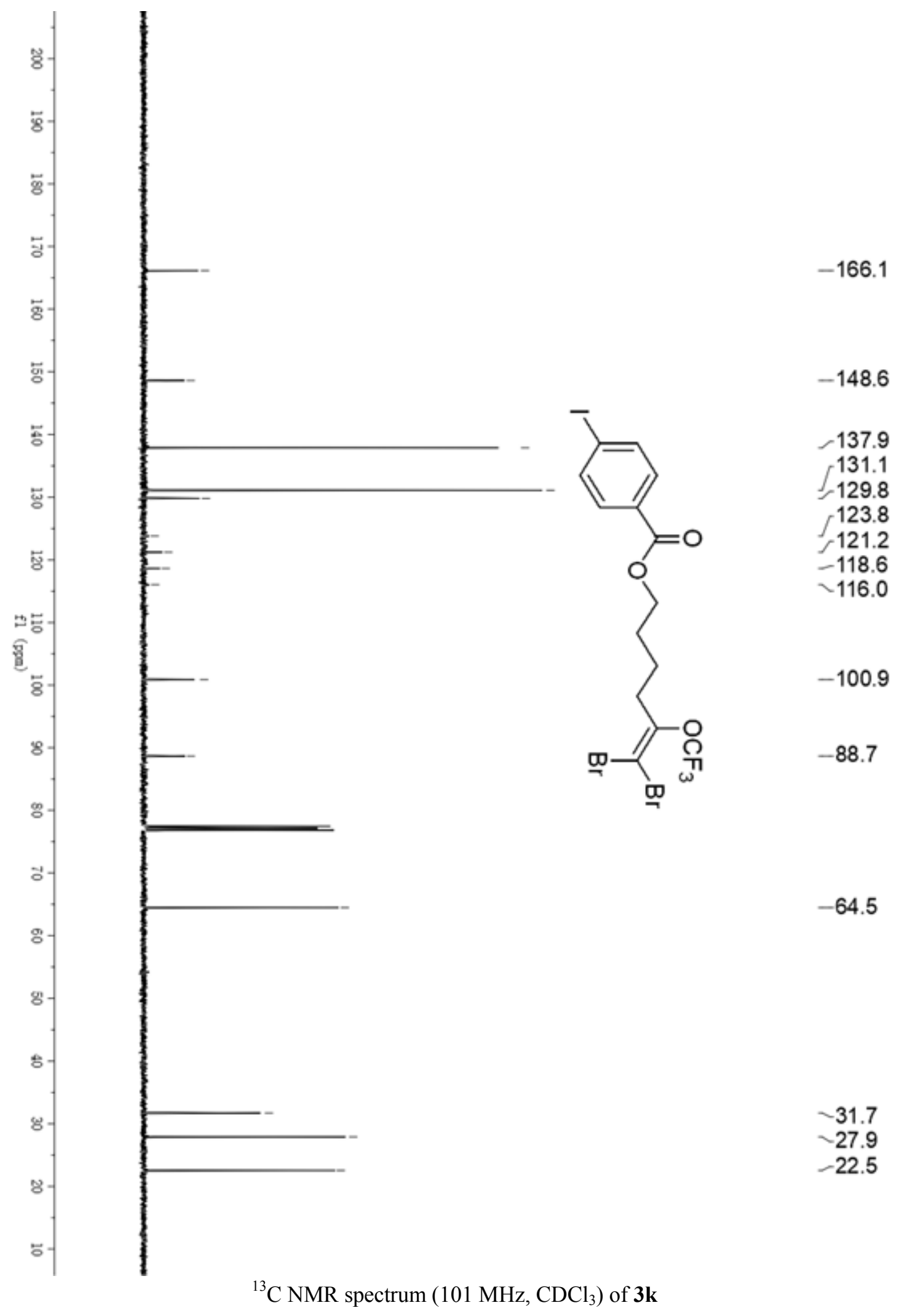




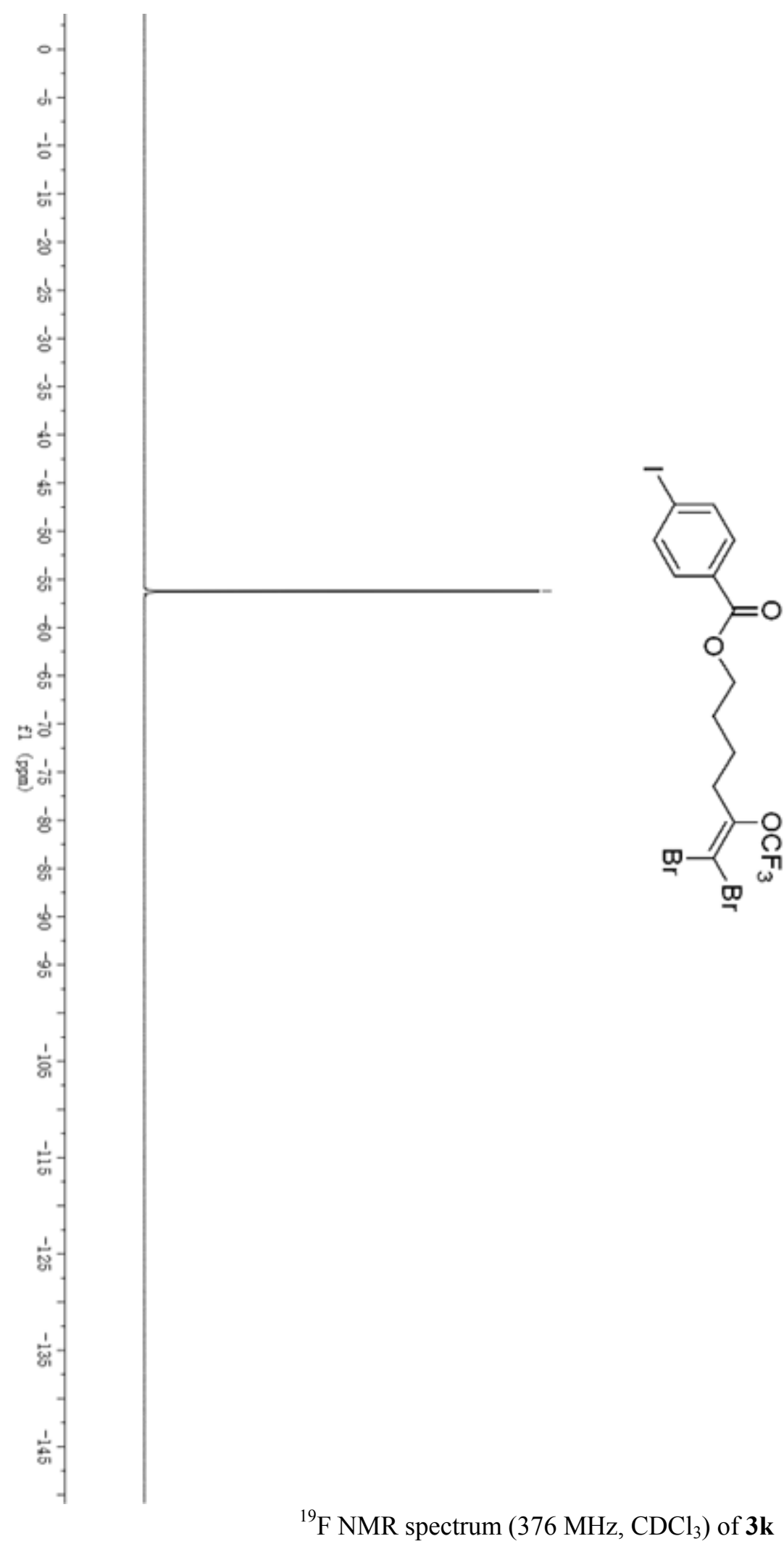

${ }^{19} \mathrm{~F}$ NMR spectrum $\left(376 \mathrm{MHz}, \mathrm{CDCl}_{3}\right)$ of $\mathbf{3 k}$ 


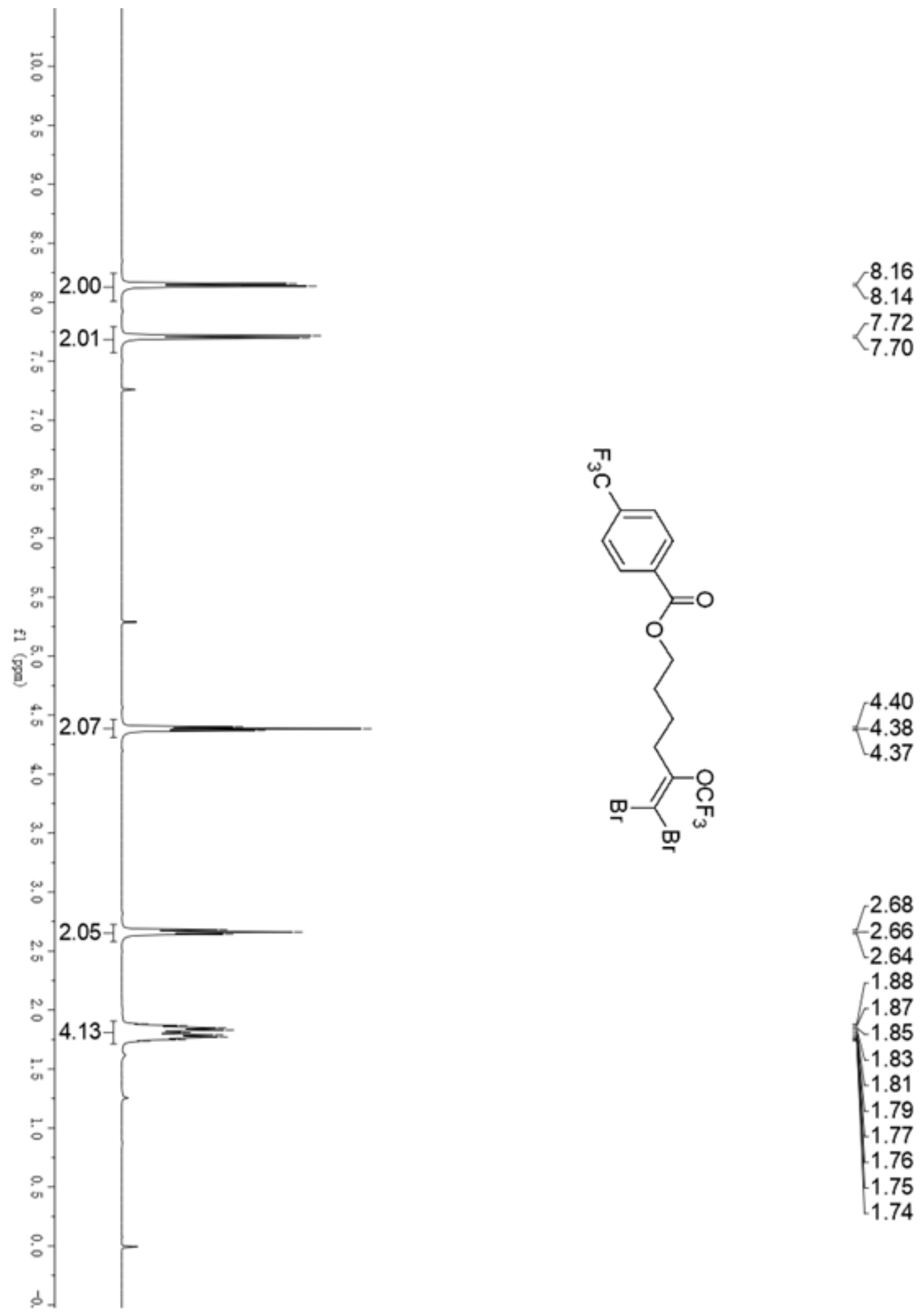

${ }^{1} \mathrm{H}$ NMR spectrum $\left(400 \mathrm{MHz}, \mathrm{CDCl}_{3}\right.$ ) of $\mathbf{3 I}$ 


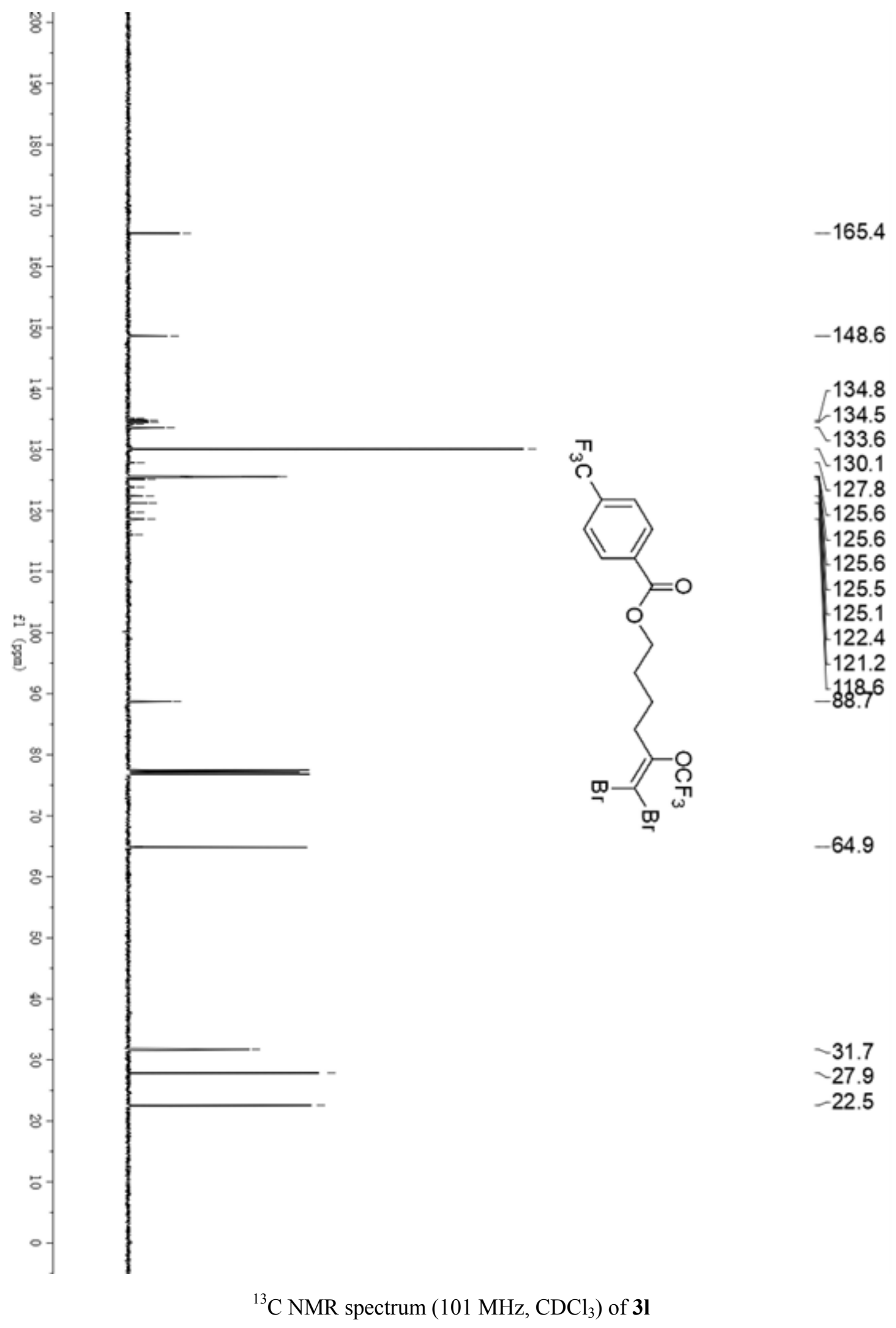




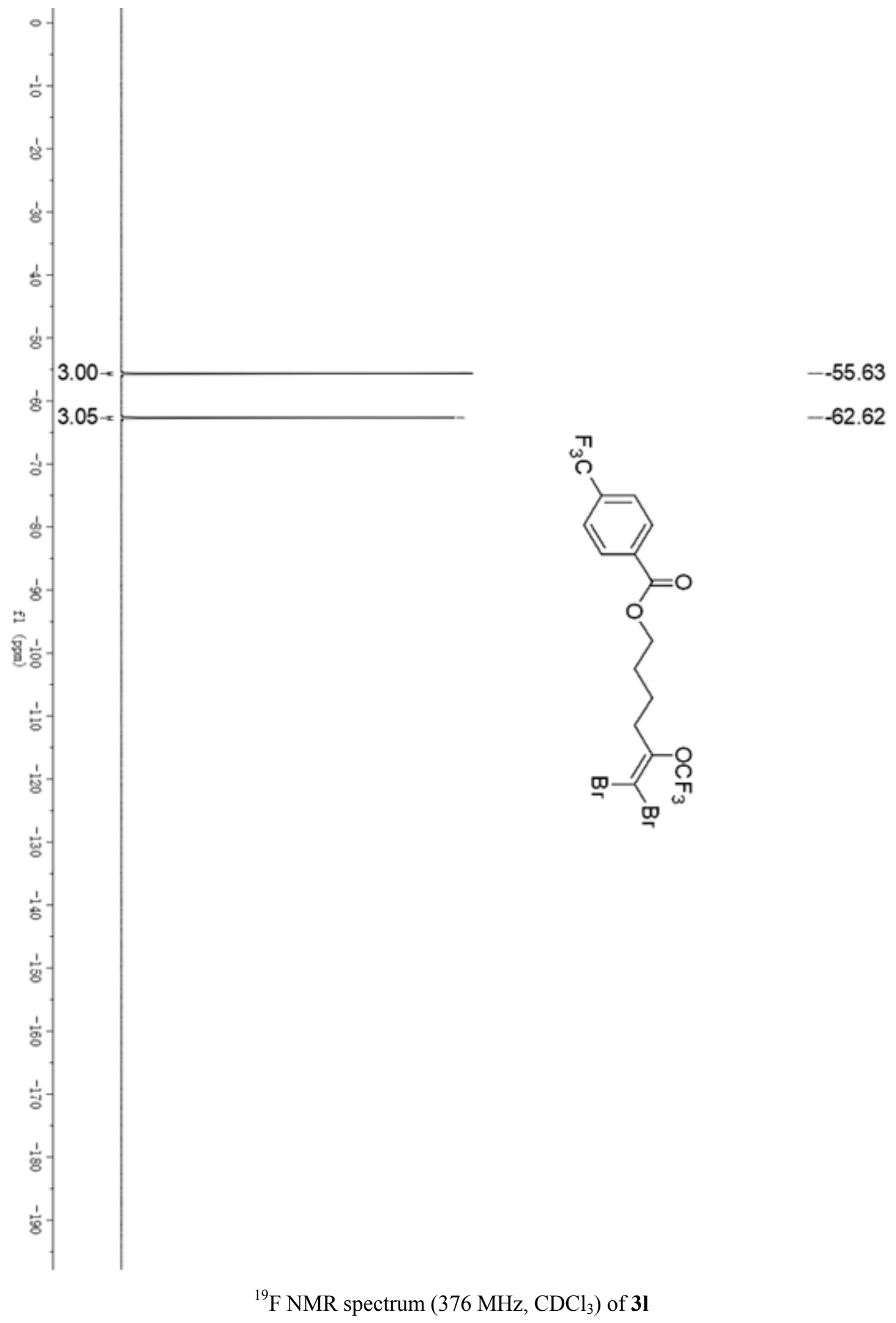



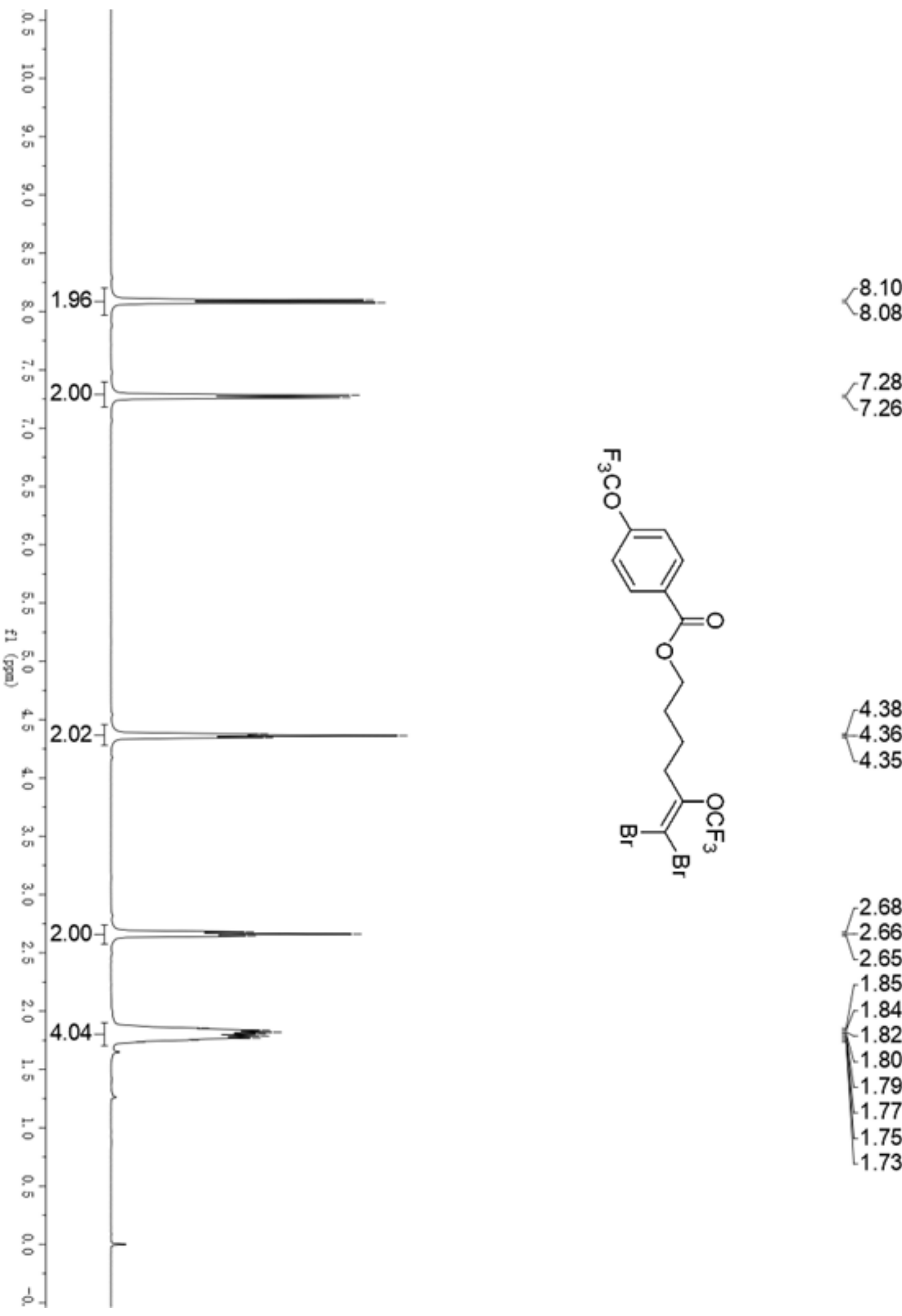

4.38

$-4.36$

4.35

${ }^{1} \mathrm{H}$ NMR spectrum $\left(400 \mathrm{MHz}, \mathrm{CDCl}_{3}\right)$ of $\mathbf{3} \mathbf{m}$

$\left\{\begin{array}{r}2.68 \\ -2.66 \\ 2.65\end{array}\right.$
$\begin{array}{r}1.85 \\ 1.84 \\ 1.82 \\ 1.80 \\ 1.79 \\ 1.77 \\ 1.75 \\ 1.73\end{array}$




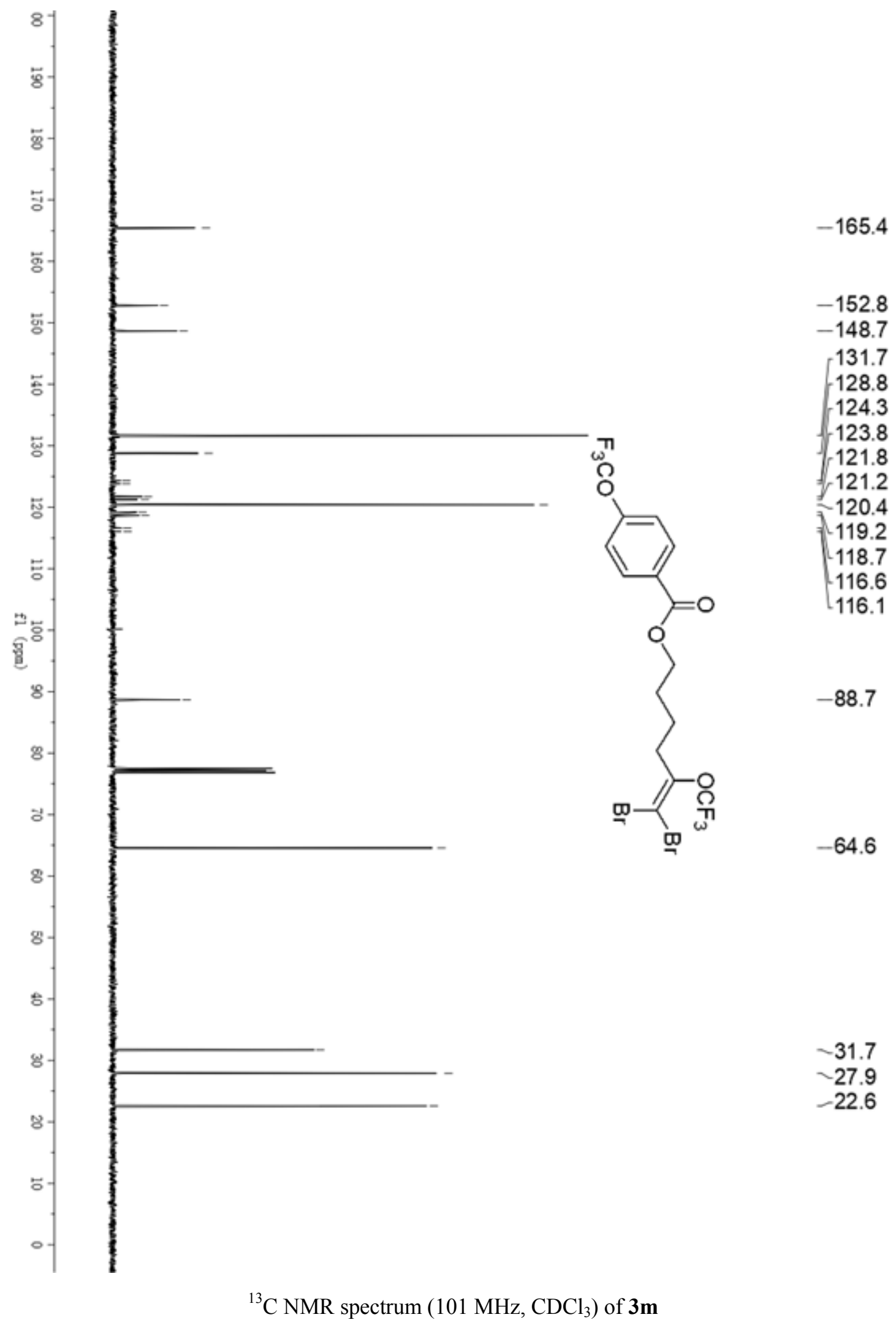




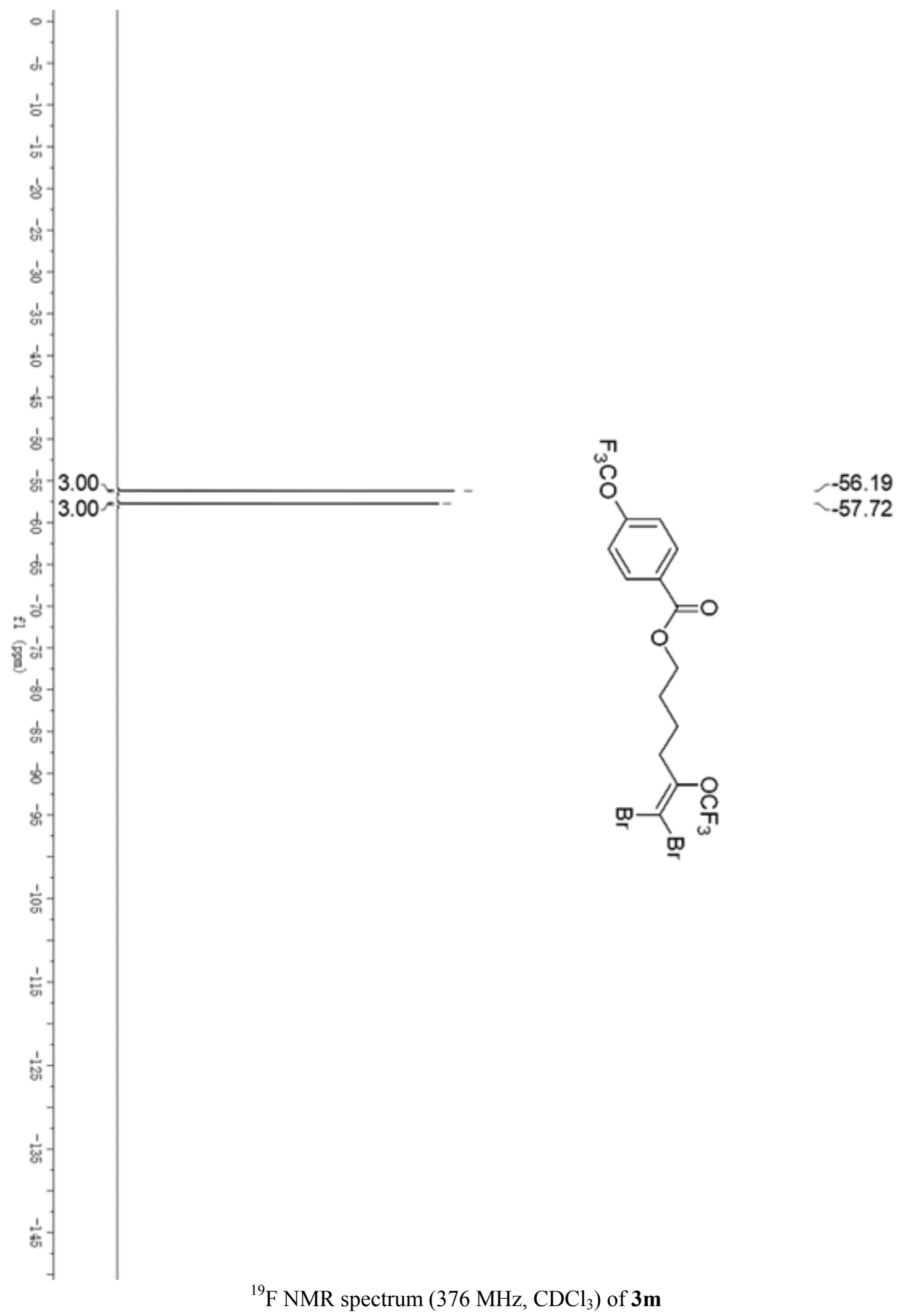




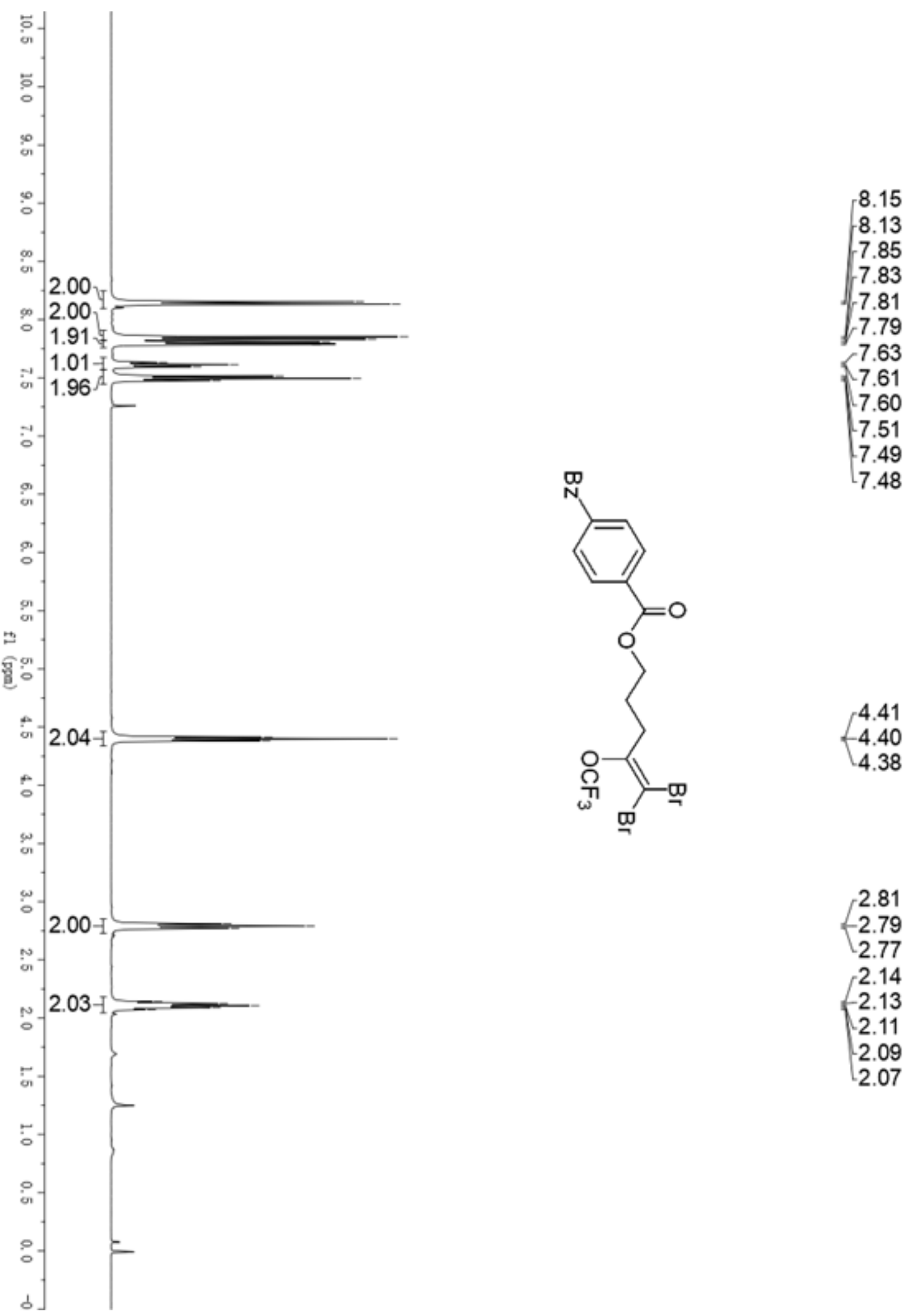

${ }^{1} \mathrm{H}$ NMR spectrum $\left(400 \mathrm{MHz}, \mathrm{CDCl}_{3}\right)$ of $\mathbf{3 n}$ 


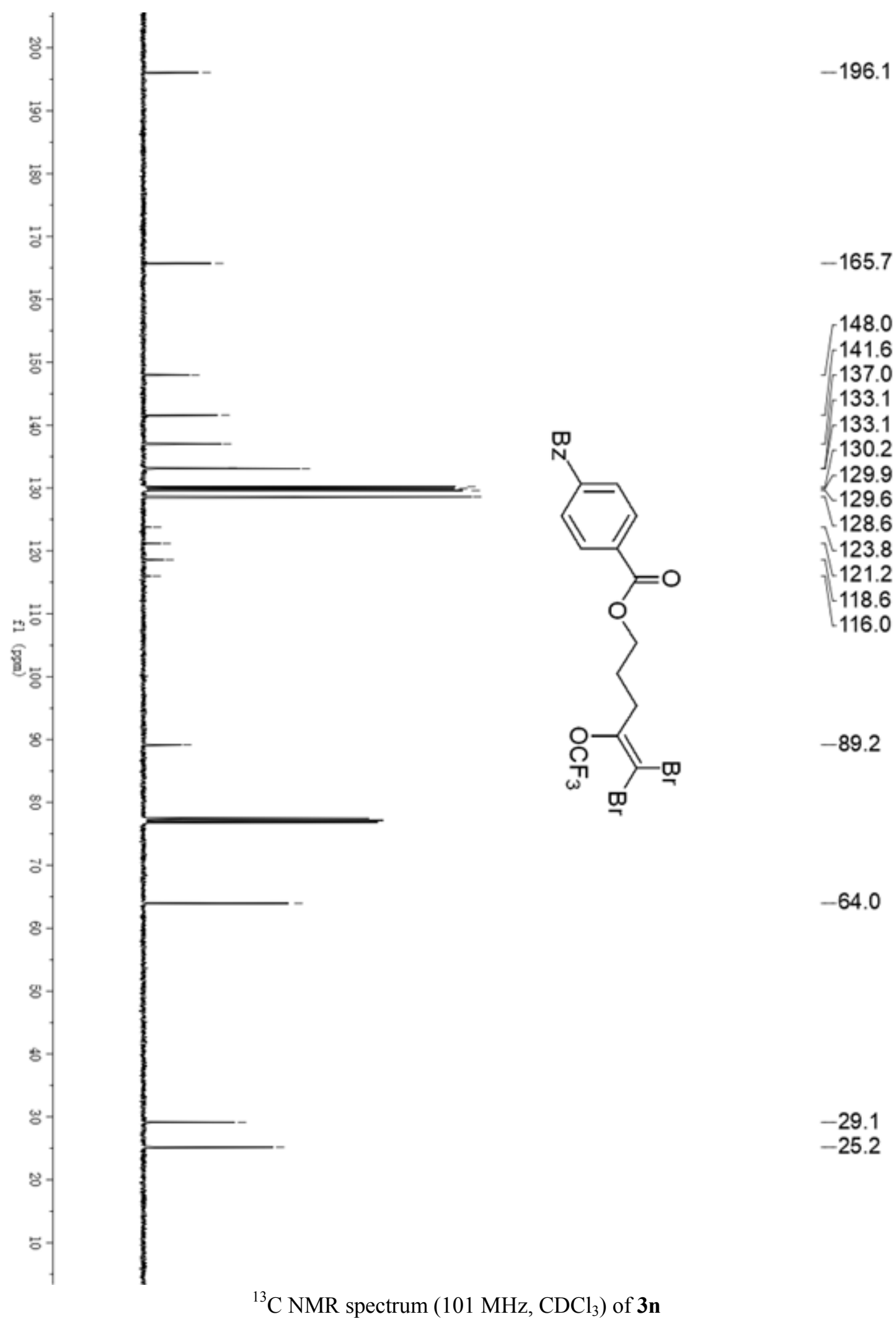




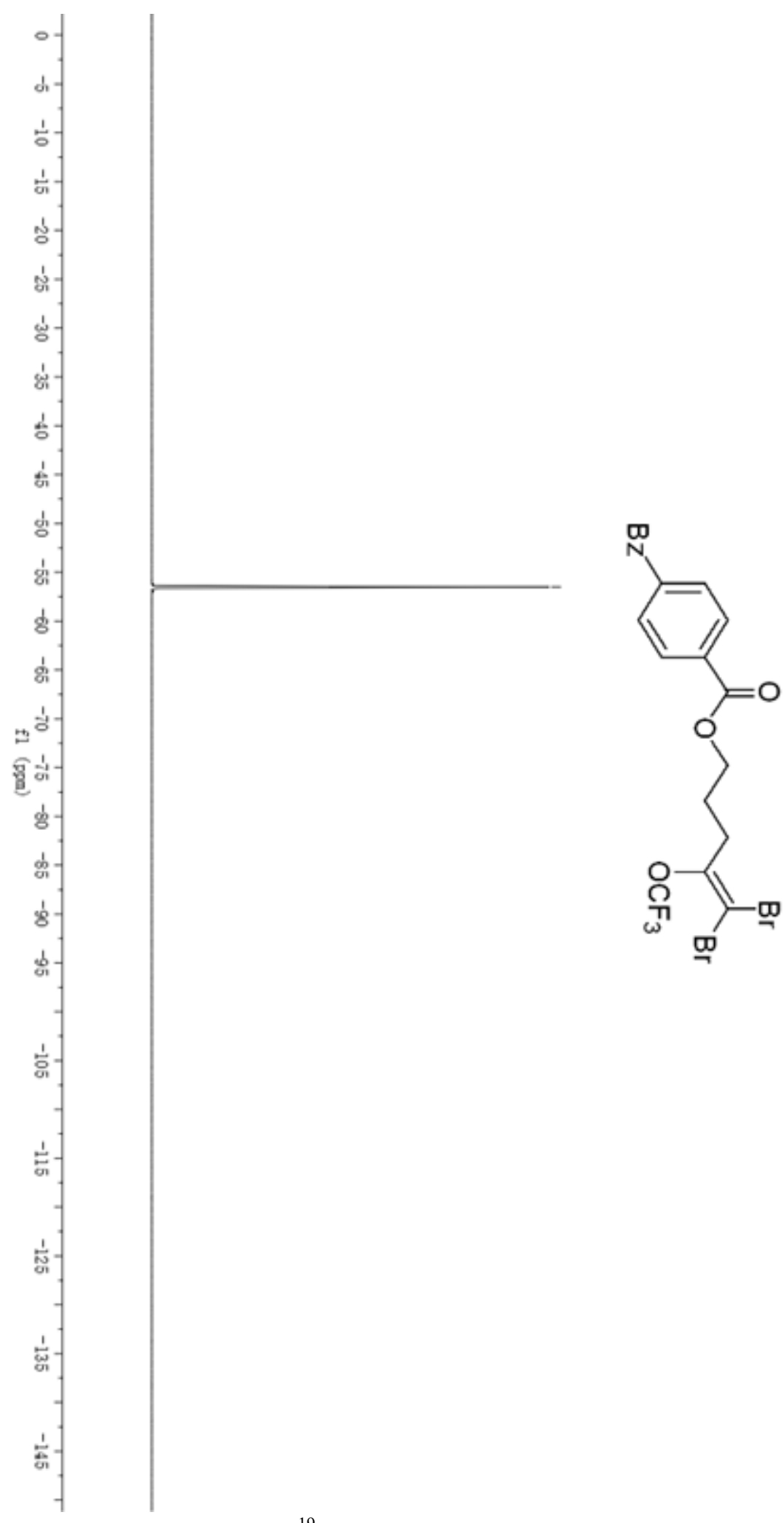

$--56.50$

${ }^{19} \mathrm{~F}$ NMR spectrum $\left(376 \mathrm{MHz}, \mathrm{CDCl}_{3}\right)$ of $\mathbf{3 n}$ 


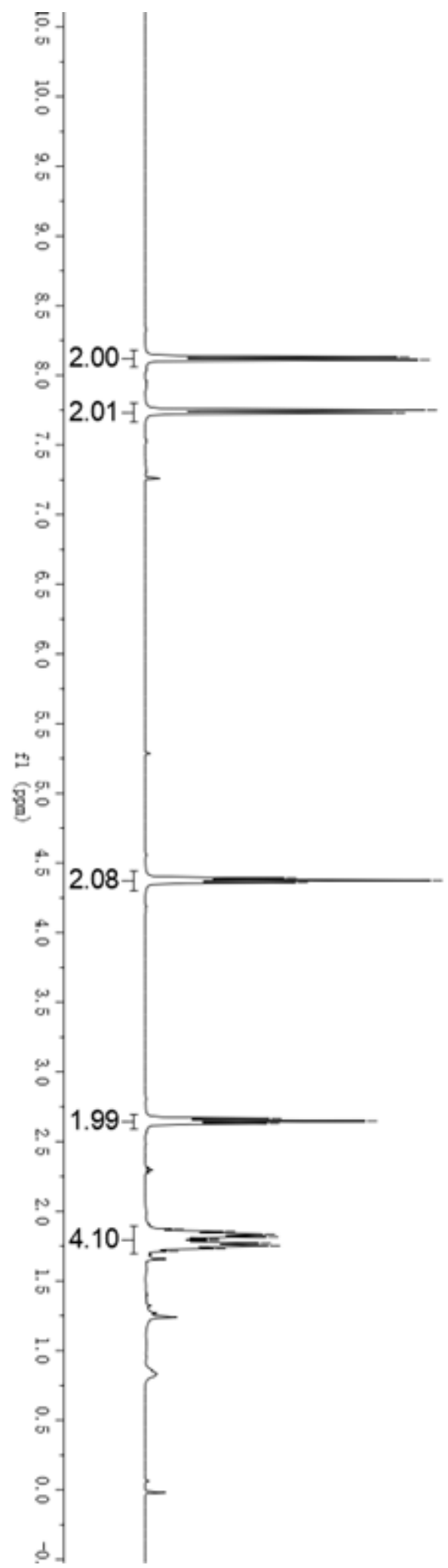

8.13

8.11

7.75

7.73

근<smiles>[CH]c1ccc(C(C)=O)cc1</smiles>

4.39

$-4.38$

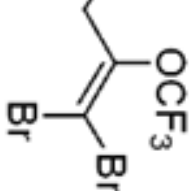

4.36

2.67

$-2.65$

2.63

1.87

1.85

1.83

1.82

1.80

1.79

1.77

$-1.75$

1.73

1.72

${ }^{1} \mathrm{H}$ NMR spectrum $\left(400 \mathrm{MHz}, \mathrm{CDCl}_{3}\right)$ of $\mathbf{3 o}$ 


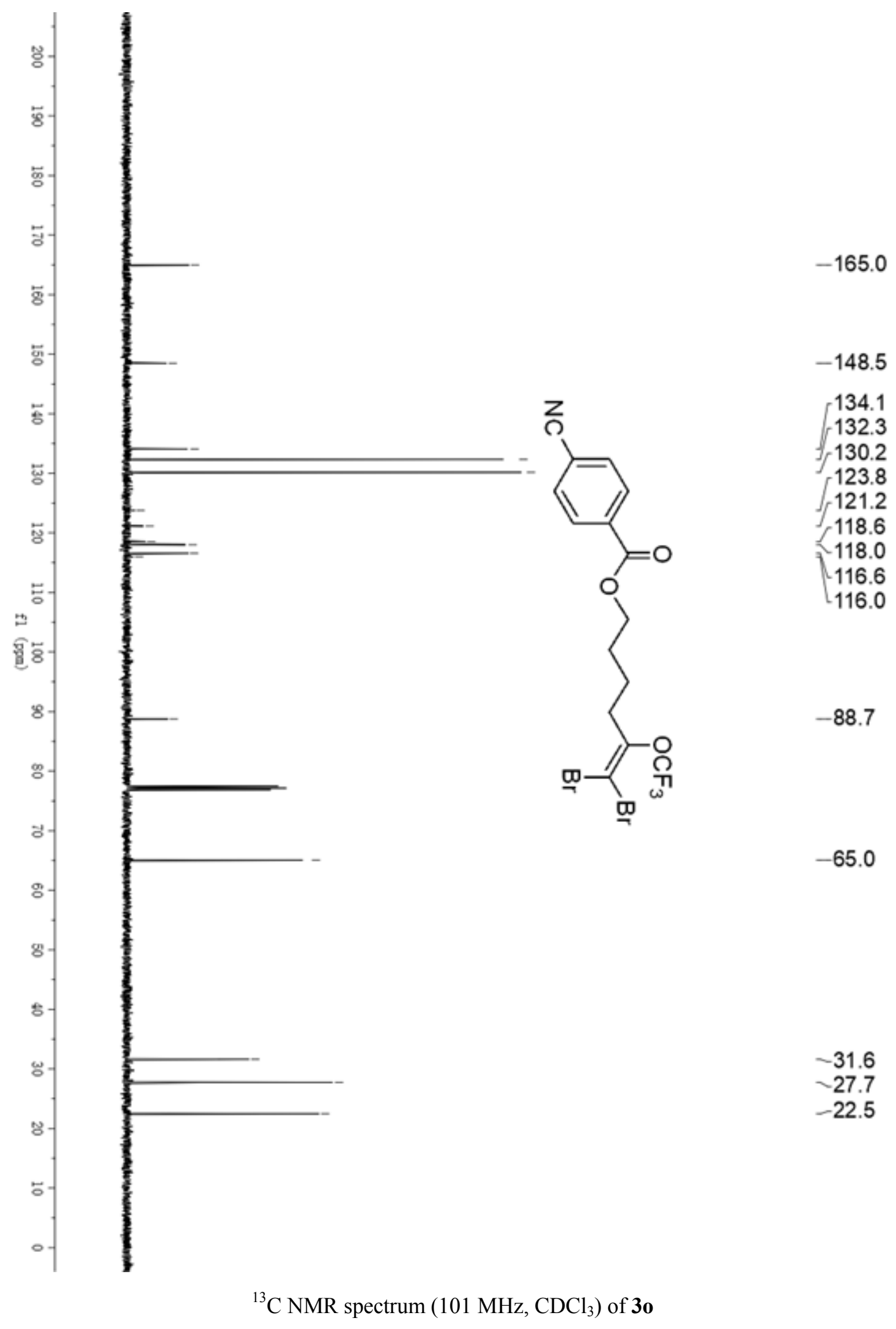




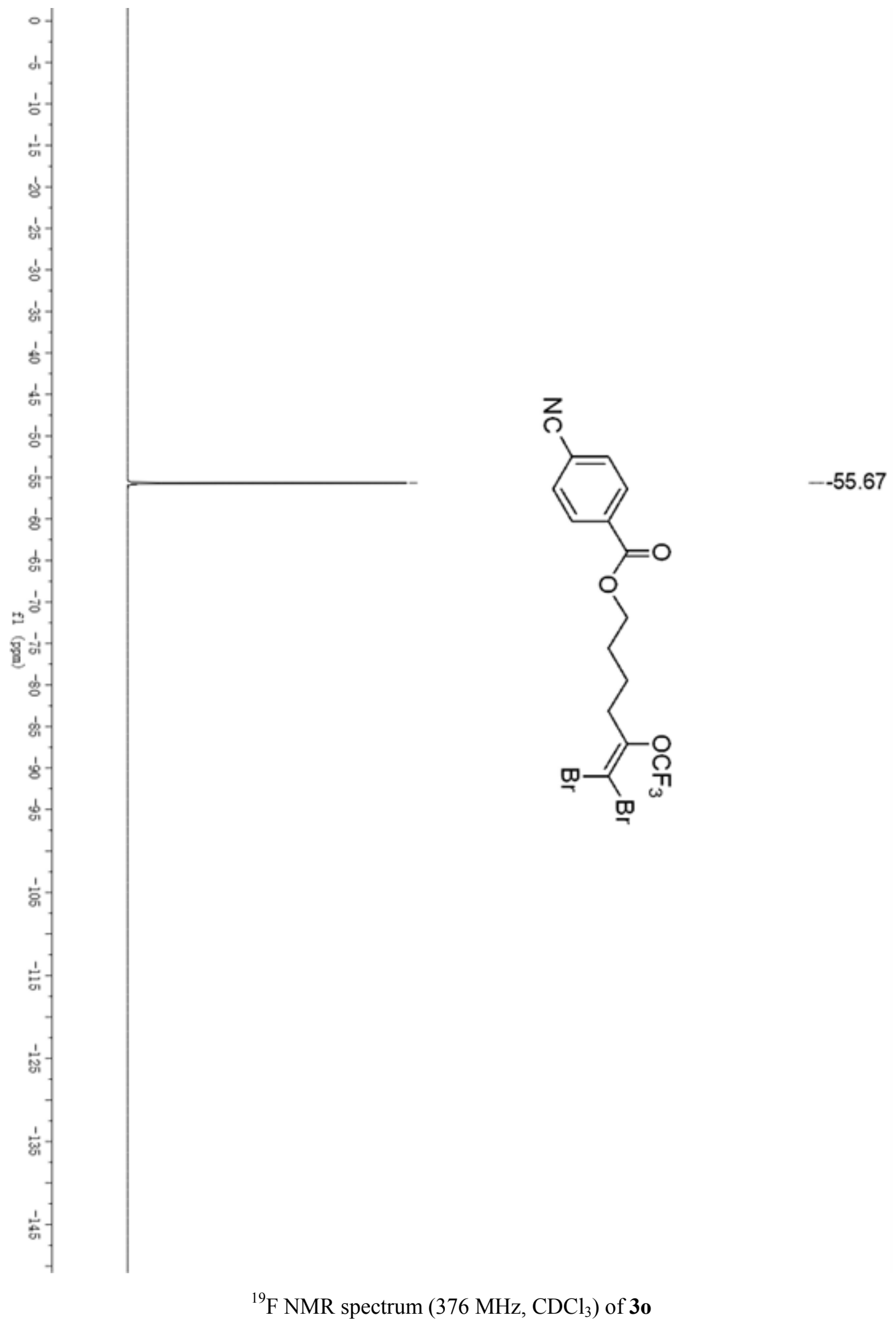




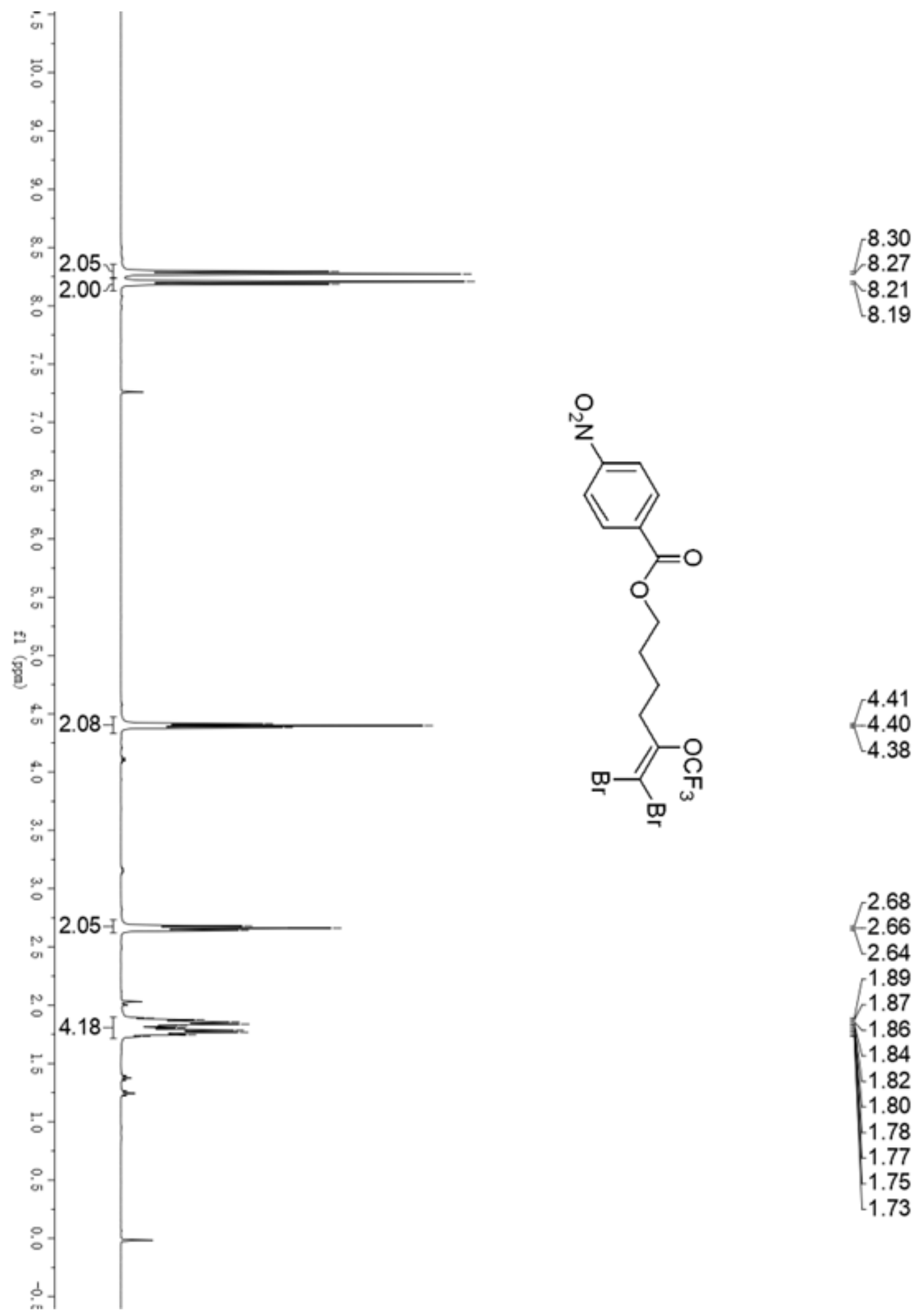

${ }^{1} \mathrm{H}$ NMR spectrum (400 MHz, $\mathrm{CDCl}_{3}$ ) of $\mathbf{3 p}$ 


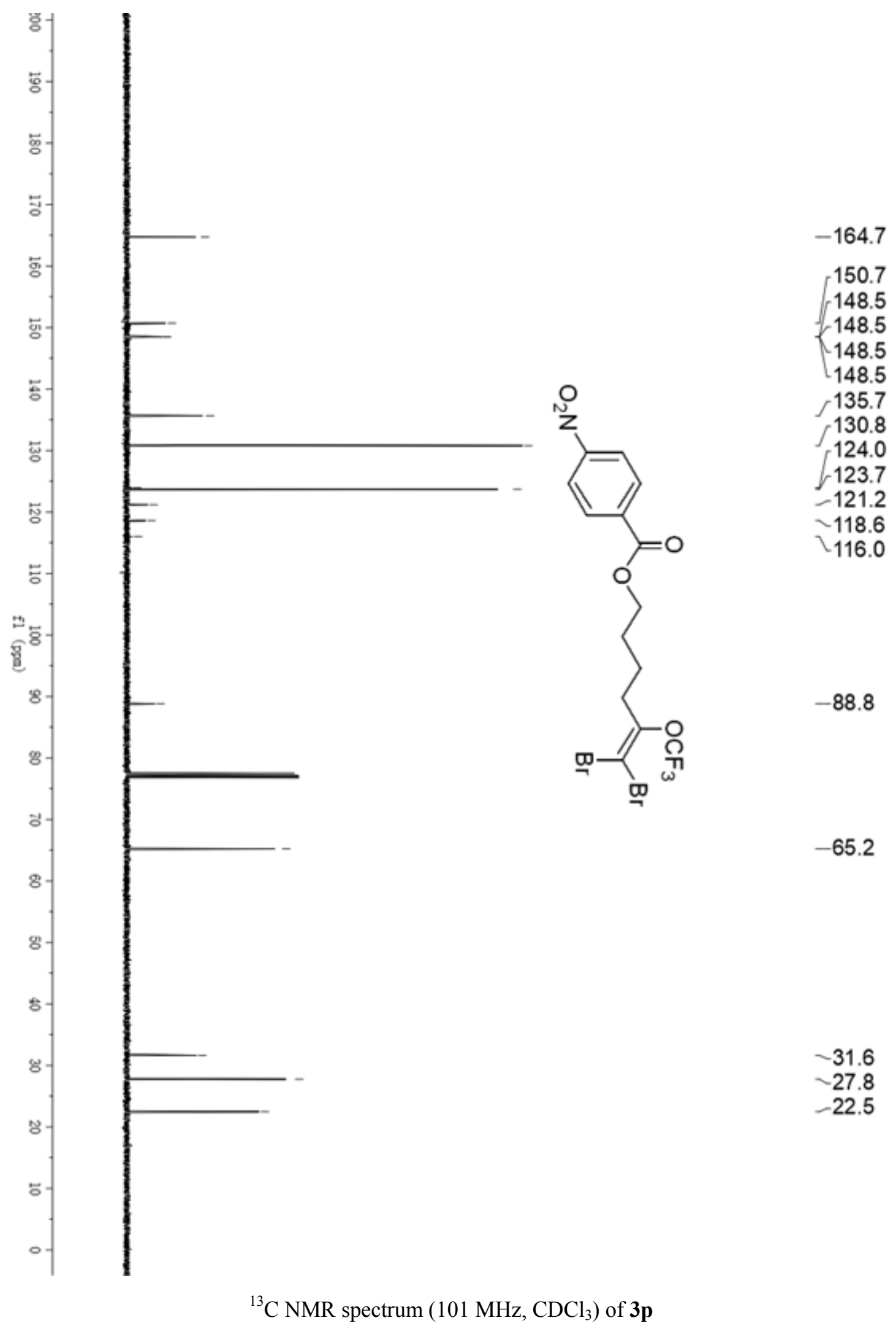




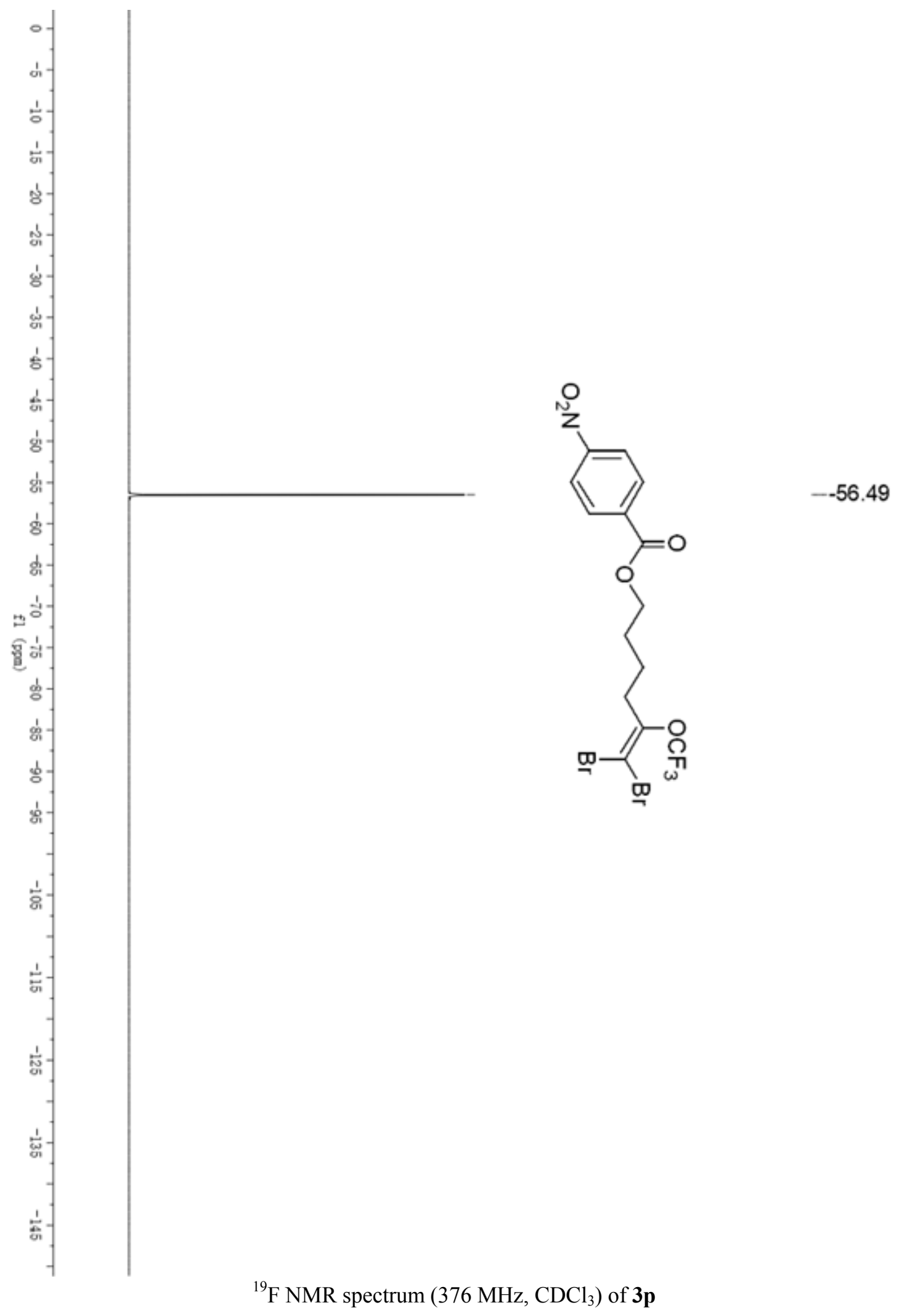




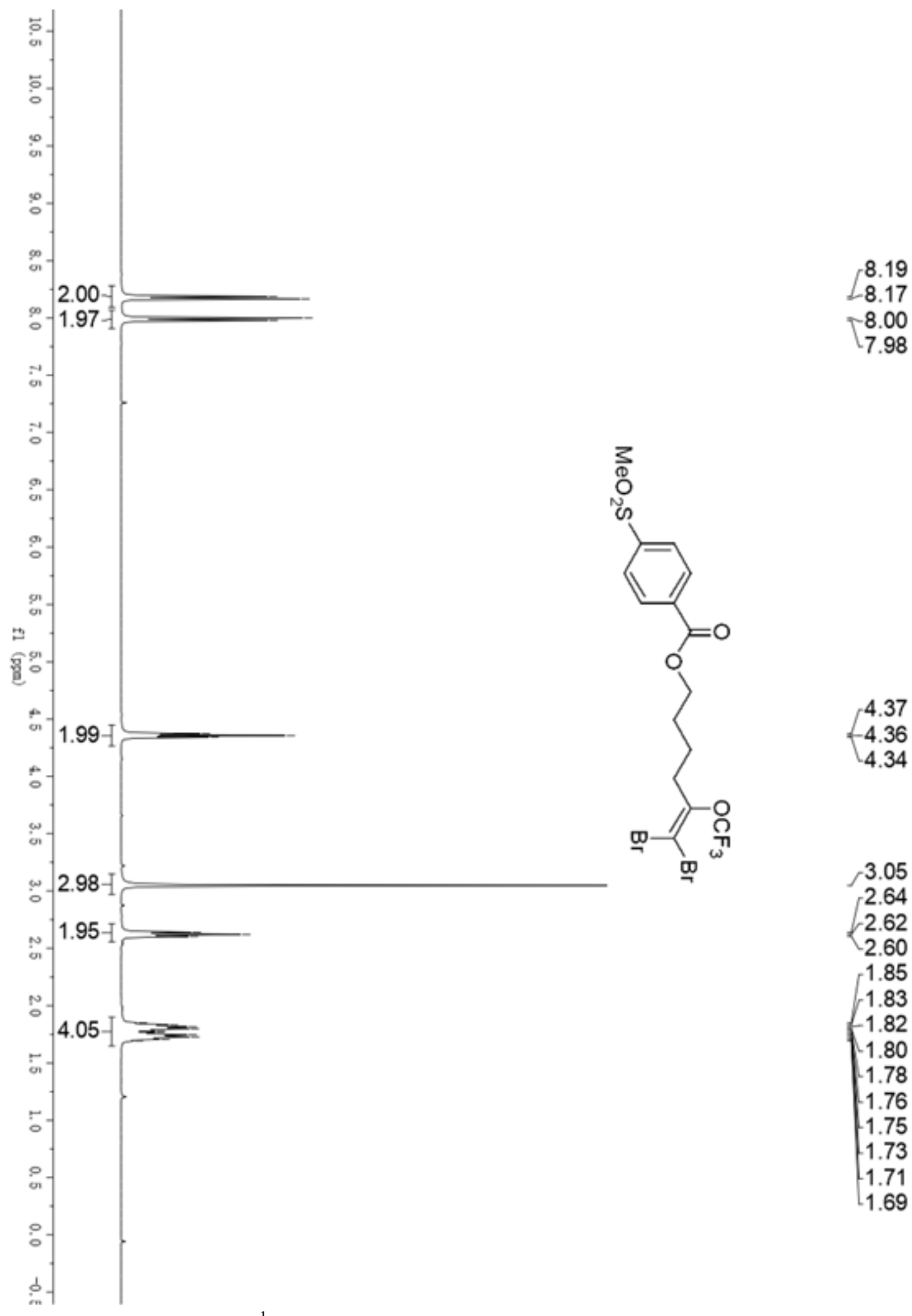

${ }^{1} \mathrm{H}$ NMR spectrum $\left(400 \mathrm{MHz}, \mathrm{CDCl}_{3}\right)$ of $\mathbf{3 q}$ 


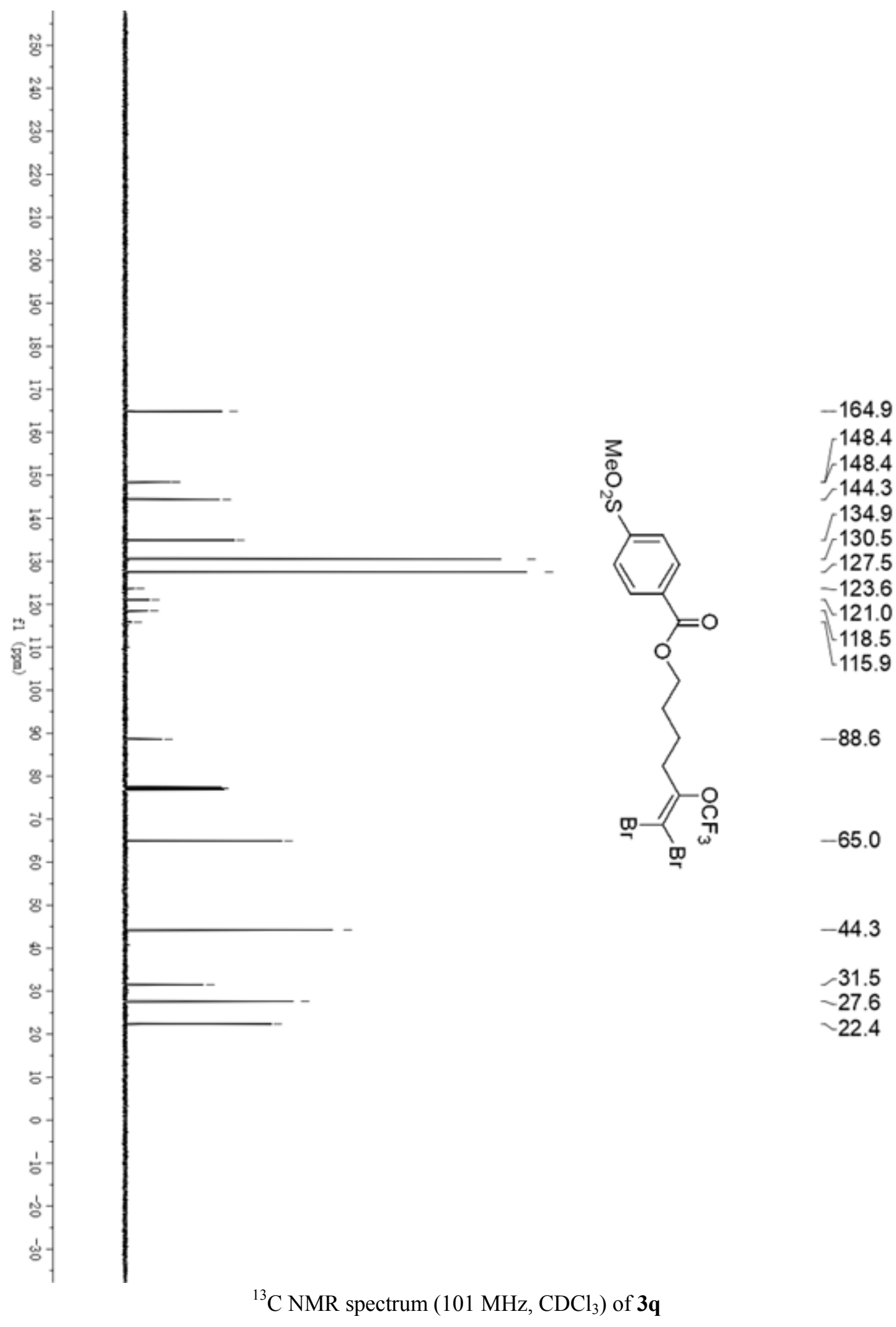




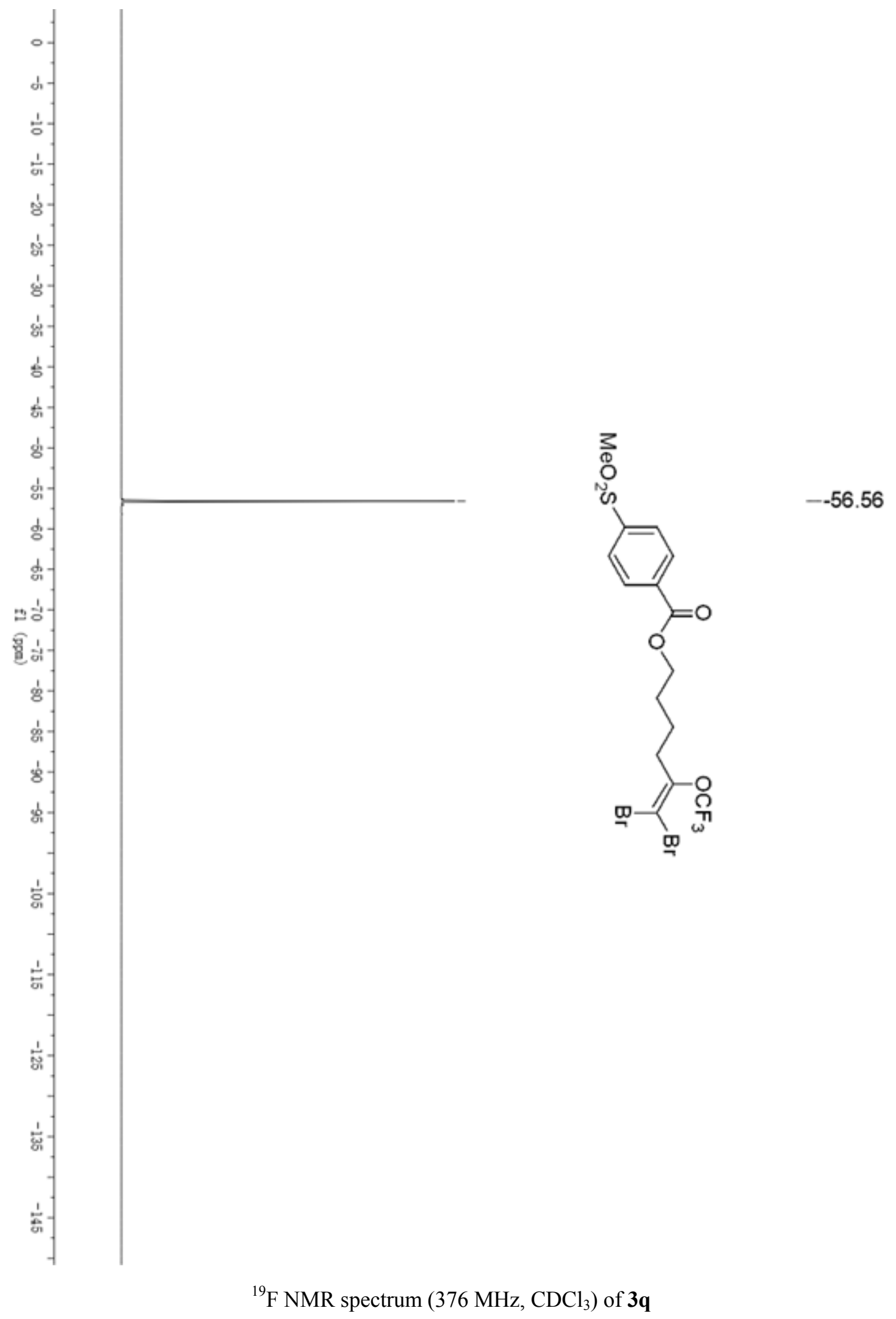




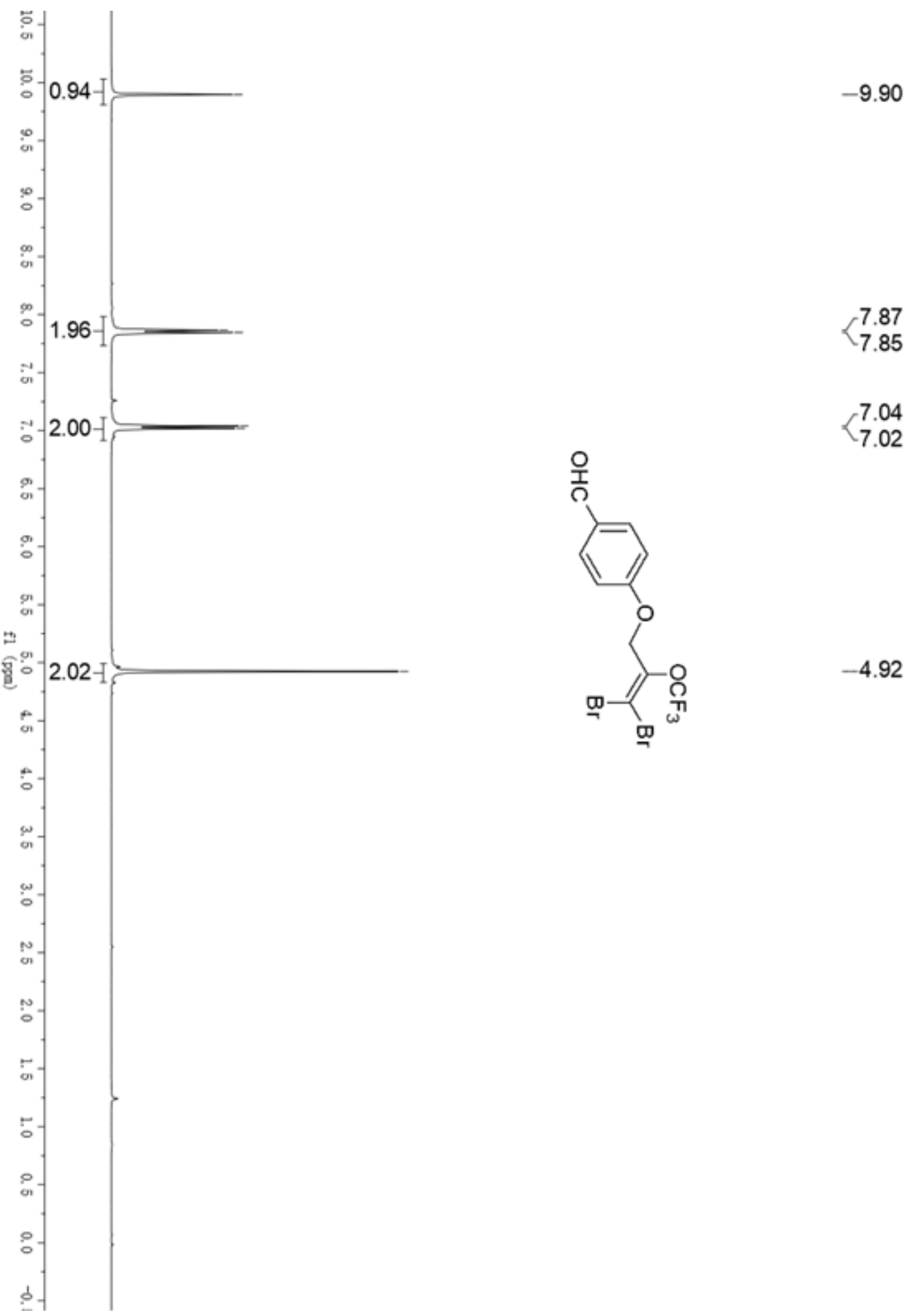

${ }^{1} \mathrm{H}$ NMR spectrum $\left(400 \mathrm{MHz}, \mathrm{CDCl}_{3}\right)$ of $\mathbf{3 r}$ 


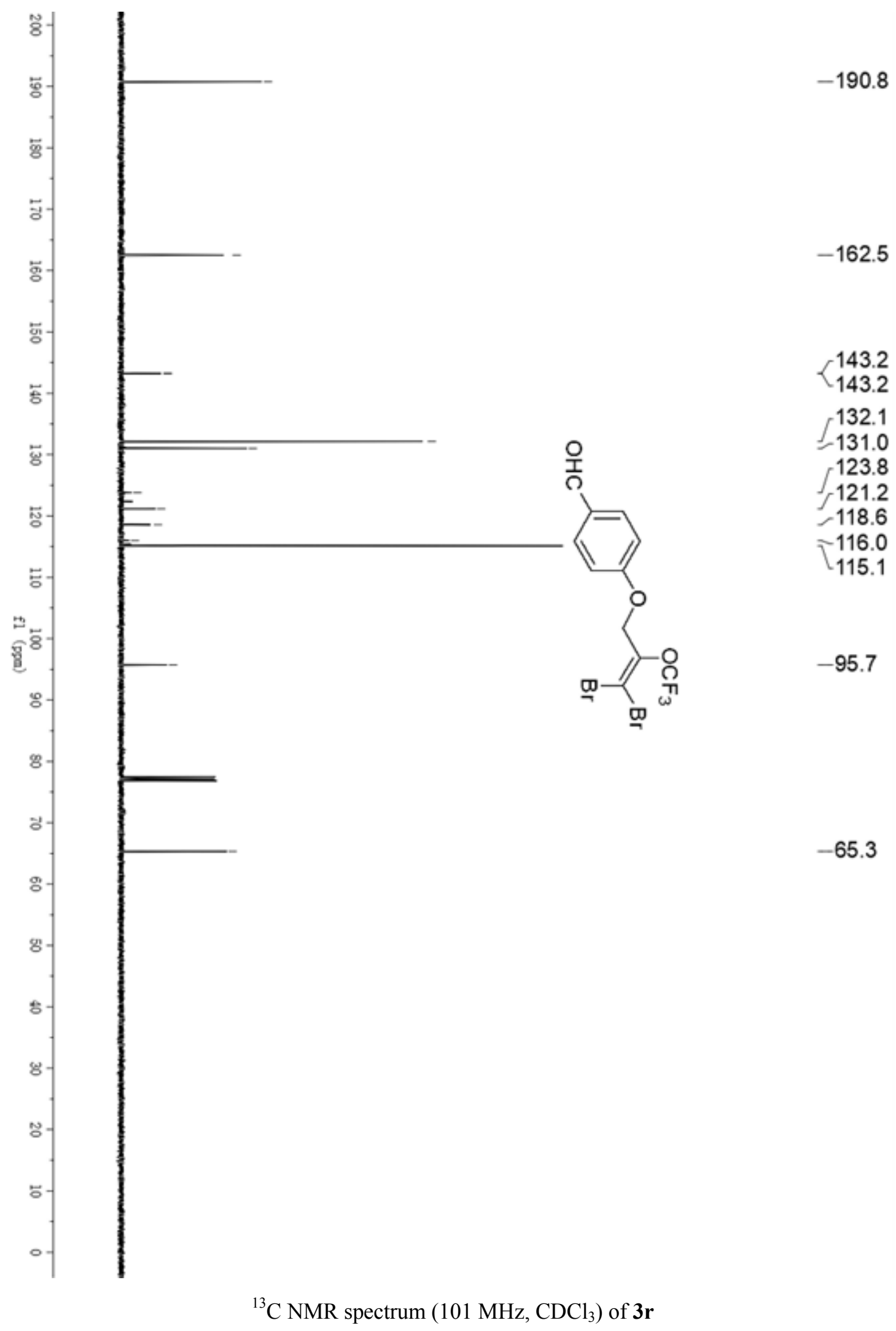




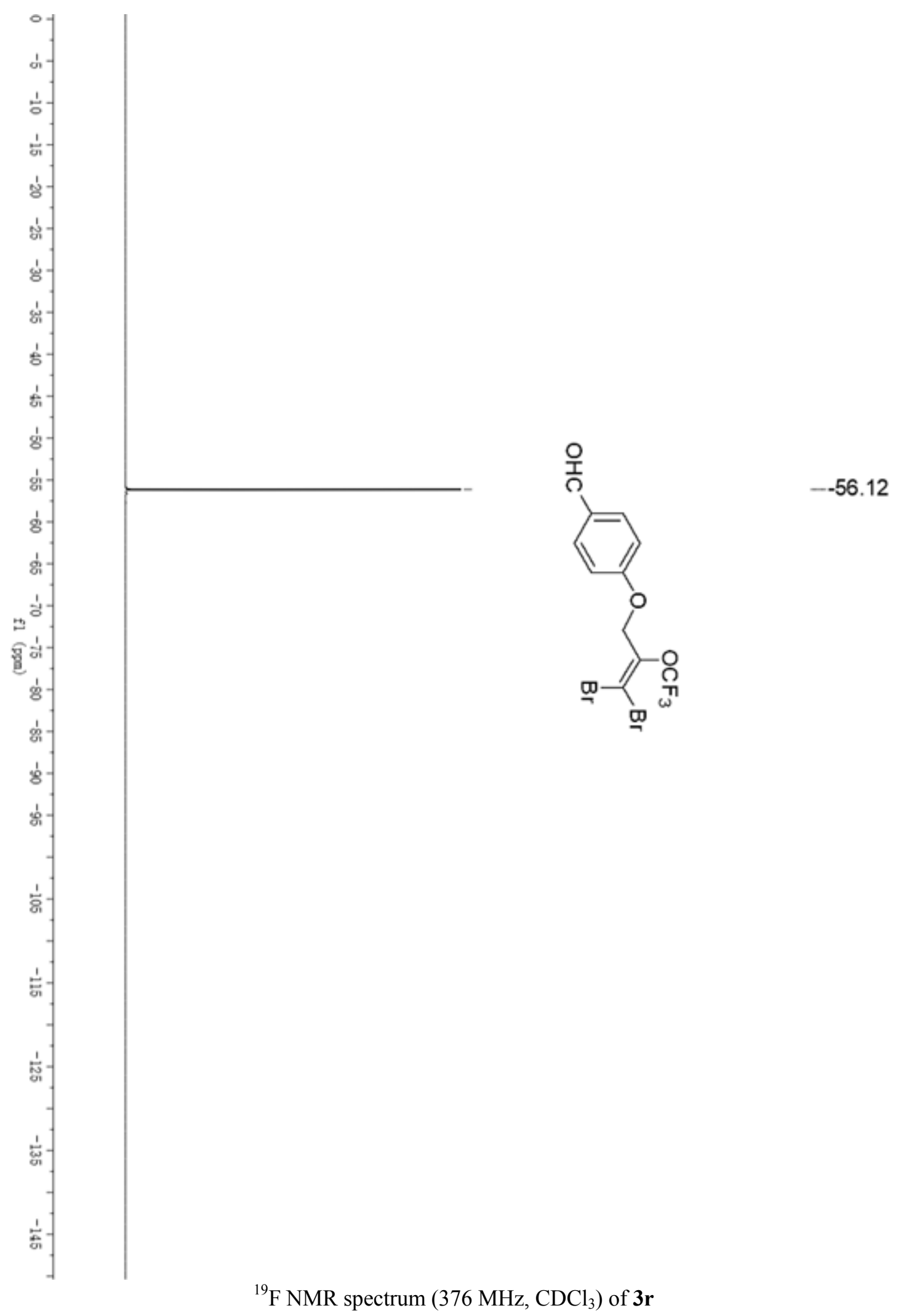




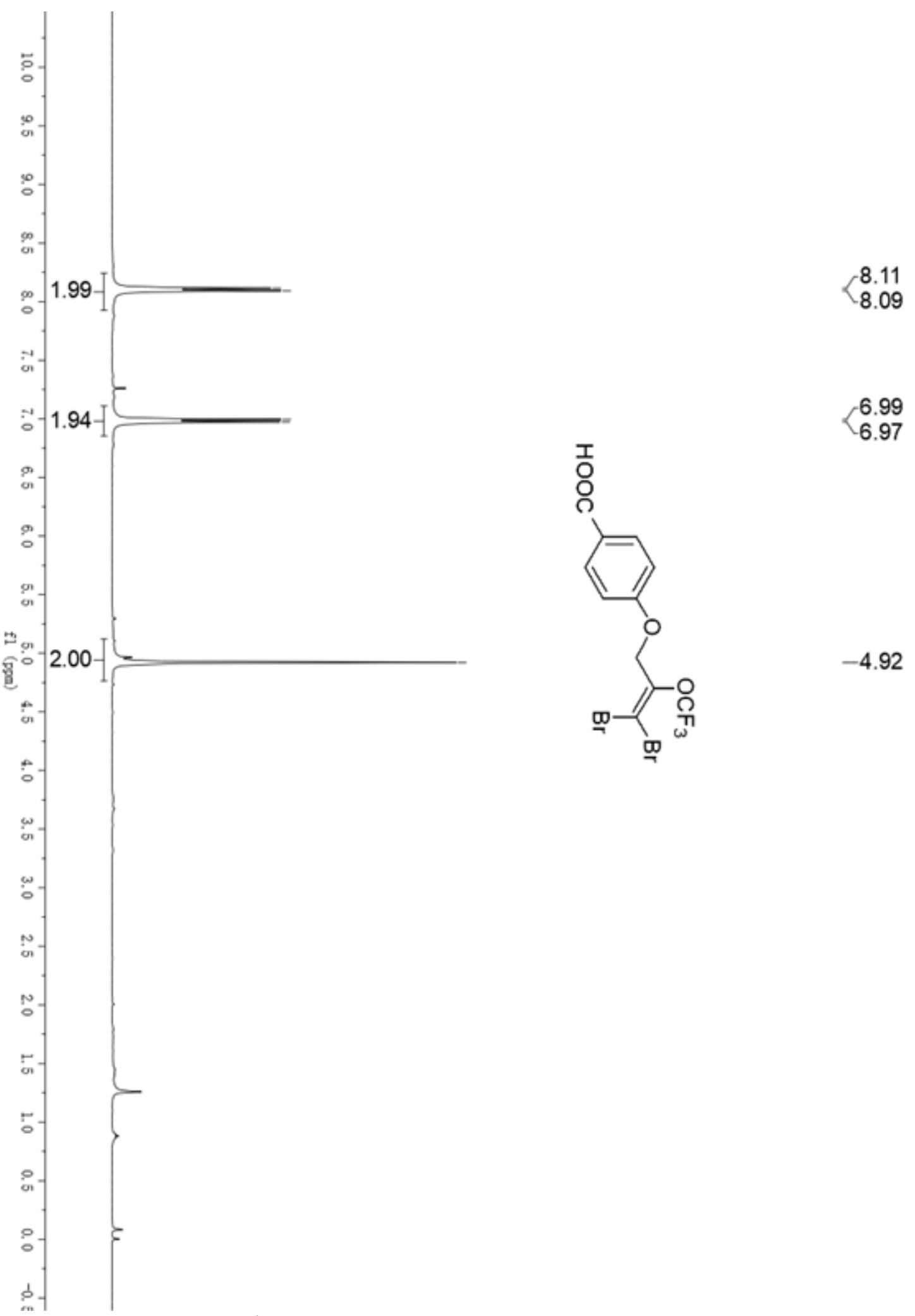

${ }^{1} \mathrm{H}$ NMR spectrum (400 MHz, $\mathrm{CDCl}_{3}$ ) of $\mathbf{3 s}$ 


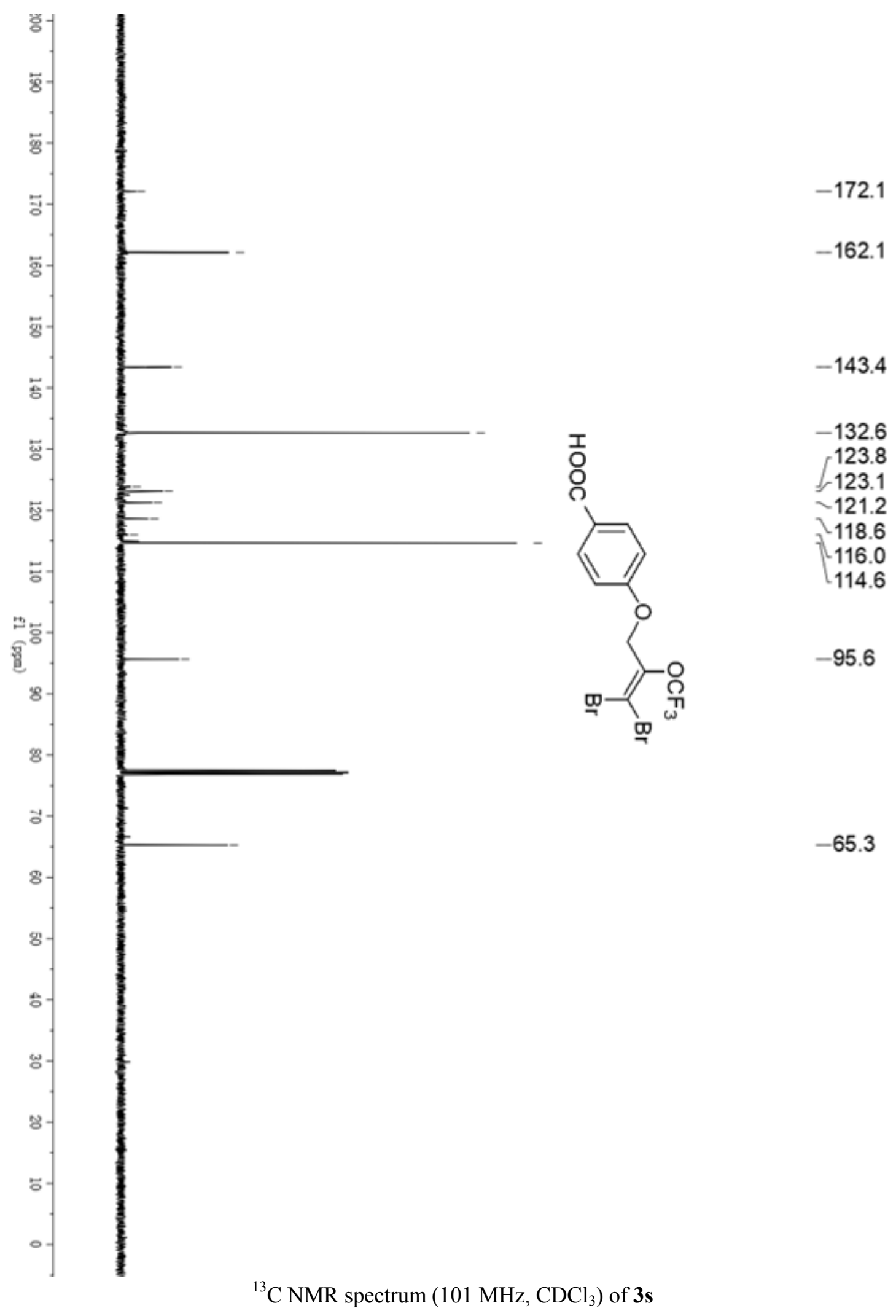




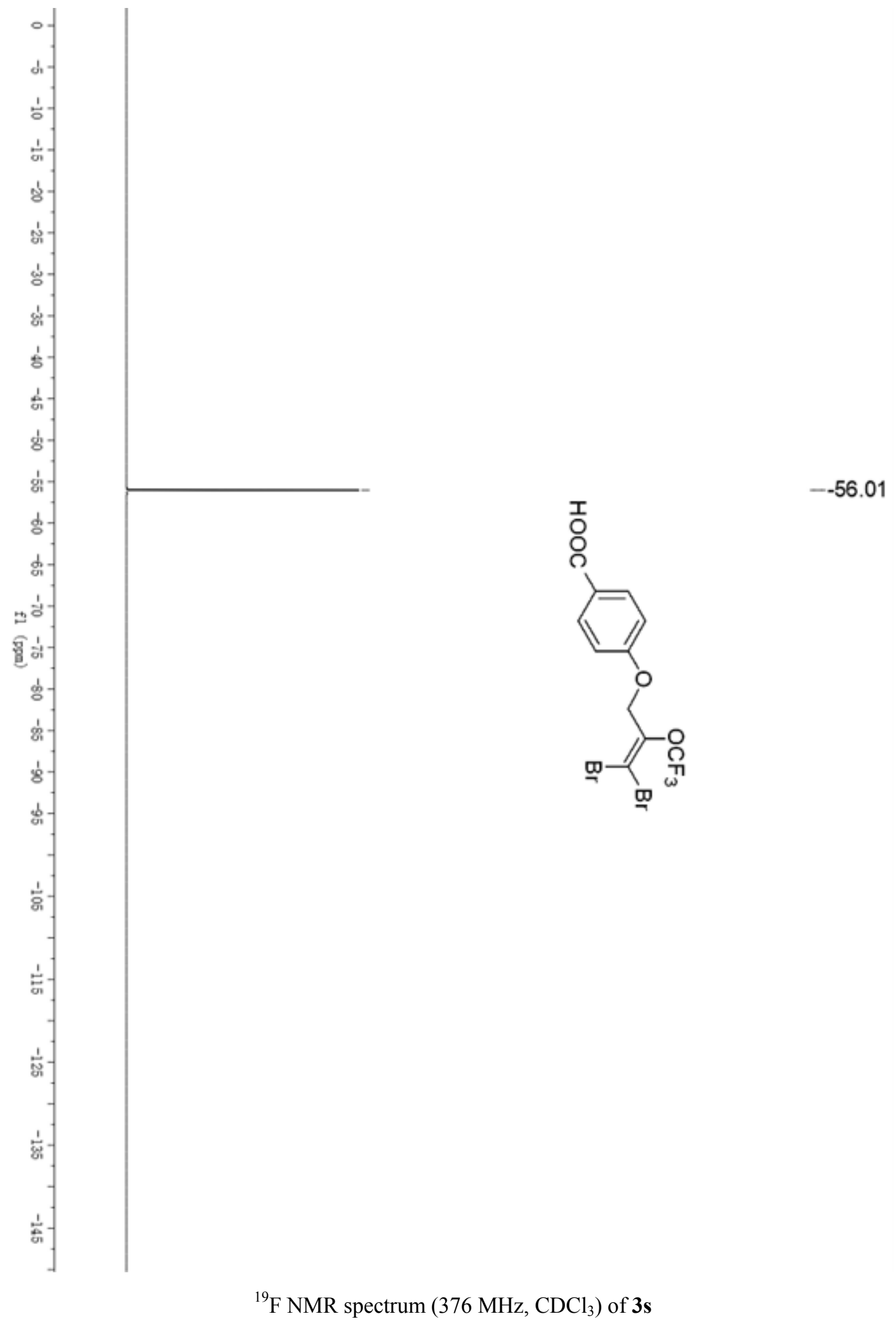




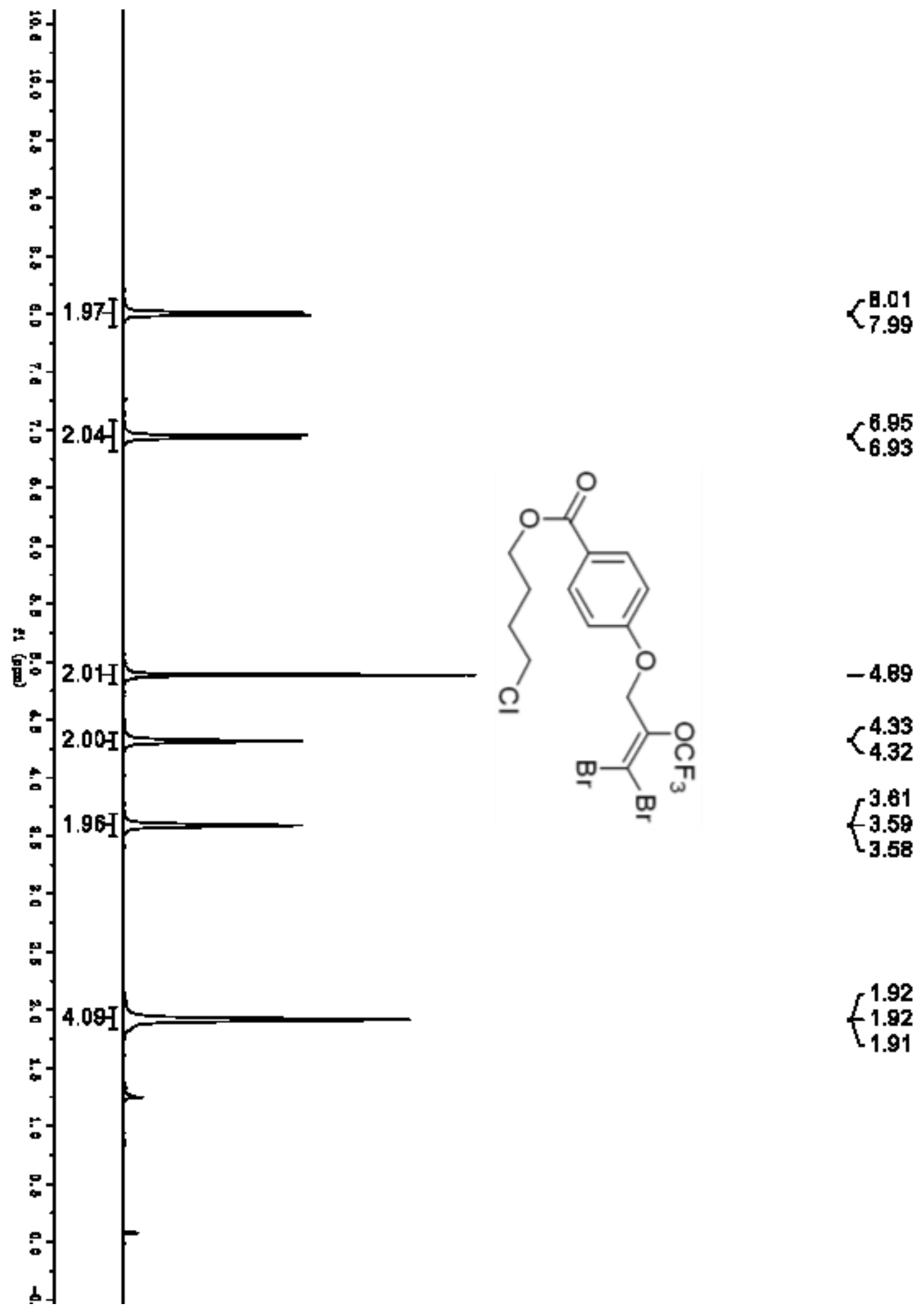

${ }^{1} \mathrm{H}$ NMR spectrum $\left(400 \mathrm{MHz}, \mathrm{CDCl}_{3}\right)$ of $\mathbf{3 t}$ 


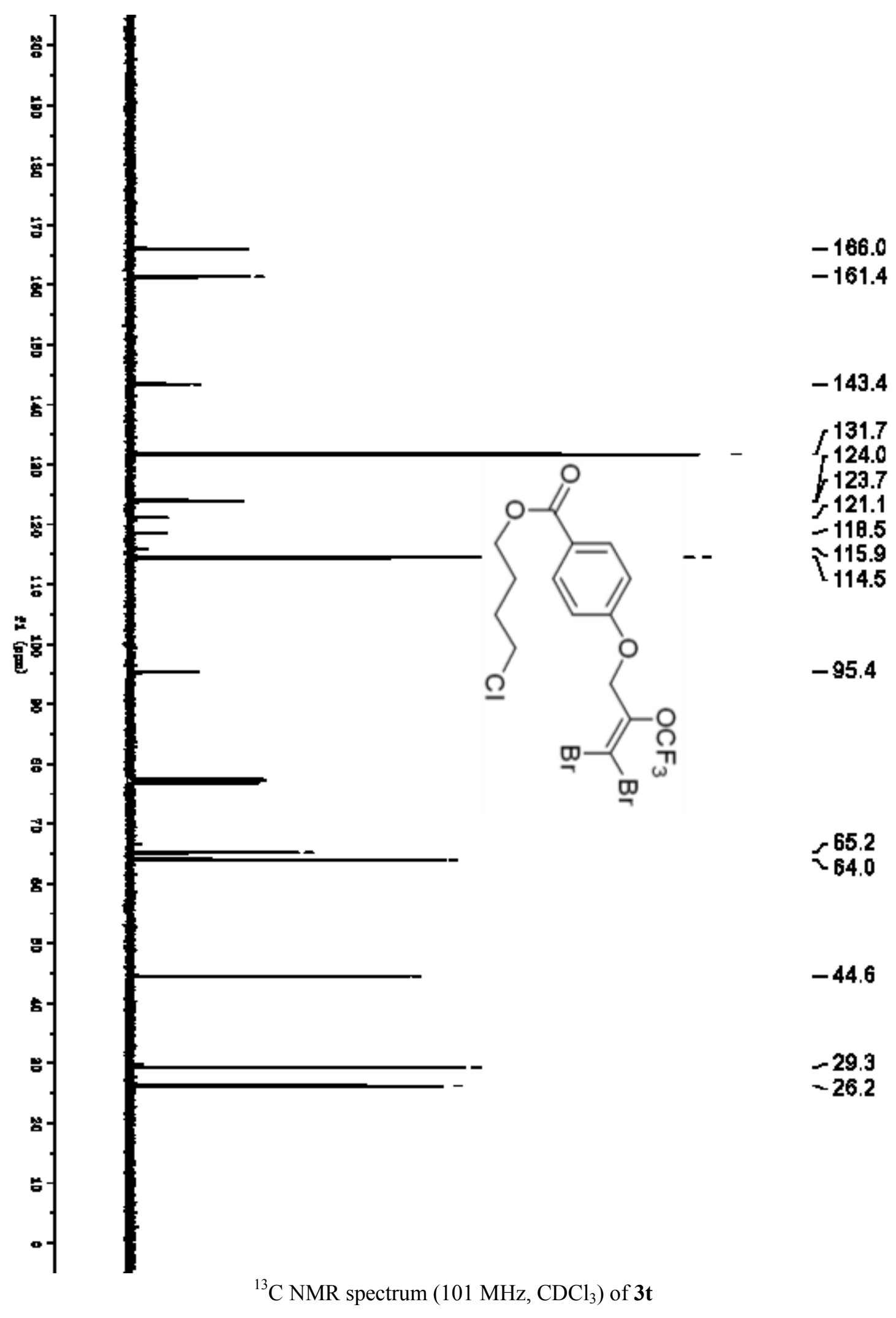




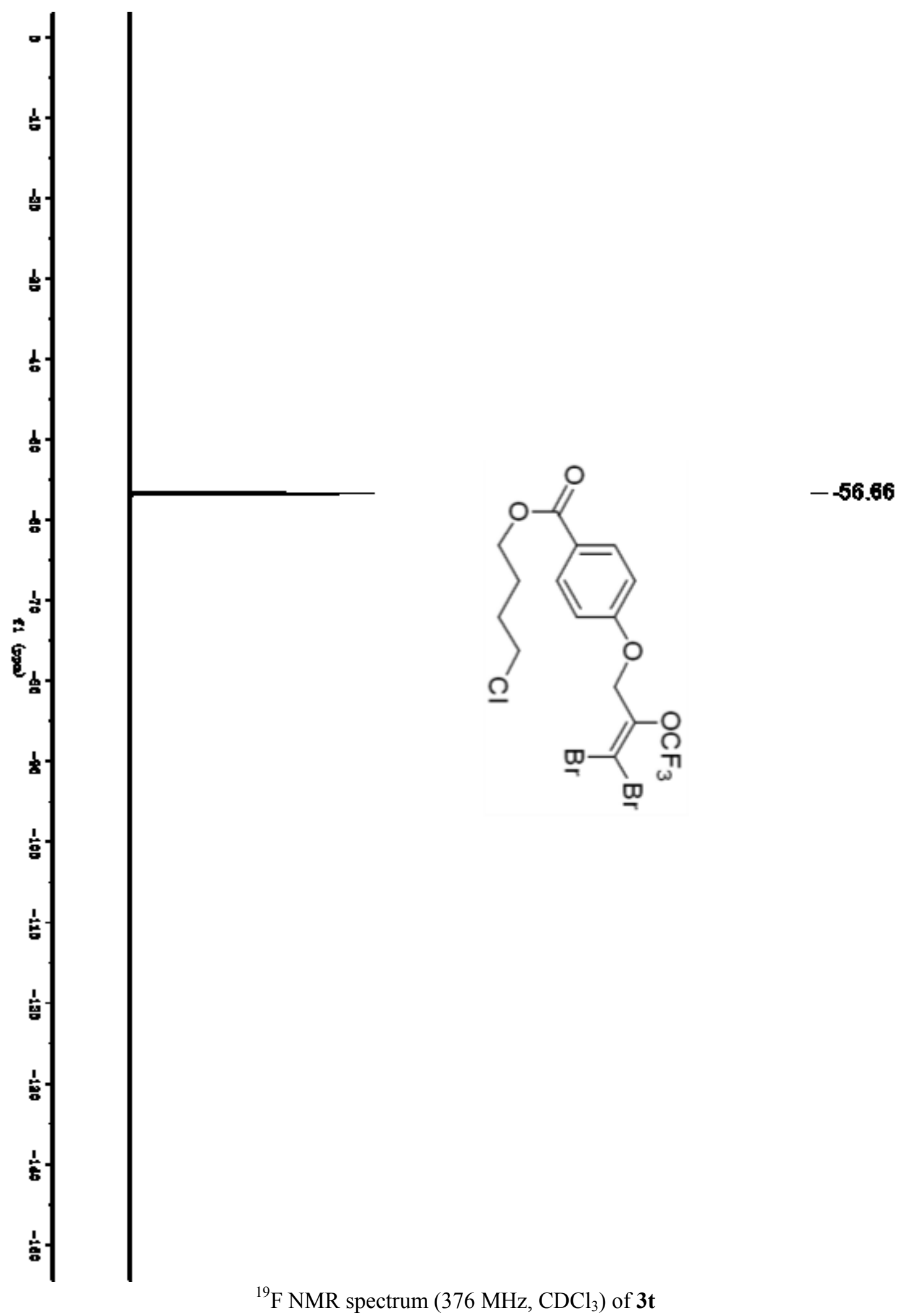




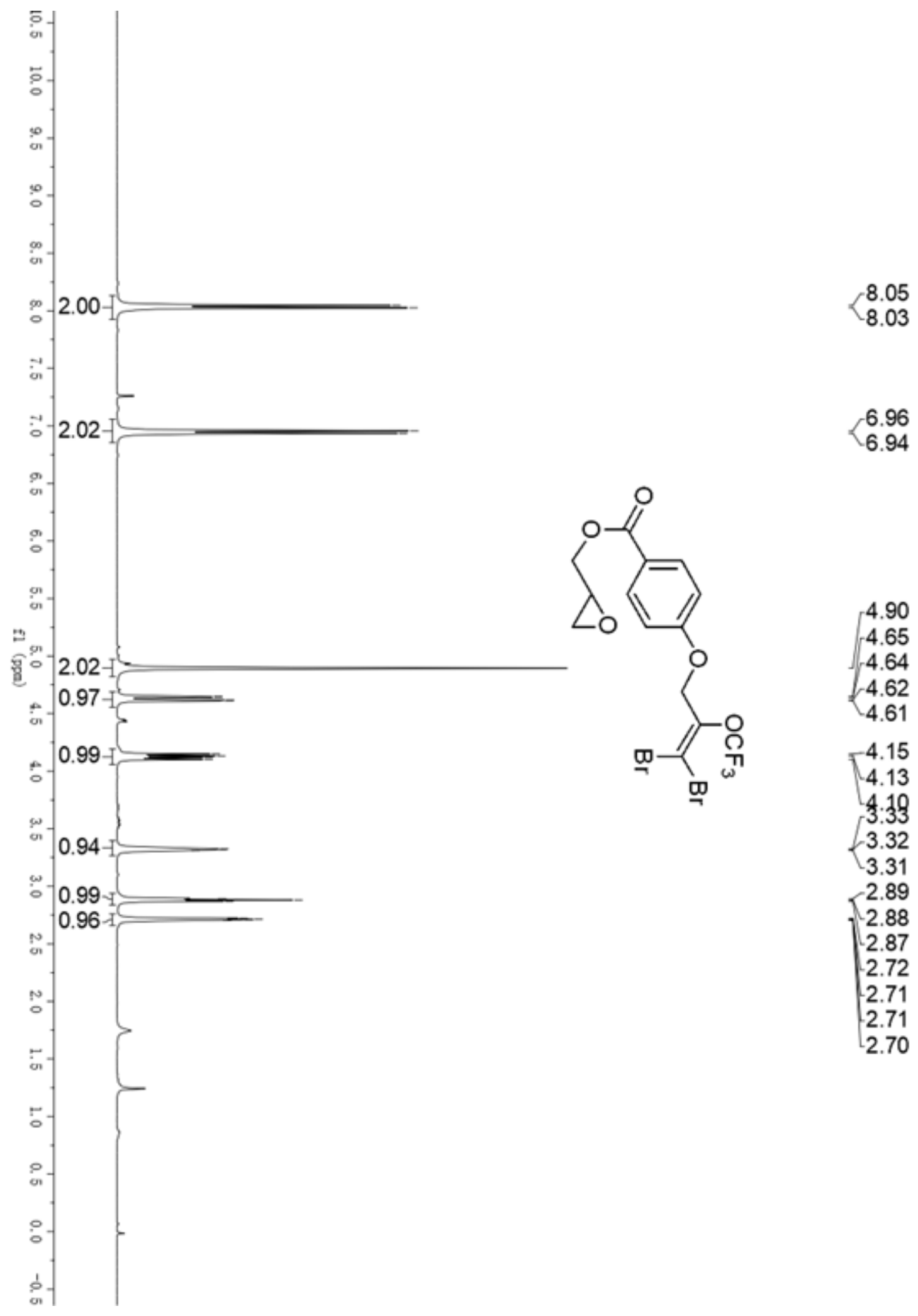

${ }^{1} \mathrm{H}$ NMR spectrum $\left(400 \mathrm{MHz}, \mathrm{CDCl}_{3}\right)$ of $\mathbf{3 u}$ 


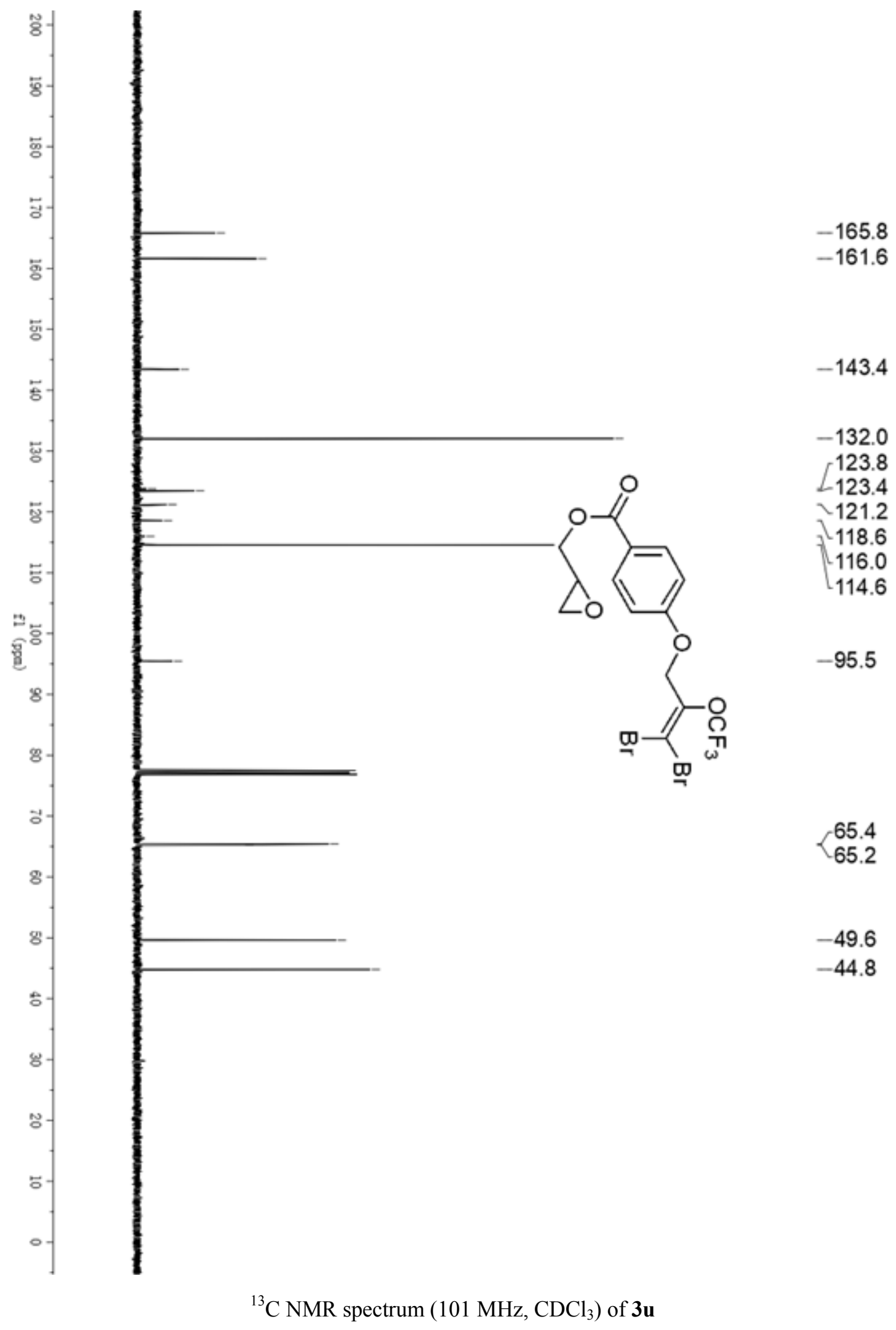




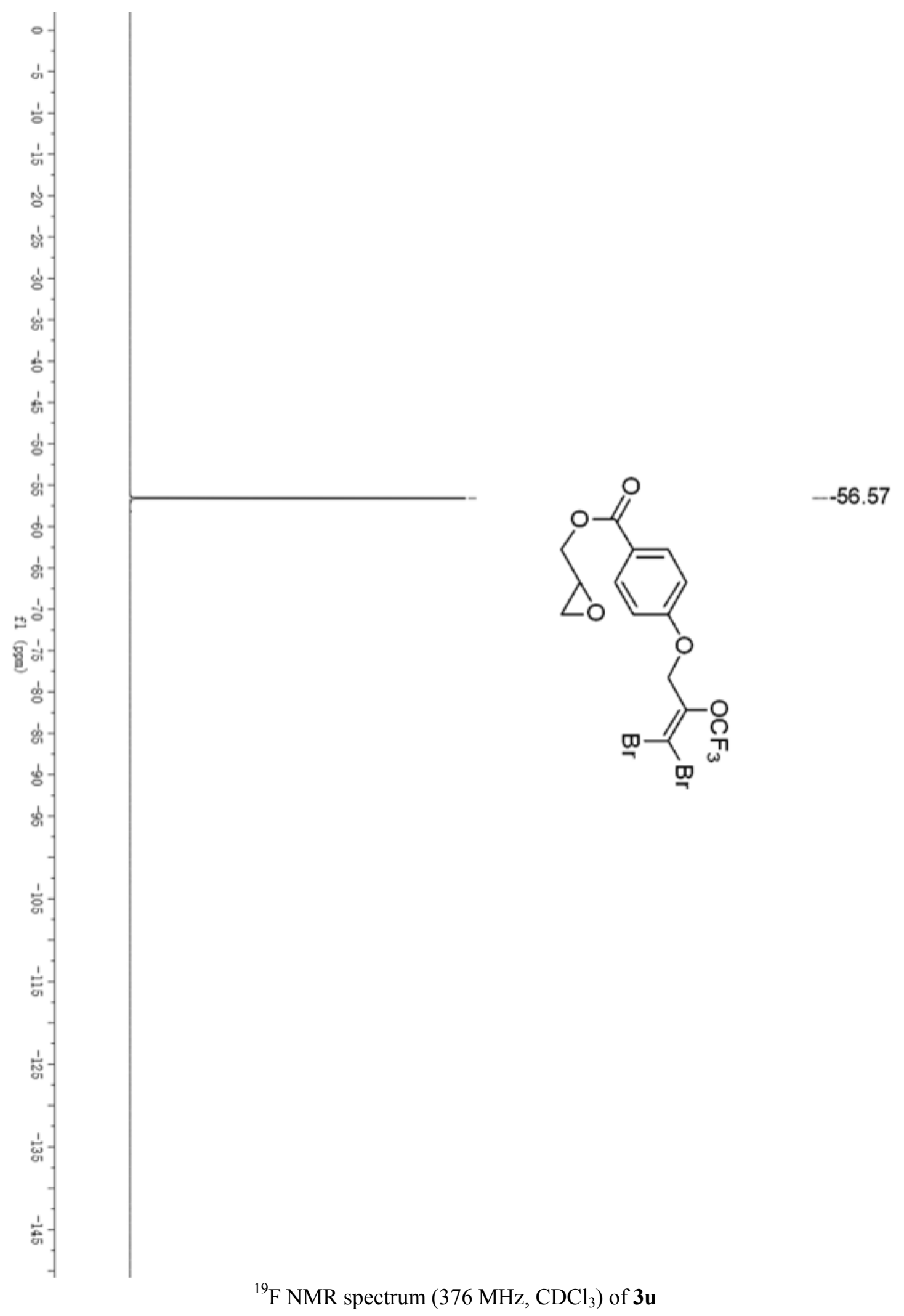




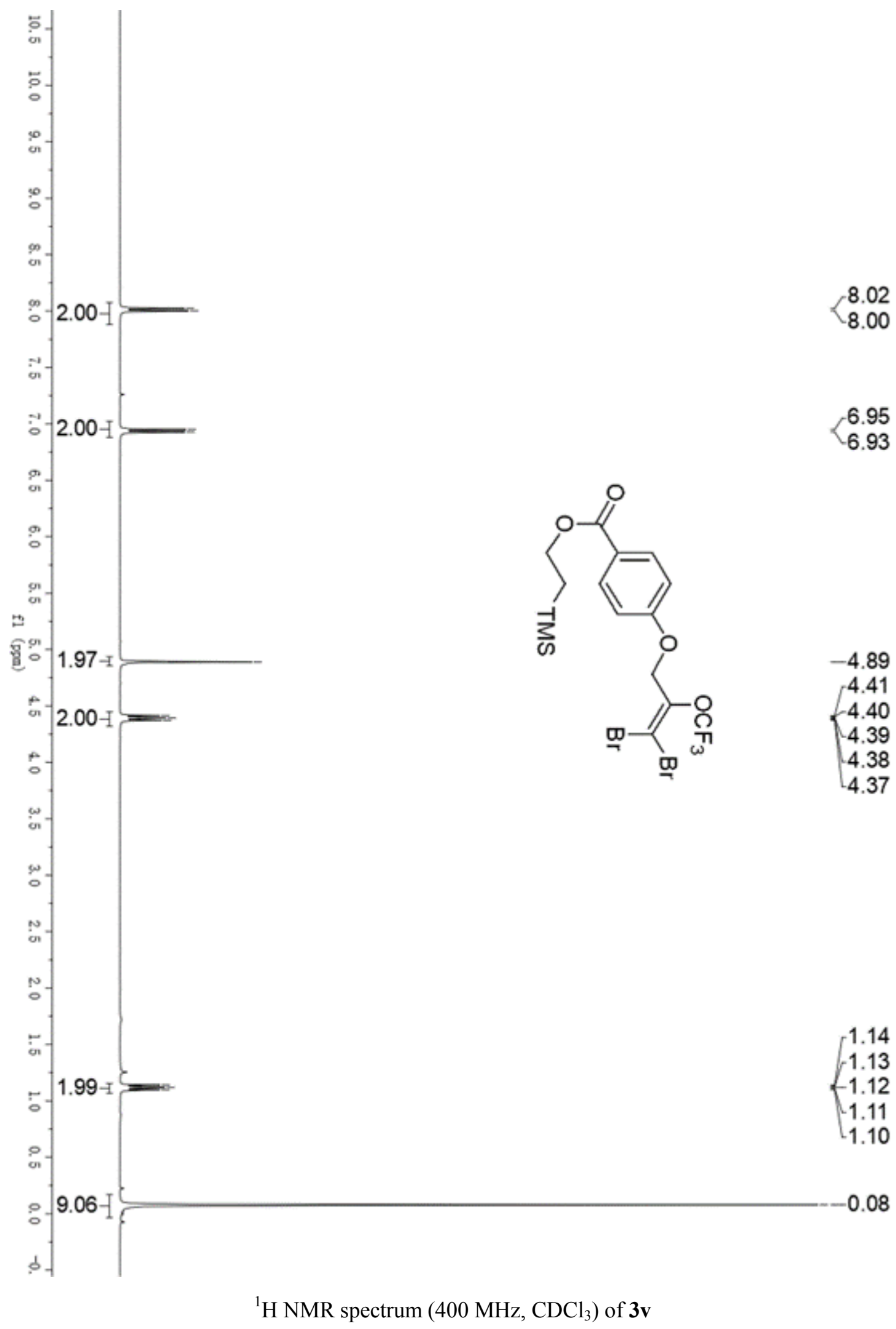




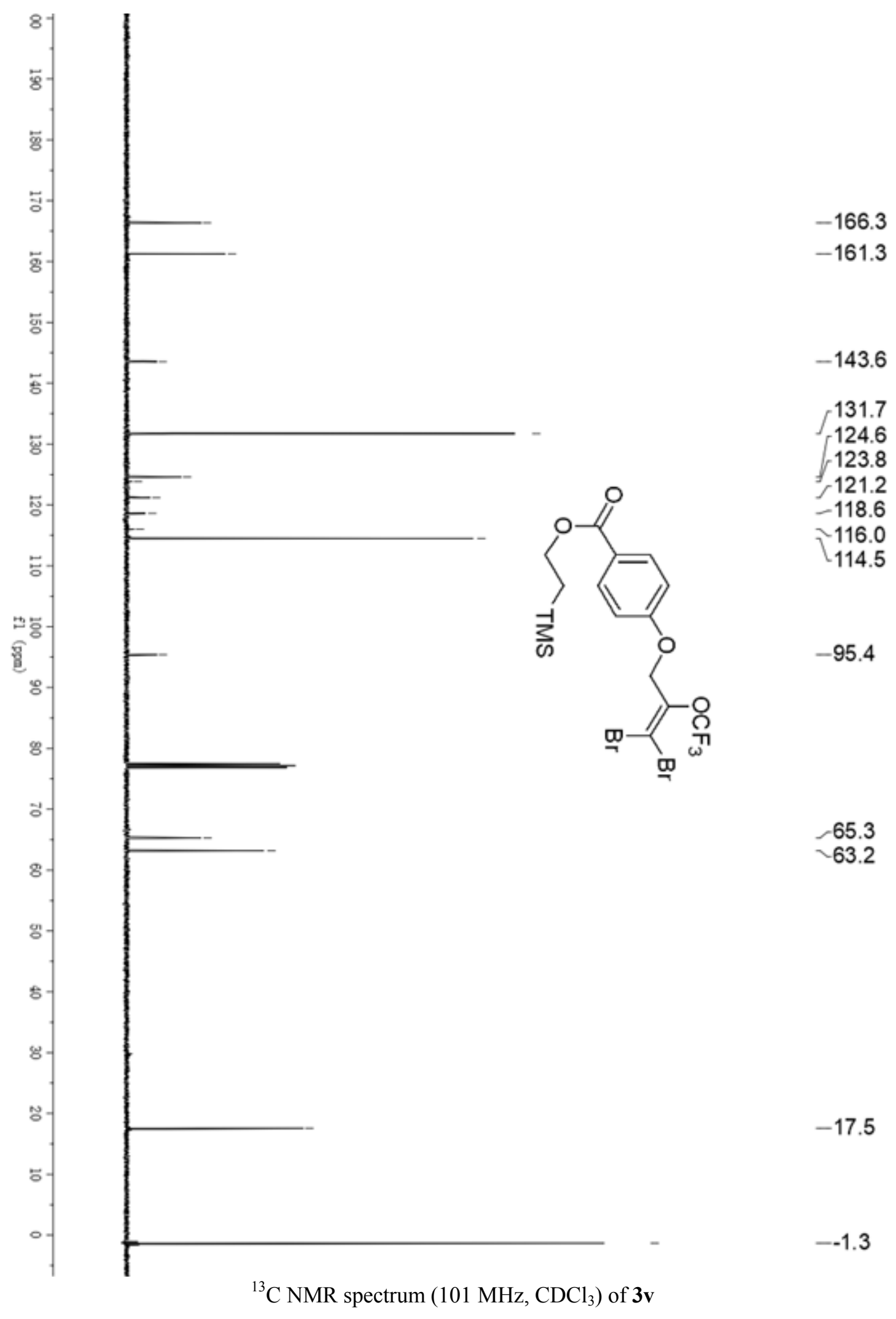




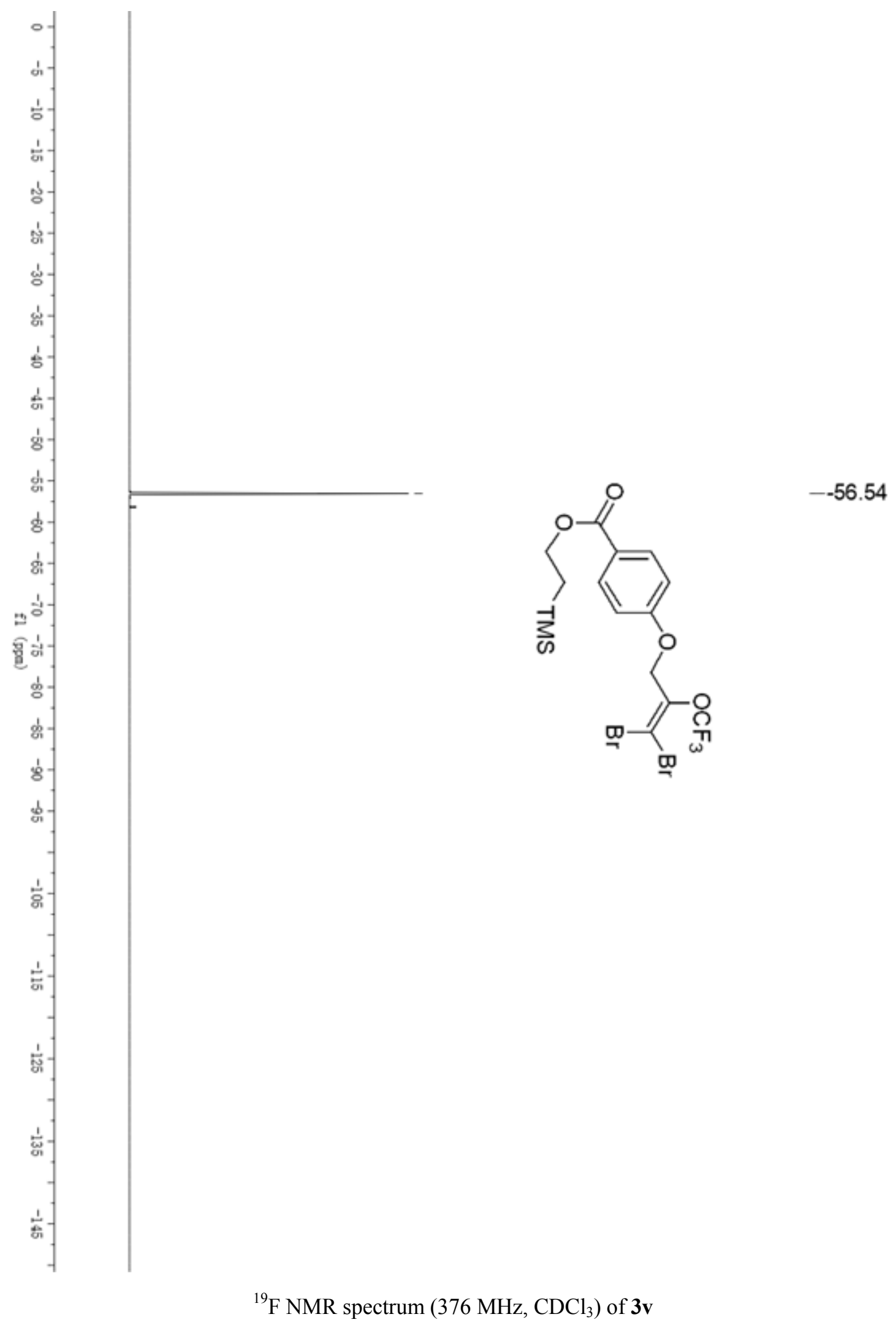




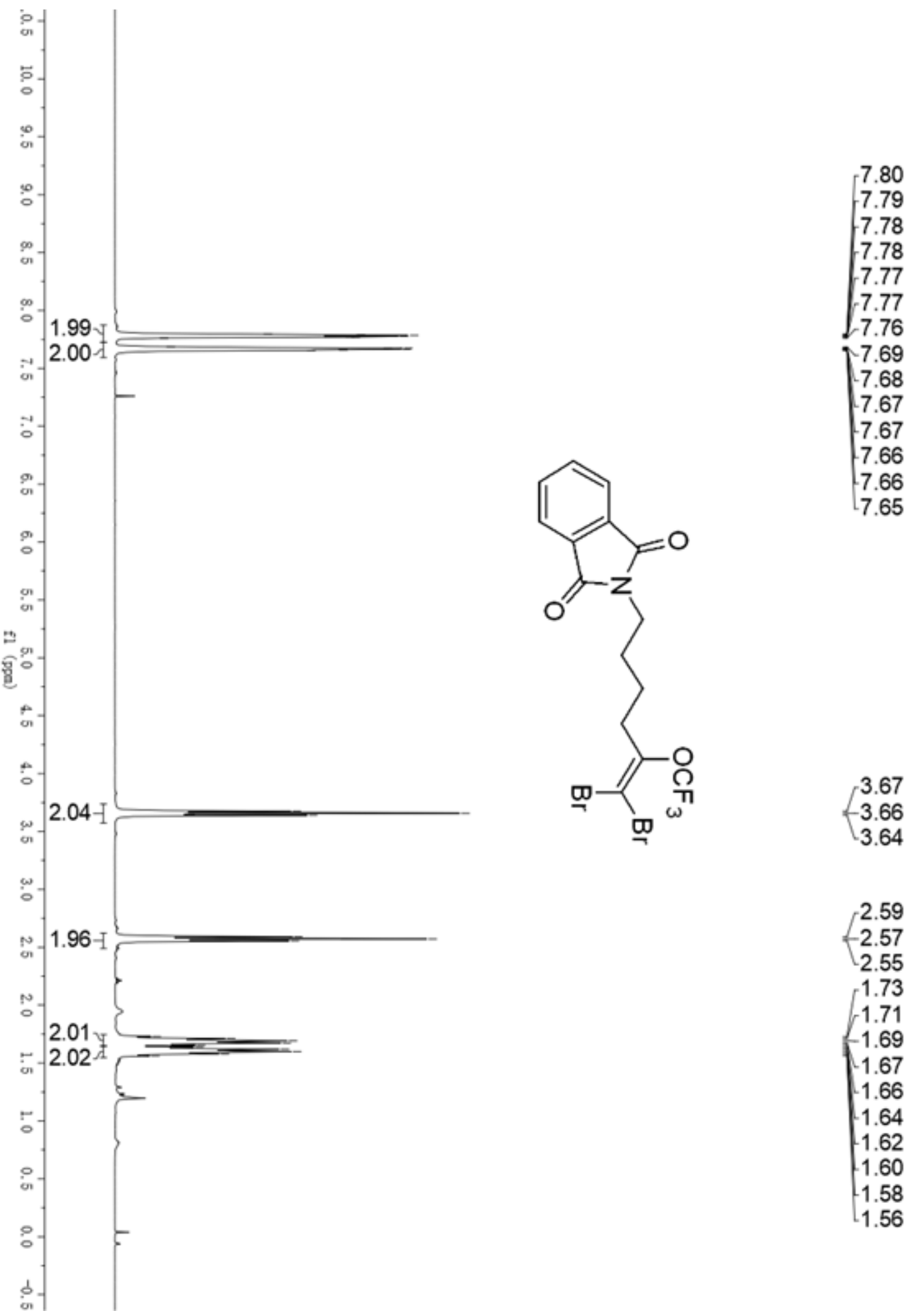

${ }^{1} \mathrm{H}$ NMR spectrum $\left(400 \mathrm{MHz}, \mathrm{CDCl}_{3}\right)$ of $\mathbf{3 w}$ 


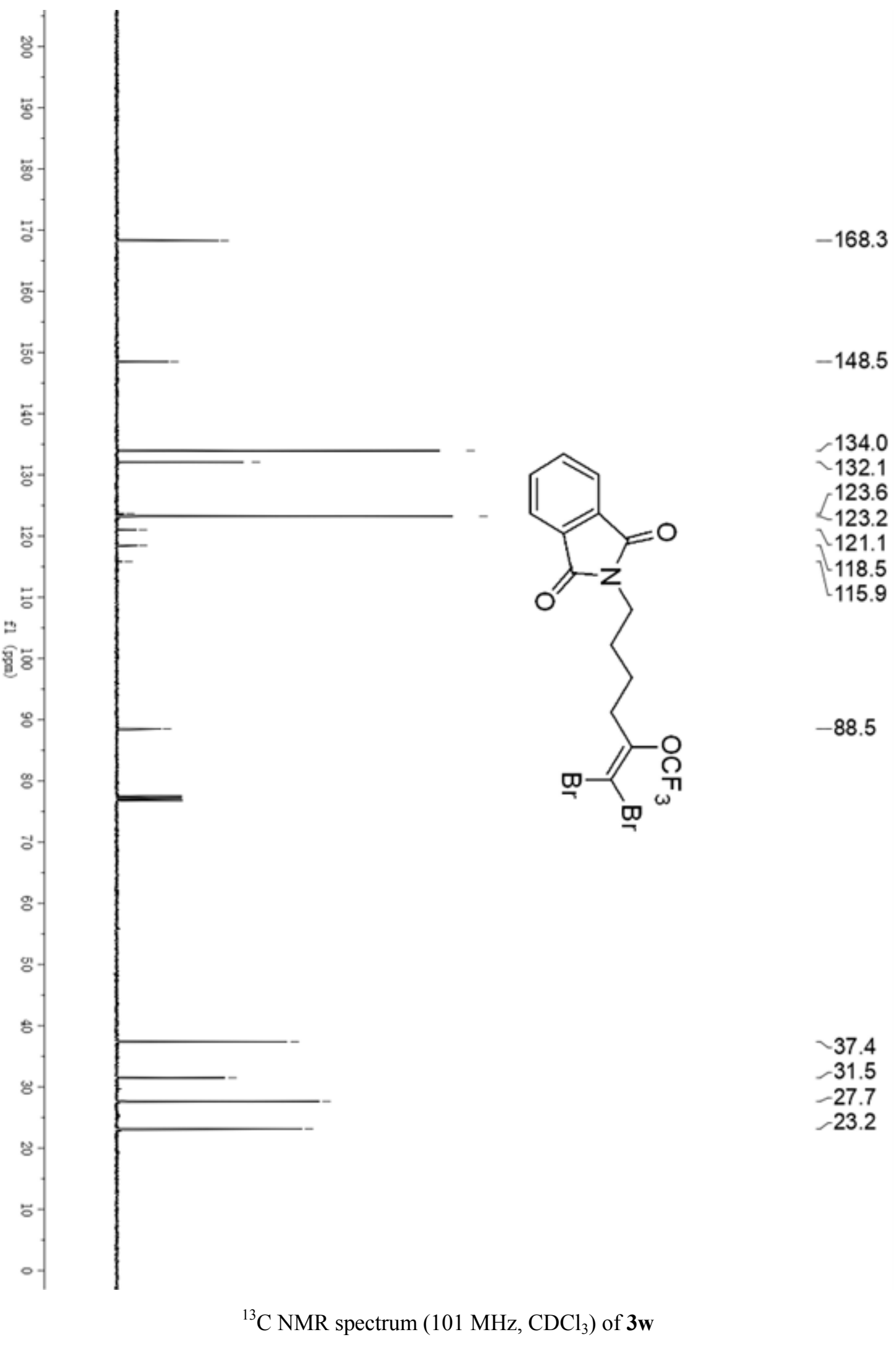




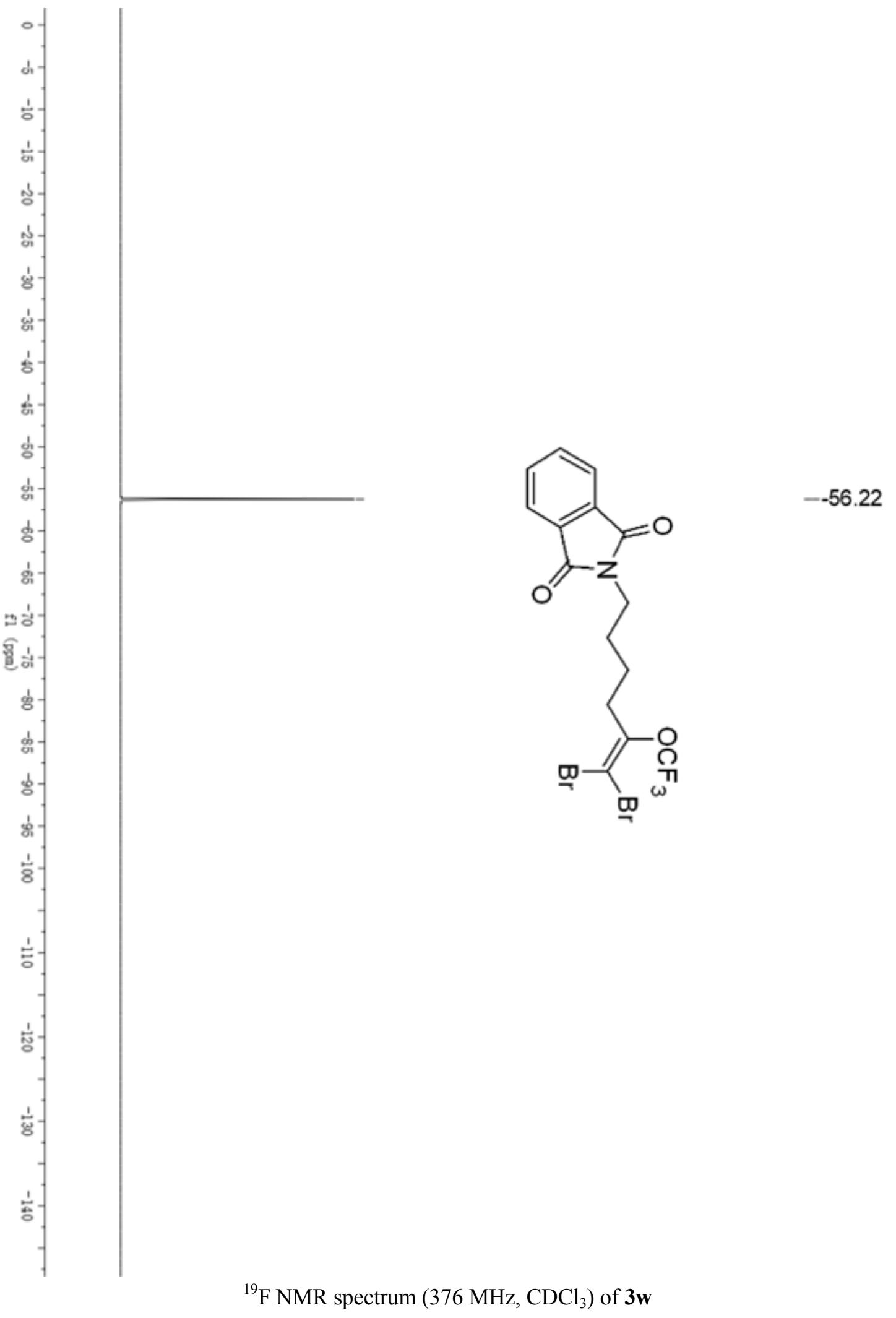




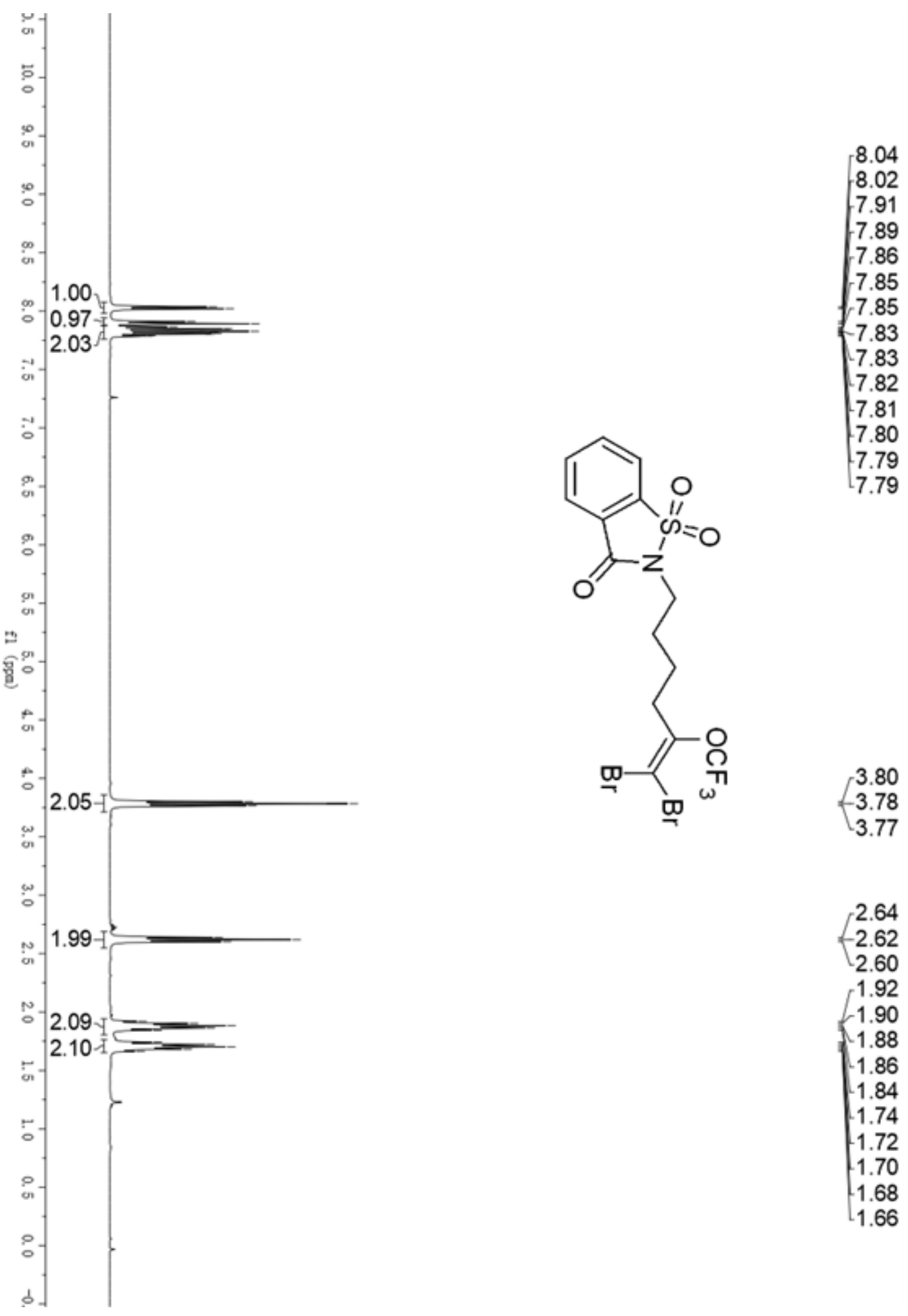

${ }^{1} \mathrm{H}$ NMR spectrum $\left(400 \mathrm{MHz}, \mathrm{CDCl}_{3}\right)$ of $\mathbf{3 x}$ 


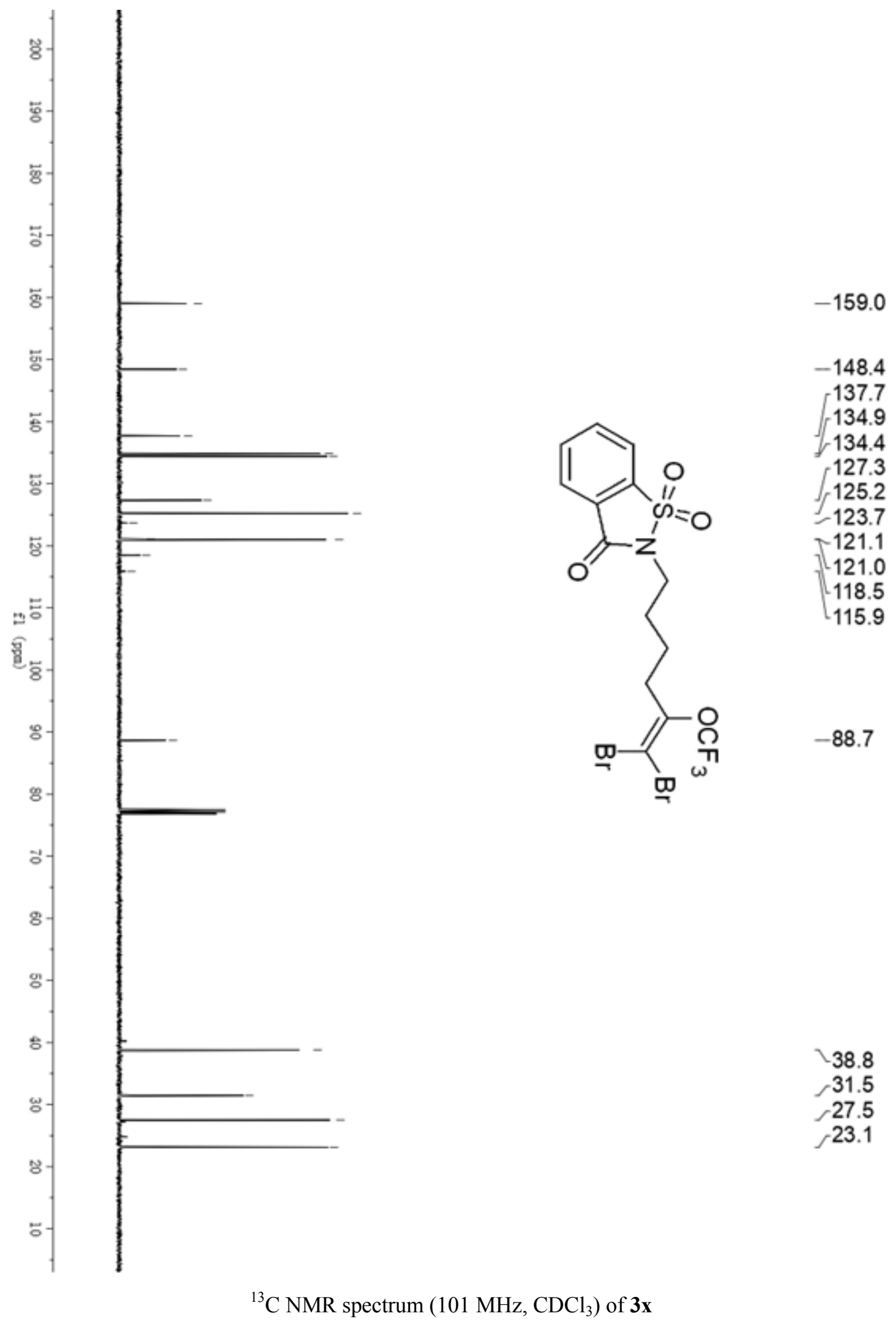



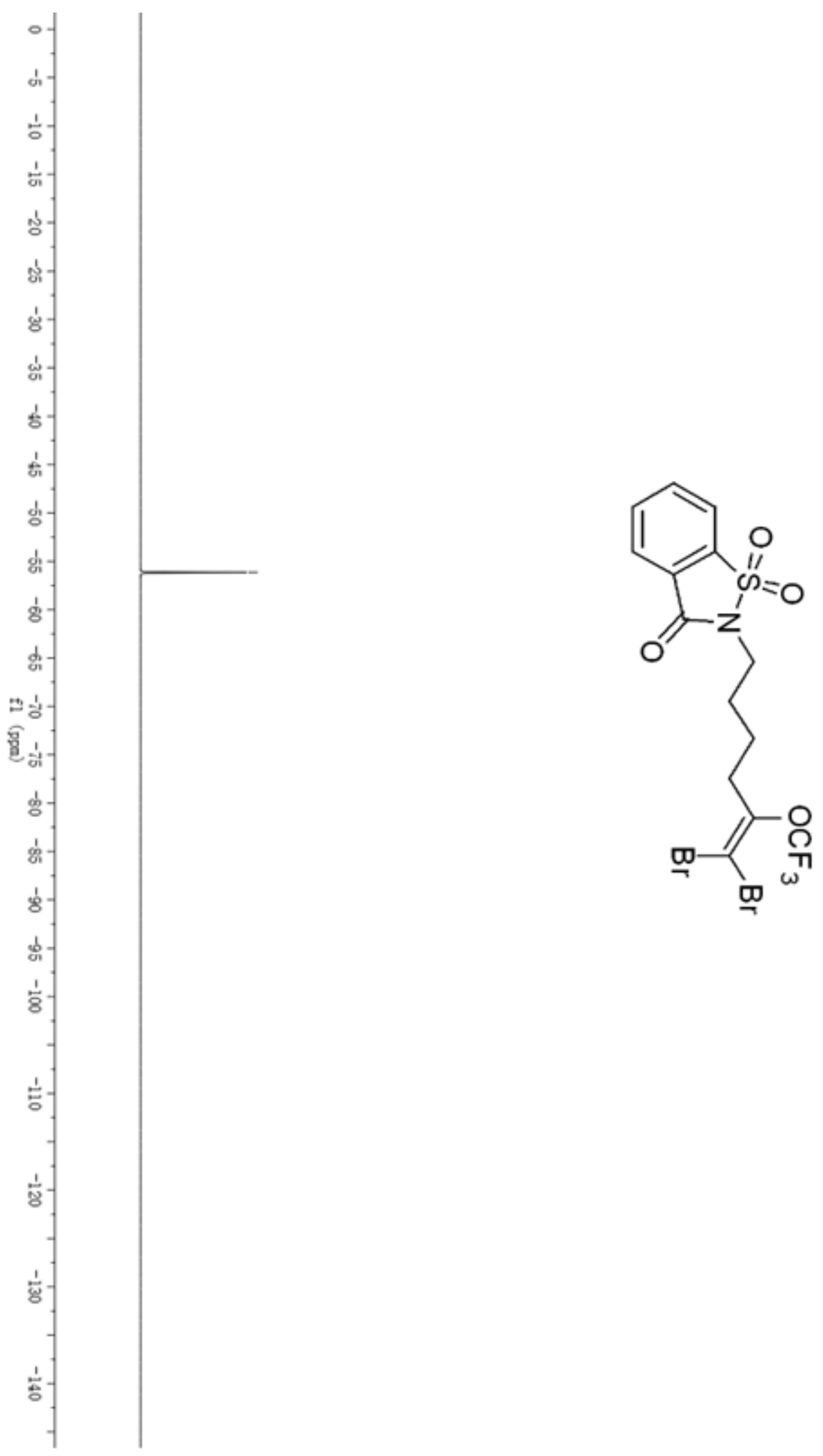

$--56.14$

${ }^{19} \mathrm{~F}$ NMR spectrum $\left(376 \mathrm{MHz}, \mathrm{CDCl}_{3}\right)$ of $\mathbf{3 x}$ 


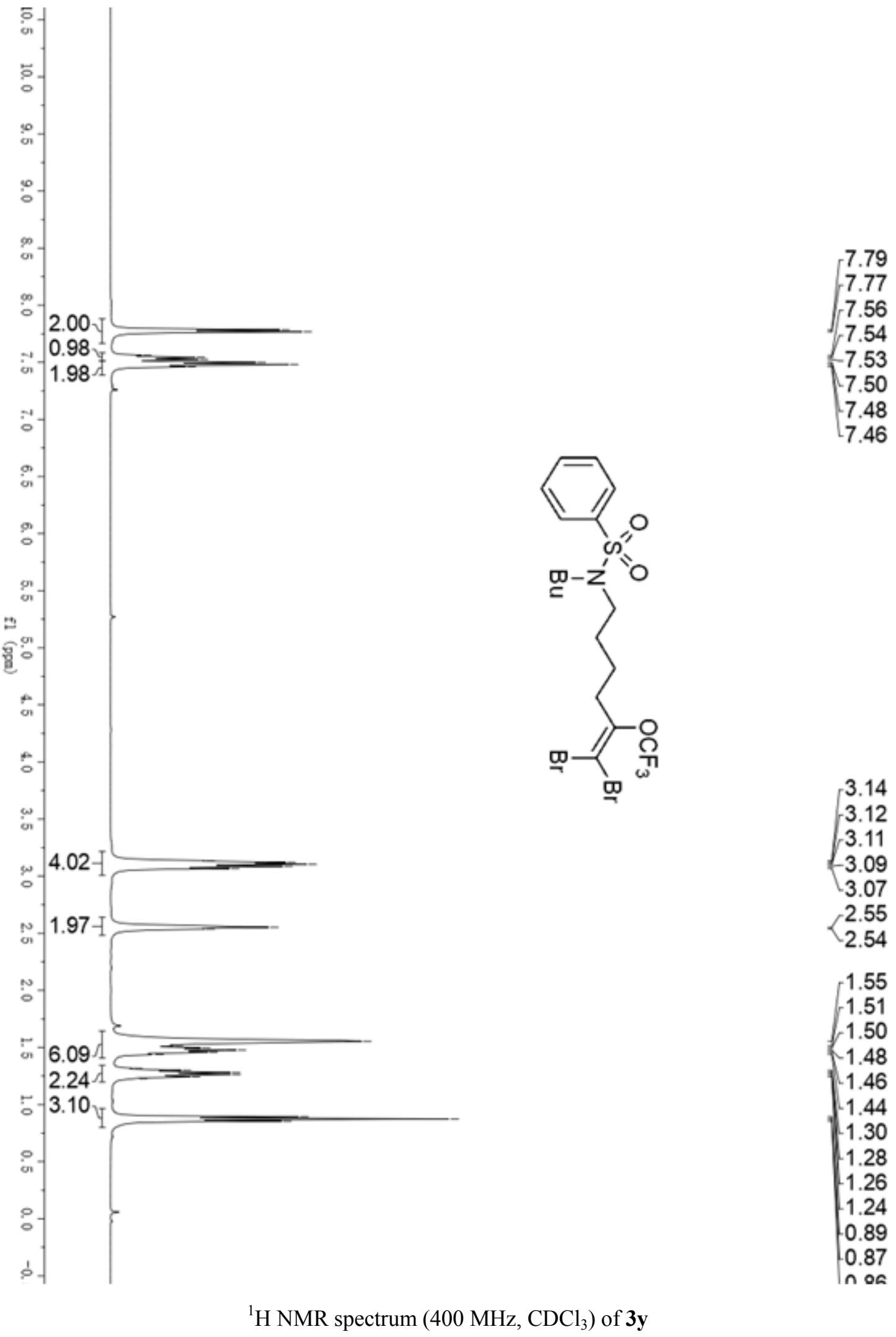




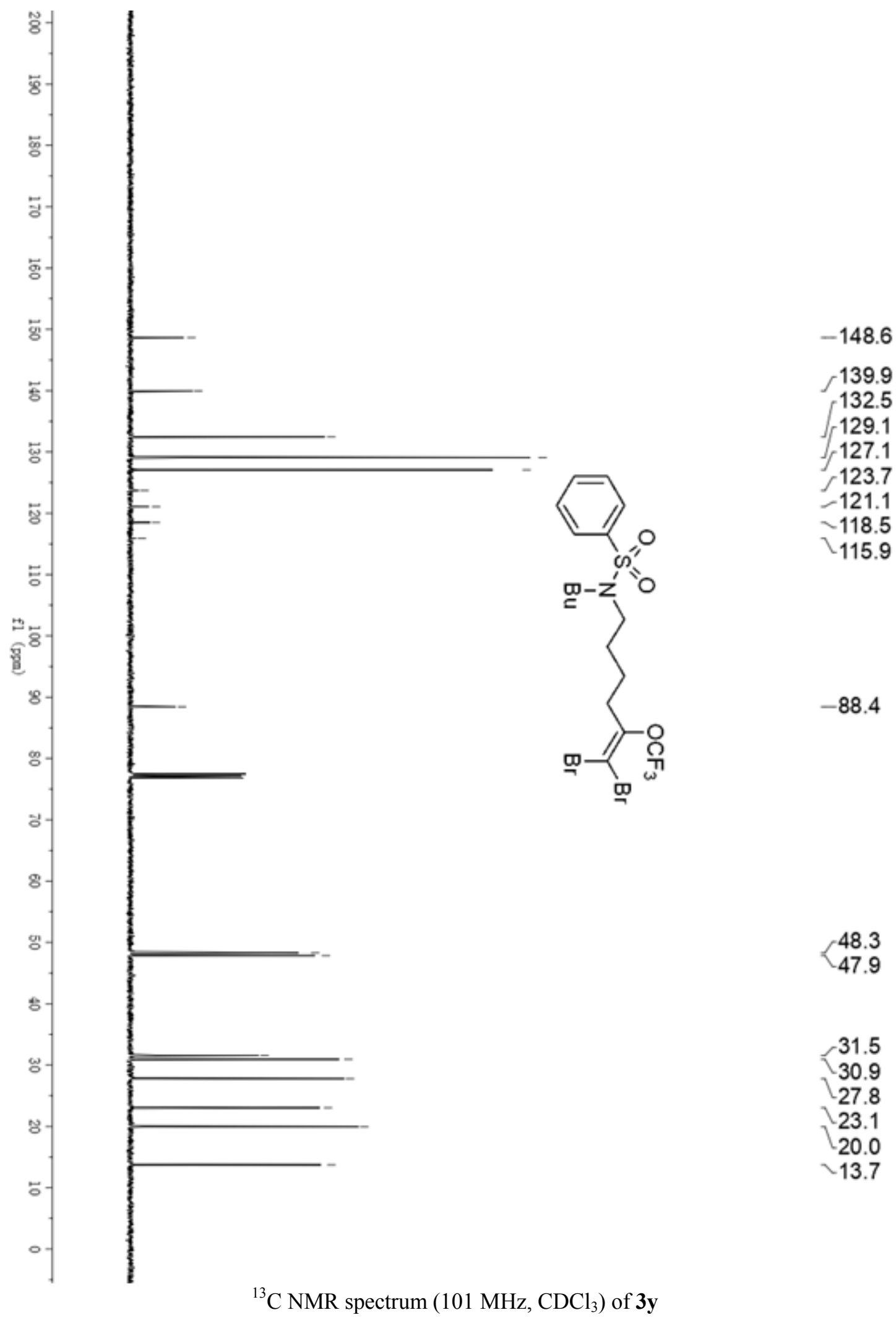




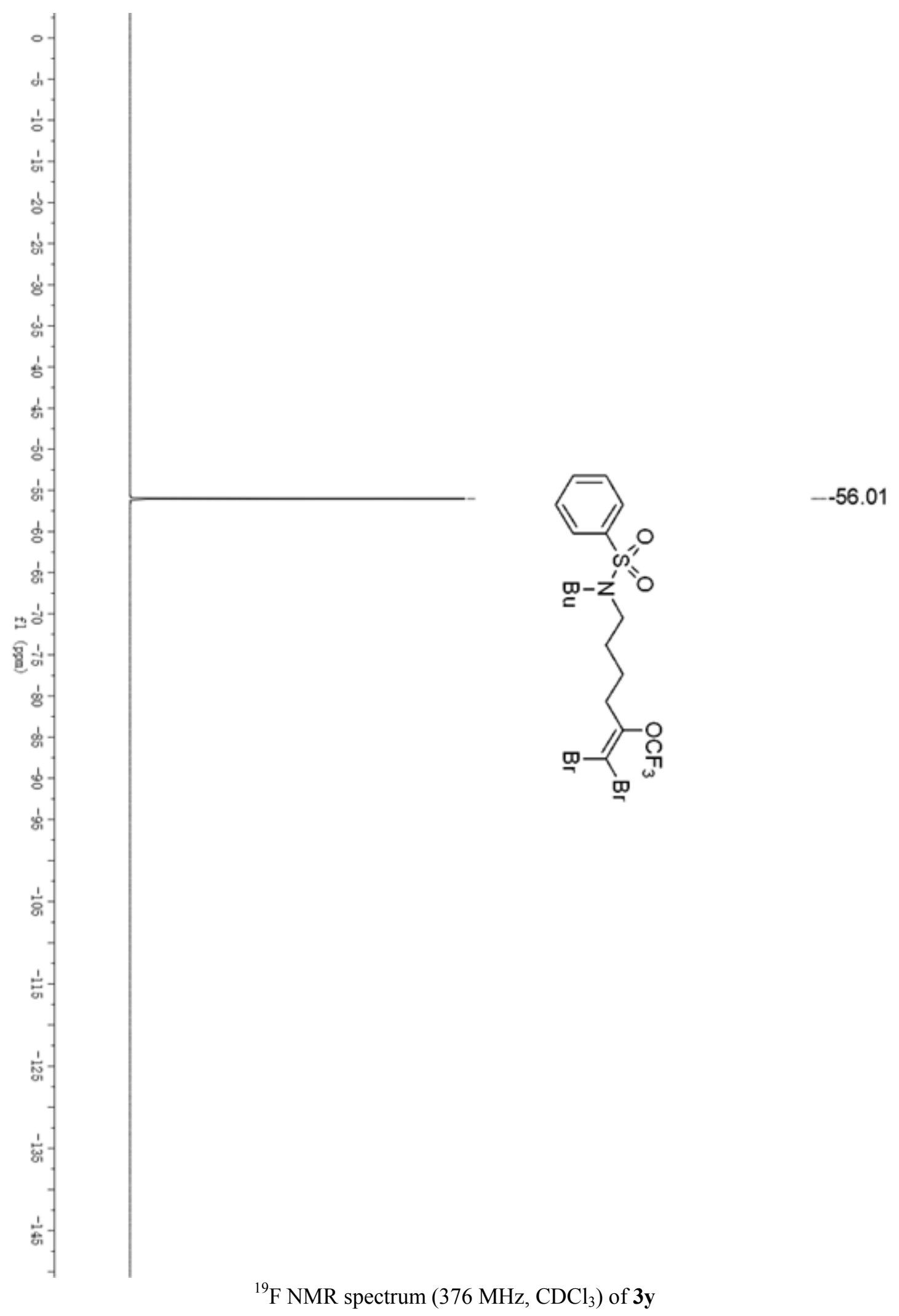




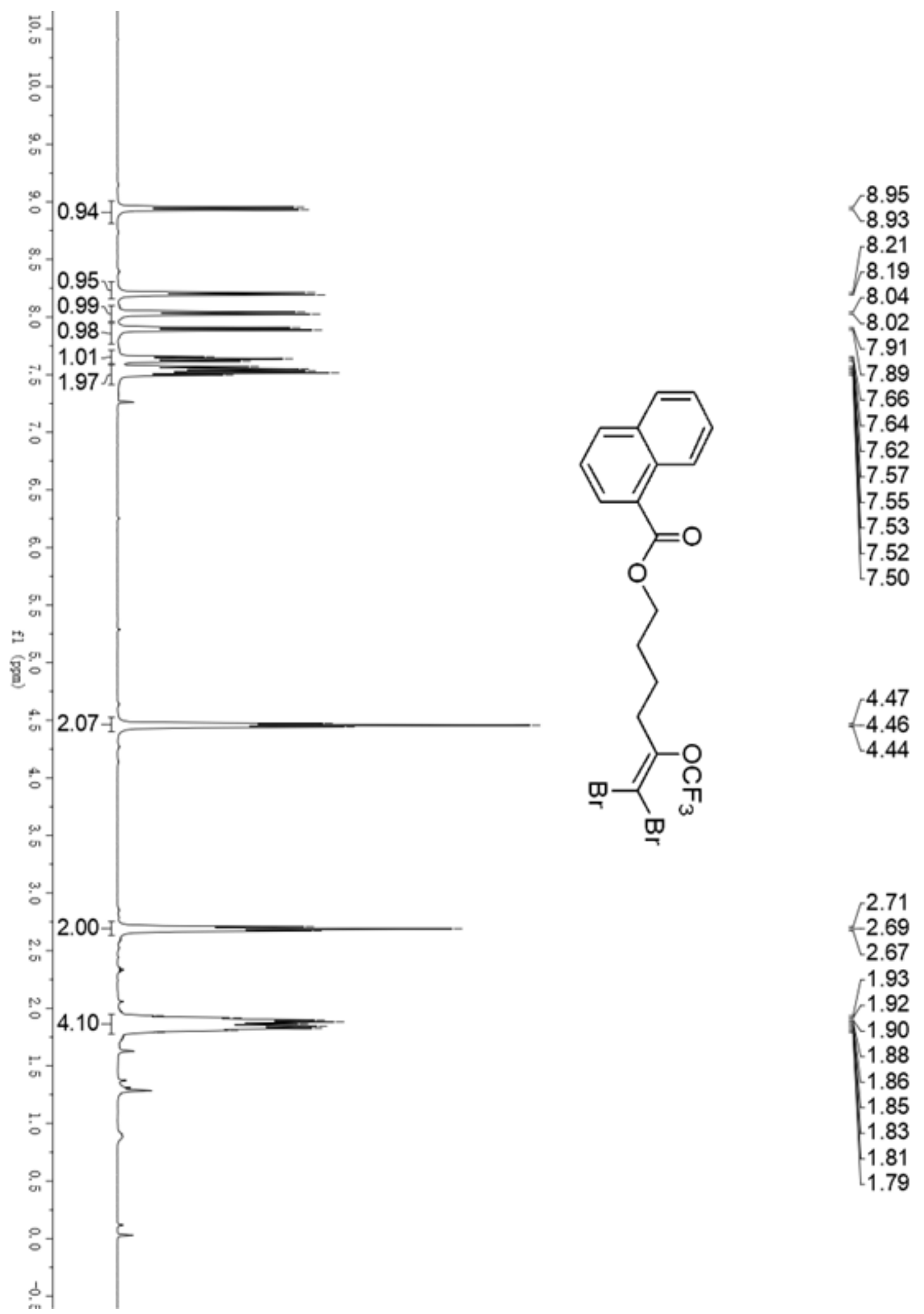

${ }^{1} \mathrm{H}$ NMR spectrum $\left(400 \mathrm{MHz}, \mathrm{CDCl}_{3}\right)$ of $\mathbf{3 z}$ 


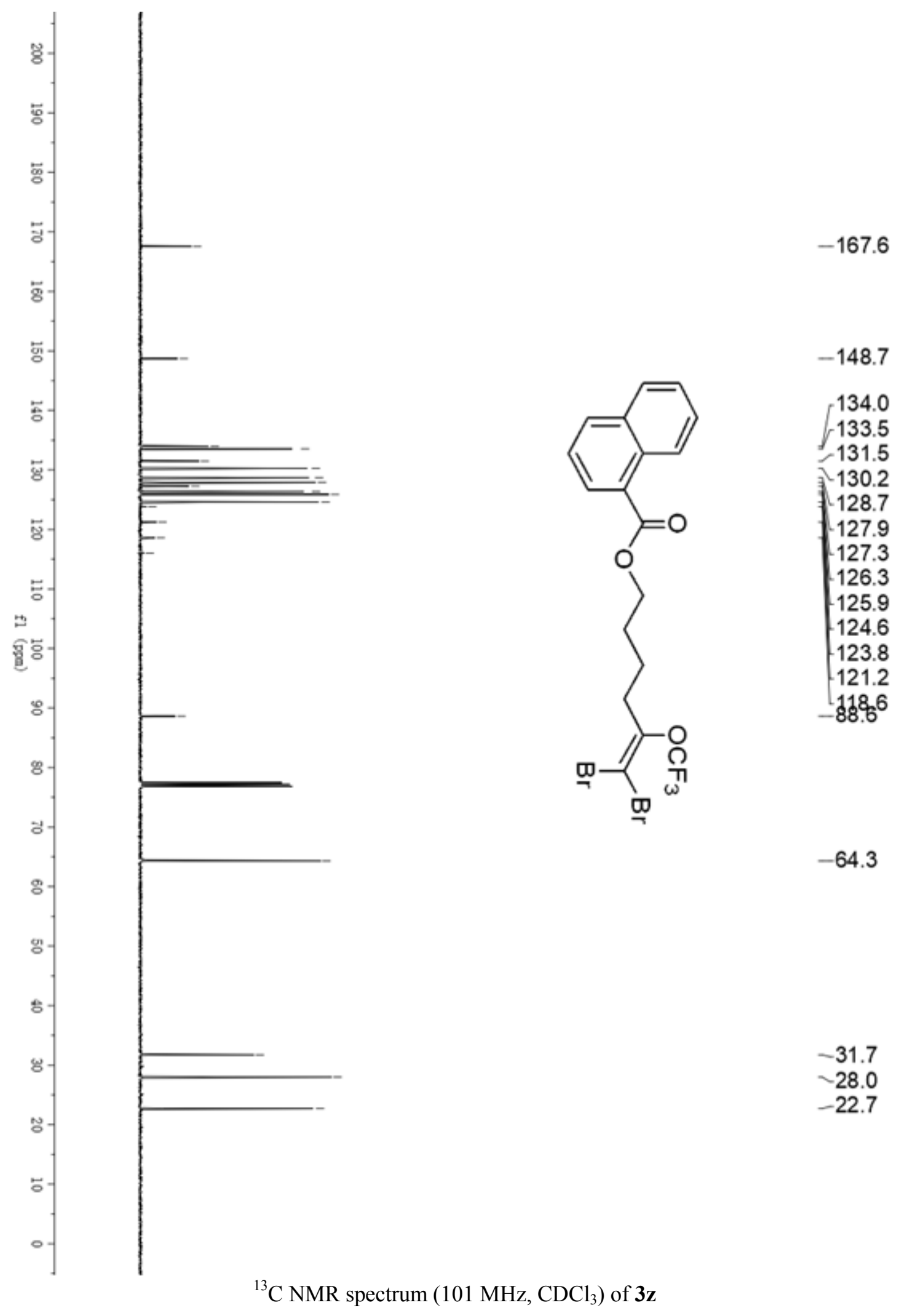




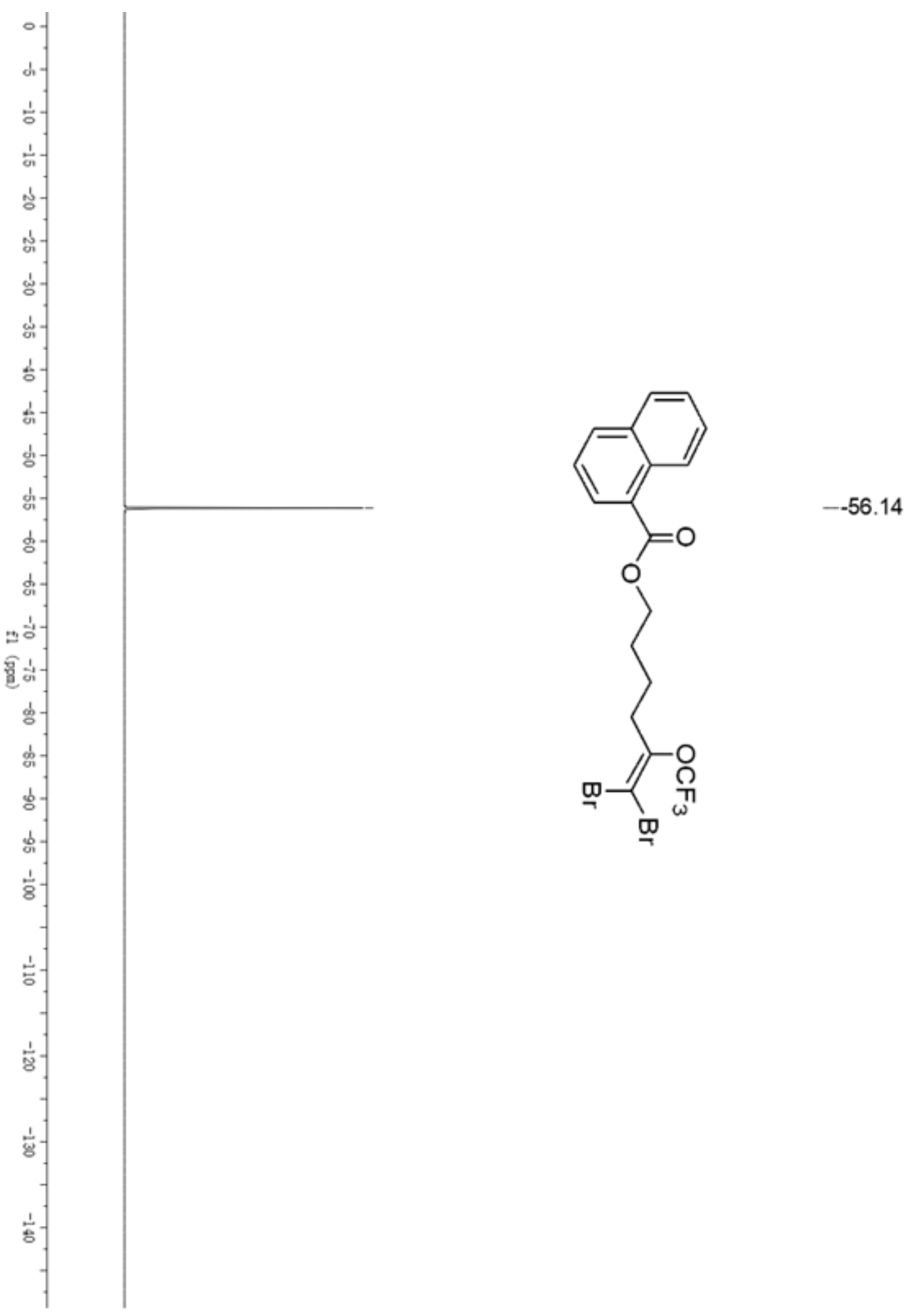

${ }^{19} \mathrm{~F}$ NMR spectrum $\left(376 \mathrm{MHz}, \mathrm{CDCl}_{3}\right)$ of $\mathbf{3 z}$ 


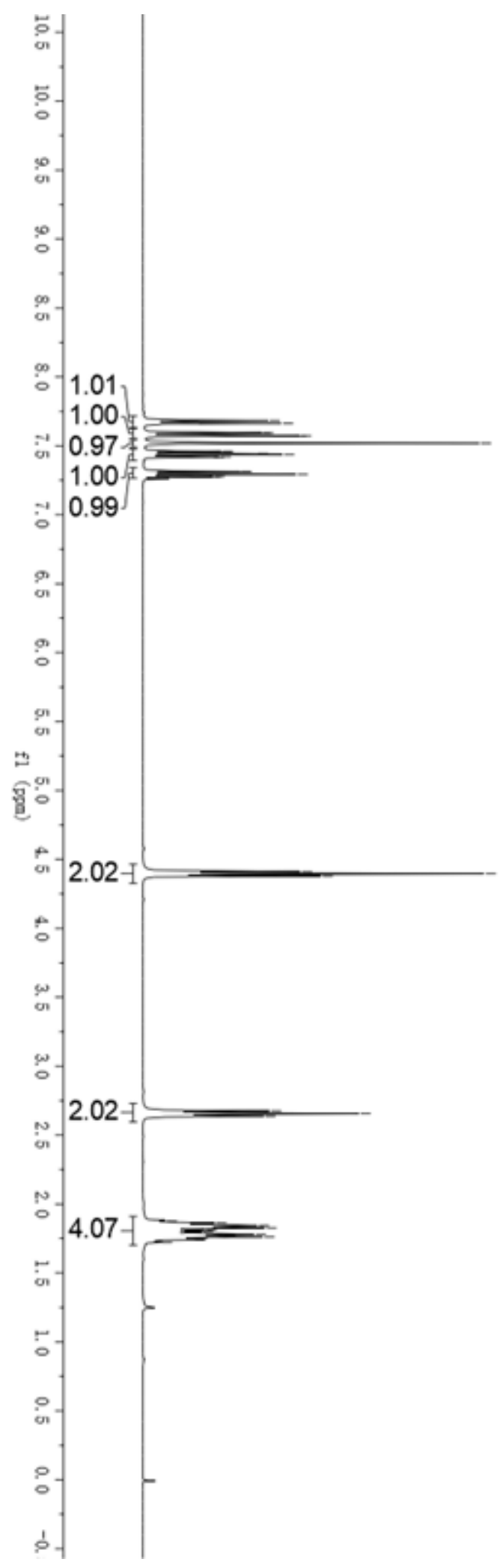<smiles>CC(O)=C(O)CCCCO</smiles> 


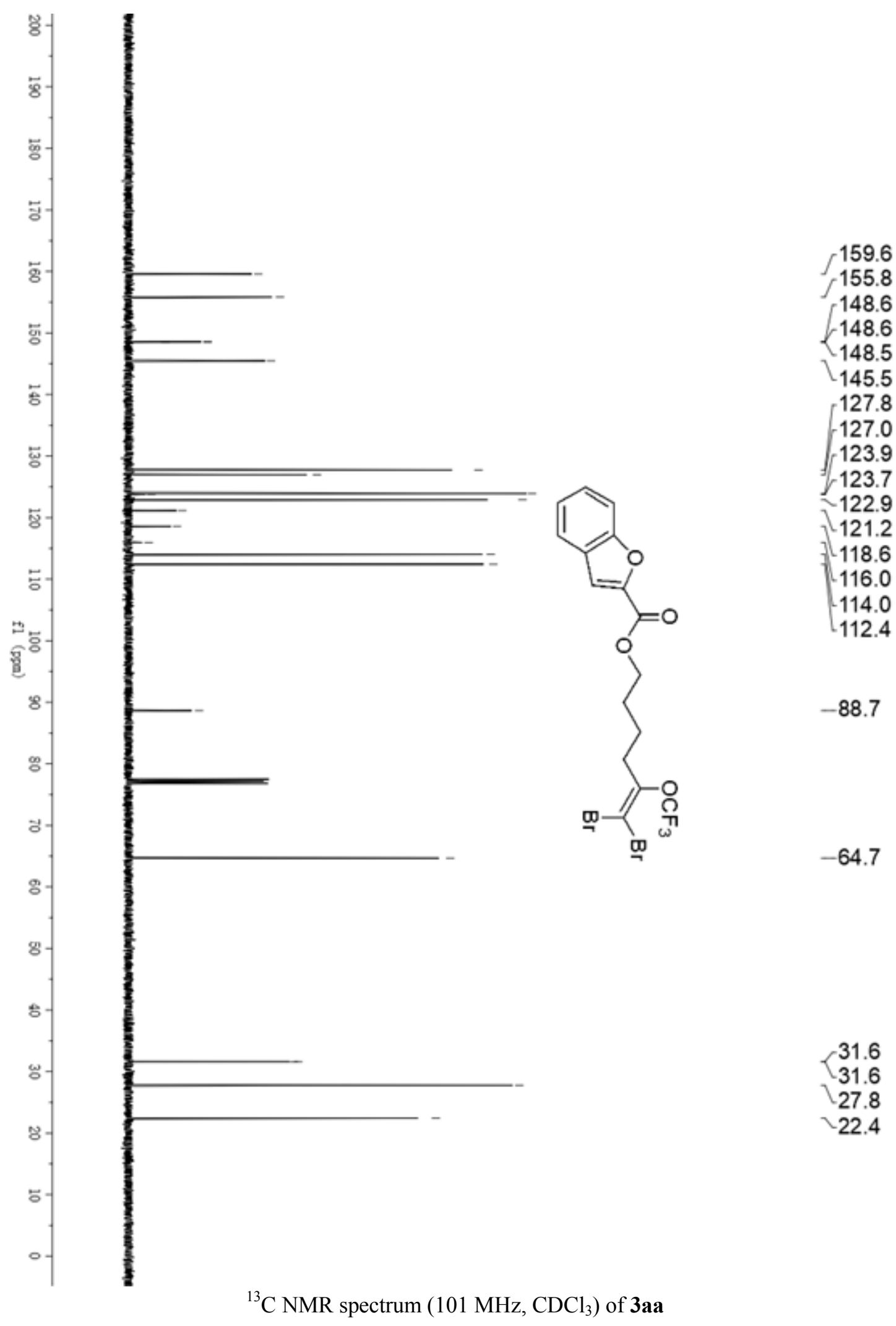




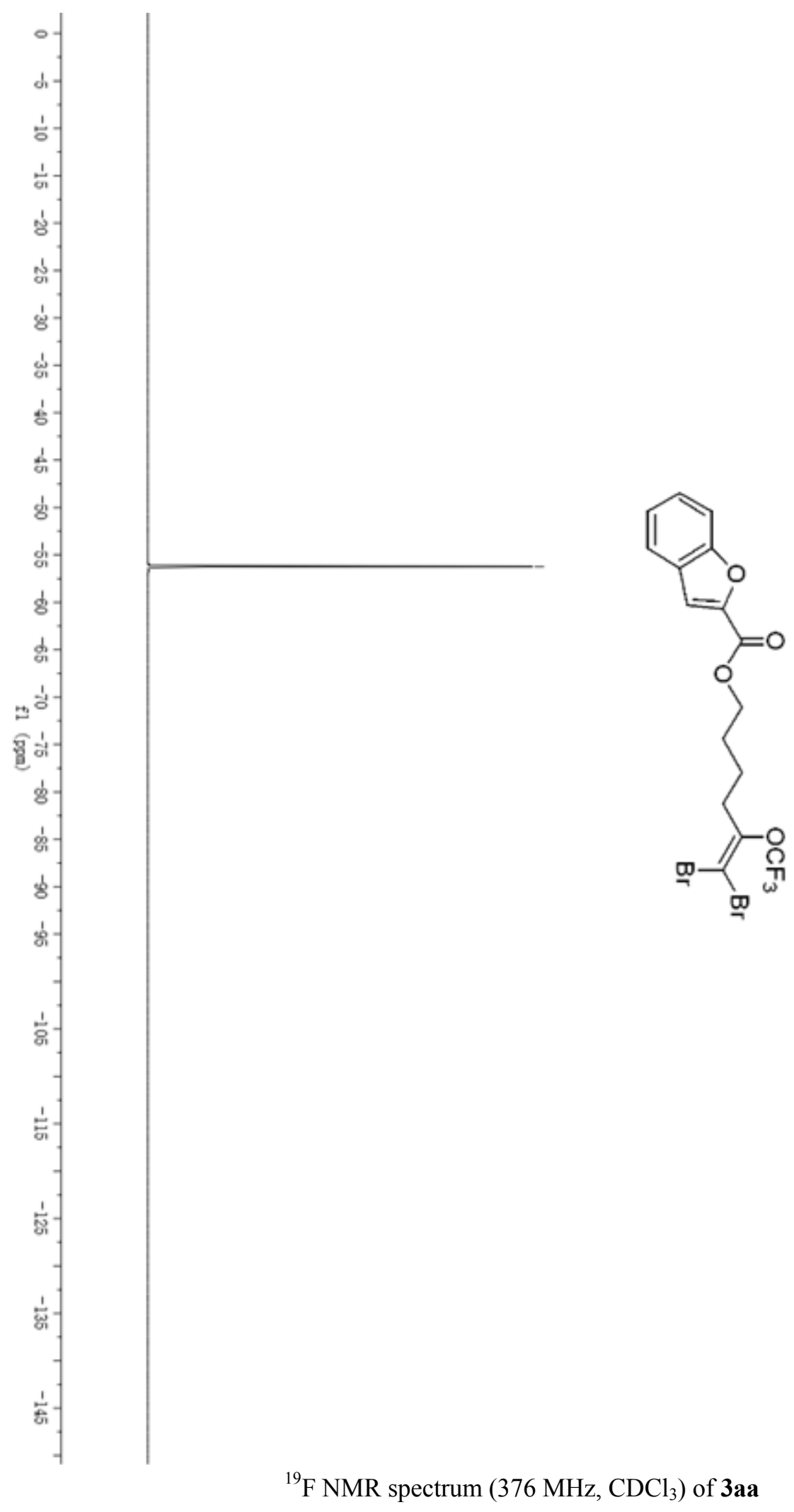




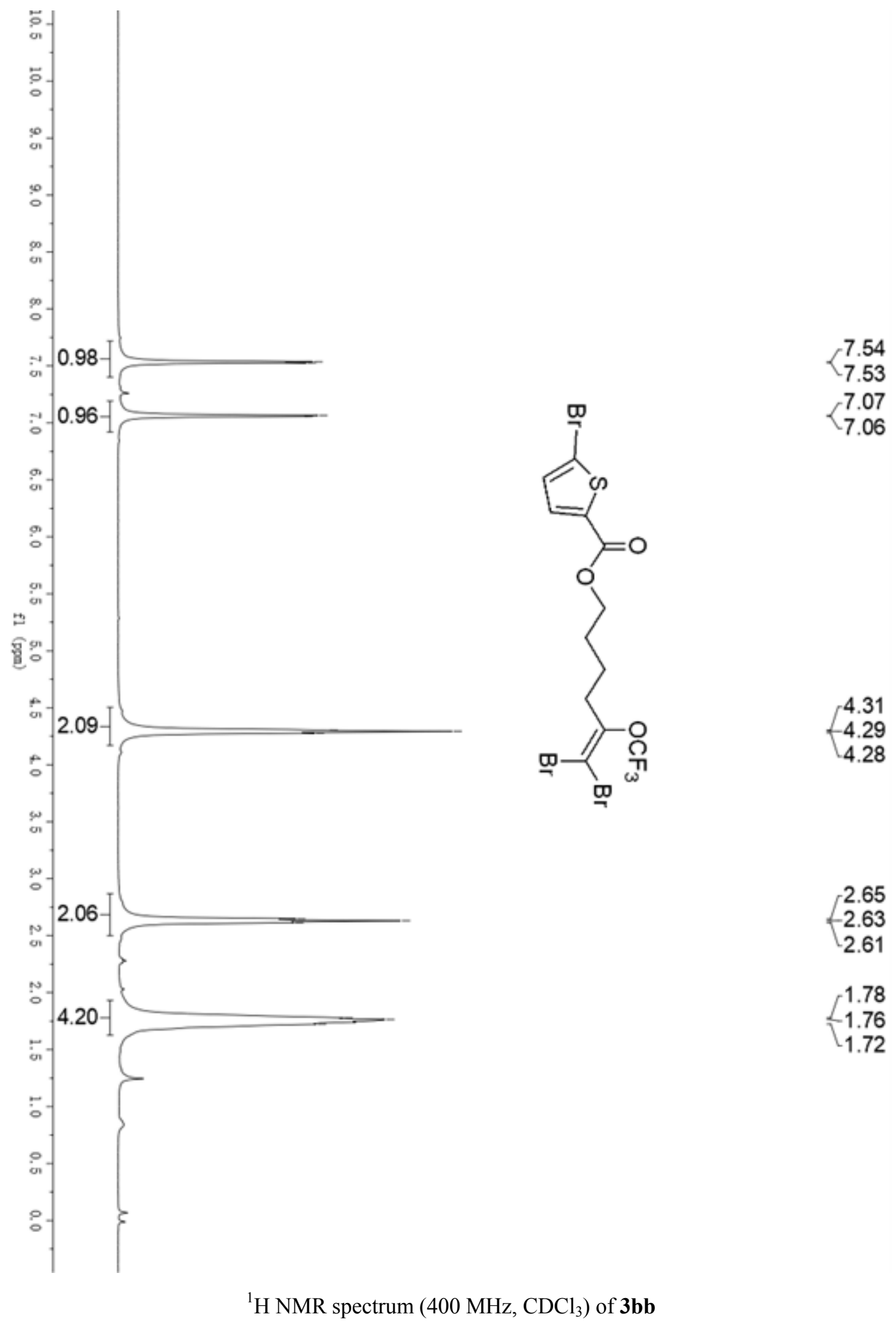




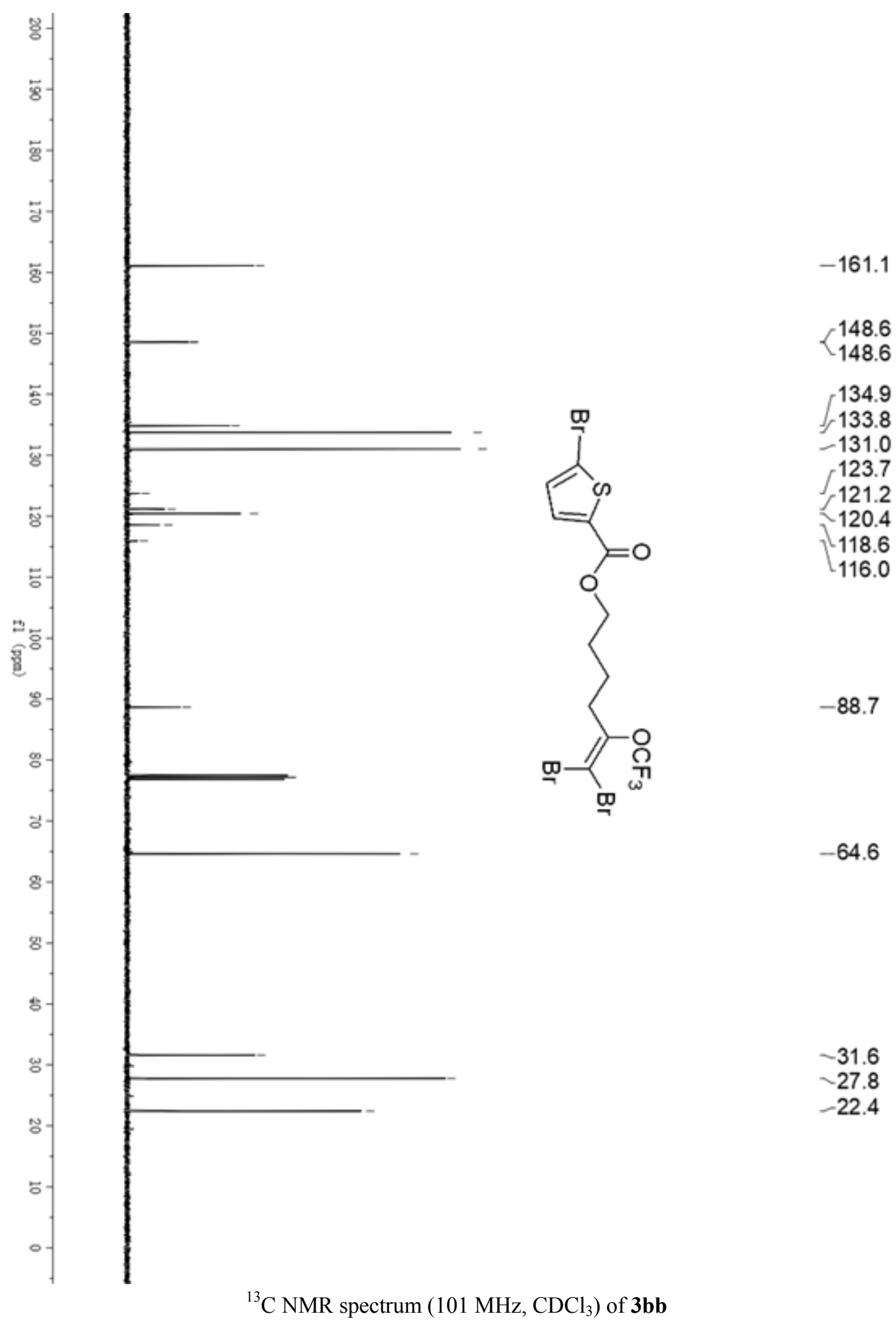




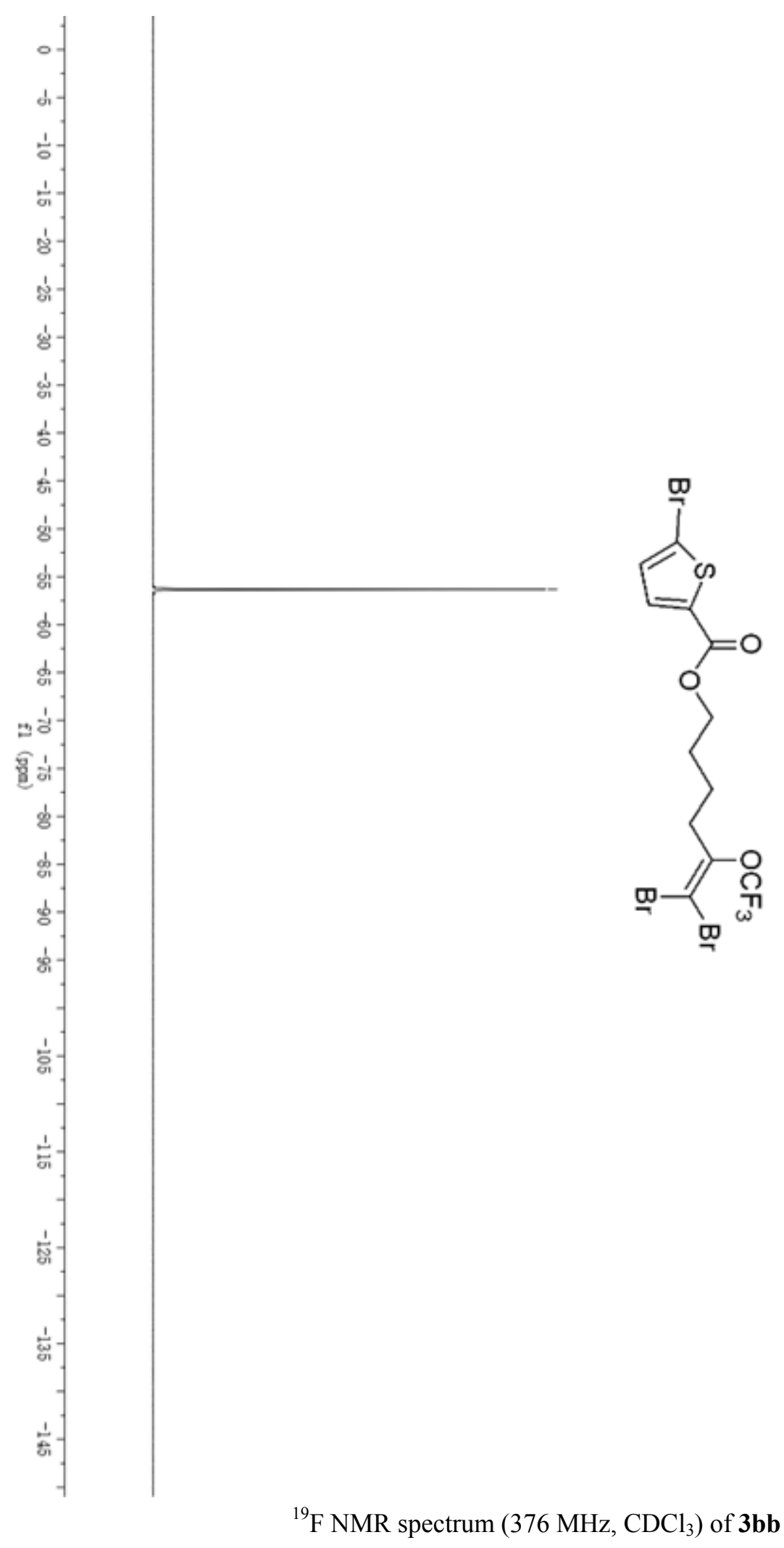

$--56.31$ 


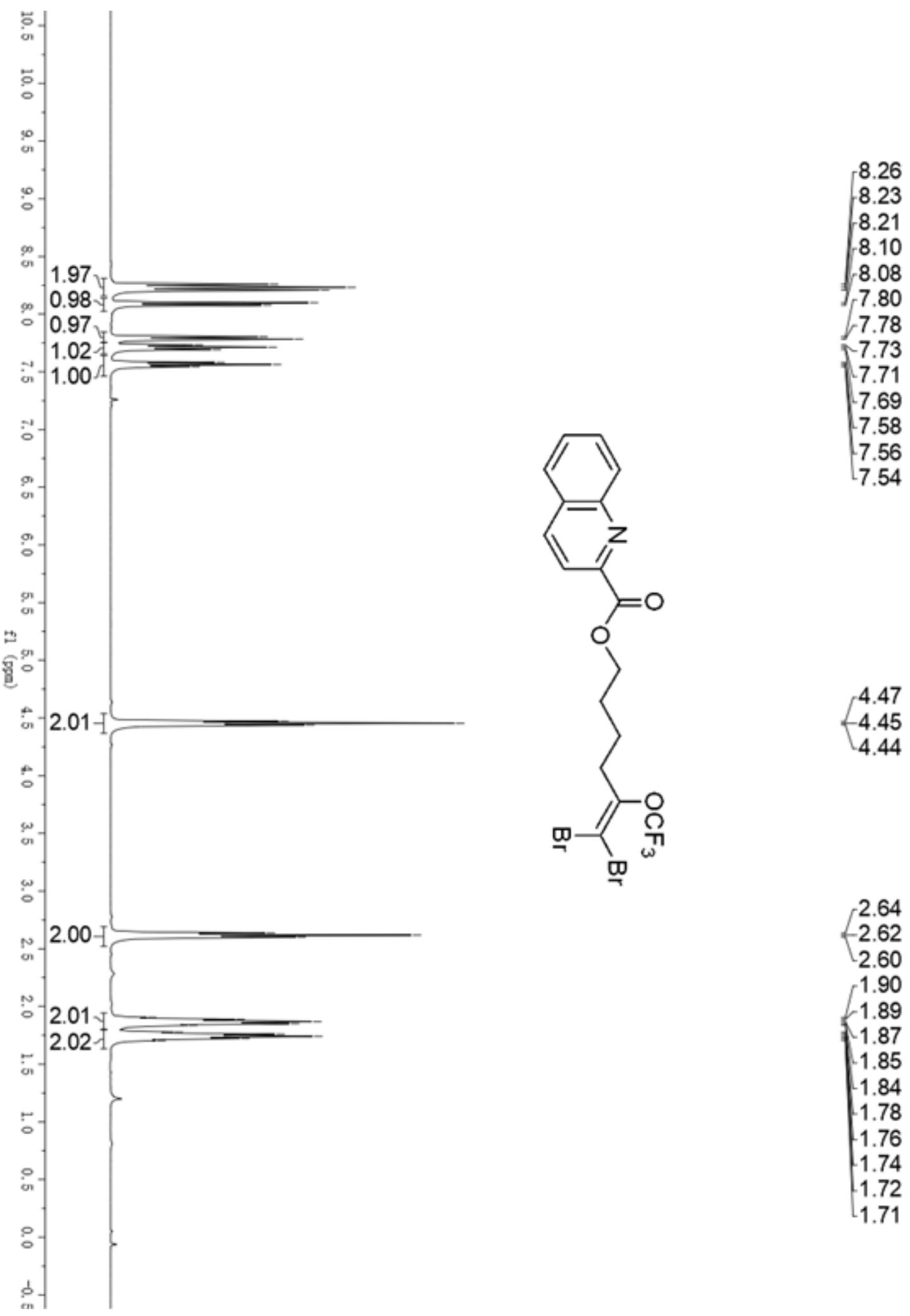

${ }^{1} \mathrm{H}$ NMR spectrum $\left(400 \mathrm{MHz}, \mathrm{CDCl}_{3}\right)$ of $\mathbf{3 c c}$ 


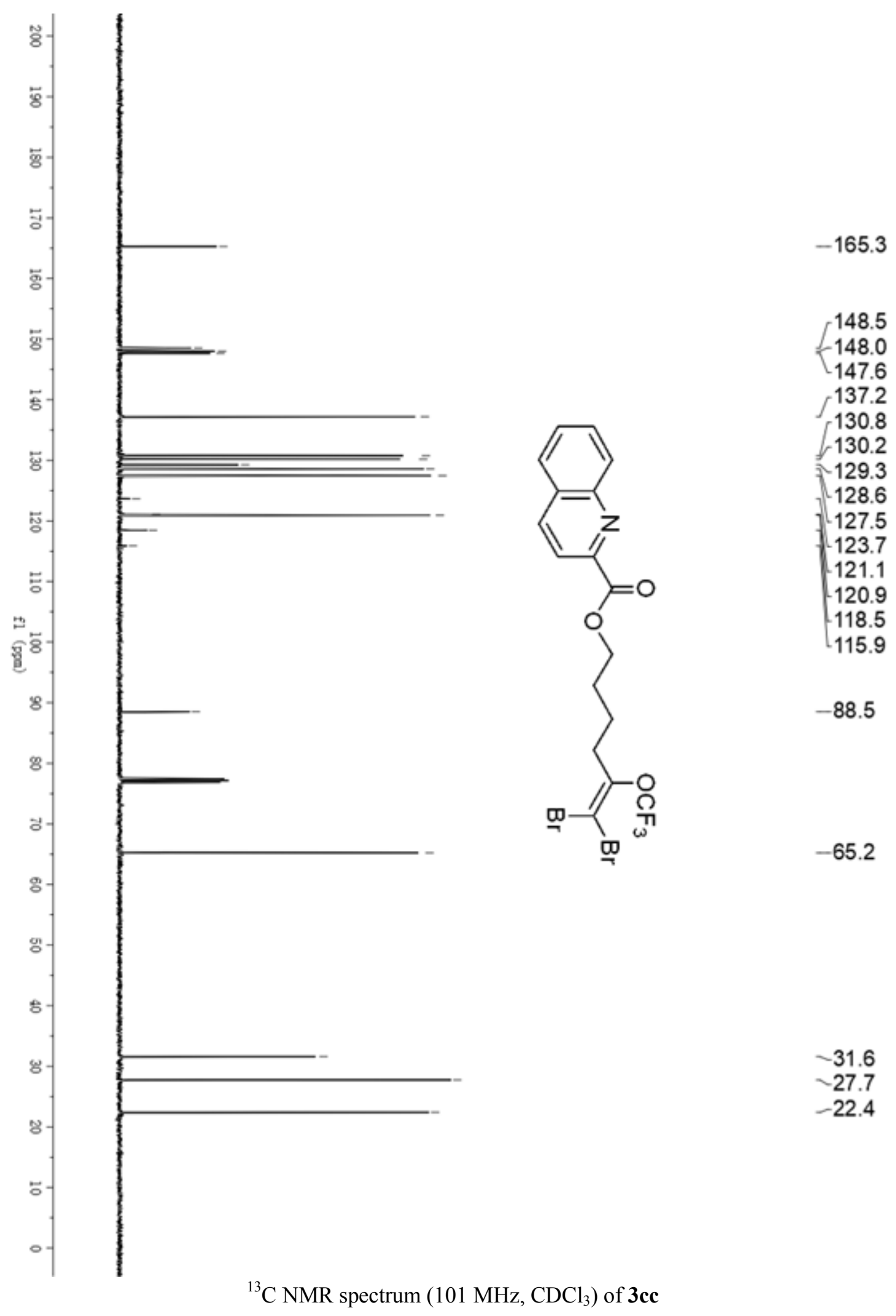




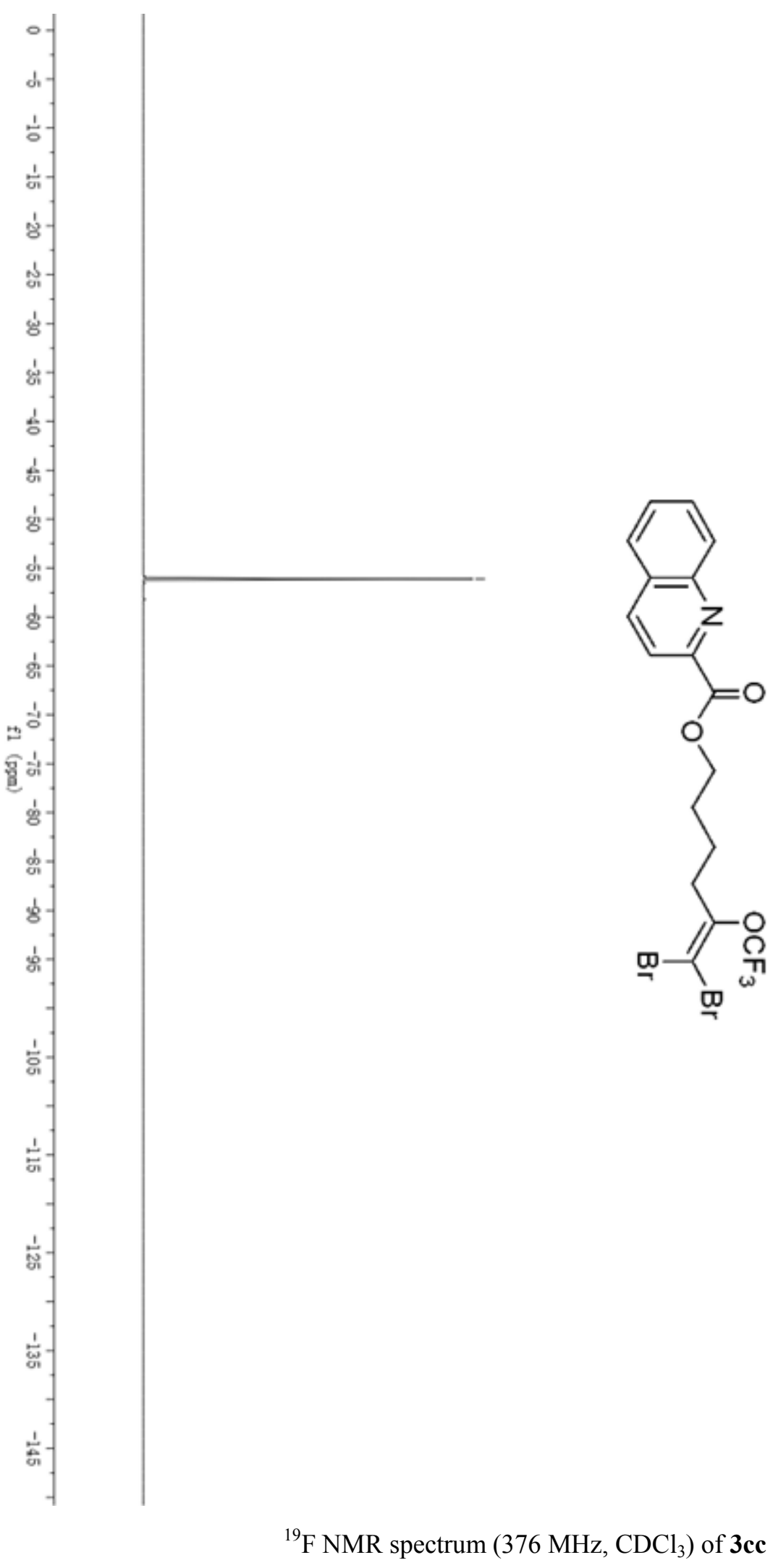




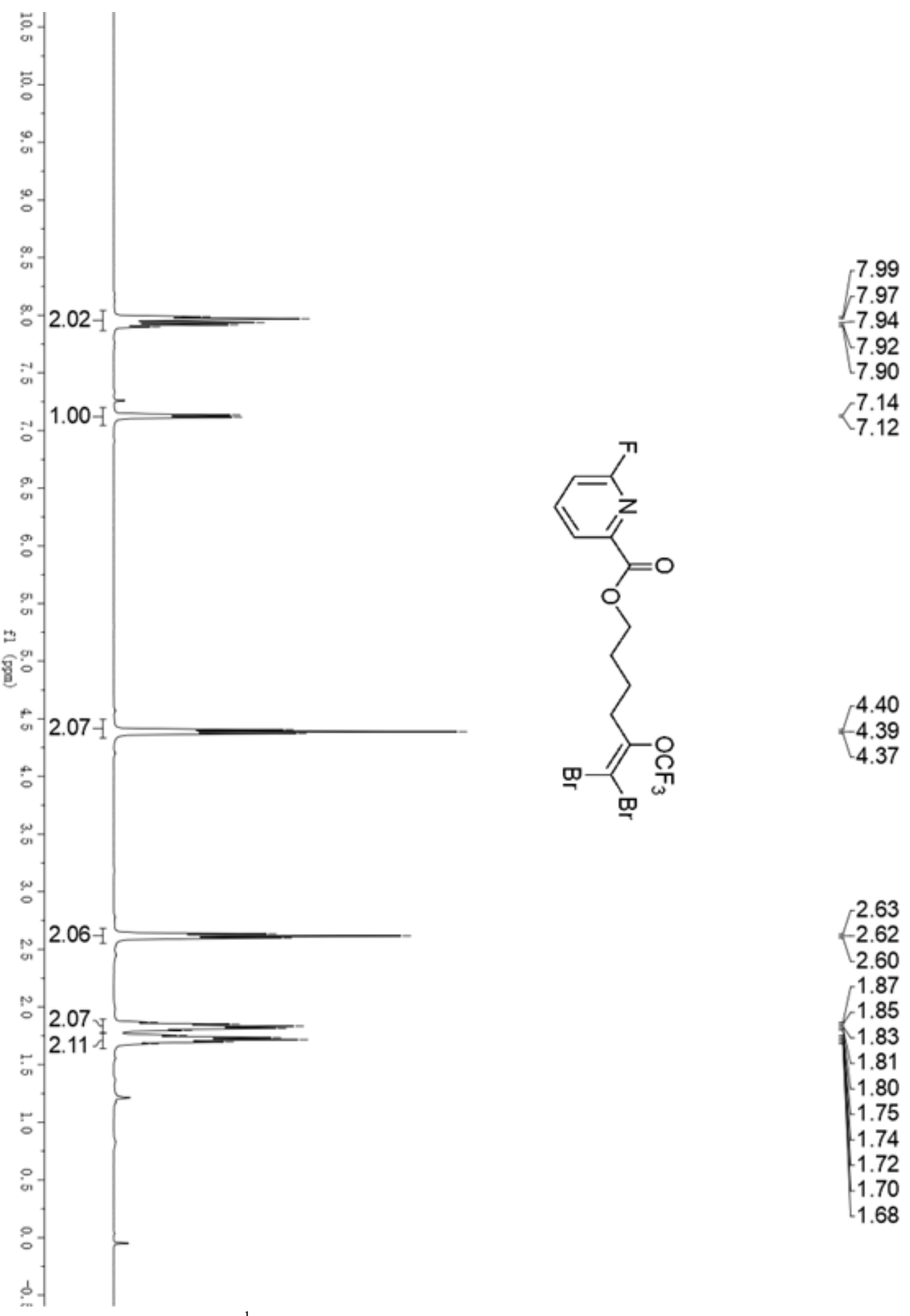

${ }^{1} \mathrm{H}$ NMR spectrum $\left(400 \mathrm{MHz}, \mathrm{CDCl}_{3}\right)$ of $\mathbf{3 d d}$ 


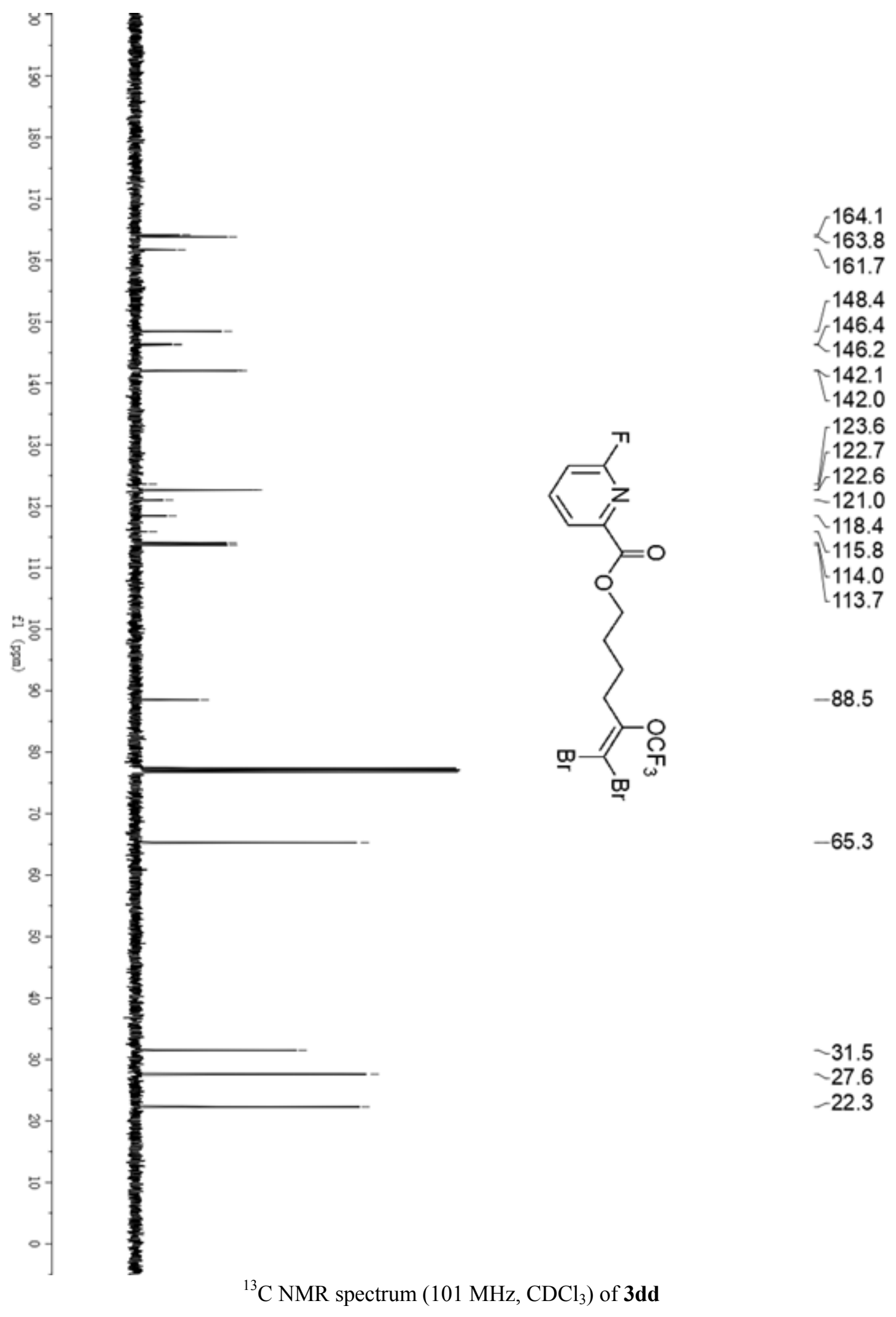




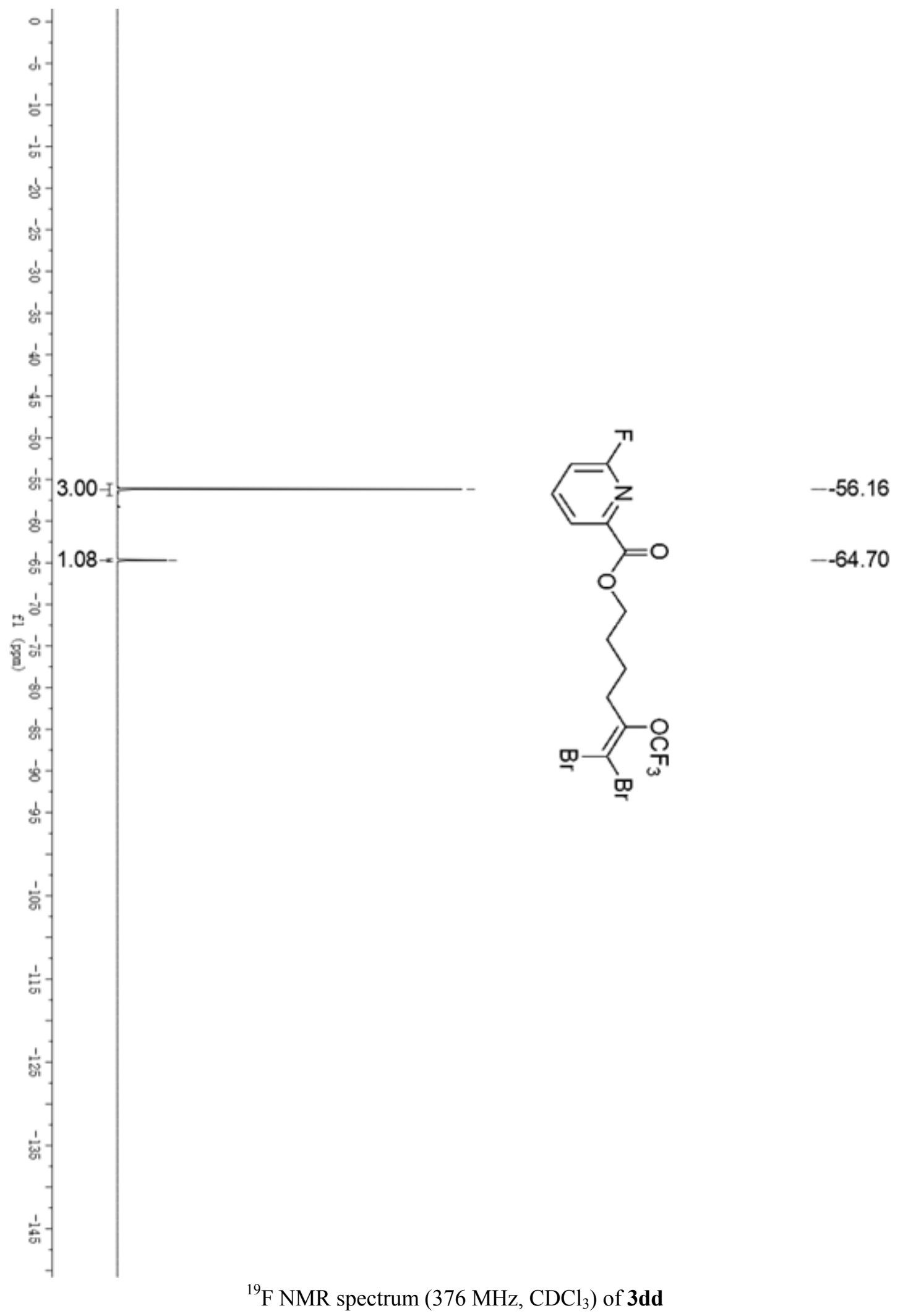




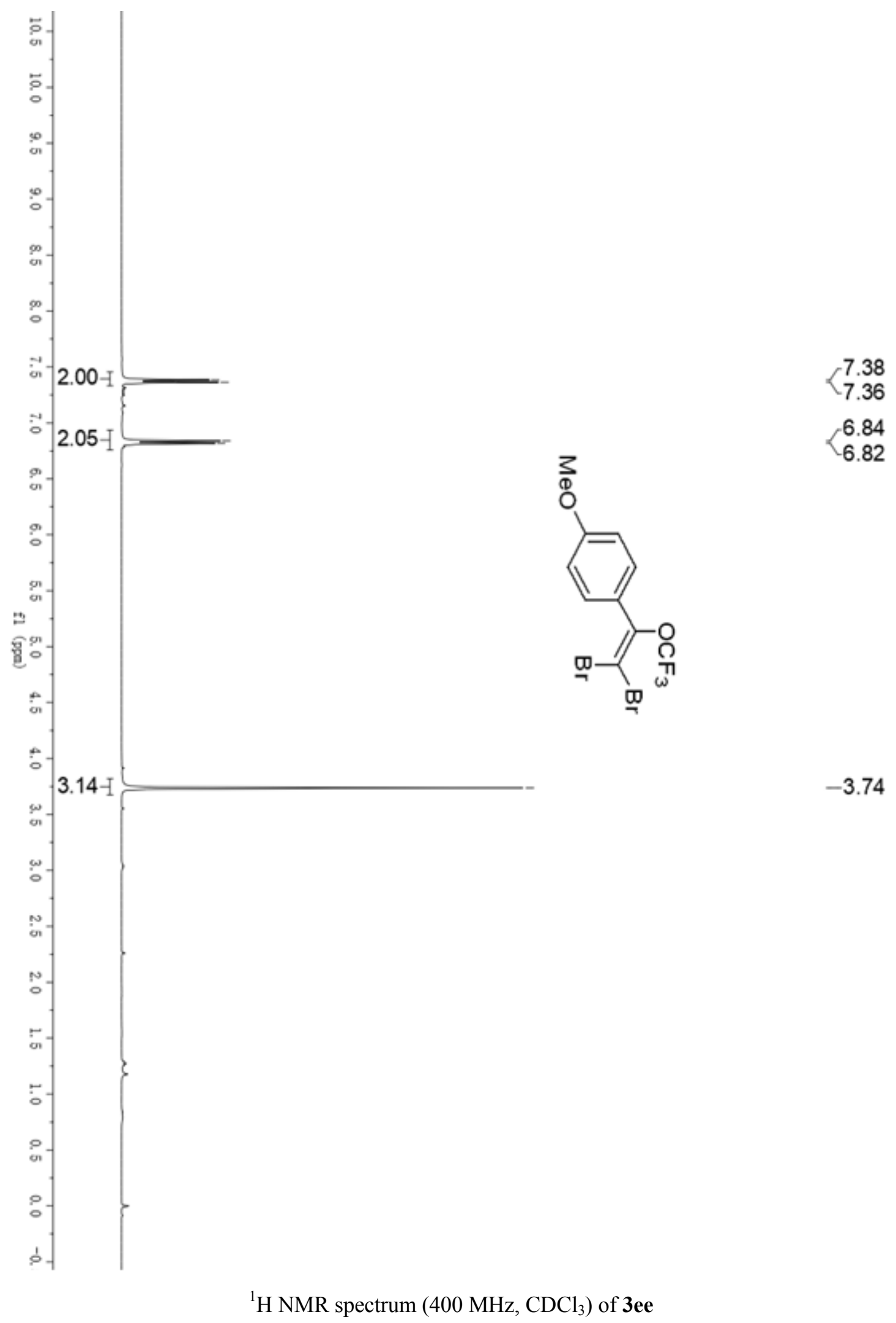




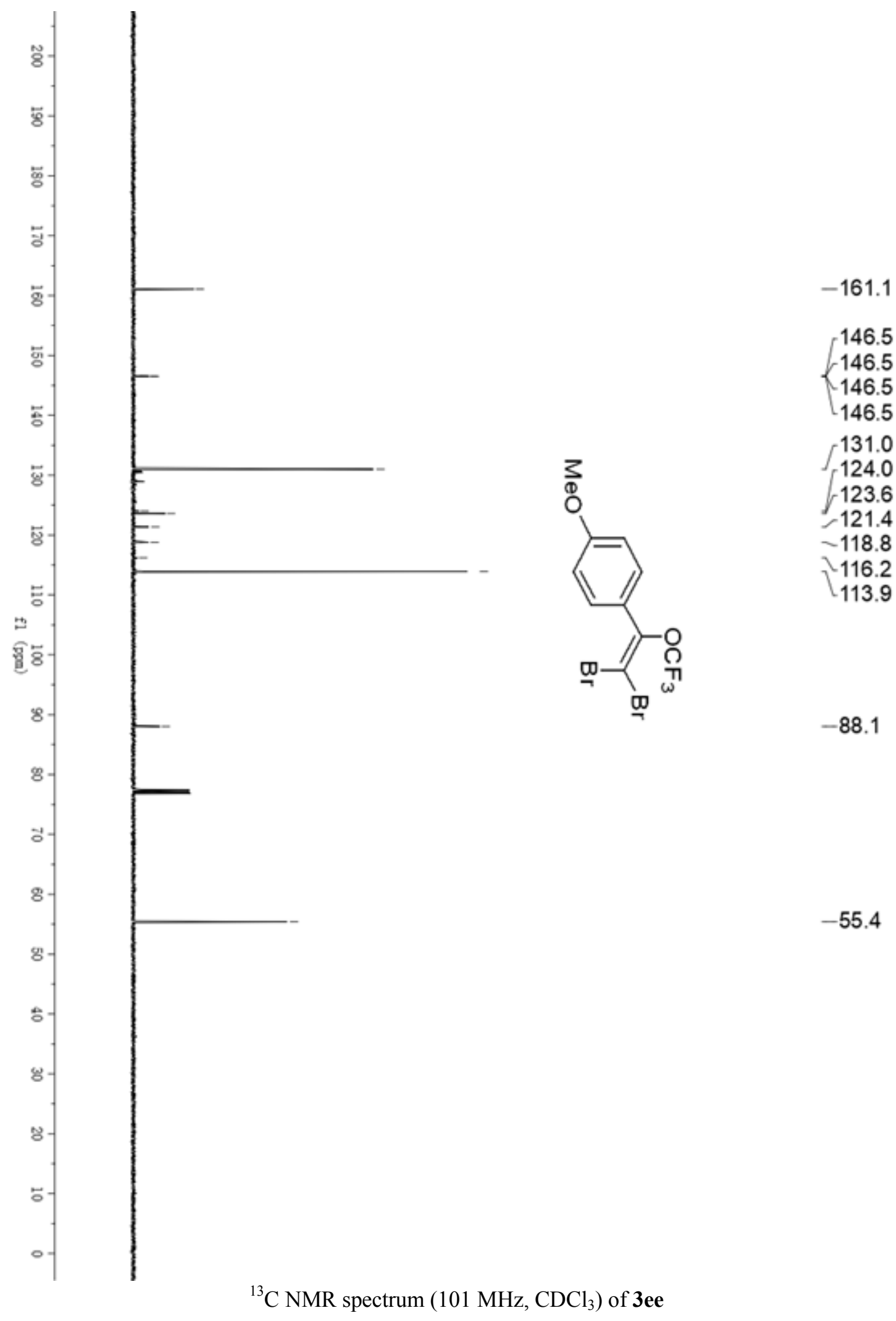




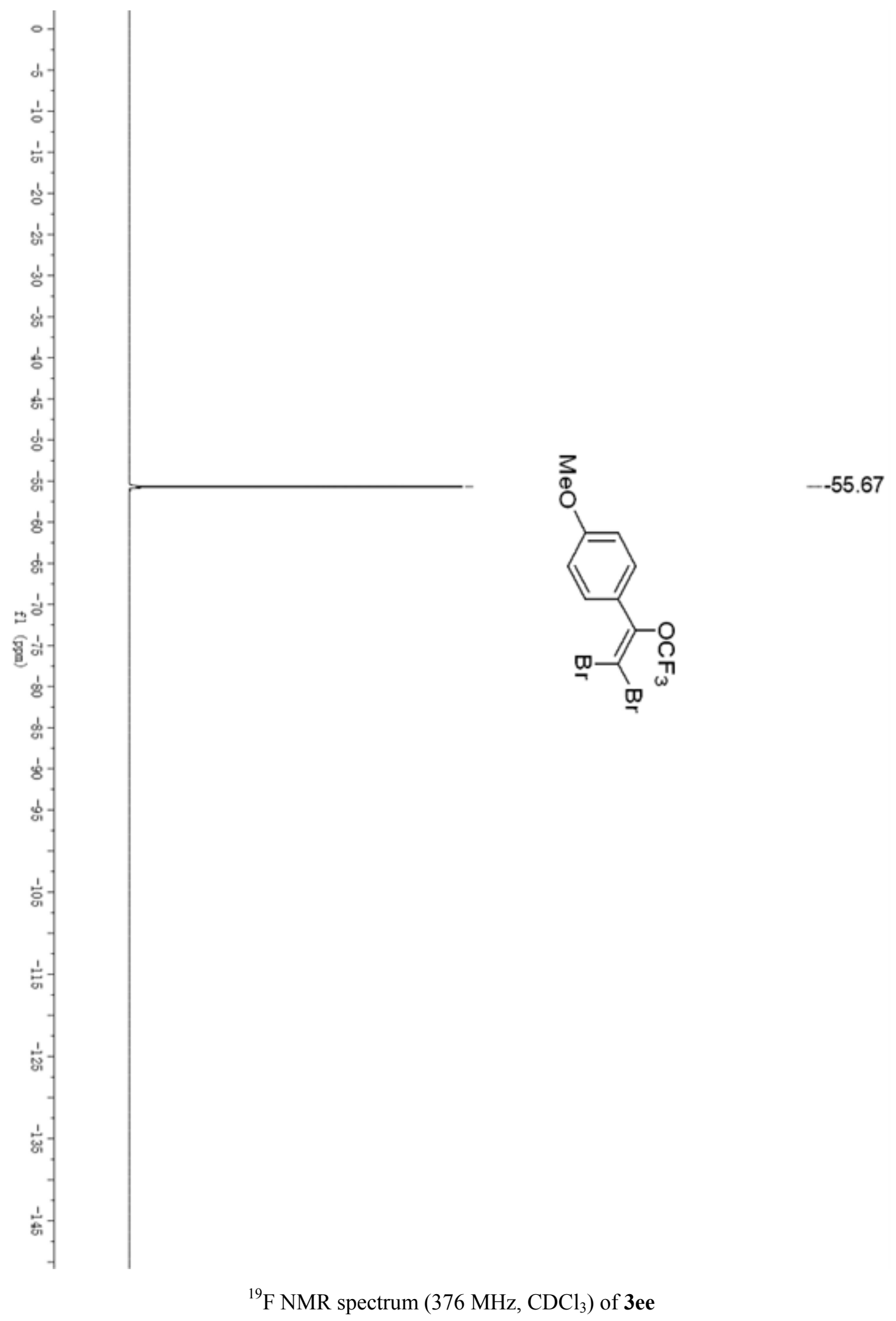




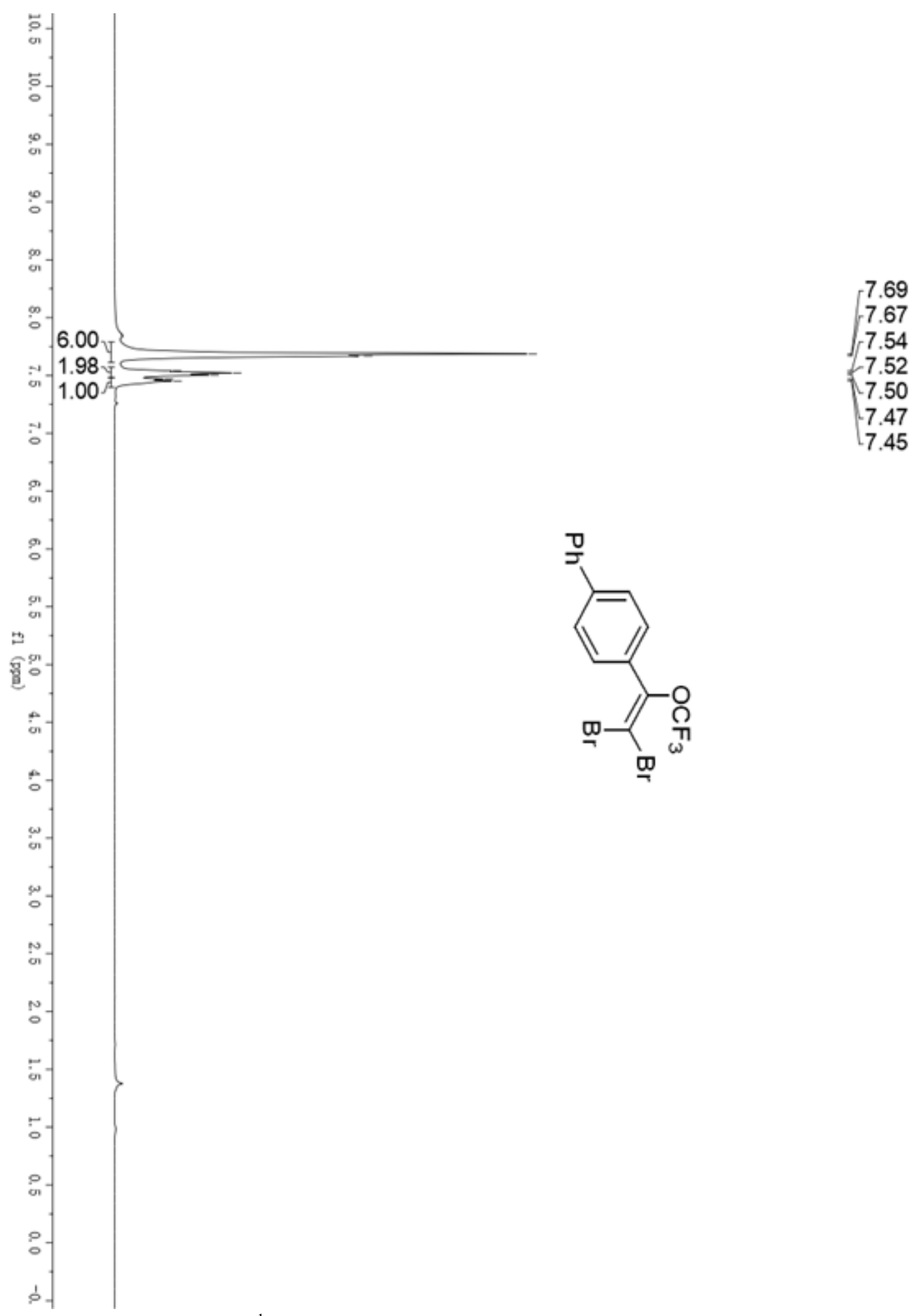

${ }^{1} \mathrm{H}$ NMR spectrum $\left(400 \mathrm{MHz}, \mathrm{CDCl}_{3}\right)$ of $\mathbf{3 f f}$ 


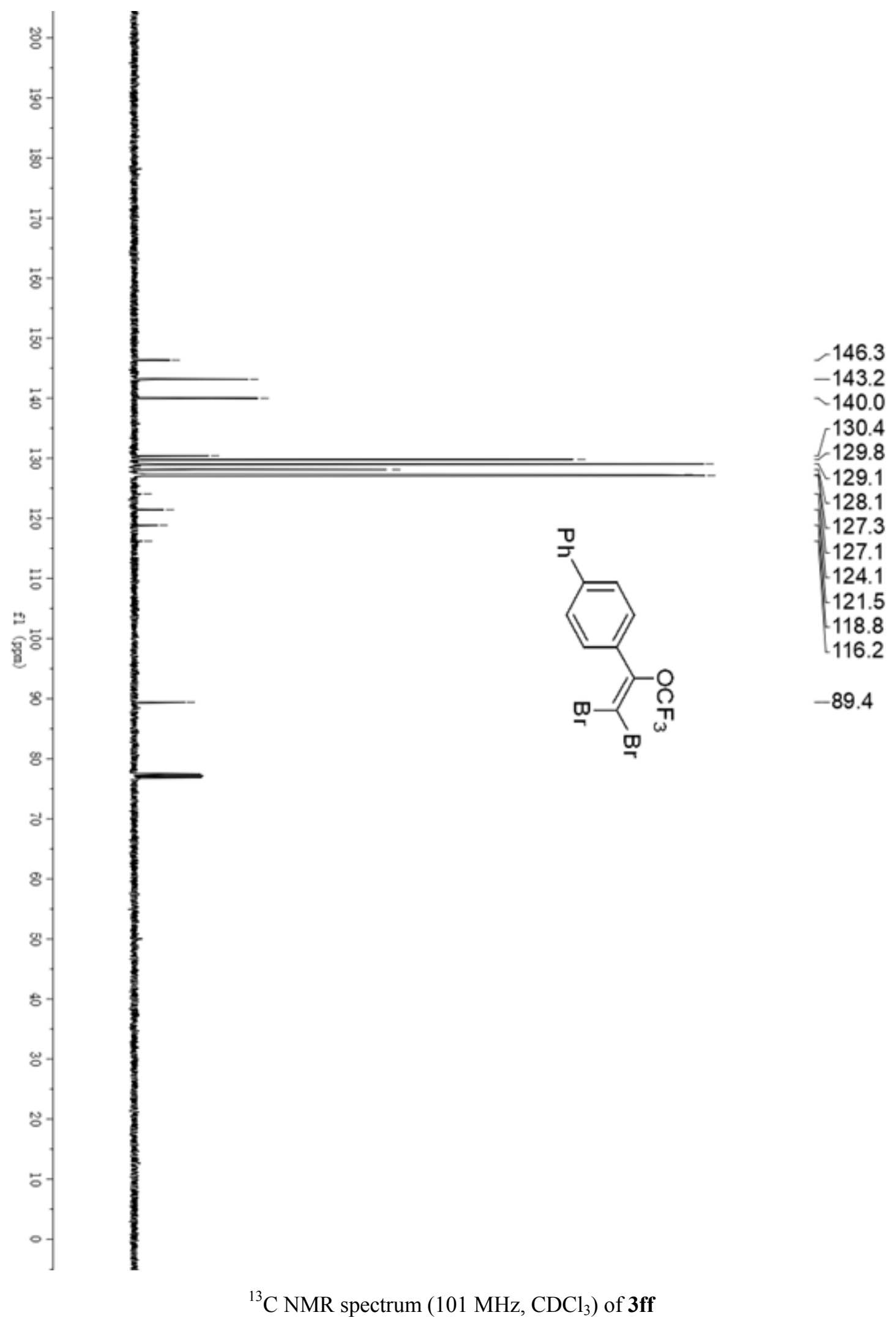




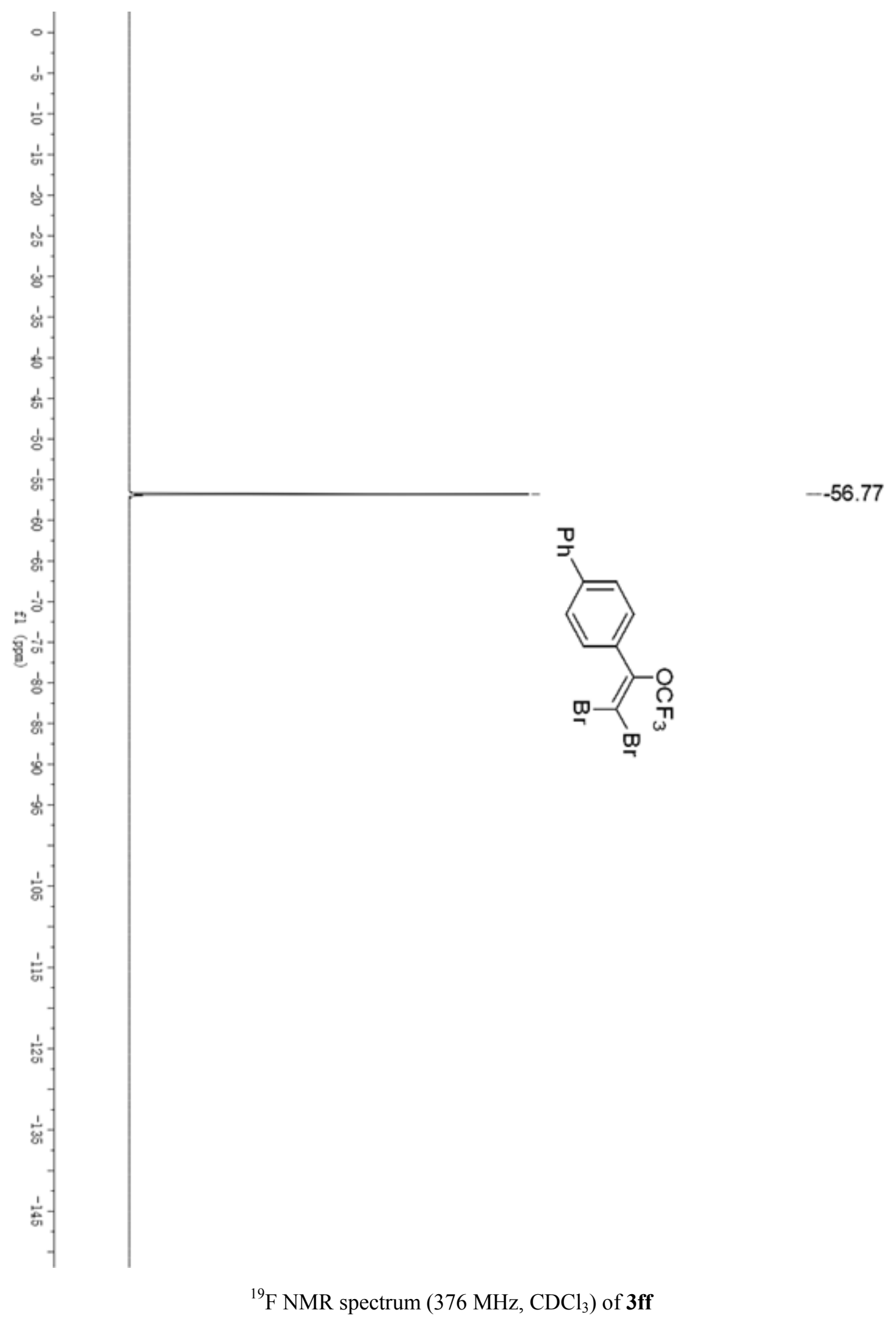




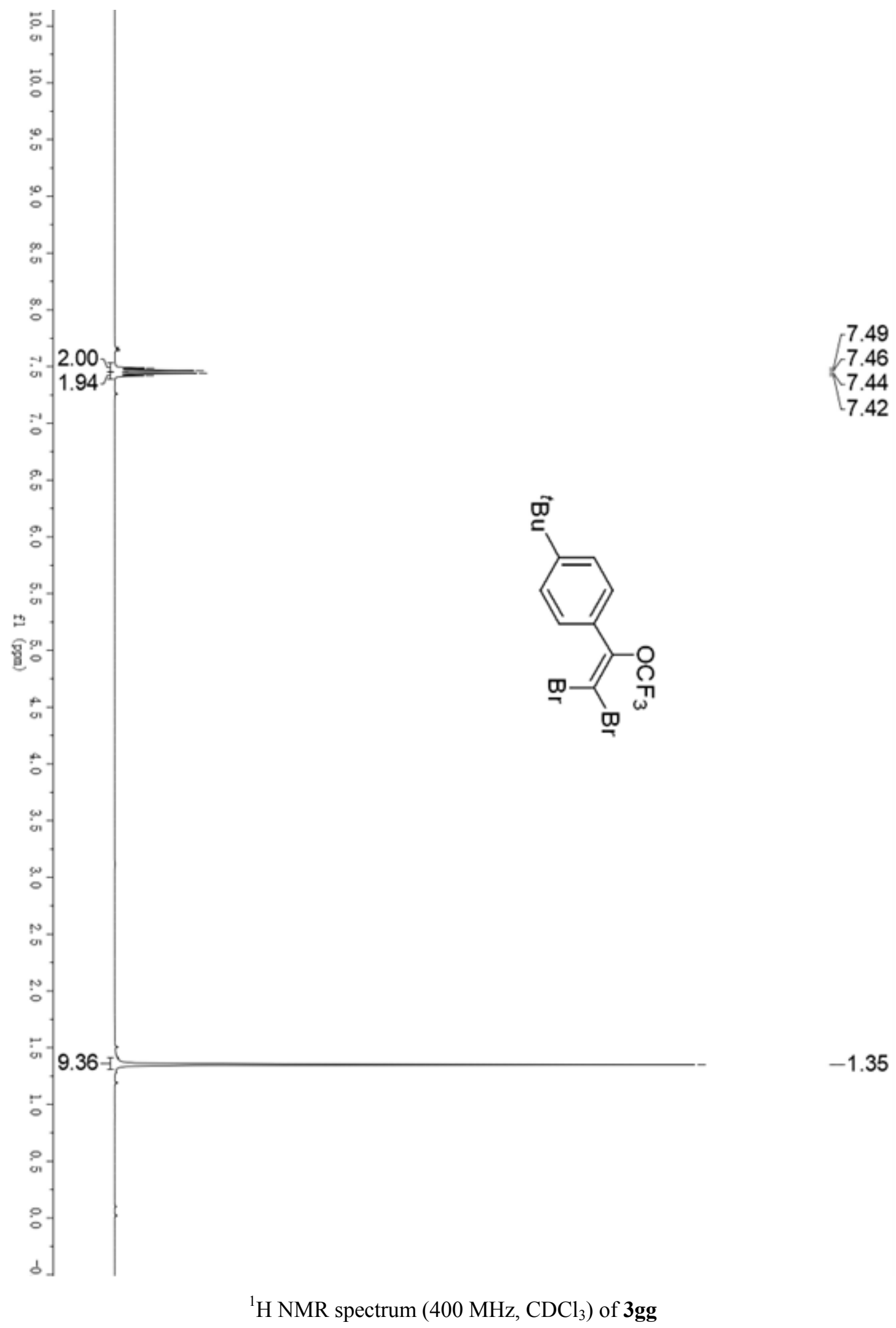




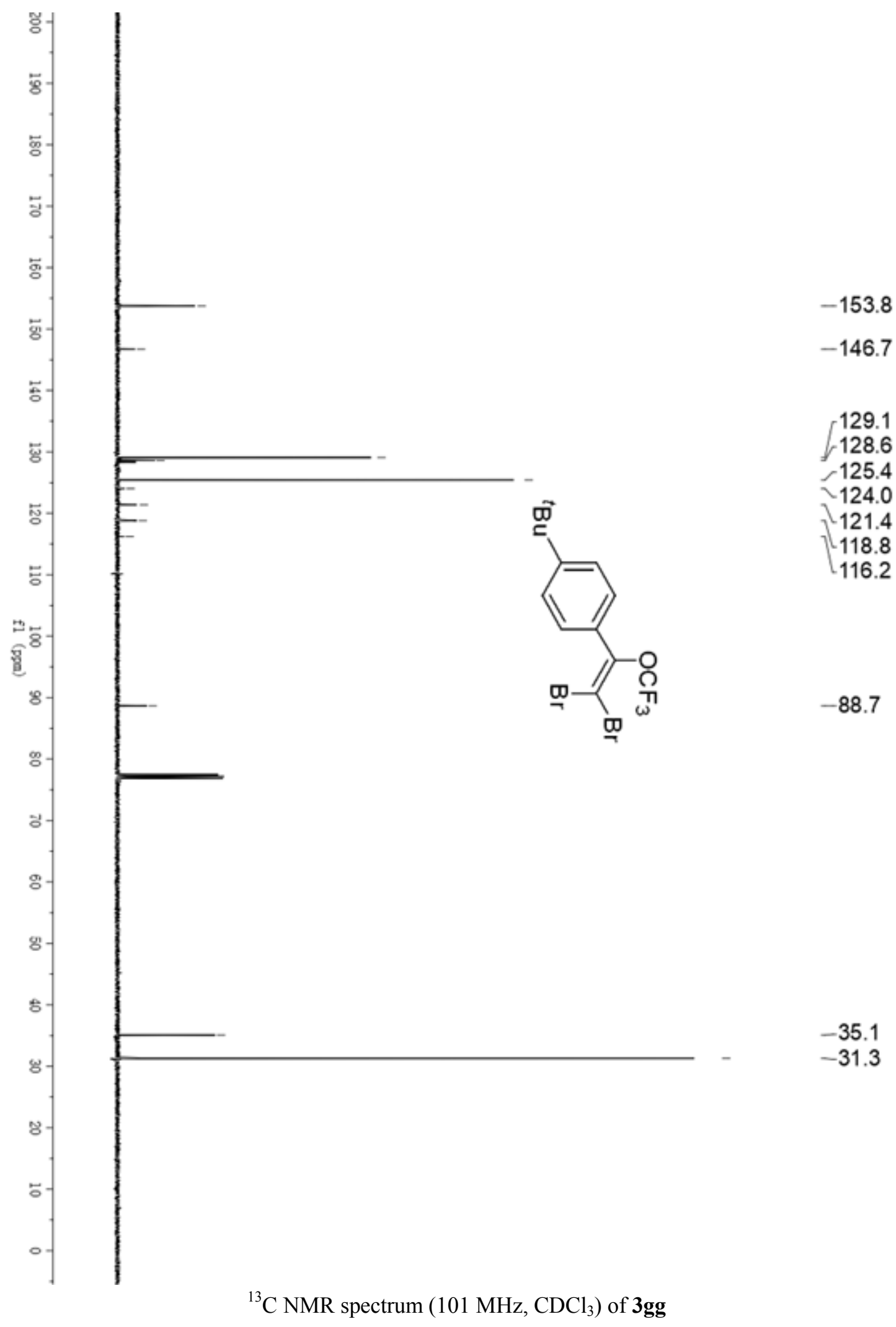




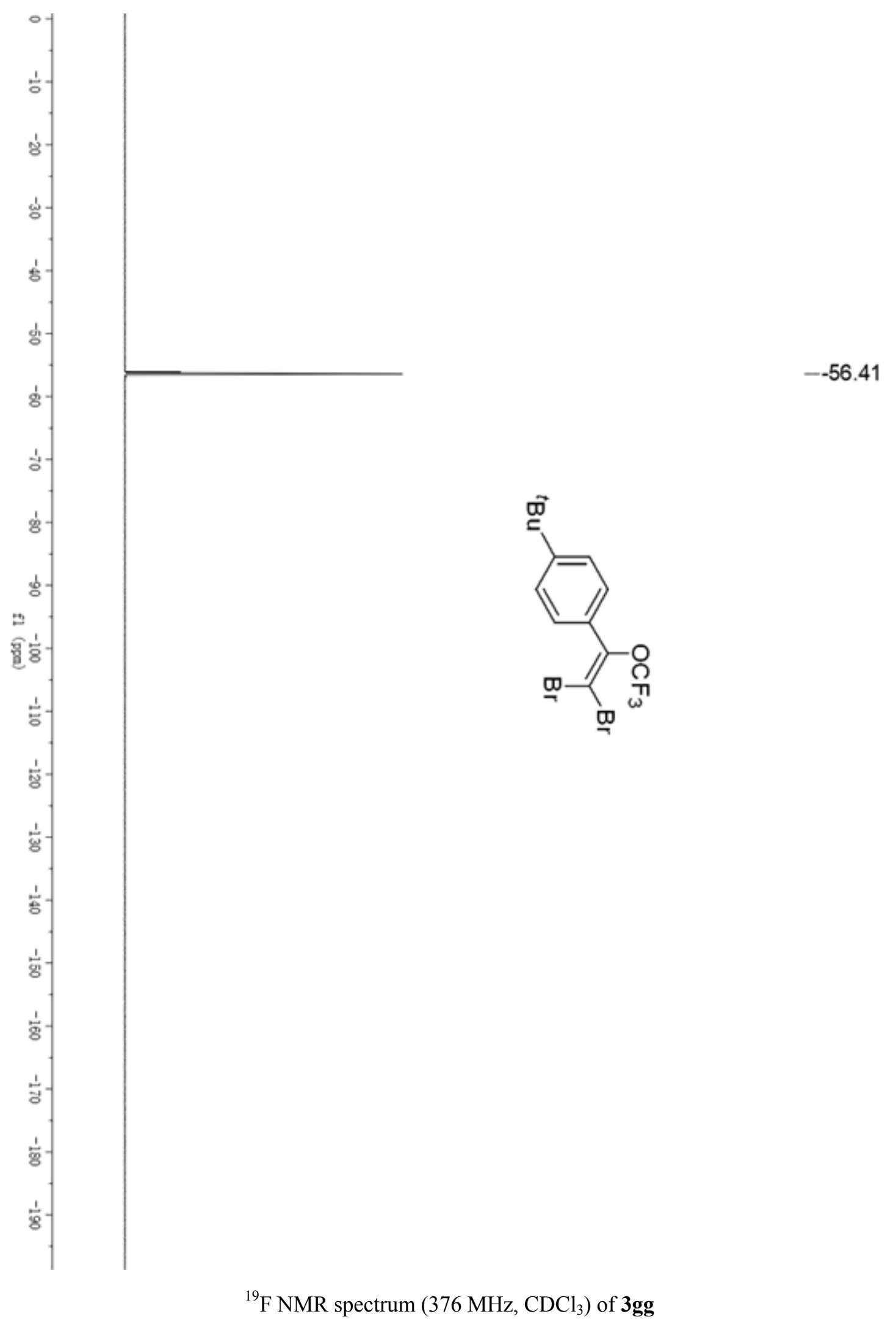




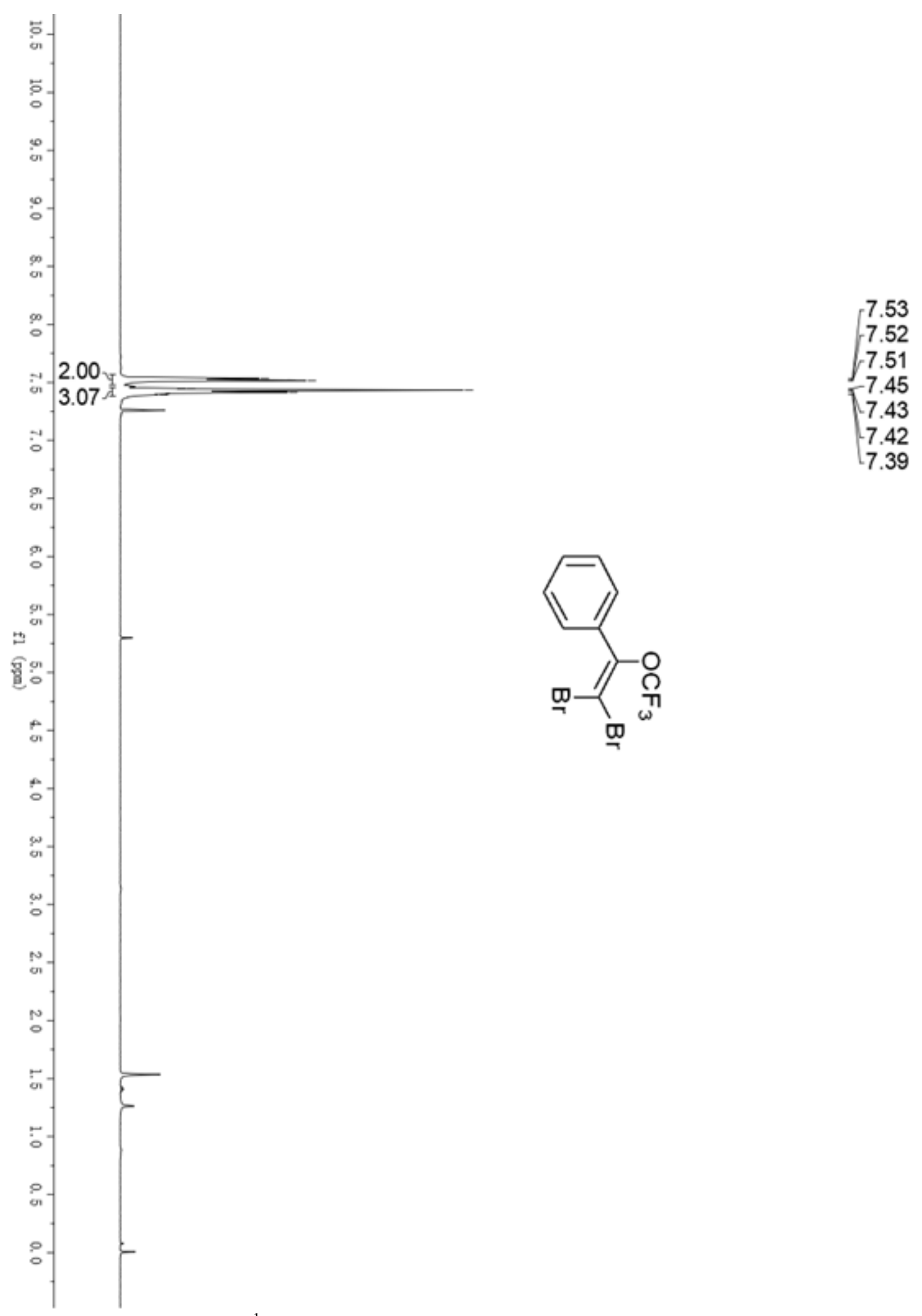

${ }^{1} \mathrm{H}$ NMR spectrum $\left(400 \mathrm{MHz}, \mathrm{CDCl}_{3}\right)$ of $\mathbf{3 h h}$ 


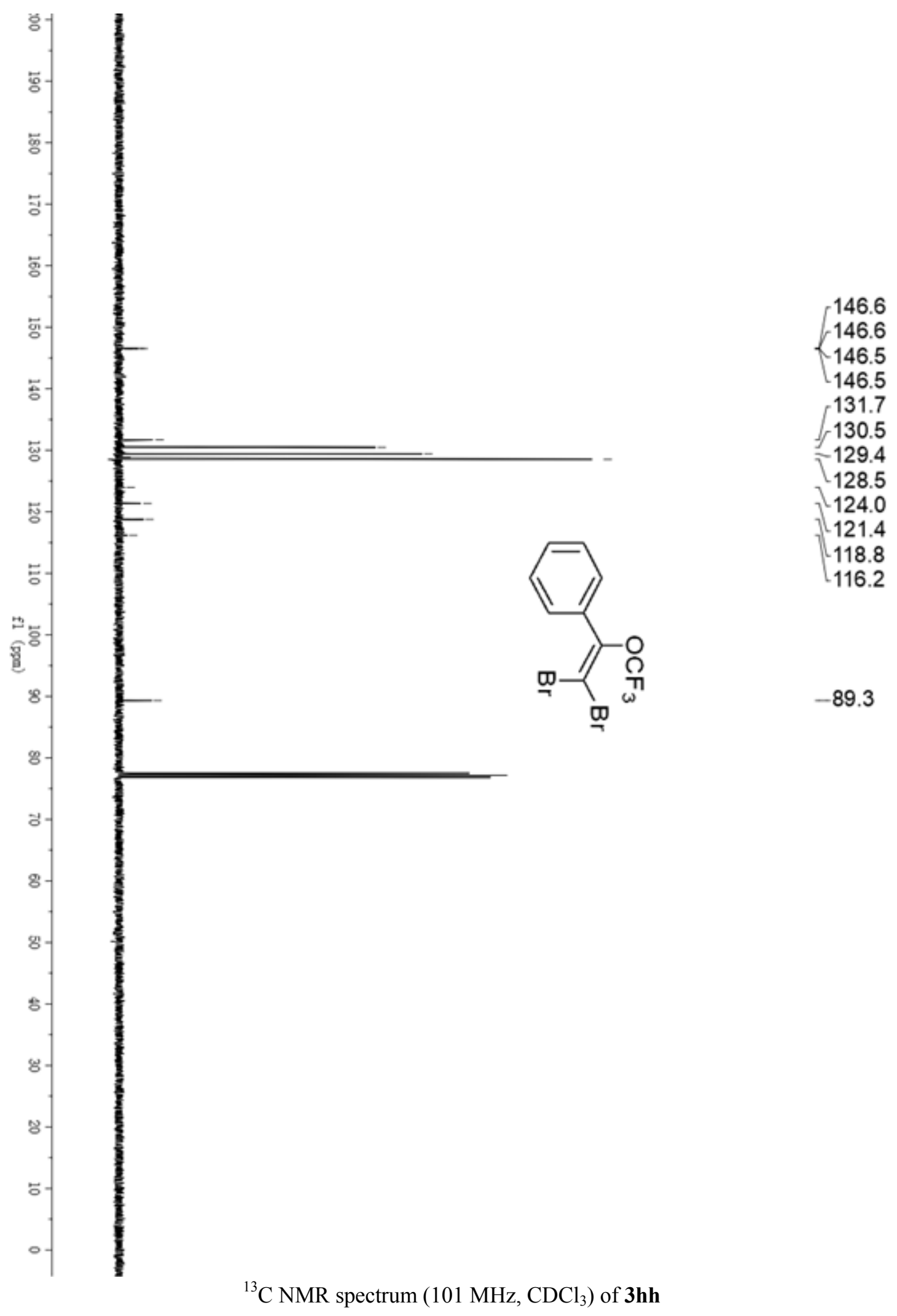




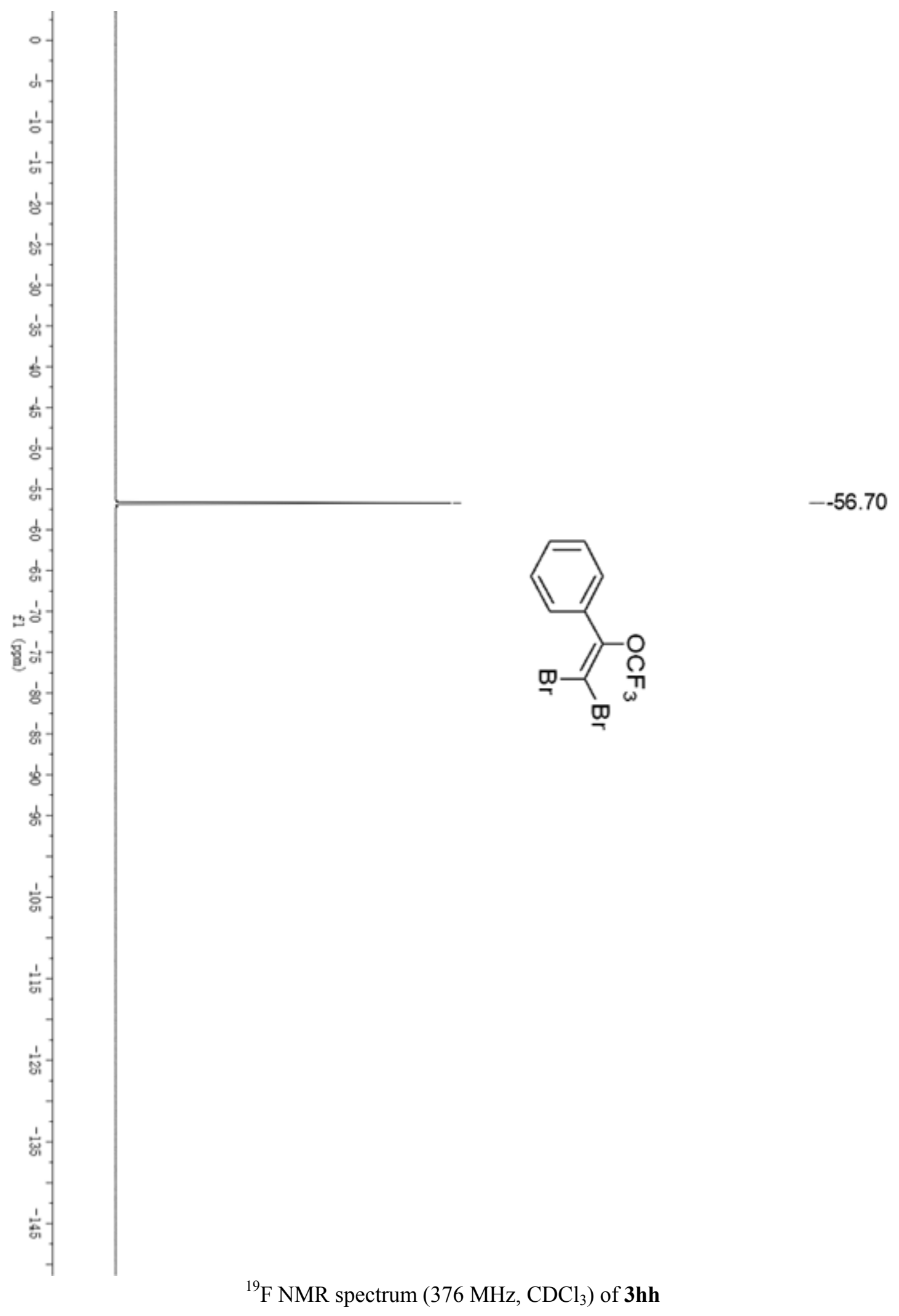




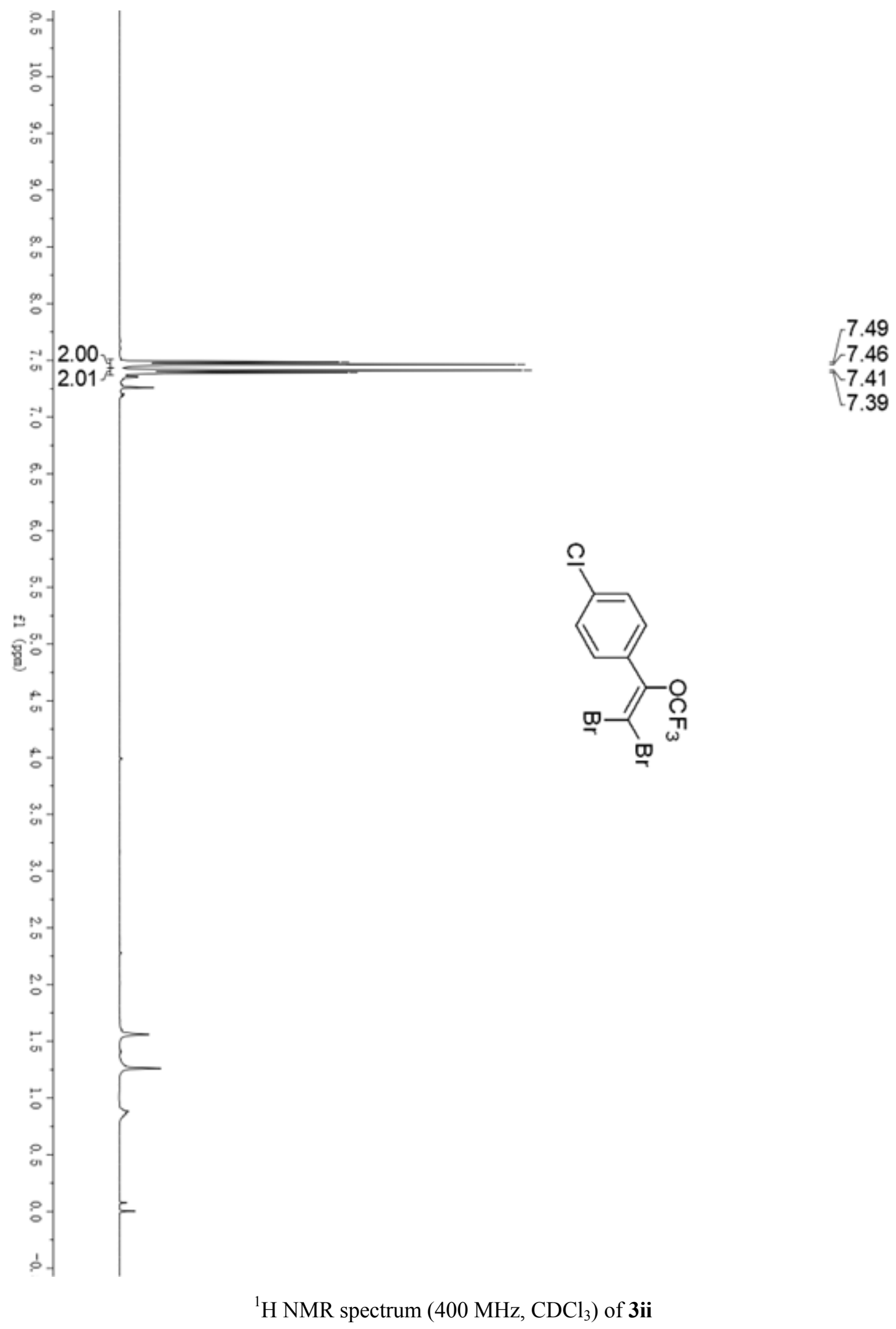




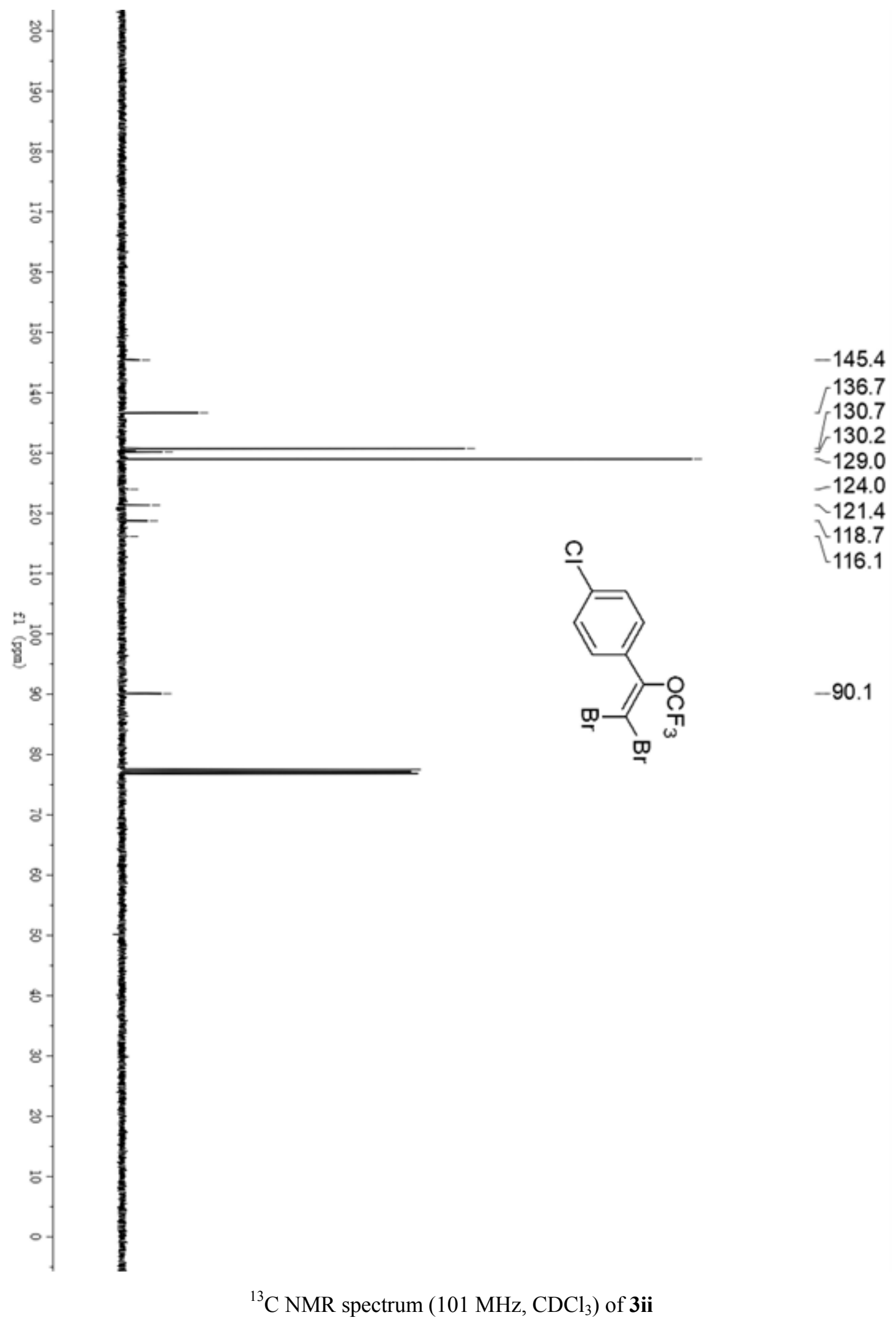




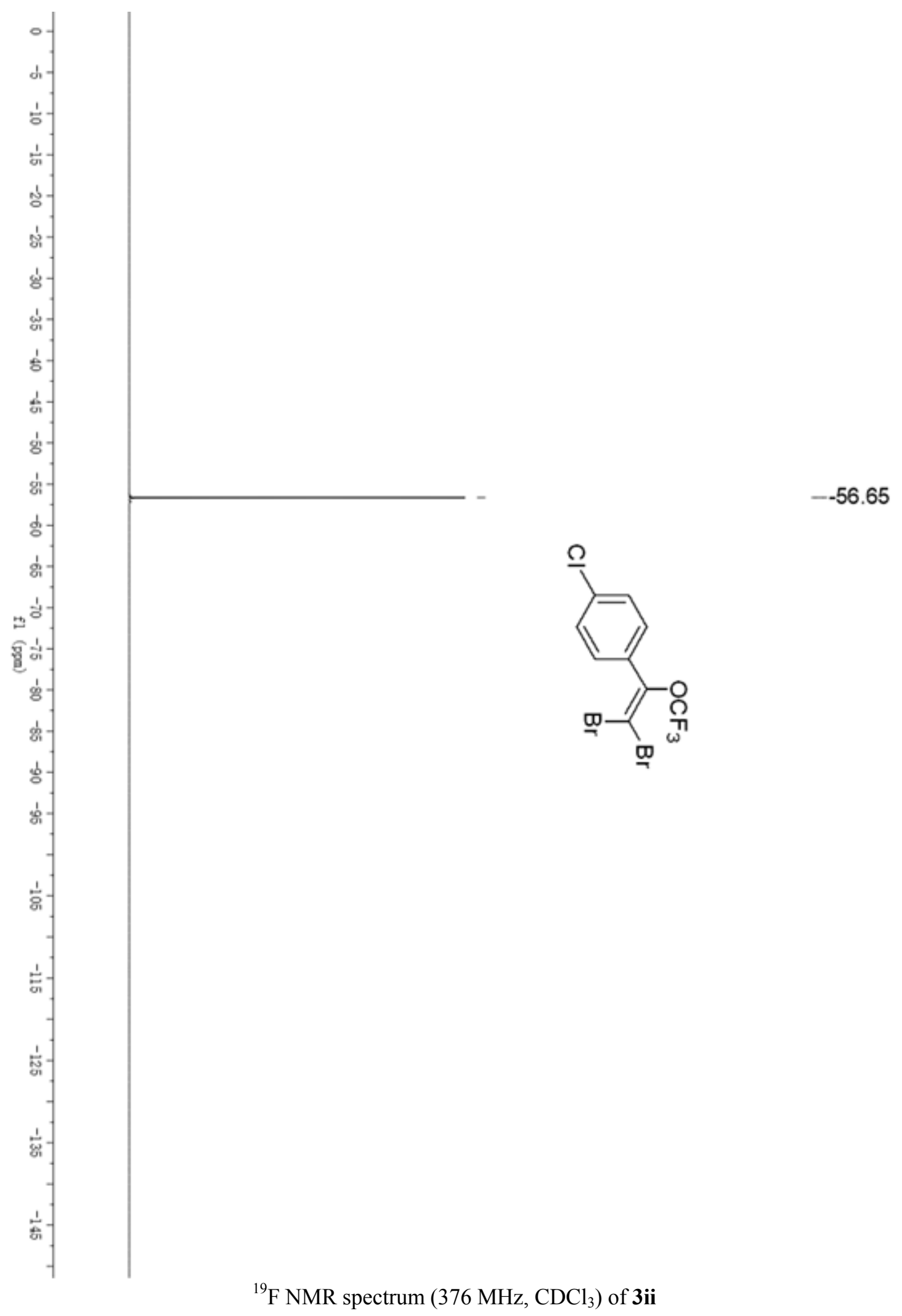




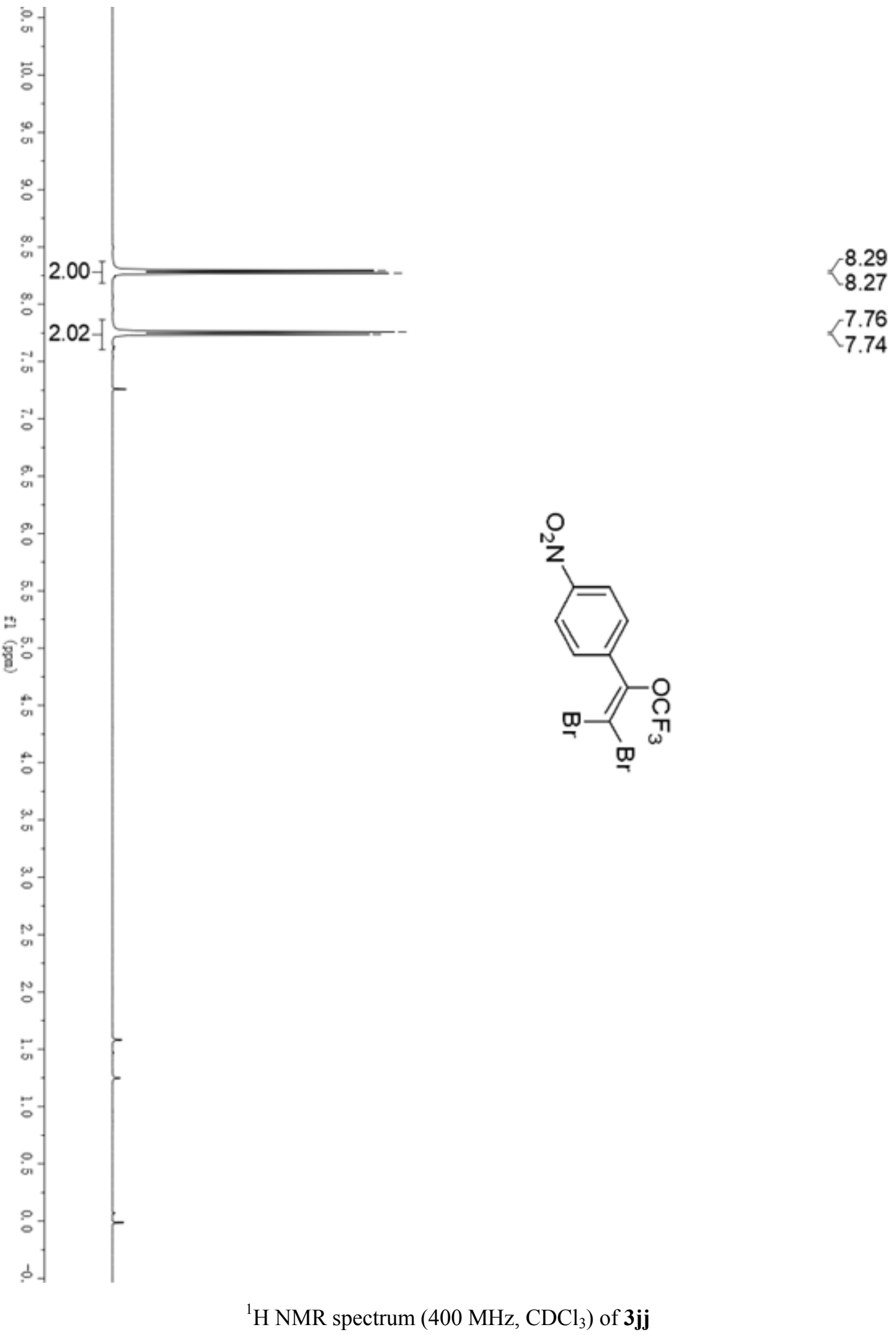




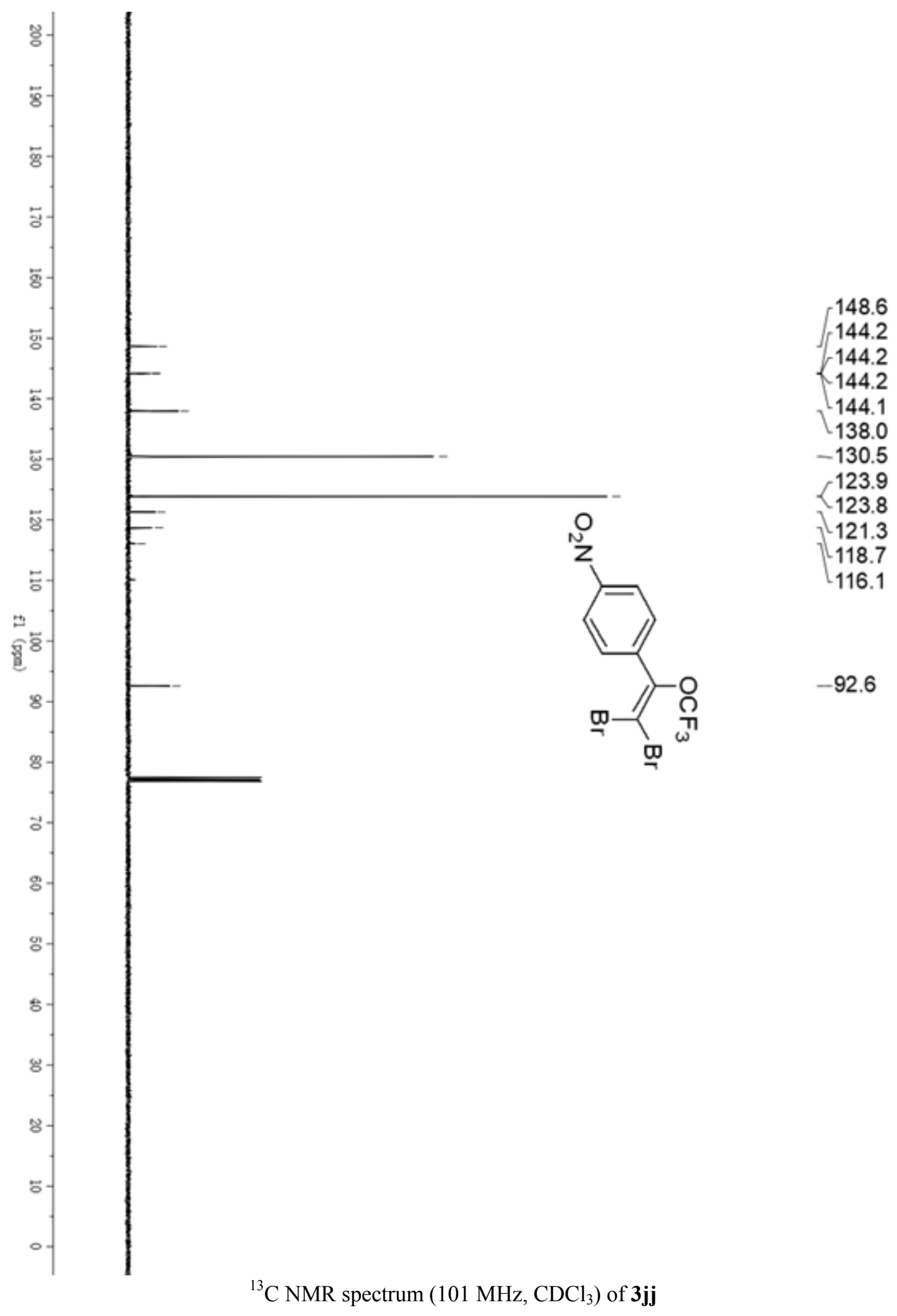




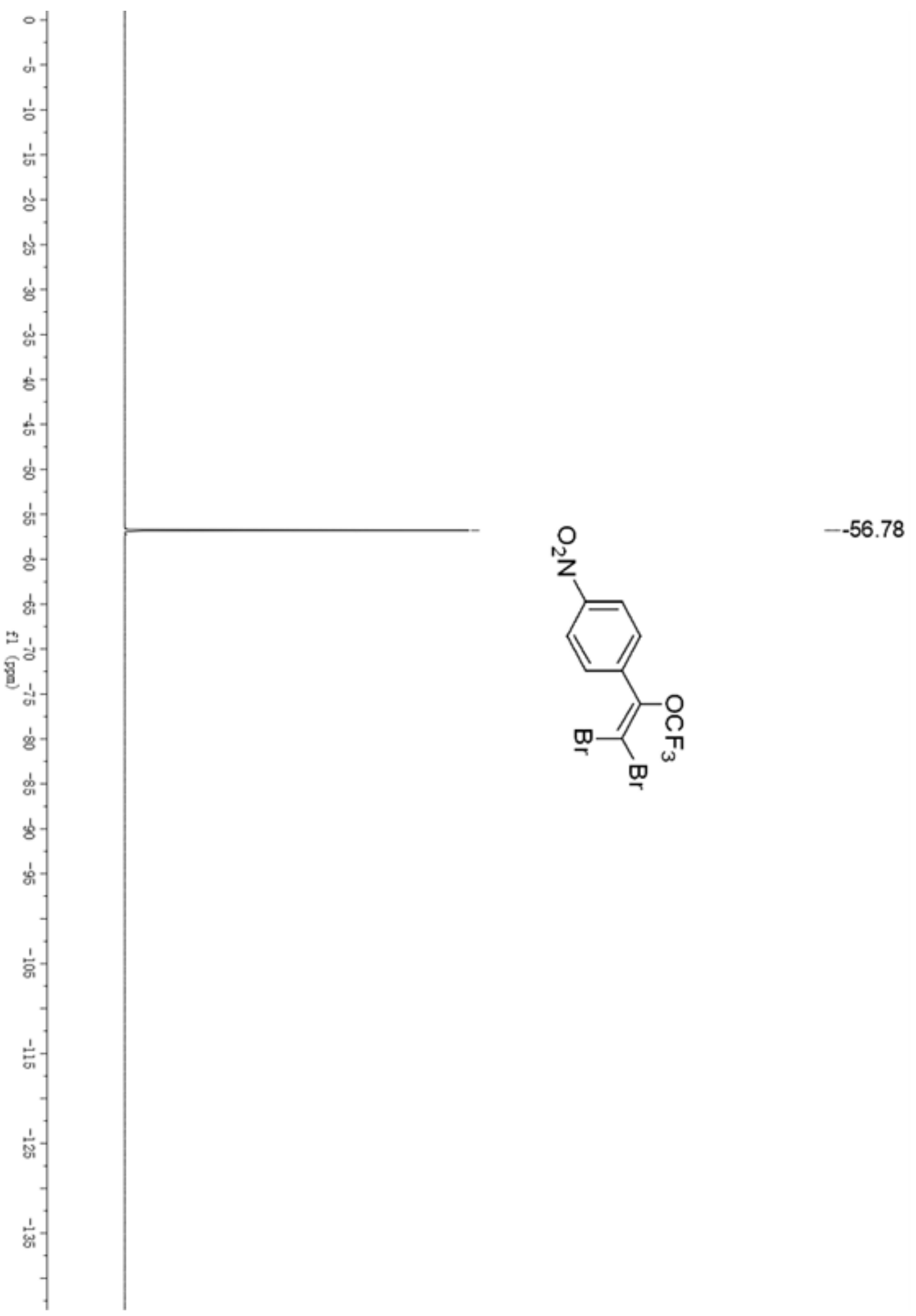

${ }^{19} \mathrm{~F}$ NMR spectrum $\left(376 \mathrm{MHz}, \mathrm{CDCl}_{3}\right.$ ) of $\mathbf{3} \mathbf{j j}$ 


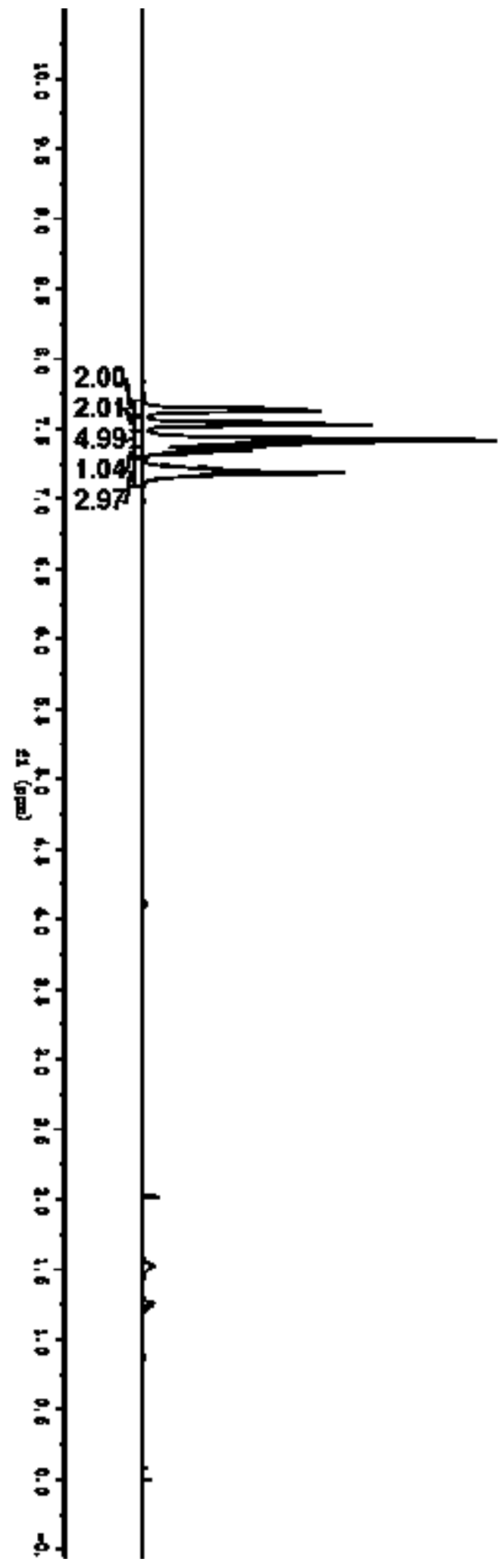

7.65

7.64

7.63

7.62

7.55

7.53

7.46

7.43

7.42

7.40

7.38

7.34

7.32

7.24

7.22

7.21

7.18

7.16

7.14

${ }^{1} \mathrm{H}$ NMR spectrum $\left(400 \mathrm{MHz}, \mathrm{CDCl}_{3}\right)$ of $\mathbf{3 k \mathbf { k }}$ 


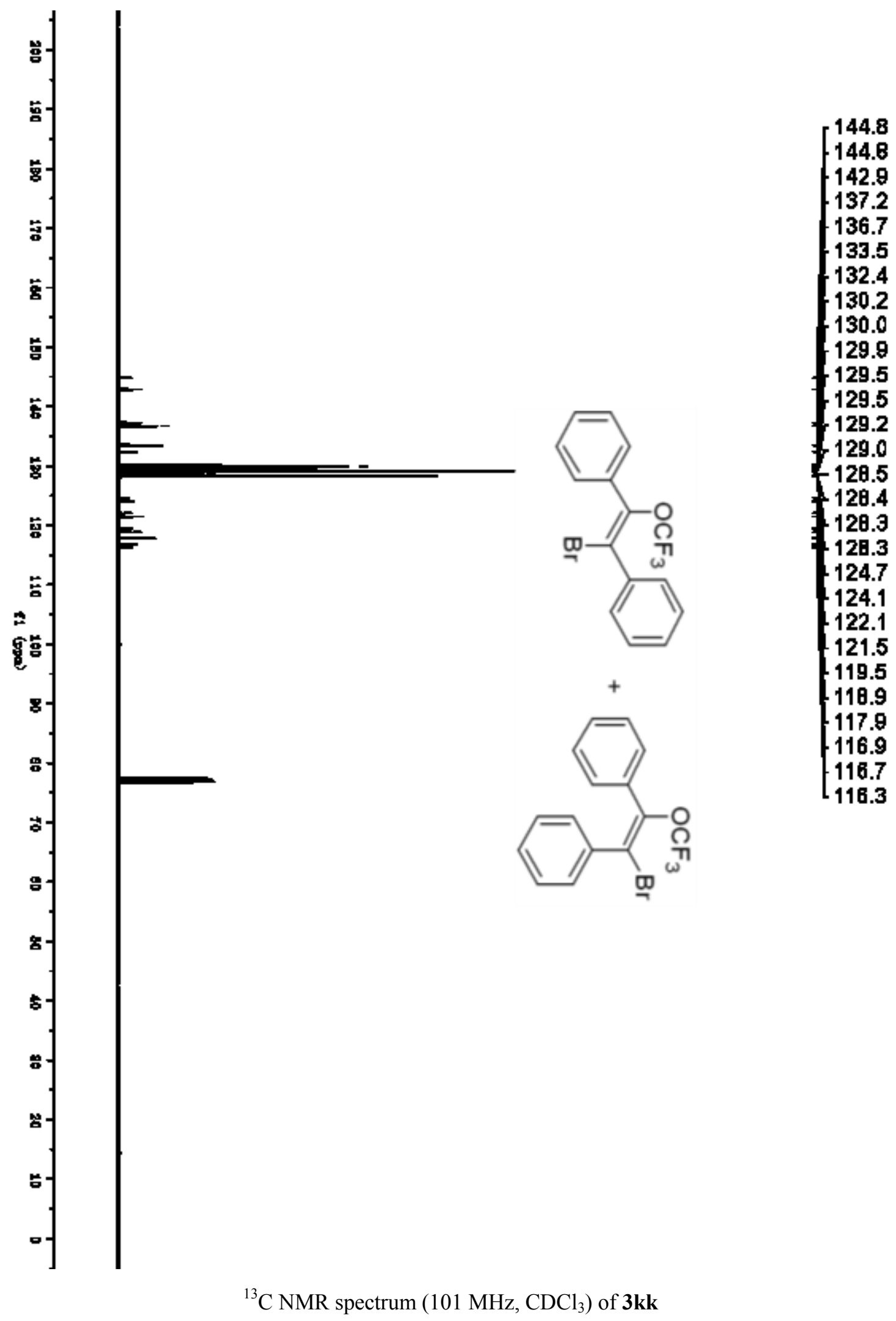




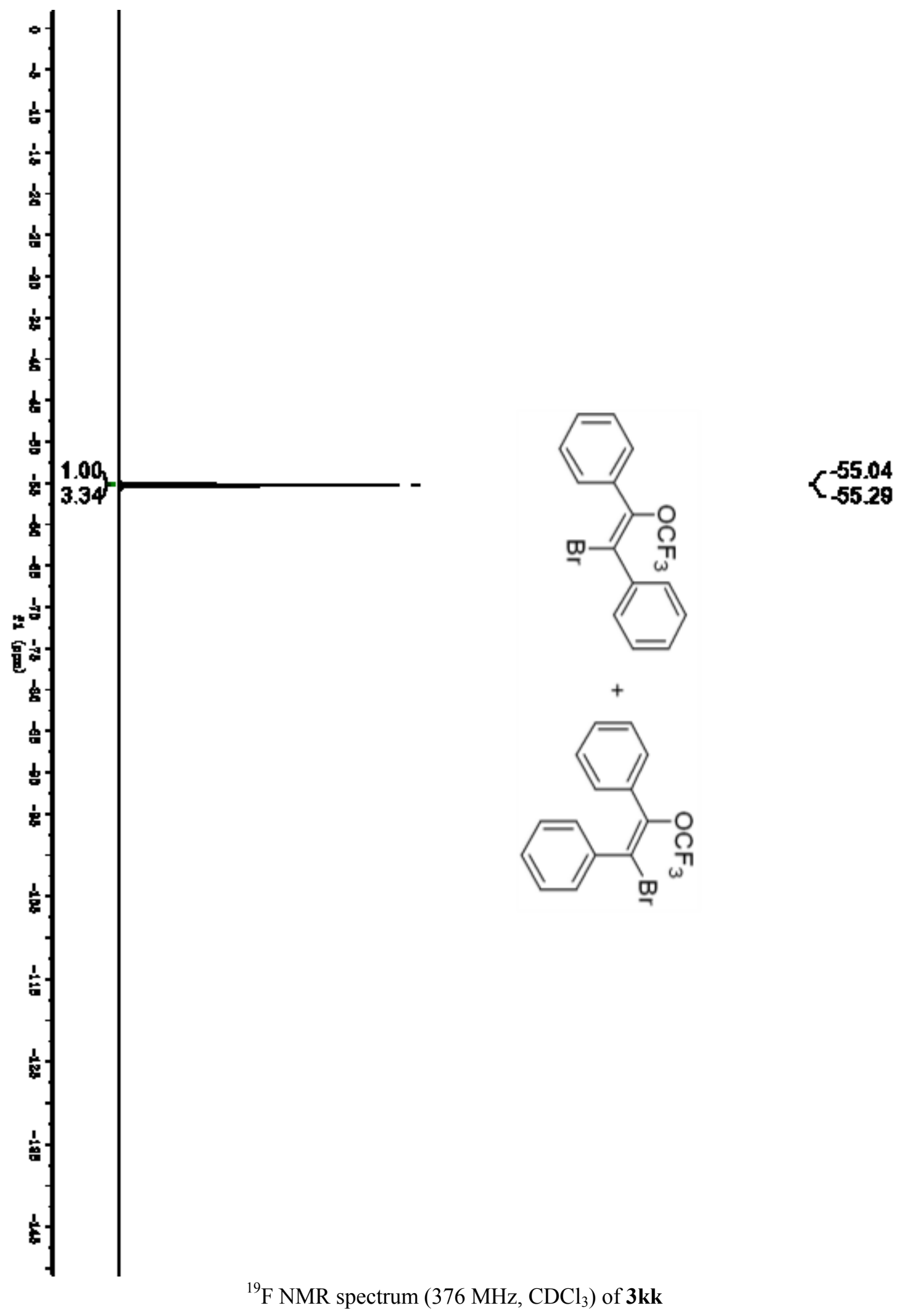




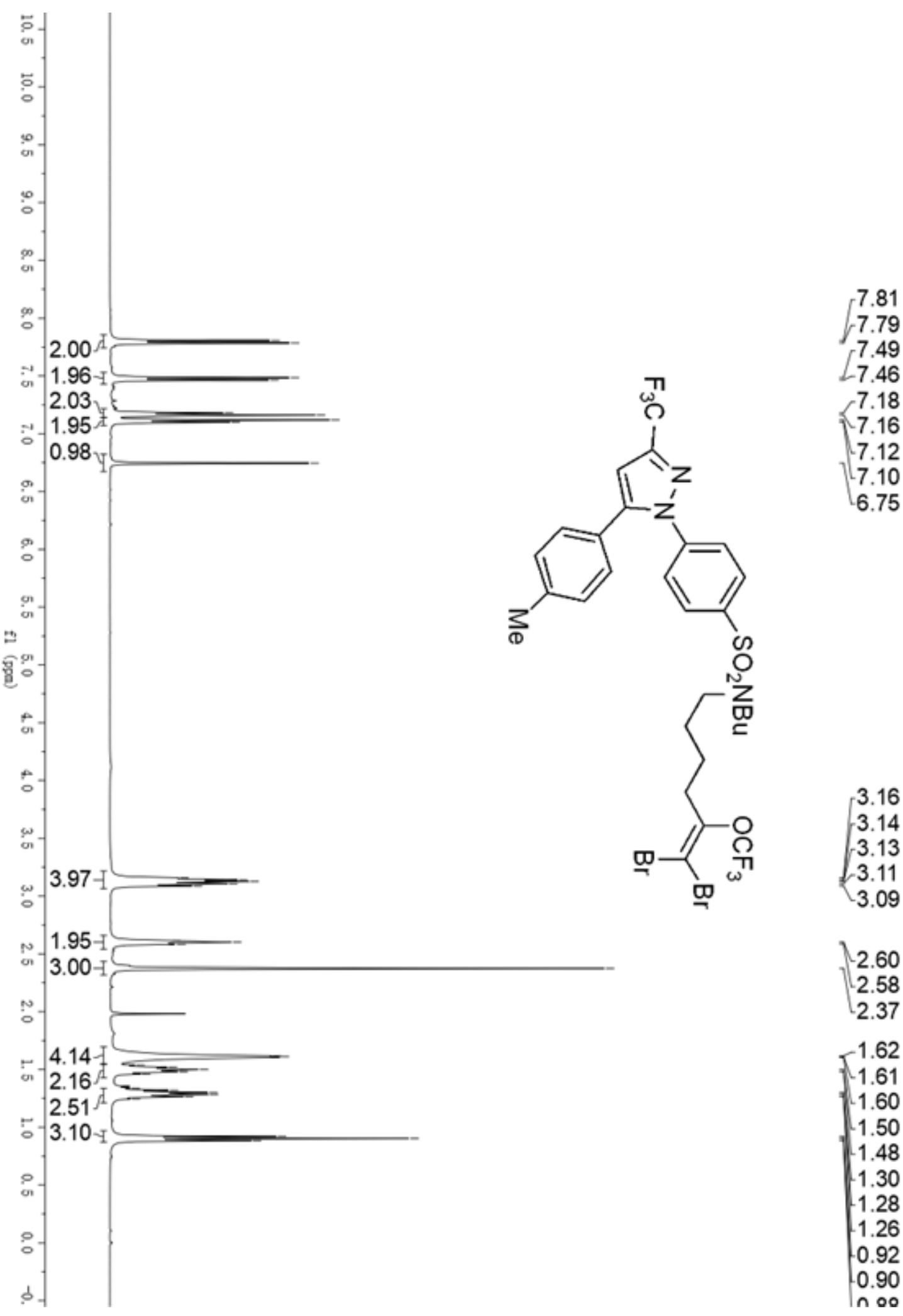

${ }^{1} \mathrm{H}$ NMR spectrum $\left(400 \mathrm{MHz}, \mathrm{CDCl}_{3}\right.$ ) of $\mathbf{3 l l}$ 


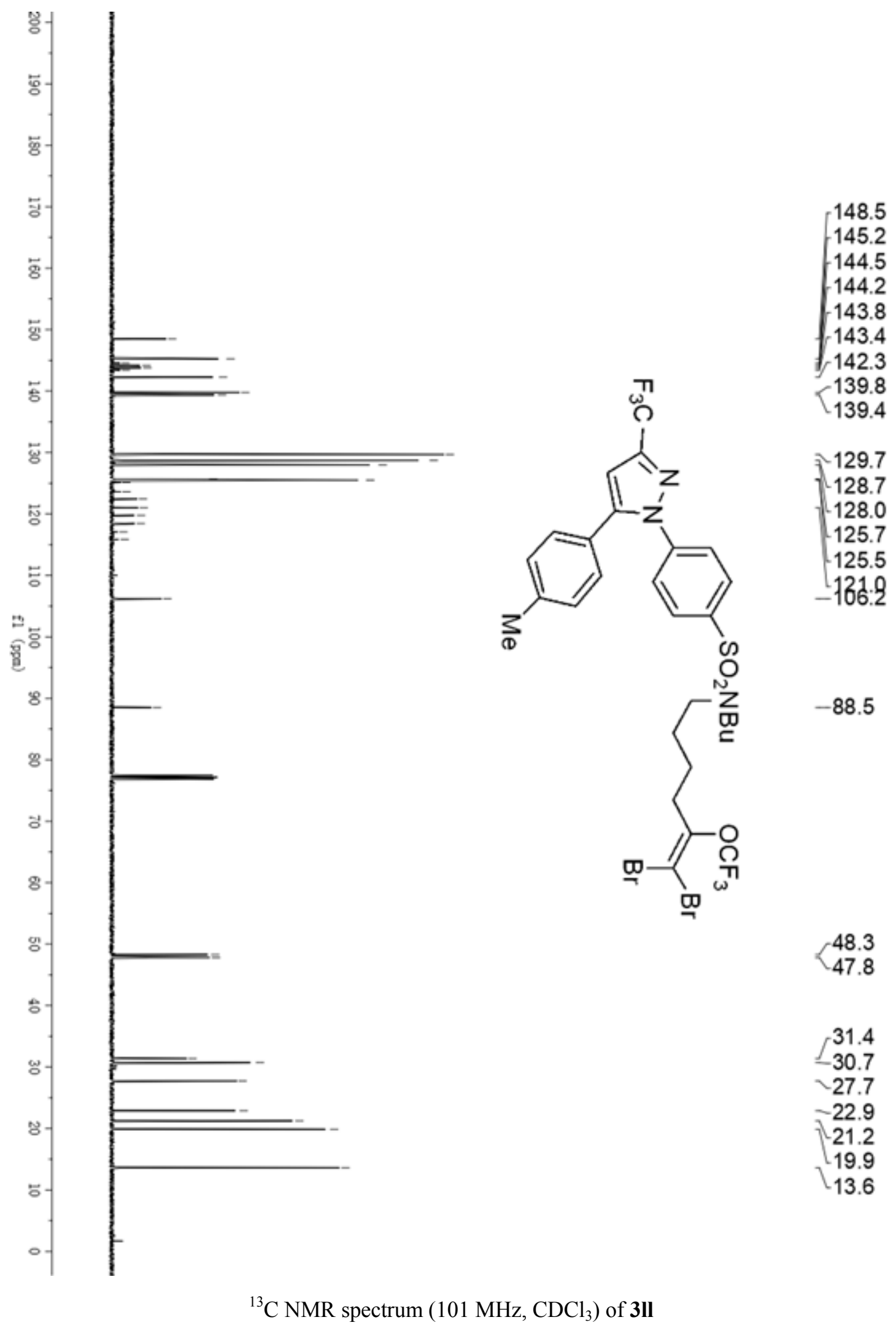




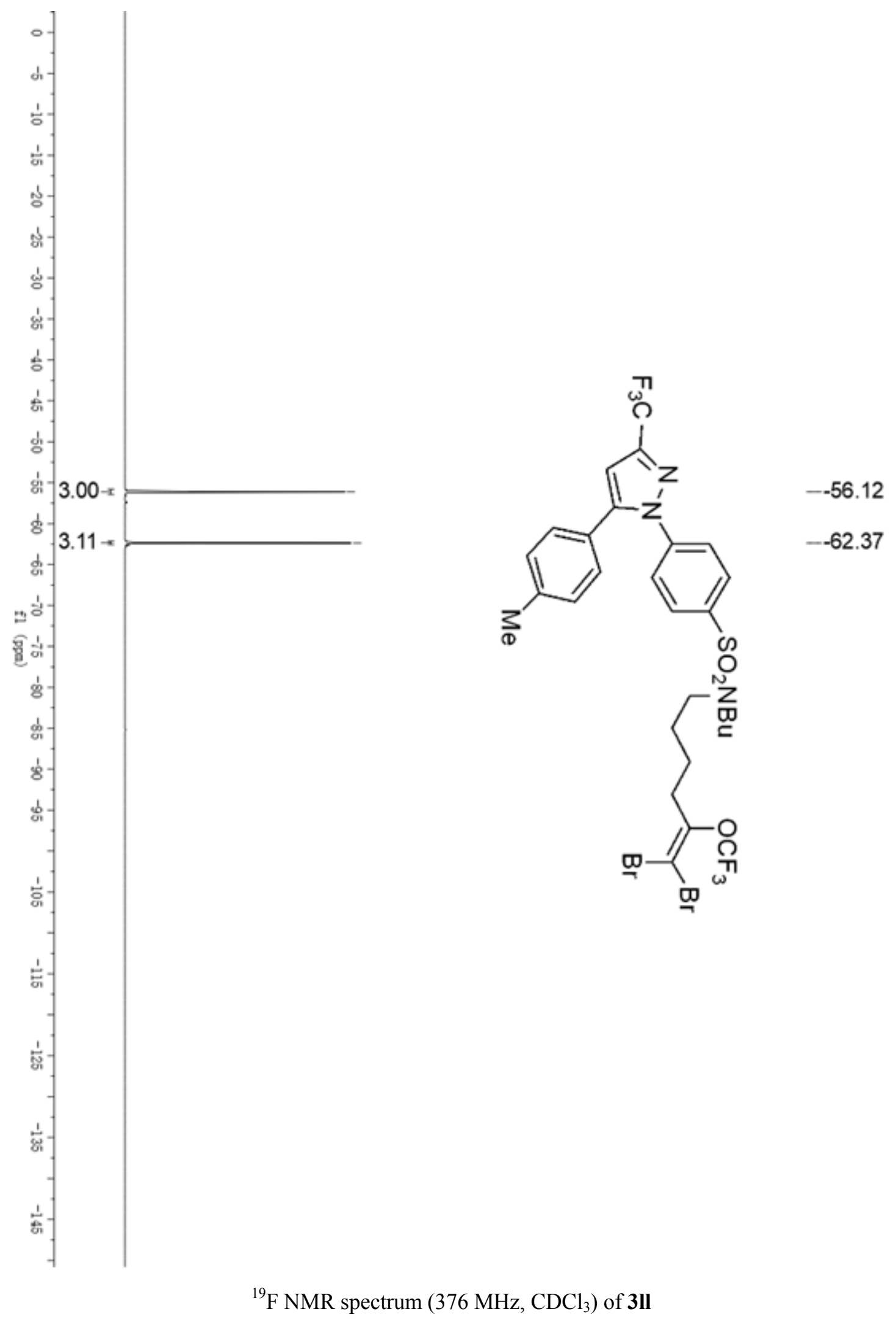




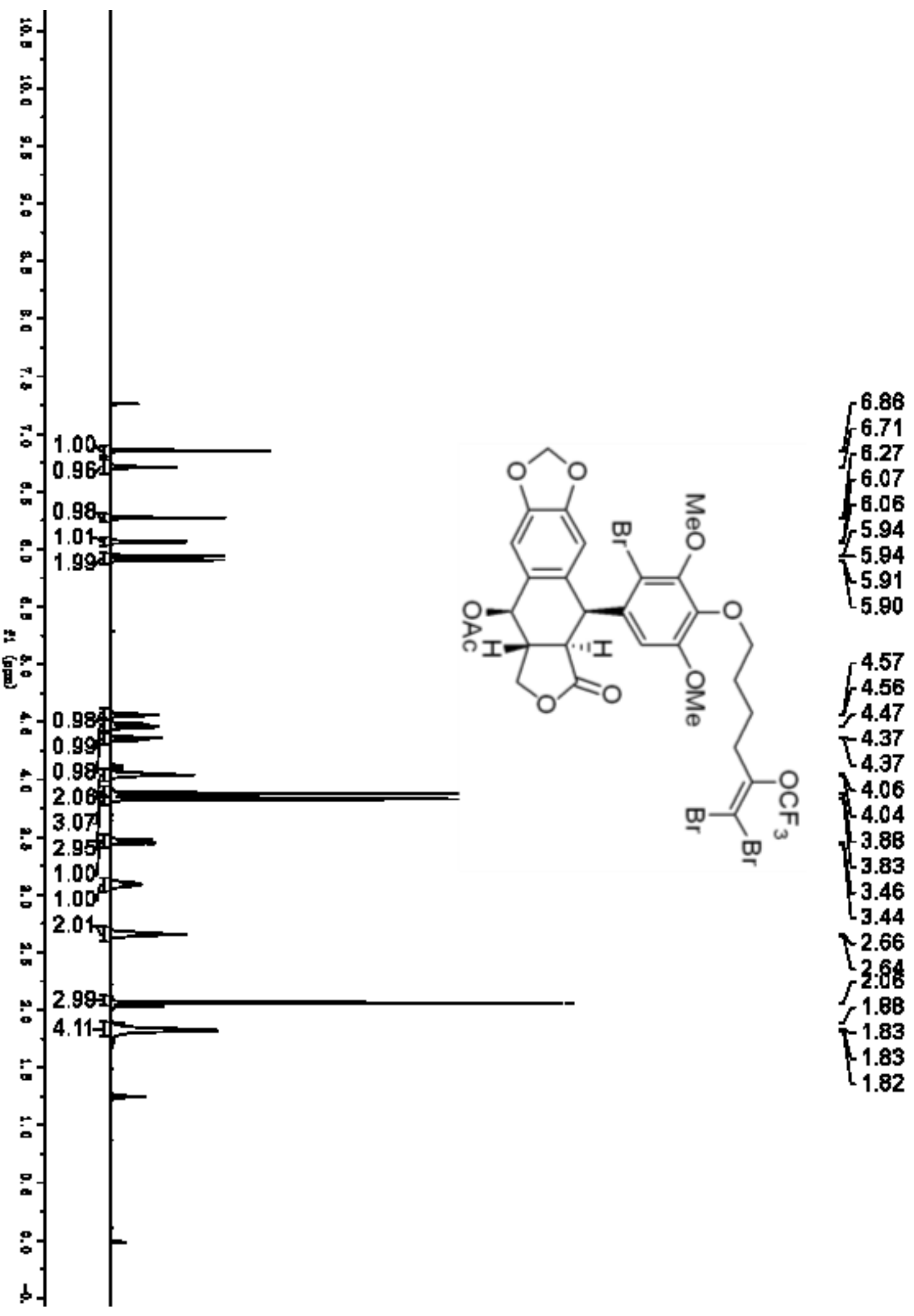

${ }^{1} \mathrm{H}$ NMR spectrum $\left(400 \mathrm{MHz}, \mathrm{CDCl}_{3}\right)$ of $\mathbf{3 m m}$ 


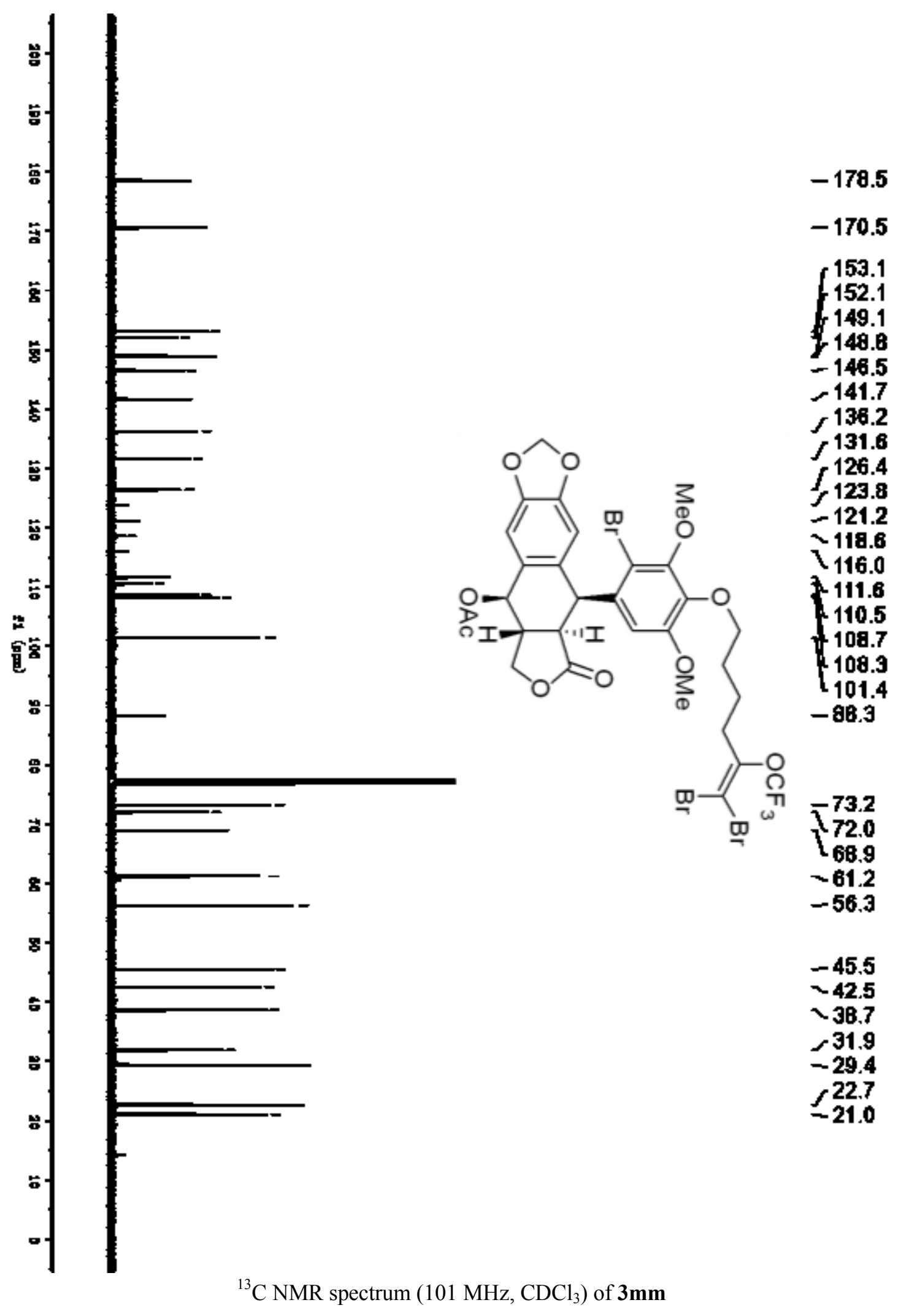




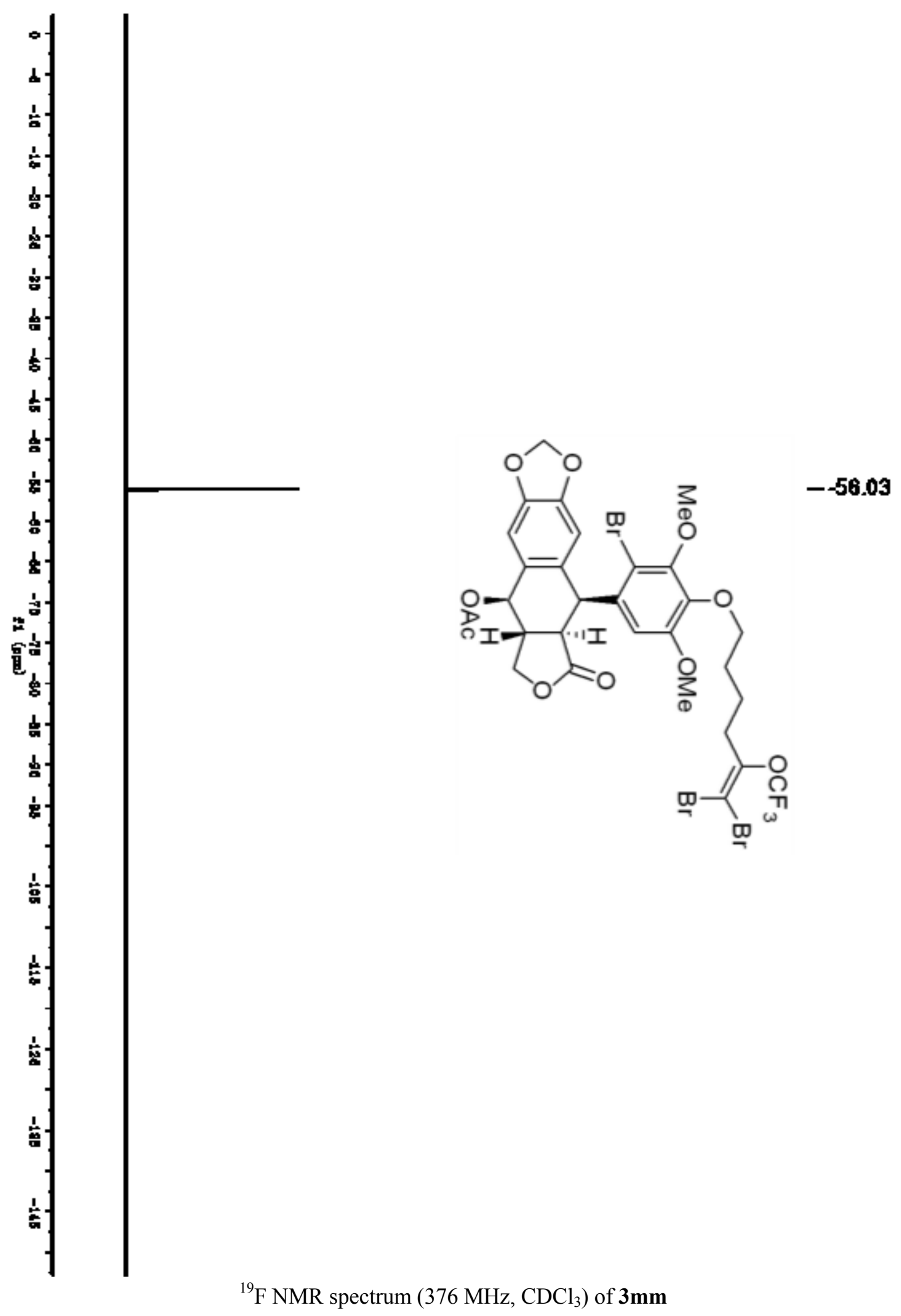



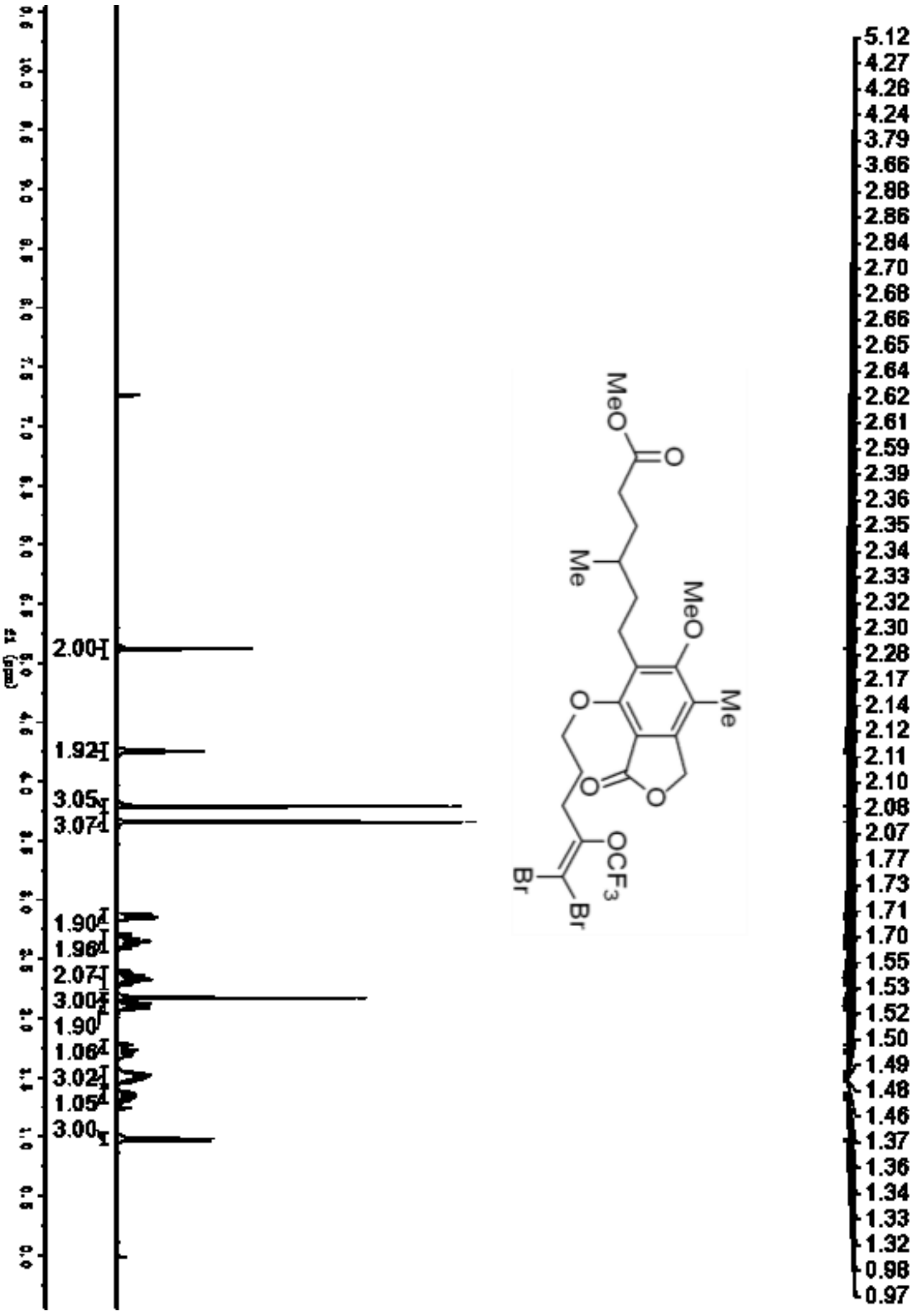

${ }^{1} \mathrm{H}$ NMR spectrum $\left(400 \mathrm{MHz}, \mathrm{CDCl}_{3}\right)$ of $\mathbf{3 n n}$ 


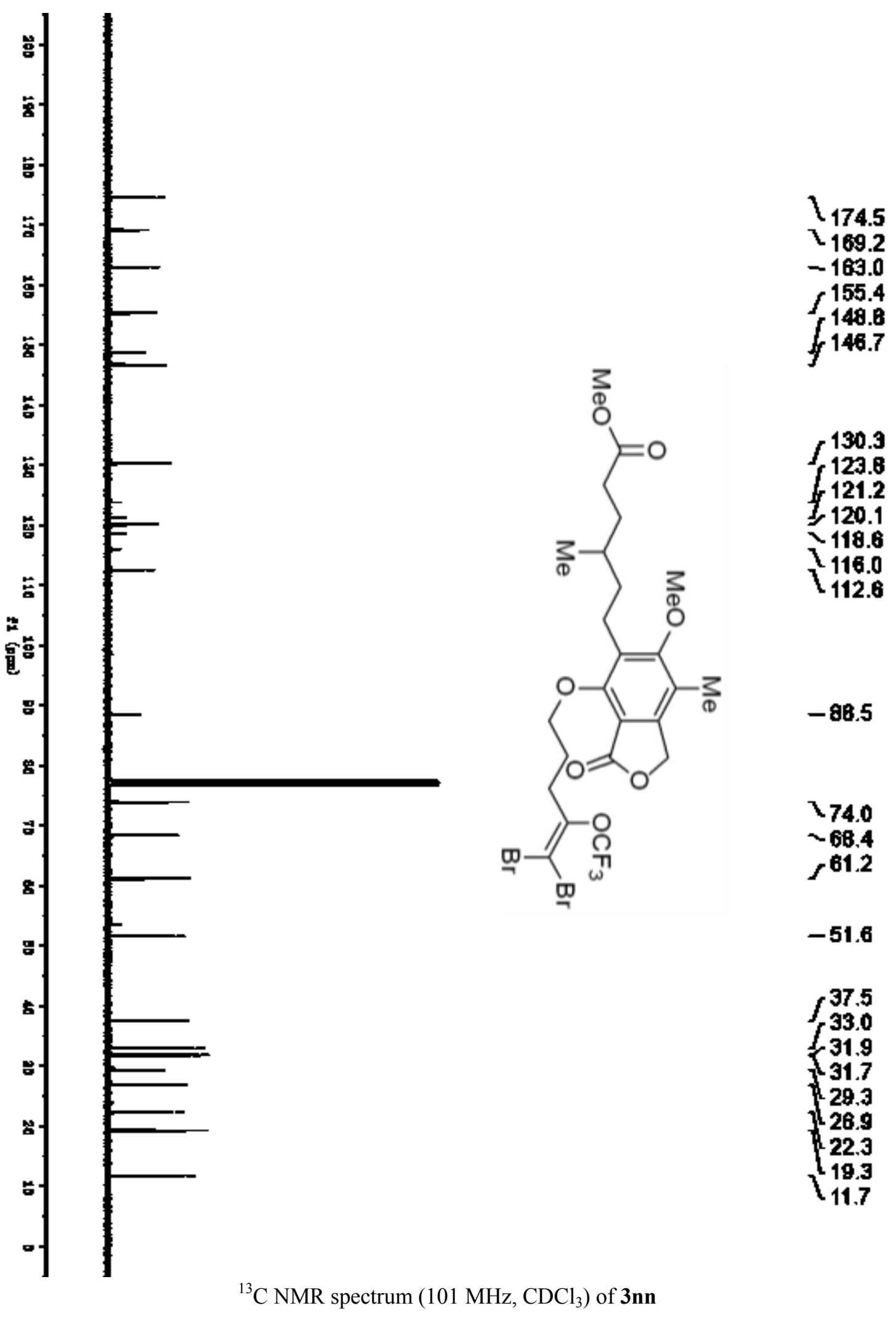




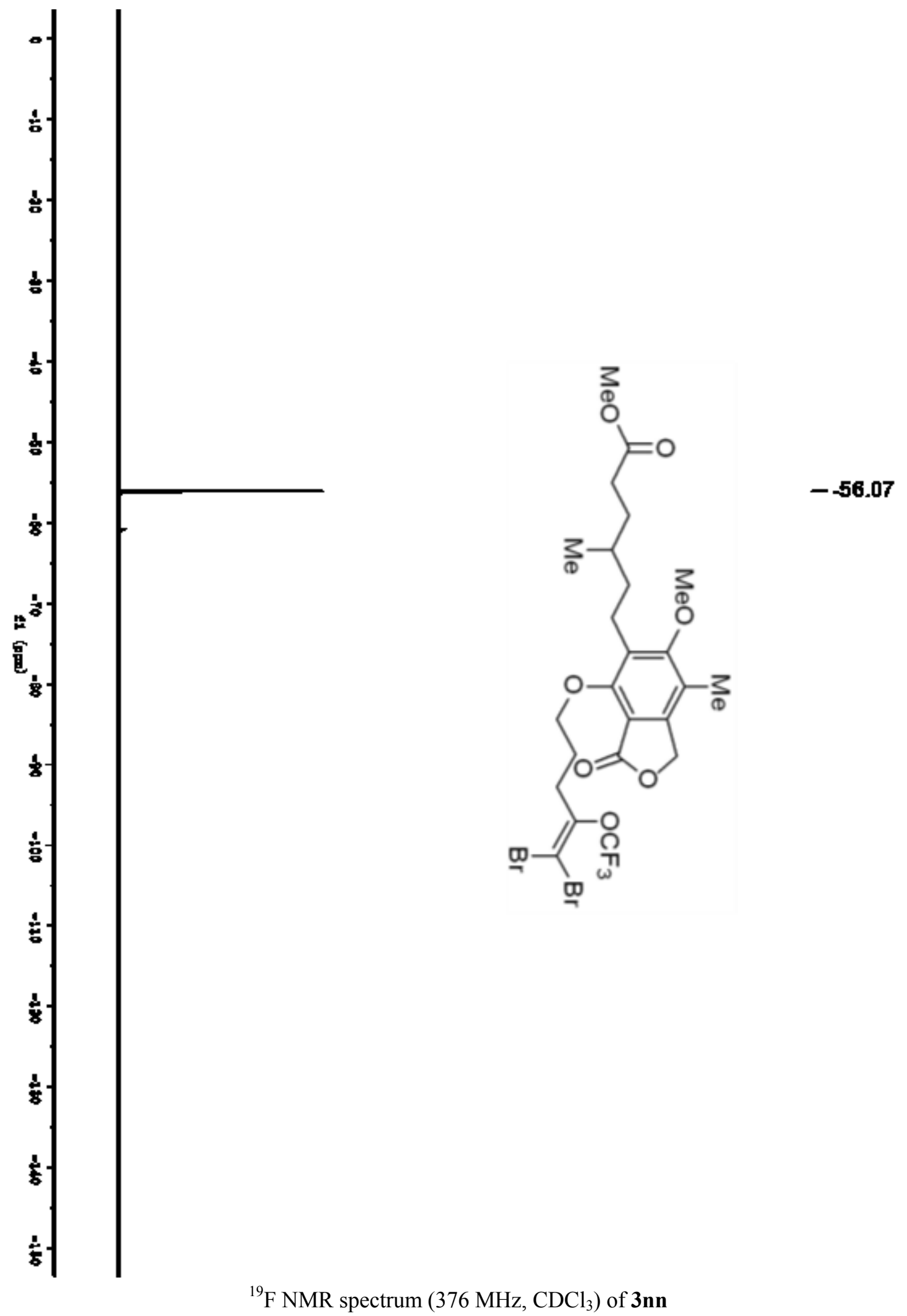




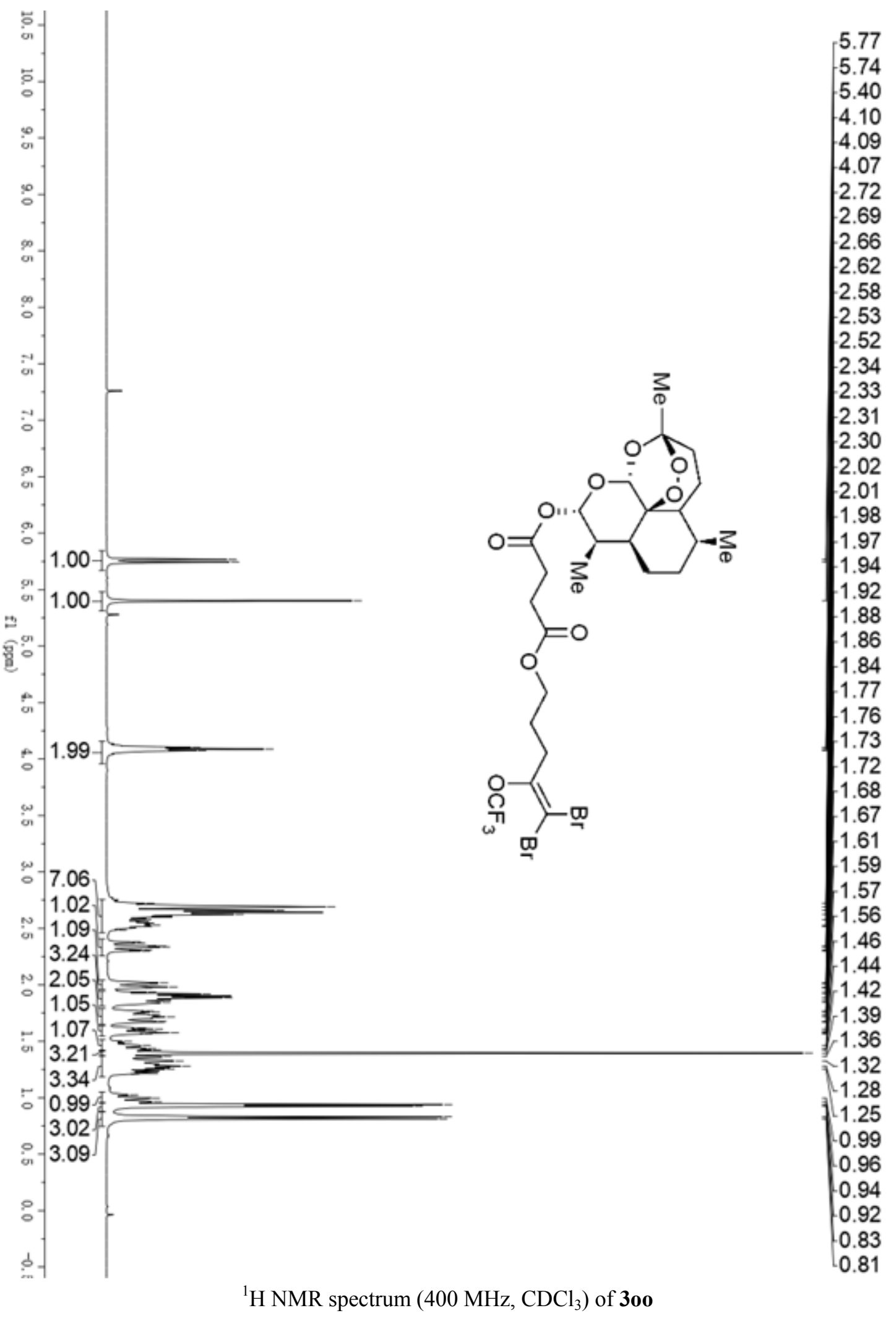




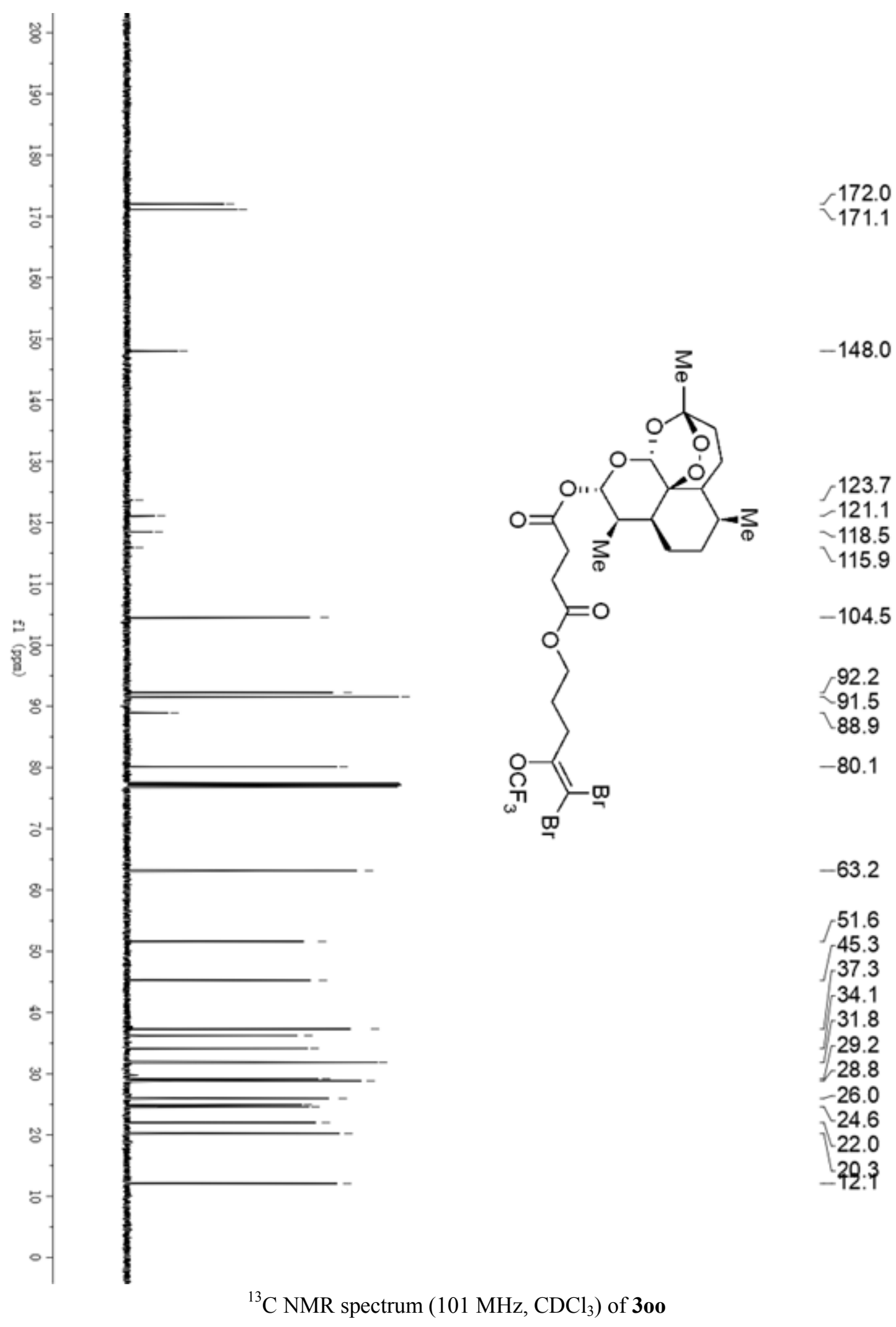




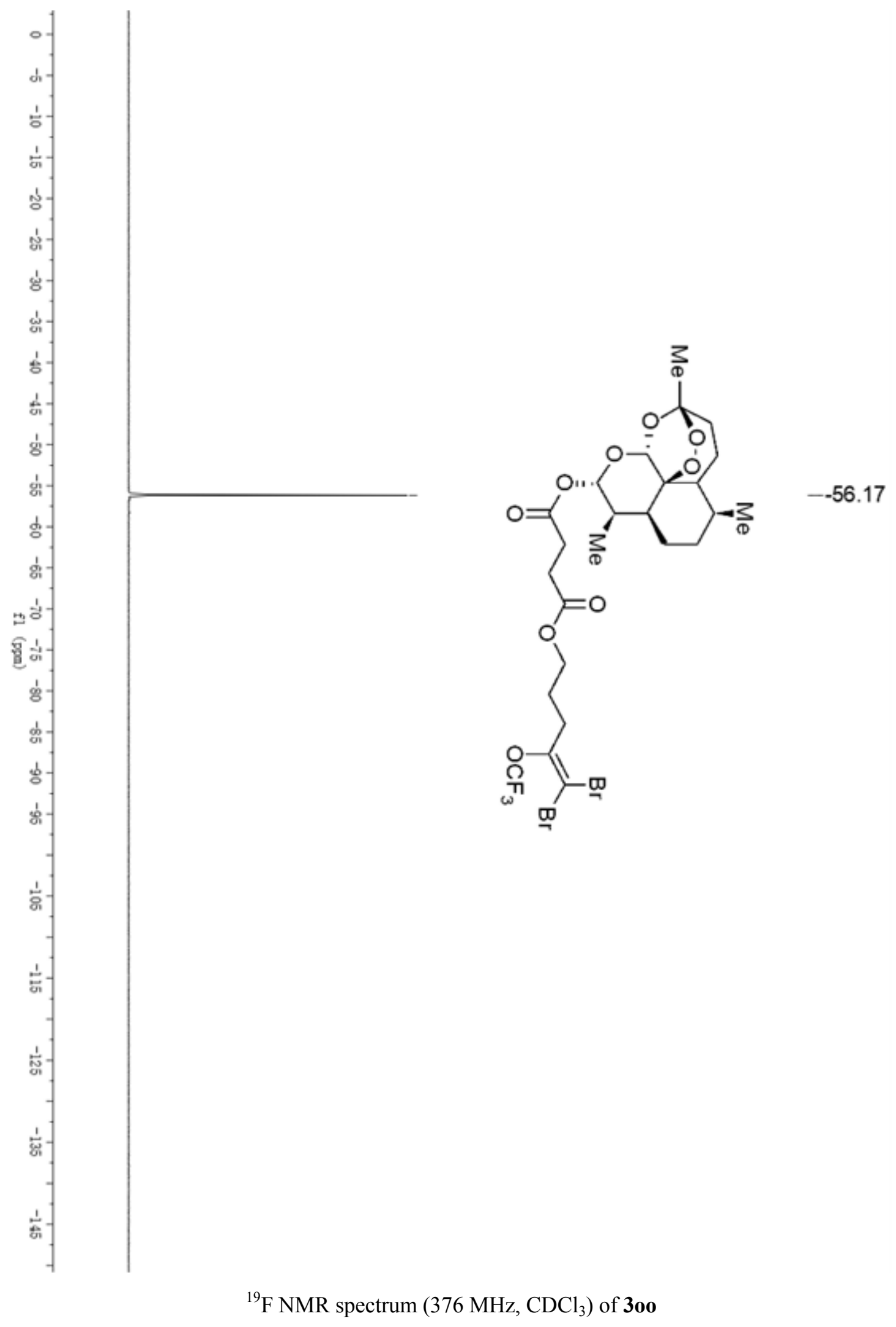




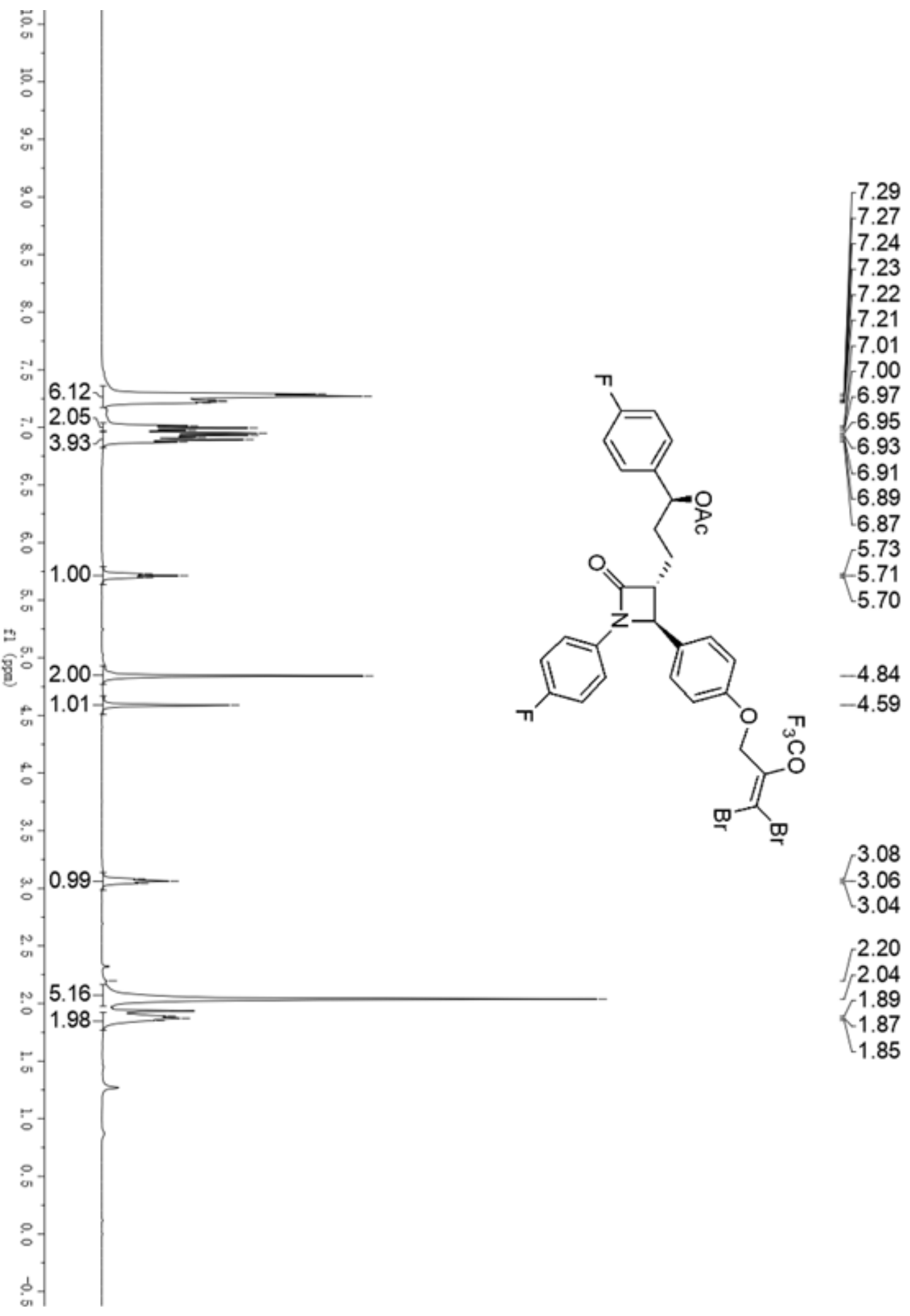

${ }^{1} \mathrm{H}$ NMR spectrum $\left(400 \mathrm{MHz}, \mathrm{CDCl}_{3}\right)$ of $\mathbf{3 p p}$ 


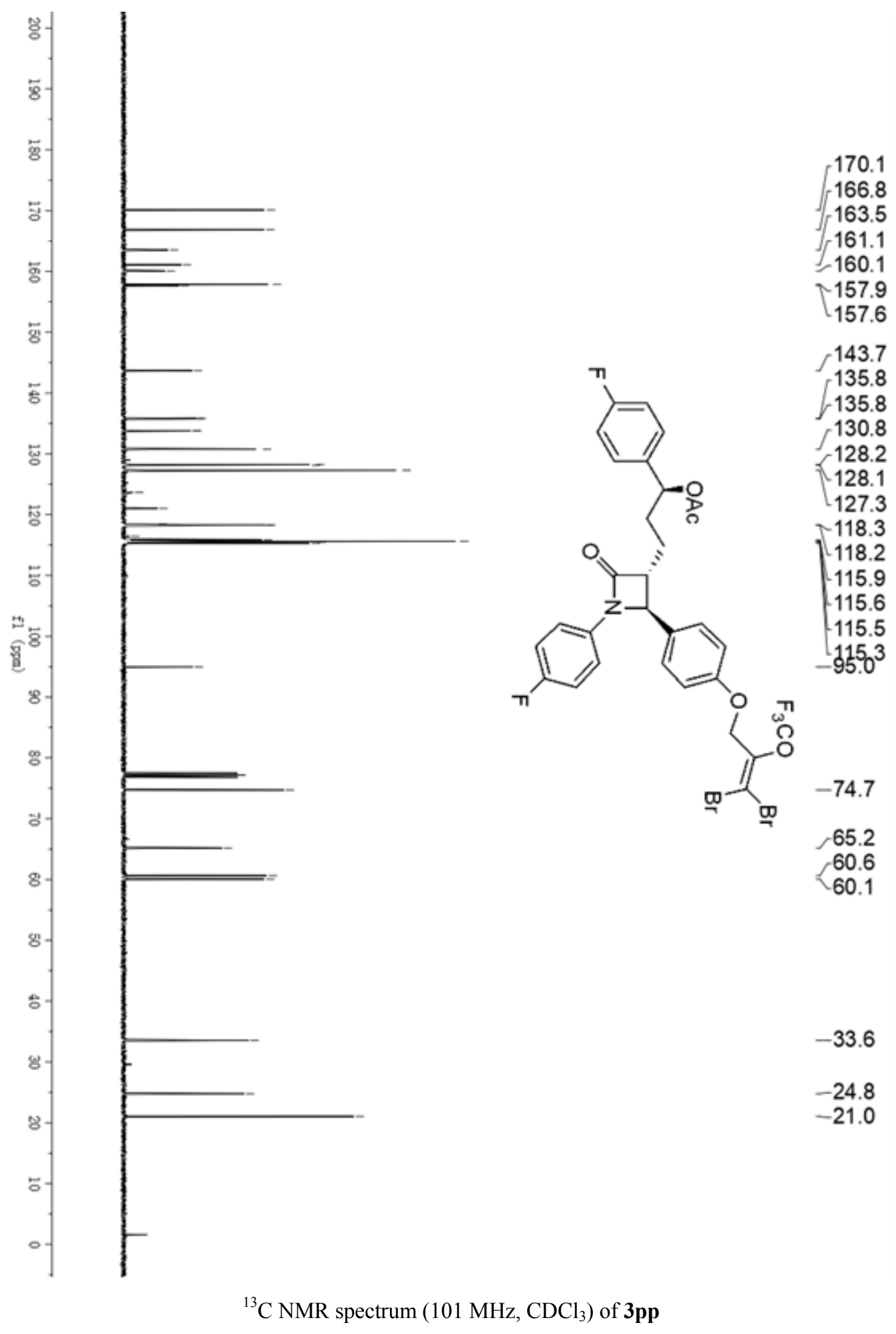




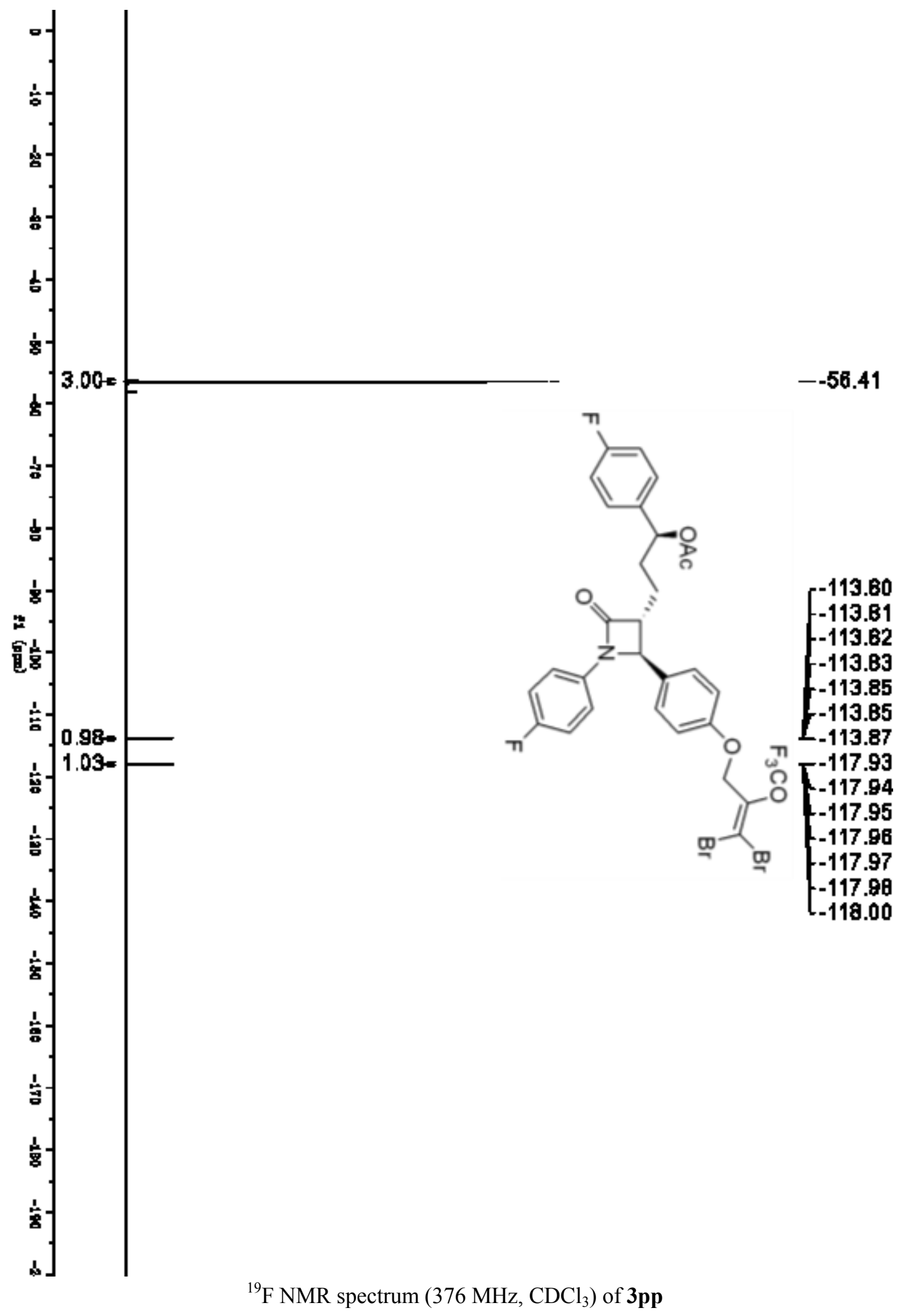




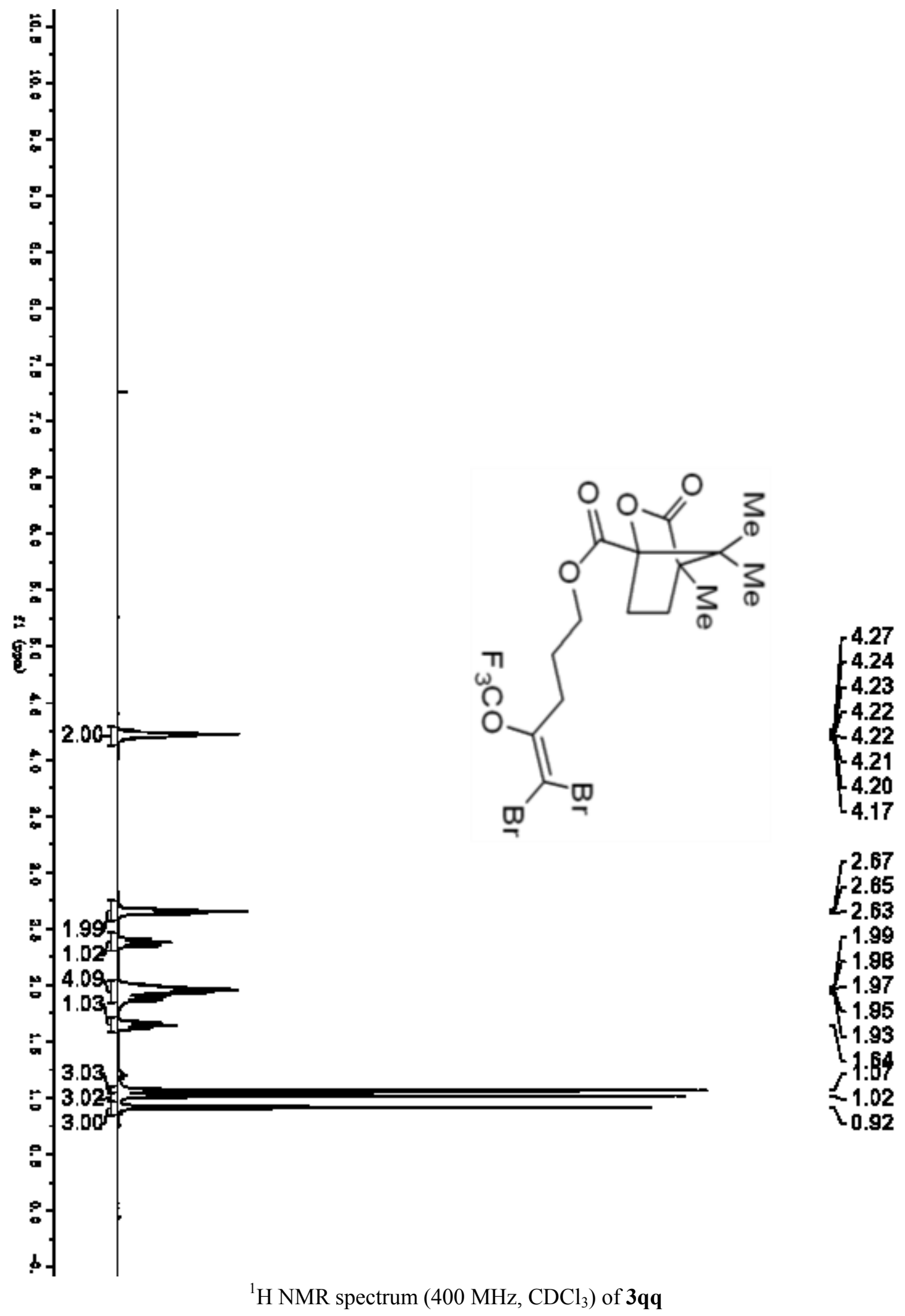




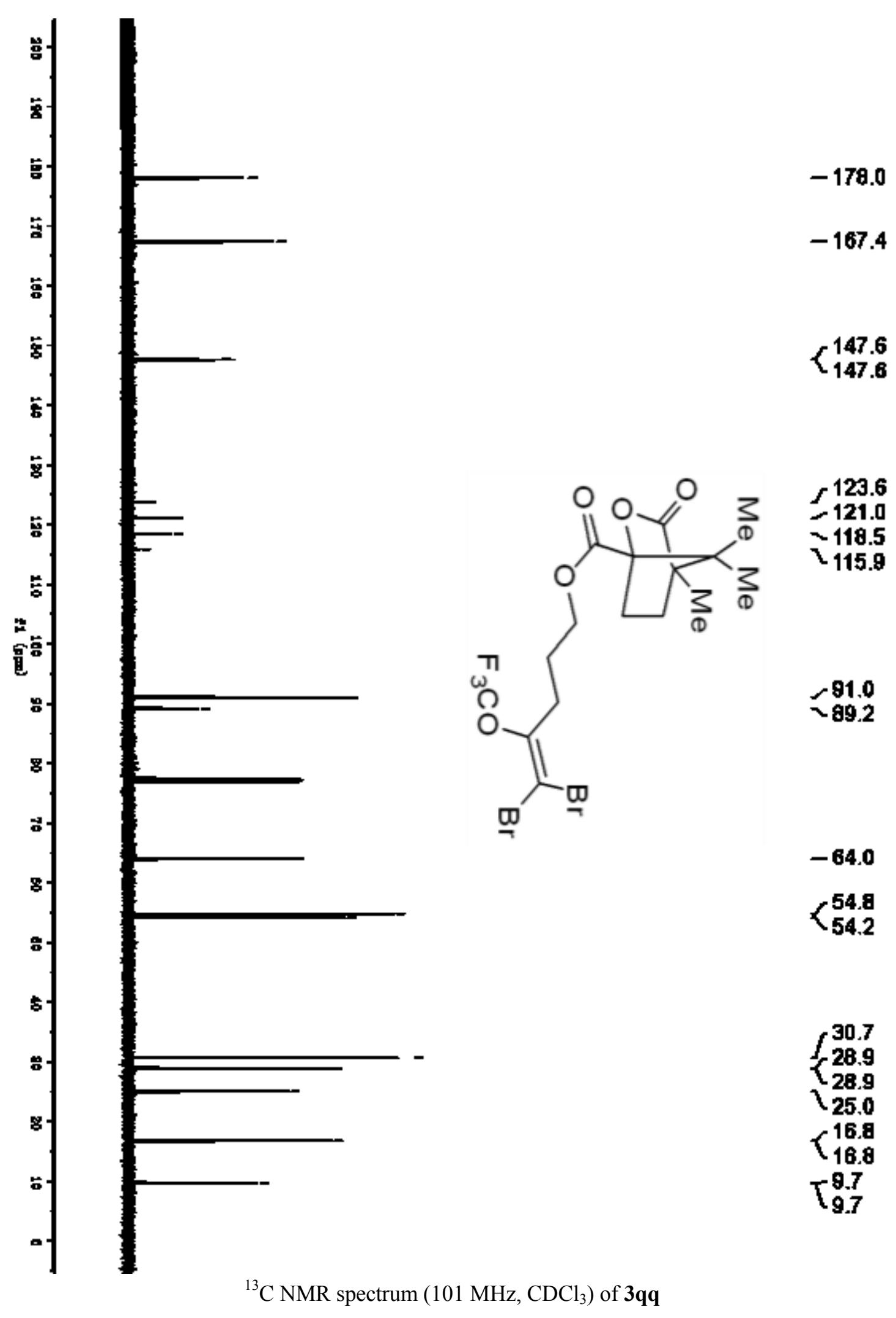




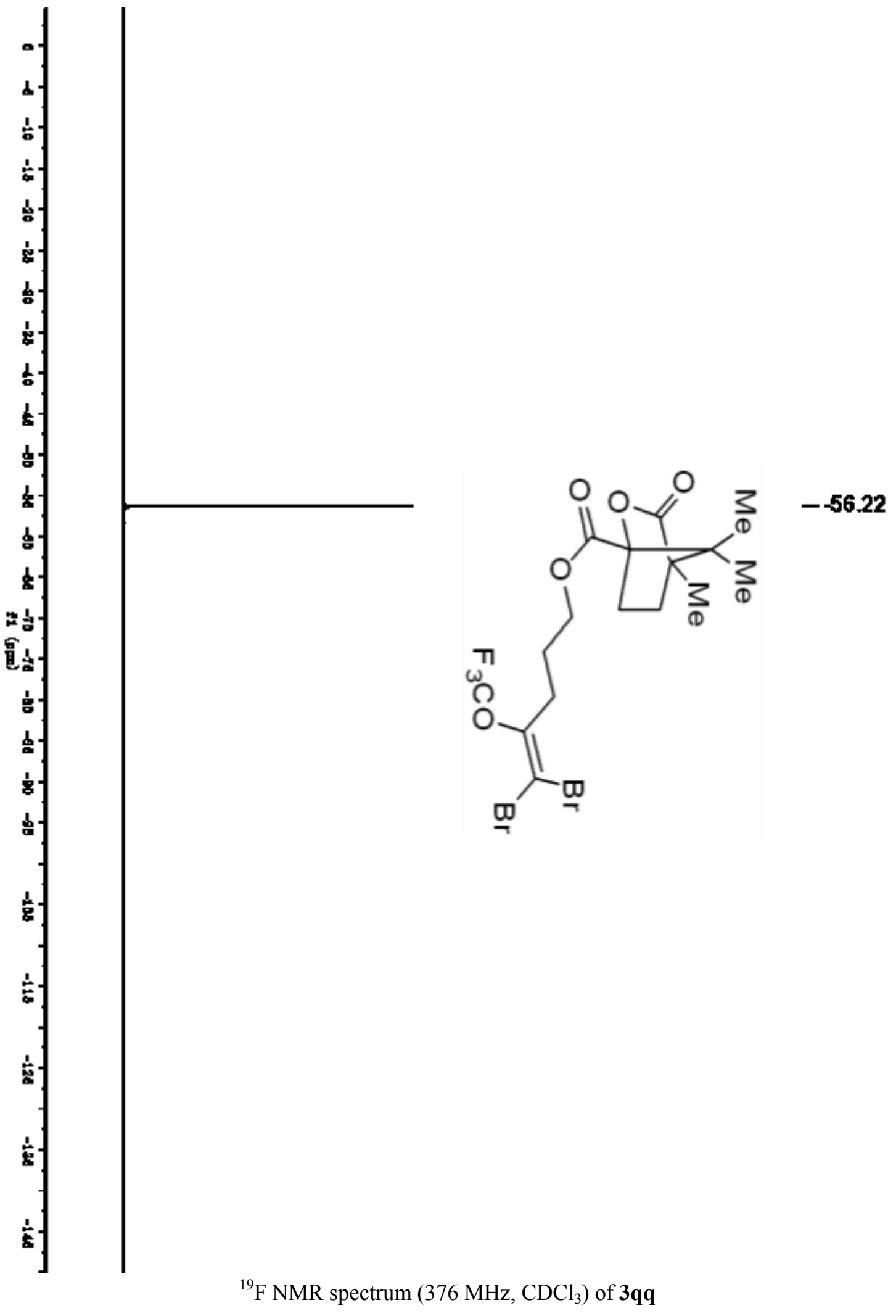




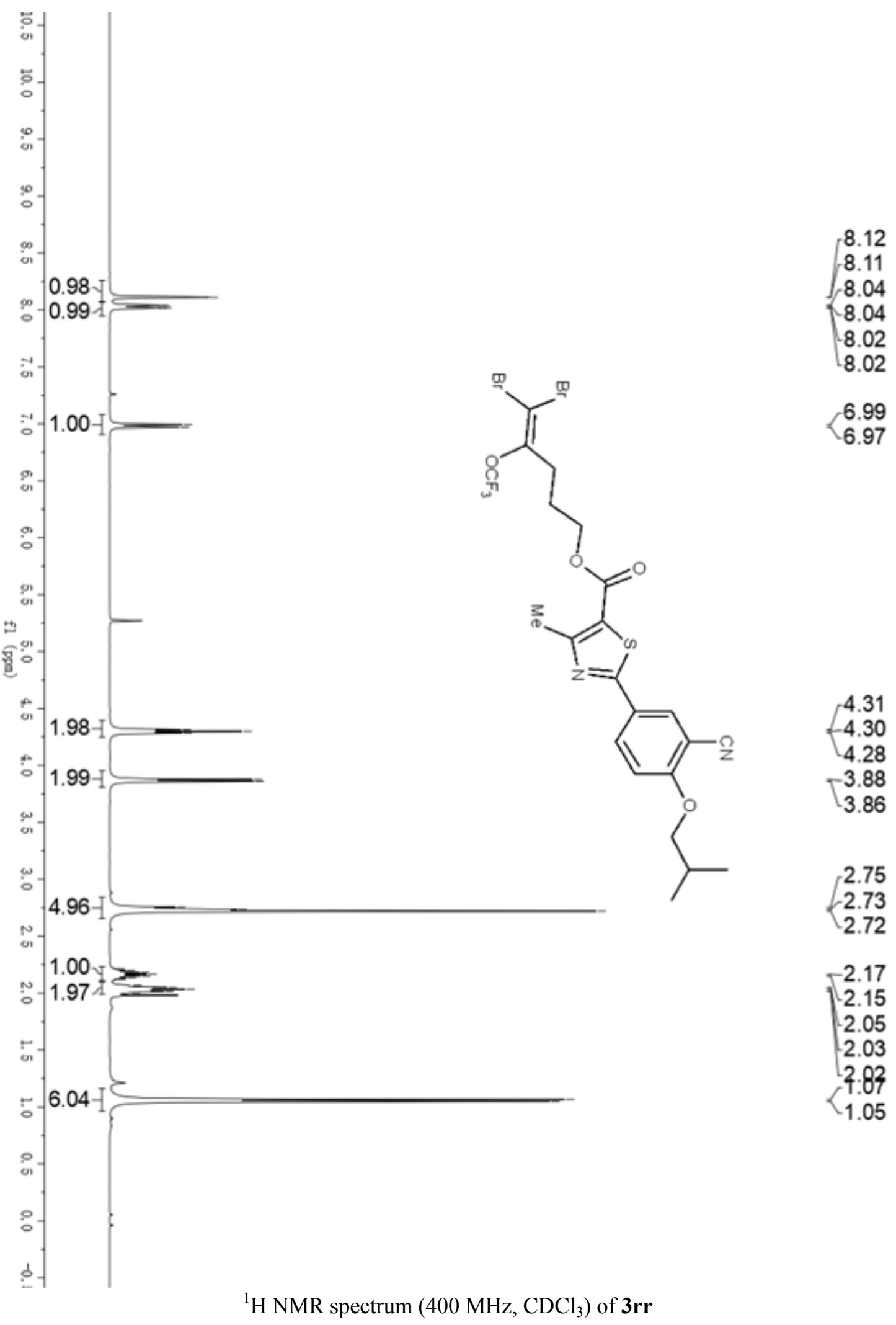




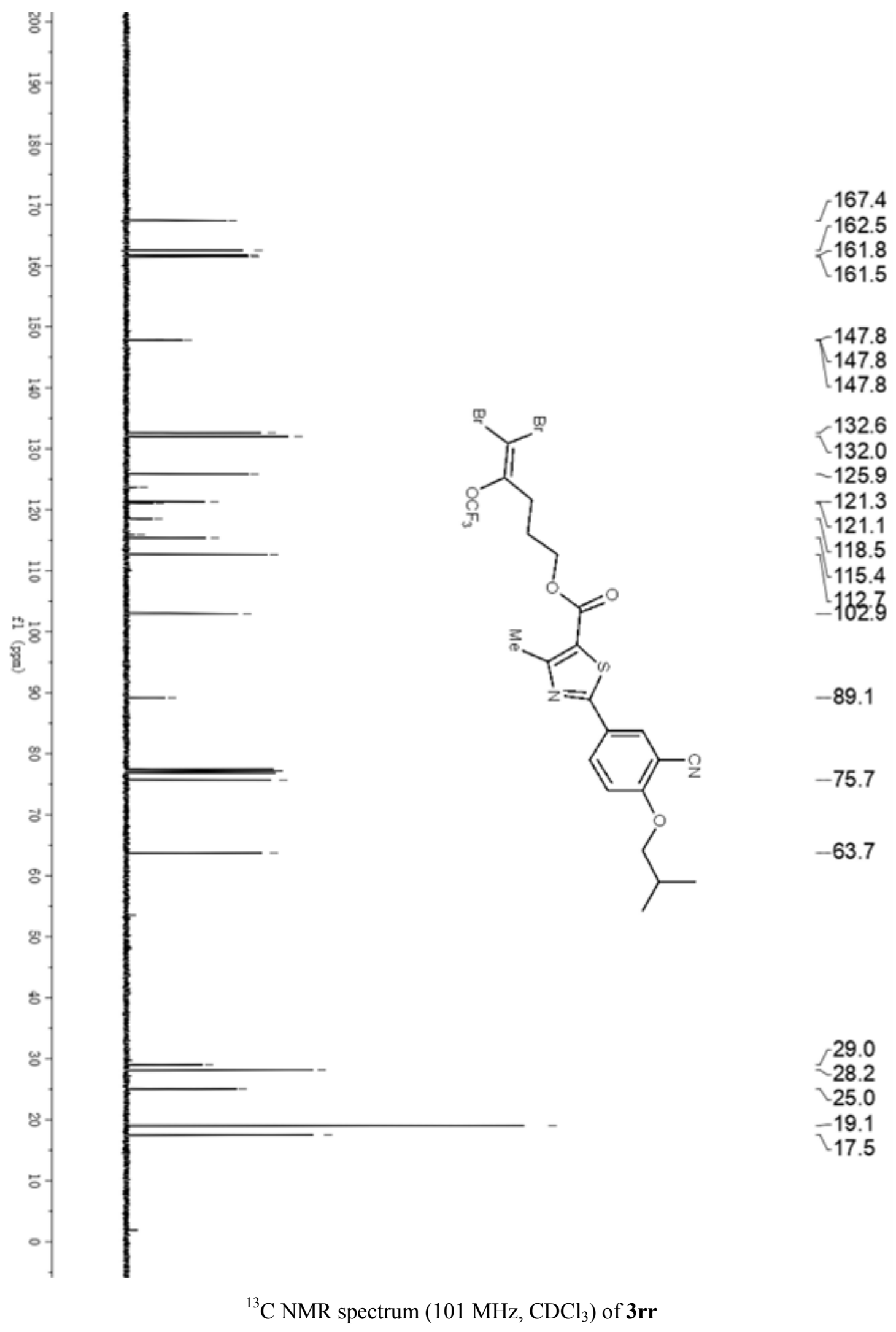




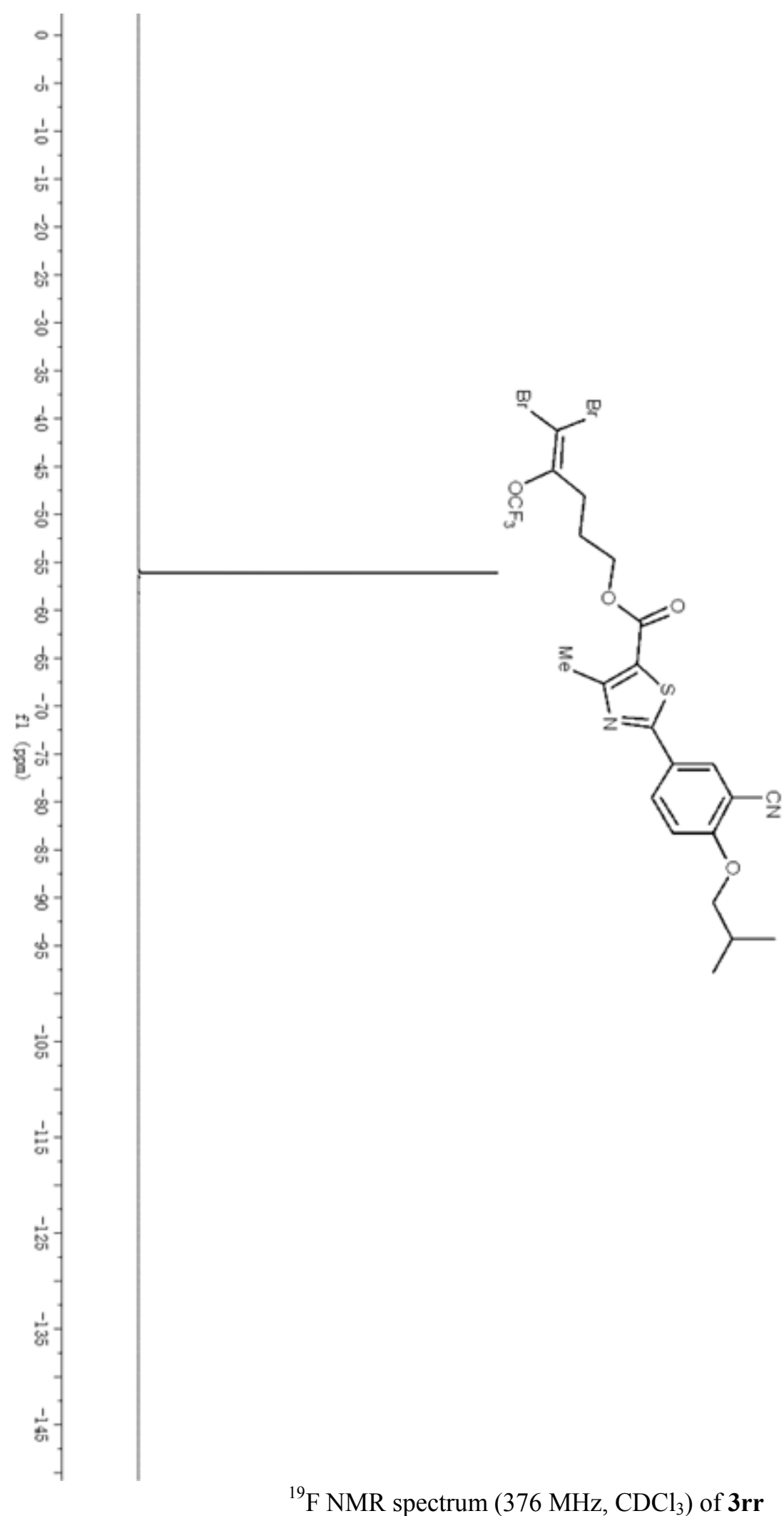

${ }^{19} \mathrm{~F} \mathrm{NMR} \mathrm{spectrum}\left(376 \mathrm{MHz}, \mathrm{CDCl}_{3}\right)$ of $3 \mathbf{r r}$ 


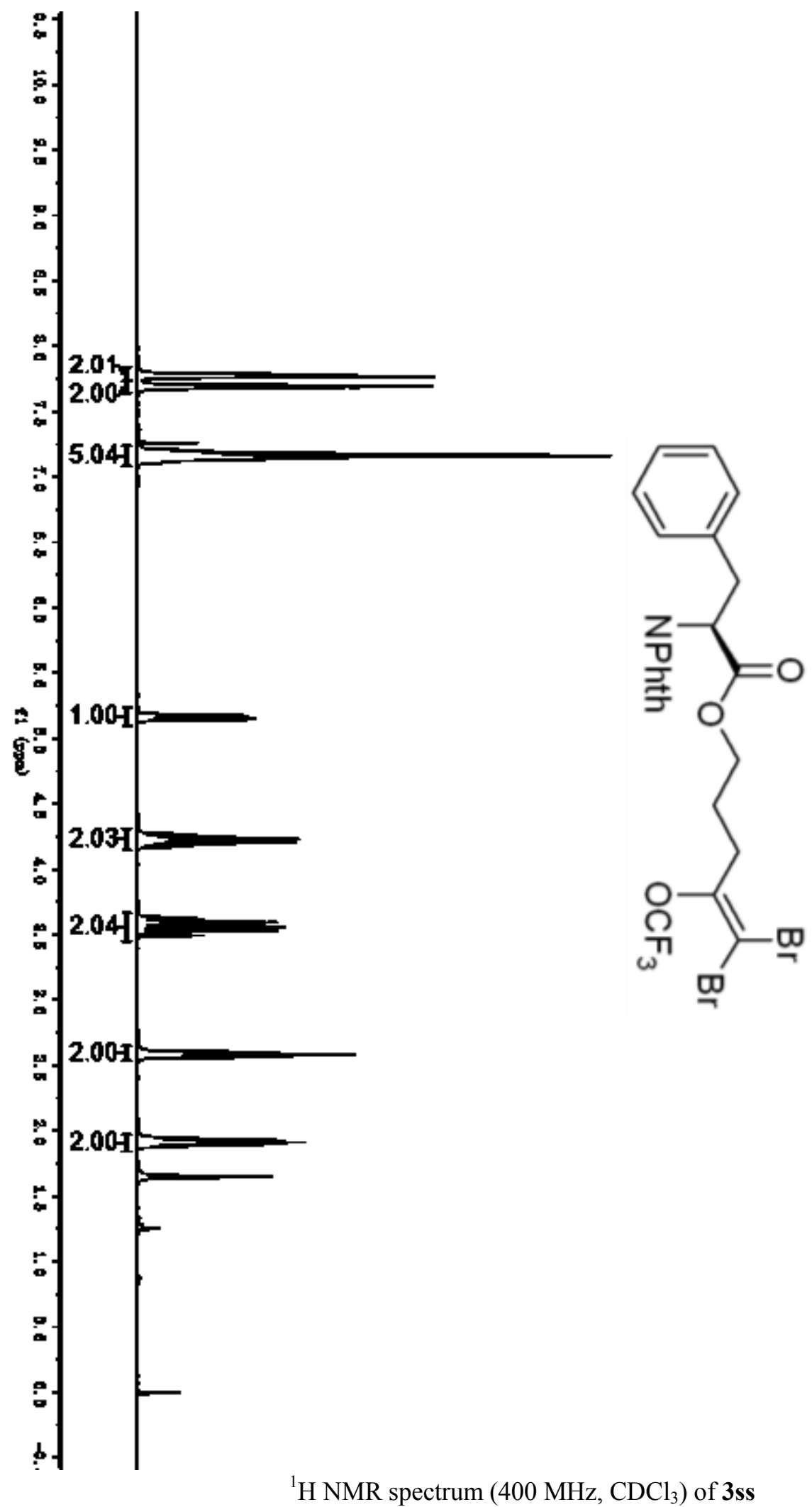

7.79
7.78

7.77

$-7.76$

7.75

$-7.71$

7.70

7.69

7.69

7.68

7.20

7.19

7.18

$-7.17$

7.16

7.14

7.13

7.12

5.18

5.17

$-5.15$

15.14

4.23

$-4.22$

4.21

$-3.59$

3.58

$+3.56$

2.53

$-2.58$

2.56

1.95
-1.93

1.91

$+1.89$

${ }^{1} \mathrm{H}$ NMR spectrum $\left(400 \mathrm{MHz}, \mathrm{CDCl}_{3}\right)$ of $\mathbf{3 s s}$ 


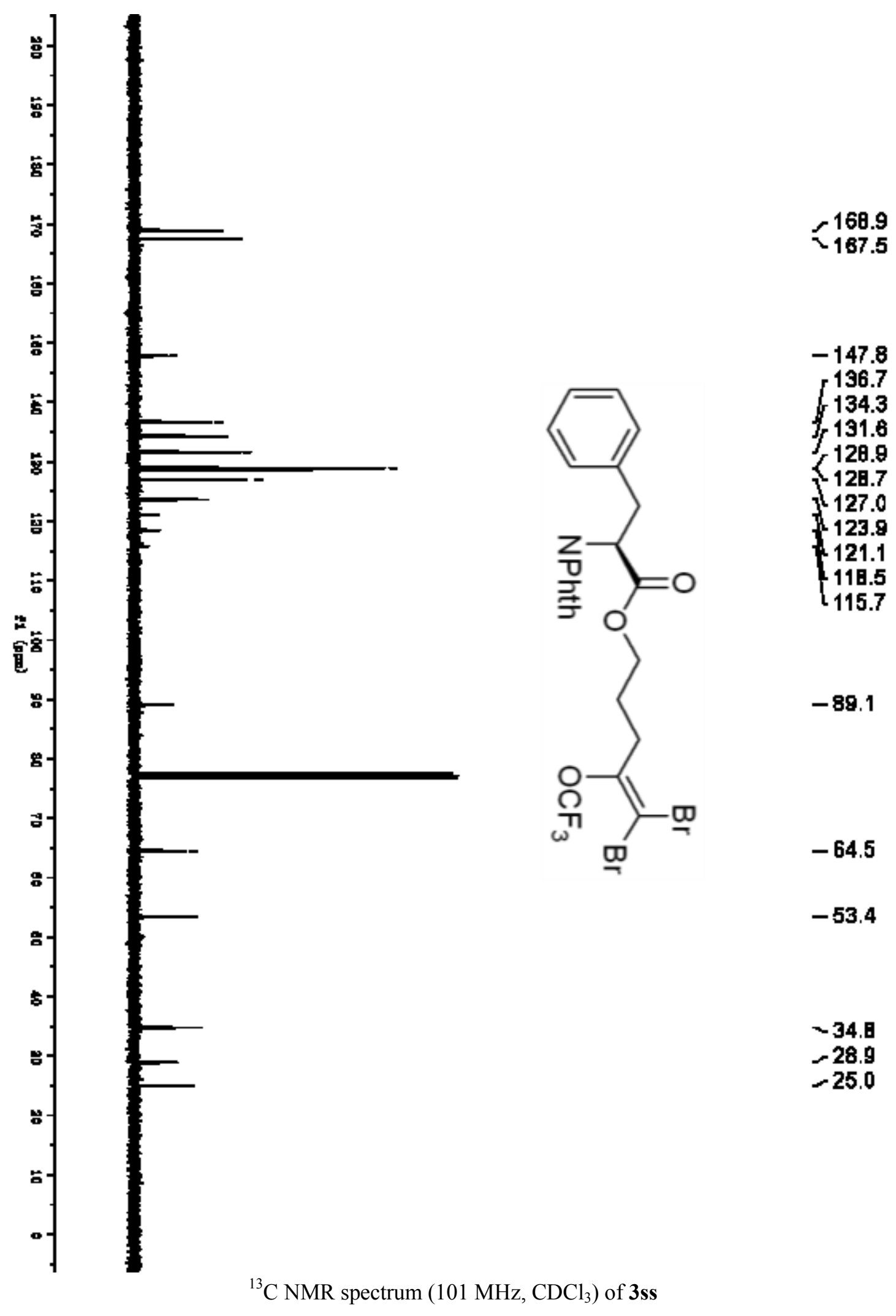




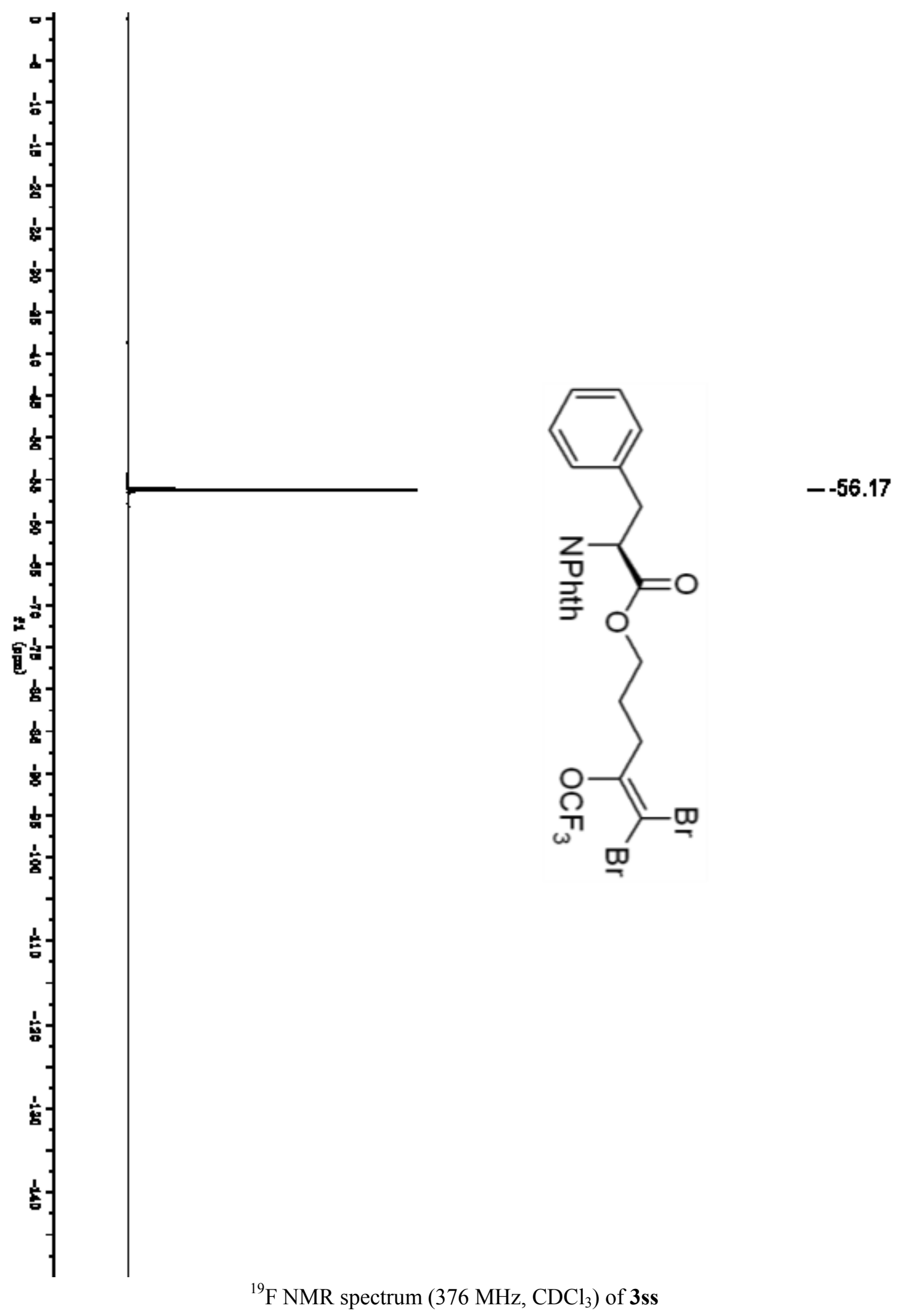



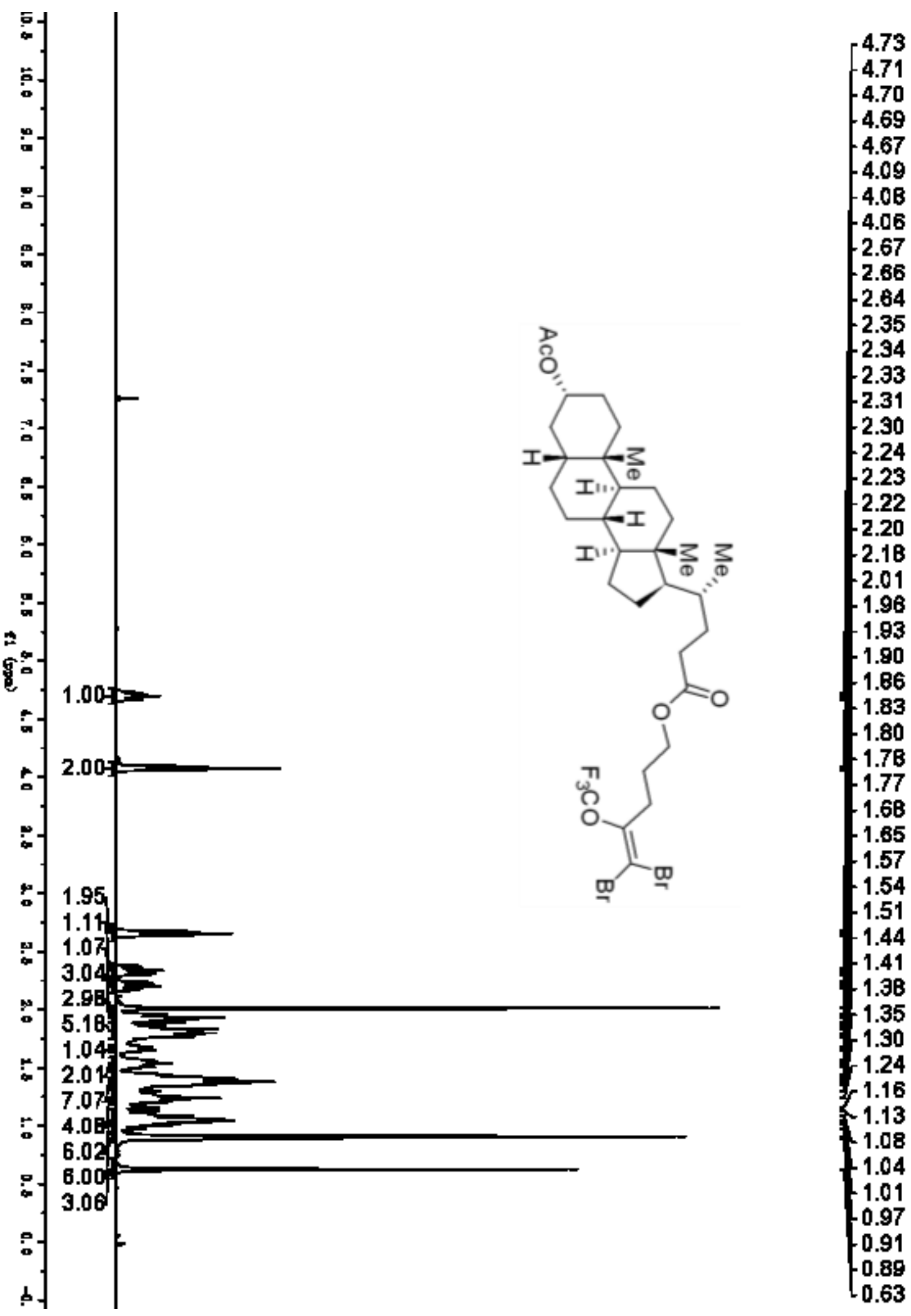

${ }^{1} \mathrm{H}$ NMR spectrum $\left(400 \mathrm{MHz}, \mathrm{CDCl}_{3}\right)$ of $\mathbf{3 t t}$ 


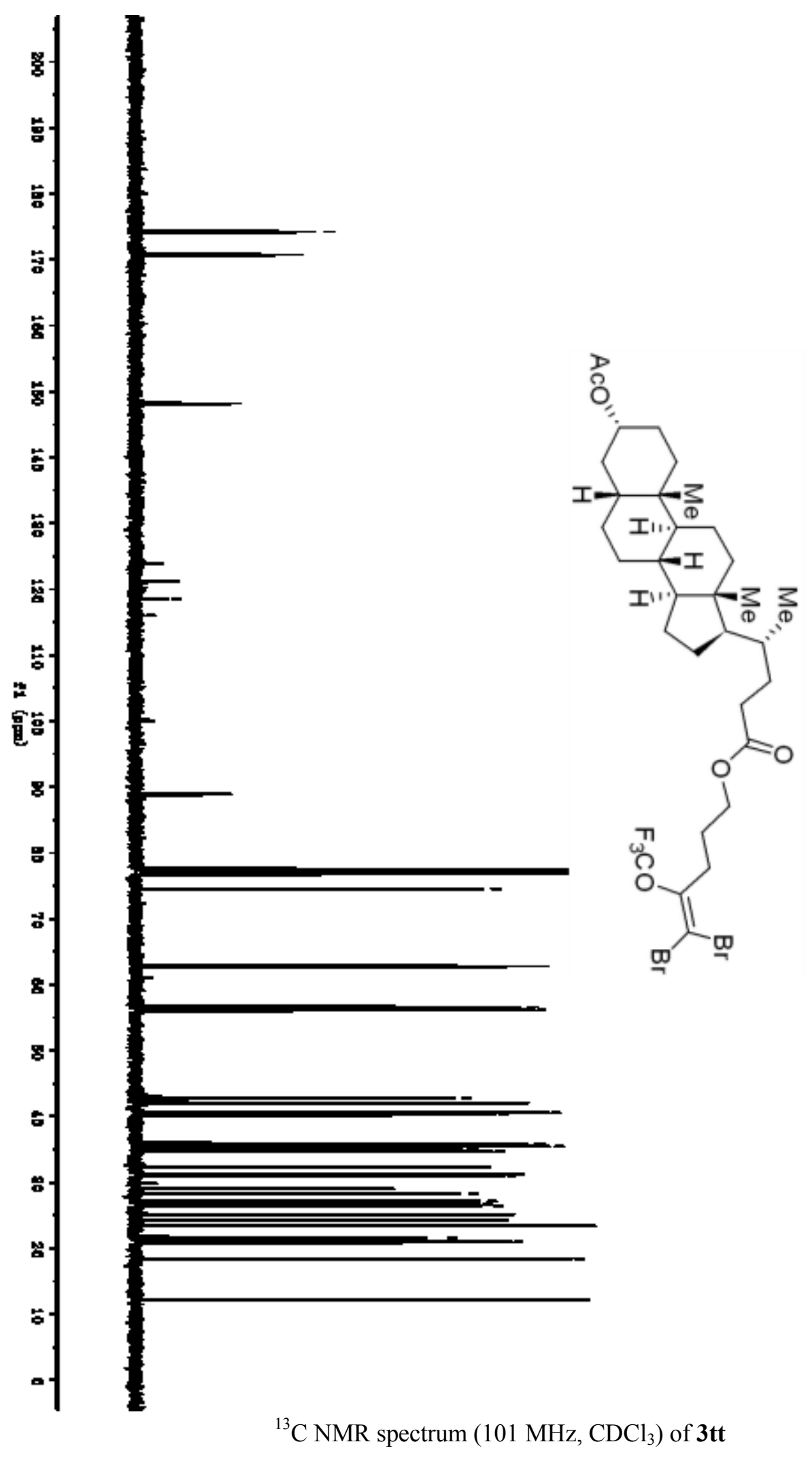

-174.2
-170.7

$-148.2$

$\leftarrow 123.8$

$-121.2$

$=118.6$

1116.0

$-88.9$

$-74.5$

$-62.8$

56.6
56.1

42.0
40.5
40.3
35.9
35.5
31.3
31.1
25.1
24.3
23.4
21.0
18.4
12. 


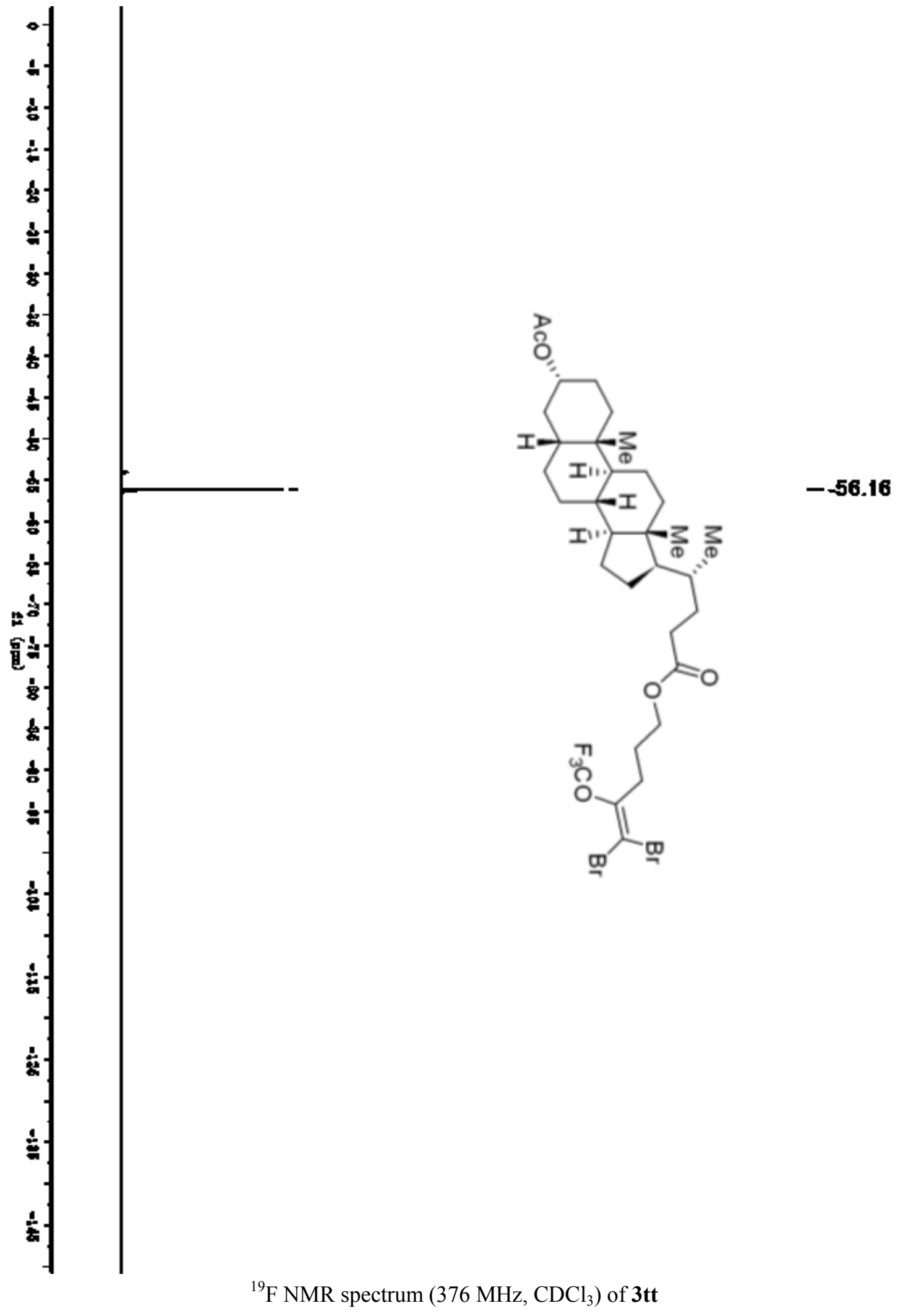




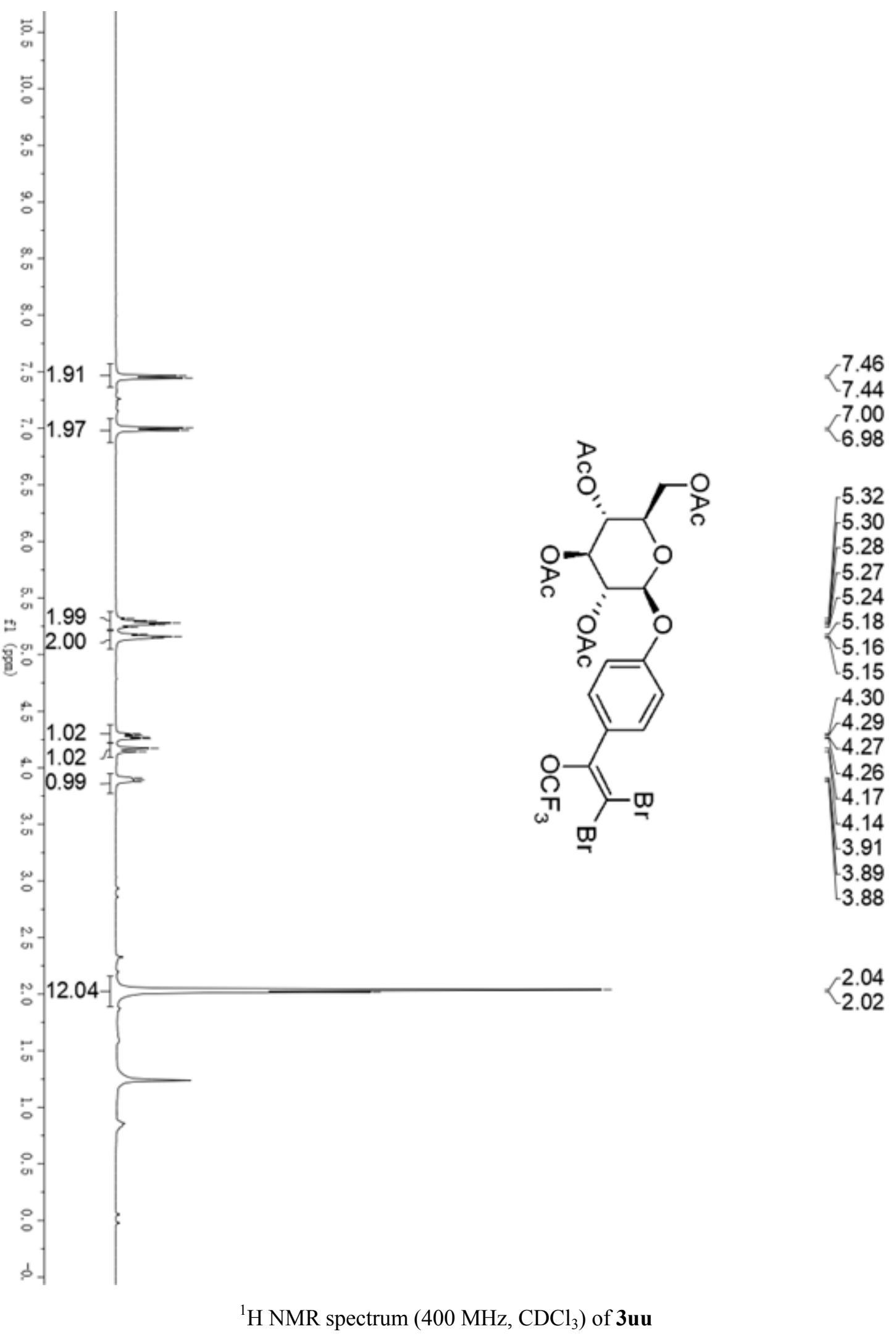




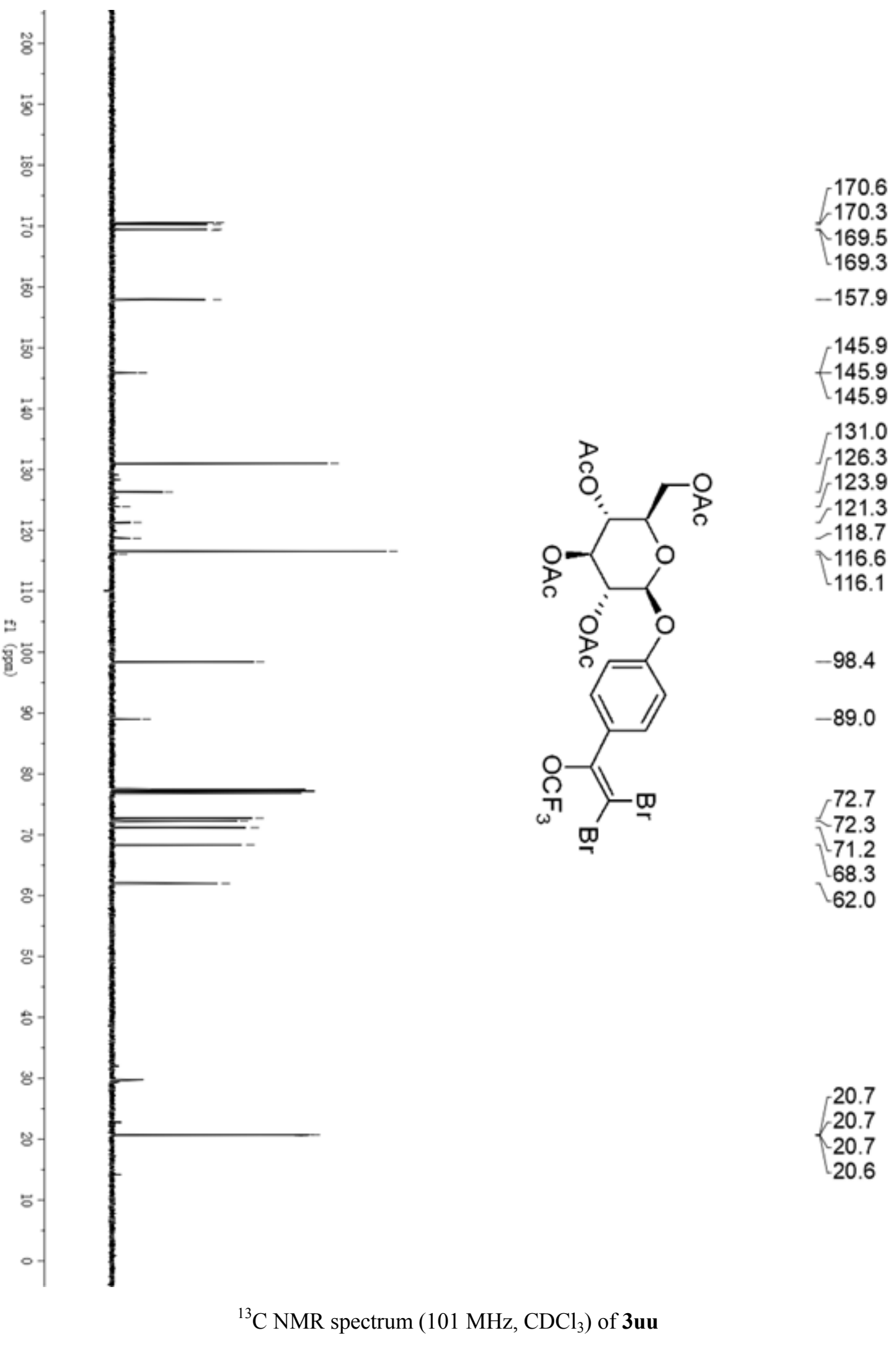




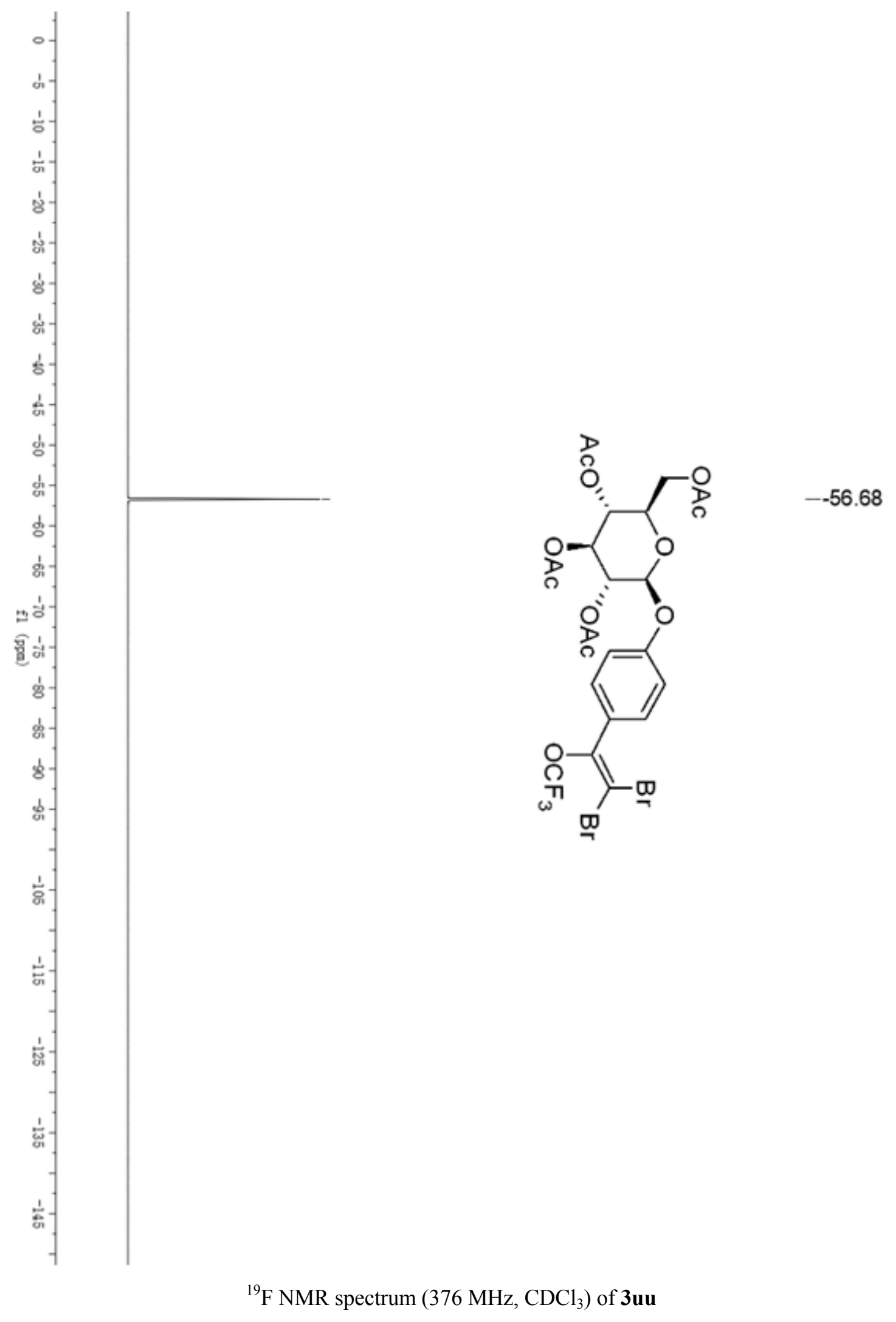




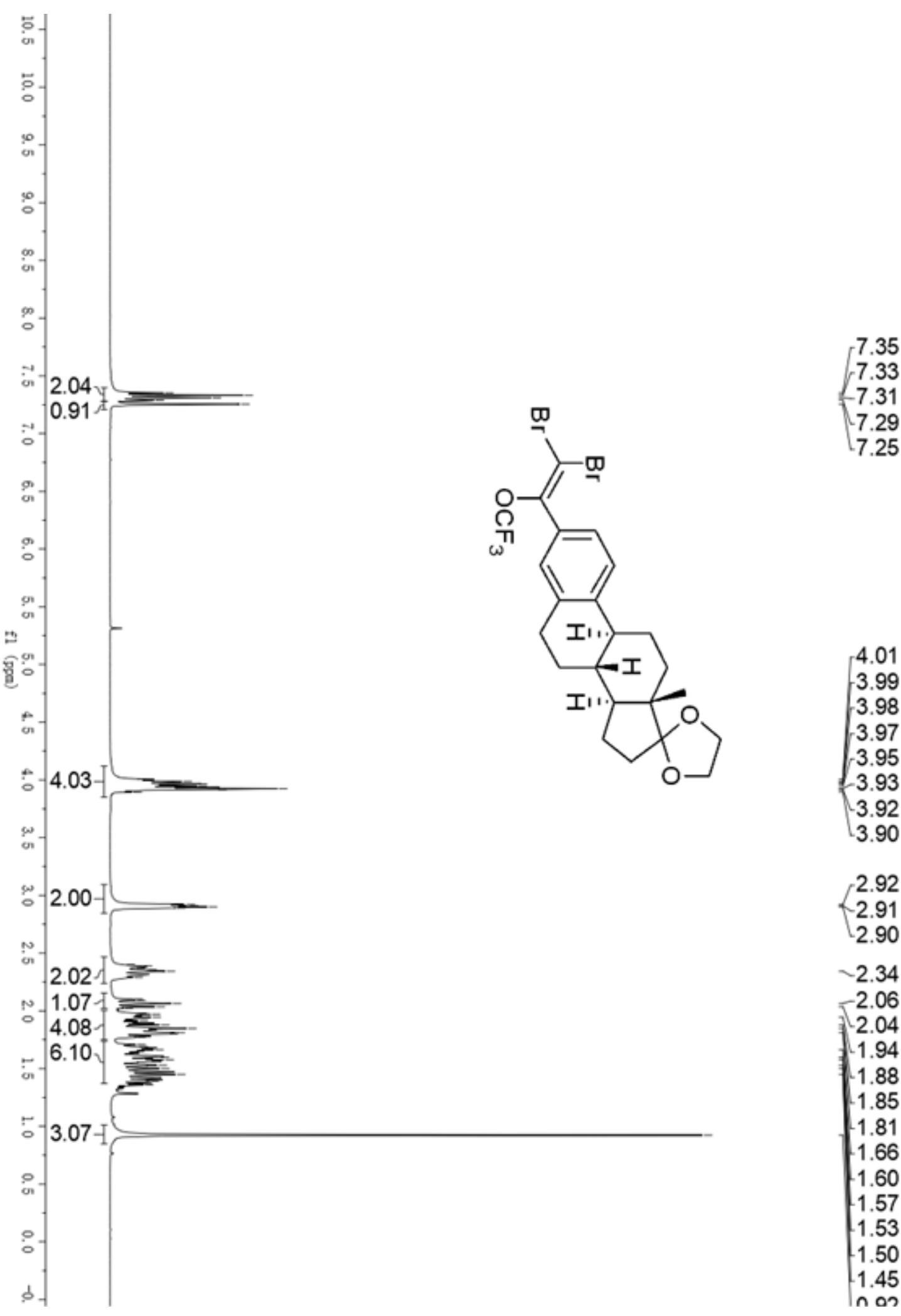

${ }^{1} \mathrm{H}$ NMR spectrum $\left(400 \mathrm{MHz}, \mathrm{CDCl}_{3}\right.$ ) of $\mathbf{3 v v}$ 


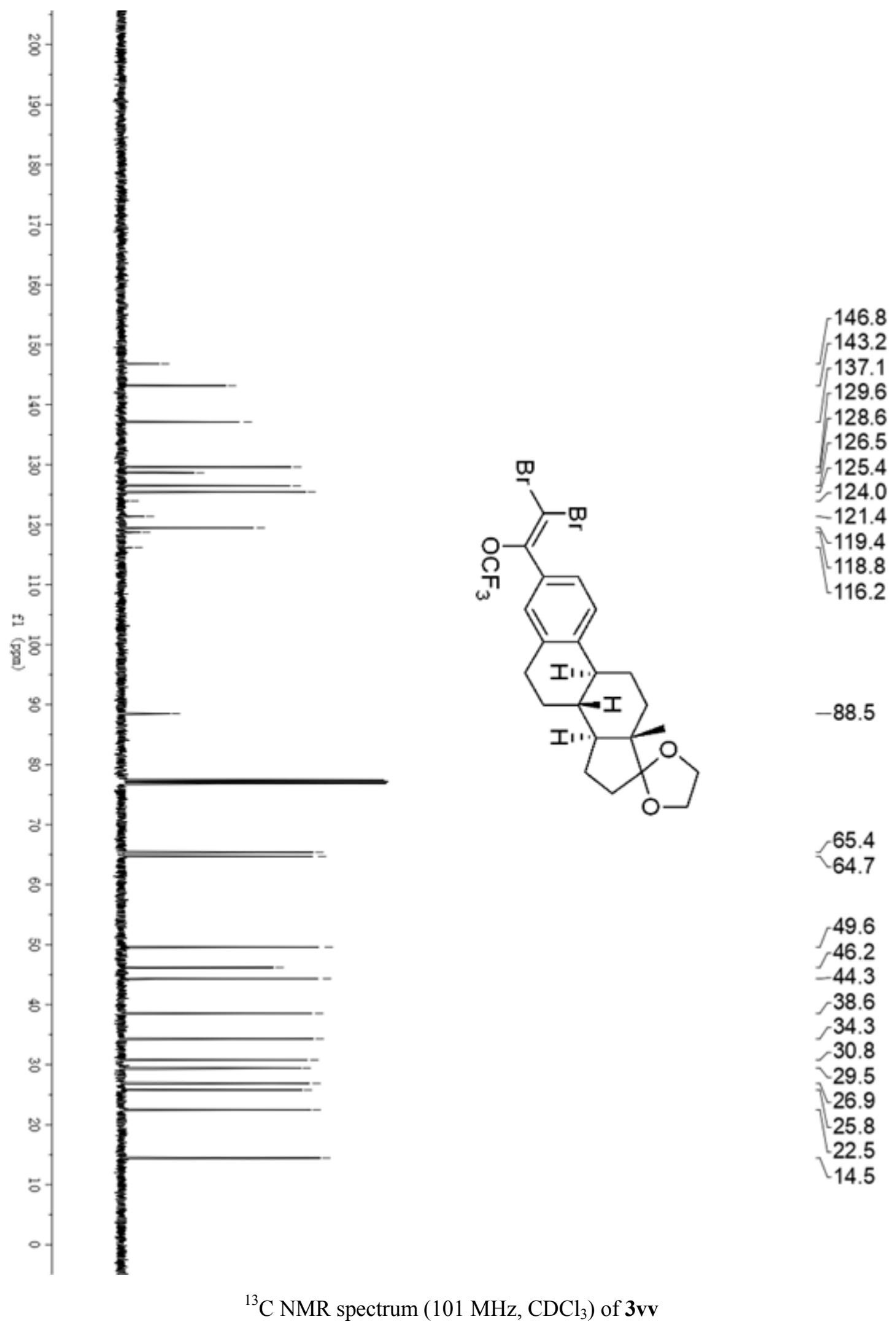




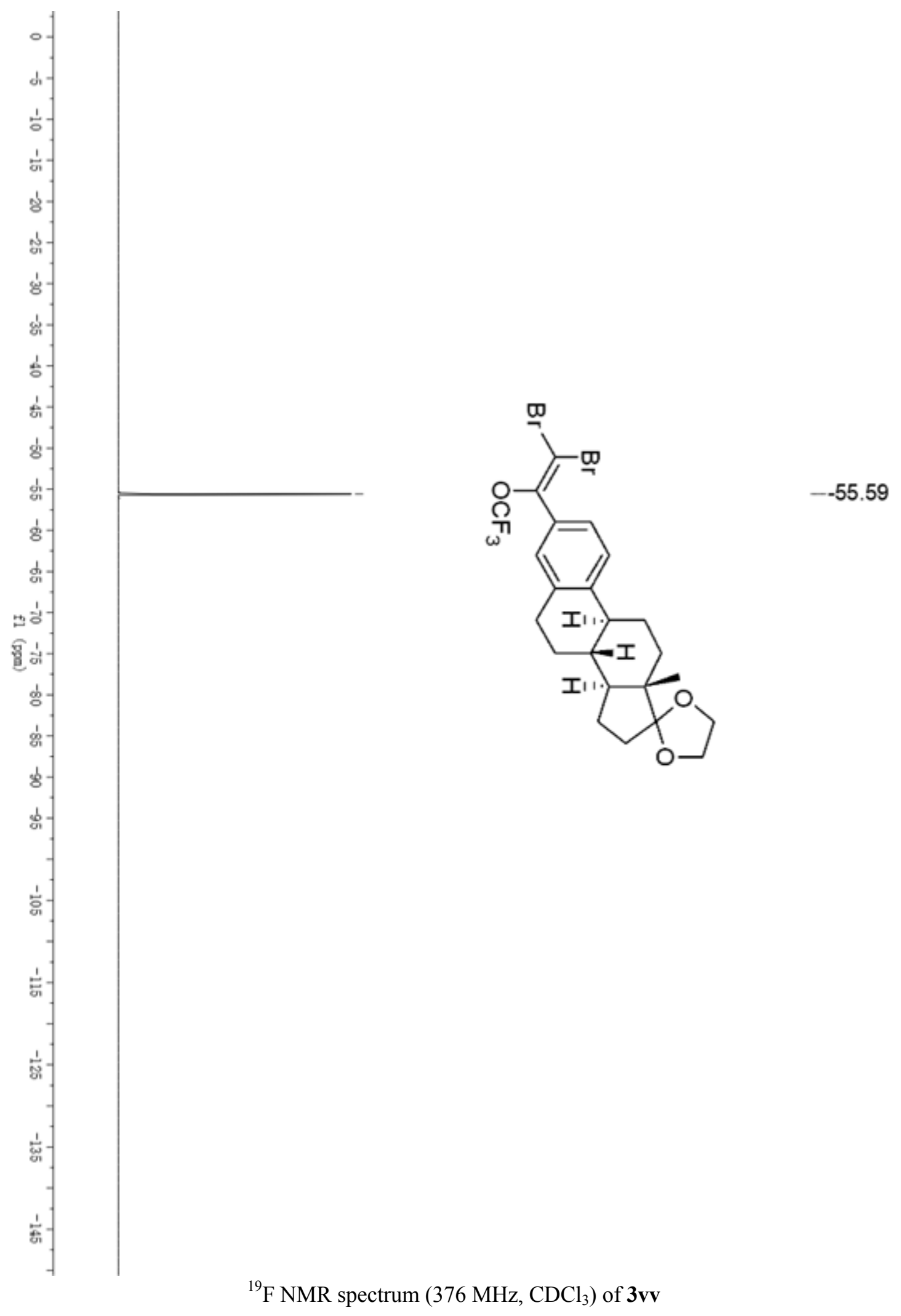




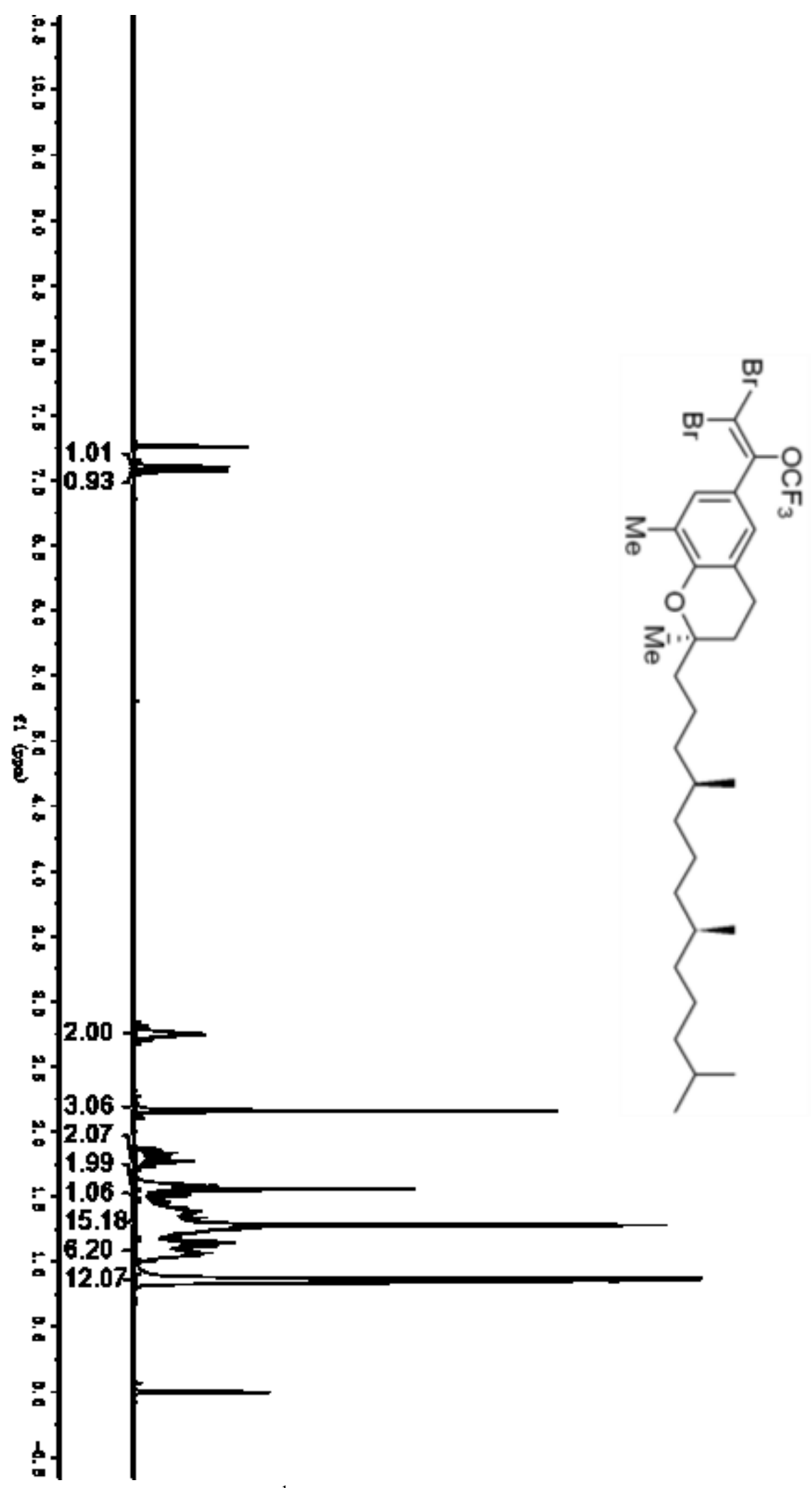

$<\begin{aligned} & 7.10 \\ & 7.07\end{aligned}$

$-2.70$

2.69

$\mathrm{l}_{2.16}$

1.58

1.28

$-1.26$

1.16
-1.14

$-1.12$

$-1.07$

$-0.87$

$-0.87$

0.86

0.85

${ }^{1} \mathrm{H}$ NMR spectrum $\left(400 \mathrm{MHz}, \mathrm{CDCl}_{3}\right)$ of $\mathbf{3 w w}$ 


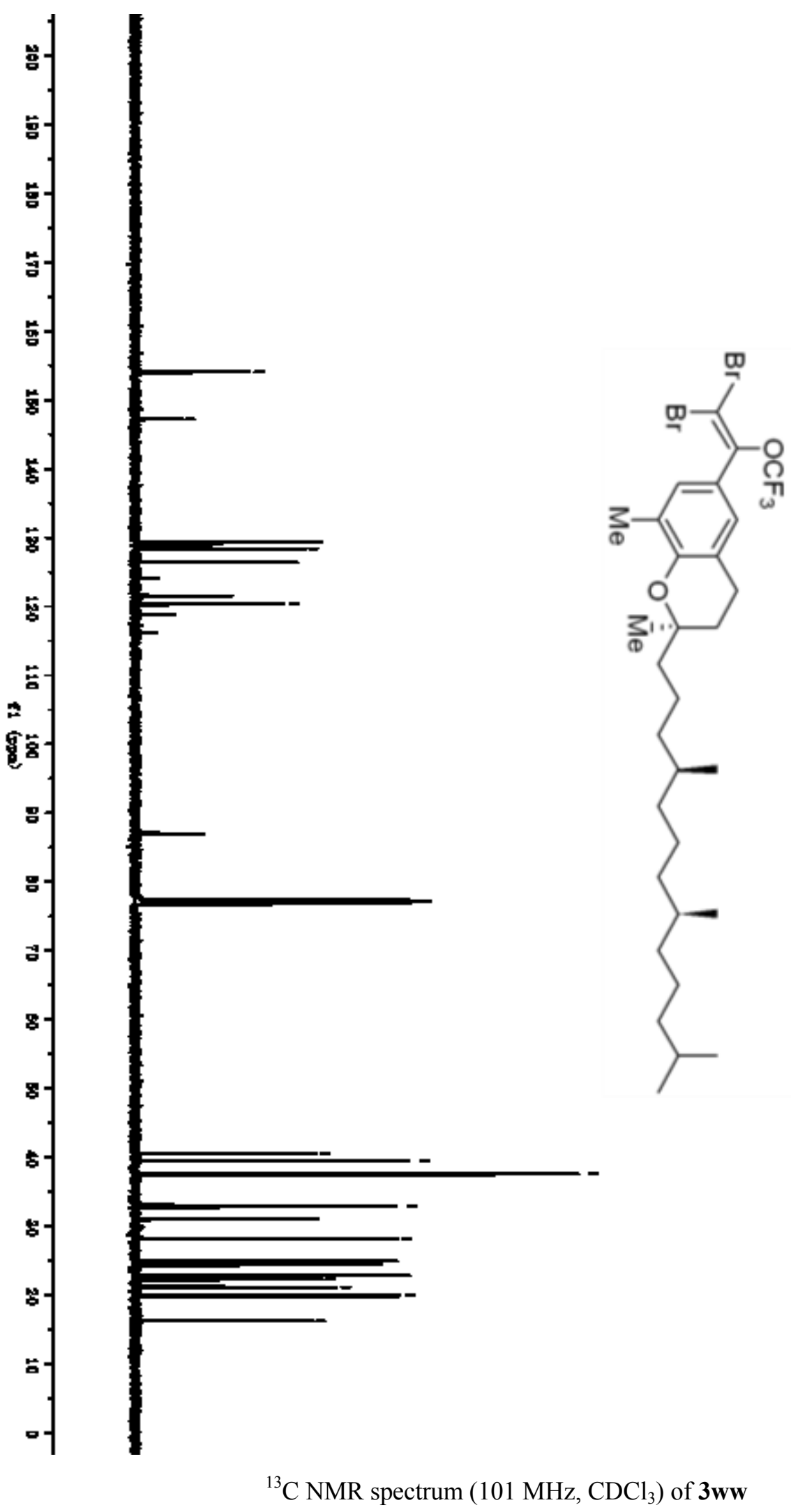

$-154.1$

$-147.2$

129.3

$-128.4$

126.4

124.0

121.5

121.4

120.3

118.8

118.2

$-87.0$

$-77.1$

40.6

39.5

37.6

37.6

37.5

33.0

32.8

28.2

25.0

24.6

124.4

22.9

22.8

22.3

21.1

19.9

19.8

${ }^{13} \mathrm{C}$ NMR spectrum $\left(101 \mathrm{MHz}, \mathrm{CDCl}_{3}\right)$ of $\mathbf{3 w w}$ 


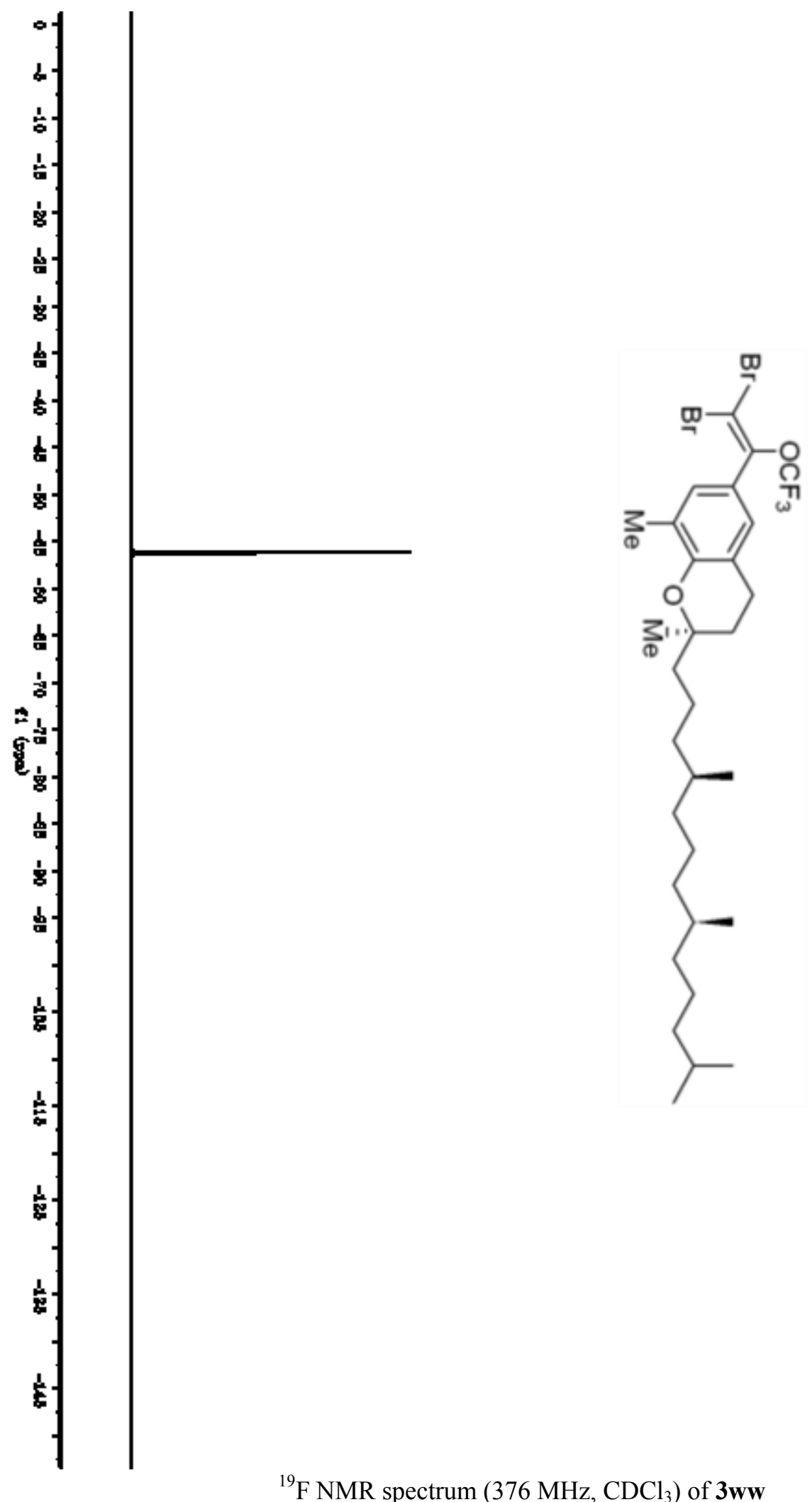

$--56.17$

${ }^{19} \mathrm{~F}$ NMR spectrum $\left(376 \mathrm{MHz}, \mathrm{CDCl}_{3}\right)$ of $\mathbf{3 w w}$ 


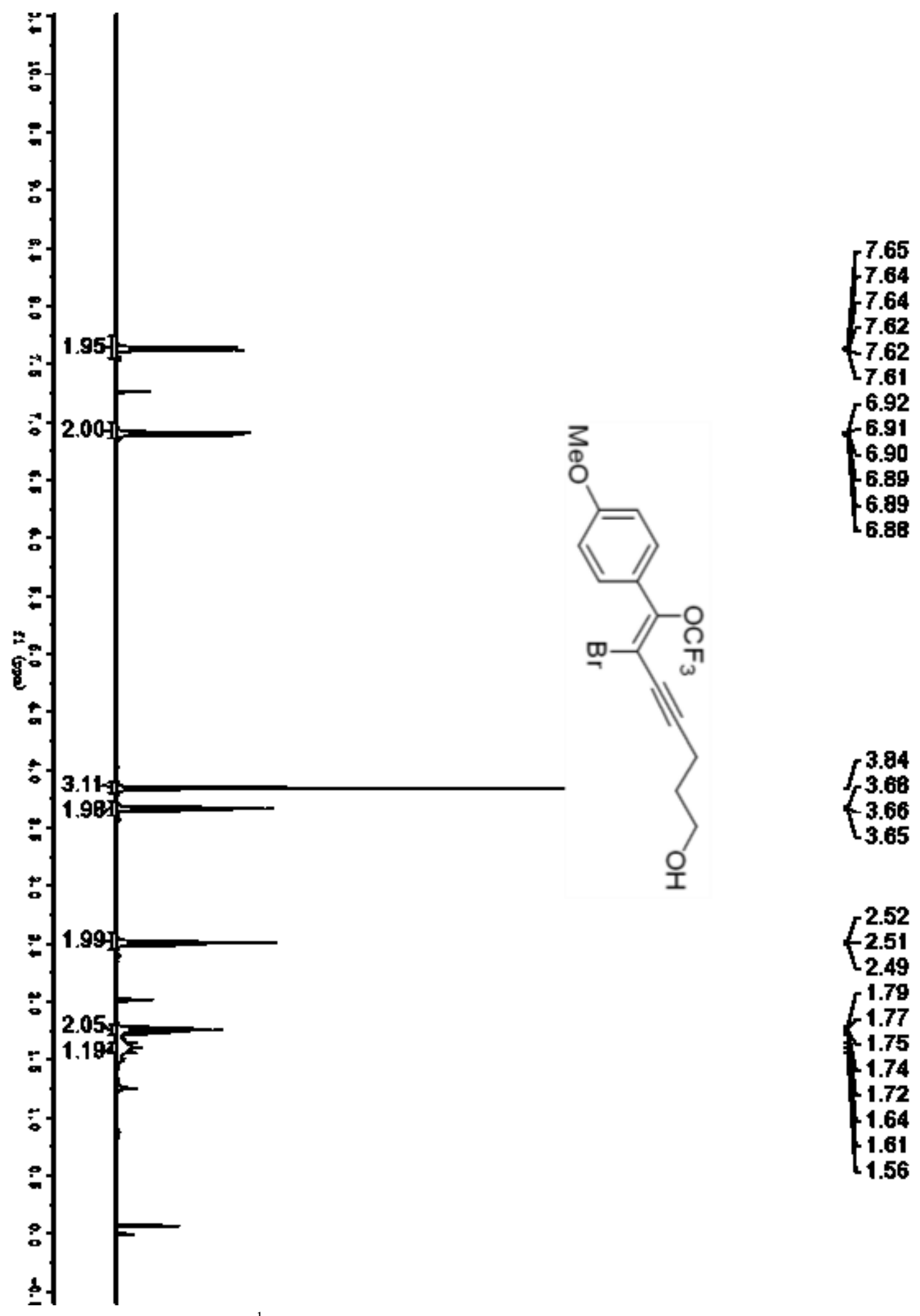

${ }^{1} \mathrm{H}$ NMR spectrum (400 MHz, $\mathrm{CDCl}_{3}$ ) of 4ee 


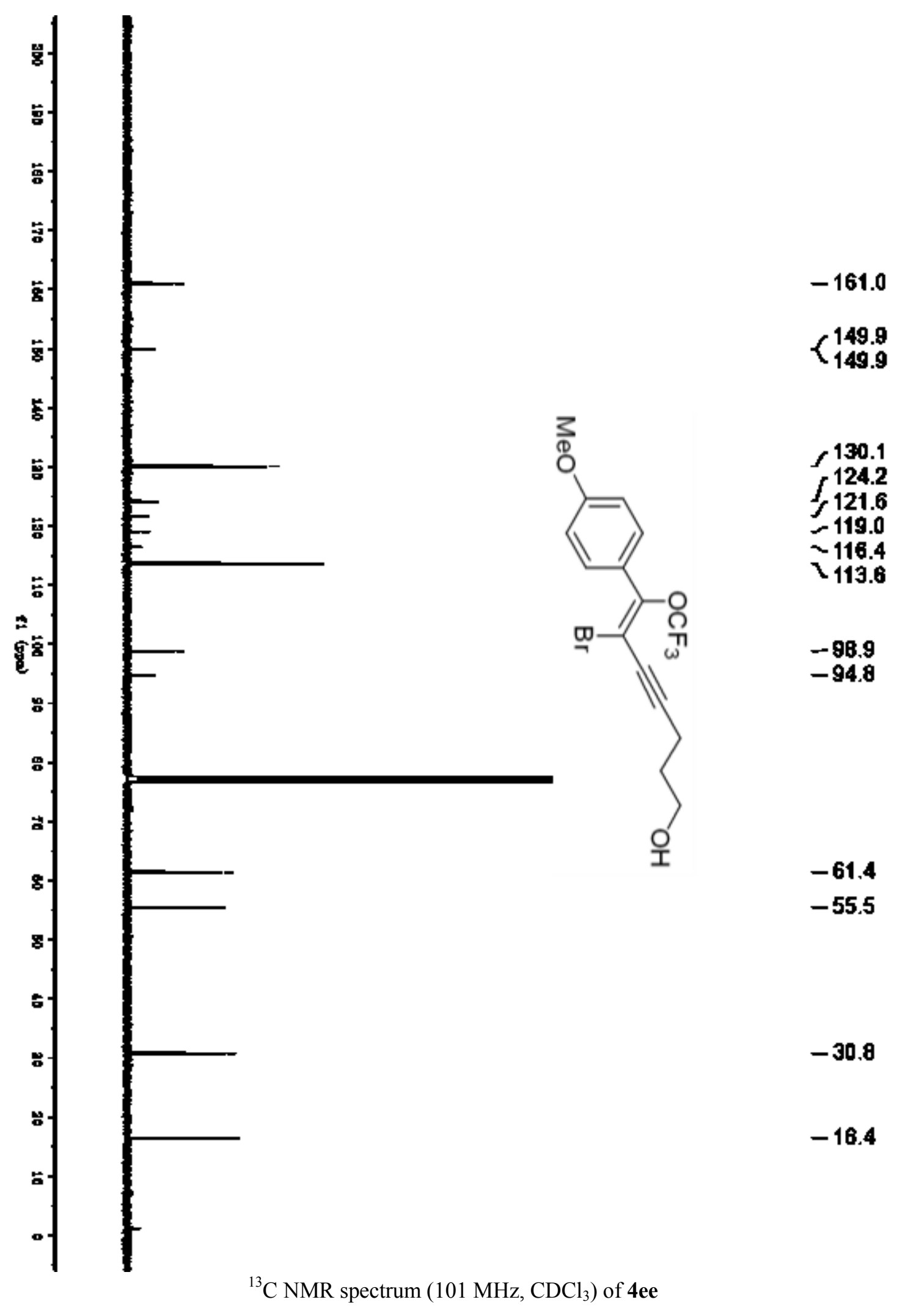




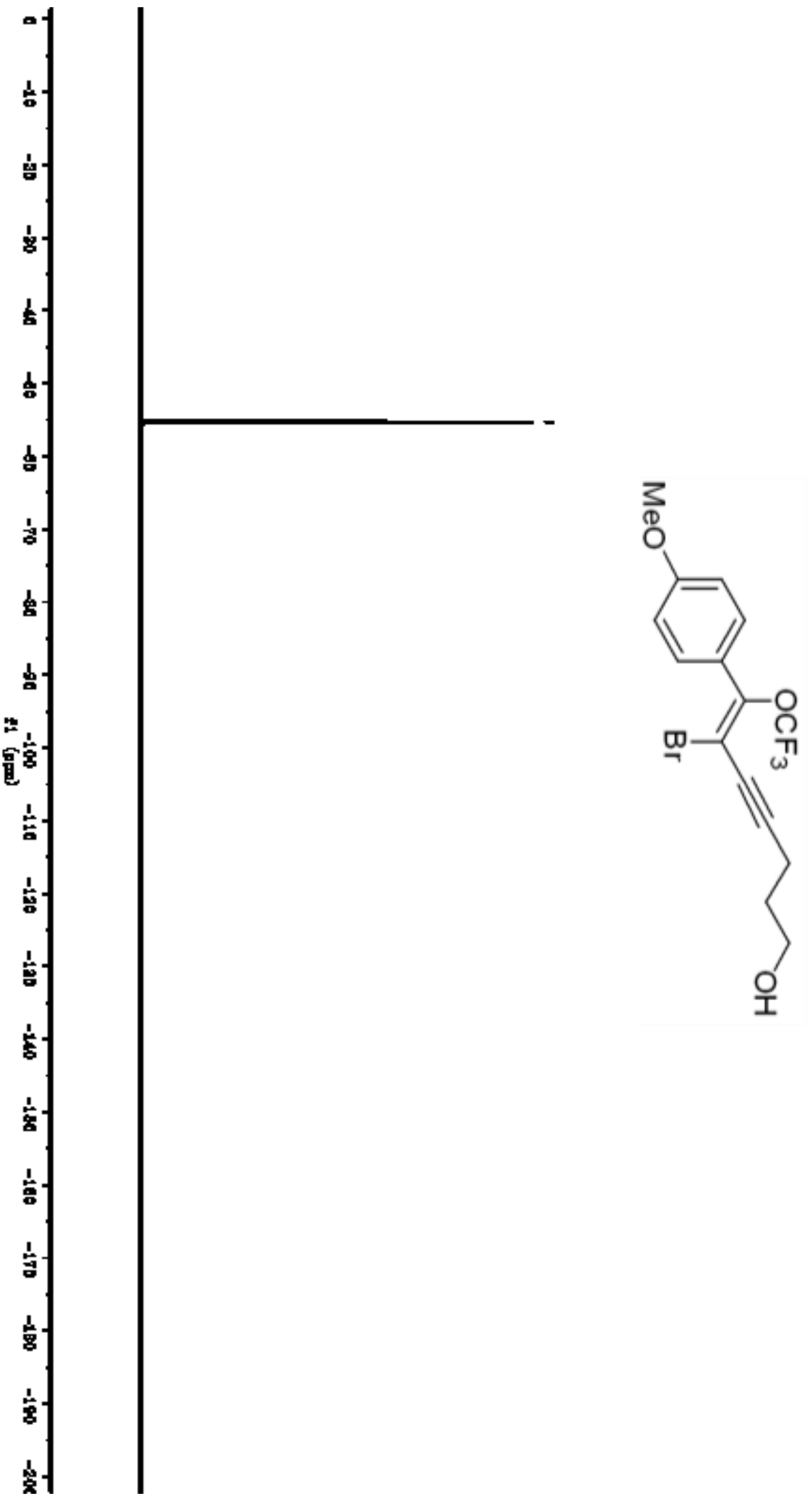

$--55.27$

${ }^{19} \mathrm{~F}$ NMR spectrum $\left(376 \mathrm{MHz}, \mathrm{CDCl}_{3}\right)$ of $4 \mathbf{e e}$ 


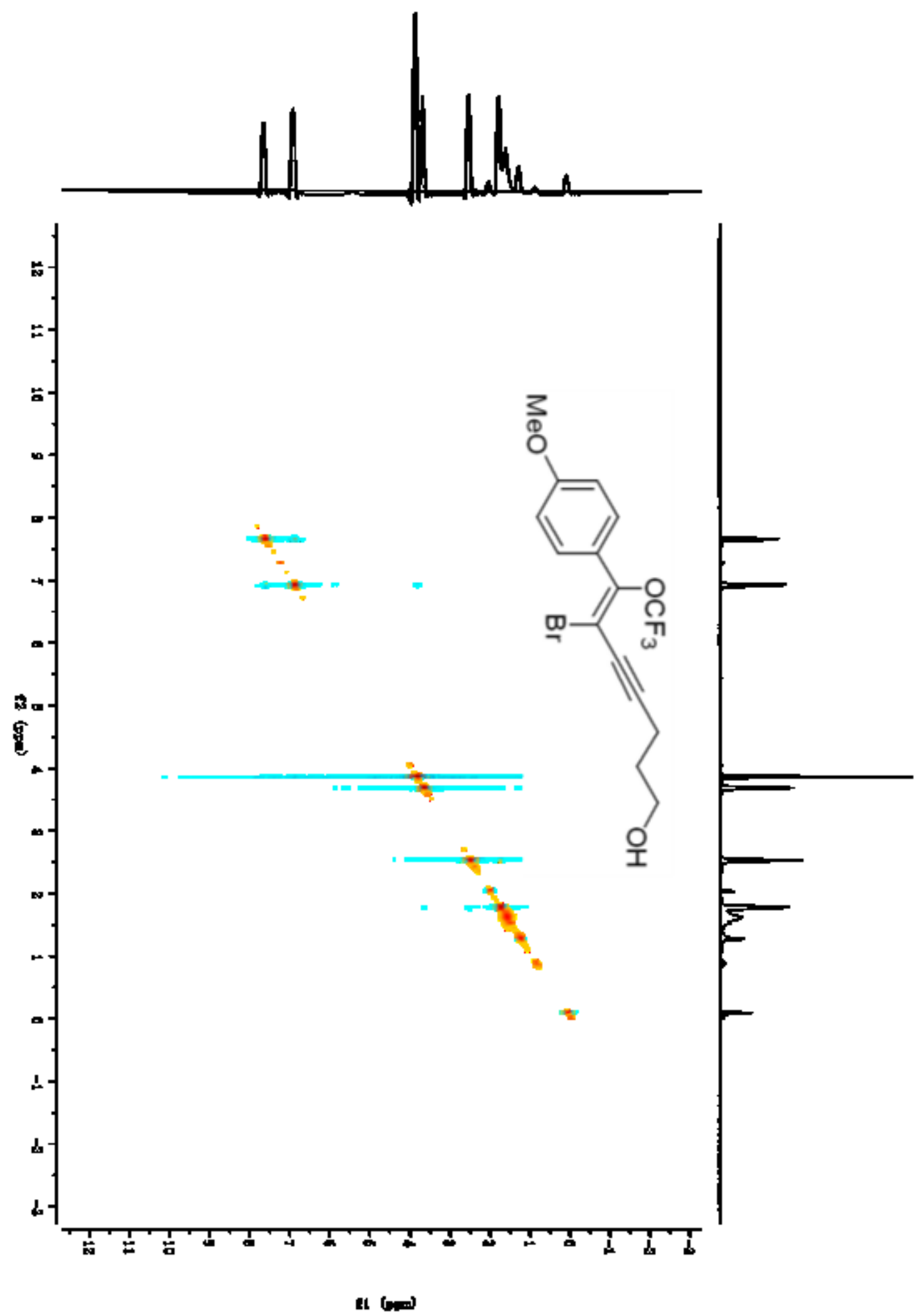

2D-NOESY NMR spectrum (400 MHz, $\left.\mathrm{CDCl}_{3}\right)$ of $4 \mathbf{e e}$ 


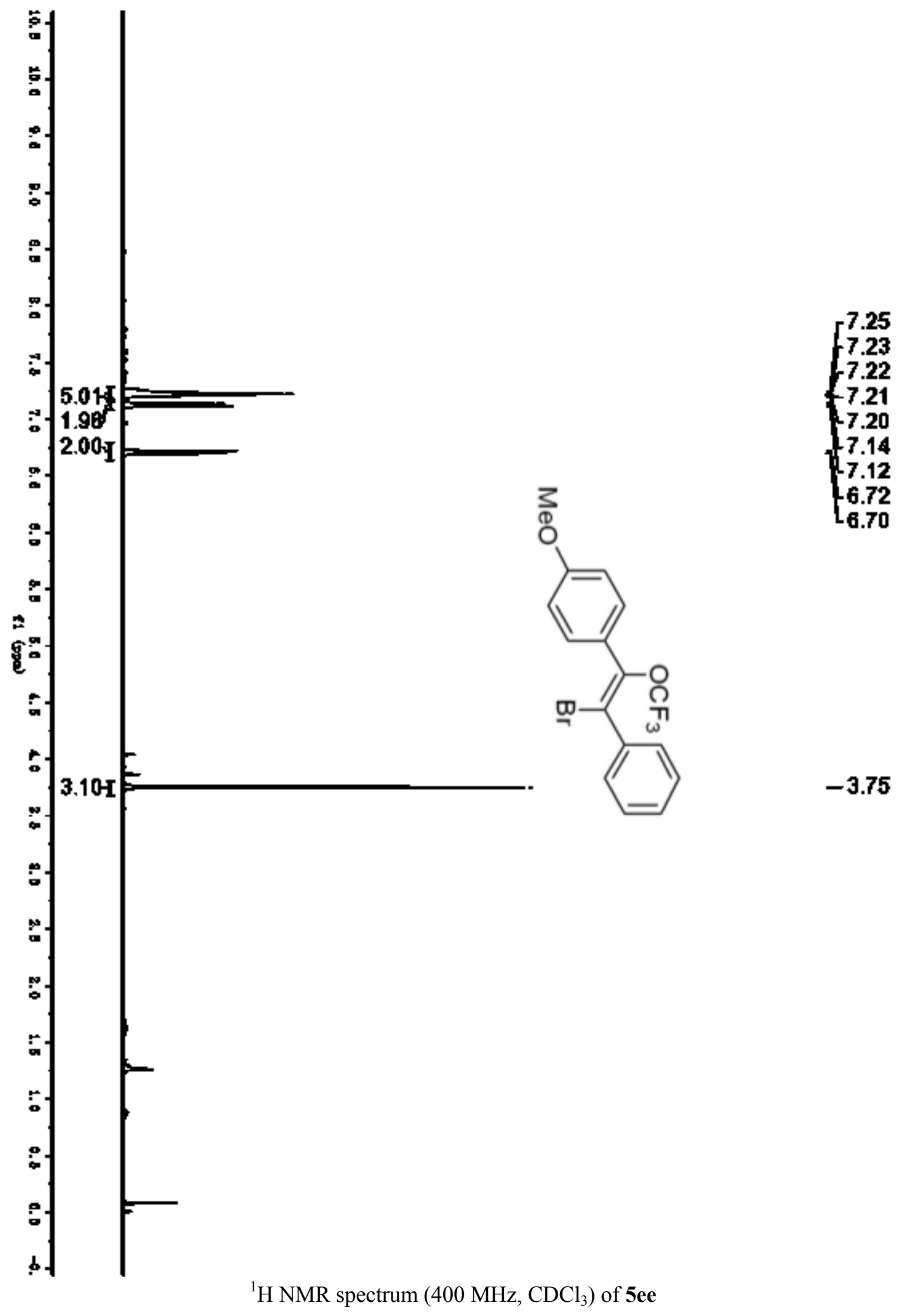




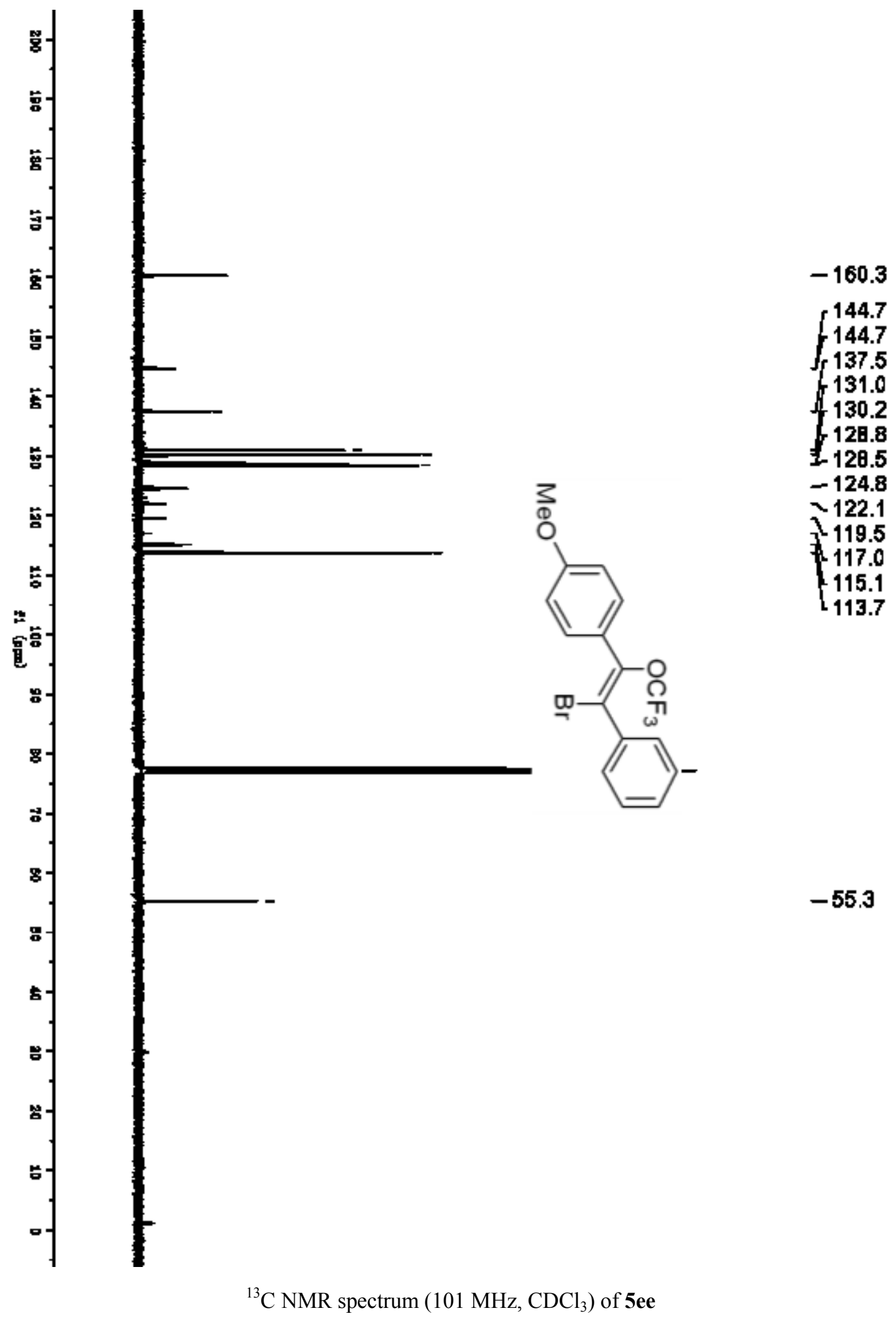




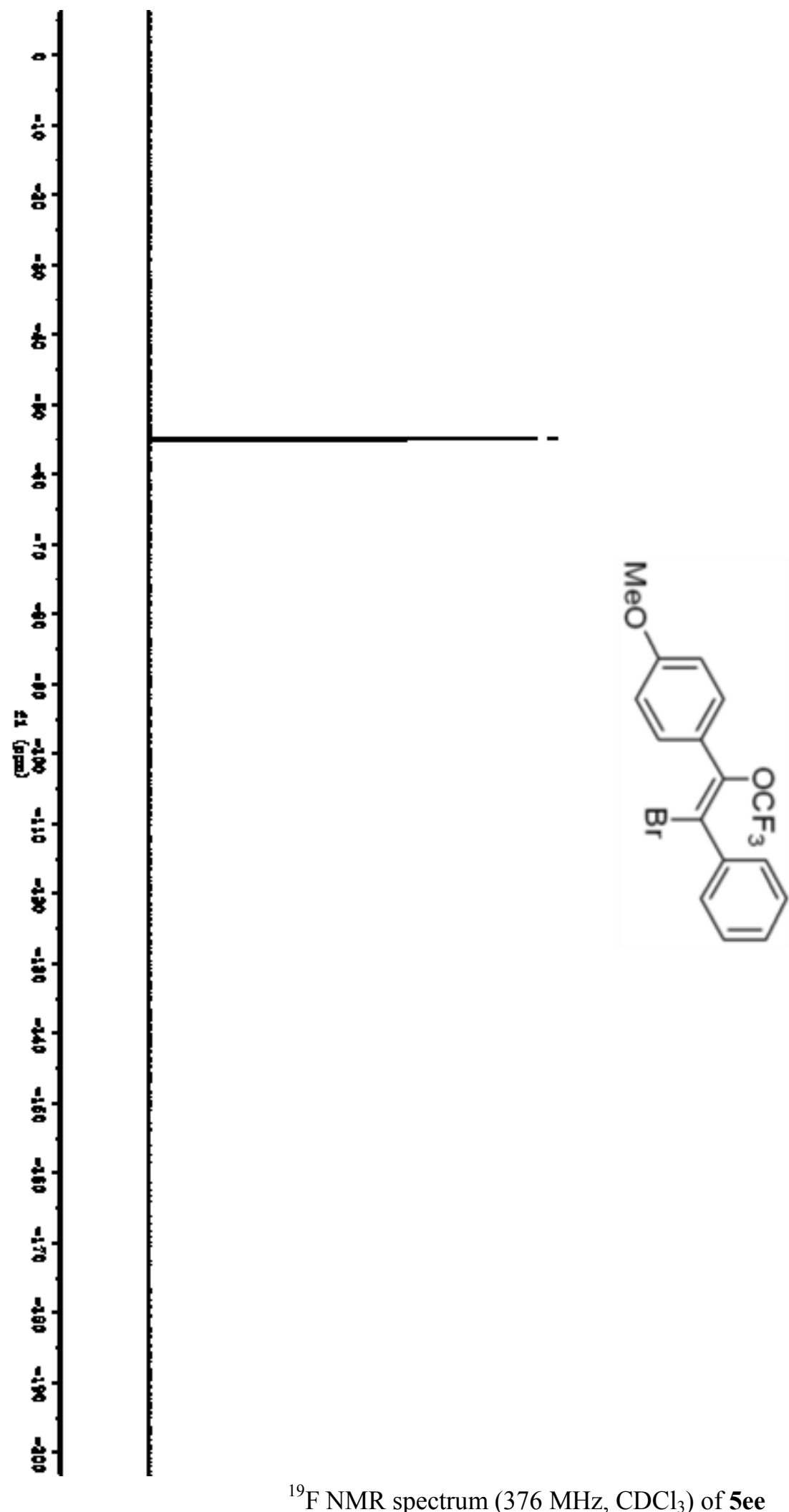

$--54.96$

${ }^{19} \mathrm{~F}$ NMR spectrum $\left(376 \mathrm{MHz}, \mathrm{CDCl}_{3}\right)$ of $5 \mathbf{e e}$ 


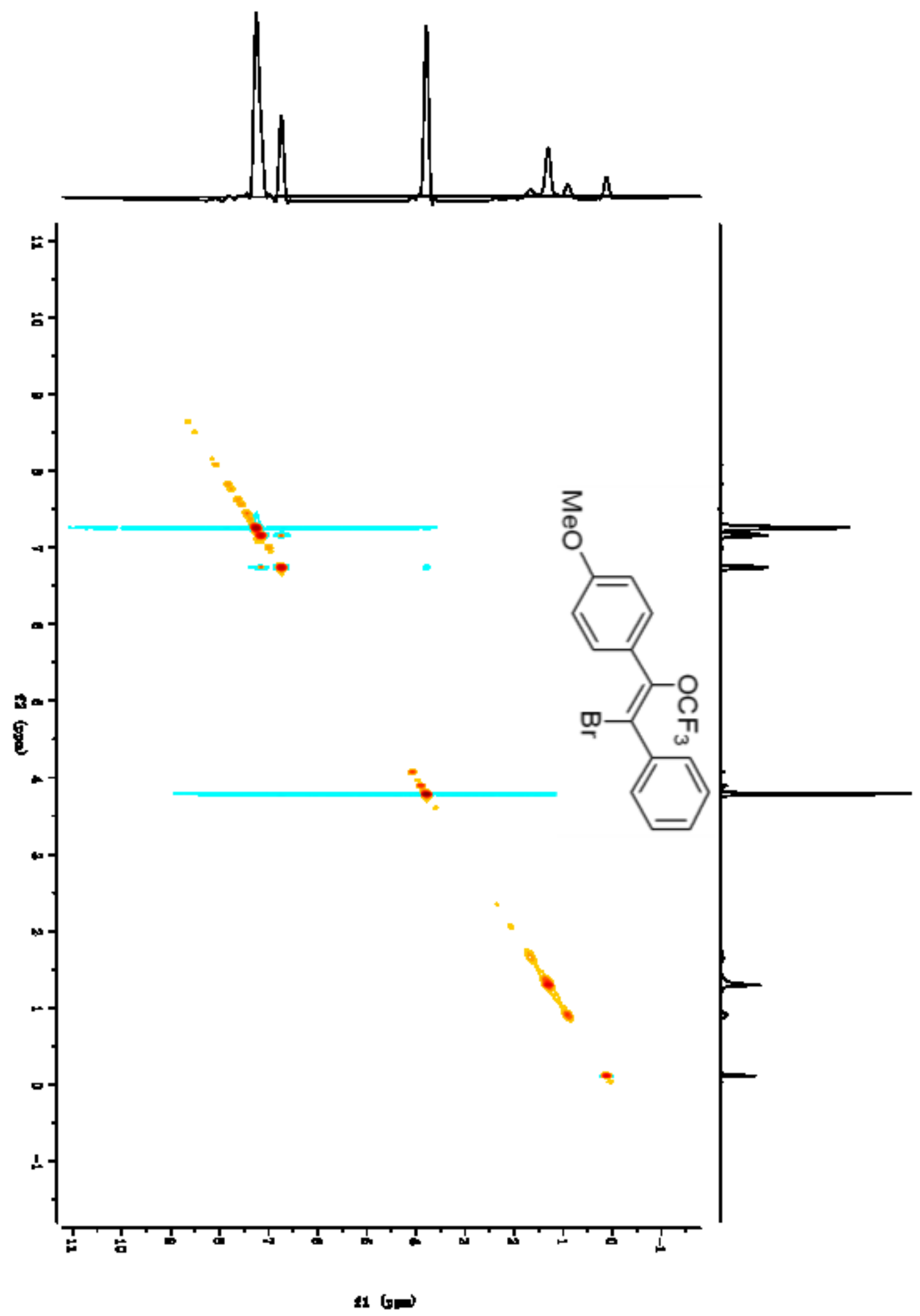

2D-NOESY NMR spectrum (400 MHz, $\mathrm{CDCl}_{3}$ ) of 5ee 


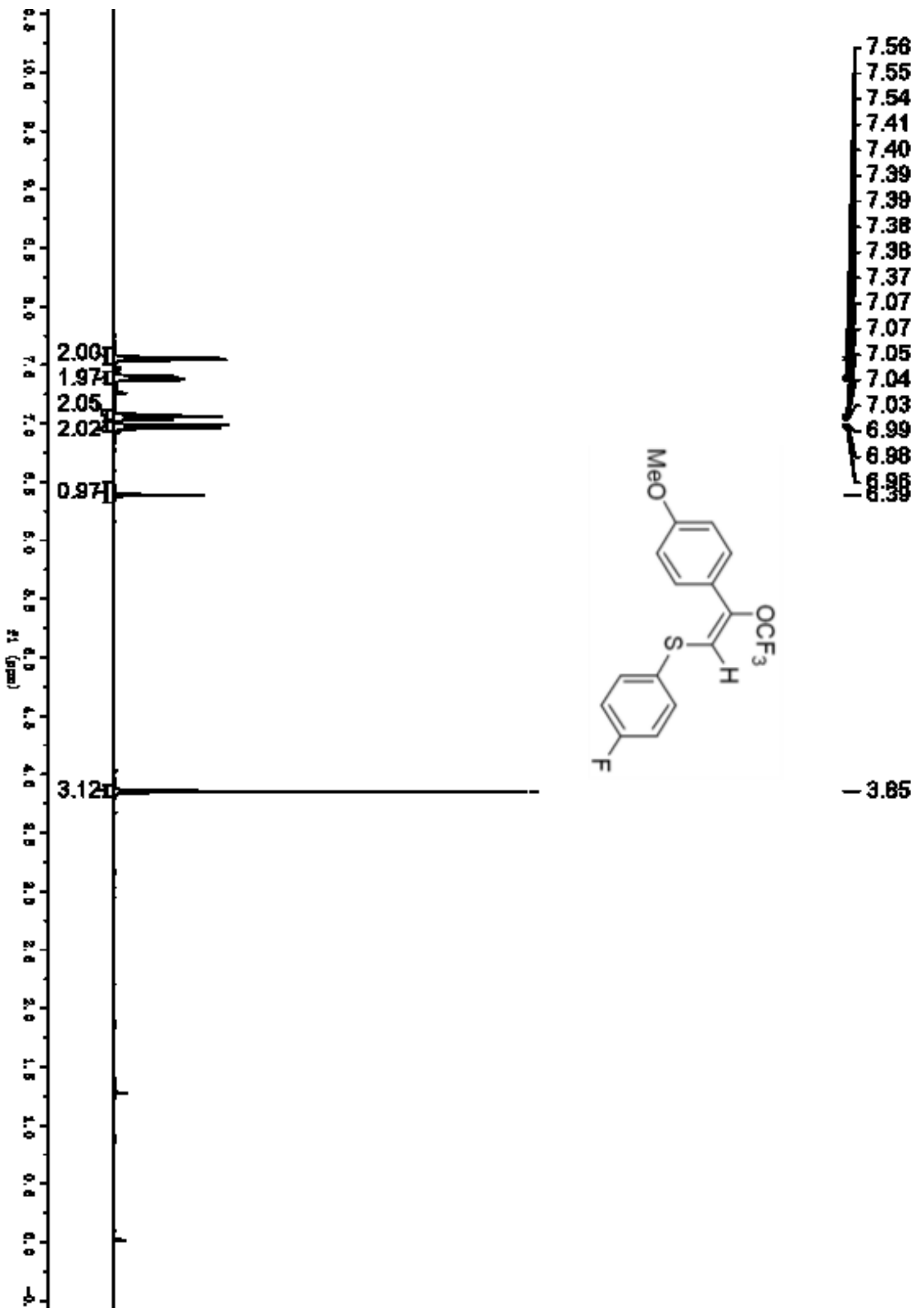

${ }^{1} \mathrm{H}$ NMR spectrum $\left(400 \mathrm{MHz}, \mathrm{CDCl}_{3}\right)$ of $\boldsymbol{E}$-6ee 


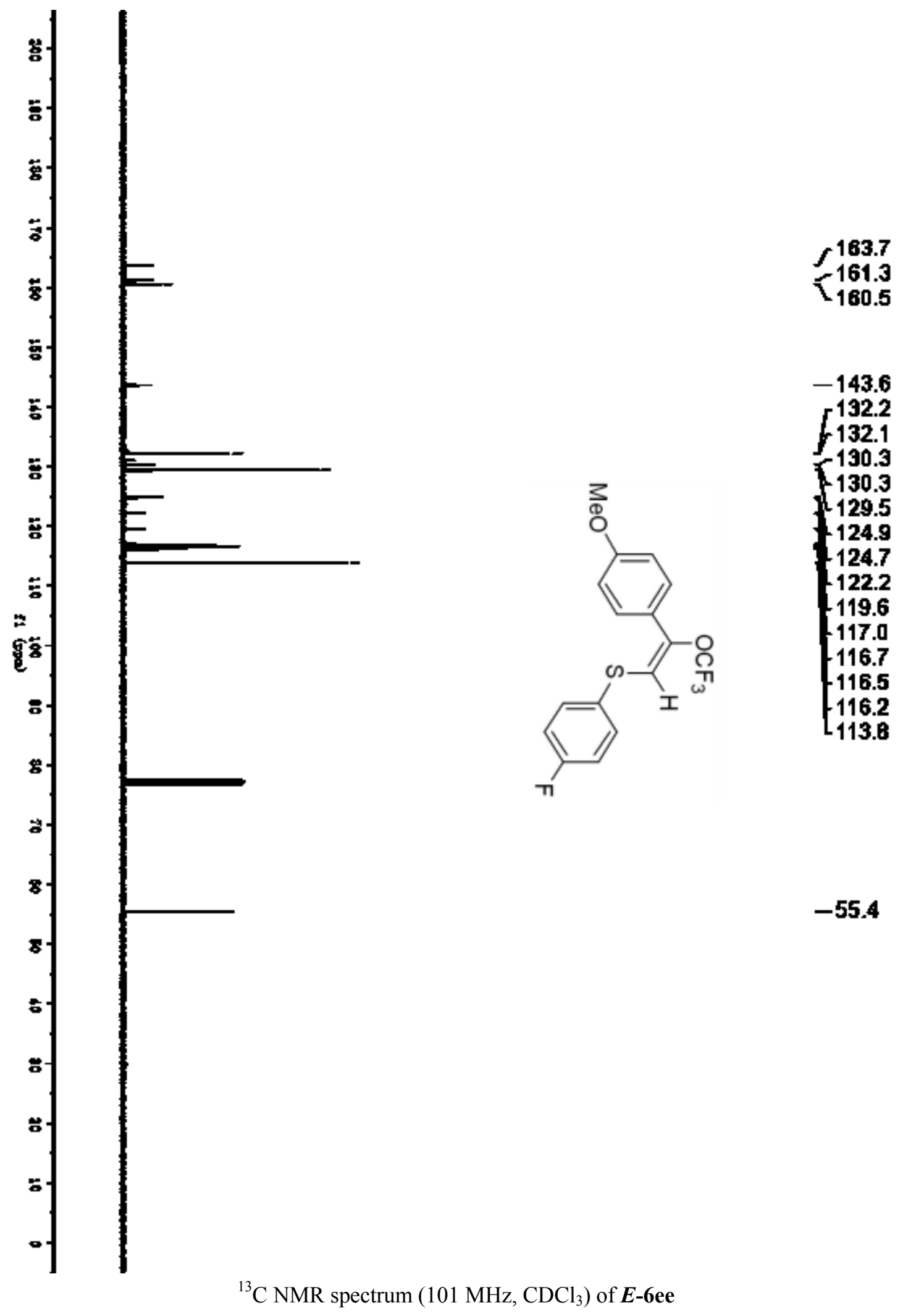




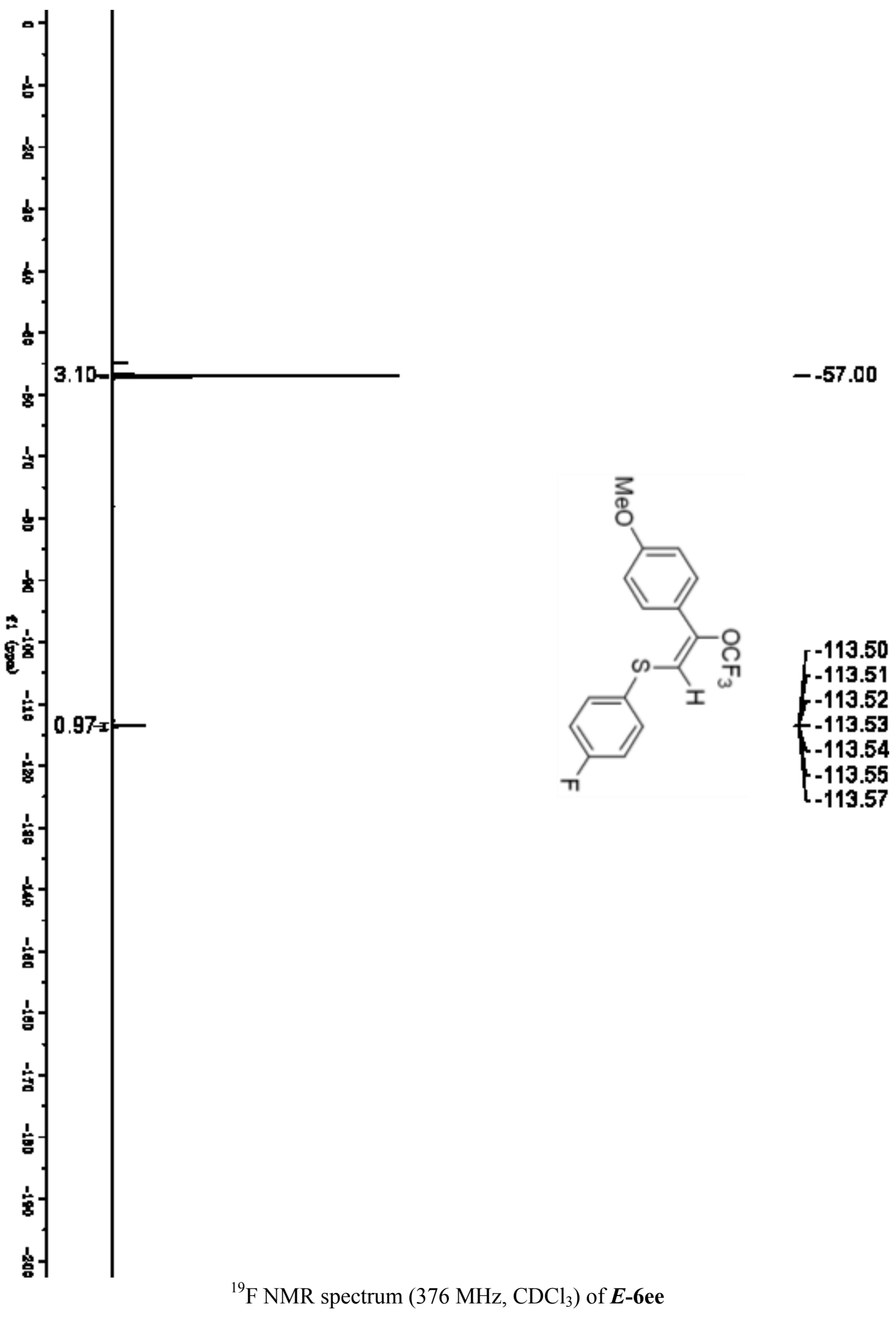




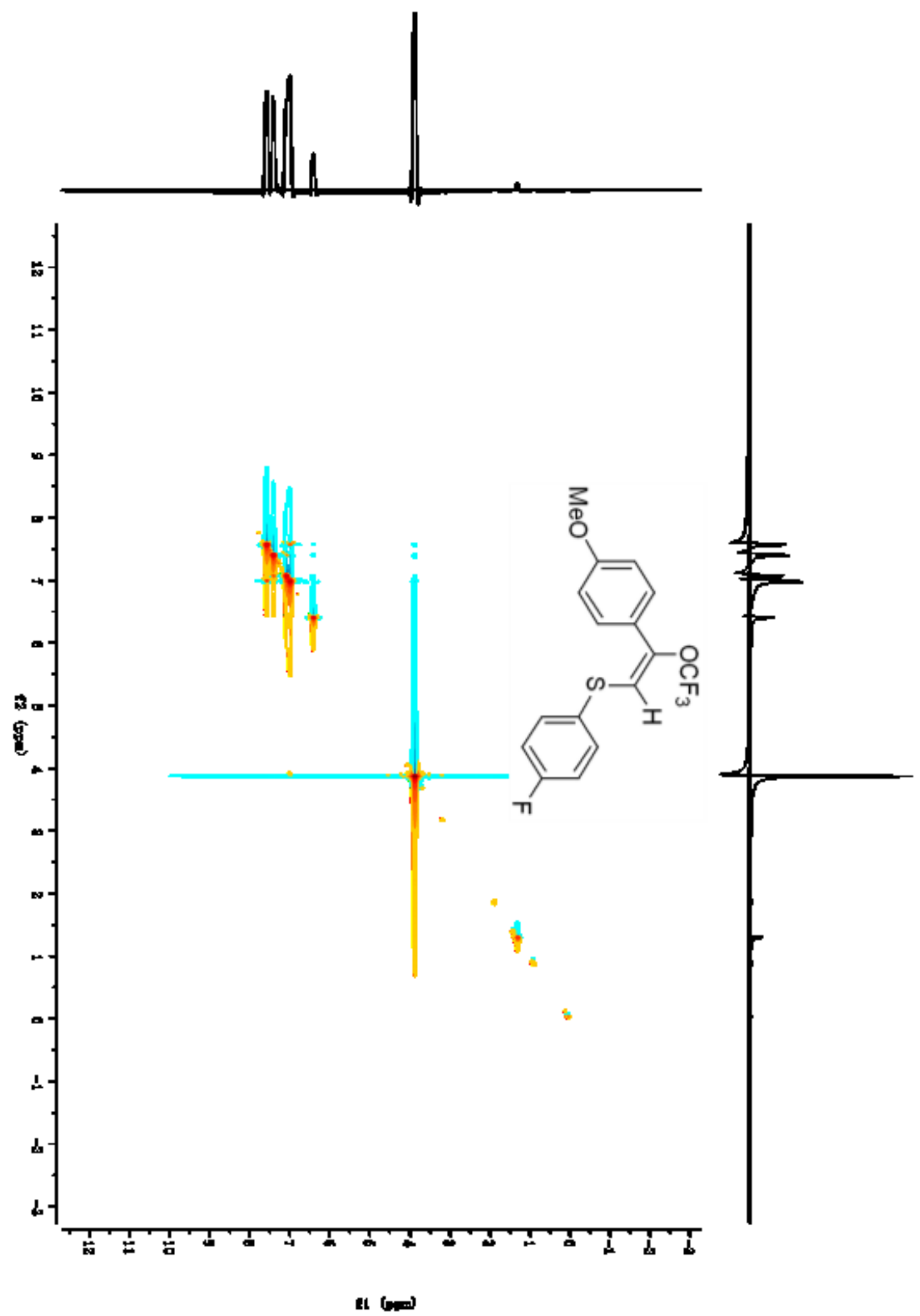

2D-NOESY NMR spectrum (400 MHz, $\mathrm{CDCl}_{3}$ ) of $\boldsymbol{E}$-6ee 


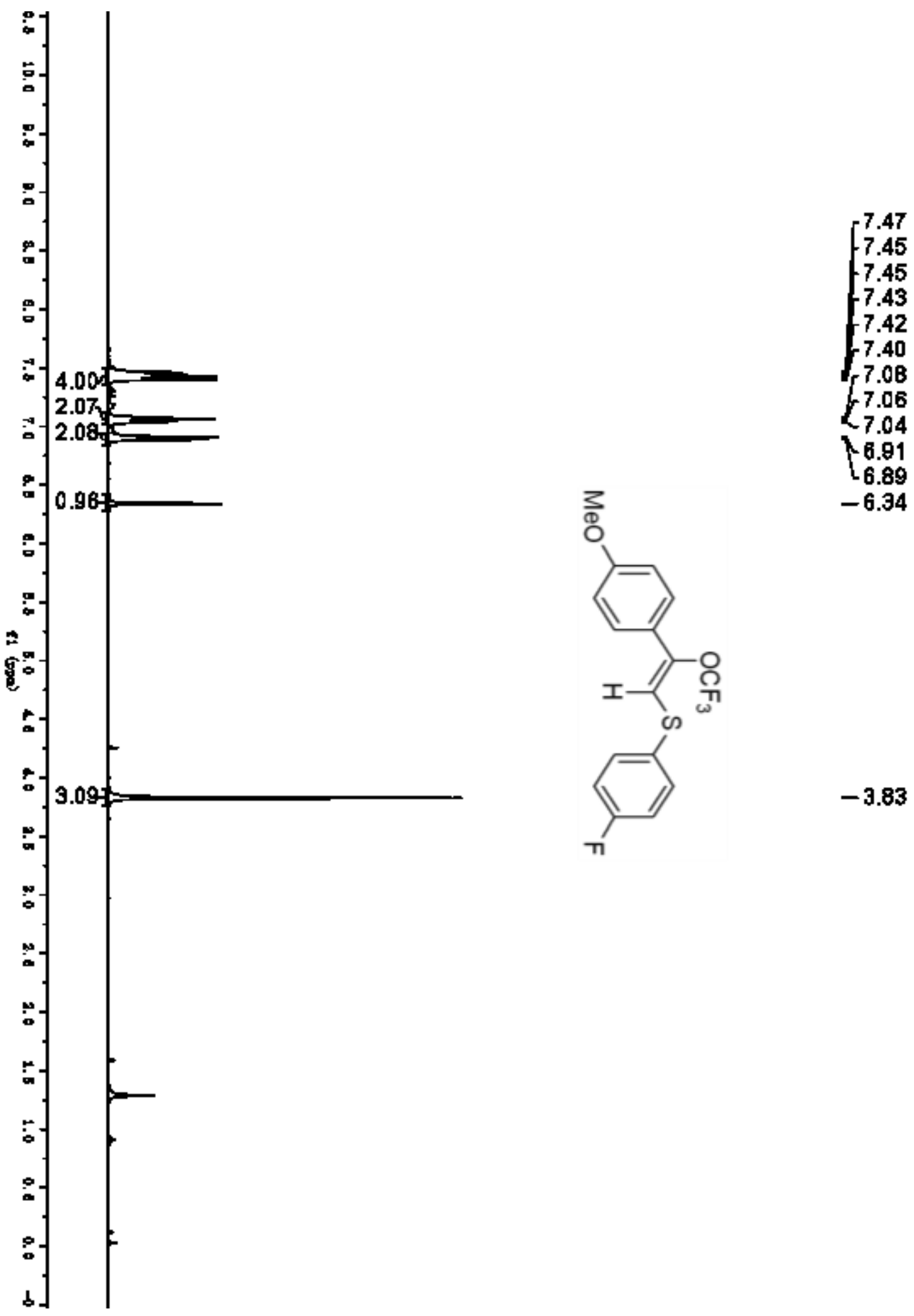

${ }^{1} \mathrm{H}$ NMR spectrum $\left(400 \mathrm{MHz}, \mathrm{CDCl}_{3}\right.$ ) of $\boldsymbol{Z}$-6ee 


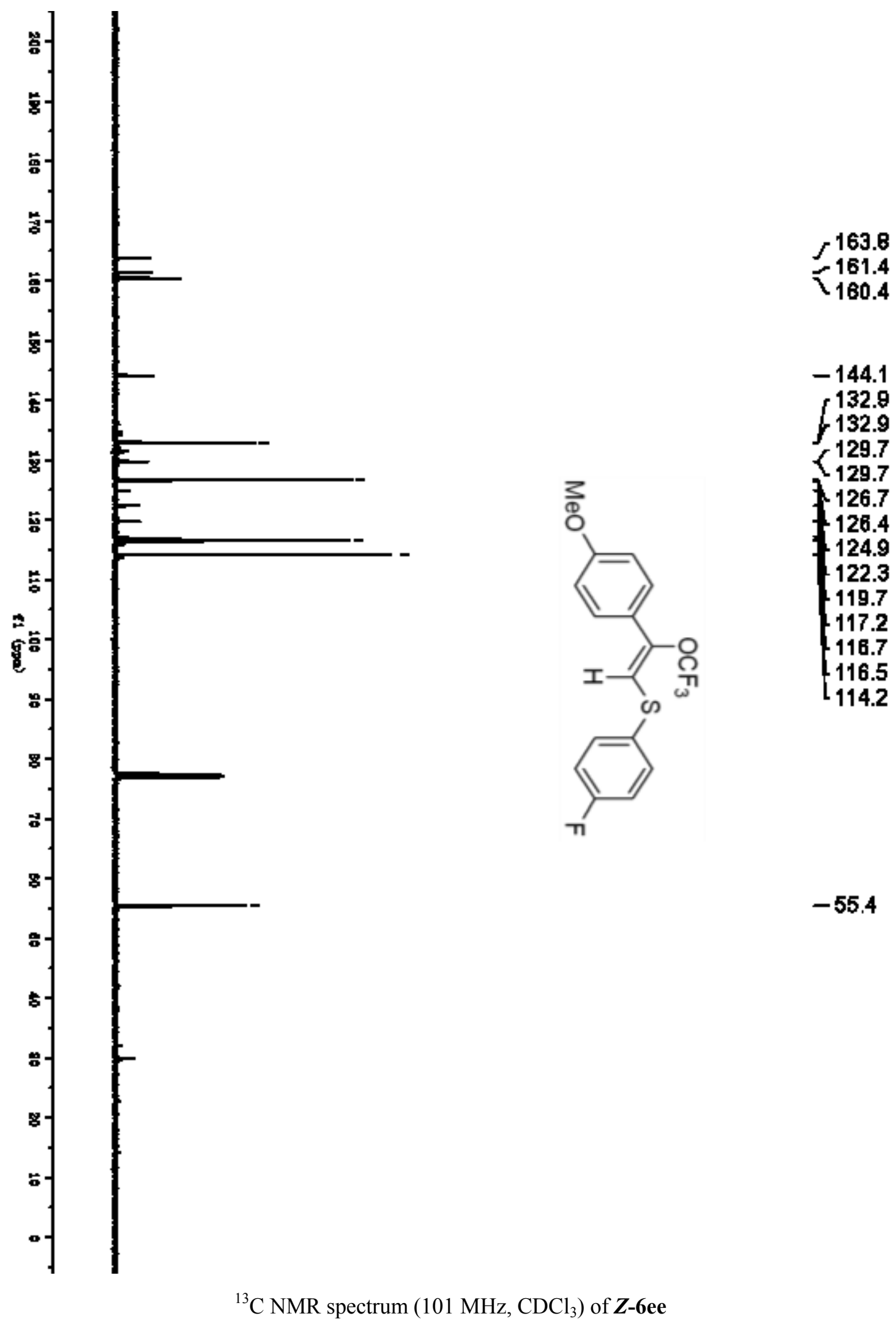




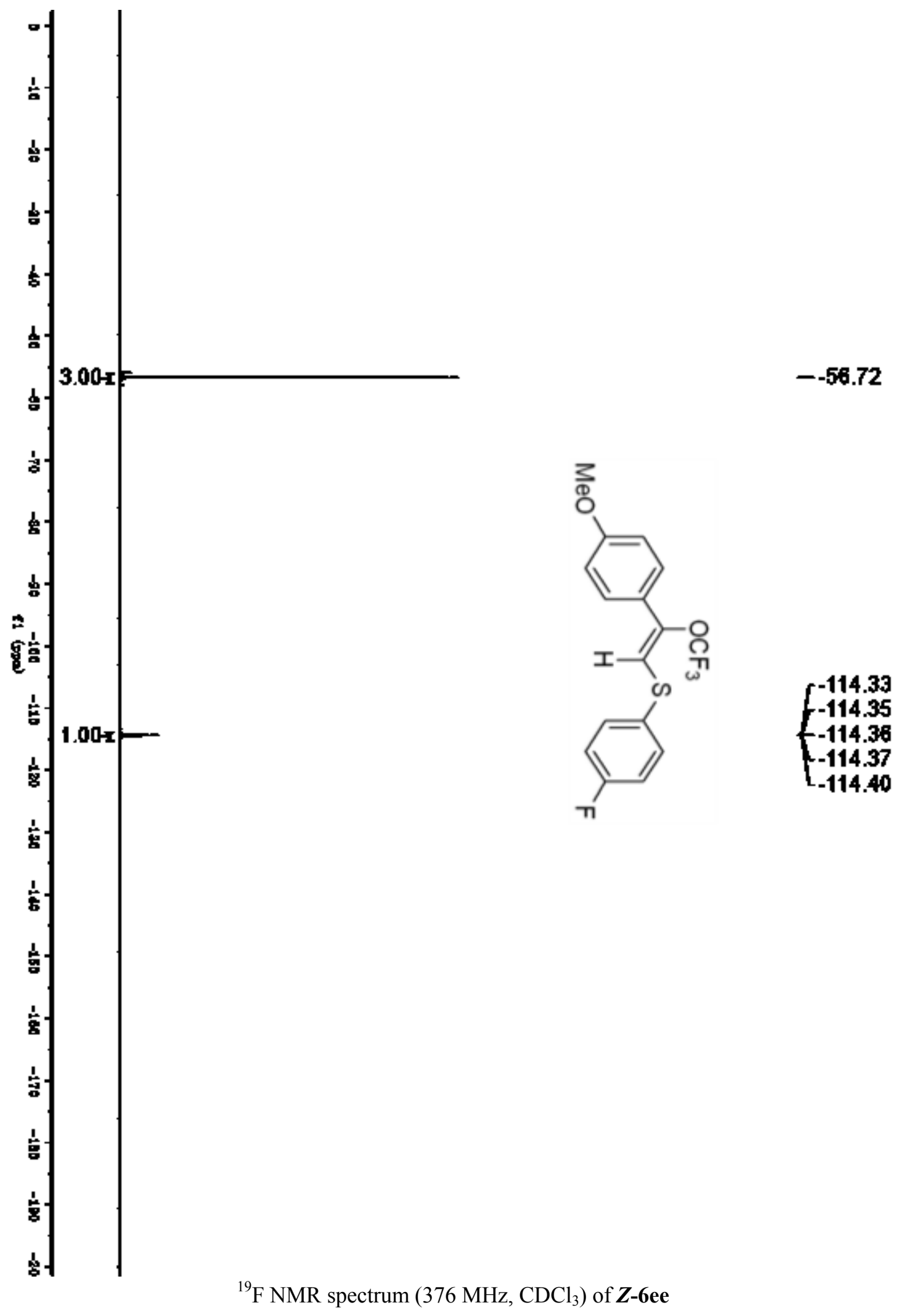




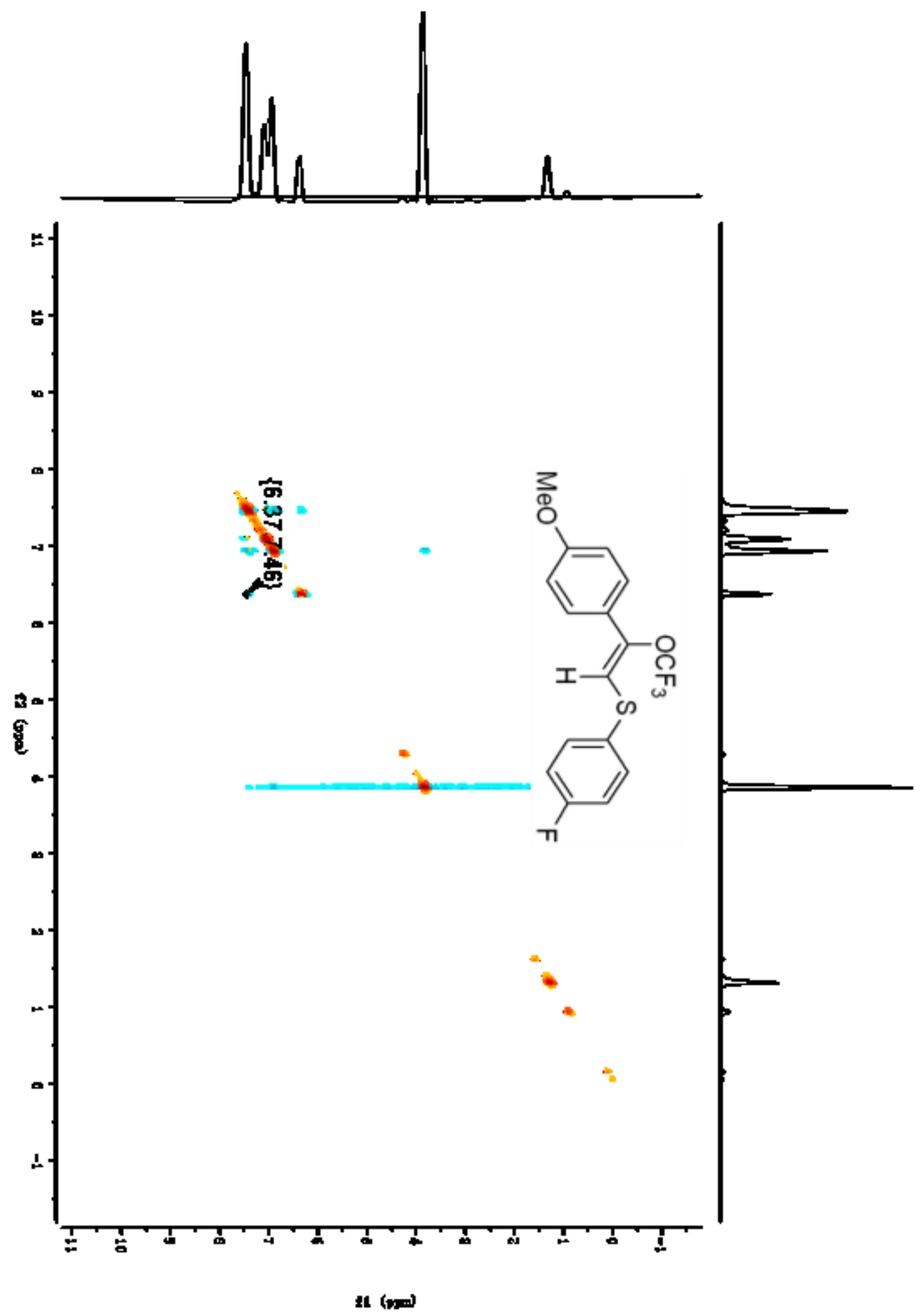

2D-NOESY NMR spectrum (400 MHz, $\mathrm{CDCl}_{3}$ ) of $\boldsymbol{Z}$-6ee 


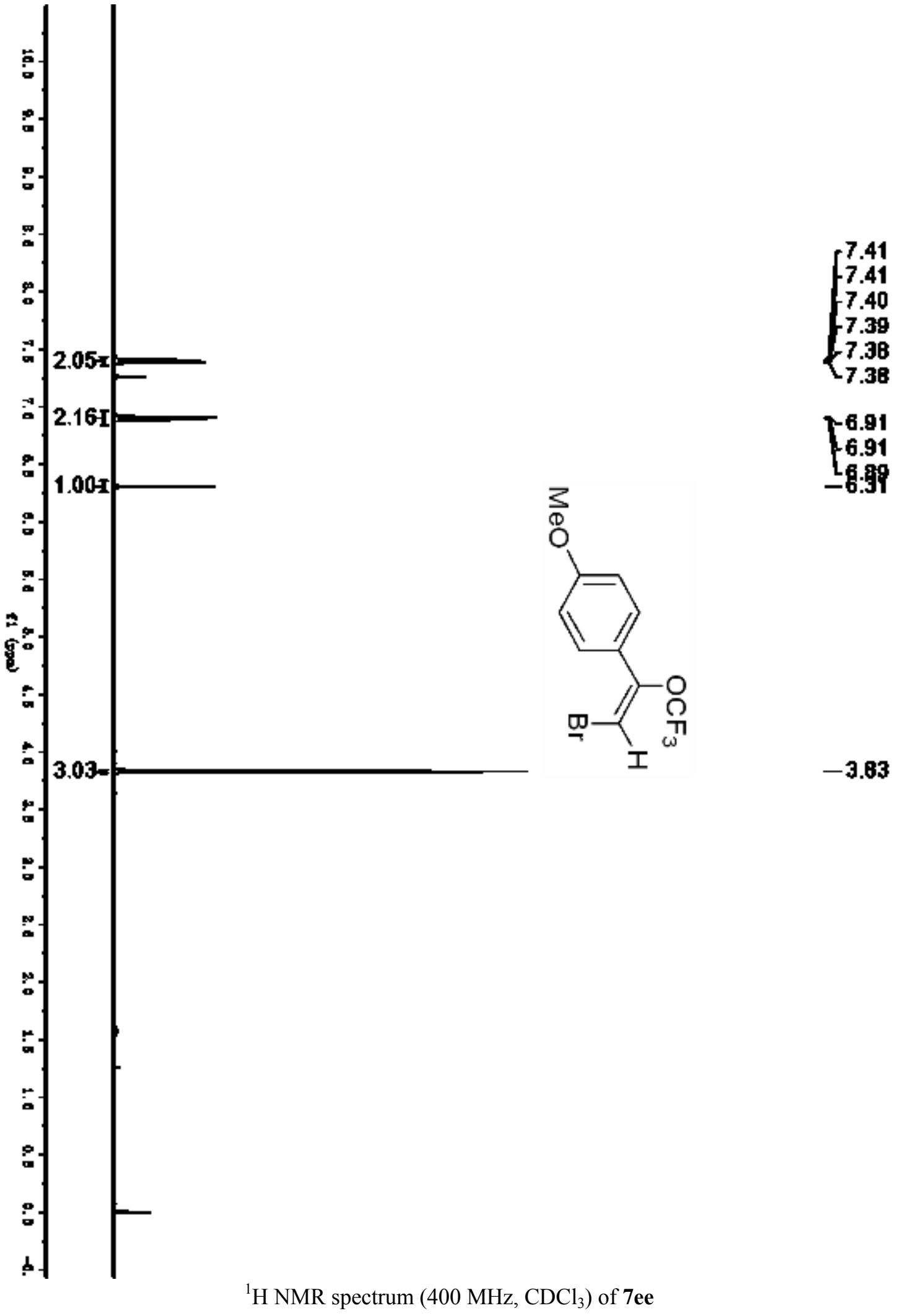




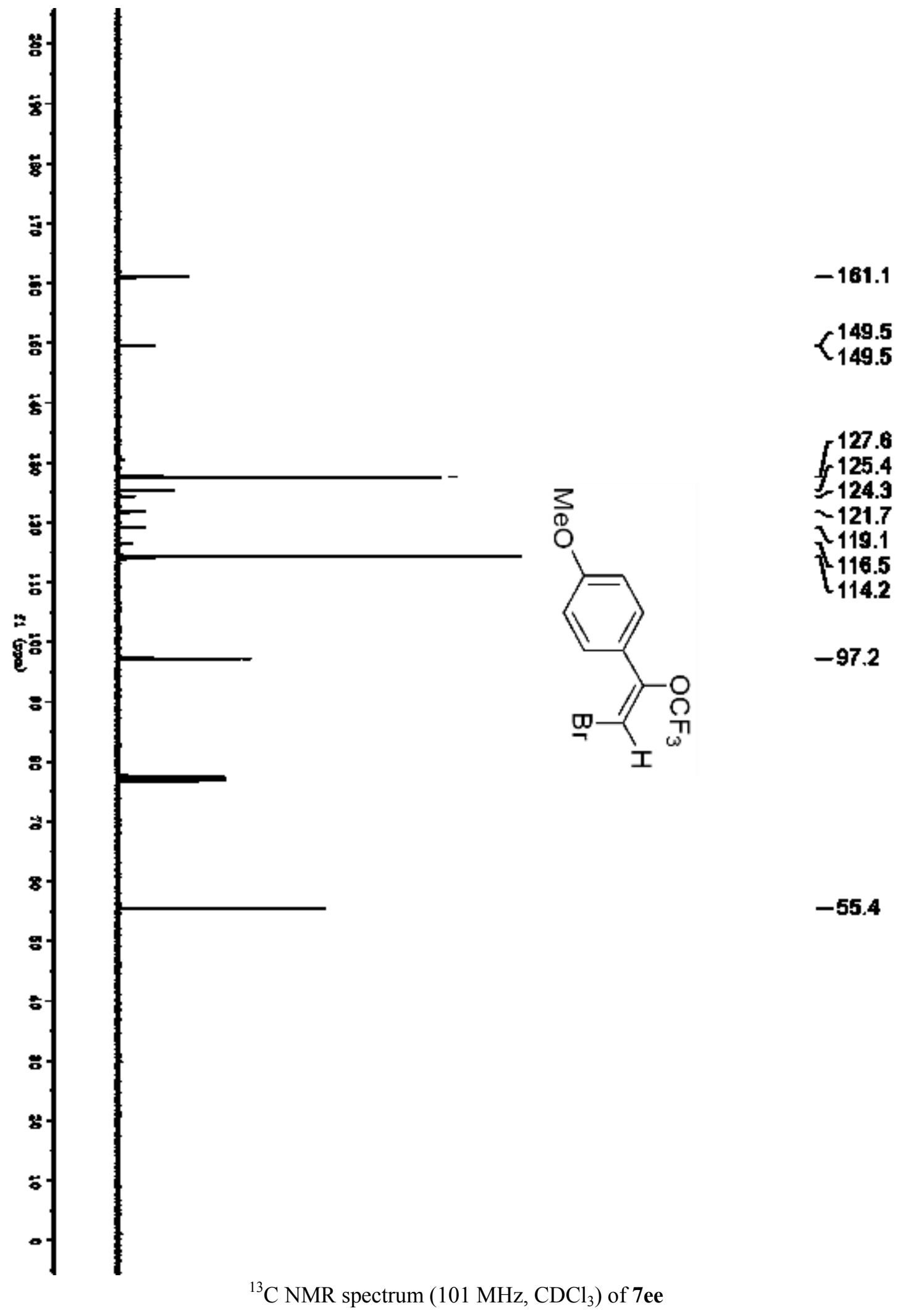




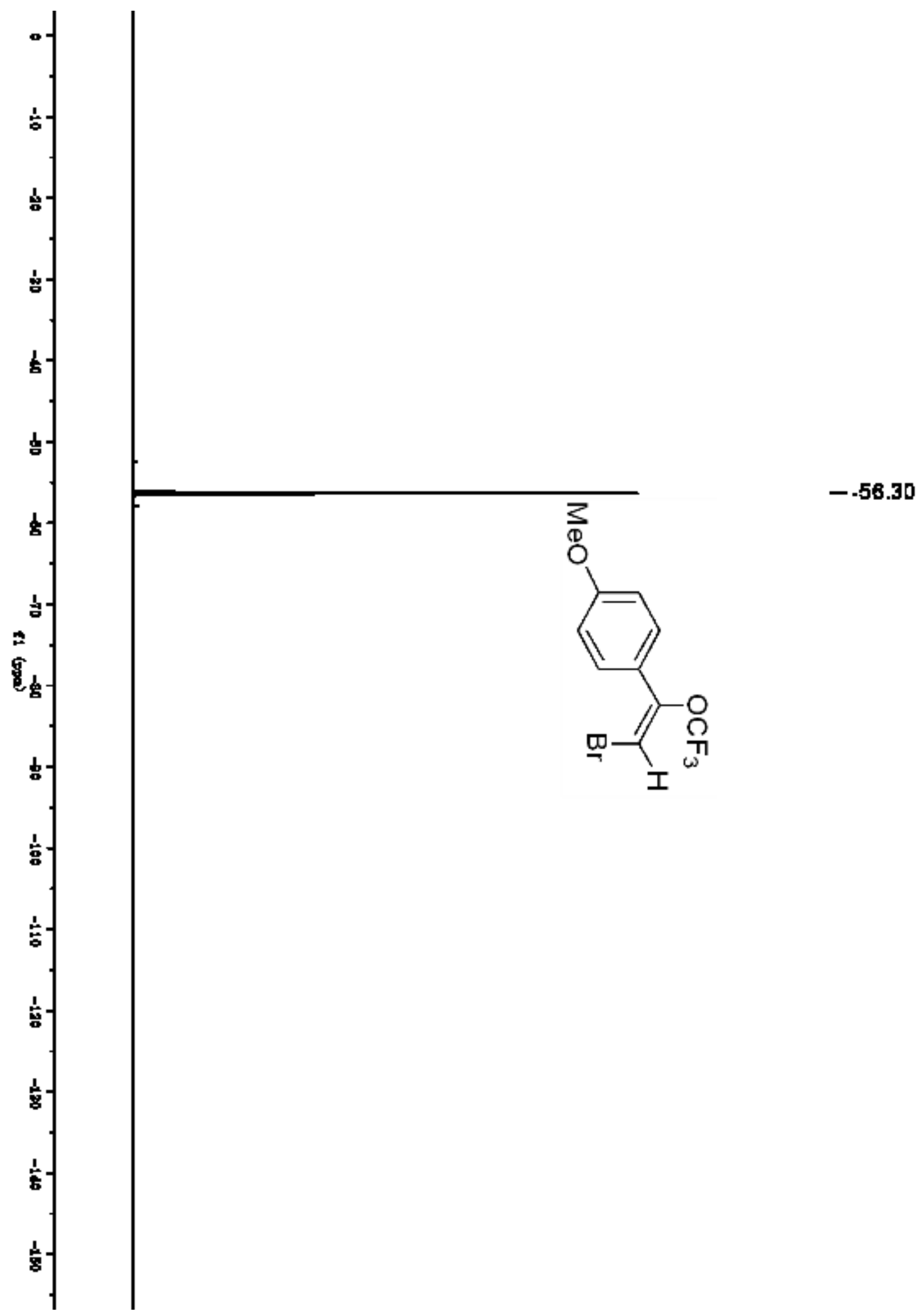

${ }^{19} \mathrm{~F}$ NMR spectrum $\left(376 \mathrm{MHz}, \mathrm{CDCl}_{3}\right)$ of $7 \mathbf{e e}$ 


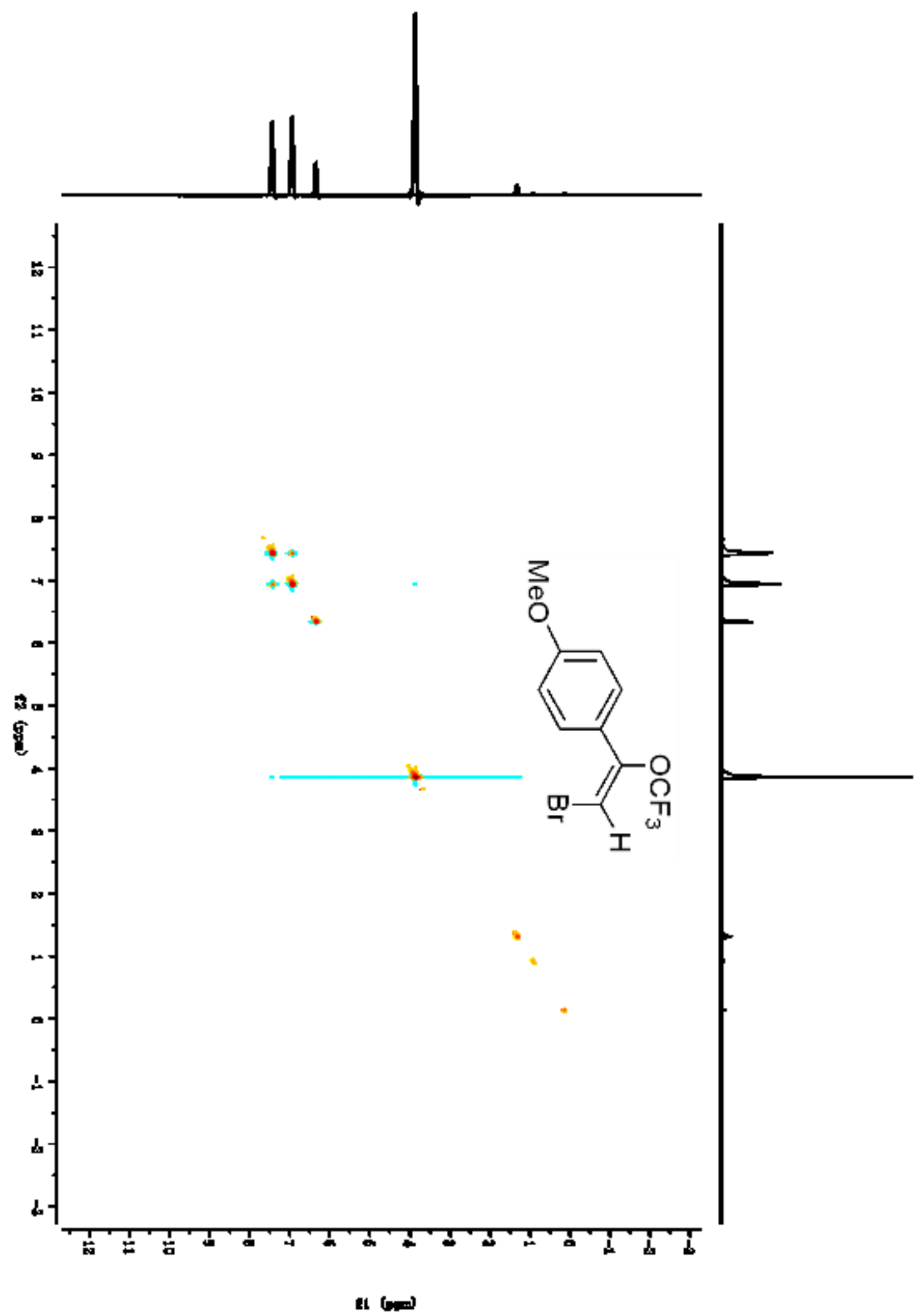

2D-NOESY NMR spectrum (400 MHz, $\mathrm{CDCl}_{3}$ ) of 7ee 


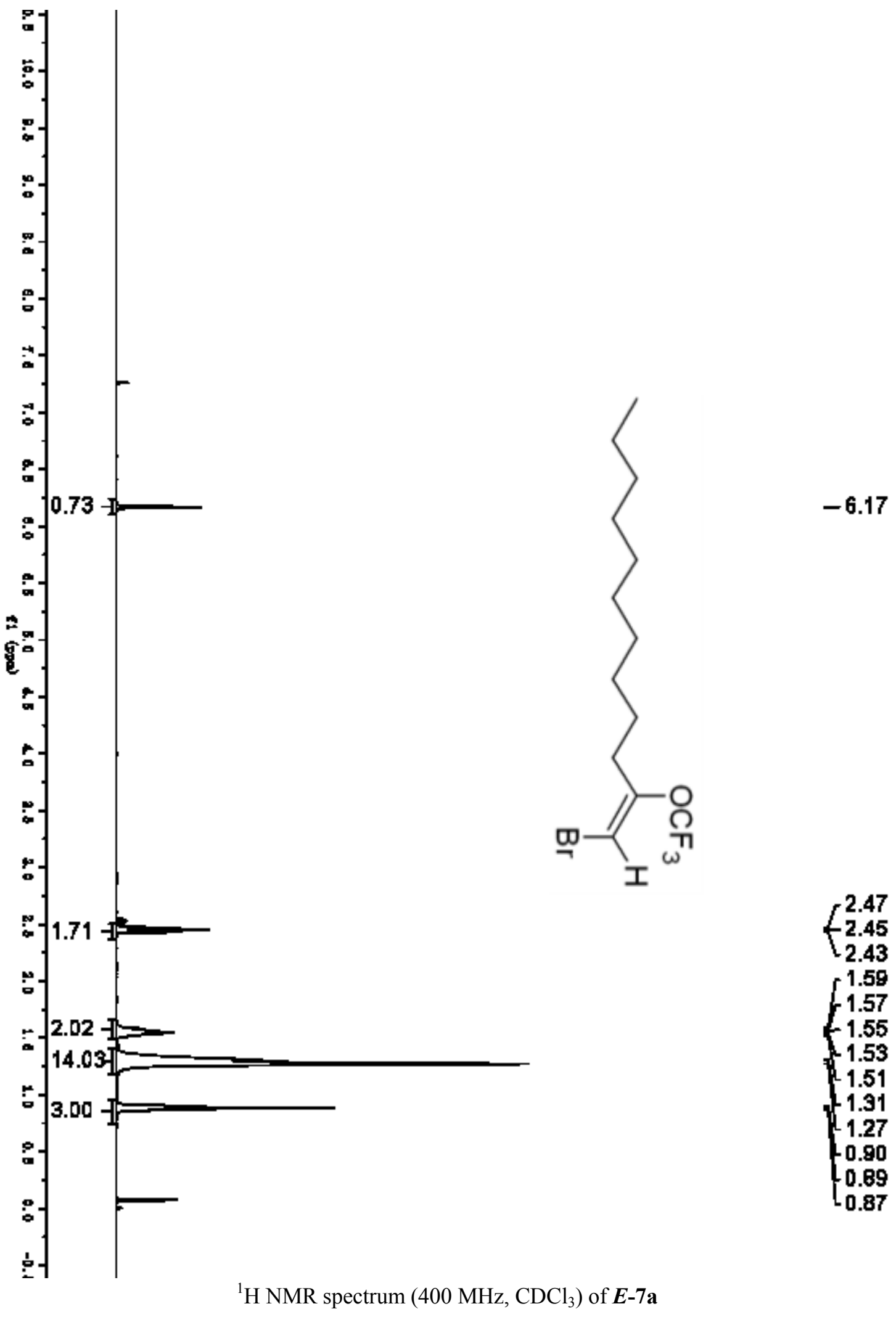




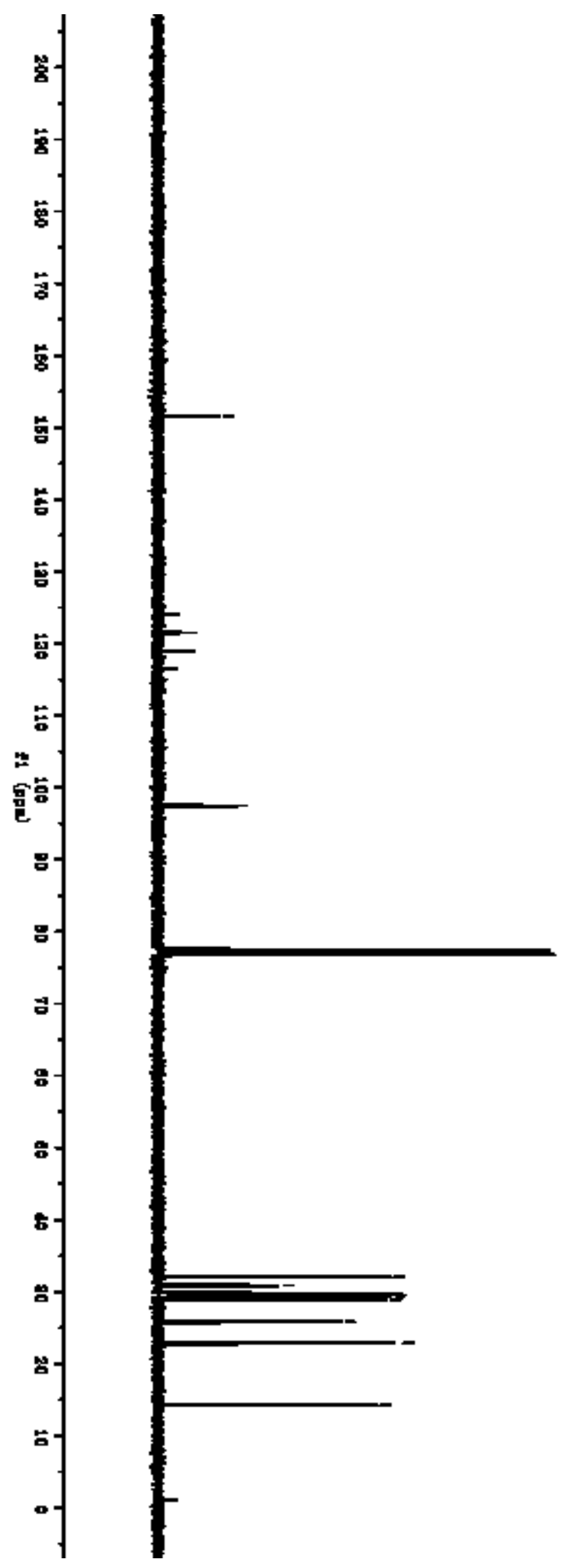

$-151.6$

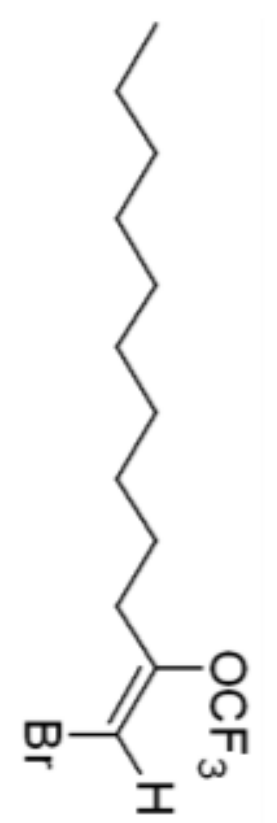

124.1

$-121.5$

$=118.9$

$\backslash 116.4$

$-97.5$

${ }^{13} \mathrm{C}$ NMR spectrum $\left(101 \mathrm{MHz}, \mathrm{CDCl}_{3}\right)$ of $\boldsymbol{E}-7 \mathbf{a}$ 


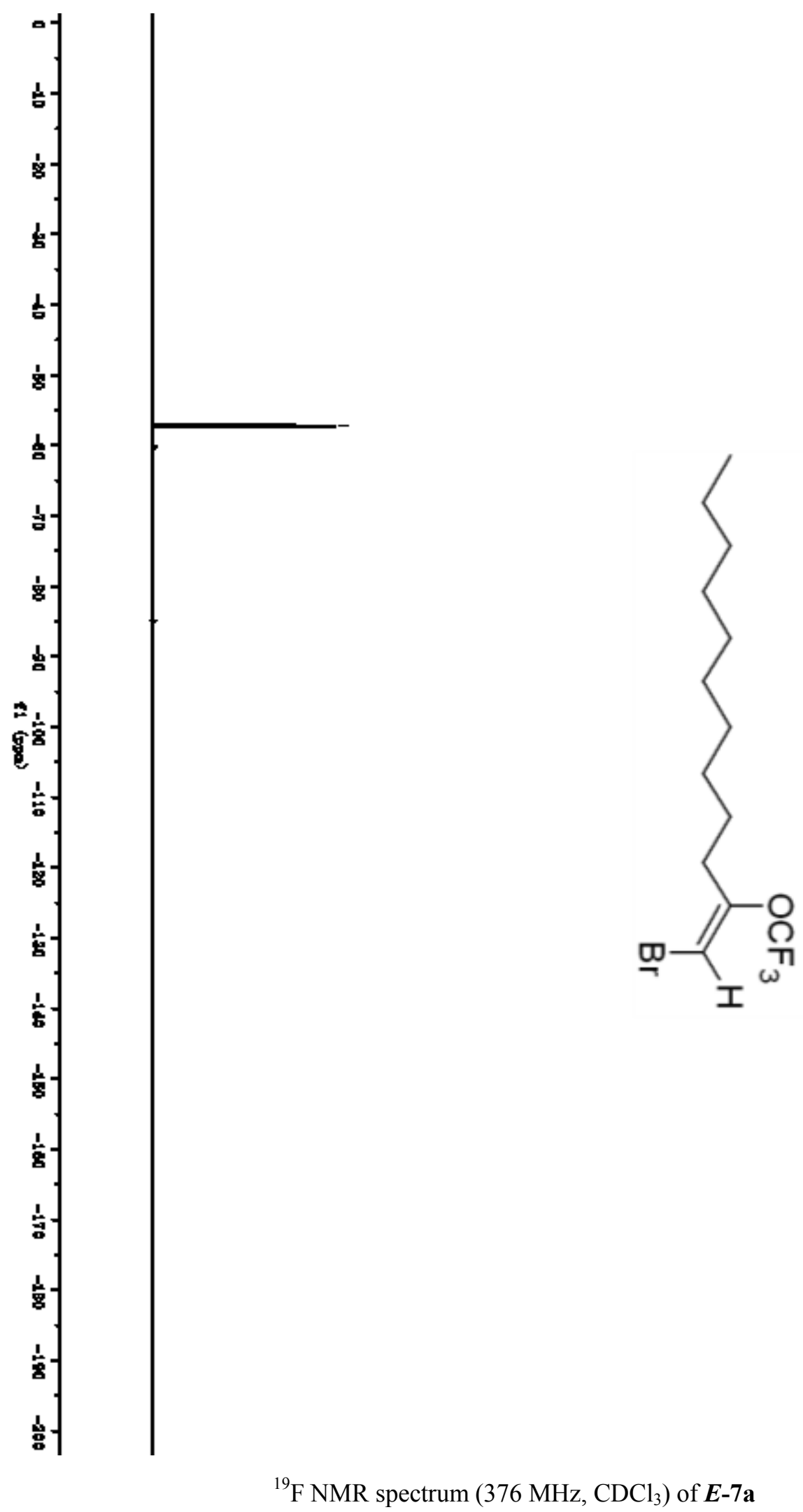

$-.57 .21$

${ }^{19}$ F NMR spectrum $\left(376 \mathrm{MHz}, \mathrm{CDCl}_{3}\right)$ of $\boldsymbol{E}-7 \mathbf{a}$ 


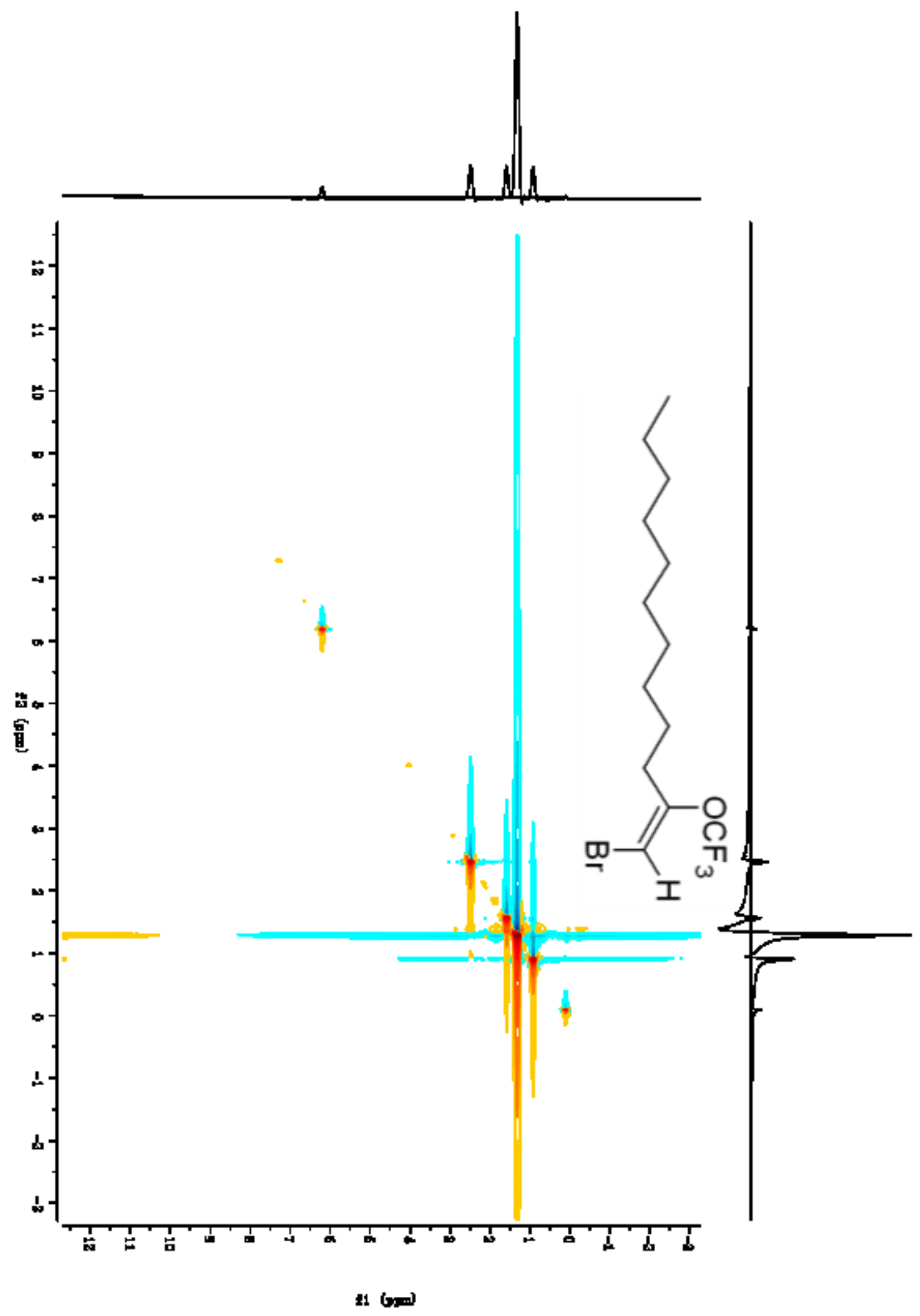

2D-NOESY NMR spectrum (400 MHz, $\mathrm{CDCl}_{3}$ ) of $\boldsymbol{E}-\mathbf{7} \mathbf{a}$ 


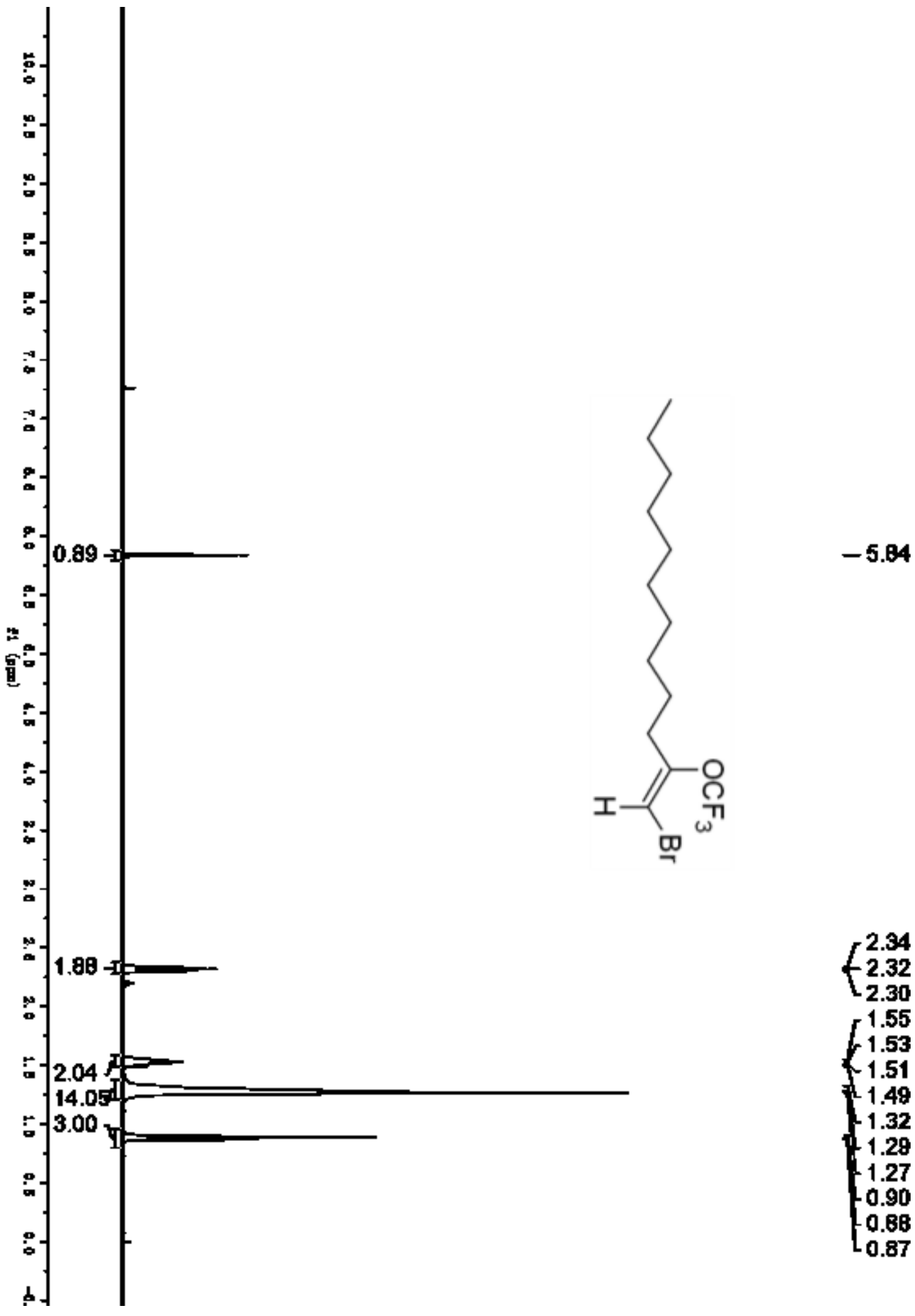

${ }^{1} \mathrm{H}$ NMR spectrum $\left(400 \mathrm{MHz}, \mathrm{CDCl}_{3}\right)$ of $\boldsymbol{Z}-\mathbf{7 a}$ 


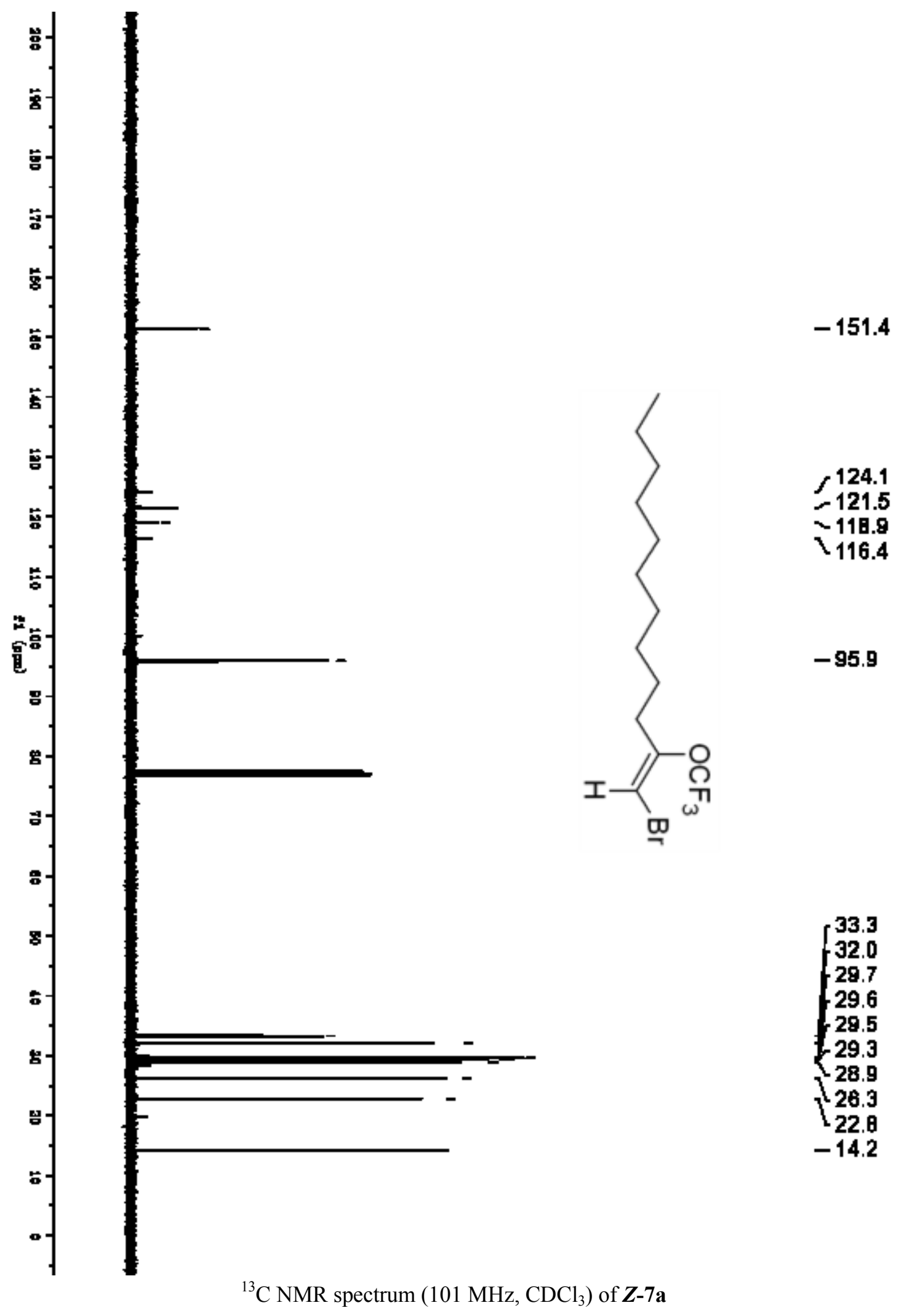




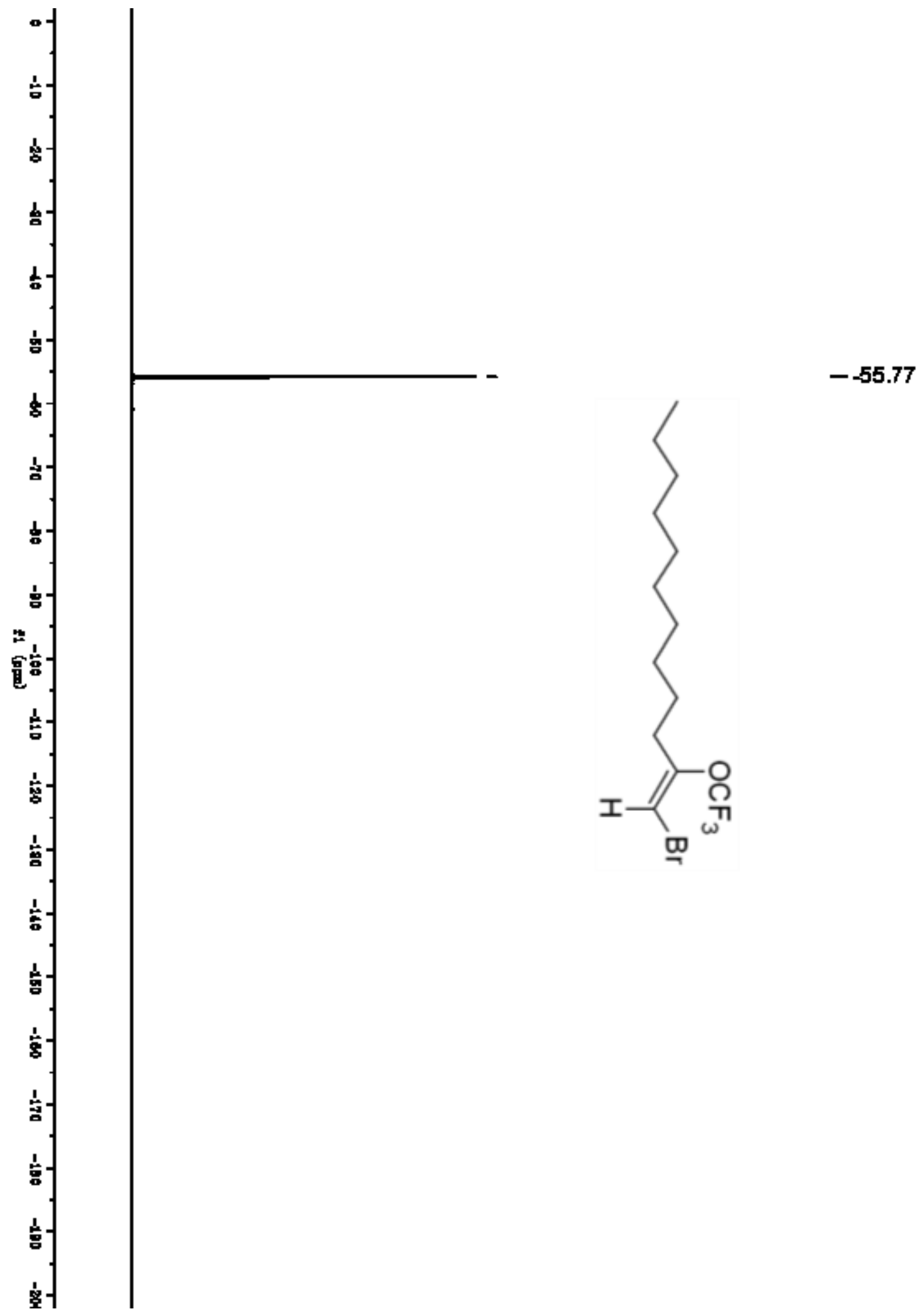

${ }^{19} \mathrm{~F}$ NMR spectrum (376 MHz, $\mathrm{CDCl}_{3}$ ) of $\boldsymbol{Z}-\mathbf{7 a}$ 


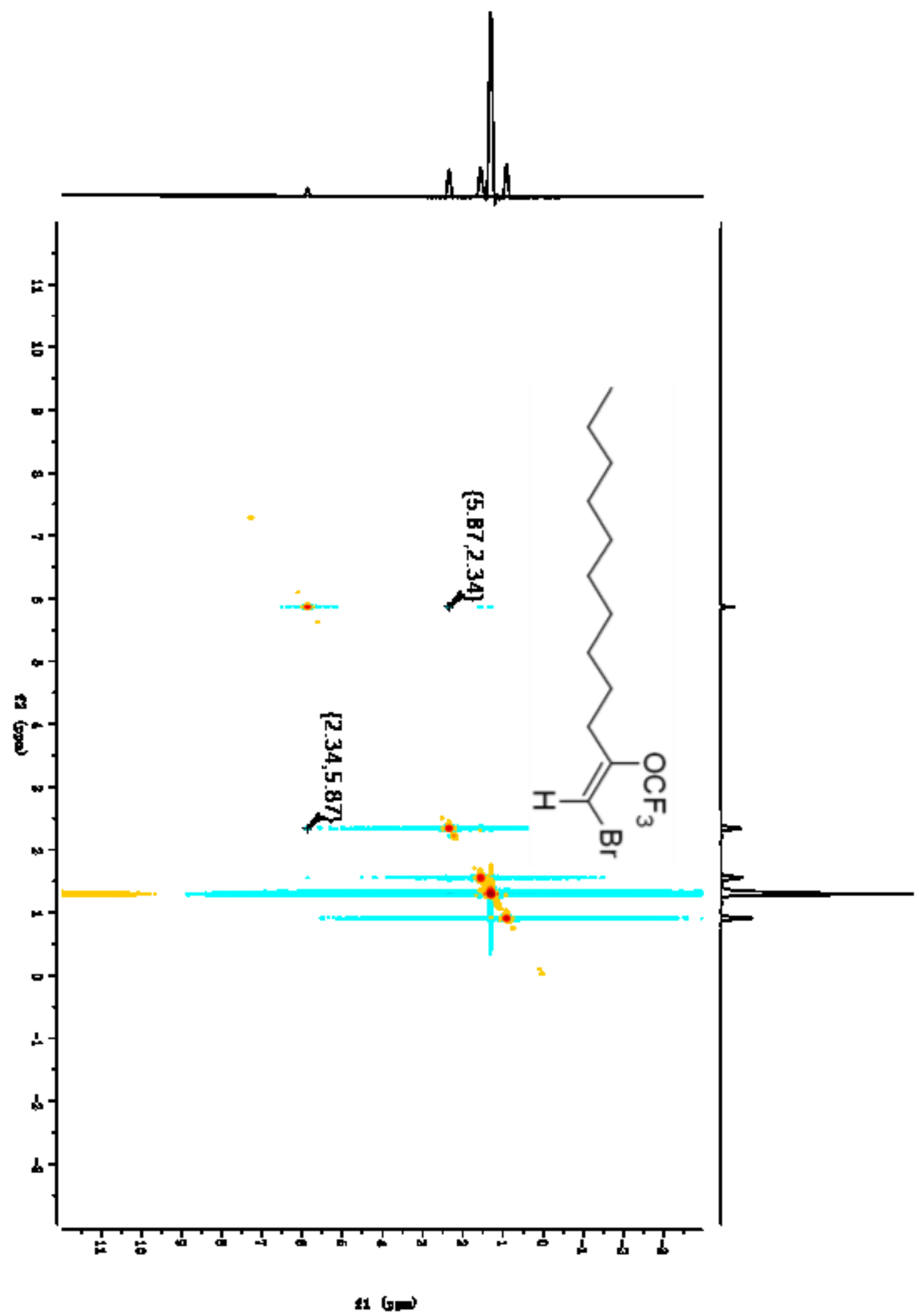

2D-NOESY NMR spectrum (400 MHz, $\mathrm{CDCl}_{3}$ ) of $\boldsymbol{Z}-\mathbf{7} \mathbf{a}$ 